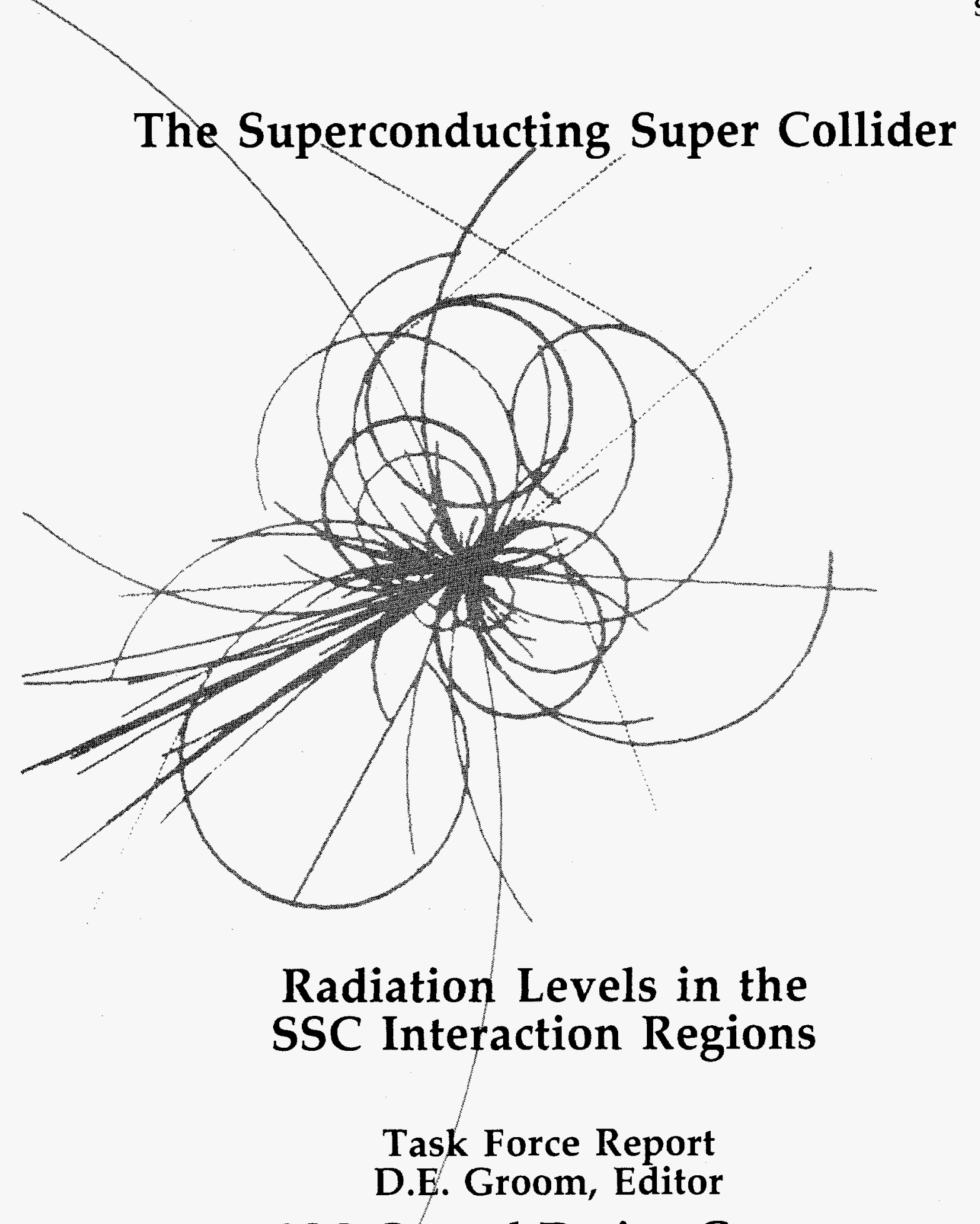

SSC Central Design Group

June 10, 1988 


\section{DISCLAIMER}

Portions of this document may be illegible in electronic image products. Images are produced from the best available original document. 
SSC-SR-1033

\title{
RADIATION LEVELS IN THE SSC INTERACTION REGIONS
}

\author{
Task Force Report \\ D. E. Groom, Editor \\ SSC Central Design Group* \\ Lawrence Berkeley Laboratory 90-4040 \\ Berkeley, California 94720 \\ 10 June 1988 \\ Reprinted with corrections 18 July 1988
}

\begin{abstract}
DISCLAIMER
This report was prepared as an account of work sponsored by an agency of the United States Government. Neither the United States Government nor any agency thereof, nor any of their employees, makes any warranty, express or implied, or assumes any legal liability or responsibility for the accuracy, completeness, or usefulness of any information, apparatus, product, or process disclosed, or represents that its use would not infringe privately owned rights. Reference herein to any specific commercial product, process, or service by trade name, trademark, manufacturer, or otherwise does not necessarily constitute or imply its endorsement, recommendation, or favoring by the United States Government or any agency thereof. The views and opinions of authors expressed herein do not necessarily state or reflect those of the United States Government or any agency thereof.
\end{abstract}

* Operated by the Universities Research Association for the Department of Energy. 


\section{SUMMARY}

The radiation environment in a typical SSC detector has been evaluated using the best available particle production models coupled with Monte Carlo simulations of hadronic and electromagnetic cascades. The problems studied include direct charged particle dose, dose inside a calorimeter from the cascades produced by incident photons and hadrons, the flux of neutrons and photons backscattered from the calorimeter into a central cavity, and neutron flux in the calorimeter.

The luminosity lifetime at the SSC is dominated by collision losses in the interaction regions, where the luminosity is equivalent to losing an entire fullenergy proton beam into the apparatus every six days. The result of an average p-p collision can be described quite simply. The mean charged multiplicity is about 110, and the particles are distributed nearly uniformly in pseudorapidity $(\eta)$ over all the angles of interest. The transverse momentum distribution is independent of angle, and for our purposes may be written as $p_{\perp} \exp \left(-p_{\perp} / B\right)$. The mean value of $p_{\perp}$ may be as high as $0.6 \mathrm{GeV} / \mathrm{c}$. Most of the radiation is produced by the very abundant low- $p_{\perp}$ particles.

The dose or neutron fluence produced by individual particles in this energy region are simulated over a wide variety of conditions, and several measurements serve to confirm the simulation results. In general, the response (a dose, fluence, the number of backscattered neutrons, etc.) for an incident particle of momentum $p$ can be parameterized in the form $N p^{\alpha}$, where $0.5<\alpha<1.0$. When a function of this form is folded with the production spectrum, the result can be written in the form $C \cosh ^{\alpha} \eta$ (e.g. the number of backscattered neutrons per rapidity interval) or $C \cosh ^{\alpha+2} \eta$ (e.g. the maximum electromagnetic or hadronic dose in a calorimeter). For example, the maximum dose due to electromagnetic showers in a calorimeter $2 \mathrm{~m}$ from the interaction point at an angle defined by $\eta=$ $-\ln \tan (\theta / 2)$ is given by $100 \cosh ^{2.9} \eta$ Gy $\mathrm{yr}^{-1}$ under standard SSC operating conditions $\left(\int \mathcal{L} d t=10^{40} \mathrm{~cm}^{-2}\right)$. This function varies by more than five orders of magnitude over the angular range covered by a typical $4 \pi$ detector.

We belive most of our results to be accurate to within a factor of two or three, sufficiently precise to serve as the basis for detailed designs. 



\section{TABLE OF CONTENTS}

Summary . . . . . . . . . . . . . . . . iii

Table of Contents . . . . . . . . . . . . . . . . . . . v

1. Introduction . . . . . . . . . . . . . . . 1

2. Particle Production at the SSC

2.1 General discussion . . . . . . . . . . . . . . . . . . . 3

2.2 Multiplicity distributions in rapidity and pseudorapidity . . . . 7

2.3 Momentum distributions . . . . . . . . . . . . . . . . . 10

2.4 Integrals . . . . . . . . . . . . . . . 15

3. Electromagnetic Cascades

3.1 Introduction . . . . . . . . . . . . . . . 21

3.2 Dose from electromagnetic showers . . . . . . . . . . . . . 21

3.3 Photoneutron production . . . . . . . . . . . . . . . . . 22

3.4 Albedo photons . . . . . . . . . . . . . . . . . . . . 22

4. Neutrons

4.1 Introduction . . . . . . . . . . . . . . . 25

4.2 Simulation and experimental data . . . . . . . . . . . 27

4.3 Albedo and maximum flux . . . . . . . . . . . . . . . . . 31

4.4 Leakage flux . . . . . . . . . . . . . . . . . . 36

4.5 Dependence upon calorimeter construction . . . . . . . . . . 37

4.6 Reflection in the central cavity . . . . . . . . . . . . . . . 37

5. Summary of Radiation Levels

5.1 Particle production model . . . . . . . . . . . . . . . . . 43

5.2 Assumptions about SSC operation . . . . . . . . . . . . . 45

5.3 Dose from minimum ionizing particles . . . . . . . . . . . . 45

5.4 Albedo photons . . . . . . . . . . . . . . . . 45

5.5 Neutron flux . . . . . . . . . . . . . . . . 47

5.6 Dose distribution . . . . . . . . . . . . . . . . . . . . . 51

References . . . . . . . . . . . . . 55 
Appendices:

1. Membership of the Task Force . . . . . . . . . . . . . 57

2. F. E. Paige, "Soft and Hard Scattering at the SSC" . . . . . . 59

3. J. Ranft, "DTUJET" . . . . . . . . . . . . . . . . . 67

4. T. K. Gaisser and T. Stanev, "Simulation of Mini-Jets in Minimum-Bias Events" . . . . . . . . . . . . 101

5. E. M. Wang, "ISAJET and PYTHIA Predictions" . . . . . . 107

6. A. Fassò, "Applicability of EGS at SSC Energies" . . . . . . . 117

7. H. Hirayama and W. R. Nelson, "Radiation Levels in Electromagnetic Calorimeter" . . . . . . . . . . . . . . 121

8. T. Stanev, "LPM Effect in the SSC Detectors" . . . . . . . . 145

9. D. E. Groom, "Flux, Current, and Lambert's Law" . . . . . . 151

10. D. E. Groom, "Measurement and Simulation of the Neutron Flux in the Tevatron Tunnel" . . . . . . . . . . 163

11. R. A. Lillie, R. G. Alsmiller, Jr., and T. A. Gabriel, "Neutron Reflection in Spherical Calorimeters" . . . . . . . 173

12. T. P. Wilcox, Jr., "Neutron Reflection in Spherical Calorimeters" 181

13. R. G. Alsmiller, Jr., F. S. Alsmiller, T. A. Gabriel, B. L. Bishop, and O. W. Hermann, "Calculated Results for Monoenergetic Protons Incident on a Uranium-Plastic Calorimeter" . . . . . 193

14. S. Ban, T. Kondo, and M. Asai, "Predictions of Neutron Yields from GHEISHA + ANISN" . . . . . . . . . . . . . 201

15. J. E. Brau and T. A. Gabriel, "Calculations of Neutron Flux in Iron and Uranium Calorimeters" . . . . . . . . . . 217

16. H. Fesefeldt, "Neutron Yields in Hadron Calorimeters" . . . . 229

17. J. S. Russ, G. R. Stevenson, and A. Fassò, "Low-Energy Neutron Measurements in an Iron Calorimeter Structure Irradiated by $200 \mathrm{GeV} / \mathrm{c}$ Pions" . . . . . . . . . . 249

18. R. Wigmans, "Estimation of Neutron Flux and Albedo from Activation Analysis Results" . . . . . . . . . . 295

19. N. V. Mokhov, "Inclusive Simulation of Hadronic and Electromagnetic Cascades in the SSC Components" . . . . . 303

20. G. R. Stevenson, "Dose to SSC Detectors Due to p-p Collisions" 310

21. D. E. Groom and G. R. Stevenson, "Backscatter and Lateral Diffusion of Hadronic Cascades in a Model Calorimeter" . . . 329 


\section{INTRODUCTION}

At or near the design luminosity of the SSC, radiation damage to some detector components will be an important consideration in the design of experiments. Although attempts have been made previously to quantify the radiation levels expected at the SSC from the particles produced in the pp collisions [1-4], this report represents the first coherent attempt to identify and quantify all of the relevant sources of potential radiation damage.

The radiation levels of interest are shown in the very schematic picture of a "detector" in Fig. 1. Potential sources of radiation damage in experiments are

- minimum ionizing particles produced in the pp collisions

- photon conversions in the beam pipe and other material

- electromagnetic showers in calorimetry

- hadronic showers in calorimetry and

Dose from Charged Particles and Photon Conversions

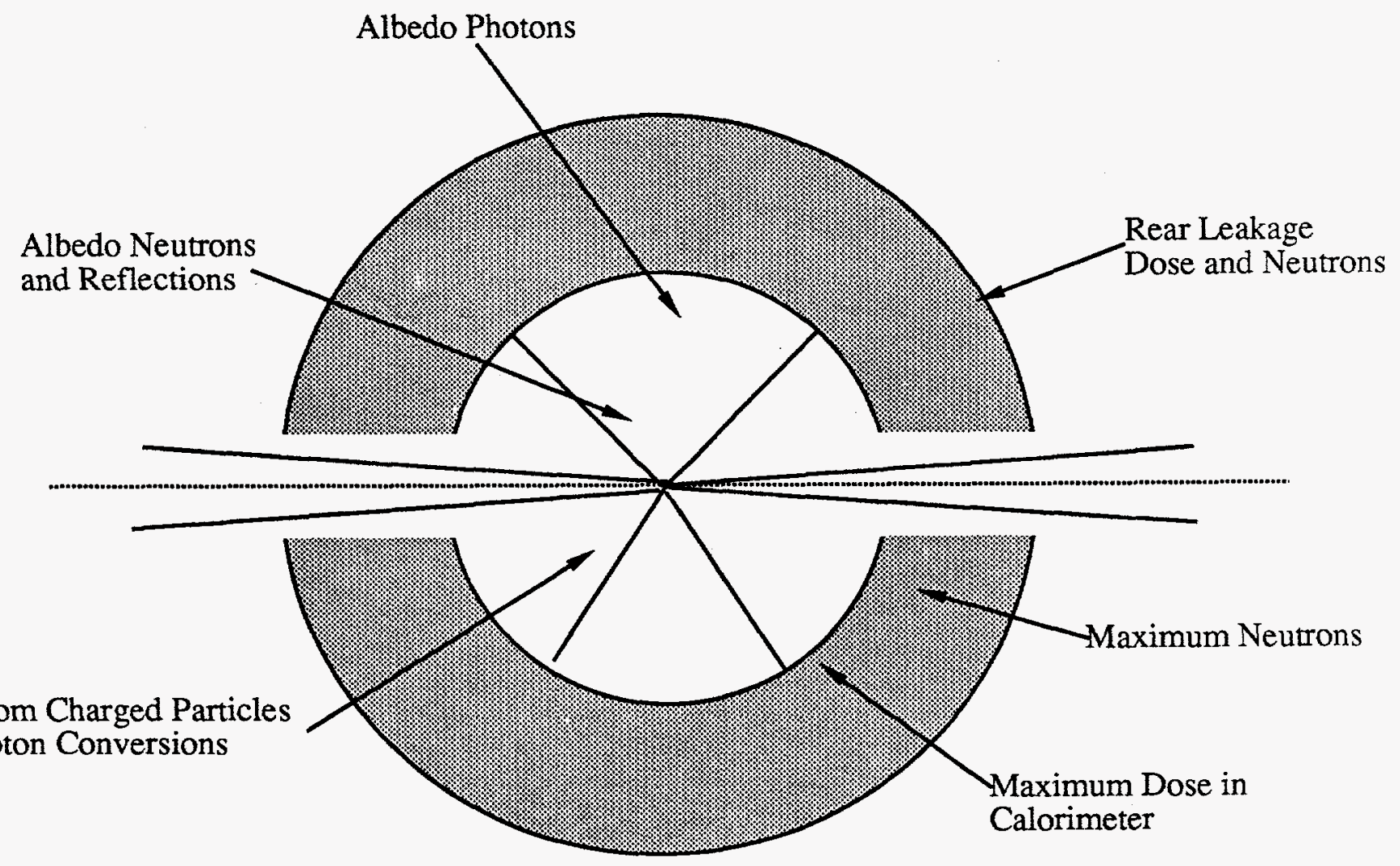

FIG. 1. A very schematic picture showing the various components of radiation levels at the SSC. 
- albedo particles, primarily neutrons and photons, from the showers in the calorimetry.

It is not within the scope of this report to discuss actual radiation damage to materials and devices; this will be the subject of additional reports. However, for the purposes of determining radiation damage one must specify the appropriate radiation levels in such a manner so that damage effects may be estimated. Radiation levels should be specified in terms of

- dose rate in grays (Gy) per unit time where $1 \mathrm{~Gy}=100 \mathrm{rads}=1$ joule $/ \mathrm{kg}$ $=6.24 \times 10^{12} \mathrm{MeV} / \mathrm{kg}$

- neutron flux in neutrons $/ \mathrm{cm}^{2}$ per unit time, ideally at some equivalent neutron energy such as $1 \mathrm{MeV}$

- total dose and neutron fluence accumulated over some reference time, e.g. one year.

For the SSC we believe that it is a conservative assumption to take dose rates and neutron rates to be uniform in time e.g., to quote doses per year at a given luminosity. Although the SSC will obviously not operate continuously at a fixed intensity for a year, the uncertainties which result from making such an assumption are much smaller than others in this Report. Our assumption in this Report is that the reference interaction region operates at a luminosity of $10^{33} \mathrm{~cm}^{-2} \mathrm{~s}^{-1}$ for $10^{7}$ seconds per year, or $10^{15}$ inelastic events per year if the cross section is $100 \mathrm{mb}$.

We note that the radiation levels given in this report are only those which arise directly or indirectly from the particles produced in pp collisions at the interaction points in the SSC. We have completely neglected contributions from single beam losses either during storage ring operation or during injection into the SSC storage rings. Unlike other storage rings, it is likely that the major source of radiation will in fact be the particles produced in the pp collisions. The beam lifetime from beam-gas scattering at the SSC is estimated to be $\approx 300$ hours, but at design luminosity the lifetime contribution from each high-luminsosity interaction region due to pp collisions will also be 300 hours. This is equivalent to full beam loss from one ring into a detector every six days. While this forms the basis for our neglect of other radiation sources, it is also well known that losses during injection are very difficult to estimate and furthermore are not usually stable in time.

Finally we would like to include a note of caution to the reader. The estimates of radiation levels presented in this report are preliminary and do not reflect the composition or character of an actual experiment. At such time as experiments for the SSC are better described, more accurate estimates can be made for specific experiments. 


\section{PARTICLE PRODUCTION AT THE SSC}

\subsection{General discussion}

Multiparticle production is a consequence of the strong interaction sector of QCD. This is not well understood theoretically-the known techniques of lattice gauge theory are grossly inadequate for calculating any $S$-matrix elements. Nevertheless, a fairly reliable extrapolation of the existing experimental data to $40 \mathrm{TeV}$ seems possible because the data vary slowly with $\log s$. For the total cross section this slow variation can be easily understood: the fact that the interaction is strong sets a lower limit of order the geometrical size $1 / m_{\pi}^{2}$, while the Froissart bound sets a rigorous upper limit of $\log ^{2} s$ on the possible growth. In Fig. 2-1 we reproduce one of the popular fits [5] to the total cross section.

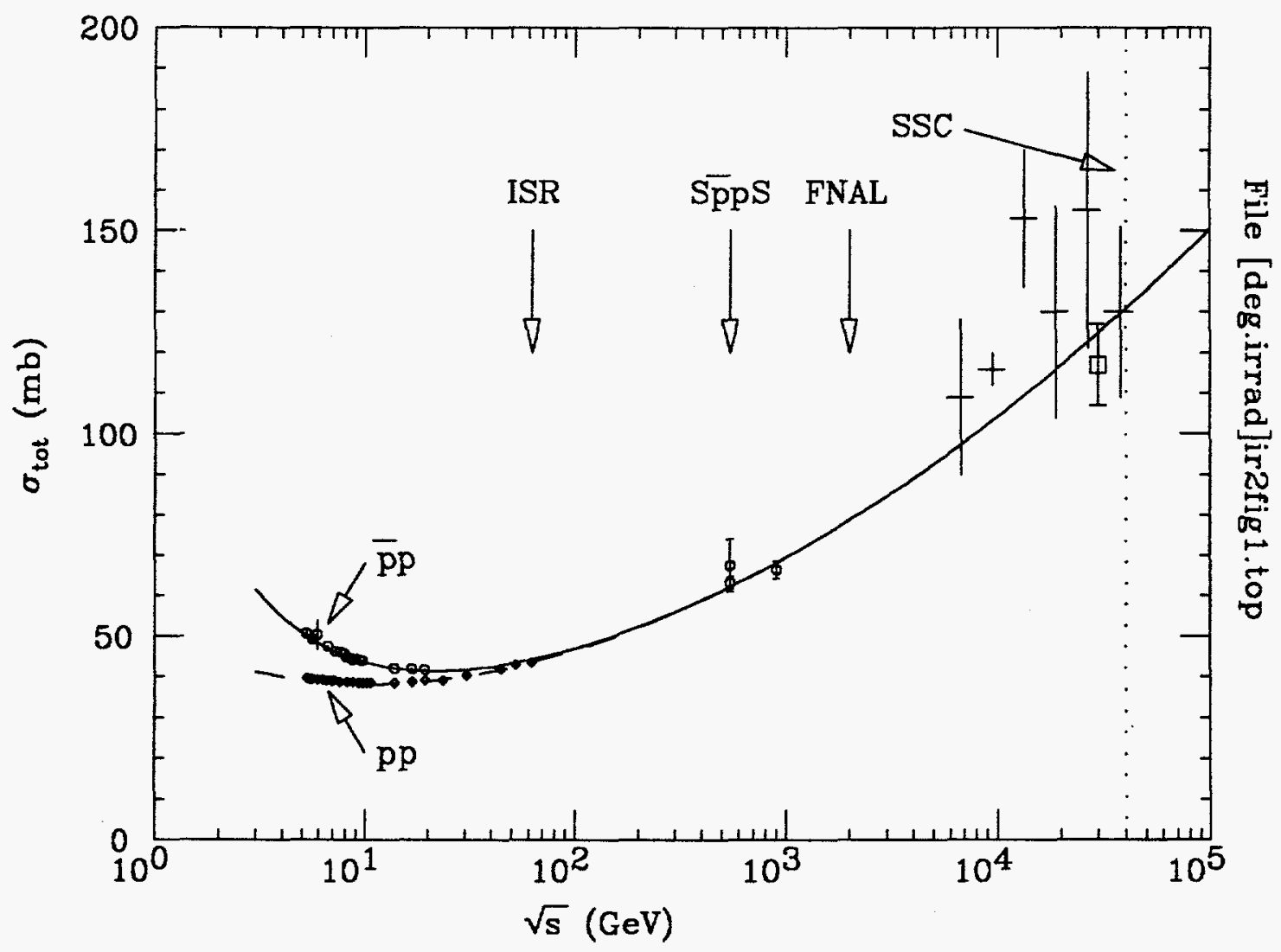

FIG. 2-1. Extrapolation of the original Amaldi fit [5] to higher energies. The highest energy fitted was $63 \mathrm{GeV}$ (ISR), so the agreement with $\mathrm{S} \overline{\mathrm{p}} \mathrm{pS}$ data must be regarded as fortuitous. Akeno air-shower results are marked by crosses, and the Fly's Eye point by the open square. Uncertainties in the cosmic ray data are discussed elsewhere [6]. 
There is no rigorous bound on the growth of the multiplicity other than the kinematic limit $\bar{n} \sim \sqrt{s}$, but the picture of particle production as the breaking of a string of confined flux in QCD naturally leads to slow variations. Given such slow variation, the uncertainty in the extrapolation to $40 \mathrm{TeV}$ is fairly small.

In the $\mathrm{S} \overline{\mathrm{p}} \mathrm{pS}$ collider energy range, however, a new phenomenon emerges. The cross sections for low- $p_{\perp}$ jets calculated in perturbative QCD become significant compared to the total cross section, whereas they are small at lower energies. In particular, with the EHLQ [7] structure functions it is found that

$$
\sigma_{\mathrm{QCD}}\left(\sqrt{s}=630 \mathrm{GeV}, p_{\perp}>3 \mathrm{GeV}\right) \approx 10 \mathrm{mb}
$$

compared to a total cross section of about $60 \mathrm{mb}$. It is difficult to identify jets at such low values of $p_{\perp}$, but the experimental data are not inconsistent [8] with the theoretical predictions. The rapid increase of the calculated QCD cross sections continues up to SSC energies, so that

$$
\sigma_{\mathrm{QCD}}\left(\sqrt{s}=40 \mathrm{TeV}, p_{\perp}>3 \mathrm{GeV}\right) \approx 200 \mathrm{mb} .
$$

Hence one might question whether the slow logarithmic variation of the total cross section and multiplicity will continue to hold.

The jet cross sections are large because the fractional momentum transfer $x \approx 2 p_{\|} / \sqrt{s}$ is small, and the Altarelli-Parisi equations predict that as $x \rightarrow 0$ the gluon distribution function behaves like [9]

$$
x G\left(x, Q^{2}\right) \sim \exp \left\{\text { const } \times\left[B \log \left(\frac{\log \left(Q^{2} / \Lambda^{2}\right)}{\log \left(Q_{0}^{2} / \Lambda^{2}\right)}\right) \log \left(\frac{1}{x}\right)\right]^{1 / 2}\right\},
$$

where the squared momentum transfer $Q^{2}$ is for hard hadronic processes proportional to the square of the jet transverse momentum, $\Lambda$ is the QCD scale parameter, and $Q_{0}^{2}$ is a reference scale - the starting point of the evolution. This behavior must fail for sufficiently small $x$ because the gluons become crowded in the proton and recombination effects, which are neglected in the Altarelli-Parisi equations, become important. However, the first correction term from this effect has been computed, and it indicates that recombination is negligible at any values of $x$ of interest at the SSC.

The cross section computed from the QCD improved parton model is actually an inclusive cross section. It corresponds to $\bar{n} \sigma_{\text {total }}$, where $\bar{n}$ is the mean number of hard scatterings per event. For $\bar{n} \ll 1$, the usual case for perturbative QCD, multiple interactions are unimportant. When $\bar{n} Z 1$, however, absorption and 
multiple interactions become important. There is a theorem [10] that absorptive corrections do not change the inclusive cross section, so the parton model calculation gives the correct multiplicity even in this case.

For the total cross section, the soft and hard contributions cannot simply be added but must be combined in a way consistent with $s$-channel unitarity. This is discussed in more detail in Appendix 2 of this Report and is implemented in the DTUJET model by Ranft (Appendix 3) and collaborators[11]. We expect the hard interactions to have only a small effect on the total cross section. Both the soft and hard interactions are distributed in impact parameter; if the soft interactions are already strong enough to make the protons black, then the hard interactions cannot increase the cross section unless their distribution in impact parameter is wider. But the distribution of the partons should be no larger than the proton size $1 / m_{\pi}^{2}$, and the interaction between the partons is pointlike. We therefore expect a smooth extrapolation of existing data to give the correct total and inelastic cross sections at SSC energies. Such extrapolations (Refs. 5 and 12, and Appendices 2 and 3) are consistent with the behaviour shown in Fig. 2-1 and give

$$
\begin{aligned}
\sigma_{\text {total }} & \approx 130 \mathrm{mb} \\
\sigma_{\text {inelastic }} & \approx 90 \mathrm{mb}
\end{aligned}
$$

with a spread of about $\pm 25 \%$ between different fits. These values are also consistent with cosmic ray data.

The uncertainties are larger for the average multiplicity. The most important uncertainty comes from the lack of knowledge of the parton distributions at small $x$. The EHLQ distributions are based on the traditional assumption that at the reference scale $Q_{0}^{2}=5 \mathrm{GeV}^{2}$,

$$
x f\left(x, Q_{0}^{2}\right) \sim \text { const }
$$

corresponding to a constant total cross section. But the Altarelli-Parisi equations then predict a more singular behavior for any $Q^{2}>Q_{0}^{2}$. Collins [13] has pointed out that a constant behavior leads to negative parton distributions if the Altarelli-Parisi equations are evolved backwards to $Q^{2}<Q_{0}^{2}$, whereas consistent behavior is obtained for

$$
x f\left(x, Q_{0}^{2}\right) \sim x^{-p}, \quad p=0.3-0.5
$$

While this behavior is not well established, it seems likely that the parton distributions should be more singular than the EHLQ ones for $x \rightarrow 0$. If one takes 
$p=0.5$ and computes the QCD improved parton model jet cross section, one finds [14]

$$
\sigma\left(\sqrt{s}=40 \mathrm{TeV}, p_{\perp}>3 \mathrm{GeV}\right) \approx 4000 \mathrm{mb},
$$

twenty times the value with the EHLQ structure functions. This is probably not a realistic value, since the central value of the rapidity ${ }^{\star}$ scales as a power of the square of the center of mass energy:

$$
\left.\frac{d \sigma}{d y}\right|_{y=0} \sim s^{p}
$$

If $p>0.5$ then the multiplicity of particles would grow faster than the total energy. But it does indicate that there is a substantial uncertainty in the calculation of the jet multiplicity. A crude limit can be set by noting that in DTUJET particles from QCD jets carry about $5 \%$ of the energy and contribute about $20 \%$ of the total multiplicity. If the fraction of energy were increased to $50 \%$ and nothing else changed, the multiplicity would increase by a factor of three.

For the EHLQ distributions the multiplicity is dominated by particles in the spectator beam jets. It is observed [8] at the $\mathrm{S} \overline{\mathrm{p}} \mathrm{p}$ that these beam jets have about twice the multiplicity for hard scattering events with $p_{\perp}>10 \mathrm{GeV}$ than for minimum bias events. For minijet events there is a transition between $p_{\perp}=0$ and $p_{\perp}>10 \mathrm{GeV}$ that is not understood. The DTUJET model (Ref. 11 and Appendix 3) seems to offer an explanation for this effect, but a detailed study of these aspects of the model is still outstanding. If the transition is real physics, it presumably occurs over a fixed range of $x$, and at SSC energies only the lower multiplicity beam jets would be important. However, if the transition simply reflects contamination from fluctuations of soft events, then a substantial fraction of SSC events will have the higher multiplicity beam jets.

$\star$ The rapidity $y$ is defined as

$$
\begin{aligned}
y & =\frac{1}{2} \ln \frac{E+p_{\|}}{E-p_{\|}} \\
& =\frac{1}{2} \ln \frac{\cos ^{2}(\theta / 2)+m^{2} / 4 p^{2}+\cdots}{\sin ^{2}(\theta / 2)+m^{2} / 4 p^{2}+\cdots} .
\end{aligned}
$$

If terms of order $(m / p)^{2}$ and higher can be neglected, the function depends only upon angle and is called the pseudorapidity $\eta$. The approximation $y \approx \eta$ breaks down for low energy particles and for angles smaller than $m / p \approx 1 / \gamma$. Because of its greater relevance to experimental design, pseudorapidity is used throughout the remainder of this report. 


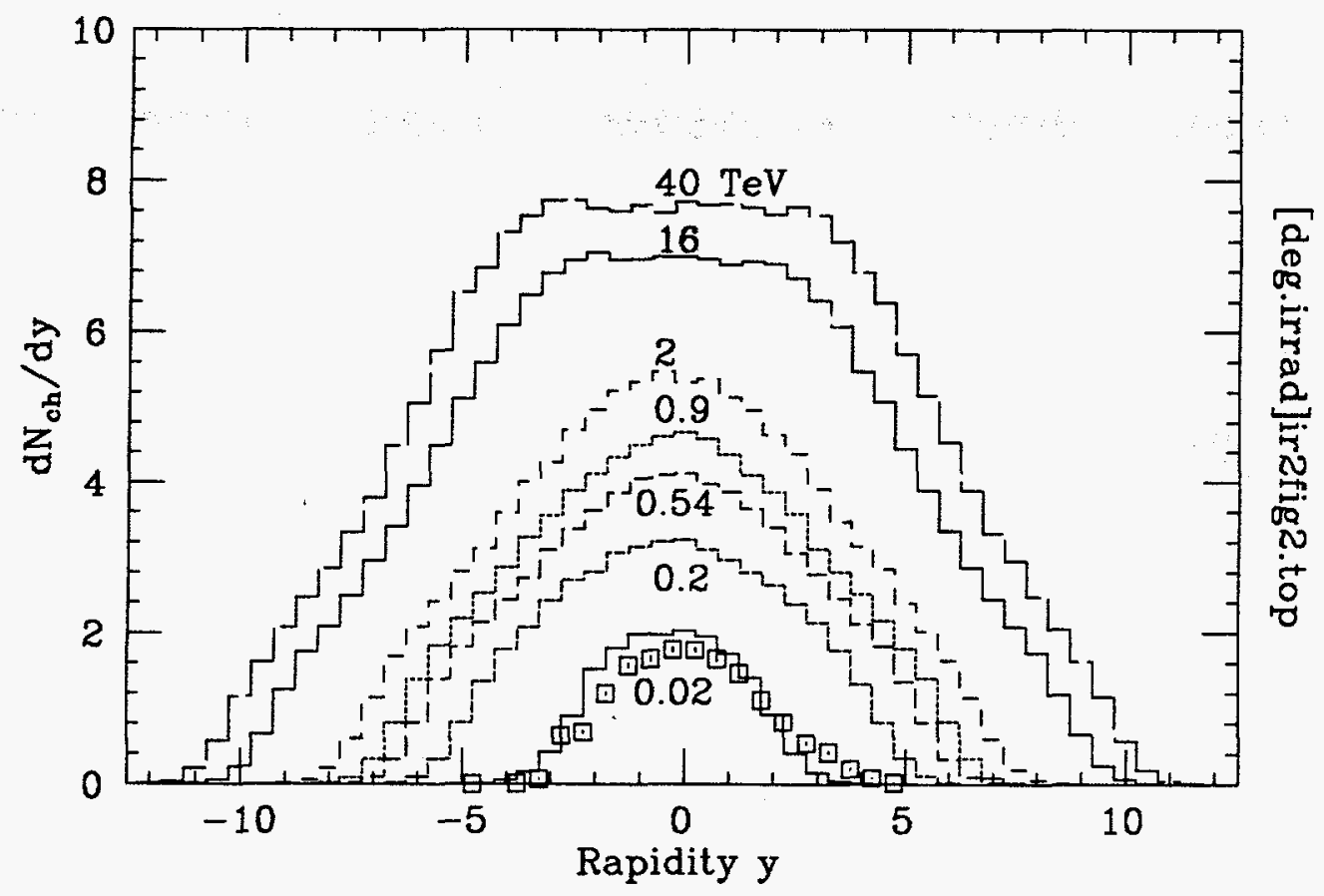

FIG. 2-2. Rapidity distributions at energies from $\sqrt{s}=20 \mathrm{GeV}$ to $40 \mathrm{TeV}$, as calculated using DTUJET.

\subsection{Multiplicity distributions in rapidity and pseudorapidity}

Results from DTUJET have been compared with those from the hard-scattering event generators ISAJET [15] and PYTHIA [16]. For ISAJET and PYTHIA we have taken equal mixtures of minimum bias events and jet events with $p_{\perp}>$ $3 \mathrm{GeV}$. In addition, comparisons have been made with a new code being developed by T. K. Gaisser and T. Stanev (see Appendix 4). The code is primarily intended for application to cosmic ray cascades. It represents the inelastic cross section as a sum of soft (energy independent) and semi-hard cross sections and uses the structure functions of Duke and Owens [17]. This code predicts an average charged multiplicity of 100 particles and a charged rapidity density of 6 . All four programs roughly agree in their predictions (Appendices 3, 4, and 5):

$$
\begin{aligned}
& \bar{N}_{\text {charged }} \approx 110 \\
& \frac{d N_{\text {charged }}}{d y} \approx 7 \text { or } 8 \quad \text { at } y=0
\end{aligned}
$$

In Fig. 2-2 we plot rapidity distributions calculated by DTUJET at energies from $\sqrt{s}=20 \mathrm{GeV}$ to $40 \mathrm{TeV}$. The shapes of these distributions are nearly Gaussian. One often assumes that the value at the maximum and the width both increase logarithmically with $s$, so that the multiplicity increases as $\ln ^{2} s$. 


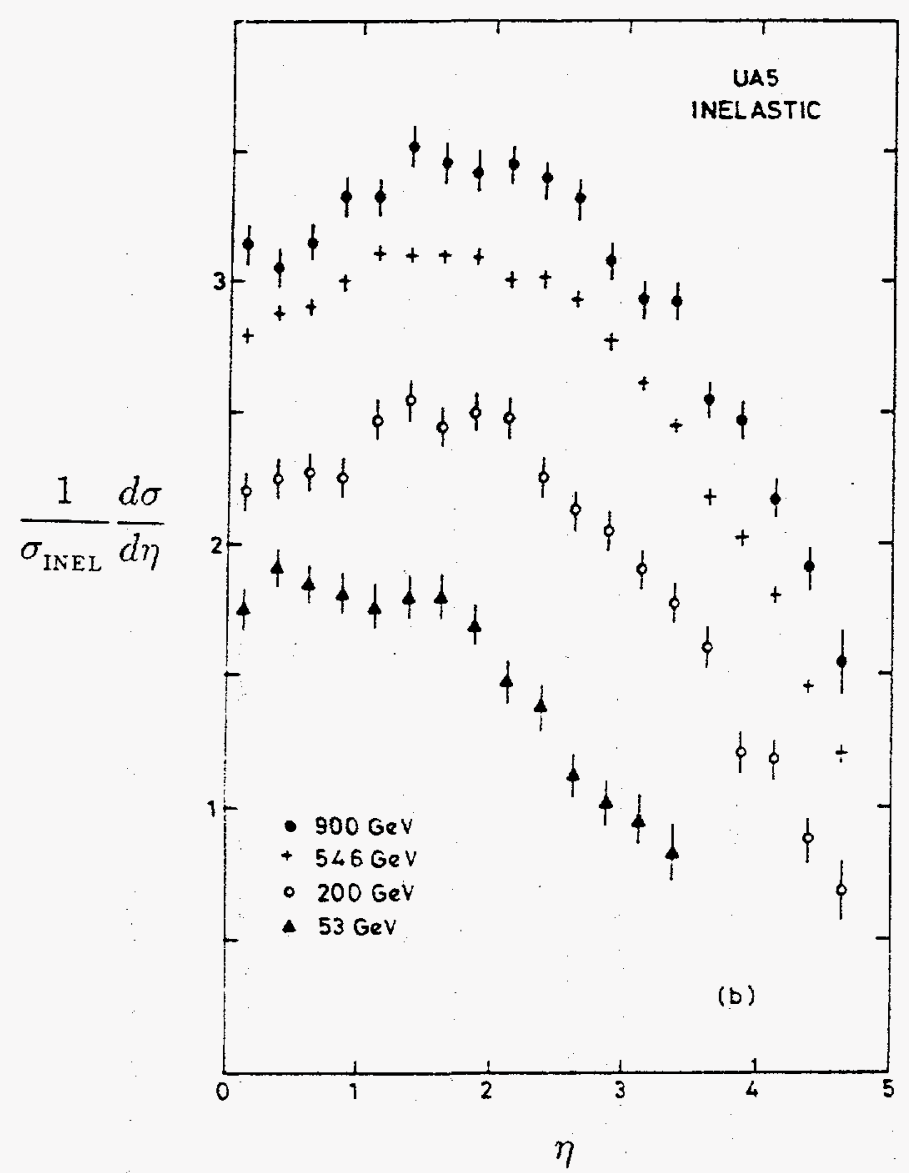

FIG. 2-3. Pseudorapidity distributions obtained by UA5 at various energies from the IRS and the SpppS collider[18].

However, the height of the function seems to increase faster than $\ln s$, and the width may increase somewhat more slowly than $\ln s$. When pseudorapidity is used instead, the shapes of the distributions look similar except for a central dip to about $10 \%$ of the maxima (the "seagull effect"), which appears for kinematical reasons. Examples of such distributions obtained by the UA5 collaboration at the Sp̄pS are shown in Fig. 2-3[18]. Disregarding the resulting double-bump structure, the pseudorapidity distribution at $40 \mathrm{TeV}$ looks rather flat in the central region $-6<\eta<6$ (i.e. for angles greater than $0.3^{\circ}$.)

The $\pi^{0}$ multiplicity is about half of the charged particle multiplicity. Since the decay photons roughly divide the energy, it is sufficient for our present purposes to describe the resulting $\gamma$-ray flux as having the same $\eta$ distribution as the charged particles but with a momentum distribution which is softer by a factor of two.

DTUJET, ISAJET, and PYTHIA all use EHLQ structure functions, and so probably at least somewhat underestimate the multiplicity of jets. 


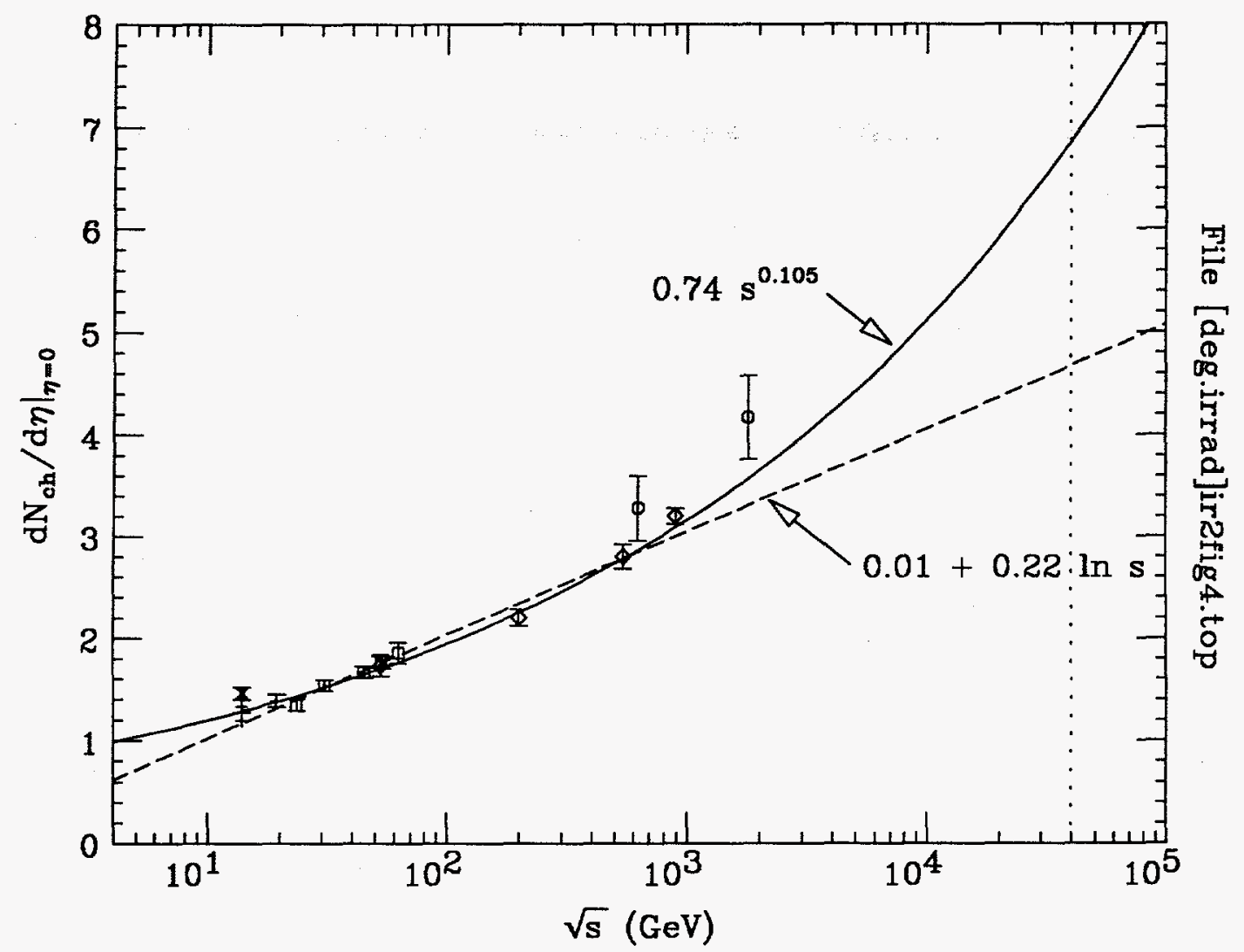

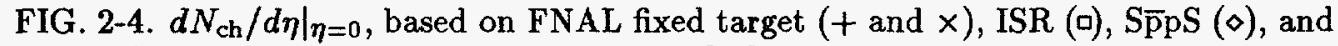
CDF (o) data. The preliminary CDF points[19] have been added to data taken from Fig. 2 in Alner et al.[18], and the two fits shown are to the data exclusive of CDF. The indicated errors for the CDF points are mostly systematic, and the error in the ratio of the two values is about 2.5 times smaller; the new data thus corroborate the increasing slope.

The input for these programs is ultimately dependent upon experimental data. The available high-energy data for the height of the pseudorapidity plateau are shown in Fig. 2-4, which is adapted from Fig. 2 of Alner et al. [18]. We have added two new points from CDF, shown by the circles at $630 \mathrm{GeV}$ and 1800 $\mathrm{GeV}[19]$, and extended the scale to include SSC energies. The errors on the CDF points are dominated by $10 \%$ systematic errors. The ratio of the values at the two energies is $1.27 \pm 0.05$, so the slope they define may be taken more seriously than the absolute normalization. The two functions shown are from Alner et al., and the CDF points are not included in the fits. An increase of slope with energy is evident. The intercept of the power-law fit at $E=40 \mathrm{TeV}$ is 6.85 . The SpppS data reported by Alner et al. show a substantial dip at $\eta=0$; the average value on the plateau appears to be $7 \%$ to $10 \%$ higher. We therefore adopt a value of 7.5 for the charged multiplicity per unit pseudorapidity interval $-6<\eta<6$. It should be evident from Fig. 2-4 that the correct value might be anywhere between 6 and 10 . 


\subsection{Momentum distributions}

For the purpose of radiation calculations we are interested in the function

$$
\frac{d^{2} N_{\mathrm{ch}}}{d \eta d p}
$$

Moreover, we are interested in this function for an "average" event-the kind which occurs $10^{8}$ times per second, not the rare physics events which may have a very different distribution. According to the above discussion, these events are described in the Monte Carlo language as some mixture of minimum bias and soft jet events. It has recently been shown that high-multiplicity events have a harder transverse momentum distribution [20]; this and other such complications do not matter because we average over all events.

The Lorentz invariant cross section

$$
F(\mathbf{p}, s)=E \frac{d^{3} \sigma_{\mathrm{tot}}(s)}{d p^{3}}
$$

may be re-written as

$$
F\left(p_{\perp}, y, s\right)=\frac{d^{2} \sigma_{\mathrm{tot}}(s)}{\pi d y d p_{\perp}{ }^{2}}
$$

since $d p_{\|} / E=d y$. According to Feynman scaling $F\left(p_{\perp}, y, s\right)$ is independent of $s$, but in practice both the $y$ dependence and $p_{\perp}$ dependence evolve slowly with $s$. Following common practice we leave the $s$ dependence implicit. So long as we are considering average behavior, $F\left(p_{\perp}, y\right)$ can be written in factored form, with the $p_{\perp}$ dependence well described by an exponential [21]:

$$
\begin{aligned}
F\left(p_{\perp}, y\right) & \approx h(y) f\left(p_{\perp}\right) \\
& \approx h(y) \frac{1}{B} \exp \left(-p_{\perp} / B\right)
\end{aligned}
$$

At ISR energies the mean value of the $p_{\perp}$ distribution for a variety of inclusive reactions is in the range $B \approx 0.22 \mathrm{GeV} / \mathrm{c}$.

The distribution given by Eq. 2-1 is differential in $p$ rather than $p^{2}$, so the distribution in $y$ analogous to Eq. 2-2 becomes

$$
\frac{d^{2} N_{\mathrm{ch}}}{d y d p_{\perp}}=H(y) \frac{p_{\perp}}{B^{2}} \exp \left(-p_{\perp} / B\right)
$$


or, since $p_{\perp}=p \sin \theta$

$$
\frac{d^{2} N_{\mathrm{ch}}}{d y d p}=H(y) \frac{p}{B^{2} \sin ^{2} \theta} \exp (-p / B \sin \theta) .
$$

If the distribution is written in this way, the mean is given by

$$
\left\langle p_{\perp}\right\rangle=2 B
$$

rather than $B$, so we should expect $\left\langle p_{\perp}\right\rangle \approx 0.5 \mathrm{GeV} / \mathrm{c}$. With the substitution of pseudorapidity for rapidity and our assumption that the height of the pseudorapidity plateau is constant over the region of interest, we finally have

$$
\frac{d^{2} N_{\mathrm{ch}}}{d \eta d p_{\perp}} \approx H f\left(p_{\perp}\right)
$$

where $f\left(p_{\perp}\right)$ is an appropriate normalized function of $p_{\perp}=p \sin \theta$.

The Monte Carlo results are compared with Eq. 2-5 in Figs. 2-5, 2-6, and 2-7. A scatter plot of $\eta$ vs. $\log p$ for Monte Carlo events (DTUJET) is shown in Fig. 2-4. The linearity of the line of maxima follows from the independence of the $p_{\perp}$ and $\eta$ distributions, and since $\eta=-\log \tan (\theta / 2)$ and $\log p=\log p_{\perp}-\log \sin \theta$. At $\eta=1$ the approximation $2 \tan (\theta / 2) \approx \sin \theta$ is $\operatorname{good}$ to within $12 \%$, and it improves rapidly with increasing $\eta$.

Slices at constant $\eta$ for similar ISAJET data are shown in Fig. 2-6, where the vertical scale is proportional to $p d N / d p$. Except for a displacement by $\log \sin \theta$ along the $\log p$ axis, these histograms are virtually identical for $\eta \lesssim 6$. This common $p_{\perp}$ distribution is shown in Fig. 2-7, where a $50 \%$ minimum bias and $50 \%$ jet-jet event admixture has been chosen to simulate average behavior. The solid curve is the exponential form of Eq. 2-3, with arbitrary vertical normalization and $B=0.213 \mathrm{GeV} / \mathrm{c}$ chosen for agreement of the maximum with that of the "data."

The $p_{\perp}$ dependence of the invariant cross section at several energies is shown in Fig. 2-8 [19]. The exponential fall-off distance $(B)$ at small $p_{\perp}$ increases with energy, and the distributions become flatter at larger $p_{\perp}$. This "skirt" has little effect on the mean, since it appears only after the cross section has decreased by many orders of magnitude. However, alternatives to the exponential form give above are often used to obtain a better parameterization in this region. The exponential and two commonly used forms are given below:

$$
f\left(p_{\perp}\right)=\frac{p_{\perp}}{B^{2}} \exp \left(-p_{\perp} / B\right)
$$




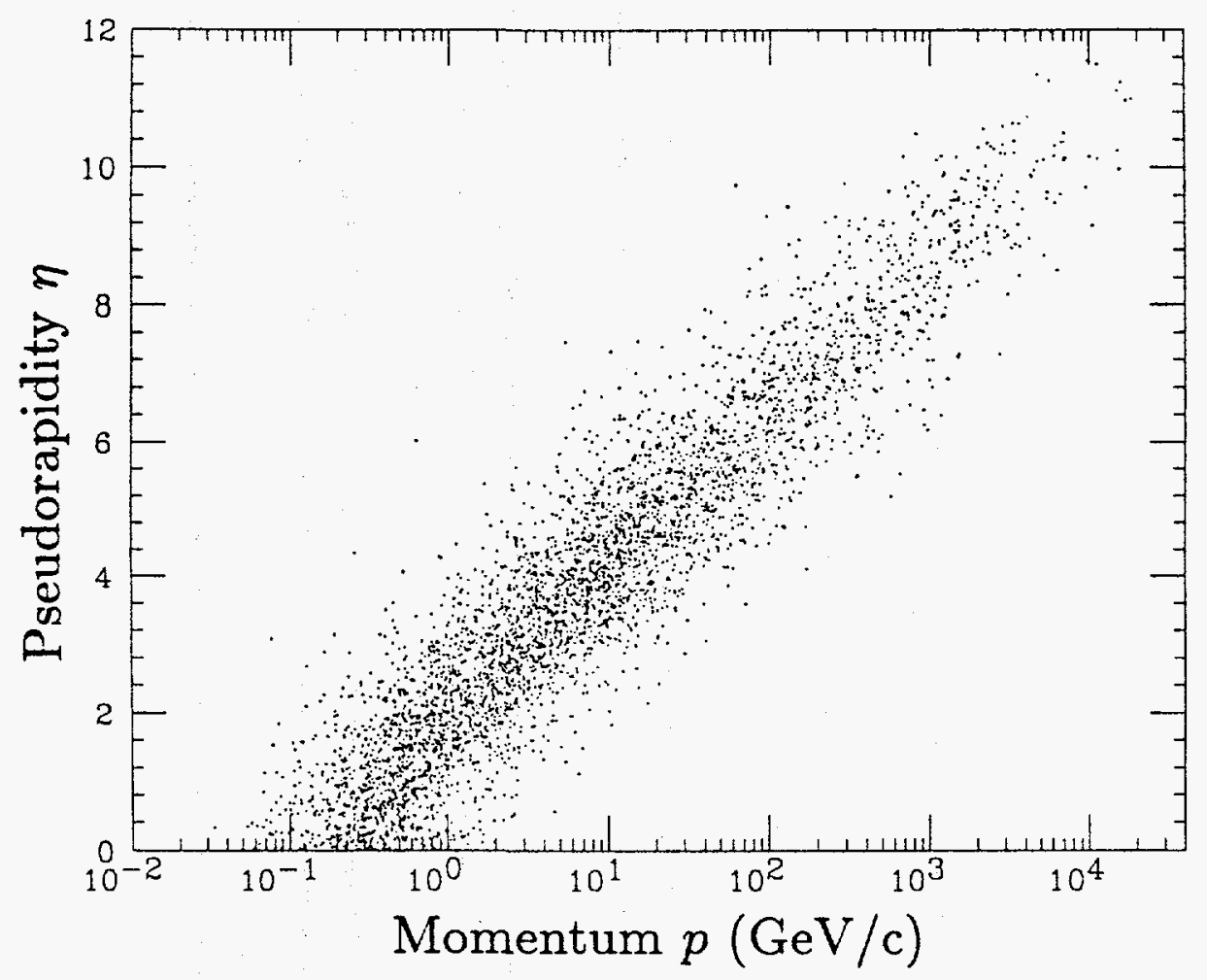

FIG. 2-5. A scatter plot of $\eta$ vs. $\log p$ for $\sqrt{s}=40 \mathrm{TeV}$ events generated by DTUJET.

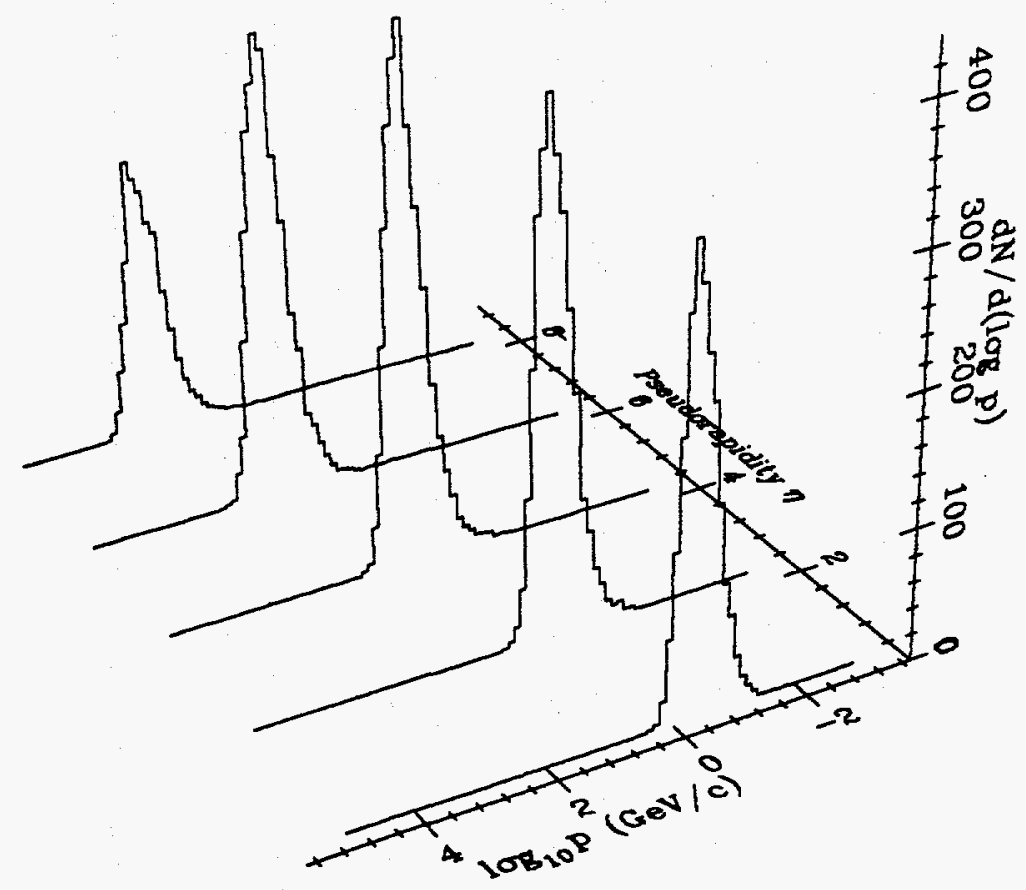

FIG. 2-6. Momentum distributions at constant $\eta$ for ISAJET events at $\sqrt{s}=40 \mathrm{TeV}$. 


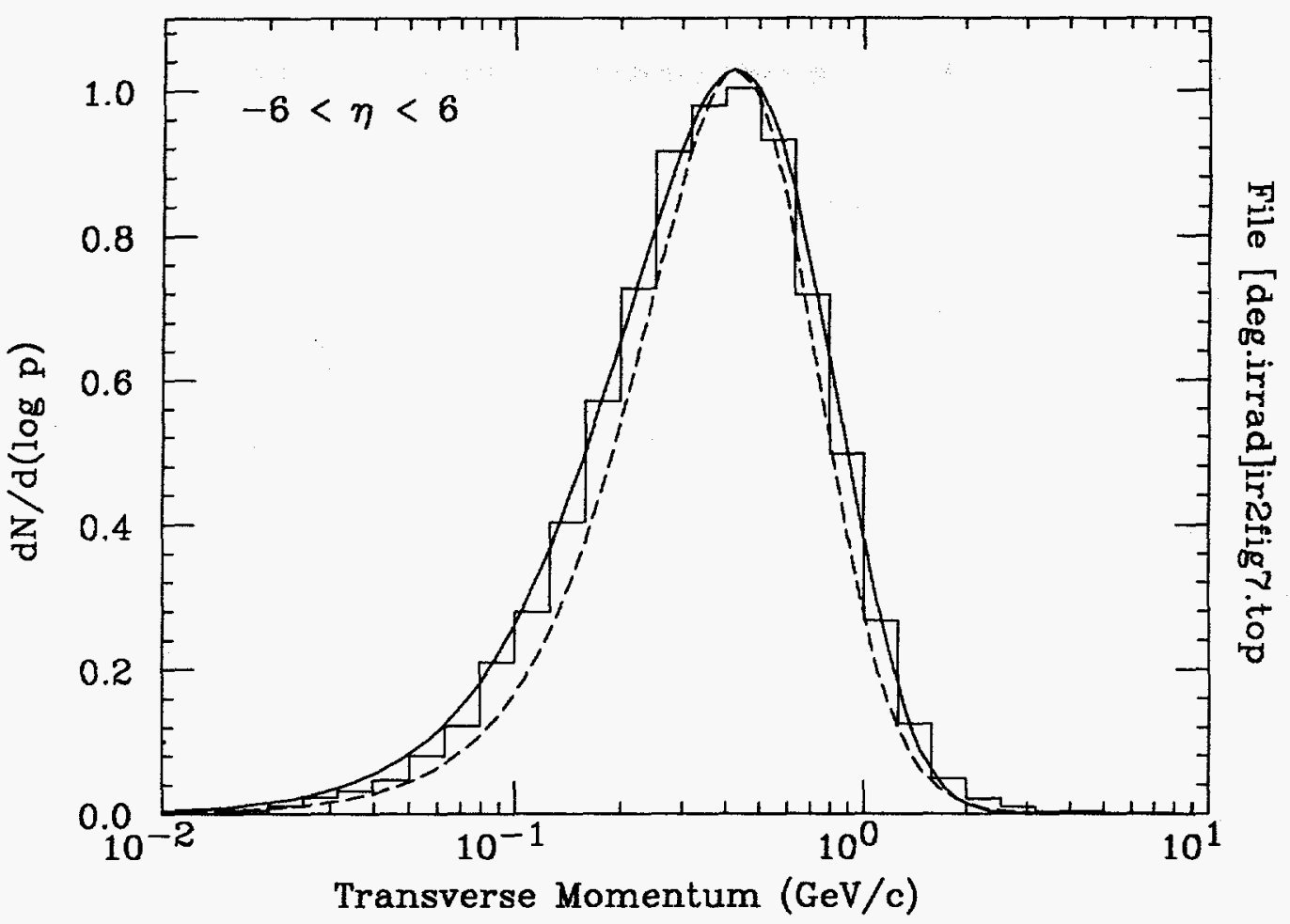

FIG. 2-7. The $p_{\perp}$ distribution of ISAJET events with $-6<\eta<6$. The solid curve is the exponential form given in Eq. 2-3, with an extra factor of $p$ because of the Jacobian involved in going to a logarithmic abscissa. The dashed curve is the rational polynomial given by $\mathrm{Eq}$. 2-7. Both are adjusted so that their maxima agree with that of the histogram $(B=0.213 \mathrm{GeV}, a=0.273 \mathrm{GeV} / \mathrm{c})$.

$$
\begin{aligned}
& f\left(p_{\perp}\right)=\frac{6 a^{6} p_{\perp}}{\left(p_{\perp}{ }^{2}+a^{2}\right)^{4}} \\
& f\left(p_{\perp}\right)=\frac{C p_{\perp}}{\left(p_{\perp}+p_{\perp 0}\right)^{m}}
\end{aligned}
$$

In Eq. 2-8 the exponent $m$ is a fitted parameter (about 9 ) and $C$ normalizes the function so that $\int f\left(p_{\perp}\right) d p_{\perp}=1$. The rational polynomial (Eq. 2-6) is used in parts of ISAJET, and a fit using this function is shown by the dashed curve in Fig. 2-7. Calculations discussed later in this section have been made using both forms 2-6 and 2-7. Far simpler results can be obtained with a fourth form,

$$
f\left(p_{\perp}\right)=\delta\left(p_{\perp}-\left\langle p_{\perp}\right\rangle\right)
$$

and, as will be seen later, these agree with results obtained with Eq. 2-6 or 2-7 to within a few percent. 


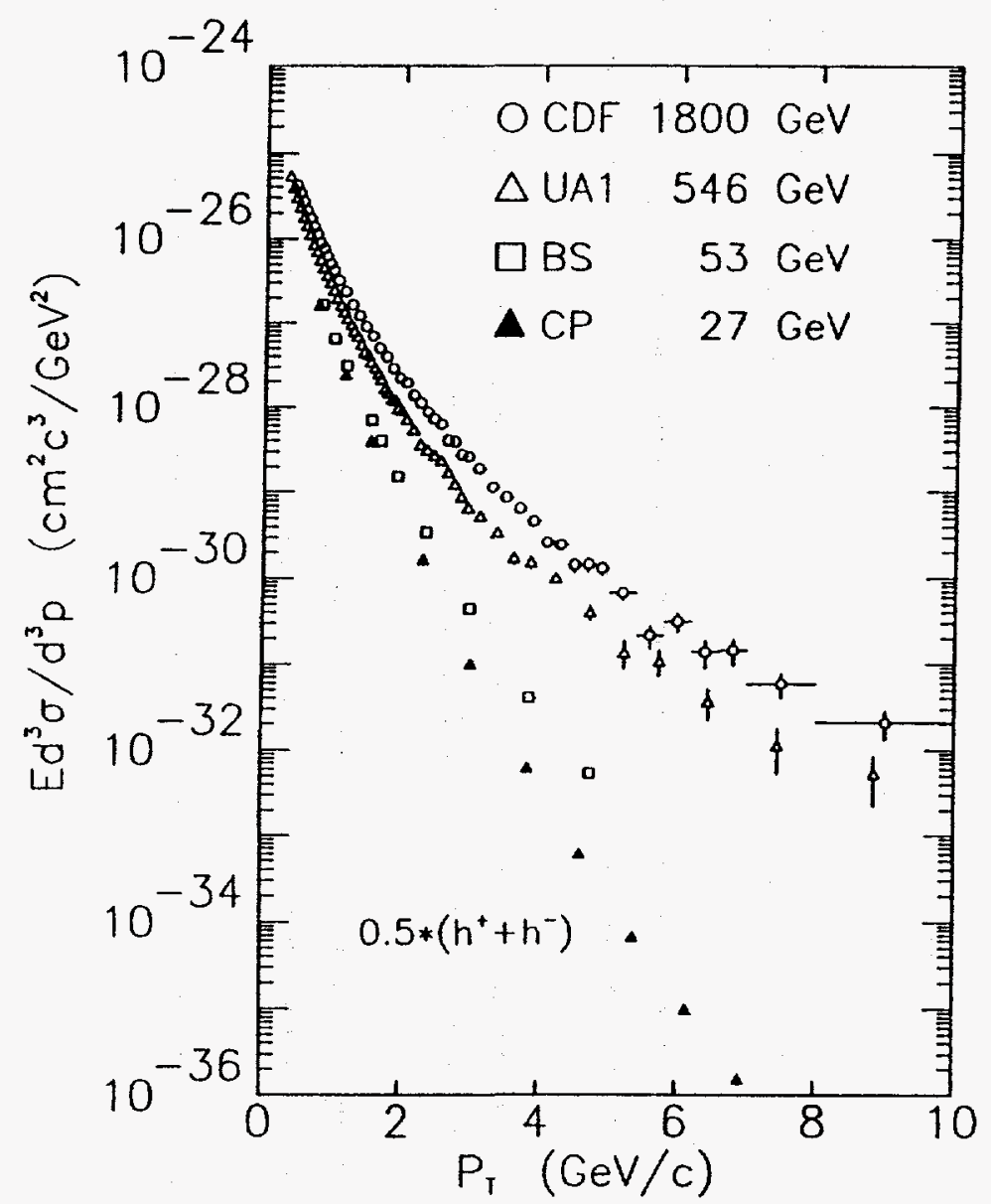

FIG. 2-8. The invariant cross section as a function of $p_{\perp}$ for various values of $\sqrt{s}[19]$. BS refers to the British-Scandanavian collaboration and $\overline{C P}$ to Chicago-Princeton results. The CDF data are preliminary.

The maximum of $f\left(p_{\perp}\right)$ for the exponential form occurs at $2 B$ (where we must remember the extra factor of $p_{\perp}$ when a logarathmic horizontal scale is used), and that of the rational polynomial at $a / \sqrt{3}$.

We reiterate that care must be taken in comparing the means of the distributions given by Eqs. 2-2 and 2-3. The mean of an invariant cross section parameterized as $\exp \left(-p_{\perp} / B\right)$ is $B$. The distribution given in Eq. 2-6 contains another factor of $p_{\perp}$, so its mean is $2 B$. Similarly, Eq. 2-7 yields $\left\langle p_{\perp}\right\rangle=(3 \pi / 16) a$. If the maxima of $d N / d(\log p)$ for the two forms are forced to agree (as in Fig. $2-7)$, then the mean of the rational polynomial is at $2(3 \sqrt{3} \pi / 16) B=2 \times 1.020 B$. Either mean may be interpreted as twice the inverse slope of the exponential in $p_{\perp}$ describing the usual invariant cross section.

Although ISAJET yields $\left\langle p_{\perp}\right\rangle=0.45 \mathrm{GeV} / \mathrm{c}$, appeal must again be made to experimental data. The available results are shown in Fig. 2-9[19]. While a curve "to guide the eye" is intentionally omitted, we conclude that $\left\langle p_{\perp}\right\rangle \approx 0.60$ $\mathrm{GeV} / \mathrm{c}$ is probably accurate to within about $20 \%$. This value is used to obtain the results of Section 5 . 


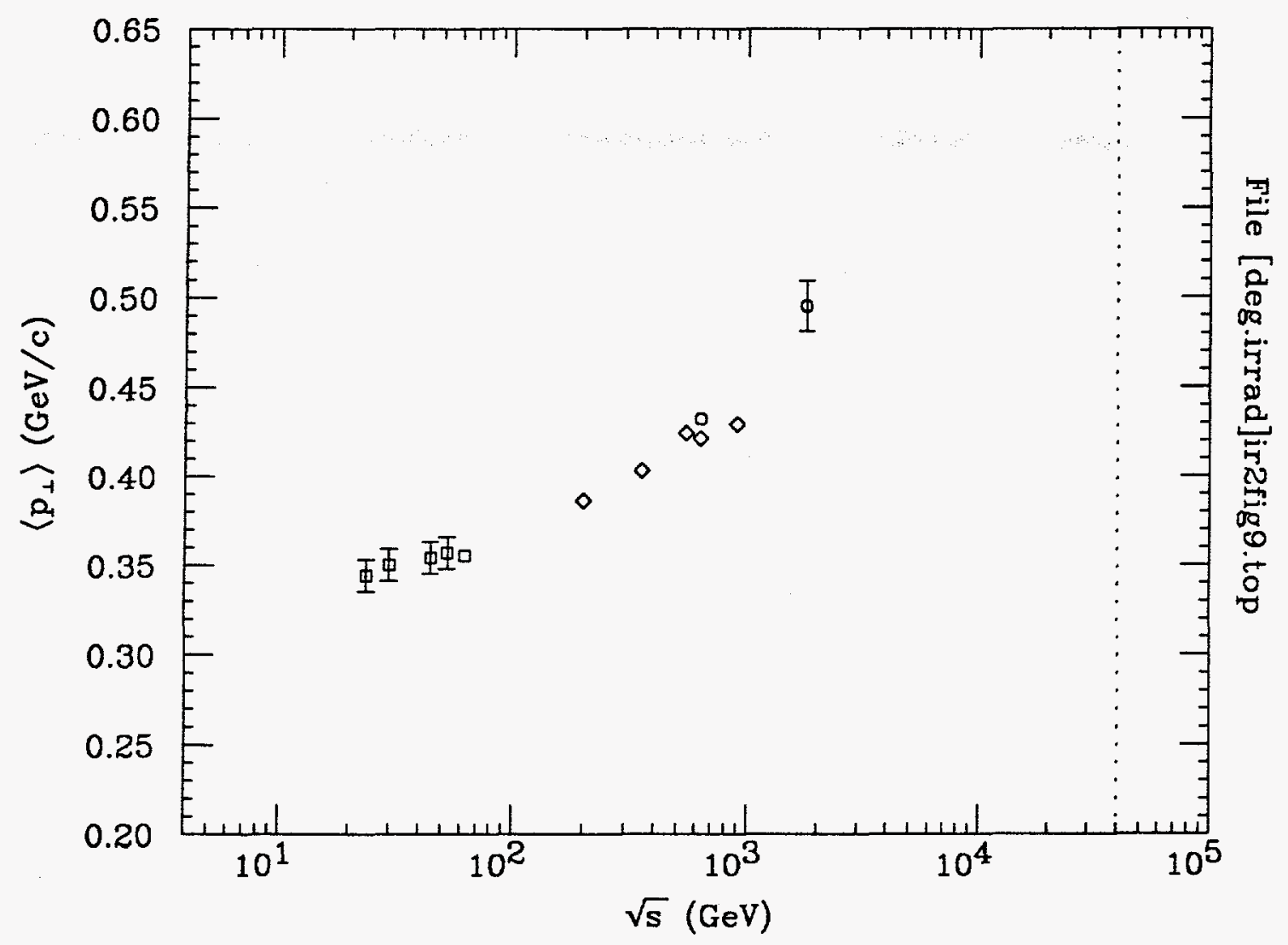

FIG. 2-9. The mean value of $p_{\perp}$ for the distribution $d^{2} N_{\mathrm{ch}} / d y d p_{\perp}$ as a function of

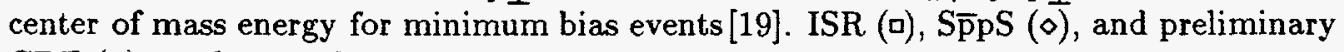
CDF (o) results are shown.

\subsection{Integrals}

In Section 4 it is shown that the available information about the flux or dose produced by a hadron or photon with momentum $p$ under various conditions can be parameterized by functions of the form

$$
N p^{\alpha}
$$

where $\alpha$ is between 0 and 1 and $N$ is evaluated at some reference momentum such as $1 \mathrm{GeV} / \mathrm{c}$. To obtain the results of Section 5 , it is necessary to integrate the product of this function and the production spectrum given in Eq. 2-5 over both momentum and pseudorapidity.

The appropriate momentum region is from a lower cutoff defined (as a function of angle) by a central solenoid and a higher cutoff defined by the highest possible momentum. The upper limit may be taken as infinite without any loss of accuracy, and the lower cutoff is zero if no solenoid is present. For an infinitely long solenoid, the lower cutoff is some fixed transverse momentum $p_{\perp 0}$, typically $0.4 \mathrm{GeV} / \mathrm{c}$. For a finite system, particles with a momentum lower than this can 
Table 2-1

Numerical values of the function $\Gamma\left(\alpha, x_{0}\right) / 2^{\alpha}$ defined by Eq. $2-10$ and shown in Fig. 2-10.

\begin{tabular}{cccccc}
\hline \hline \multirow{2}{*}{$\alpha$} & \multicolumn{5}{c}{$\Gamma\left(\alpha, x_{0}\right) / 2^{\alpha}$ for $x_{0}=$} \\
& 0.0 & 1.0 & 2.0 & 3.0 & 4.0 \\
\hline 0.00 & 1.0000 & 0.7358 & 0.4060 & 0.1991 & 0.0916 \\
0.05 & 0.9874 & 0.7398 & 0.4154 & 0.2065 & 0.0960 \\
0.10 & 0.9764 & 0.7444 & 0.4251 & 0.2141 & 0.1006 \\
0.15 & 0.9670 & 0.7494 & 0.4351 & 0.2220 & 0.1054 \\
0.20 & 0.9592 & 0.7548 & 0.4455 & 0.2303 & 0.1105 \\
0.25 & 0.9527 & 0.7607 & 0.4563 & 0.2389 & 0.1158 \\
0.30 & 0.9477 & 0.7672 & 0.4675 & 0.2479 & 0.1214 \\
0.35 & 0.9439 & 0.7741 & 0.4791 & 0.2573 & 0.1273 \\
0.40 & 0.9414 & 0.7816 & 0.4911 & 0.2671 & 0.1335 \\
0.45 & 0.9401 & 0.7896 & 0.5035 & 0.2772 & 0.1400 \\
0.50 & 0.9400 & 0.7982 & 0.5164 & 0.2878 & 0.1469 \\
0.55 & 0.9410 & 0.8073 & 0.5299 & 0.2989 & 0.1541 \\
0.60 & 0.9432 & 0.8171 & 0.5438 & 0.3105 & 0.1616 \\
0.65 & 0.9465 & 0.8275 & 0.5582 & 0.3225 & 0.1696 \\
0.67 & 0.9481 & 0.8318 & 0.5642 & 0.3275 & 0.1729 \\
0.70 & 0.9509 & 0.8385 & 0.5732 & 0.3351 & 0.1779 \\
0.75 & 0.9563 & 0.8502 & 0.5888 & 0.3482 & 0.1867 \\
0.80 & 0.9629 & 0.8626 & 0.6051 & 0.3619 & 0.1960 \\
0.85 & 0.9705 & 0.8757 & 0.6219 & 0.3763 & 0.2057 \\
0.90 & 0.9793 & 0.8896 & 0.6395 & 0.3912 & 0.2160 \\
0.95 & 0.9891 & 0.9042 & 0.6577 & 0.4069 & 0.2268 \\
1.00 & 1.0000 & 0.9197 & 0.6767 & 0.4232 & 0.2381 \\
\hline \hline
\end{tabular}

strike the endcaps. In practice, cutoffs at 0 and $p_{\perp 0}$ bracket the answer. In the integrals discussed below we take the useful range of lower cutoffs for integrals over $p_{\perp}$ to be $0<p_{\perp 0}<1 \mathrm{GeV} / \mathrm{c}$.

For the distribution given by Eq. 2-6, the momentum integral ${ }^{*}$ is

$$
\int N p^{\alpha} \frac{d^{2} N_{\text {ch }}}{d \eta d p_{\perp}} d p_{\perp}=N H \int_{p_{\perp 0}}^{\infty}\left(\frac{p_{\perp}}{\sin \theta}\right)^{\alpha} \frac{p_{\perp}}{B^{2}} \mathrm{e}^{-p_{\perp} / B} d p_{\perp}
$$

* For notational clarity Eq. 2-10 specifies an incident flux of charged hadrons. The same thing is true for the incident photon flux. 


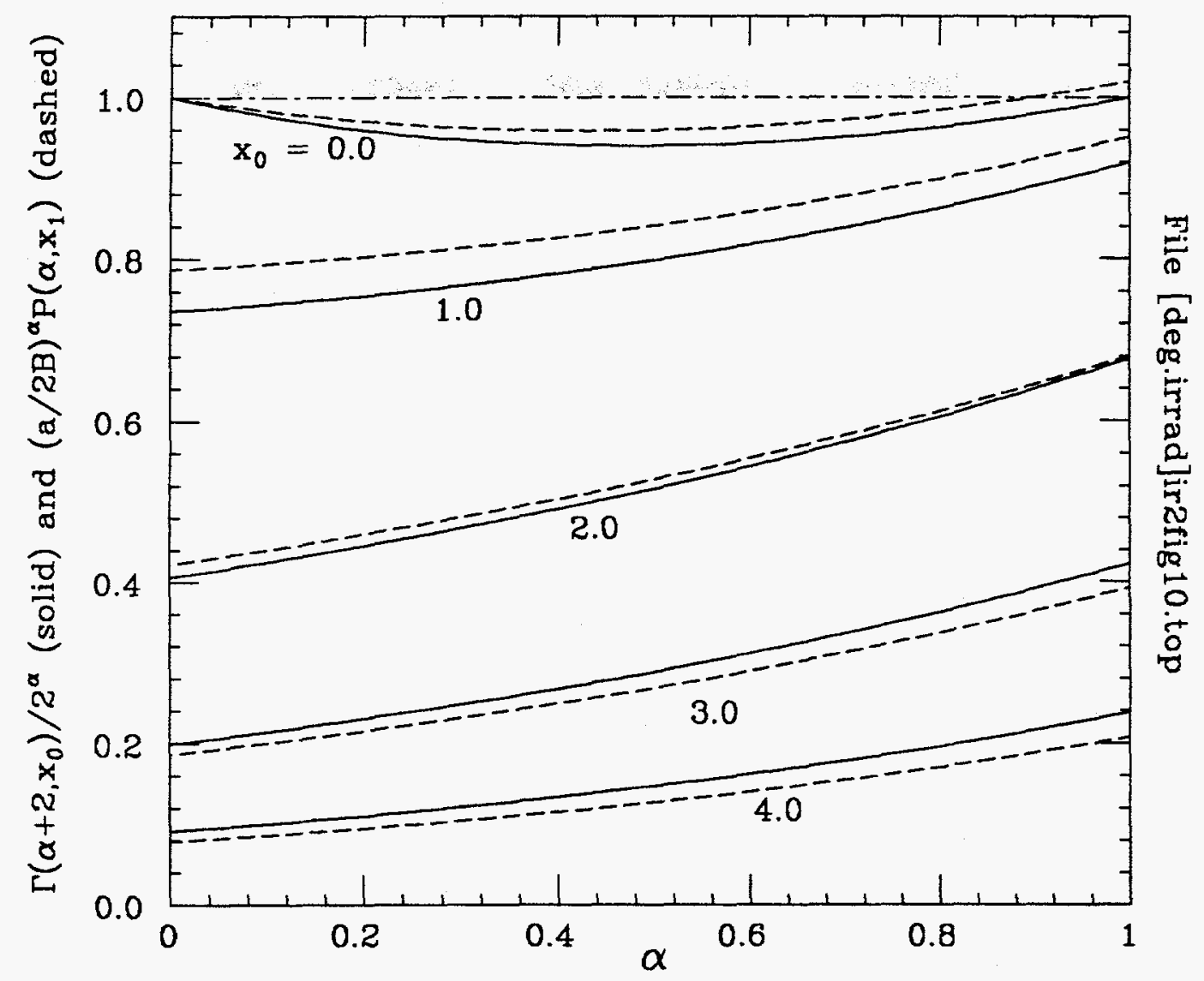

FIG. 2-10. The functions $\Gamma\left(\alpha+2, x_{0}\right) / 2^{\alpha}$ (solid curves) and $(a / 2 B)^{\alpha} F\left(\alpha, x_{1}\right)$ (defined by Eq. 2-11; dashed curves) for useful values of the lower cutoffs $x_{0}$ and $x_{1}=(a / B) x_{0}$. If the $p_{\perp}$ distribution is a $\delta$-function centered at $2 B$ and this momentum is above the momentum cutoff imposed by the solenoid, the result is unity (dot-dashed).

$$
\begin{aligned}
& =N H\left(\frac{2 B}{\sin \theta}\right)^{\alpha} \frac{1}{2^{\alpha}} \int_{x_{0}}^{\infty} x^{\alpha+1} \mathrm{e}^{-x} d x \\
& =N H\left(\frac{2 B}{\sin \theta}\right)^{\alpha}\left[\Gamma\left(\alpha+2, x_{0}\right) / 2^{\alpha}\right]
\end{aligned}
$$

where $x=p_{\perp} / B$ and $\Gamma\left(\alpha+2, x_{0}\right)$ is an incomplete gamma function, e.g. as defined by Eq. 6.5.3 in Abramowitz and Stegun [23]. The function $\Gamma\left(\alpha+2, x_{0}\right) / 2^{\alpha}$ is shown in Fig. 2-10 and given in Table 2-1 for the useful range of $x_{0}$.

* The new IMSL Version 10.0 function $\operatorname{GAMDF}(\mathrm{X}, \mathrm{A})$ may be used to call the probability function $P(a, x)$ as defined by Abramowitz and Stegun 6.5.1 [23], so that the incomplete gamma function $\Gamma(a, x)$ (Abramowitz and Stegun 6.5.5) may be calculated as GAMMA $(A) *(1.0-$ $\operatorname{GAMDF}(X, A)$ ). However, note that although $\Gamma(a, 0)=\Gamma(a)$ the IMSL routine bombs for $x=0$. 
Similarly, with the input distribution function given by Eq. 2-7, we obtain

$$
\begin{aligned}
\int N p^{\alpha} \frac{d^{2} N_{\mathrm{ch}}}{d \eta d p_{\perp}} d p_{\perp} & =N H \int_{p_{\perp 0}}^{\infty}\left(\frac{6 a^{6} p_{\perp}}{\sin \theta}\right)^{\alpha} \frac{p_{\perp} d p_{\perp}}{\left(p_{\perp}^{2}+a^{2}\right)^{4}} \\
& =N H\left(\frac{a}{\sin \theta}\right)^{\alpha} \int_{x_{1}}^{\infty} \frac{6 x^{\alpha+1} d x}{\left(x^{2}+1\right)^{4}} \\
& \equiv N H\left(\frac{a}{\sin \theta}\right)^{\alpha} F\left(\alpha, x_{1}\right) \\
& =N H\left(\frac{2 B}{\sin \theta}\right)^{\alpha}\left[(a / 2 B)^{\alpha} F\left(\alpha, x_{1}\right)\right]
\end{aligned}
$$

where $x=p_{\perp} / a$. The quantity in the square brackets is shown by the dashed curves in Fig. 2-10. It is in reasonable agreement with $\Gamma\left(\alpha+2, x_{0}\right) / 2^{\alpha}$. The main difference is at $x_{0}=1.0$ or $p_{\perp 0} \approx 0.2 \mathrm{GeV} / \mathrm{c}$, where Fig. 2.7 indicates the maximal difference should occur. In any case, the agreement is quite adequate for our present purposes.

What happens if the $p_{\perp}$ distribution used in Eq. 2-10 is simply replaced by a $\delta$ function evaluated at $p_{\perp}=\left\langle p_{\perp}\right\rangle=2 B$, as given in Eq. 2-9? (That is, all particles at angle $\theta$ are assumed to have momentum $\left.\left\langle p_{\perp}\right\rangle / \sin \theta\right)$. Then $\Gamma\left(\alpha+2, x_{0}\right) / 2^{\alpha}$ is simply replaced by unity if the particles can cross the solenoidal field, and otherwise by zero. At worst, $\Gamma(\alpha+2,0) / 2^{\alpha}$ is overestimated by $6 \%$ in the no-field case.

A final transformation to rate per unit solid angle may be useful. Since

$$
\frac{d}{d \Omega}=\frac{1}{2 \pi \sin ^{2} \theta} \frac{d}{d \eta}
$$

we may rewrite Eq. 2-10 as

$$
R \int N p^{\alpha} \frac{d^{2} N_{\mathrm{ch}}}{d \Omega d p_{\perp}}=R N H \frac{(2 B)^{\alpha}}{2 \pi \sin ^{(2+\alpha)} \theta}\left[\Gamma\left(\alpha, x_{0}\right) / 2^{\alpha}\right],
$$

where $R$ is the event rate. For example, if 1 hadron $\mathrm{cm}^{-2}$ is incident on a uranium/scintillator calorimeter, the neutron flux at shower maximum is about 18 $\mathrm{cm}^{-2} p^{0.67}$. With $R=10^{8} \mathrm{~s}^{-1}, H=7.5,2 B=\left\langle p_{\perp}\right\rangle=0.60 \mathrm{GeV} / \mathrm{c}$, no solenoidal field, and $\theta=2^{\circ}(\eta=4.0)$, we find that the flux at $1 \mathrm{~m}$ is $9.9 \times 10^{8} \mathrm{~cm}^{-2} \mathrm{~s}^{-1}$.

At this stage we have finished most of the problem. However, if we wish to know the rate at which albedo neutrons are injected into the central cavity of a 


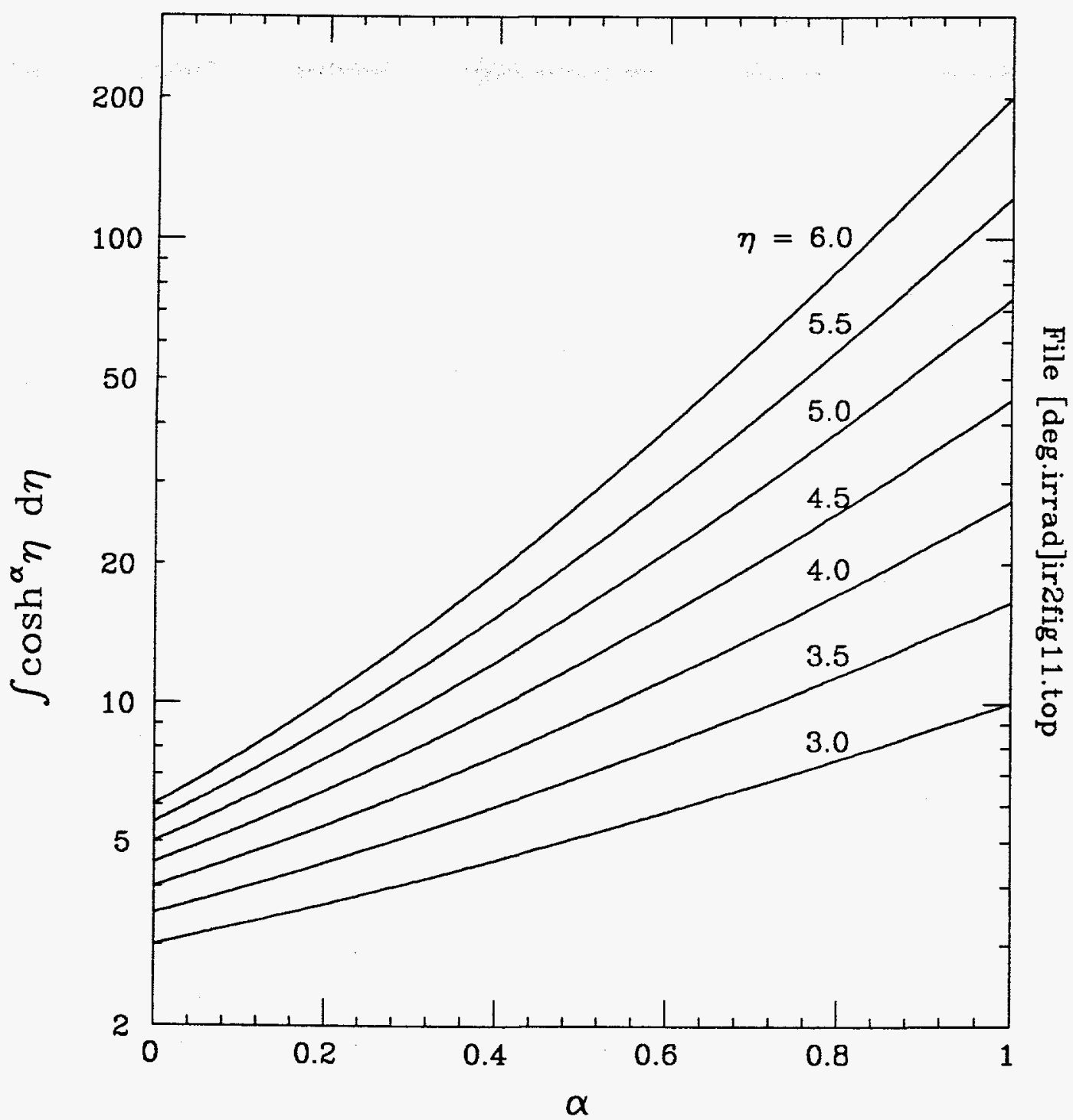

FIG. 2-11. The integral of $\cosh ^{\alpha} \eta$ between 0 and $\eta_{0}$ for values of the upper limit relevant to $4 \pi$ or forward detector design.

detector, the integral over $\eta$ is also necessary:

$$
\begin{aligned}
\int N p^{\alpha} \frac{d^{2} N_{\mathrm{ch}}}{d \eta d p_{\perp}} d p_{\perp} d \eta & =N H(2 B)^{\alpha} \Gamma\left(\alpha, x_{0}\right) / 2^{\alpha} 2 \int_{0}^{\eta_{0}} \cosh ^{\alpha} \eta d \eta \\
& \equiv N H(2 B)^{\alpha} \Gamma\left(\alpha, x_{0}\right) / 2^{\alpha} 2 I\left(\alpha, \eta_{0}\right)
\end{aligned}
$$

where 
Table 2-2

Numerical values of $\int_{0}^{\eta_{0}} \cosh ^{\alpha} \eta d \eta$.

\begin{tabular}{rrrrrrrrr}
\hline \hline \multirow{2}{*}{$\alpha$} & \multicolumn{7}{c}{$I\left(\alpha, \eta_{0}\right)$ for $\eta_{0}=$} \\
\hline 0.00 & 3.00 & 3.5 & \multicolumn{1}{c}{4.0} & \multicolumn{1}{c}{4.5} & \multicolumn{1}{c}{5.0} & \multicolumn{1}{c}{5.5} & \multicolumn{1}{c}{6.0} \\
0.05 & 3.15 & 3.72 & 4.00 & 4.50 & 5.00 & 5.50 & 6.00 \\
0.10 & 3.31 & 3.95 & 4.63 & 5.34 & 6.09 & 6.88 & 7.71 \\
0.15 & 3.48 & 4.21 & 5.00 & 5.85 & 6.77 & 7.76 & 8.83 \\
0.20 & 3.66 & 4.50 & 5.42 & 6.44 & 7.56 & 8.81 & 10.18 \\
0.25 & 3.86 & 4.81 & 5.88 & 7.10 & 8.48 & 10.04 & 11.82 \\
0.30 & 4.08 & 5.16 & 6.41 & 7.86 & 9.55 & 11.52 & 13.80 \\
0.35 & 4.31 & 5.54 & 7.00 & 8.74 & 10.81 & 13.27 & 16.21 \\
0.40 & 4.56 & 5.96 & 7.66 & 9.74 & 12.28 & 15.37 & 19.16 \\
0.45 & 4.84 & 6.42 & 8.41 & 10.89 & 14.00 & 17.89 & 22.77 \\
0.50 & 5.14 & 6.94 & 9.25 & 12.22 & 16.03 & 20.92 & 27.21 \\
0.55 & 5.46 & 7.51 & 10.20 & 13.75 & 18.42 & 24.57 & 32.67 \\
0.60 & 5.81 & 8.14 & 11.28 & 15.53 & 21.25 & 28.98 & 39.41 \\
0.65 & 6.19 & 8.84 & 12.51 & 17.58 & 24.59 & 34.31 & 47.74 \\
0.67 & 6.36 & 9.15 & 13.04 & 18.49 & 26.10 & 36.74 & 51.61 \\
0.70 & 6.61 & 9.62 & 13.90 & 19.96 & 28.56 & 40.76 & 58.08 \\
0.75 & 7.06 & 10.49 & 15.47 & 22.72 & 33.26 & 48.60 & 70.92 \\
0.80 & 7.56 & 11.45 & 17.26 & 25.93 & 38.85 & 58.13 & 86.89 \\
0.85 & 8.09 & 12.53 & 19.30 & 29.66 & 45.50 & 69.74 & 106.80 \\
0.90 & 8.68 & 13.72 & 21.62 & 34.01 & 53.43 & 83.90 & 131.67 \\
0.95 & 9.32 & 15.06 & 24.27 & 39.08 & 62.90 & 101.19 & 162.76 \\
1.00 & 10.02 & 16.54 & 27.29 & 45.00 & 74.20 & 122.34 & 201.71 \\
\hline \hline
\end{tabular}

$$
I\left(\alpha, \eta_{0}\right)=\int_{0}^{\eta_{0}} \cosh ^{\alpha} \eta d \eta
$$

and where we have used the identity $\sin \theta \cosh \eta=1$. This integral shown in Fig. 2-11 and given in Table 2-2.

Again, an example may be useful. For albedo neutrons, $\alpha \approx 0.5$ and $N \approx 5$. Then for a rate of $10^{8} \mathrm{~s}^{-1}$, a pseudorapidity cutoff at 4.0 (a detector extending to within $7 \mathrm{~cm}$ of the beam at $2 \mathrm{~m}$ ),$H=7.5$, and no field, we find that $5.1 \times 10^{10}$ neutrons $\mathrm{s}^{-1}$ are injected into the interior of the detector. 


\section{ELECTROMAGNETIC CASCADES}

\subsection{Introduction}

In this section we describe briefly the radiation levels associated with electromagnetic cascades. The properties of prime interest are

- maximum dose from electromagnetic showers in calorimetry

- material and energy dependence of the dose

- photoneutron production

- albedo photons.

Using the Monte Carlo program EGS4, Hirayama and Nelson in Appendix 7 have calculated the dose from electromagnetic showers. A somewhat similar calculation using FLUKA has been done by Stevenson and is given in Appendix 20. We will compare these estimates in Section 5. Photoneutron production and the number of albedo photons produced are also described by Hirayama and Nelson. We summarize their results below.

Since most existing simulation codes were developed before SSC energies were available, one must question their reliability at these energies. Are the QED formulae still accurate? Are the algorithms used to compute them in codes such as EGS valid at the highest energies? Do new radiative effects, such as the supression of bremsstrahlung by coherent effect at highly relativistic energies (the LPM effect), play an important role? Fortunately, the answers to all of these questions appear to be negative. The QED questions are considered by Fassò in Appendix 6, and the LPM effect is discussed by Stanev in Appendix 8.

\subsection{Dose from electromagnetic showers}

Imagine an electron (or photon) normally incident on a sampling calorimeter. The electromagnetic shower will have some typical longitudinal shape (see Fig. 4 in Appendix 7) and transverse shape (see Fig. 9 in this Appendix). The maximum dose absorbed by the sampling medium will occur in the vicinity of the shower maximum. It will depend somewhat on the sampling medium, the type of absorber, and the sampling fraction. Some of this dependence has been explored in Appendix 7 (Fig. 3). The result is that for typical media and with similar sampling fractions there is little dependence on the sampling medium. Using Fig. 3 from Appendix 7 as a guide, we plot the maximum dose in Gy per incident photon $\mathrm{cm}^{-2}$ in Fig. 2.1. ${ }^{\star}$ The maximum dose in these units is well described by

* The simulation actually generates the lateral integral of energ. deposition at a given depth per incident particle, in e.g. $\mathrm{GeV} \mathrm{cm} \mathrm{cm}^{-1}$. This is easily shown to be equivalent to the energy deposition at this depth for an incident flux of one particle $\mathrm{cm}^{-2}$. 


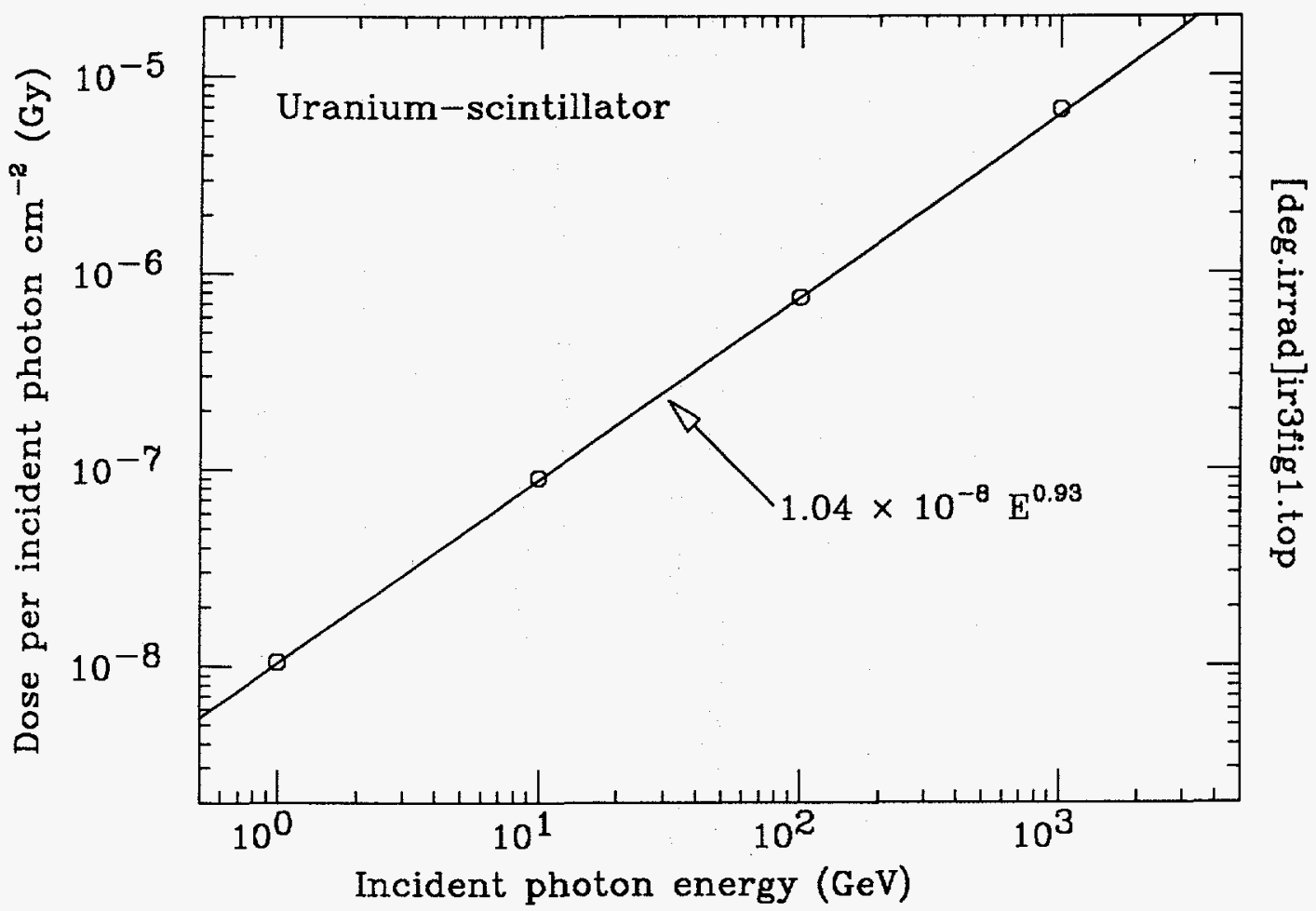

FIG. 3.1. The maximum electromagnetic dose in Gy per incident photon $\mathrm{cm}^{-2}$ in the sampling medium of a uranium/scintillator calorimeter vs. incident photon energy.

the function $\left(1.04 \times 10^{-8}\right) E^{0.93}$, where the photon energy $E$ is measured in $\mathrm{GeV}$. We will use this formula in Section 5 to estimate the maximum electromagnetic dose absorbed in a typical calorimeter.

\subsection{Photoneutron production}

Photonuclear reactions within the calorimeter absorber may produce low energy $(<20 \mathrm{MeV})$ neutrons. In Appendix 7 Hirayama and Nelson have made estimates of the number of photoneutrons produced vs. incident photon energy. They have also explored the differences between uranium and lead absorber. As expected, more photoneutrons are produced in uranium. The number of photoneutrons produced is less than $10 \%$ of the number produced in a hadronic cascade for particles of equal incident energy. Hence in our estimates of neutron fluxes and fluences we have neglected the contribution from photoneutrons.

\subsection{Albedo photons}

Again imagine a photon or electron normally incident upon a sampling calorimeter. A small fraction of the shower energy will "leak out" the front face of the calorimeter. As shown in Fig. 13 of Appendix 7, the spectrum of the albedo 


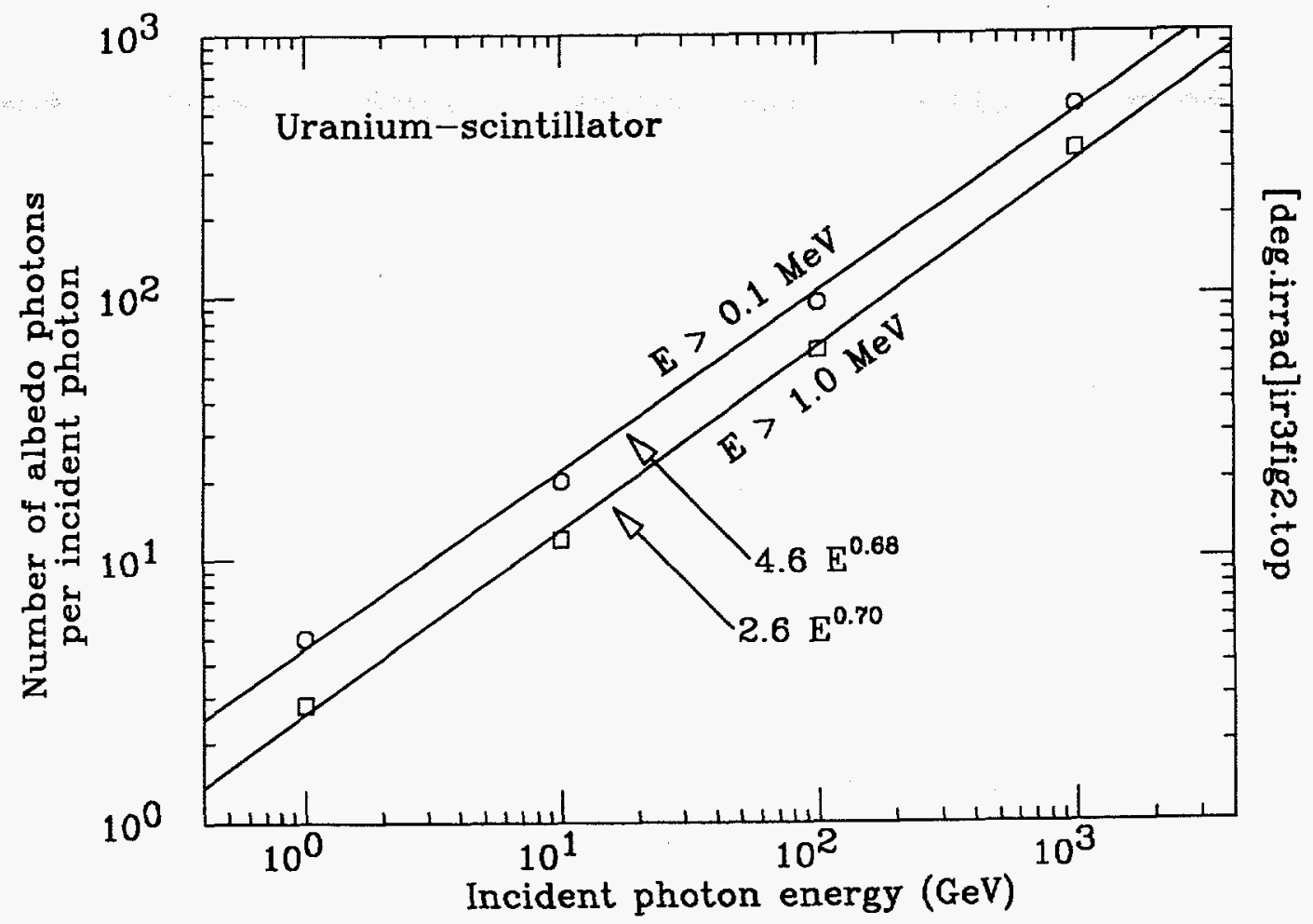

FIG. 3.2. The number of albedo photons for two different lower limits on the albedo photon energy vs. incident photon energy. The fits are given in the text.

photons decreases rapidly with energy, with a characteristic energy of $\sim 1 \mathrm{MeV}$. Again the number of albedo photons is roughly independent of the absorbing material, although uranium yields somewhat more high energy photons than lead (see Fig. 11 in Appendix 7). The number of albedo photons for albedo photon energies greater than $0.1(1.0) \mathrm{MeV}$ vs. incident particle energy in $\mathrm{GeV}$ is shown in Fig. 3.1, and is well fit by the expression $4.6 E^{0.68}\left(2.6 E^{0.70}\right)$. We will use these fits in Section 5 to describe the photon albedo within a typical calorimeter configuration. 



\section{NEUTRONS}

\subsection{Introduction}

As a hadronic cascade develops in matter, a large number of secondary particles with progressively lower energies are produced. At high energies $(Z 1 \mathrm{GeV})$ the $\pi^{+}, \pi^{-}, \mathrm{p}$, and $\mathrm{n}$ spectra are similar, but at lower energies the charged particles tend to "range out" and cease to propagate the cascade. The net result is a large number of low-energy neutrons.

Hadronic spectra typical of cascades in dense matter are shown in Fig. 4-1. In this example, the cascades are generated in a soil backwall with incident 500 $\mathrm{GeV}$ protons [24]. The authors argue that the spectra are very nearly independent of position, except on the cascade axis and near the beginning of the cascade. Moreover, the shapes are almost independent of incident hadronic species or energy. All of the spectra are essentially identical above $1 \mathrm{GeV}$. At $100 \mathrm{MeV}$

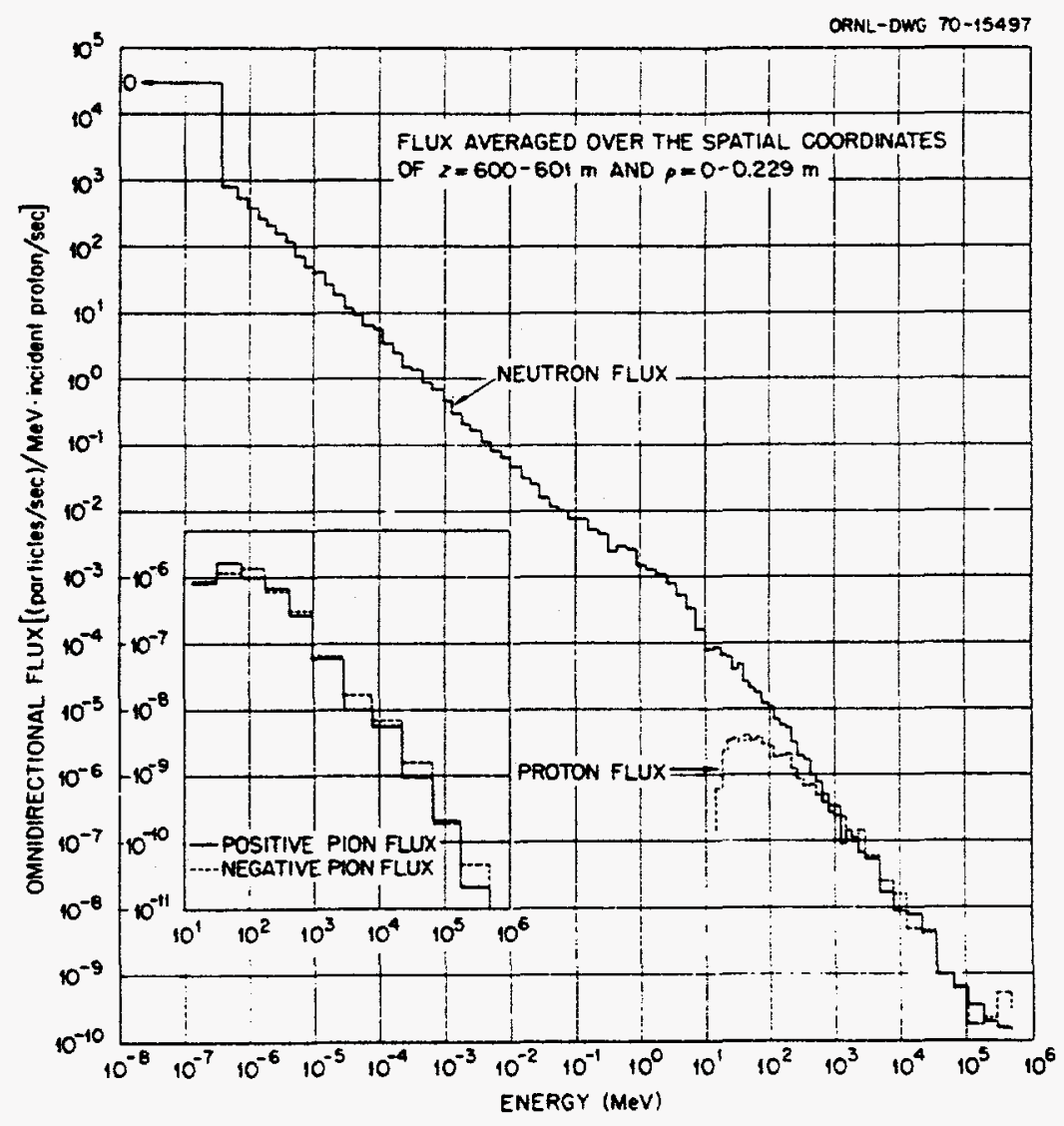

FIG. 4-1. Kinetic energy spectrum of hadrons as calculated by T. A. Gabriel and R. T. Santoro for the soil backwall of a target-tunnel configuration [24]. This spectrum was assumed to be a representative "universal" spectrum by Van Ginneken and Awschalom in calculating the effects of low energy particles not followed by CASIM [25]. 
there are about five times as many neutrons as protons. There is no significant proton or pion flux below $10 \mathrm{MeV}$.

It has since become usual to plot $d \varphi / d(\log E)$ rather than $d \varphi / d E$ when a logarithmic abscissa is used, so that the area under the curve is equal to the total flux $\varphi$. When this is done, the shoulder near $1 \mathrm{MeV}$ becomes a prominent peak. The details depend upon the nuclear physics in the particular material involved, but this feature occurs in all combinations of materials commonly used in calorimeters. At even lower energies $(\$ 10 \mathrm{eV})$ the neutron spectrum again rises because of thermalization.

Silicon is damaged by neutrons with energies above $\sim 160 \mathrm{keV} .{ }^{\star}$ This "threshold" and the spectral peaking for neutrons near $1 \mathrm{MeV}$ conspire to make this region the only one of importance in considering radiation damage to electronics. In a gas-filled device containing hydrogen (e.g. in $\mathrm{CH}_{4}$ ) elastic n-p scattering can create ionizing recoils which can be important, and even thermal neutrons can wreak some havoc in such detectors, for example, via $\mathrm{n}+\mathrm{H} \rightarrow \mathrm{D}+\gamma$. Further studies of the effects of neutron irradiation on the operation of gas-filled detectors are badly needed; at this time very little can be said. Accordingly, this report focuses on neutrons in the spectral peak near $1 \mathrm{MeV}$. The area under this peak is insensitive to the exact lower cutoff, which we usually take to be around $0.1 \mathrm{MeV}$.

The neutron flux problem divides into several parts. A detector may consist of an essentially empty central cavity containing tracking devices which is surrounded by a calorimeter. When a hadron initiates a cascade in the calorimeter, some "albedo" neutrons re-emerge into the tracking volume, where they can harm silicon microstrip or pixel devices and other detectors. This problem may be exacerbated by a large factor because the neutrons bounce from the walls many times before they are absorbed. In the calorimeter itself, the flux reaches a much larger value at the cascade maximum, where it can damage whatever material is used to sample the energy deposition (scintillator, silicon, ...). Neutron flux beyond the maximum can also be important if electronics are to be placed within or even behind the calorimeter.

According to Section 2, the probability of producing a secondary particle with momentum $p$ at angle $\theta$ is well described by

$$
F(p) \propto p \mathrm{e}^{-p \sin \theta / B},
$$

where $B \approx 0.2 \mathrm{GeV} / \mathrm{c}$. At $\sqrt{s}=40 \mathrm{TeV}$ the expected charged particle multiplicity is 110 , so that the average particle has 20 or $30 \mathrm{GeV}$. At central angles,

\footnotetext{
* A brief review of silicon damage mechanisms is given in Ref. 26.
} 
the above expression indicates far lower energies. Accordingly, it is thus the very large number of very low energy hadrons $(1 \mathrm{GeV}$ to $100 \mathrm{GeV}$ ) which produce most of the neutron flux in a detector. In this Section, we critique the simulation and experimental results on the subject, and attempt to parameterize the data in a useful way. In Section 5, these estimates are folded with the production spectra to yield estimates of the radiation environment in an SSC detector.

\subsection{Simulation and experimental data}

Calculated and measured neutron flux data for a variety of calorimeter designs are given in Appendices 13 through 18. The content of these papers is summarized in Table 4-1. Most investigators report both maximum flux and albedo flux (neutrons which re-emerge from the front face). In the case of the experimental results, these quantities are inferred from the radioactivation of suitably chosen materials placed in the test calorimeter. All of the results have important qualifications, such as energy cutoffs having to do with activation thresholds or computational details and the finite transverse size of the calorimeters.

Usually neutron fluxes and albedos are presented as integrals over a transverse plane, e.g. $2 \pi \int \varphi(r) r d r=600$ neutrons, where $r$ is the distance from the shower axis. To calculate radiation effects, one would like to calculate the total flux (inside a calorimeter or backscattered from it) at a given place. This should be done by calculating the incident particle flux (a function of $\eta$ and $p$ or $E$ ) at a surface element a distance $r$ away (yielding the contribution of particles of a certain energy striking this element to the neutron flux at the point of interest), then integrating over incident particle energy and all contributing surface elements. By using the flux integrated over a plane, we essentially replace $\varphi(r)$ by its integral times a delta function, leaving only the integral over energy of the incident flux times this quantity. The procedure is valid if the width of the cascade is small compared with distances over which the incident flux changes appreciably.

That this is often the case is shown in Fig. 4-2. In this FLUKA87 simulation a high-energy proton enters an iron cylinder along its axis. The height indicates the density of nuclear interactions in the iron, while the radial and longitudinal $(z)$ coordinates are plotted in the horizontal plane. The radial bins are only 0.5 $\mathrm{cm}$ wide, yet the interaction density falls by more than an order of magnitude in only a few bins. The neutron flux distribution is somewhat broader, but not by much. Very roughly, $\varphi(r)$ is of the form $\exp (-r / \lambda) / r$, and for iron $\lambda \approx 10 \mathrm{~cm}$.

In this report we use the integrals of the neutron flux with the caveat that the procedure is not valid in places where the incident flux changes appreciably over a few centimeters. Problems which can result from tnis approach are discussed in Appendix 21. 
Table 4-1

Summary of neutron flux calculations (Appendices 13-16) and measurements (17 and 18$)$ by members of the Task Force.

\begin{tabular}{|c|c|c|c|c|c|}
\hline App. & Authors & Calorimeter & Hadron & Energy $(\mathrm{GeV})$ & Reported \\
\hline 13 & Alsmiller et al. & $\mathrm{U} / \mathrm{scint}$ & $\mathrm{p}$ & $1,5,10,20,50,100$ & Albedo, flux \\
\hline 14 & Ban et al. & $\begin{array}{l}\mathrm{U} / \text { scint } \\
\mathrm{Pb} / \text { scint } \\
\mathrm{U} / \text { argon } \\
\mathrm{Pb} / \text { argon }\end{array}$ & $\begin{array}{c}\pi^{+}, \mathrm{p} \\
\pi^{+}, \mathrm{p} \\
\pi^{+} \\
\pi^{+}\end{array}$ & $\begin{array}{l}1,3,10,100^{\star} \\
1,3,10,100^{\star} \\
1,3,10,100^{\star} \\
1,3,10,100^{\star}\end{array}$ & $\begin{array}{l}\text { Albedo } \\
\text { Albedo } \\
\text { Albedo } \\
\text { Albedo }\end{array}$ \\
\hline 15 & Brau, Gabriel & $\begin{array}{l}\mathrm{Fe} / \mathrm{scint} \\
\mathrm{Fe} / \mathrm{Si} \\
\mathrm{U} / \mathrm{scint} \\
\mathrm{U} / \mathrm{Si}\end{array}$ & $\begin{array}{l}\pi^{-} \\
\pi^{-} \\
\pi^{-} \\
\pi^{-}\end{array}$ & $\begin{array}{l}0.4,3,20 \\
0.4,3,20 \\
0.4,3,20 \\
0.4,3,20\end{array}$ & $\begin{array}{l}\text { Albedo, flux } \\
\text { Albedo, flux } \\
\text { Albedo, flux } \\
\text { Albedo, flux }\end{array}$ \\
\hline 16 & Fesefeldt & $\begin{array}{l}\text { U/TMS } \\
\text { U/scint } \pi \\
\text { U/scint } \\
\mathrm{Pb} / \text { scint } \\
\mathrm{Fe} / \text { scint } \\
\mathrm{U} / \mathrm{PWC} \\
\mathrm{Pb} / \mathrm{PWC} \\
\mathrm{Fe} / \mathrm{PWC}\end{array}$ & $\begin{array}{c}\pi^{-} \\
\pi^{-}, \overline{K^{-}}, \overline{\mathrm{p}}, \mathrm{p} \\
\pi^{-} \\
\pi^{-} \\
\pi^{-} \\
\pi^{-} \\
\pi^{-} \\
\pi^{-}\end{array}$ & $\begin{array}{l}0.1 \text { to } 200 \\
0.1 \text { to } 200 \\
0.1 \text { to } 200 \\
0.1 \text { to } 200 \\
0.1 \text { to } 200 \\
0.1 \text { to } 200 \\
0.1 \text { to } 200 \\
0.1 \text { to } 200\end{array}$ & $\begin{array}{l}\text { Flux } \\
\text { Albedo } \\
\text { Albedo, flux } \\
\text { Albedo, flux } \\
\text { Albedo, flux } \\
\text { Albedo, flux } \\
\text { Albedo, flux } \\
\text { Albedo, flux }\end{array}$ \\
\hline 17 & Russ, Stevenson & $\mathrm{n} \mathrm{Fe}$ & $\frac{2}{3} p+\frac{1}{3} \pi^{+}$ & 200 & Albedo, flux \\
\hline 18 & Wigmans & $\begin{array}{l}\mathrm{U} \\
\mathrm{U} / \sin t \\
\mathrm{U} \\
\mathrm{U} / \sin t\end{array}$ & $\begin{array}{c}\mathrm{p} \\
\mathrm{p} \\
\pi^{-} \\
\pi^{-}\end{array}$ & $\begin{array}{l}0.591 \\
0.591 \\
300 \\
300\end{array}$ & $\begin{array}{l}\text { Albedo, flux } \\
\text { Albedo, flux } \\
\text { Albedo, flux } \\
\text { Albedo, flux }\end{array}$ \\
\hline
\end{tabular}

* For pions, these are momenta in $\mathrm{GeV} / \mathrm{c}$.

$\dagger$ For several plate/TMS thicknesses, and with and without a $1 \lambda$ xenon front absorber.

$\ddagger$ For $3 \mathrm{~mm} \mathrm{U}$ plates with both $2.5 \mathrm{~mm}$ and $7.5 \mathrm{~mm}$ scintillator sheets.

The importance of energy thresholds for the tallied flux may be estimated from the spectra given in several of the Appendices. For example, in Fig. 43 (a) we show albedo neutron spectra for the $1 \mathrm{GeV}$ and $20 \mathrm{GeV}$ points from Appendix 13, and for the $20 \mathrm{GeV}$ point from Appendix 15. Both are for a uranium $(3 \mathrm{~mm})$ and scintillator $(3 \mathrm{~mm})$ calorimeter. The normalized integrals of the two Alsmiller et al. spectra are shown in Fig. 4-3(b). Although there are appreciable differences between the spectra (we believe), the normalized integrals 


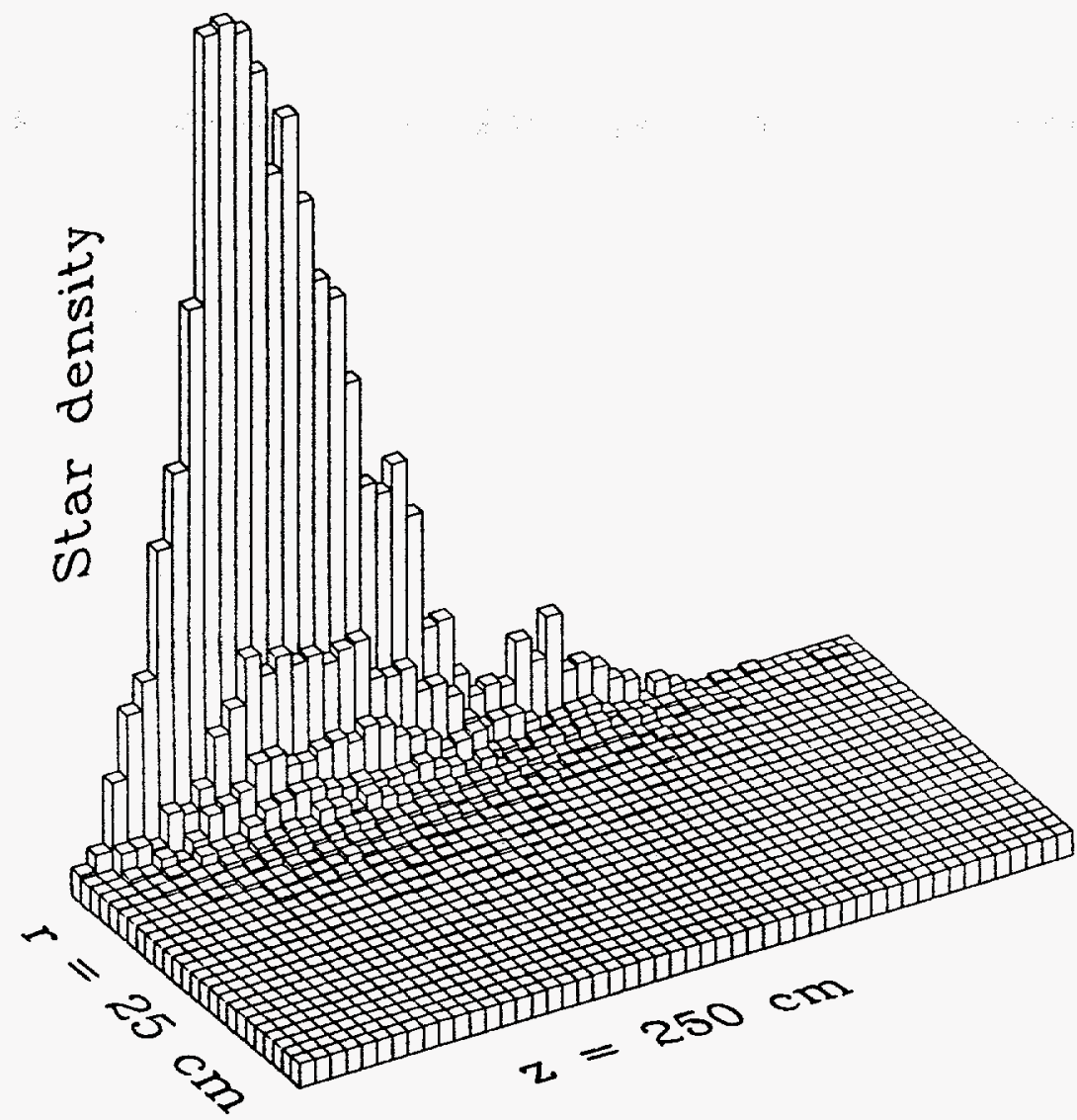

FIG. 4-2. FLUKA87 calculation of the average star (nuclear interaction) density in cascades generated by $20 \mathrm{TeV}$ protons incident along the axis of an iron cylinder. Height indicates star density, while the horizontal plane represents radial and longitudinal variables. All scales are linear.

agree everywhere within $10 \%$ or $20 \%$, and we may correct for modest cutoff effects on the basis of these curves. For example, Wigmans (Appendix 18) reports a 1.5 $\mathrm{MeV}$ fission threshold for his measurements, so his results must be corrected by about (1/0.46) to compare with calculations using a $100 \mathrm{keV}$ threshold. (It is implicit that we are interested in the silicon-damage region from $\sim 160 \mathrm{keV}$ up.)

Corrections can be made for the finite transverse size of calorimeters by (a) extrapolating data using fitted functions, as Russ and Stevenson do, or (b) calculating fluxes integrated over the transverse plane for finite and infinite planes, as Brau and Gabriel do. We use both methods.

Finally, there is the problem of comparing results calculated or measured using different calorimeter configurations. In particular, experimental results are in such short supply that they must all be evaluated. Here guidance comes from the simulations: If the same program yields twice the flux in a massive uranium calorimeter as in a uranium/scintillator calorimeter, then we are justified 


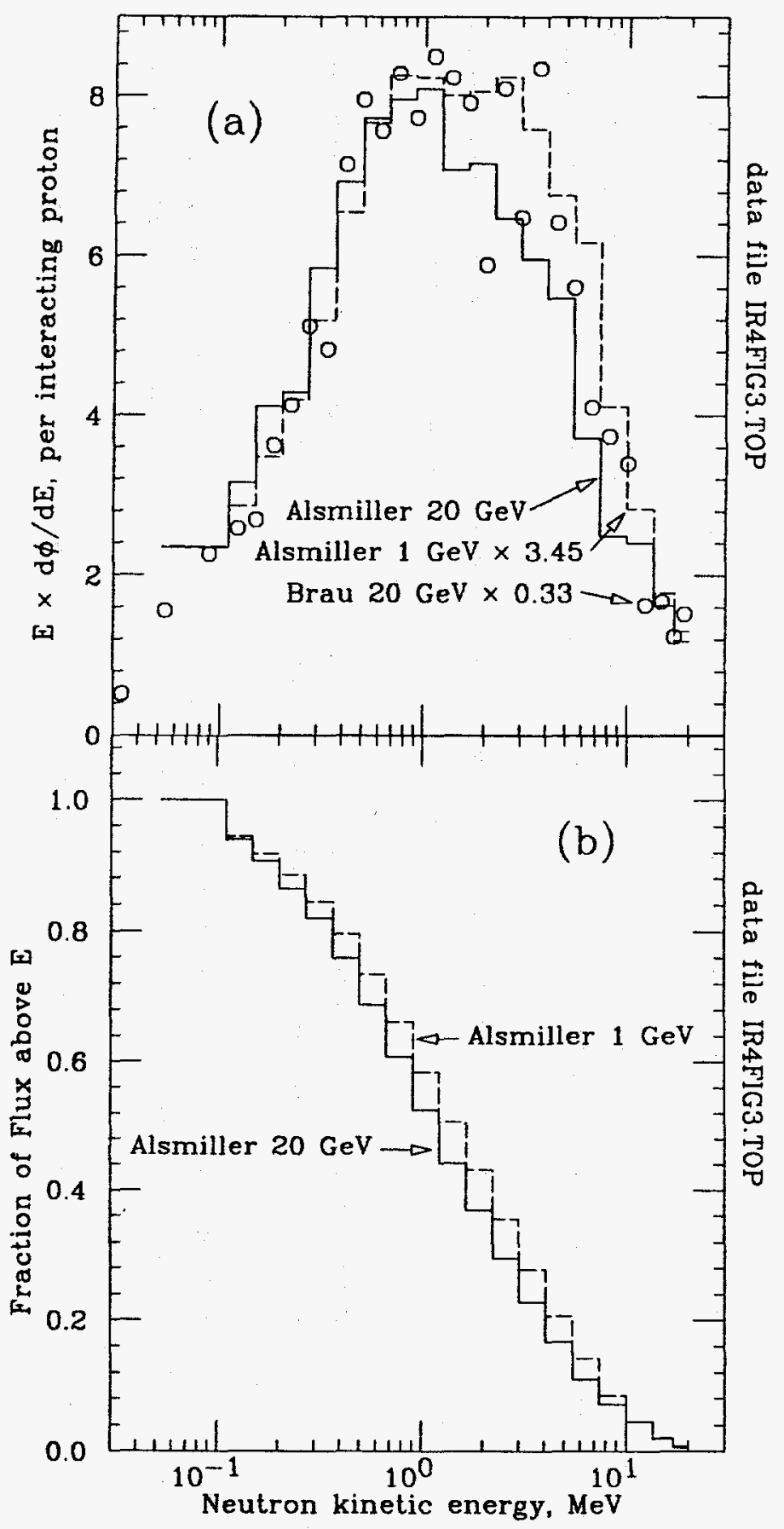

FIG. 4-3. The spectra of albedo neutrons from a uranium/scintillator calorimeter. (a) shows spectra at $1 \mathrm{GeV}$ and $20 \mathrm{GeV}$ as calculated by Alsmiller et al. (Appendix 13 and private communication) (dashed and solid histograms) and at $20 \mathrm{GeV}$ as calculated by Brau and Gabriel (Fig. 9 of Appendix 15) (circles). Normalizations are arbitrarily chosen so that the peaks are commensurate. Normalized integrals of the Alsmiller et al. spectra are shown in (b). 
in correcting by this factor elsewhere. There is little sensitivity to incident hadron species, at least above kinetic energies of a few $\mathrm{GeV}$, so this difference may be ignored. We restrict our attention to calorimeter configurations studied by many groups, which for purposes of this Report means U/scint with some comments about how the results scale to other configurations.

\subsection{Albedo and maximum flux}

In this section we critique the reported data and present summaries of corrected albedo and shower maximum results. The goal is to parameterize the kinetic dependence of these quantities for use in further calculations. Because most groups treated the case of a fine-sampling uranium/scintillator calorimeter ( $3 \mathrm{~mm}$ plates of each), we use this design as a reference.

In the case of albedo (or backscattered) neutrons, we are interested in the number of neutrons injected into the central cavity of a detector. From this the flux inside the cavity can be calculated. For a single particle incident on a calorimeter this number is the integral of the current (not flux) over the transverse dimensions, or $\int I d a$. At the cascade maximum the flux is relevant. As has been stated above, the integral of the flux or current over the transverse dimensions for one incident hadron is equal to the flux or current which would obtain at all transverse positions for one incident hadron per unit area.

1. Alsmiller et al.

The albedo data are summarized in Fig. 2 of Appendix 13. The HETC82 points cannot be connected by a smooth line, and there has been extensive discussion of whether the $1 \mathrm{GeV}$ point is high or the $5 \mathrm{GeV}$ point low. The difficulty arises because HETC handles intranuclear cascades differently depending upon whether the incident hadron has energy above or below $3 \mathrm{GeV}$. Calculations using the old version of HETC82, which uses a different algorithm, resulted in the $\times$ 's being added to the figure but do little to resolve the problem. The authors conclude that the $1 \mathrm{GeV}$ point and high-energy points are correct, and that truth at $5 \mathrm{GeV}$ lies halfway between the two points. We indicate the HETC 82 points by A's in our summary Fig. 4-4, and the HETC82(OLD) points by A"s. At $1 \mathrm{GeV}$ they are coincident. The reported fluxes are scaled downward by a factor of two to convert to currents.

The albedo neutron spectra obtained with HETC82 at 5, 10,20,50, and $100 \mathrm{GeV}$ are indistinguishable; the $20 \mathrm{GeV}$ case is shown in Fig. 4-3. However, the $1 \mathrm{GeV}$ spectrum, also shown in the figure, seems to show a shoulder on the high-energy side. The extra area under this shoulder contributes enough to raise the $1 \mathrm{GeV}$ point above a smooth curve defined by the other 


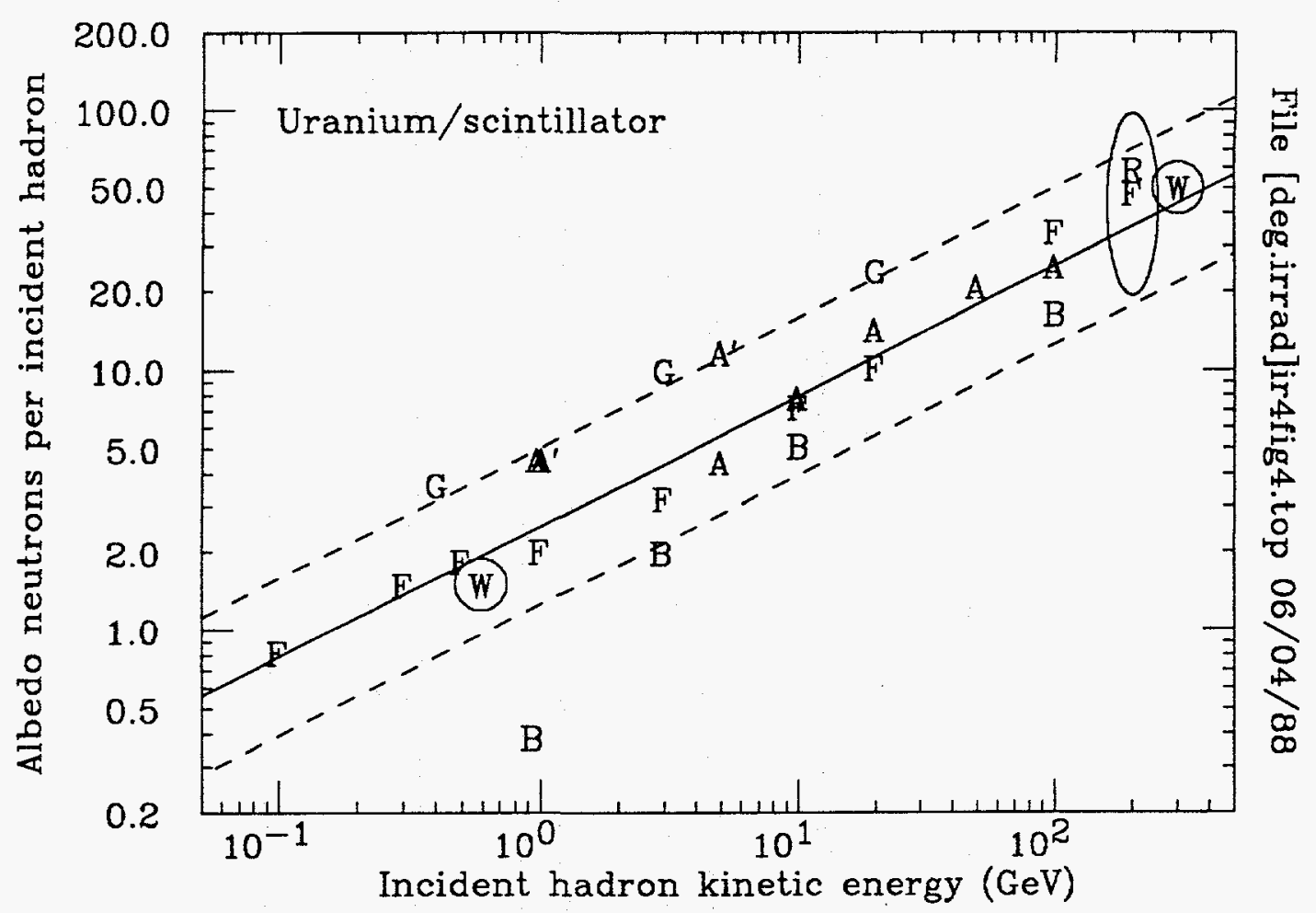

FIG. 4-4. Summary of selected albedo data for a fine-sampling uranium/scintillator calorimeter. The solid curve is $\int I d a=2.5\left(E_{k} /(1 \mathrm{GeV})\right)^{0.50}$, while the dashed curves are a factor of two higher and lower. For pions, which are easily the most abundant hadron, $E^{0.50}$ may be replaced with $p^{0.50}$ without loss of accuracy.

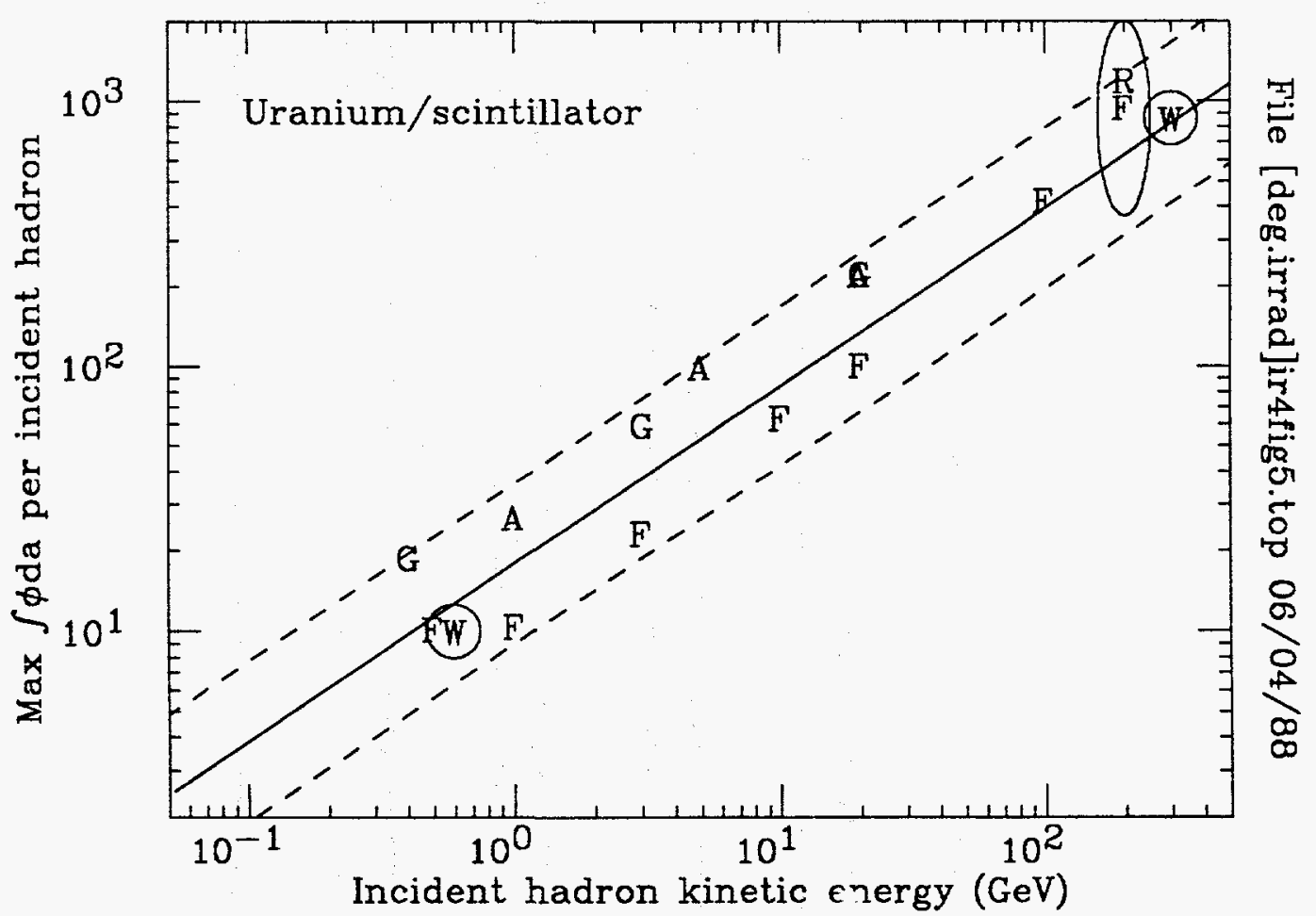

FIG. 4-5. Summary of selected maximum neutron flux data for a fine-sampling uranium/scintillator calorimeter. The solid curve is $\int \varphi d a=18\left(E_{k} /(1 \mathrm{GeV})\right)^{0.67}$, while the dashed curves are a factor of two higher and lower. As above, $E_{k}$ may be replaced by $p$ for practical calculations. 
points. The authors disagree with this conclusion, and note (their Fig. 3) that the new and old codes yield similar spectra at $5 \mathrm{GeV}$. However, the spectra obtained by Brau and Gabriel using essentially HETC82(OLD) (Appendix 15, Fig. 9) show similar "squared off" spectra.

Maximum flux data may be read from Fig. 4 of Appendix 13: $\int \varphi d a=27$, 100 , and 225 per incident proton at kinetic energies 1,5 , and $20 \mathrm{GeV}$, respectively. These results are indicated by A's in Fig. 4-5.

2. Ban, Kondo, and Asai

This group made calculations in two ways. In both cases the high-energy cascade was propagated using GHEISHA (Version 7.03). In the first case, it was used to transport neutrons down to $0.1 \mathrm{MeV}$, while in the second the neutron transport code ANISN was used for neutron energies below $15 \mathrm{MeV}$.

Albedo calculations were made at $1,3,10$, and $100 \mathrm{GeV}$ in both ways for incident protons and $\pi^{-}$'s. In general, results with GHEISHA + ANISN were a factor of two below those with GHEISHA alone. With a given code, proton and $\pi^{-}$results agree. To avoid the confusion of adding 16 points to Fig. 4-4, we (a) average $\mathrm{p}$ and $\pi^{-}$results, and (b) plot only GHEISHA + ANISN results, in accord with the author's opinion that these results are more dependable. Since they report the number of albedo neutrons, their results are plotted without further correction. These points are indicated by B's in Fig. 4-4.

Their computational method yielded the total number of neutrons in the detector, but not a peak flux.

\section{Brau and Gabriel}

Maximum flux and albedo results are summarized in Figs. 11 and 12 of Appendix 15. Those for uranium/scintillator are indicated by G's in Figs. 4-4 and 4-5. As mentioned above, the code was essentially the same as HETC82 (OLD) and so the agreement with the points marked as A"s is not surprising. A smooth curve drawn through the HETC 82 high-energy results (the four higher A's) is systematically below the Brau-Gabriel results, and presumably reflects improved code. However, since none of the energies are really high compared to the $3 \mathrm{GeV}$ transition where trouble occurs, no strong conclusion can be made.

The flux results for iron/silicon are about $10 \%$ lower than for $\mathrm{U} /$ scintillator, and the albedo results are lower by a factor of $(1 / 1.5)$. The iron/silicon case is closest to the iron test calorimeter used to obtain the results reported in Appendix 17, and we accordingly use these factors to correct the reported results to the $\mathrm{U} /$ scintillator reference case. 


\section{Fesefeldt}

Our main difficulty with these extensive results has to do with the old current/flux problem. On pp. 6 and 7 of Appendix 16, he equates interaction rate with "the number of neutrons $I_{N}(z)$ passing through this distance," which we interpret as a current. According to our discussion in Appendix 9, the interaction rate is $\varphi / \lambda$, where $\varphi$ is the flux and $\lambda$ the capture length. On p. 12 flux is calculated by multiplying $I_{N}$ by two, which we feel is unnecessary. Accordingly, for purposes of plotting in Figs. 4-4 and 4-5 his results are lowered by a factor of two from those given in his Tables 2 and $3 .{ }^{\star}$ The points are marked with F's in the figures.

Our earlier statement about neutron flux being independent of incident hadron species is based to a large extent on Fig. 11(b) of this Appendix. Deviations can be understood as the results of (a) mass being available for low-energy cascade contributions, e.g. in the cases of the $\mathrm{K}^{-}$and $\overline{\mathrm{p}}$, and (b) energy loss differences at low energies, e.g. the proton-pion difference at $100 \mathrm{MeV}$. To keep Fig. 4-4 uncluttered, we have plotted the average of the albedos calculated for incident protons and pions except at $100 \mathrm{MeV}$, where the pion result is used.

In general, the number of neutrons produced in a hadronic cascade rises less rapidly than energy. This comes about because there are more highenergy cascade steps in a higher energy cascade, and in each of them a large fraction of the energy (about $1 / 3$ ) is "bled off" into the electromagnetic component through $\pi^{0}$ production. We would expect the flux to go about as $E^{0.8}$, on the basis of other simulation results as well as a few experimental data [26,27]. According to Fig. 8 of this Appendix, the number of neutrons produced per $\mathrm{GeV}$ of incident energy is independent of energy, except below a few $\mathrm{GeV}$ where the expected mass effects appear. The linear behavior with energy in the energy region shown in the figure can perhaps be understood as a sort of compensation: With increasing energy more of the energy goes into electromagnetic showers, but in fission reactions more neutrons are produced. However, this conclusions is also at some variance with that of other authors.

5. Russ and Stevenson

Layers of material prepared for activation analysis were interleaved with iron and exposed to a $200 \mathrm{GeV} / \mathrm{c}$ positive (mostly $\pi^{+}$) hadron beam, and

* H. Fesefeldt strongly disagrees. Our conclusion was reached with difficulty and only after consulting more expert colleagues. If it is incorrect, the overall albedo number and maximum flux estimates summarized in Eqns. 4-1 and 4-2 should be scaled upward by factors of about 1.5 . 
the activation was measured as a function of radius in materials with different neutron activation thresholds. The results were interpreted in terms of neutron flux above these thresholds.

The activation of an indium front plate provides a measurement of albedo flux between $0.5 \mathrm{MeV}$ and $5 \mathrm{MeV}$. Extrapolation of the observed radial distribution suggests that the observed laterally integrated flux must be multiplied by 1.8 to correct for side leakage. From the activation of an aluminum plate at the same position, it is estimated that $30 \%$ of the flux lies above $5 \mathrm{MeV}$. The total (corrected) laterally integrated flux above 0.5 $\mathrm{MeV}$ is $60 \pm 30$. On the basis of Fig. $4-3$ (b) we divide by 0.76 to correct for the $0.5 \mathrm{MeV}$ threshold, and we multiply by 1.5 to normalize to a uranium/scintillator calorimeter on the basis of Brau and Gabriel's results. Finally, we divide by two to convert the radially integrated flux to the number of albedo neutrons per incident $200 \mathrm{GeV} / \mathrm{c}$ hadron. The final result is 59 neutrons, and the error is now considerably larger than 30 . This result is indicated by the $R$ in Fig. 4-4, and the vertical size of the ellipse gives a rough indication of the error.

A similar procedure yields the flux at cascade maximum. An interpolation of the indium data presented in Fig. A17-30a indicates a maximum at about 300 . Higher energy neutrons add another 50 or so, and the side loss factor, energy correction, and conversion to uranium/scintillator combine to yield 1200 for the transversely integrated flux. The difficulty in assigning an error to the result is obvious. The point is indicated by the $\mathrm{R}$ in Fig. $4-5$, and the vertical size of the ellipse indicates a fairly arbitrary error of \pm 800 .

6. Wigmans

In Appendix 18 Wigmans interprets his extensive study of fission products in test calorimeters [28] for the needs of this Report. The results of interest for this section are for massive uranium and for a "fine-sampling uraniumplastic scintillator calorimeter." The latter had $3 \mathrm{~mm}$ U plates and $2.5 \mathrm{~mm}$ scintillator sheets, which is essentially our reference case. For a $300 \mathrm{GeV} \pi^{-}$ exposure on massive uranium, $\int \varphi d a$ was 600 at shower maximum and the radially integrated albedo flux was 100 per incident hadron. A $0.591 \mathrm{GeV}$ proton exposure yielded 7 neutrons at the maximum and 3 albedo neutrons (flux) in massive uranium, while the same experiment in the U/scintillator calorimeter resulted in 5 at the maximum and 1.5 albedo. Threshold for the fission reaction used for this part of the study was $1.5 \mathrm{MeV}$, so according to Fig. 4-3 all results should be multiplied by $(1 / 0.5)$ to correct to a 100 $\mathrm{keV}$ threshold. The corrected $0.591 \mathrm{GeV}$ results are plotted in Figs. 4-4 
and 4-5 as W's inside circles of arbitrary size. The $300 \mathrm{GeV}$ results present more of a problem because they are available only for massive uranium. However, using the factors measured at $0.591 \mathrm{GeV}(5 / 7$ for the flux maximum and $1 / 2$ for albedo), we infer that the appropriate corrected numbers for $U /$ scintillator are 860 at the maximum and 100 albedo. Again, the circles around the W's are of arbitrary size, and albedo number rather than flux is plotted. The large uncertainty in scaling the numbers from massive uranium to uranium/scintillator must be emphasized.

The scatter of the points in Figs. 4-4 and 4-5 is surprisingly small, given the diversity of the simulations and experiments and the difficulty of interpreting and comparing the results. Since meaningful error bars cannot be assigned to most of the points, a sensible analytic fit to these data is impossible. In each case the solid curve has simply been drawn by eye, and the parallel dashed curves shown in the Figures indicate changes by a factor of two in either direction. We find

$$
\int I d a \approx 2.5\left(\frac{E}{1 \mathrm{GeV}}\right)^{0.5} \approx 2.5\left(\frac{p}{1 \mathrm{GeV} / \mathrm{c}}\right)^{0.5}
$$

for the number of albedo neutrons and

$$
\int \varphi d a \approx 18\left(\frac{E}{1 \mathrm{GeV}}\right)^{0.67} \approx 18\left(\frac{p}{1 \mathrm{GeV} / \mathrm{c}}\right)^{0.67}
$$

for the flux at the cascade maximum inside a fine-sampling uranium/scintillator calorimeter. Momentum is a more convenient variable than energy, and the substitution is justified because very few of the data falls below $1 \mathrm{GeV}$ and most of the hadrons incident on an SSC calorimeter are pions.

\subsection{Leakage Flux}

The only information about neutron flux deep in a uranium/scintillator calorimeter comes from Fig. 4 of Appendix 13. Parallel data for massive uranium are presented in Fig. 1 of Appendix 18 and for a U/TMS in Fig. A16-10 of Appendix 16. These data are difficult to scale to the $\mathrm{U} / \mathrm{scintillator}$ case, and in the case of the Wigmans results are well above the $O(10 \mathrm{GeV})$ region of interest. The Alsmiller et al. simulations are for 1,5 , and $20 \mathrm{GeV}$. The results extend to just over 5 inelastic interaction lengths $\left(5 \lambda_{I}\right)$, since for the $3 \mathrm{~mm} / 3 \mathrm{~mm} \mathrm{U} /$ scintillator calorimeter $\lambda_{I}=18.6 \mathrm{~cm}$. The laterally integrated fluxes obtained from their figure in this way are given in Table 4-2.

These data are not well described by power laws. At low energies one is far down on an exponential tail, which at a given depth rises rapidly with energy. 
Table 4-2

Laterally integrated neutron flux inside a uranium/scintillator calorimeter; as read from Fig. 4 of Appendix 13.

\begin{tabular}{cccc}
\hline \hline \multirow{2}{*}{ Depth } & \multicolumn{3}{c}{$\int \varphi$ da at $E=$} \\
& $1 \mathrm{GeV}$ & $5 \mathrm{GeV}$ & $20 \mathrm{GeV}$ \\
\hline $3 \lambda_{I}$ & 4.6 & 43. & 140. \\
$4 \lambda_{I}$ & 1.7 & 25. & 86. \\
$5 \lambda_{I}$ & 0.8 & 13. & 50. \\
\hline \hline
\end{tabular}

At higher energies, these depths are not far from cascade maximum, and so the energy dependence decreases toward the $E^{0.7}$ characteristic of the maximum flux. However, one can approximate the momentum distribution by particles produced at a given rapidity by a $\delta$-function, as discussed in Section 2, to relate the tabulated energies to rapidity and thus establish the total flux. This procedure is carried out in Section 5.

\subsection{Dependence upon calorimeter construction}

In the above discussion, we have concentrated on a uranium/scintillator calorimeter because it was the most widely studied. The calculations indicate that (a) more neutrons are produced in uranium than in other materials, and (b) the addition of a hydrogenous moderator such as scintillator reduces the flux, in contrast with high- $A$ readout materials such as silicon. The available data are summarized in Table 4-3. Unfortunately, there is no common model calorimeter to which all of the data can be normalized. In each case we have chosen uranium with the highest- $A$ material studied for normalization, but for each study it was different. The results indicate more scatter than might be expected. In particular, Fesefeldt's albedo results for U/scintillator and $\mathrm{Fe} / \mathrm{PWC}$ calorimeters appear to be high. In general, it might be concluded that the use of scintillator rather than high- $A$ readout materials reduces the flux by a factor of about two, and that further reductions may occur if lead is used instead of uranium, even with a high- $A$ readout material.

\subsection{Reflection in the central cavity.}

A realistic detector contains a central volume which is very nearly empty. It is surrounded by calorimeters over most of the solid angle; for coverage to $\eta=3\left(5.7^{\circ}\right)$ the calorimeters subtend $99.5 \%$ of the available solid angle. As discussed above, hadrons from the interaction point produce albedo neutrons when they strike the calorimeter, and additional neutrons may come from more distant endcaps. These neutrons may be reflected one or more times before being 
Table 4-3

Relative neutron flux at $3 \mathrm{GeV}$ incident kinetic energy for a variety of model calorimeters.

\begin{tabular}{lccc}
\hline \hline Calorimeter & Ban et al.* $\begin{array}{c}\text { Brau and } \\
\text { Gabriel }\end{array}$ & Fesefeldt \\
\hline 1. Albedo flux & & & \\
U/silicon & - & 1 (def.) & - \\
U/argon & 1 (def.) & - & - \\
U/PWC & - & - & 1 (def.) \\
U/scint. & 0.68 & 0.33 & 0.44 \\
$\mathrm{~Pb} /$ argon & 0.13 & - & - \\
$\mathrm{Pb} / \mathrm{PWC}$ & - & - & 0.37 \\
$\mathrm{~Pb} /$ scint. & 0.10 & - & 0.24 \\
$\mathrm{Fe} /$ silicon & - & 0.23 & - \\
$\mathrm{Fe} / \mathrm{PWC}$ & - & - & 0.32 \\
$\mathrm{Fe} /$ scint. & - & 0.10 & 0.19 \\
\hline $\mathrm{Maximum}$ flux & & & \\
$\mathrm{U} /$ silicon & - & $1($ def. $)$ & - \\
U/argon & - & - & - \\
$\mathrm{U} / \mathrm{PWC}$ & - & - & $1($ def. $)$ \\
$\mathrm{U} /$ scint. & - & 0.25 & 0.51 \\
$\mathrm{~Pb} /$ argon & - & - & - \\
$\mathrm{Pb} / \mathrm{PWC}$ & - & - & 0.29 \\
$\mathrm{~Pb} /$ scint. & - & - & 0.23 \\
$\mathrm{Fe} /$ silicon & - & 0.29 & - \\
$\mathrm{Fe} / \mathrm{PWC}$ & - & - & 0.57 \\
$\mathrm{Fe} /$ scint. & - & 0.09 & 0.26 \\
\hline \hline
\end{tabular}

${ }^{\star}$ GHEISHA only.

absorbed, so the flux in the cavity will be higher than the flux expected from the injected neutrons alone.

Neutron reflection in spherical calorimeters is studied in Appendices 11 (Oak Ridge) and 12 (Livermore). Both groups simulated a variety of model calorimeters, all of which had spherical cavities with $2 \mathrm{~m}$ radii. Scaling of the results to different geometries is discussed in Appendix 9, and we rely upon the formalism presented there in analyzing the results.

The simplest problem consists of an isotropic neutron source uniformly distributed on the inside surface of the cavity. Both groups considered spheres of vac- 
uum, concrete, uranium, and uranium/scintillator. The vacuum case was run to check overall normalization, since according to Appendix 9 the flux in the absence of reflection should be $\varphi_{0}=\mathcal{N} / \pi R^{2}$. All of the Oak Ridge and Livermore results have been normalized to $\mathcal{N}=1$ injected neutron, so $\varphi_{0}=0.795 \times 10^{-5} \mathrm{~cm}^{-2}$ for $R=2 \mathrm{~m}$. The concrete case was included to permit comparisons with calculations and measurements made for the SSC arcs [26], and the other two are reference calorimeter compositions used elsewhere in this report. The results are summarized in Table 4-4.

\section{Table 4-4}

Neutron reflection inside a closed spherical shell calorimeter with a $200 \mathrm{~cm}$ inside radius. Lawrence Livermore Laboratory (LLNL) and Oak Ridge National Laboratory (ORNL) results are tabulated for a variety of shell compositions for an isotropic 1 $\mathrm{MeV}$ source (normalized to one neutron) on the inside of the shell. The notation $f_{>}$means the fraction of the flux with kinetic energy in excess of $0.1 \mathrm{MeV}$, and $\varphi_{0}=\left[\pi \times(200 \mathrm{~cm})^{2}\right]^{-1}=0.795 \times 10^{-5} \mathrm{~cm}^{-2}$ is the expected flux in the absence of reflection.

\begin{tabular}{llccccc}
\hline \hline & \multicolumn{3}{c}{ LLNL } & \multicolumn{3}{c}{ ORNL } \\
& \multicolumn{1}{c}{$10^{5} \varphi$} & $f_{>}$ & $\varphi f_{>} / \varphi_{0}$ & $10^{5} \varphi$ & $f_{>}$ & $\varphi f_{>} / \varphi_{0}$ \\
\hline Void & $0.795 \pm 0.002$ & 1.00 & 1.00 & 0.787 & 1.00 & 0.99 \\
Concrete & $3.08 \pm 0.03$ & 0.55 & 2.13 & 2.90 & 0.49 & 1.77 \\
Uranium & $2.36 \pm 0.05$ & 0.82 & 2.43 & 2.21 & 0.89 & 2.47 \\
U/scint. & $2.38 \pm 0.17$ & 0.62 & 1.87 & 1.33 & 0.81 & 1.35 \\
\hline \hline
\end{tabular}

Reflection is complicated by energy loss, which occurs through moderation and inelastic processes. Up to half of the reflected flux has been thermalized, and is of little interest. We count a neutron as contributing to the reflected flux if its energy exceeds some threshold, usually taken as $0.1 \mathrm{MeV}$ because of our preoccupation with damage to silicon. It is convenient to define $a$ as the mean number of times a neutron reflects back into the cavity before being lost due to absorption or falling below the energy threshold. In the case of an isotropic source, the flux is then $(1+a) \varphi_{0}$. The fraction above $0.1 \mathrm{MeV}$ is labeled " $f_{>}$" in Table $4-4$, and $\varphi f_{>} / \varphi_{0}$ is equal to the flux enhancement factor $(1+a)$. It is about two for all of the materials considered. It is larger if no moderator (hydrogen) is present, and it was shown in a previous section that more neutrons are injected in the first place if uranium is present.

It is of interest to compare these results with those obtained in the tunnel study (Ref. 26 and Appendix 10). It is shown in section 8 of Appendix 9 that the enhancement factor $(1+a)$ is replaced by $(1+8 a r / \pi R)$ for the tunnel case, 
where $R$ is the tunnel radius and $r$ is the distance from the magnet string (neutron source) to the observation point. The comparison is further complicated because of the use of a large scoring volume in the simulation. It consists of a cylindrical shell extending from $r_{1}=50 \mathrm{~cm}$ to $r_{2}(=R)=152 \mathrm{~cm}$. Flux is obtained by dividing the total track length scored in the volume (say of length $L>>r_{2}$ ) by the volume:

$$
\frac{\text { Total track length }}{\pi L\left(r_{2}^{2}-r_{1}^{2}\right)}=\frac{\text { Total track length } / \Delta r}{2 \pi L\langle r\rangle}
$$

where $\Delta r=\left(r_{2}-r_{1}\right)$ and $\langle r\rangle=\left(r_{2}+r_{1}\right) / 2$. Since the total track length for the direct component is proportional to the cylinder thickness $\Delta r$, the direct flux scored in the simulation is the same as that at a small detector a distance $\langle r\rangle$ from the magnet string. In this particular case $\langle r\rangle=101 \mathrm{~cm}$. (As elsewhere in this report, the reflected flux is assumed to be independent of position.)

Simulations are made with and without concrete tunnel walls, so that the ratio of the results should yield $(1+8 a\langle r\rangle / \pi R)$. At $875 \mathrm{GeV}$ the ratio was 3.03 , and at $20 \mathrm{TeV}$ it was 2.97 , for an average of 3.00 . We thus infer

$$
1+a=2.18
$$

for concrete.

From Table $4-4$ we obtain $(1+a)=2.13$ (Livermore) and 1.77 (Oak Ridge). However, the tunnel results are obtained using a $40 \mathrm{keV}$ cutoff; when this is done for the spherical cavity results the factors increase to about 2.25 and 1.84 . A comparison of all three results would suggest that the Oak Ridge spherical cavity results are somewhat low, but the reasons for the discrepancy remain obscure. It is also interesting to note that $a \approx 1$ leads to an enhancement of two in the case of a spherical cavity but an enhancement of nearly five in the tunnel.

The Oak Ridge and Livermore results for massive uranium are in good agreement, but in the uranium/ scintillator case the difference is fairly large. The larger number (1.87) is thought to be more reasonable.

These results were obtained using a $1 \mathrm{MeV}$ source, ${ }^{\star}$ while a typical spectrum is shown in Fig. 4-3. The Livermore group made a separate study in which the source energy was varied from $0.1 \mathrm{MeV}$ to $20 \mathrm{MeV}$. The total flux (not cut at 0.1 $\mathrm{MeV}$ ) was fairly insensitive to source energy below $10 \mathrm{MeV}$, so we conclude that results obtained with a $1 \mathrm{MeV}$ source are representative.

* In the Oak Ridge case $1 \mathrm{MeV}$ was actually bracketed by two adjacent energy groups. The effects of this difference have been studied by both sets of authors, with the conclusion that the resulting differences are fairly small. 
It might be hoped that some of this reflected flux might escape through the holes near the beam line. For an isotropic flux the probability of a neutron hitting a hole is proportional to the fractional solid angle subtended by the hole. Stated differently, the probability of not hitting one of the two holes subtending half-angle $\theta_{0}$ is $\cos \theta_{0}$. If the probability of a reflection is $P$, then $P$ should be replaced by $P^{\prime}=P \cos \theta_{0}$ if holes are present. Since $a=P /(1-P)$, the new mean number of reflections $a^{\prime}=P^{\prime} /\left(1-P^{\prime}\right)$ can easily be calculated.

Simulations are reported in Appendix 12 for a uranium/scintillator calorimeter without holes and with holes for $\theta_{0}=5.7^{\circ}, 10^{\circ}, 20^{\circ}, 30^{\circ}$, and $40^{\circ}$. The results are normalized to $\Omega / 4 \pi\left(=\cos \theta_{0}\right)$ direct neutrons, and so fluxes must be scaled by the reciprocal of this factor before calculating the "experimental" values for $P^{\prime} . P^{\prime} / \cos \theta_{0}$ should be independent of angle. The results are summarized in Table 4-5, and the function $P^{\prime} / \cos \theta_{0}$ is shown in Fig. 4-6. As in the Table 4-4, $f_{>}$is the flux fraction above $0.1 \mathrm{MeV}$. The column headed " $10^{5} \varphi f_{>} / \cos \theta_{0}$ " is the flux above the threshold energy corrected to a source over the entire cavity. $(1+a)$ is this quantity divided by $\varphi_{0}$, the flux in the case of no reflection. According to the above discussion $P^{\prime} / \cos \theta_{0}$ should be independent of angle. The slope of the least-squares linear fit is $0.184 \pm 0.046$, with $\chi^{2}=6$ for four degrees of freedom. The agreement with expectation is adequate, particularly in view of the weight attached to the final point.

There is finally the problem of a nonisotropic source. For albedo neutrons the number injected goes about as $p^{0.5} \approx\left\langle p_{\perp}\right\rangle^{0.5} / \sin ^{0.5} \theta$, and the number striking unit area is proportional to $1 / \sin ^{2} \theta$. The source distribution is thus reasonably described by $1 / \sin ^{2.5} \theta$, with a cutoff at $\theta_{0} \approx 5.7^{\circ}$. The flux distribution inside a standard calorimeter for this source distribution is shown in the final figure of Appendix 12. As expected, there are enhancements near the hot spots at the ends, and a relatively low flux near the calorimeter $90^{\circ}$ from the beam line. 
Table 4-5

Data reduction for comparisons of Livermore simulation of neutron reflection in spherical shell uranium/scintillator calorimeters with expectation. $\theta_{0}$ is the halfangle of the hole in each end, $\varphi$ the flux in the cavity per injected $1 \mathrm{MeV}$ neutron, and $f>$ the fraction of neutrons with energy above $0.1 \mathrm{MeV} . a^{\prime}$ is the mean number of reflections and $P^{\prime}$ the probability of a reflection. From elementary considerations, the last column is expected to be nearly constant.

\begin{tabular}{cccccccc}
\hline \hline$\theta_{0}$ & $10^{5} \varphi$ & $\Delta \varphi / \varphi$ & $f_{>}$ & $10^{5} \varphi f_{>} / \cos \theta_{0} 1+a^{\prime}$ & $P^{\prime}$ & $P^{\prime} / \cos \theta_{0}$ \\
\hline $0.0^{\circ}$ & 2.38 & 0.07 & 0.62 & 1.49 & 1.87 & 0.464 & $0.464 \pm 0.039$ \\
$5.7^{\circ}$ & 2.12 & 0.02 & 0.67 & 1.43 & 1.80 & 0.444 & $0.447 \pm 0.012$ \\
$10.0^{\circ}$ & 2.20 & 0.05 & 0.65 & 1.45 & 1.82 & 0.449 & $0.456 \pm 0.028$ \\
$20.0^{\circ}$ & 1.86 & 0.01 & 0.69 & 1.37 & 1.72 & 0.419 & $0.446 \pm 0.006$ \\
$30.0^{\circ}$ & 1.57 & 0.02 & 0.73 & 1.32 & 1.66 & 0.399 & $0.460 \pm 0.012$ \\
$40.0^{\circ}$ & 1.17 & 0.01 & 0.76 & 1.16 & 1.46 & 0.314 & $0.410 \pm 0.007$ \\
\hline \hline
\end{tabular}

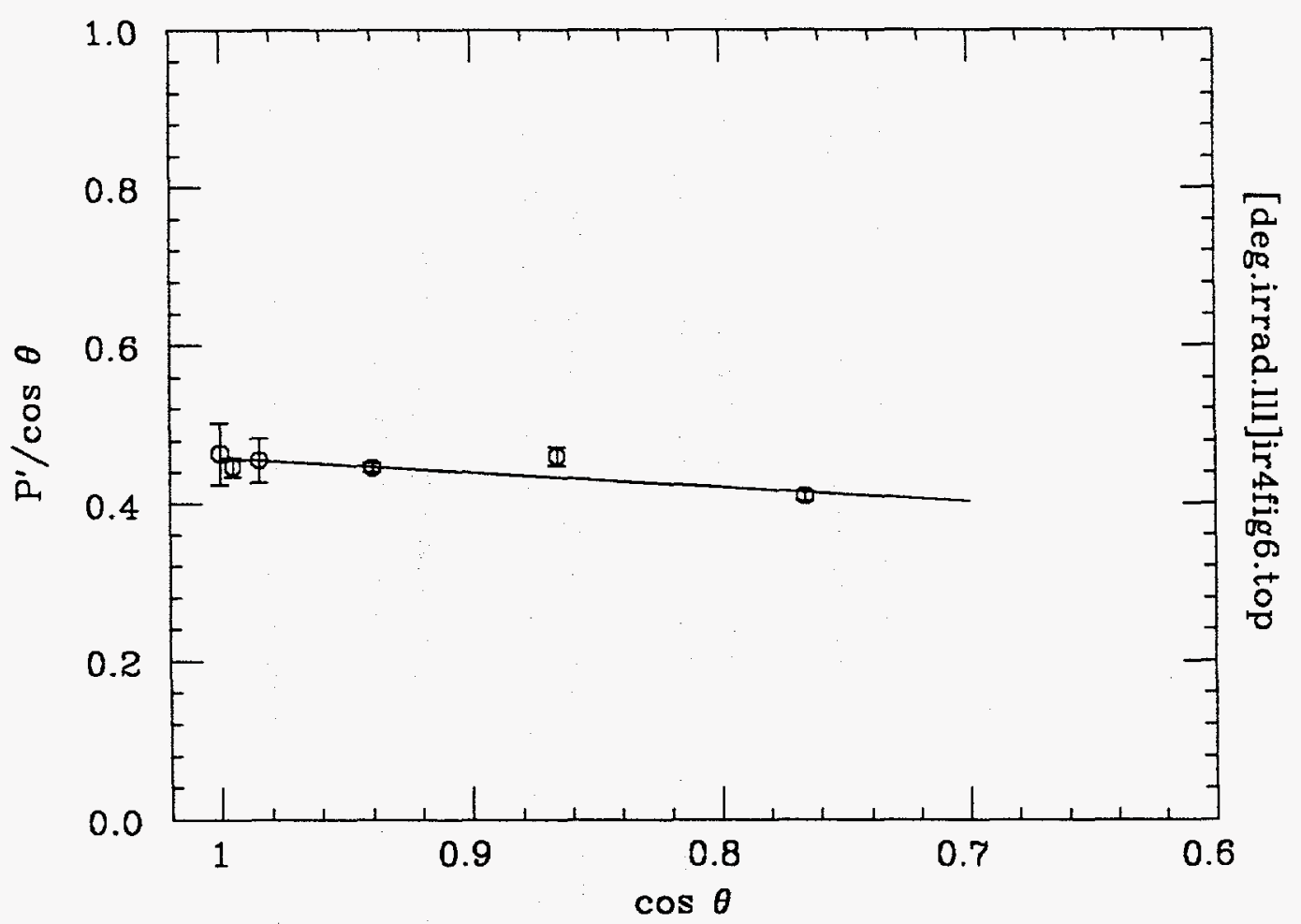

Fig. 4-6. Variation of $P^{\prime} / \cos \theta_{0}$ with $\cos \theta_{0}$, as reduced from Livermore simulations of neutron reflection in a uranium/scintillator spherical shell with axial conical holes with half-angle $\theta_{0}$. 


\section{SUMMARY OF RADIATION LEVELS}

In this Section we summarize the results of the Task Force and present them in such a way that they can be used to determine radiation levels in experimental detectors. It is of course impossible to solve the general problem of radiation levels in all parts of an arbitrary detector, so at such time as a given configuration is known additional detailed calculations of radiation levels will be required. We also note once again that there are substantial uncertainties (factors of 2-3) in many of our estimates. Improved estimates of radiation levels will require more experimental measurements of doses and neutron fluences and comparison of such measurements with Monte Carlo calculations. Finally, we comment that the radiation levels described in this report do not include contributions from accidental beam losses, losses during injection into the SSC storage rings or "halo" from upstream interactions, all of which could adversely affect detectors. Although these contributions are expected to be small relative to the effects of the particles from the p-p collision (at or near the design luminosity), ${ }^{\star}$ our estimates should be taken as approximate lower bounds to the expected radiation levels

\subsection{Particle production model}

It was concluded in Section 2 that for $|\eta|<6$ (or $\theta>0.3^{\circ}$ ) the height $H$ of the rapidity plateau is about 7.5 for charged particles and about half this for $\pi^{0}$ 's. We therefore also take $H=7.5$ for photons, which come primarily from $\pi^{0}$ decay, and assume that their mean momentum is half that of the charged particles. We are also warned that the plateau could be as high as 10 at the SSC. In this case our estimates should be increased by about $30 \%$.

In Section 2 it was also shown that if the dose or fluence resulting from single incident particles of momentum $p$ could be represented as $N p^{\alpha}$, then integration over the incident momentum spectrum at a given angle could be replaced by evaluation at the mean momentum with an error of less than $10 \%$. This is true for values of the exponent between zero and one and in the absence of a transverse momentum cutoff due to a possible central solenoid. The mean momentum is the mean transverse momentum divided by $\sin \theta$ :

$$
\langle p\rangle=\left\langle p_{\perp}\right\rangle / \sin \theta=\left\langle p_{\perp}\right\rangle \cosh \eta
$$

If we approximate the pseudorapidity and momentum distribution for the average

* In contrast to the situation at existing colliders, proton collisions at the interaction points will be the main source of beam loss at the SSC. Each IR operating at the design luminosity will contribute $(300 \mathrm{hr})^{-1}$ to the reciprocal lifetime, which is about same as that of beamgas losses around the entire ring. This is equivalent to dumping a full-energy beam into the apparatus every 6 days. It is difficult to imagine other significant sources of radiation in the detectors which would not damage the machine or substantially decrease its performance. 
event by $d^{2} N_{\text {charged }} / d \eta d p=H \delta(p-\langle p\rangle)$, the result of folding with the singleparticle response $N p^{\alpha}$ is

$$
\text { Dose or fluence }=H N\left\langle p_{\perp}\right\rangle^{\alpha} \cosh ^{\alpha} \eta \text {. }
$$

As discussed in Section 2, the mean transverse momentum $\left\langle p_{\perp}\right\rangle$ may be taken as $0.6 \mathrm{GeV} / \mathrm{c}$ for charged particles and $0.3 \mathrm{GeV} / \mathrm{c}$ for photons.

Eq. 5-1 describes dose or fluence as a function of pseudorapidity. If dose or fluence per unit solid angle or per unit area is of interest instead, then an additional $\cosh ^{2} \eta$ appears because $d \eta / d \Omega=1 / 2 \pi \sin ^{2} \theta$. In this case, $\cosh \eta$ is raised to the power $\alpha^{\prime}=\alpha+2$. In general we may write

$$
\text { Dose or fluence }=A\left\langle p_{\perp}\right\rangle^{\alpha} \cosh ^{\alpha^{\prime}} \eta
$$

where $\alpha^{\prime}=\alpha$ or $\alpha+2$, depending upon the problem. Most of the figures in this section show a dose or fluence of this form. For convenience, the parameters $A,\left\langle p_{\perp}\right\rangle, \alpha$, and $\alpha^{\prime}$ used in making the figures are listed in Table 5-1.

Table 5-2

Coefficients of functions of the form $A\left\langle p_{\perp}\right\rangle^{\alpha} \cosh ^{\alpha^{\prime}} \eta$ shown in the figures of this Section. $\alpha$ is the slope of the momentum power law fitted to whatever single-particle response is being considered. $\alpha^{\prime}$ is the same as $\alpha$ if the dose or fluence is a function of pseudorapidity and greater by two if it is a function of solid angle or area. $A$ is the product of the rapidity plateau height (taken as 7.5), the amplitude of the single-particle response, and (if required) the number of collisions per year. $\left\langle p_{\perp}\right\rangle$ is in units of $\mathrm{GeV} / \mathrm{c}$.

\begin{tabular}{ccllcll}
\hline \hline Figure & $A$ & Units & $\left\langle p_{\perp}\right\rangle$ & $\alpha$ & \multicolumn{1}{c}{$\alpha^{\prime}$} & \multicolumn{1}{c}{ Remarks } \\
\hline $5-2$ & 19.5 & per evt & 0.3 & 0.70 & 0.70 & $E_{\gamma}>1.0 \mathrm{MeV}$ \\
& 34.5 & per evt & 0.3 & 0.68 & 0.68 & $E_{\gamma}>0.1 \mathrm{MeV}$ \\
$5-3$ & 18.8 & per evt & 0.6 & 0.5 & 0.5 & \\
$5-4$ & $5.4 \times 10^{11}$ & $\mathrm{~cm}^{-2} \mathrm{yr}^{-1}$ & 0.6 & 0.67 & 2.67 & $r=200 \mathrm{~cm}$ \\
& $5.4 \times 10^{7}$ & $\mathrm{~cm}^{-2} \mathrm{yr}^{-1}$ & 0.6 & 0.67 & 2.67 & $r=20 \mathrm{~m}$ \\
$5-6$ & 300. & $\mathrm{~Gy} \mathrm{yr}^{-1}$ & 0.3 & 0.93 & 2.93 & $r=200 \mathrm{~cm}$ \\
$5-8$ & 11.3 & $\mathrm{~Gy} \mathrm{yr}^{-1}$ & 0.6 & 0.89 & 2.89 & $r=200 \mathrm{~cm}$ \\
\hline \hline
\end{tabular}




\subsection{Assumptions about SSC operation}

Unless otherwise noted, we have assumed the following in order to calculate doses and fluences:

- $10^{8}$ events per second

- $10^{7}$ seconds of operation per year

- $10^{15}$ events per year.

\subsection{Dose from minimum ionizing particles}

Previous estimates have been made of the radiation dose from charged particles from the primary p-p collisions $[2,3]$. These estimates apply, for example, to tracking devices within the cavity of a calorimeter. They do not apply to the dose within the calorimeter; this is considered in a later section.

If $d N_{\text {charged }} / d \eta=H$, then

$$
\frac{d N_{\text {charged }}}{d \Omega}=\frac{H}{2 \pi \sin ^{2} \theta}
$$

The charged particle flux through a normal area element $d a$ at a distance $r$ from the interaction point is

$$
\frac{d N_{\text {charged }}}{d a}=\frac{H}{2 \pi} \frac{\left(1.0 \times 10^{8} \mathrm{~s}^{-1}\right)}{r^{2} \sin ^{2} \theta}
$$

However, $H / 2 \pi \approx 1.2$, and $r \sin \theta=r_{\perp}$, where $r_{\perp}$ is the perpendicular distance from the beam line to the element. Then the flux is simply

$$
\frac{d N_{\text {charged }}}{d a}=\frac{1.2 \times 10^{8} \mathrm{~s}^{-1}}{r_{\perp}^{2}}
$$

for $r$ in $\mathrm{cm}$. In a light material $d E / d x \approx 1.8 \mathrm{MeV} \mathrm{g}^{-1} \mathrm{~cm}^{2}$, so $1 \mathrm{~Gy}$ corresponds to $3 \times 10^{9}$ particles $/ \mathrm{cm}^{2}$. The results are shown in Fig. $5-1$.

If a solenoidal field is present the low-momentum particles will make multiple passes through a given point in the central cavity. Depending upon the field and radius, the dose will be increased, typically by a factor of two.

\subsection{Albedo photons}

The data of Appendix 7 were replotted in Section 3 to show that the number of albedo photons per normally incident photon (or electron) with energy $E$ could be represented by the power laws 


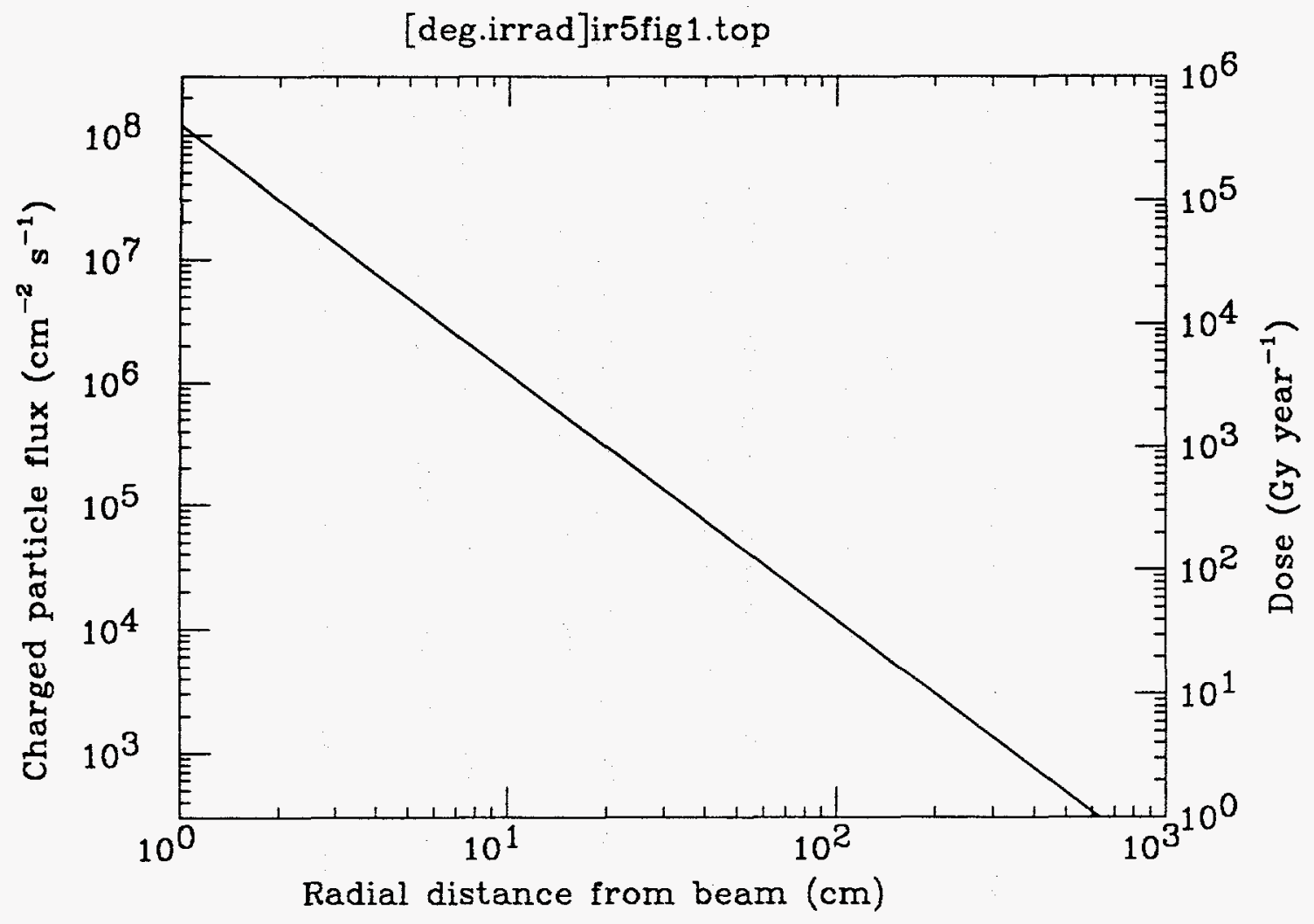

FIG. 5-1. The charged particle flux and annual dose as a function of perpendicular distance from the beam under standard SSC operating conditions.

$2.6 E^{0.70}$ for albedo photon energies $>1.0 \mathrm{MeV}$,
$4.6 E^{0.68}$ for albedo photon energies $>0.1 \mathrm{MeV}$

where $E$ is in $\mathrm{GeV}$. Using the particle production model discussed above, we obtain the number of albedo photons per event per rapidity interval:

$8.4 \cosh ^{0.70} \eta$ for albedo photon energies $>1.0 \mathrm{MeV}$

$15.2 \cosh ^{0.68} \eta$ for albedo photon energies $>0.1 \mathrm{MeV}$

These functions are shown in Fig. 5-2.

It is also shown in Appendix 7 that these results do not depend significantly on calorimeter composition, at least for uranium and lead calorimeters with either liquid argon or scintillator. The results do depend on the angle of incidence, with more albedo photons being generated for non-normal incidence.

Making use of the results presented in Fig. 2-11 and Table 2-2, one may integrate these results over rapidity to estimate the total number of albedo photons injected into a typical calorimeter cavity. Suppose that photons with $|\eta|<3$ strike the inside of the cavity, and that the inside radius of the cavity (a "spherical calorimeter") is $200 \mathrm{~cm}$. From Table $2-2$ we obtain $\int_{-3}^{3} \cosh ^{0.68} \eta d \eta=2 \times 6.41$, or 


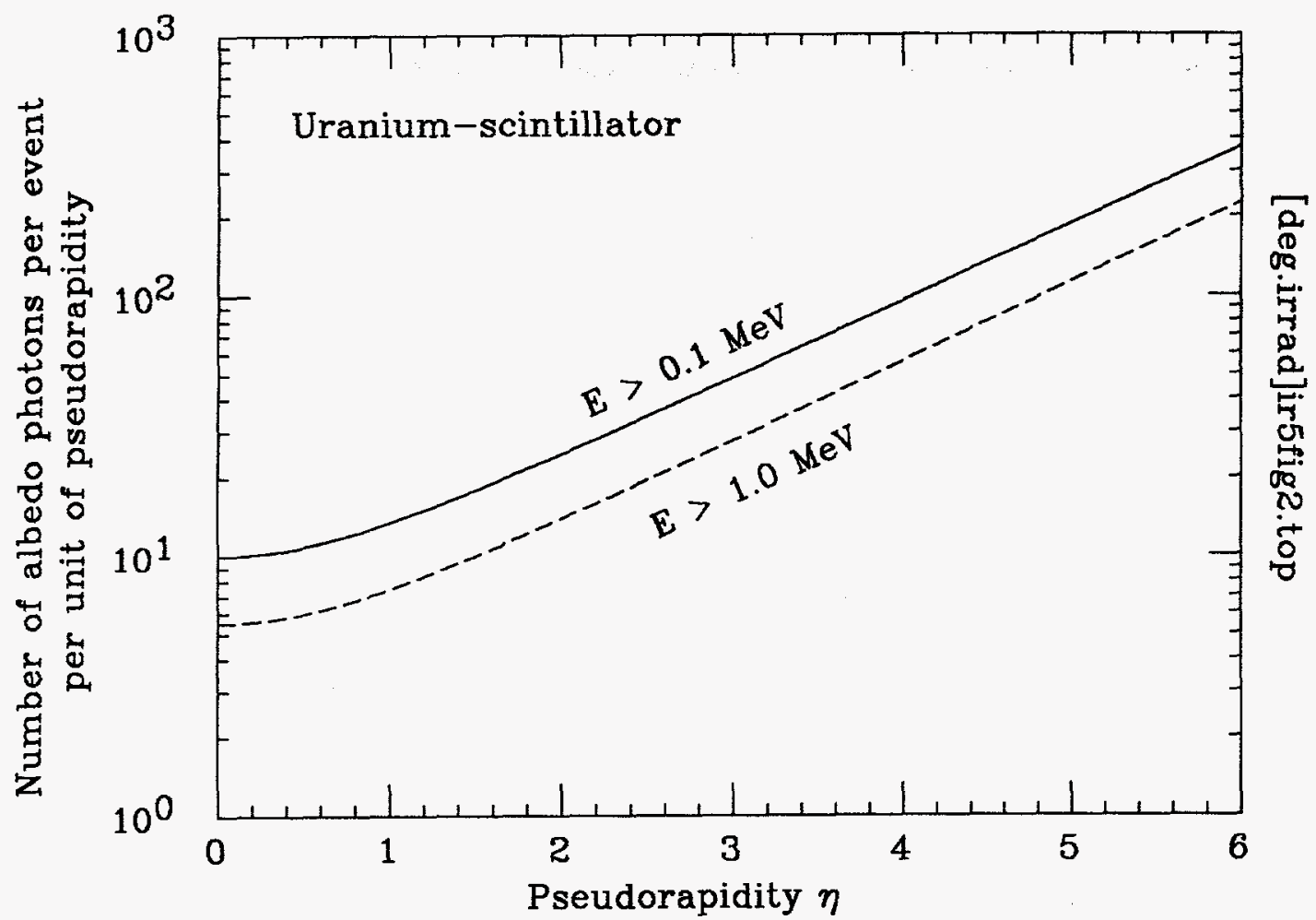

FIG. 5-2. The number of albedo photons per event for two different lower cutoffs on the albedo photon energy.

12.82 , so that 195 photons/event with $E<0.1 \mathrm{MeV}$ are injected into the cavity. Then

- the total number is $\approx 200$ per event, or about $2 \times 10^{17}$ per year

- according to Appendix 9, the flux near the center of a cavity with radius $r$ is $\mathcal{N} / \pi r^{2}$, where $\mathcal{N}$ particles per unit time are injected and secondary reflection is neglected. In the present case $r=200 \mathrm{~cm}$ and $\mathcal{N}=2 \times 10^{10} \mathrm{~s}^{-1}$, so $\varphi=1.6 \times 10^{5} \mathrm{~cm}^{-2} \mathrm{~s}^{-1}$

- there will be secondary reflection via Compton scattering. Quantitative estimates have not been made, but on the basis of other studies we would estimate an enhancement factor of less than two for scattered photons with energy above the stated threshold.

In this example the albedo photon flux is larger than the direct flux from the interaction region for distances greater than $30 \mathrm{~cm}$ from the beam line.

\subsection{Neutron flux}

Neutron production is discussed in detail in Section 4 and in Appendices 10 and 13-18. The estimates of neutron fluxes are uncertain by at least a factor 
of two for a given material, and additional uncertainty arises when comparing calorimeters of different composition. Here we show results for a fine-grained uranium/scintillator calorimeter. Interpolation to other combinations of materials is discussed in Section 4.5, where the results reported in the Appendices are compared in Table 4-3. The content of this Table is summarized here in Table 52. We emphasize that the error in the ratios given in this Table may be as large as a factor of two.

Table 5-2

The relative albedo neutron flux and the neutron flux at cascade maximum.

\begin{tabular}{ll}
\hline U-LiqAr or Si & 1.0 \\
Pb-LiqAr or Si & 0.5 \\
U-Scint. & 0.3 \\
Pb-Scint. & 0.15 \\
\hline
\end{tabular}

In quoting the number of neutrons one must also state the range of neutron energies included. For the numbers given here this range is $0.1<\mathrm{E}<20 \mathrm{MeV}$.

\section{Albedo neutrons}

In Section 4 it was shown that the number of albedo neutrons produced by the normal incidence of one pion per unit area ${ }^{\dagger}$ upon a uranium/scintillator calorimeter could be represented by $2.5 p^{0.5}$, where $p$ is the pion's momentum in $\mathrm{GeV} / \mathrm{c}$. The number of albedo neutrons produced per event per pseudorapidity interval is then $7.5 \times 2.5 \times(0.60)^{0.5} \cosh ^{0.5} \eta$, or $14.5 \cosh ^{0.5} \eta$, as shown in Fig. 5-3.

Following the above discussion for the albedo photon case, one may use the results from Section 2 and Appendix 9 to estimate the neutron flux inside the detector cavity. We again consider a spherical cavity with inside radius $200 \mathrm{~cm}$ over the interval $|\eta|<3$ (or $\theta>6^{\circ}$ ). Since $\int_{-3}^{3} \cosh ^{0.5} \eta d \eta=10.28$, the total number of albedo neutrons injected into the cavity of a uranium/scintillator calorimeter is 150 per event, or $\mathcal{N}=1.5 \times 10^{10} \mathrm{~s}^{-1}$. Then near the center of the cavity

$$
\begin{aligned}
\varphi & =\frac{\mathcal{N}}{\pi r^{2}}(1+a) \\
& =1.2 \times 10^{5}(1+a) \mathrm{cm}^{-2} \mathrm{~s}^{-1}
\end{aligned}
$$

for an annual fluence of $1.2 \times 10^{12}(1+a) \mathrm{cm}^{-2}$ at $r=2 \mathrm{~m}$, where $a$ is the mean

* A number of different cutoffs were used in the Appendices. In Section 4 an attempt was made to correct all results to this range before comparisons or parameterizations were made.

$\dagger$ As had been mentioned, this is easily shown to be the same as the lateral integral of the fluence $\int \varphi d a$ for one incident particle. 


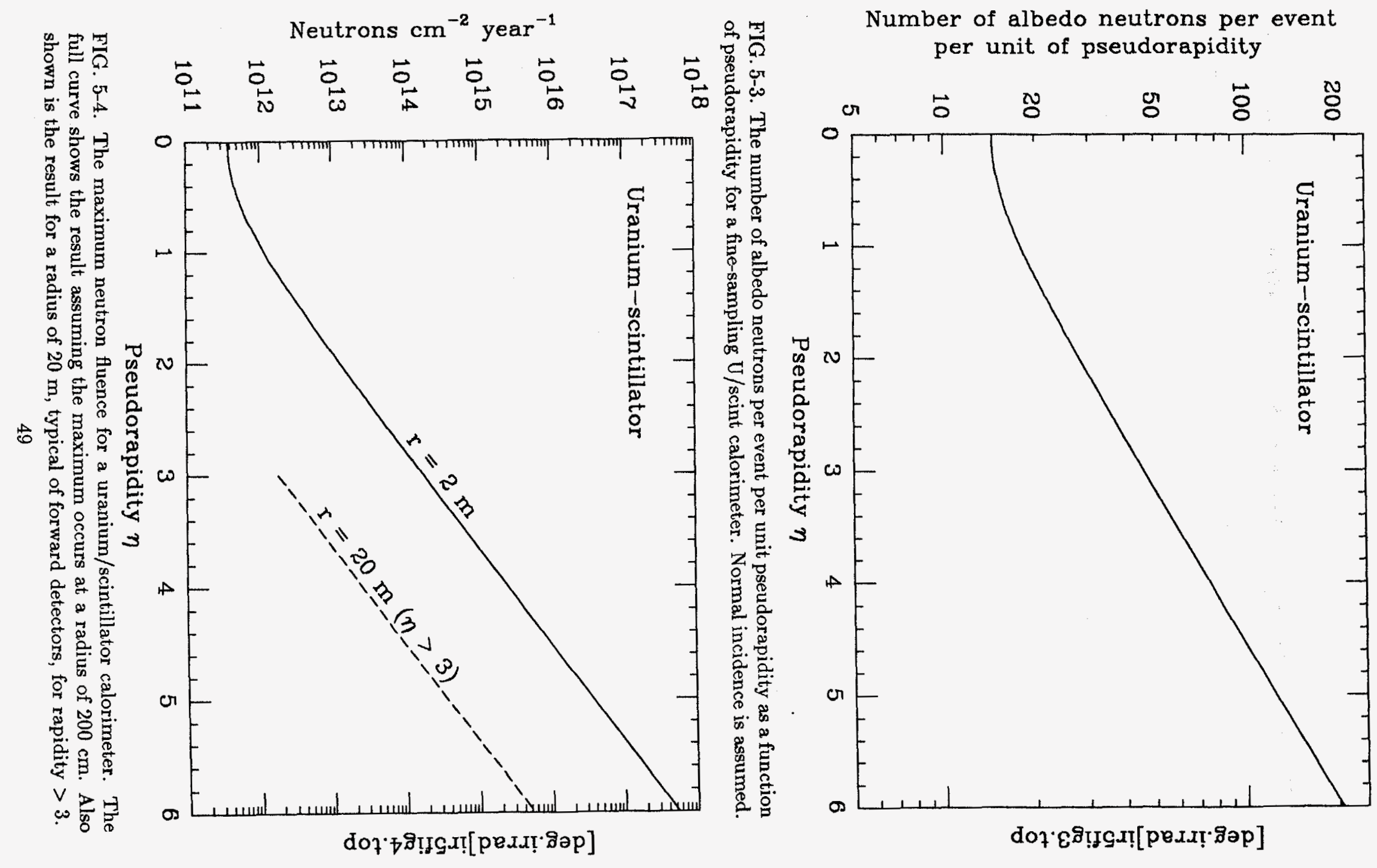


number of reflections before a neutron is absorbed or loses so much energy that it can no longer do damage. On the basis of the simulations reported in Appendices 11 and 12 , as summarized in Section $4,(1+a) \approx 2$ for spherical calorimeters. (The results may be scaled to non-spherical calorimeters as per the discussion in Appendix 9.) Our estimate becomes $2.4 \times 10^{12} \mathrm{~cm}^{-2} \mathrm{yr}^{-1}$ inside a spherical cavity with a $2 \mathrm{~m}$ radius. It is important to remember that this fluence scales inversely as the square of a characteristic linear dimension of the cavity.

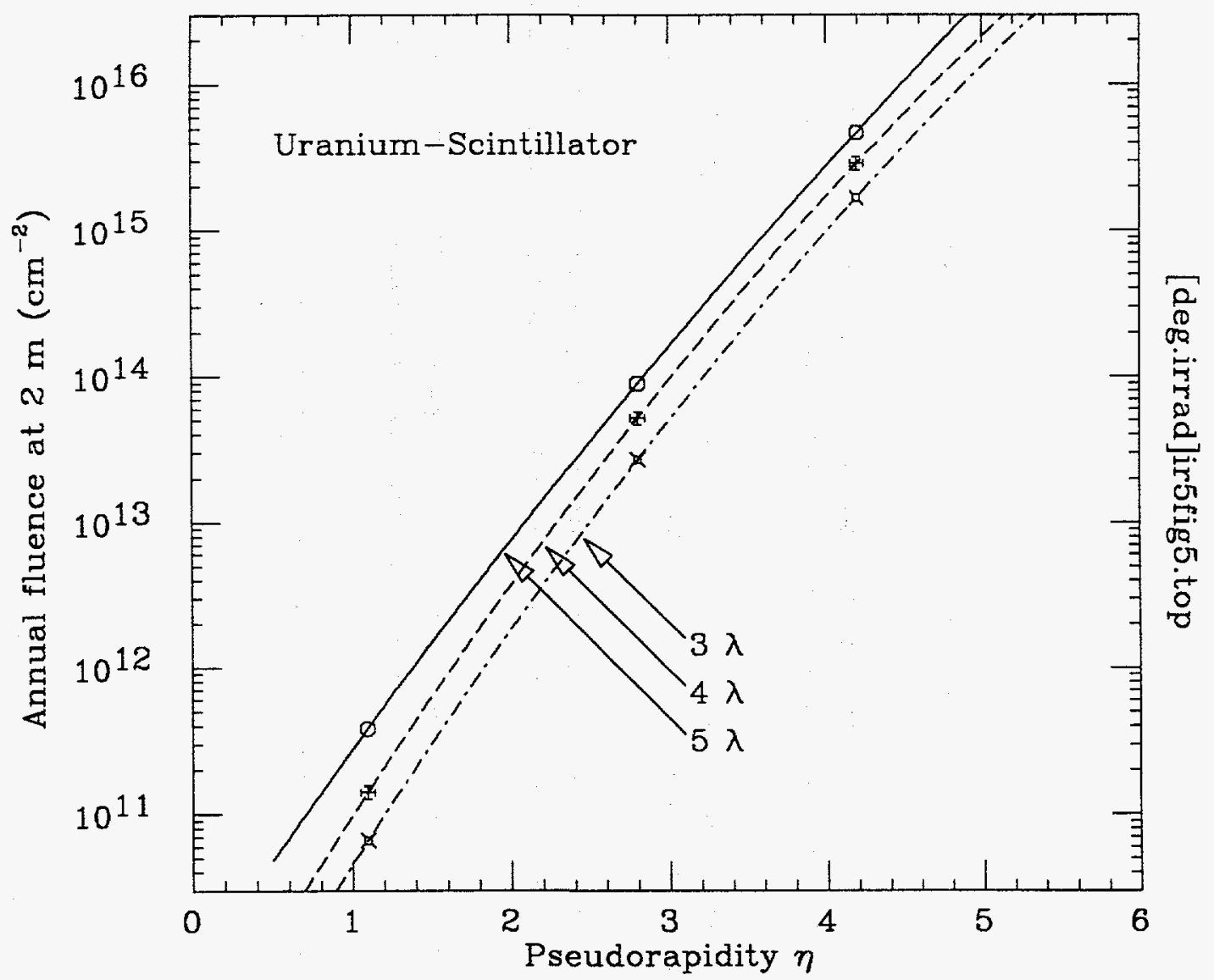

FIG. 5-5. Annual fluence at the rear of a uranium/scintillator calorimeter at $200 \mathrm{~cm}$ from the interaction point as a function of pseudorapidity and calorimeter depth, in interaction lengths.

\section{Maximum neutron flux in a calorimeter}

In Section 4 it was shown that the maximum neutron flux within a uranium/scintillator calorimeter could be represented by $18 p^{0.67}$ for $p$ in $\mathrm{GeV} / \mathrm{c}$ and an incident flux of 1 particle $\mathrm{cm}^{-2}$. If we assume that the maximum flux occurs at a radius of $200 \mathrm{~cm}$, the annual fluence is $3.8 \times 10^{11} \cosh ^{2.67} \eta \mathrm{cm}^{-2}$, as shown in fig. 5-4. 


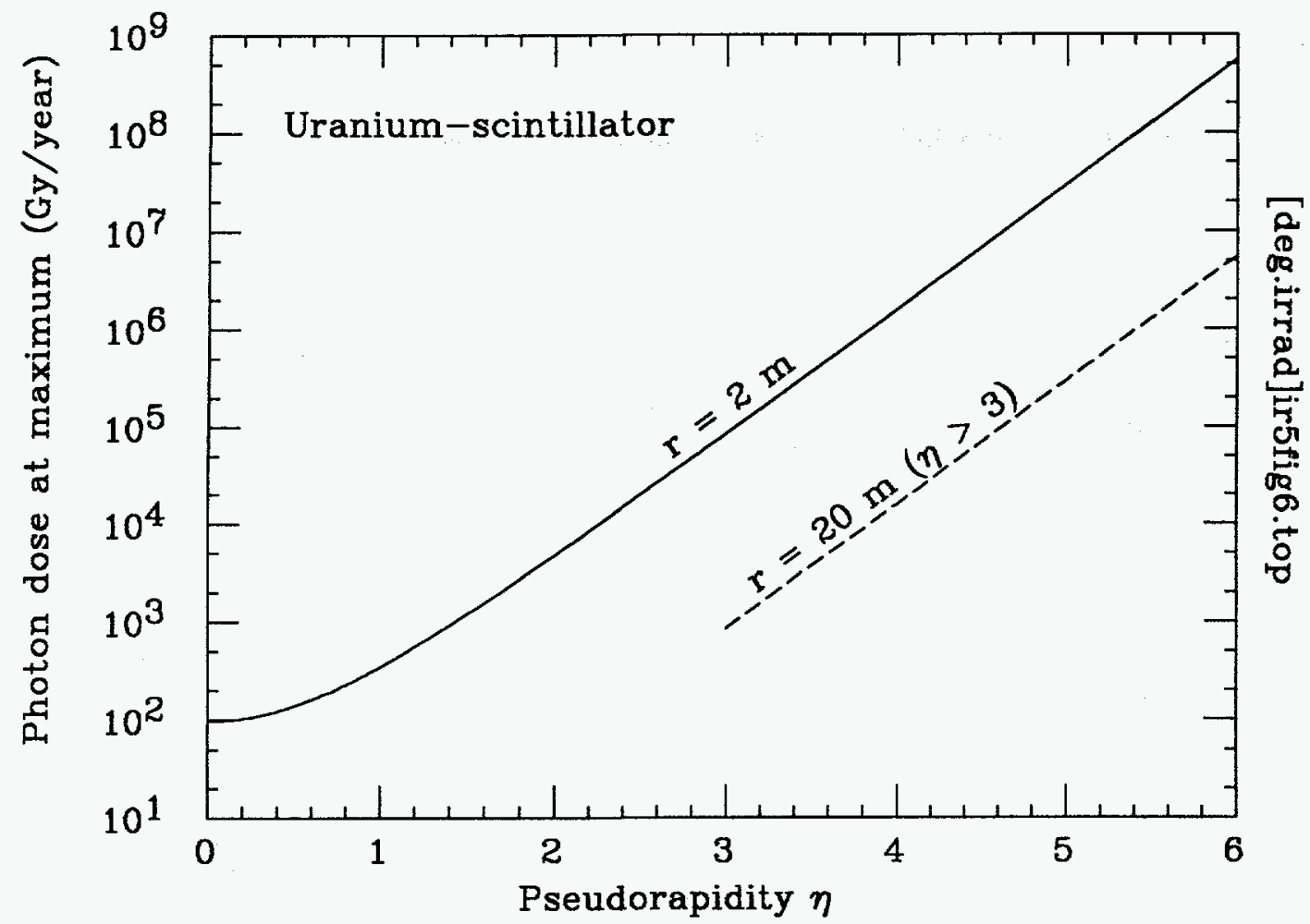

FIG. 5-6. The maximum dose from incident photons. The full curve assumes the maximum occurs at $200 \mathrm{~cm}$. The other curve is calculated for $20 \mathrm{~m}$, typical of forward detectors.

\section{Rear leakage of neutrons from a calorimeter}

Finally, there is the question of rear leakage from a "thin" calorimeter. We follow the same procedure, using the data from Appendix 13 as summarized in Table 4-2. The annual fluence of charged hadrons is $1.2 \times 10^{15} \cosh ^{2} \eta / r^{2}$. We again take $r=200 \mathrm{~cm}$ (this time to the end of the calorimeter), and relate pseudorapidity to the tabulated energies via $\cosh \eta=p /\left\langle p_{\perp}\right\rangle$ to calculate the points shown in Fig. 5-5. The curves are parabolae drawn through the points.

Because of the way the points are obtained, an increase in $\left\langle p_{\perp}\right\rangle$ has the effect of moving the points downward and to the left, with the net effect that the curves are raised. Increasing $\left\langle p_{\perp}\right\rangle$ by $30 \%$ is almost equivalent to reducing the calorimeter thickness by $1 \lambda$. This is hardly surprising, since particles with a harder spectrum produce more penetrating cascades.

\subsection{Dose distribution}

\section{Maximum electromagnetic dose in a calorimeter}

Estimates of the total dose from both electromagnetic and hadronic showers have been given in Appendix 19. Estimates of the dose from incident photons alone have been described in Section 3 and in Appendix 7. We first consider the 


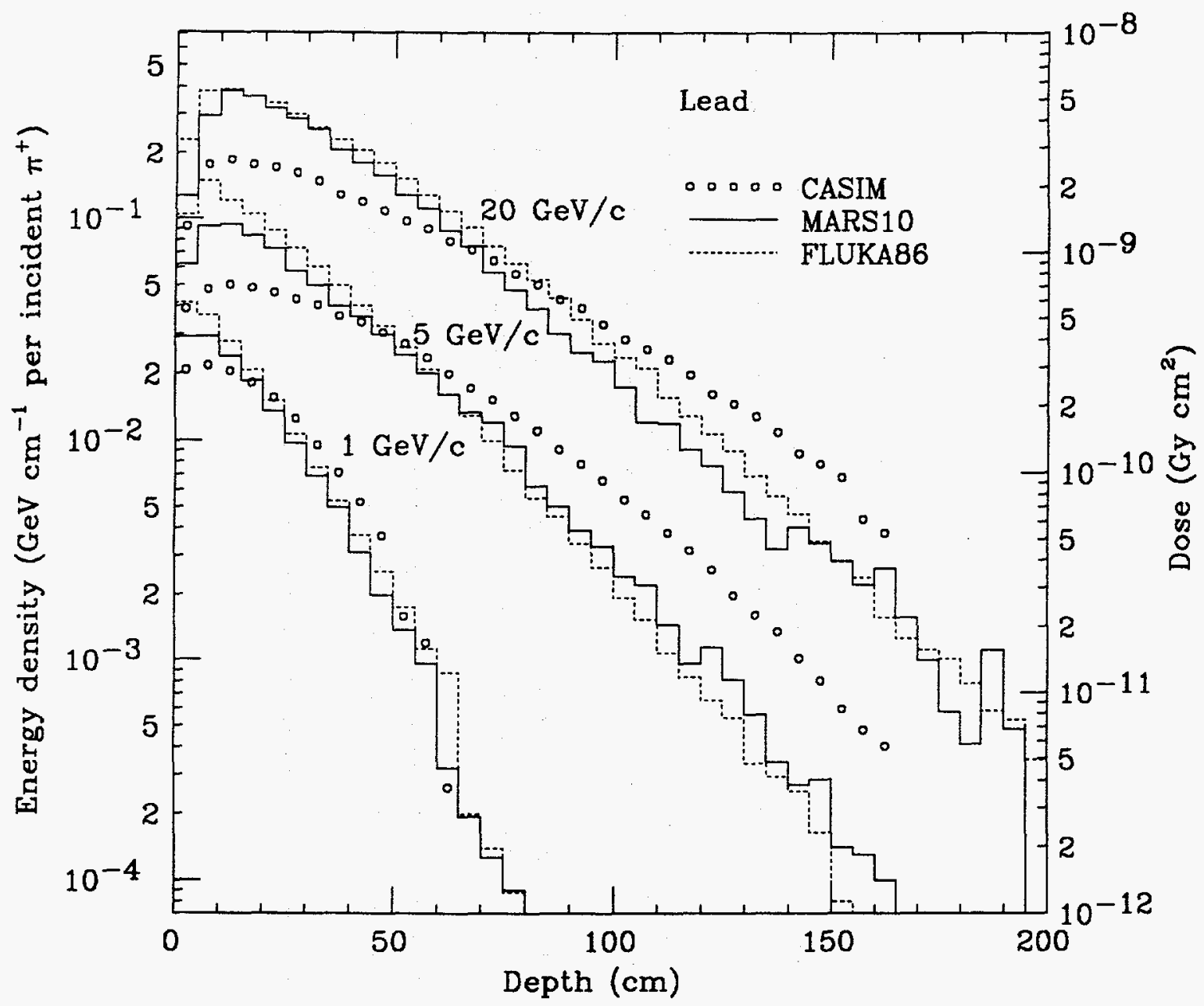

FIG. 5-7. The dose from hadronic showers from incident pions of the energies shown. The dose is given in Gy per incident particle $\mathrm{cm}^{-2}$ vs. depth in solid lead.

dose from incident photons alone. In Appendix 20 it is shown that this does not depend strongly on the calorimeter composition. In Section 3 we used the results of Appendix 7 to represent the maximum dose as

Dose $\left(\mathrm{Gy} /\right.$ incident photon $\left./ \mathrm{cm}^{2}\right) \approx\left(1 \times 10^{-8}\right) \mathrm{E}^{0.93}$

with $E$ in $\mathrm{GeV}$. Using this formula and the methods described above, the maximum dose from incident photons is found to be $97 \cosh ^{2.93} \eta$ at $r=200 \mathrm{~cm}$. The results are shown in Fig. 5-6.

Maximum hadronic dose in a calorimeter

N. Mokhov has compared the results of several cascade codes in an attempt to obtain a good maximum dose estimate for hadronic cascades (Appendix 19). The simulations were made for a lead target, since A. Van Ginneken has pointed out that the density, radiation length, and nuclear interaction length of lead 


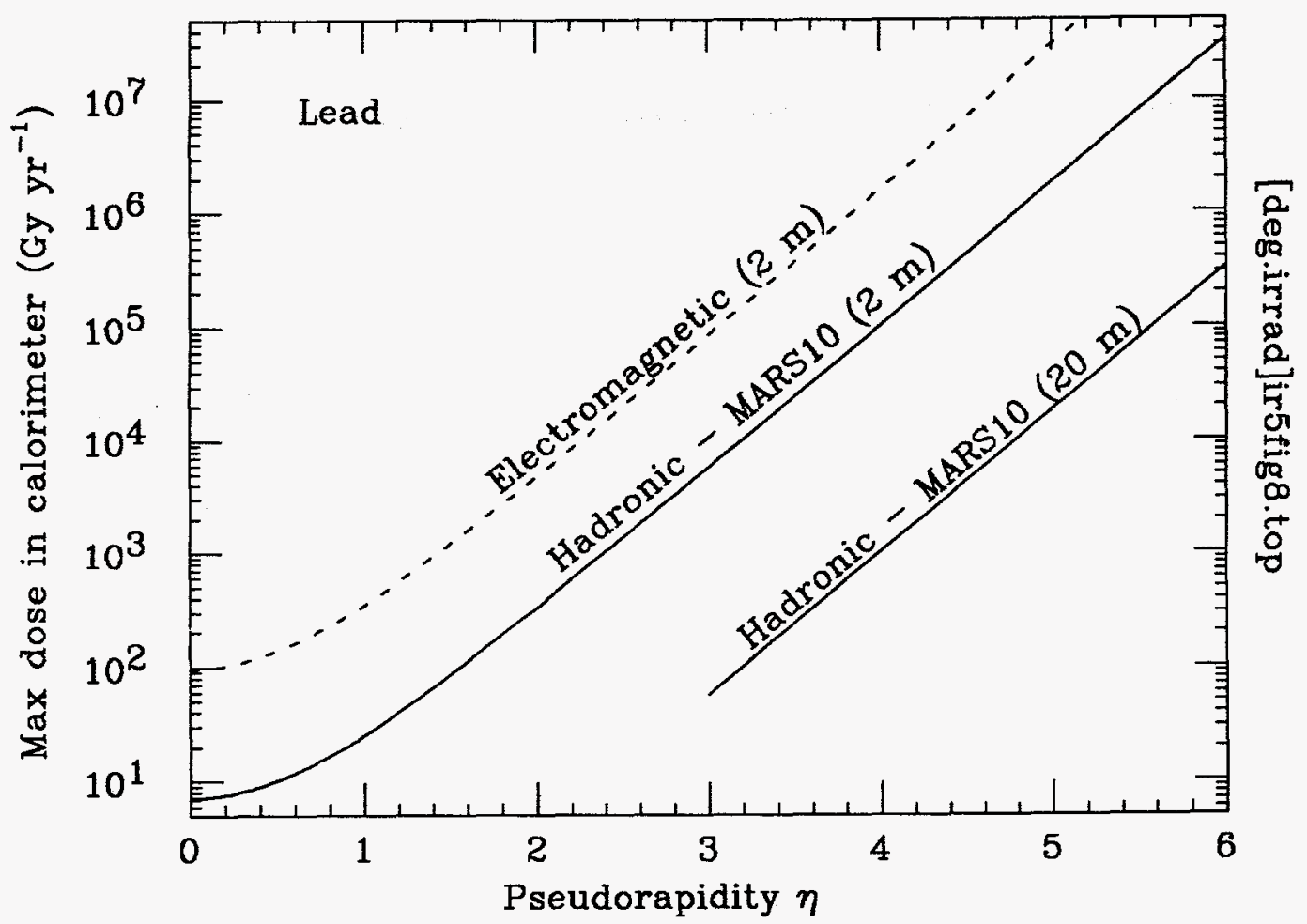

FIG. 5-8. The maximum hadronic dose as a function of pseudorapidity for a lead sphere, assuming that the maximum dose occurs at the indicated radius. The electromagnetic maximum dose (U/scint.) is copied from Fig. 5-6 for comparison purposes.

are almost identical to those of the fine-sampling uranium/scintillator we have taken as a reference. Mokhov's results are summarized in Fig. 5-7. It is felt that CASIM underestimates the dose near the maximum and overestimates it in the tails. A higher energy threshold than is used in MARS10 is thought to be largely responsible for the higher maximum dose obtained with FLUKA86. At any rate, we use the MAR10 results for our present purposes. According to the fits shown in Fig. A19-3, the laterally integrated dose per incident hadron is well described

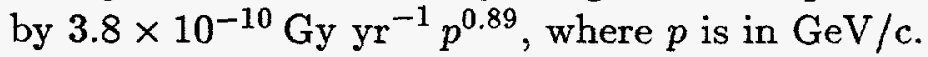

This is 27 times smaller than the maximum dose induced by a photon with the same momentum. Unlike electromagnetic showers which are well localized, hadronic showers deposit energy much more evenly throughout a block of material-roughly in the ratio of absorption length to radiation length, which in this case is 30 . Thus the maximum dose in a typical SSC calorimeter will arise from electromagnetic showers created by incident photons.

Folding with our assumed production spectrum yields $7.2 \cosh ^{2.89} \eta$ for the maximum dose due to cascades initiated by hadrons, as shown in Fig. 5-8. An electromagnetic dose curve from Fig. 5-6 has been added for comparison. 


\section{Discussion}

Calculations of both the electromagnetic and total dose for spherical shell calorimeters of different materials are given in Appendix 20. The results differ from those given above because of lateral leakage in the cascades and the effects of backscattered hadrons. The situation is analyzed in Appendix 21. At small angles lateral diffusion feeds energy from smaller to somewhat larger angles, while at large angles particles backscattered from the "hot spots" near the beam line are more important than those coming from the interaction region.

We have so far neglected the effects of a magnetic field. If a solenoidal field is present, it introduces a cutoff at $p_{\perp}=p_{\perp 0}$ which must be taken into account in calculated the effects of charged particles on calorimeters. The formalism for doing this is introduced in Section 2, and the relevant numerical integral is parameterized in terms of $x_{0}=2 p_{\perp 0} /\left\langle p_{\perp}\right\rangle$. However, this is useful only at wide angles, where doses are small in any case. If a particle leaves the end of the solenoid before reaching the coil, then $p_{\perp 0}$ must be reduced accordingly. For all practical purposes, the field does little to reduce radiation effects produced by charged hadrons. 


\section{REFERENCES}

1. Physics at the Superconducting Super Collider, Eds. P. Hale and B. Winstein (June 1984).

2. Proceedings of the 1984 Summer Study on the Design and Utilization of the Superconducting Super Collider, Eds. R. Donaldson and J. Morfin, Snowmass, Colorado (1984).

3. Report of the Task Force on Detector Research and Development for the SSC, SSC Central Design Group Report SSC-SR-1021 (June 1986).

4. Proceedings of the Workshop on Physics at Future Accelerators, Vols. I and II of CERN Report 87-07 (1987).

5. M. M. Block and R. N. Cahn, Phys. Lett. 188B, 143 (1987); U. Amaldi et al., Phys. Lett. 66B, 390 (1977).

6. D. E. Groom, "The Total p-p Cross Section at $40 \mathrm{TeV}$," SSC Central Design Group Report SSC-N-154 (1986).

7. E. Eichten, I. Hinchliffe, K. Lane, and C. Quigg, Rev. Mod. Phys. 56, 579 (1984).

8. A. Di Ciaccio, et al.(UA1 Collaboration) in Multiparticle Dynamics 1986, ed. by M. Markytan, W. Majerotto, and J. MacNaughton (Seewinkel, Austria, 1986, ISBN 9971-50-177-5) p. 679.

9. T. A. DeGrand, Nucl. Phys. B151, 485 (1979).

10. V. A. Abramovskii, O.V. Kanchelli and V.N. Gribov, in XVI International Conf. on High Energy Physics (Batavia, IL, 1973) Vol. 1, p. 389

11. J. Ranft, P. Aurenche, F. W. Bopp, A. Capella, K. Hahn, J. Kwiecinski, P. Maire, and J. Tran Thanh Van, SSC-Report to be published 1987; P. Aurenche, F. W. Bopp, and J. Ranft, in Multiparticle Dynamics 1986, ed. by M. Markytan, W. Majerotto, and J. MacNaughton (Seewinkel, Austria, 1986, ISBN 9971-50-177-5) p. 589.

12. A. Capella, J. Tran Thanh Van and J. Kwiecinski, Phys. Rev. Lett. 58, 2015 (1987).

13. J. C. Collins, in Supercollider Physics, ed. by D. E. Soper (Eugene, OR, 1985, ISBN 9971-50-051-5) p. 62.

14. F. Olness, private communication.

15. F. E. Paige and S. D. Protopopescu, in Physics of the Superconducting Supercollider, ed. by R. Donaldson and J. Marx (Snowmass CO, 1986) p. 320.

16. H. U. Bengtsson and T. Sjostrand, ibid., p. 311.

17. D. W. Duke and J. F. Owens, Phys. Rev. D30, 49-54 (1984).

18. G. J. Alner et al. (UA5), Z. Phys. C 33, 1-6 (1986).

19. S. Errede and A. Para, private communications (May 1988).

20. T. Alexopoulos et al. (C0), Phys. Rev. Lett. 60, 1622 (1988).

21. M. L. Perl, High Energy Hadron Physics, John Wiley \& Sons, New York (1974), p. 189.

22. M. Jacob, in Proceedings of the Sixteenth International Conference on High Energy Physics, 1972, (National Accelerator Laboratory, Batavia IL 1973).

23. M. Abramowitz and I. A. Stegun, Handbook of Mathematical Functions, National Bureay of Standards Applied Mathematics Series \# 55 (1964).

24. T. A. Gabriel and R. T. Santoro, "Calculation of the Long-Lived Activity in Soil Produced by $500 \mathrm{GeV}$ Protons," Oak Ridge National Laboratory Report ORNL-TM-3262 (1970). 
25. A. Van Ginneken and M. Awschalom, High Energy Particle Interactions in Large Targets. Vol. 1, Hadronic Cascades, Shielding, Energy Deposition, Fermilab, Batavia IL (undated and unnumbered report).

26. T. A. Gabriel, F. S. Alsmiller, R. G. Alsmiller, Jr., B. L Bishop, O. W. Hermann, and D. E. Groom, "Preliminary Simulation of the Neutron Flux Levels in the Fermilab Tunnel and Proposed SSC Tunnel," SSC Central Design Group Report SSC-110 (1987).

27. R. H. Thomas and G. R. Stevenson, Radiological Safety Aspects of the Operation of Proton Accelerators, IAEA Technical Report Series (1987) (to be published).

28. C. Leroy, Y. Sirois, and R. Wigmans, Nucl. Inst. and Meth. A252, 4 (1986).

29. A. Van Ginneken, Fermilab Report FN-272, Fermilab, Batavia, IL, USA (1975). 


\section{APPENDIX 1 MEMBERSHIP OF THE TASK FORCE}

The members of the SSC Task Force on Radiation in the SSC Interaction Regions are as follows:

Frances S. Alsmiller

R. G. (Tut) Alsmiller, Jr. Syuichi Ban

James E. Brau Kenneth W. Edwards Alberto Fassò Harm Fesefeldt Tony A. Gabriel Murdock G. D. Gilchriese Donald E. Groom Hideo Hirayama Heinrich Kowalski Hobart W. Kraner Nikolai V. Mokhov

David R. Nygren Frank E. Paige Johannes Ranft James Russ

Helmut Schönbacher Todor Stanev

Graham R. Stevenson Andreas Van Ginneken Edward M. Wang Richard Wigmans Thomas P. Wilcox, Jr.
Oak Ridge National Laboratory

Oak Ridge National Laboratory

Radiation Safety Group, KEK (Japan)

University of Tennessee

SSC Central Design Group

CERN

Technische Hochschule, Aachen

Oak Ridge National Laboratory

SSC Central Design Group [Chairman]

SSC Central Design Group

Radiation Safety Group, KEK (Japan)

Deutches Elecktronen-Synchrotron

Brookhaven National Laboratory

Institute for High Energy Physics,

Serpukhov, U.S.S.R.

Lawrence Berkeley Laboratory

Brookhaven National Laboratory

Karl Marx Universität (GDR)

Carnegie-Mellon University

CERN

Bartol Research Foundation

CERN

Fermi National Accelerator Laboratory

Lawrence Berkeley Laboratory

Nikhef, Amsterdam

Lawrence Livermor? National Laboratory 



\title{
APPENDIX 2 \\ SOFT AND HARD SCATTERING AT SSC
}

\author{
Frank E. Paige \\ Physics Department \\ Brookhaven National Laboratory \\ Upton, NY 11973, USA
}

The interplay between soft and semi-hard scattering at SSC energies is discussed. It is argued that hard scattering has a negligible effect on the total cross section, although it does affect the multiplicity.

\section{Introduction}

The traditional picture of multiparticle production is that the total cross section, the multiplicity, and related quantities all vary slowly with energy. Indeed, this is sometimes called " $\log s$ physics." For the total cross section one can give a simple geometrical argument for this behavior. Since the interaction is strong, there must be a strong absorption over the area of the proton, which is set by $1 / m_{\pi}^{2}$, so

$$
\sigma_{\text {total }} \geq \text { const. }
$$

One can also prove from unitarity and analyticity the Froissart bound [1] on the rate of growth of the cross section:

$$
\sigma_{\text {total }} \leq \text { const } \times \log ^{2} s .
$$

Hence the cross section cannot vary too rapidly. The experimental data up to $\sqrt{s}=900 \mathrm{GeV}$ are fully consistent with the form [4]

$$
\sigma_{\text {total }}=A+\beta\left[\log ^{2}\left(\frac{s}{s_{0}}\right)-\frac{\pi^{2}}{4}\right]
$$

in agreement with this simple picture.

There is no similar rigorous bound on the multiplicity except the kinematic one $\bar{n}<$ const $\times \sqrt{s}$, but any picture of particle production as coming from the breaking of a confining tube of QCD flux naturally leads to an expectation of slow variations. This again is consistent with all available data.

In the $S \bar{p} p S$ energy range the cross section for low- $p_{\perp}$ minijets is calculated to be a significant fraction of the total cross section. With the EHLQ [2] structure 
functions it is found that

$$
\sigma\left(\sqrt{s}=630 \mathrm{GeV}, p_{\perp}>3 \mathrm{GeV}\right) \approx 10 \mathrm{mb}
$$

compared to a total cross section of about $65 \mathrm{mb}$. The experimental data are not inconsistent [3]with this value, although it is not really clear that the jets being observed actually correspond to QCD hard scattering. The calculated QCD cross sections continue to increase rapidly up to SSC energies, so that

$$
\sigma\left(\sqrt{s}=40 \mathrm{TeV}, p_{\perp}>3 \mathrm{GeV}\right) \approx 200 \mathrm{mb}
$$

This paper discusses the implications of such a large hard-scattering cross section for the total cross section and for multiparticle production at the SSC.

\section{Hard-Soft Interplay}

This section is based on work with G. Marchesini, but very similar ideas have been advanced by Aurenche, Bopp, and Ranft [5], by Sjostrand and van Zij1[6], and by Kajantie, Landshoff, and Lindfors [7].

It is wrong to calculate the total cross section by adding the cross sections for hard and soft scattering. Indeed, this would violate the Froissart bound, since the hard cross section grows faster than any power of $\log s$ as $s \rightarrow \infty$. The hard and soft processes must be combined in way which respects unitarity, so that at any impact parameter $b$ the imaginary part of the amplitude is less than unity: a proton cannot be blacker than black. The simplest way to insure this is to use the eikonal representation. Then the elastic amplitude is

$$
F\left(s, t=-\vec{q}^{2}\right)=2 i s \int d^{2} b e^{i \vec{q} \cdot \vec{b}}\left[1-e^{-A(s, b)}\right]
$$

where $A(s, b)$ is called the eikonal function. If $A(s, b)$ grows like a power of $s$ and if it falls exponentially in $b$, as expected in any theory with massive particles,

$$
A(s, b) \sim s^{\alpha} e^{-\mu b}
$$

then proton becomes black disk of radius $R \sim \log s$ and

$$
\sigma_{\text {total }} \sim \log ^{2} s
$$

This is a heuristic justification of the Froissart bound. 
It is instructive to consider a toy model in which the soft and hard contributions to eikonal are added:

$$
A(s, b)=A_{\text {soft }}(s, b)+A_{\text {hard }}(s, b) .
$$

The soft contribution is determined by fitting existing data. If the fit were exact, it would of course include both hard and soft contributions, but since it dominated by low-energy $(\sqrt{s} \leq 63 \mathrm{GeV})$ data, it effectively determines only the soft contribution. The hard contribution is calculated from the perturbative QCD cross section for jets above some minimum threshold, here taken to be $p_{\perp}>2$, 3 , or $4 \mathrm{GeV}$. This model obviously cannot be exact, since it involves an arbitrary separation between soft and hard scattering, but it does insure that at least the trivial constraints of unitarity are satisfied.

A fit of the eikonal type to the total and elastic cross sections has been made by Bourrely, Sofer, and Wu (BSW) [8]. The form of the fit was motivated by the analysis by Cheng and $\mathrm{Wu}[9]$ of the high energy behavior of a massive Abelian gauge theory (massive QED). In this theory the leading-log series comes from an iteration of vector boson exchange in the $t$ channel and gives a total cross section which grows like a power of $s$,

$$
\sigma \sim \frac{s^{c}}{\log ^{d} s}, \quad c=\mathcal{O}\left(\alpha^{2}\right),
$$

violating the Froissart bound. Cheng and Wu argued that unitarity should be restored by treating this cross section as an eikonal. BSW fit the available data with this form, obtaining

$$
\begin{aligned}
A_{\text {soft }}(s, b) & =S(s) F\left(b^{2}\right)+R\left(s, b^{2}\right) \\
S_{\text {soft }}(s) & =s^{c} /(\log s)^{c^{\prime}}+u^{c} /(\log u)^{c^{\prime}} \\
c & =0.167, \quad c^{\prime}=0.748 \\
\tilde{F}_{\text {soft }}(t) & =f \frac{1}{\left(1-t / m_{1}^{2}\right)^{2}\left(1-t / m_{2}^{2}\right)^{2}}\left(\frac{a^{2}+t}{a^{2}-t}\right)
\end{aligned}
$$

where $R$ is an irrelevant Regge term.

The connection between $A_{\text {hard }}(s, b)$ and the perturbative QCD cross section is provided by the analysis of Abramovskii, Kanchelli, and Gribov (AKG)[10]. The object is to interpret the elastic amplitude in terms of $s$-channel intermediate states, or cuts:

$$
\operatorname{Disc} F(s, 0)=2 i \operatorname{Im} F(s, 0)=\sum_{n}|\langle p p|S| n\rangle|^{2} .
$$

First neglect $A_{\text {hard }}$ and consider the expansion of $F$ in powers of $A_{\text {soft }}$. The term 
with $N$ powers of $A_{\text {soft }}$ corresponds to $N$ exchanges, and $\operatorname{Disc} F(s, 0)$ is given by a sum over all combinations with $K$ of these cut and $N-K$ not cut. If $A_{\text {soft }}$ is an elementary Pomeron (or a QCD string, or a tower in the analysis of Cheng and $\mathrm{Wu}$ ), then it gives an average multiplicity $\bar{n}$ with only Poisson fluctuations. Thus the $K$ cut Pomerons contribute a multiplicity $K \bar{n}$, and the $N-K$ uncut ones produce absorptive corrections. (A cut through only part of a Pomeron leaves a large mass hanging from a single line and so is suppressed by a power of s.) By a simple combinatorial analysis, it is found that terms with $N>1$ do not contribute to the average multiplicity: the absorption exactly cancels the extra multiplicity from the multiple cuts.

For perturbative QCD calculations, only a single power of $A_{\text {hard }}$ is important, and it must be cut to produce the hard scattering final state. Hence

$$
\int d^{2} b \operatorname{Re} A(s, b)=\frac{1}{2} \sigma_{\mathrm{QCD}} .
$$

The sum over all cut and uncut soft exchanges does not change the cross section because some soft scattering must occur with unit probability $[11,12]$. When the hard cross section becomes large, then higher powers of $A_{\text {hard }}$ become important. Events contain multiple hard scatterings, and hard scattering contributes to the absorption. As before, the sum over all cut and uncut exchanges does not change the multiplicity $\bar{n} \sigma_{\text {total }}$, which is given by the perturbative QCD formula.

Once the discontinuity of $A_{\text {hard }}$ is determined, the phase can be calculated from the requirements that $-i A_{\text {hard }}$ be real analytic in the cut $s$ plane and symmetric under $s \leftrightarrow u$. A simple approximation to this phase is provided by the derivative analyticity relation, which for this case is

$$
\begin{aligned}
\operatorname{Im} A & =-\cot (\pi \alpha / 2) \operatorname{Re} A, \\
\alpha & =\frac{\partial \log \sigma}{\partial \log s}+1 .
\end{aligned}
$$

This approximation is badly behaved at low $s$, where the QCD cross section is varying rapidly but is very small, but it is reasonable over most of the interesting region.

To complete the toy model, the $b$ dependence for hard scattering must be specified. This is not known, but the partons are presumably distributed over an area no larger than the size of the proton, and the hard interaction itself is short range, so it is reasonable to assume

$$
\begin{aligned}
\operatorname{Re} A(s, b) & =\frac{1}{2} \sigma_{\mathrm{QCD}}(s) F_{\text {hard }}(b) \\
F_{\text {hard }}(b) & =k_{b}^{2} F_{\text {soft }}\left(k_{b} b\right)
\end{aligned}
$$


where $k_{b} \geq 1$ is a parameter. In what follows the values $k_{b}=1, \sqrt{2}$, and 2 are used; the central value is rather close to the ratio of the average transverse momenta for beam jet particles in hard scattering and minimum bias events.

Given this toy model, the addition of hard scattering to the eikonal function makes a small change in $\sigma_{\text {total }}$ for all choices of $k_{b}$ and the $p_{\perp}$ cutoff; see Figs. 1 and 2. At $\sqrt{s}=40 \mathrm{TeV}$ for $k_{b}=\sqrt{2}$ and $p_{\perp}>3 \mathrm{GeV}$,

$$
\begin{aligned}
\sigma_{\text {total }} & =125 \mathrm{mb} \rightarrow 133 \mathrm{mb} \\
\sigma_{\text {elastic }} & =38 \mathrm{mb} \rightarrow 43 \mathrm{mb}
\end{aligned}
$$

which is surely less than the uncertainty in the fit to the soft amplitude. The value of $\rho$, the ratio of the real to the imaginary part of the elastic amplitude, is also increased, but it is still well below the black disk limit, $\rho=.5$. At $\sqrt{s}=630 \mathrm{GeV}$ the increase of $\rho$ is more significant, but the value is still below the recently reported measurement [13]. Hard scattering should not significantly affect the total cross section at the SSC.

\section{Small $x$ Behavior}

The QCD cross sections used so far have been calculated using the QCDimproved parton model with the EHLQ [2] parton distributions. While $p_{\perp}$ is presumably large enough for QCD perturbation theory to be valid, it is not clear that the parton distributions are correct for $x \sim 10^{-4}$, the relevant region for the SSC. Neither the QCD evolution nor the input parton distributions are tested in this region.

The EHLQ parton distributions are a fit to a numerical solution to the Altarelli-Parisi equations, corresponding to a sum of ladder graphs (in an axial gauge) which give the leading log approximation to QCD. This approximation ignores the recombination of partons, which becomes important when the partons become dense in the proton. The first correction [14] comes from the interaction of two ladders. It is suppressed by a power of $Q^{2}$, and for the EHLQ distributions it is found to be numerically small for all values of $x$ and $Q^{2}$ of interest at the SSC.

The behavior of the parton distributions $x f\left(x, Q_{0}^{2}\right)$ as $x \rightarrow 0$ at the initial scale $Q_{0}^{2}$ is unknown. EHLQ make traditional assumption that

$$
x f\left(x, Q_{0}^{2}\right) \rightarrow \text { const, } \quad x \rightarrow 0,
$$

which corresponds to a constant total cross section. But the validity of this assumption is suspect because the Altarelli-Parisi equations imply a more singular behavior for any $Q^{2}>Q_{0}^{2}$. 


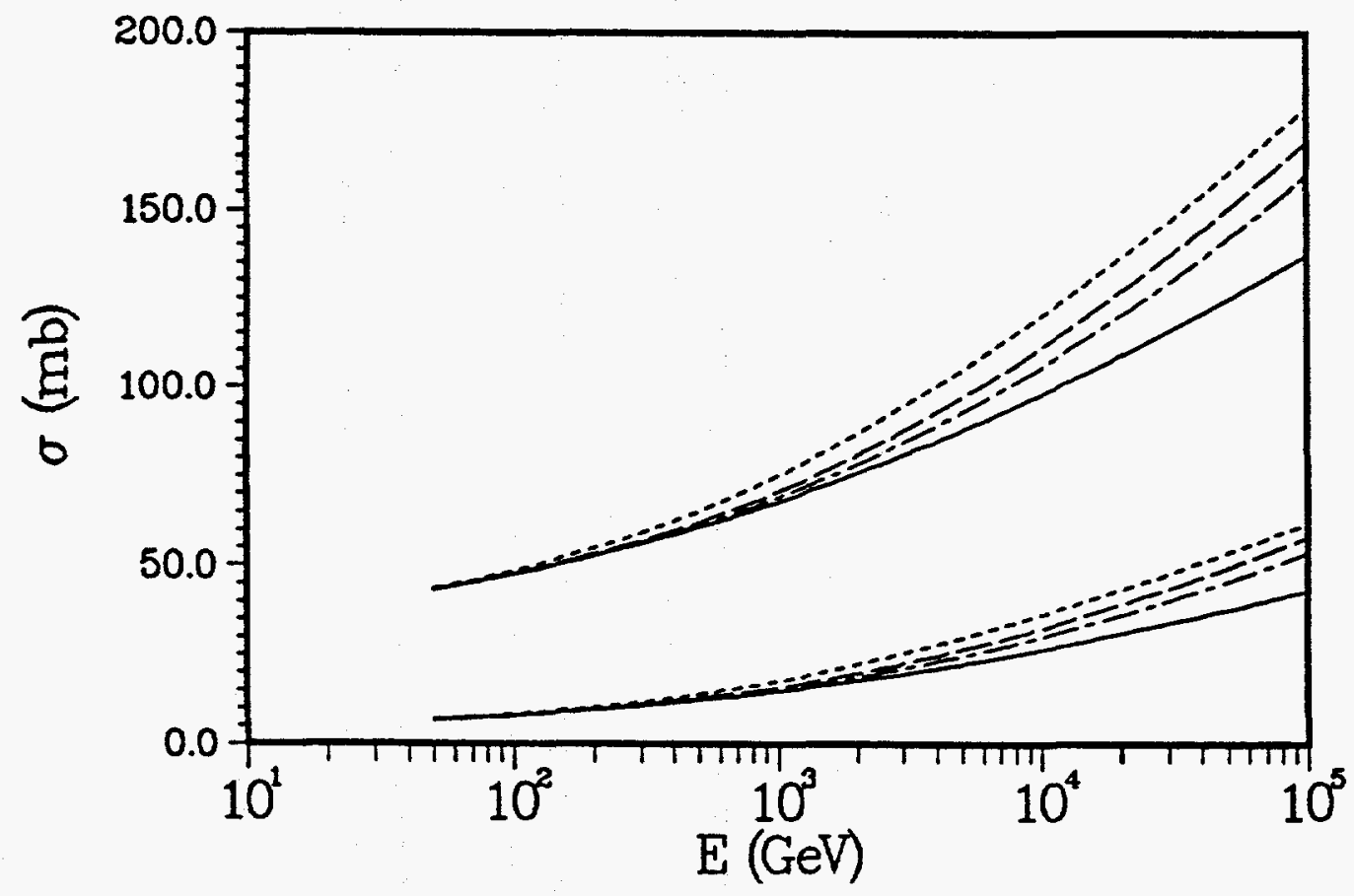

FIG. 1. $\sigma_{\text {total }}$ and $\sigma_{\text {elastic }}$ vs. $\sqrt{s}$ for $k_{b}=1$. Solid curves: BSW fit; Dotted curves: Including $A_{\text {hard }}\left(p_{\perp}>2 \mathrm{GeV}\right)$; Dashed curves: Including $A_{\text {hard }}\left(p_{\perp}>3 \mathrm{GeV}\right)$; Dotdashed curves: Including $A_{\text {hard }}\left(p_{\perp}>4 \mathrm{GeV}\right)$.

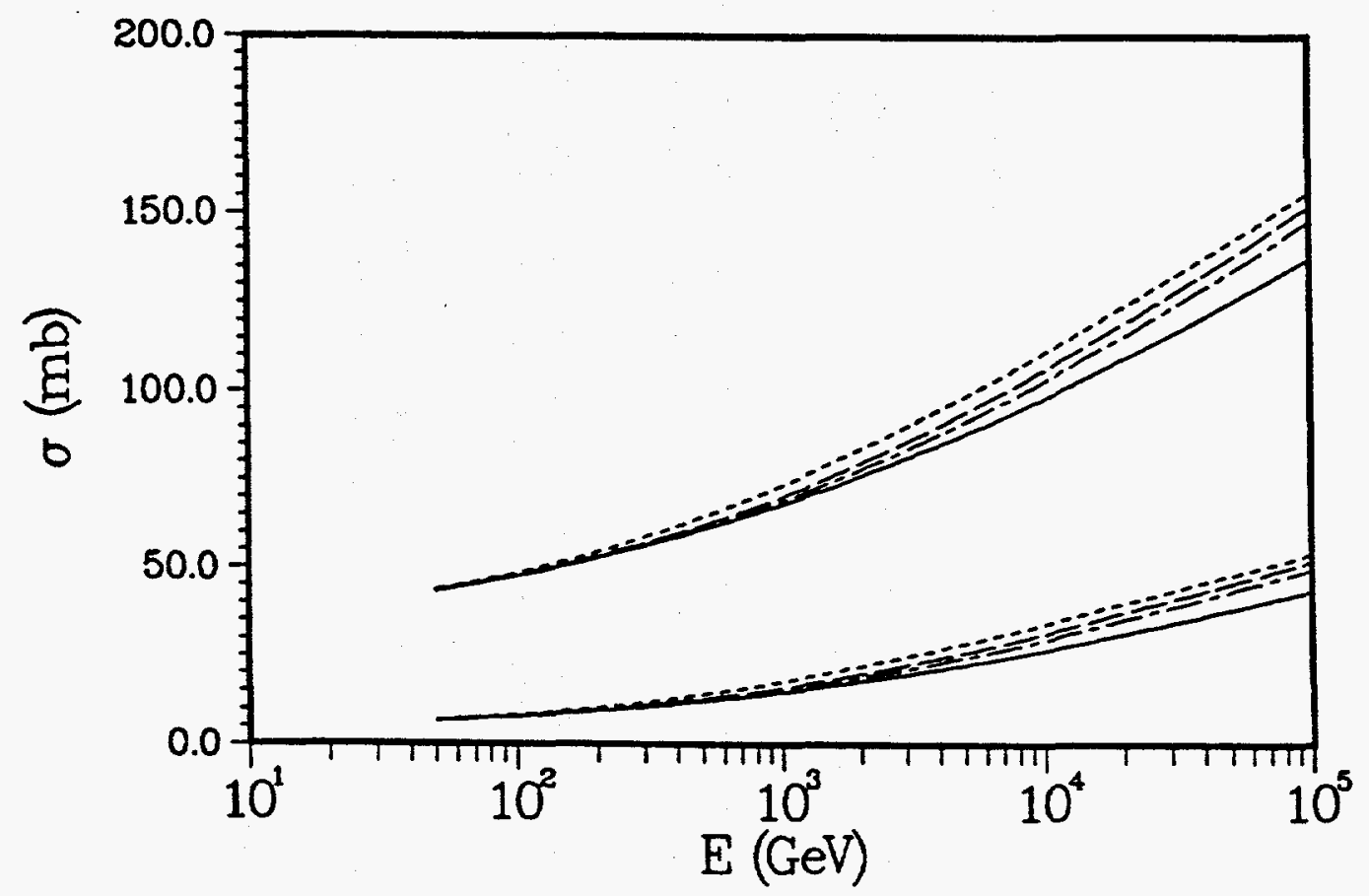

FIG. 2. Same as Fig. 1 for $k_{b}=\sqrt{2}$. 
Collins [15] has noted that a constant behavior is unstable: if it is assumed, then a backwards Altarelli-Parisi evolution implies that $x f\left(x, Q^{2}\right)$ becomes negative for $Q^{2}<Q_{0}^{2}$. Stable results are obtained if

$$
x f\left(x, Q_{0}^{2}\right) \sim \frac{1}{x^{p}}, \quad p=.3-.5
$$

A similar behavior is suggested, but not definitely established, by the Lipatov equation. Using this form with $p=.5$ gives [16]

$$
\sigma\left(p_{\perp}>3 \mathrm{GeV}\right) \approx 4000 \mathrm{mb},
$$

a factor of 20 greater than the EHLQ value. This is probably an overestimate: $p=.5$ is at the upper limit, and recombination effects on the parton distributions have been neglected. Nevertheless, it is clear that there is a large uncertainty in the QCD minijet cross section at SSC energies.

The small- $x$ behavior of the parton distributions can be tested by measuring Drell-Yan pairs with $Q \sim 10 \mathrm{GeV}$ at the Tevatron, since for large rapidity $y$ one $x$ becomes small. Hopefully this will be done soon, but until it is, one must leave a large safety factor in the QCD cross sections.

\section{Structure of Minijet Events}

The EHLQ structure functions give a QCD jet cross section of about $200 \mathrm{mb}$, or about 2 hard scatterings per inelastic collision. Hence the multiplicity is dominated not by the hard scattering jets but by the associated beam jets.

The transverse energy flow $d E_{T} / d y$ on same side as a jet and not near it has been measured at the SppS [3]. It is found to be approximately constant both for jets with $p_{\perp}>20 \mathrm{GeV}$ and for other hard interactions, with a value about twice that for minimum bias events. The increase over minimum bias events comes both from a higher multiplicity and from a higher $\bar{k}_{T} \approx .45 \mathrm{GeV}$. It is not due to initial state radiation, which is too small and scales with $p_{\perp}$. Hence it must be a nonperturbative effect related to the interactions of the spectators.

Between minimum bias events and minijet events with $p_{\perp} \sim 10 \mathrm{GeV}$, the beam jets show [3] an approximately linear dependence on $p_{\perp}$. This may be real dynamics, but it unclear what could set such a scale. Or it may simply reflect contamination with fluctuations of soft events. This interpretation is suggested by the fact that the variable

$$
F=\frac{E_{T}(\Delta R<.2)}{E_{T}(\Delta R<1 .)},
$$

which measures the collimation of the jets, shows a nonuniform behavior below 
$10 \mathrm{GeV}$. To understand these phenomena, more experimental data are needed, including

- Beam jets for interactions with $Q \sim 10 \mathrm{GeV}$ that cannot be fluctuations, including high- $p_{\perp}$ single particles and $\ell^{+} \ell^{-}$pairs.

- The energy dependence of beam jets from the S̄ppS to the Tevatron.

In any case the uncertainty from the associated beam jets is less than that from the behavior of the parton distributions.

\section{Conclusion}

Since multiparticle production involves the strong interaction sector of QCD, it is not well understood theoretically, and any extrapolation to SSC energies is therefore uncertain. There is, however, good reason to expect that the smooth $\log s$ behavior seen in existing data will continue to higher energies.

\section{References}

1. M. Froissart, Phys. Rev. 123, 1053 (1961).

2. E. Eichten, I. Hinchliffe, K. Lane, and C. Quigg, Rev. Mod. Phys. 56, 579 (1984).

3. UA1 Collaboration, A. Di Ciaccio, et al., in Multiparticle Dynamics 1986, ed. by M. Markytan, W. Majerotto, and J. MacNaughton (Seewinkel, Austria), ISBN 9971-50-177-5) p. 679 (1986).

4. M. M. Block and R. N. Cahn, Phys. Lett. 188B, 143 (1987).

5. P. Aurenche, F. W. Bopp, and J. Ranft, in Multiparticle Dynamics 1986, op. cit., p. 589.

6. T. Sjostrand and M. van Eijk, LU TP 87-5 (1987).

7. K. Kajantie, P. V. Landshoff, and J. Lindfors, HU-TFT-87-35 (1987).

8. C. Bourrely, J. Sofer, and T. T. Wu, Phys. Rev. Lett. 54, 757 (1985).

9. H. Cheng and T. T. Wu, Phys. Rev. Lett. 24, 1456 (1970).

10. V. A. Abramovskii, O. V. Kanchelli and V.N. Gribov, in XVI International Conf. on High Energy Physics (Batavia, Ilinois), Vol. 1, p. 389 (1973).

11. J. L. Cardy and G. A. Winbow, Phys. Lett. 52B, 95 (1974).

12. C. E. DeTar, S. D. Ellis, and P. V. Landshoff, Nucl. Phys. B87, 176 (1975).

13. P. Kluit, talk at EPS High Energy Physics Conference, (Uppsala, Sweden, June 1987).

14. A. H. Mueller and J. Qiu, Nucl. Phys. B268, 427 (1986).

15. J. C. Collins, in Supercollider Physics, ed. by D. E. Soper (Eugene, Oregon), ISBN $9971-$ 50-051-5) p. 62 (1985).

16. F. Olness, private communication. 


\title{
APPENDIX 3
}

\author{
DTUJET \\ DUAL TOPOLOGICAL UNITARIZATION OF \\ SOFT AND HARD HADRONIC PROCESSES: \\ PREDICTIONS OF A NEW MONTE-CARLO EVENT \\ GENERATOR FOR BEAM-BEAM COLLISIONS \\ $\mathrm{AT} \sqrt{s}=40 \mathrm{TeV}$ \\ J. Ranft, * \\ SSC Central Design Group, LBL 90-4040, Berkeley CA 94720 \\ in collaboration with \\ P. Aurenche ${ }^{\dagger}$ F. Bopp, ${ }^{\circ}$ A. Capella, ${ }^{x}$ K. Hahn, ${ }^{*}$ \\ J. Kwiecinski, ${ }^{\triangle}$ P. Maire ${ }^{\dagger}$ and J. Tran Thanh $\operatorname{Van}^{x}$
}

\section{Introduction}

The dual topological unitarization of hard and soft hadronic cross sections is a new model of hadronic multiparticle production, which will be described in more detail in a forthcoming SSC-Report [1].

During the last years several groups have studied soft hadronic multiparticle production in the framework of the DTU-model[2-6]. These models and in particular the Monte-Carlo formulation of this model in the form of the dual multi-chain fragmentation model $[5,6]$ are the first starting point for the new model described here.

Experimental observations made it clear, that at collider energies the soft and hard components of hadronic multiparticle production are closely related. These observations are the discovery at the CERN-SPS-collider of correlations between the average transverse momenta of hadrons produced and the multiplicity density in rapidity [7] and the observation of 'minijets' in hadronic collisions and changes of the properties of the underlying soft events in data samples with jets or minijets [8].

Both of these properties were understood within the dual multichain fragmentation model $[6,9]$ by introducing transverse momenta (in addition to intrinsic transverse momenta) with magnitudes, which could only be interpreted to be

\footnotetext{
^ Permanent address: Sektion Physik, Karl-Marx-Universität, Leipzig, G.D.R.

$\dagger$ LAPP, Annecy, France

- Universität Siegen, F.R.G.

$x$ LPTHE, Orsay, France

$\triangle$ Institute of Nuclear Physics, Krakow, Poland
} 
due to hard constituent scattering for the partons at the ends of the fragmenting chains.

The need for an uniform treatment of hard and soft hadronic collisions is furthermore underlined by the fact that the perturbative QCD cross sections for hard constituent scattering rise strongly with energy reaching for transverse momenta greater than $2 \mathrm{GeV} / \mathrm{c}$ values around $200 \mathrm{mb}$ at the energy of the SSC. Since this is larger than the total hadronic cross section at these energies, we expect in average hadronic events in this energy range one or several hard constituent scatterings. At those energies one expects that unitarity corrections should play an important role. Those corrections then inevitably lead to several semi-hard interactions resulting in the increase of the average number of jets.

The perturbative hard constituent scatterings are also one of the processes responsible for the rise of the hadronic cross sections. This was studied quantitatively in papers by Capella, Tran Thanh Van and Kwiecinski [10] and Durand and $\mathrm{Pi}[11]$ where the consequences for the total and inelastic cross sections of the unitarization of soft and hard scattering cross sections were studied. This model as formulated in [10] is the second starting point for the model to be described here.

In Section 2 the basic ideas of the model will be outlined. In Section 3 the total and inelastic hadronic cross-sections are studied within the model, compared to data at present energies and predicted in the SSC energy range. In Section 4 the properties of the two component (hard and soft) multi-Pomeron events according to the model are presented. In Section 5 the model is formulated on the parton level, where the partons are understood as the quarks, antiquarks and diquarks at the ends of the hard and soft multiparticle strings. In Section 6 finally the properties of the model are presented after the fragmentation of all strings into hadrons and the decay of all hadronic resonances. The model is compared to data in the ISR and SPS collider energy range. Subclasses of events are studied with and without hard jets. In Section 7 the predictions of the model in the form of the hadronic Monte-Carlo event generator DTUJET for $40 \mathrm{TeV}$ collisions in the SSC interaction regions are presented in a form suitable to facilitate the study of radiation effects on the collider detectors. Such consequences can also be investigated using events generated by DTUJET. The code DTUJET is described in a SSC Report [12]. 


\section{Unitarization of Soft and Hard Hadron Cross Sections, A Model for Hadron Cross Sections and Multiparticle Production}

We start by describing shortly the dual multistring fragmentation model for soft hadron production. In first approximation the proton is made out of valence quarks $q_{v}$ and diquarks $q q_{v}$. In hadronic collisions (to be definite we consider only $p \bar{p}$ collisions) the color is rearranged between the constituents of the hadrons and two high mass color singlet chains or strings are formed $S_{1}=\left(q q_{v}-\bar{q} \bar{q}_{v}\right)$ and $S_{2}=\left(q_{v}-\bar{q}_{v}\right)$, see Fig. 1a. Via unitarity, the square of diagram 1a generates the imaginary part of the $\bar{p} p$ elastic amplitude, which is dominated at high energy by Pomeron exchange, see Fig. 1b.

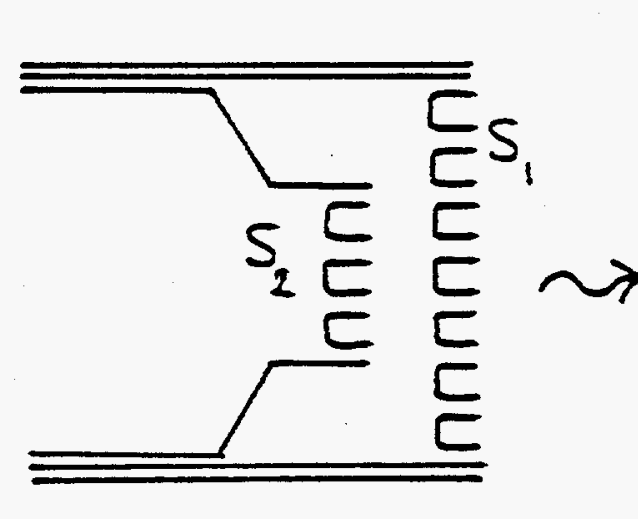

a)

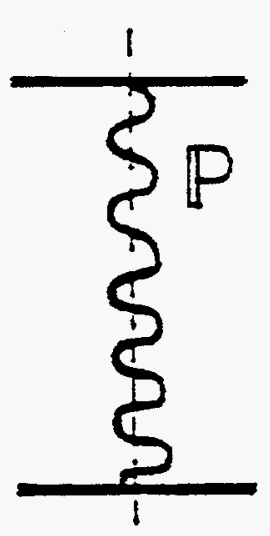

b)

FIG. 1. The s-channel content a) of the imaginary part of the Pomeron exchange in the elastic amplitude b).

In the next level of approximation, the effect of gluons, sea quarks and antiquarks in the incoming hadrons are taken into account. An extra $q \bar{q}$ pair in each of the colliding hadrons leads to the production of 4 strings as shown in Fig. 2. When calculating the elastic cross section, this contribution leads to two-Pomeron exchange.

The weights for the production of 2 strings (one Pomeron exchange), 4 strings (2-Pomeron exchange) and so on are related to the amplitudes for $\mathbf{n}$-Pomeron exchange given by the Gribov (eikonal) model. Using the AGK cutting rules [13] one obtains the probabilities for $\mathrm{N}$-cut Pomerons or equivalently $2 \mathrm{~N}$ string production as used in the dual multichain fragmentation models [2-6]. 


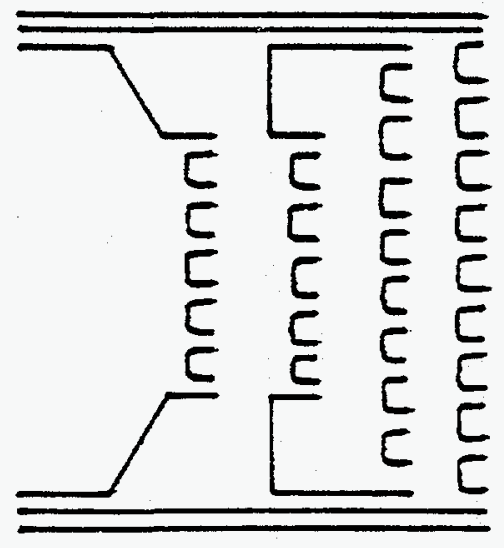

FIG. 2. Production of four strings in proton-antiproton interaction.

At the energy of present and future hadron colliders the hard scattering of quarks and gluons as calculated in QCD perturbation theory [14] becomes a second important mechanism of multiparticle production besides the soft particle production mechanism as described above. In the QCD-parton model the cross section for hard constituent scattering is given by

$$
\sigma_{h}(s)=\sum_{i j} \int d x_{1} \int d x_{2} \int d \hat{t} \frac{d \sigma_{i j}}{d \hat{t}} F_{i}\left(x_{1}, Q^{2}\right) F_{j}\left(x_{2}, Q^{2}\right)
$$

where the $F_{i}\left(x_{i} Q^{2}\right)$ are the parton structure functions of the hadron and the $d \sigma_{i j} / d \hat{t}$ is the perturbative hard scattering cross section of the constituents $i$ and $j$. The cross section (1) makes sense only at scales $Q^{2}$, which are sufficiently large. In practice, this means, that the cross section (1) can only be calculated for transverse momenta of the scattered constituents (jets, minijets) greater than $2 \mathrm{GeV} / \mathrm{c}$. We will use in this paper a minimum transverse momentum for $\sigma_{n}(\mathrm{~s})$ of $p_{\perp, \min }=2 \mathrm{GeV} / \mathrm{c}$. The dash dotted curve in Fig. 3 gives as function of the c.m.s. energy $\sqrt{s}$ the cross section $\sigma_{h}$ as calculated for all constituent subprocesses [14] using the parameterization of the hadron structure functions as given by Eichten, Hinchliffe, Lane and Quigg[15] (EHLQ-1). This cross section rises in the energy region of interest practically as a power of $s$.

It should be stressed that there is considerable theoretical and experimental uncertainty about this rise of $\sigma_{h}$ with the energy. Structure functions like the ones used here postulate a $1 / x$ dependence for gluon- and sea-quark-structure 


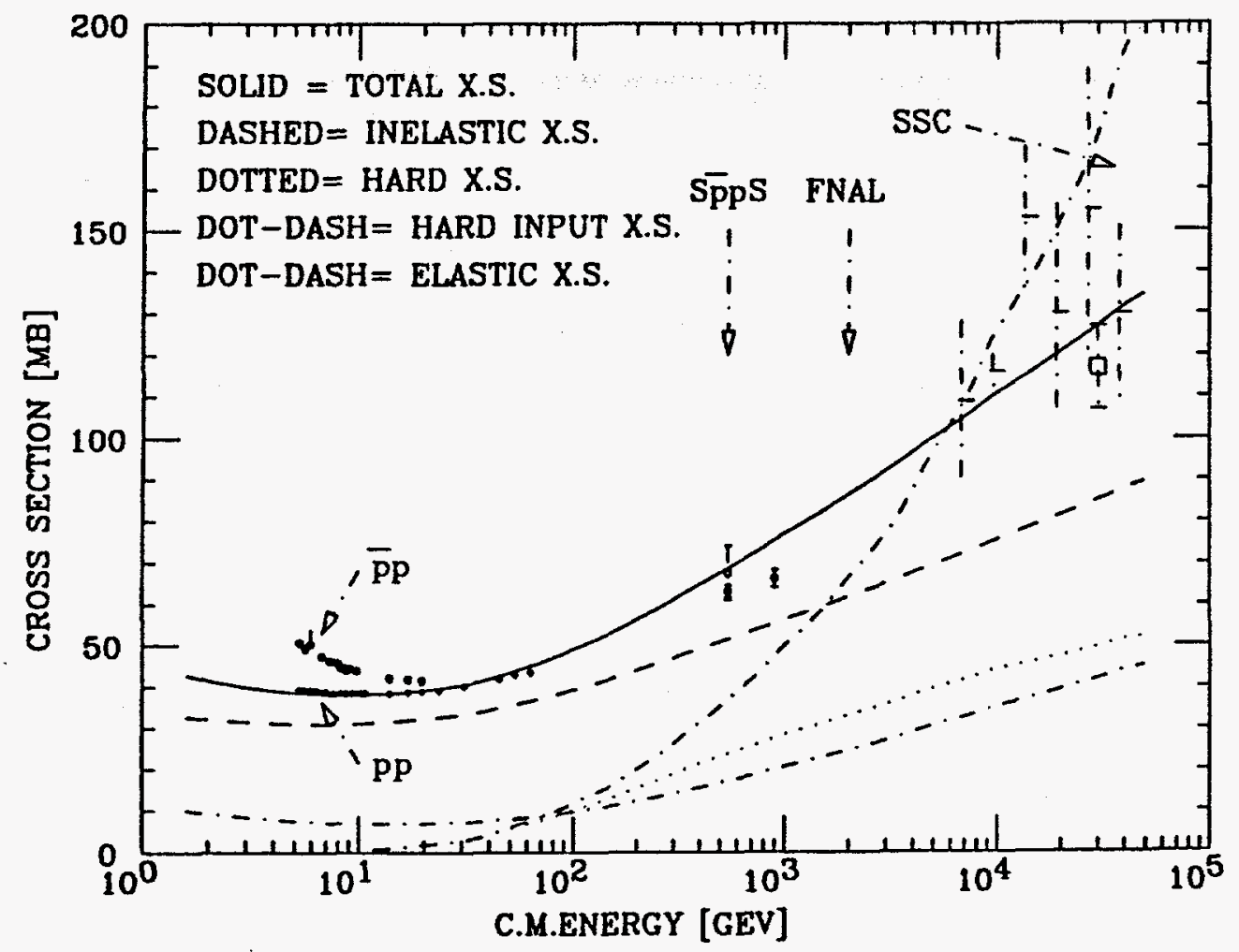

FIG. 3. $\sigma_{\text {total }}, \sigma_{\text {inelastic }}$ and the minijet cross section $\sigma_{h, i n e l}$ as calculated by the unitarization of hard and soft cross sections. We show also the input perturbative hard cross section $\sigma_{h}$ for the production of minijets with $p_{\perp}>2 \mathrm{GeV} / \mathrm{c}$. The total cross section calculated is compared with data at accelerator and cosmic ray energies compiled by Groom [25].

functions at some reference scale $Q_{o}^{2} \approx 5 \mathrm{GeV}^{2}$ but of course the $\mathrm{QCD}$ evolution makes the gluon distribution much steeper at larger $Q^{2}$ values. At the $x$-values of $10^{-4}$ which become important for the production of minijets at SSC energies, the structure functions cannot be determined experimentally at present accelerator energies and there is presently no practical method available which would permit to calculate the structure functions from QCD. It has been argued that the $x$ dependence of the structure functions at these small $x$-values indeed differ from $x^{-1}$. Collins [16] presented arguments for a $x^{-1.5}$ behavior.

The simultaneous unitarization of both hadronic cross sections, the soft and the hard cross sections, was first performed in papers by Capella et al. [10] and Durand et al.[11]. In these models one has two kinds of exchanged Pomerons, soft Pomerons, corresponding to a pair of soft strings in the inelastic cross section and hard Pomerons, corresponding to two hard scattered chains. 
This generalization of the AGK approach leads in our model to a decomposition of the scattering amplitude in terms of contributions with the exchange of say $\ell$ soft and $m$ hard Pomerons corresponding to inelastic events with $2 \ell$ soft and $2 m$ hard strings (large $p_{\perp}$ jets or minijets).

\section{Unitarization of Soft and Hard Hadron Scattering and \\ Total and Inelastic Hadron-Hadron Cross Sections}

In Appendix A we give the expression obtained by Capella et al. [10] for the total and inelastic hadronic cross-sections in terms of the bare (input) hard and soft cross sections $\sigma_{h}$ and $\sigma_{s}$. We use for $\sigma_{h}$ the cross section as obtained by numerical integration of (1) and summing over all constituent subprocesses. The cross section $\sigma_{h}$ obtained is given in Fig. 3 (dot-dashed curve).

For the soft cross sections we use[10]:

$$
\sigma_{s}=a s^{\alpha-1}
$$

with $\alpha=1.076$ and $a=37.8 \mathrm{mb}$. For $\sigma_{T P}$ the expression is

$$
\sigma_{T P}=c\left[\ln \left(b+2 \alpha^{\prime} \ln s\right)-\ln \left(b+2 \alpha^{\prime} \ln 20\right)\right]
$$

with $\alpha^{\prime}=0.24 \mathrm{GeV}^{-2}, b=3.51 \mathrm{GeV}^{-2}$ and $c=40 \mathrm{mb}$. For the slopes we take $b_{s}=b+\alpha^{\prime} \ln s, b_{h}=b$ and $b_{T P}=b_{s}$.

In Fig. 3 we present the total proton-proton cross section obtained from (A1) and compare to date at energies up to cosmic ray energies near the energy of the SSC. This cross section agrees also very well with parameterizations like the ones presented by Block and Cahn [17]. We give in Fig. 3 the inelastic and elastic cross sections $\sigma_{i n e l}$ and $\sigma_{e l}$ and the inelastic hadronic cross section corresponding to events with one or more than one hard jets or minijets $\sigma_{h, \text { inel }}$.

It is remarkable that the cross sections $\sigma_{h, \text { inel }}$ and even $\sigma_{\text {inel }}$ are smaller than the bare hard cross sections $\sigma_{h}$ (or the bare soft cross section $\sigma_{s}$ ).

We expect in average inelastic collisions

$$
\left\langle n_{h}\right\rangle=\sigma_{h} / \sigma_{\text {inel }}
$$

hard Pomerons ( $2 n_{h}$ hard strings) and

$$
\left\langle n_{s}\right\rangle=\sigma_{s} / \sigma_{\text {inel }}
$$

soft Pomerons ( $2 n_{s}$ soft strings). Only in the fraction

$$
\left\langle F_{h}\right\rangle=\sigma_{h, \text { inel }} / \sigma_{\text {inel }}
$$


of all inelastic collisions jets or minijets are expected. The average number of hard Pomerons in this fraction of collisions

$$
\left\langle n_{h, h}\right\rangle=\sigma_{h} / \sigma_{h, i n e l}
$$

is even larger than $\left\langle n_{h}\right\rangle$.

The unitarization scheme leads to rather stable hadronic cross sections when changing the input cross sections $\sigma_{s}$ and $\sigma_{h}$. If we for instance introduce a $K$ factor $K=1.5$ into the calculation of $\sigma_{h}$, we get at $\sqrt{s}=40 \mathrm{TeV}$ an increase of $\sigma_{h}$ from $194 \mathrm{mb}$ to $291 \mathrm{mb}$. This leads to increases of $\sigma_{t o t}$ from 132 to $139 \mathrm{mb}$, of $\sigma_{\text {inel }}$ from 88 to $90 \mathrm{mb}$ and of $\sigma_{h \text {,inel }}$ from 52 to $59 \mathrm{mb}$. Such an increase would however change the structure of the inelastic events more strongly. The increase of $\sigma_{h}$ leads roughly to an corresponding increase of the numbers of jets or minijets $\left\langle n_{h}\right\rangle$ and $\left\langle n_{h, h}\right\rangle$ per event.

\section{The Two Component Multi-Pomeron Model for Inelastic Hadron Interactions}

Due to the alternating sign of the terms with rising powers $\ell$ and $m$, the cross section $\sigma_{\text {inel }}$ as given in (A-2) cannot be interpreted as a sum over cross-sections corresponding to $\ell$ soft and $m$ hard Pomerons. To obtain the cross sections for $n_{s}$ soft and $n_{h}$ hard Pomerons, the cross section $\sigma_{i n e l}$ in (A-2) has to be resummed into a sum with only non-negative terms. In Appendix B such an expression is given. The cross section $\sigma_{i j}$ in (B-1) is the cross section for $n_{s}=i$ cut soft and $n_{h}=j$ cut hard Pomerons. As explained in Appendix B, the corresponding formulae become transparent in the impact parameter representation.

This expression $\sigma_{n_{s}}, n_{h}$ simplifies considerably if one evaluates the sum in the approximation of vanishing $\sigma_{T P}$. In the collider energy range, where we are mostly interested, this is a good approximation since $\sigma_{T P} \ll \sigma_{s}, \sigma_{h}$. All results presented in this paper correspond to this approximation. In the energy range up to $40 \mathrm{TeV}$ and with the input cross sections as given in Fig. 3 and in (2) at least the first 50 terms have to be computed in the remaining alternating sums in (B-1).

Many properties of the model can be understood already at this level of multi-Pomeron cross sections. In Fig. 4 we present as function of the numbers of soft and hard Pomerons $n_{s}$ and $n_{h}$ the results of the Monte Carlo sampling of 1000 events at some energies between the CERN-SPS collider and the SSC.

We note, the width of the distribution as well as the average values of the numbers $n_{h}$ and $n_{s}$ increase with energy. At low energy, the hard cross sections $\sigma_{h}$ and $\sigma_{h, \text { inel }}$ approach zero and we get as the low energy limit $\sigma_{i n e l}=\sigma_{n_{s}=1, n_{h}=0}$. 


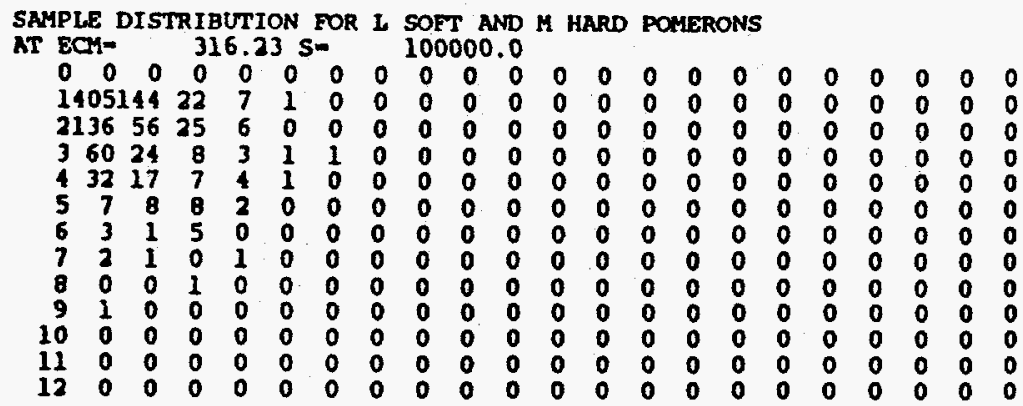

SAMPLE DISTRIEUTION FOR L SOFT AND M HARD POKERONS AT ECH- $1000.00 \mathrm{~s}-1000000.0$

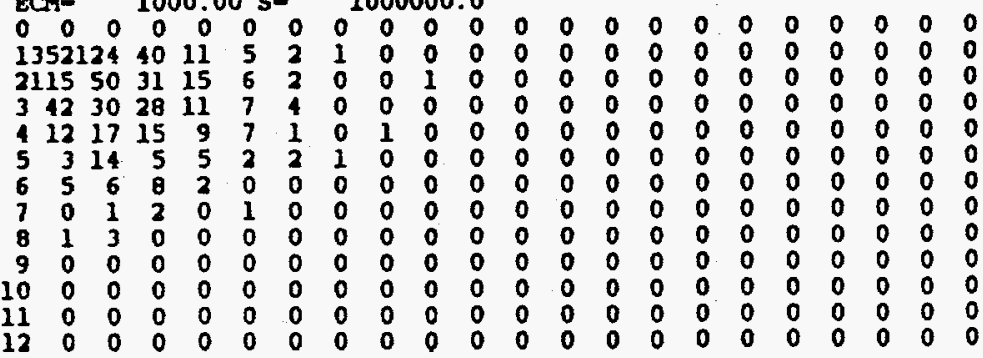

SAMPLE DISTRIBUTION FOR I SOFT AND M HARD POMERONS AT ECM- $3162.28 \mathrm{~s}-10000000.0$

$\begin{array}{llllllllllllllllllllll}0 & 0 & 0 & 0 & 0 & 0 & 0 & 0 & 0 & 0 & 0 & 0 & 0 & 0 & 0 & 0 & 0 & 0 & 0 & 0 & 0 & 0 \\ 1319144 & 38 & 22 & 13 & 2 & 3 & 0 & 0 & 1 & 0 & 0 & 0 & 0 & 0 & 0 & 0 & 0 & 0 & 0 & 0 \\ 2 & 96 & 45 & 37 & 19 & 14 & 5 & 3 & 1 & 0 & 1 & 0 & 0 & 0 & 0 & 0 & 0 & 0 & 0 & 0 & 0 & 0 \\ 3 & 37 & 26 & 18 & 17 & 7 & 1 & 3 & 2 & 1 & 0 & 0 & 0 & 0 & 0 & 0 & 0 & 0 & 0 & 0 & 0 & 0 \\ 1 & 14 & 15 & 11 & 6 & 1 & 3 & 3 & 1 & 1 & 0 & 0 & 0 & 0 & 0 & 0 & 0 & 0 & 0 & 0 & 0 & 0 \\ 5 & 6 & 7 & 6 & 9 & 4 & 4 & 2 & 1 & 0 & 0 & 0 & 0 & 0 & 0 & 0 & 0 & 0 & 0 & 0 & 0 & 0 \\ 6 & 4 & 1 & 3 & 3 & 3 & 1 & 0 & 1 & 0 & 0 & 0 & 0 & 0 & 0 & 0 & 0 & 0 & 0 & 0 & 0 & 0 \\ 7 & 1 & 2 & 0 & 0 & 2 & 1 & 0 & 0 & 0 & 0 & 0 & 0 & 0 & 0 & 0 & 0 & 0 & 0 & 0 & 0 & 0 \\ 8 & 0 & 0 & 1 & 0 & 0 & 0 & 0 & 0 & 0 & 0 & 0 & 0 & 0 & 0 & 0 & 0 & 0 & 0 & 0 & 0 & 0\end{array}$

\section{SAMPLE DISTRIBUTION FOR L SOFT AND M HARD POMERONS}

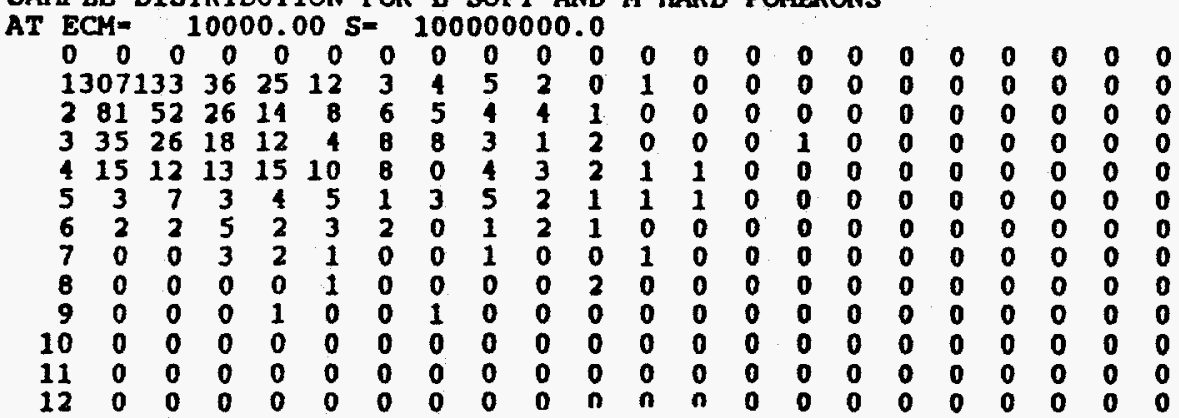

SAMPLE DISTRIBUTION FOR L SOFT AND M HARD POMERONS

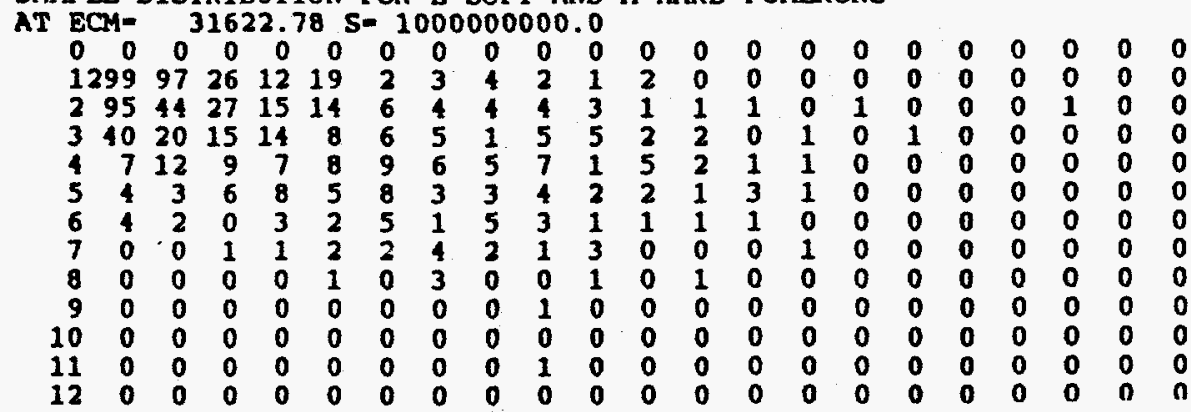

FIG. 4. Distribution in the number $n_{s}=L$ of soft (down the page) and the number $n_{h}=M$ of hard (across the page) Pomerons, as calculated by sampling 1000 events at each energy for the distribution given in Appendix B. 
With rising energies about $50 \%$ of the total inelastic cross section remains in the fraction with $n_{h}=0$ (no hard scattering), but the width of the distribution in $n_{s}$, the number of soft Pomerons and the average values $\left\langle n_{s}\right\rangle$ increase with energy. Also for $n_{h} \neq 0$ the shape of the distribution in $n_{s}$ becomes wider and flatter.

\section{Formulation of the Model on the Level of the Partons at the End of the Strings}

Up to here we have selected the main parameters of the model by choosing the input soft and hard cross sections $\sigma_{s}$ and $\sigma_{h}$ and obtaining a good fit to the hadronic total and inelastic cross sections. From this we obtain without further input the multi-Pomeron cross-sections $\sigma_{n_{s}, n_{k}}$.

Next we have to choose the methods to sample the partons at the ends of the hard and soft strings in events with $n_{s}$ soft and $n_{h}$ hard Pomerons.

The hard scattering cross section (1) before performing the integrations and sums is just the distribution function for the $x$ values of the partons which get engaged in the hard scattering. We need of course distribution function for $n_{h}$ hard scatterings and simultaneously $n_{s}$ soft scatterings.

To demonstrate, that in most situations one hard scattering corresponds to one hard Pomeron and therefore to two additional strings, we give in Fig. 5 some examples of events with one soft (valence-valence) pair of strings and one hard scattering. * In the case, that gluons are involved in the hard scattering, we have to transform the gluons into quark-antiquark pairs before forming strings, which can fragment into hadrons. In some situations, for instance for valence quarkvalence quark hard scattering, we need from each interacting proton one gluon or sea quark-antiquark pair in order to form color singlet strings involving the diquarks.

To sample the hard scattering of partons in the code DTUJET we use the method and parts of the FORTRAN-code of the Lund event generator PYTHIA [18]. We fragment gluons in the final state of the hard scattering always into quark pairs to prepare for the string fragmentation as indicated in Fig. 5.

Besides sampling the partons involved in hard scattering we have also to sample the partons at the ends of the soft strings. In an event with $2 n_{s}$ soft chains and $n_{h}$ hard Pomerons (to be definite we consider as an example the case of hard gluon-gluon scattering) we have to sample the partons for each of the primary hadrons from exclusive parton distributions for $2 n_{s}$ soft (valence quark,

* One should have in mind, however, that assignment of strings to a given parton configuration is ambiguous and model dependent. 


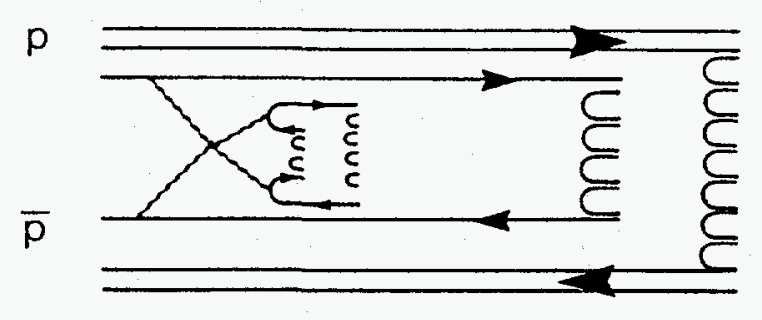

a)

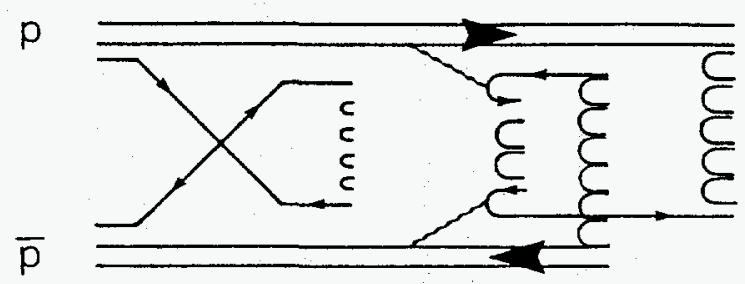

b)

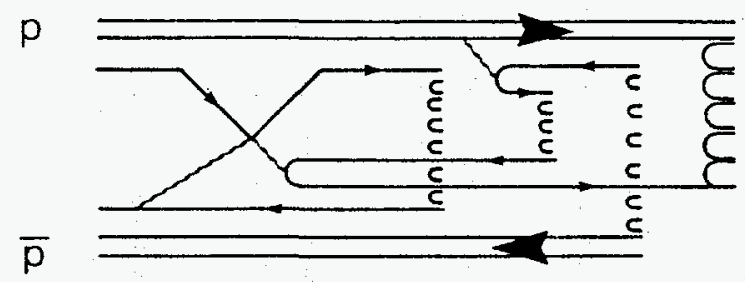

c)

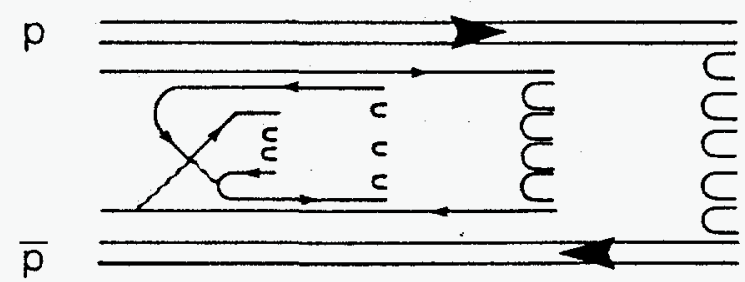

d)

FIG. 5. Examples for string configurations in events with one soft and one hard Pomeron for different hard scattering subprocesses. a) gluon-gluon, b) valence quark-valence quark, c) valence quark-gluon and d) sea quark-gluon.

valence diquark and $\left(2 n_{s}-2\right) / 2$ quark-antiquark pairs or gluons which fragment subsequently into quark-antiquark pairs) and $n_{h}$ hard partons (gluons). This exclusive parton distribution has the form

$$
\begin{aligned}
\rho\left(x_{1}, \ldots, x_{2 n_{s}}, x_{2 n_{s}+1}, \ldots, x_{2 n_{s}+n_{h}}\right) \\
\sim \frac{1}{\sqrt{x}_{1}}\left(\prod_{2}^{2 n_{s}-1} \frac{1}{x_{i}}\right) x_{2 n_{s}}^{1.5} \prod_{2 n_{s}+1}^{2 n_{s}+n_{h}} \mathrm{~g}\left(x_{i}\right) \delta\left(1-\sum_{1}^{2 n_{s}+n_{h}} x_{i}\right)
\end{aligned}
$$


The form of the soft parton distributions for small $x$-values is obtained by dual Regge arguments [19]. For valence partons

$$
\begin{array}{ccc}
\rho\left(x_{q}\right) \sim \frac{1}{x_{q}^{\alpha_{R}}} & , \quad \alpha_{R}=\frac{1}{2} \\
f\left(x_{q q}\right) \sim \frac{1}{x_{q q}^{\alpha_{E}}} \quad, \quad \alpha_{E}=-1.5
\end{array}
$$

where $\alpha_{R}$ is the leading $(q \bar{q})$ Regge trajectory and $\alpha_{E}$ the exotic ( $\left.q q \bar{q} \bar{q}\right)$ trajectory. The $x$ distribution of soft sea quarks is $f(x) \sim 1 / x$ for $x \rightarrow 0$. The distributions $g\left(x_{i}\right)$ are the distribution functions for the $x$ values of partons from the hard scattering, they follow from (1).

In the present version of the model we do not use a method sampling exactly from (8), instead we use a rejection method, where the $x$ values of the hard and soft partons are first sampled independently and we reject the event if the $x$-value remaining for the diquark is inconsistent. In the dominating configuration the valence diquarks get much larger $x$-values than all other partons, we find, that only very few configurations sampled by this approximate method have to be rejected. A method to sample exactly from (8) is presently being worked out.

To judge the importance of the different hadron production mechanisms in the model, we subdivide the strings and the partons at their ends into three classes. Only in some situations the subdivision is ambiguous. The three classes are:

- soft valence strings

- soft sea strings

- hard strings

In Table 1 we give the energy fractions carried by the strings (or partons) of the three classes for typical collision energies. This table indicates, that for the hard scattering cross sections $\sigma_{h}$ as used here, the soft valence strings remain up to SSC energies the dominant mechanisms carrying around $90 \%$ of the total collision energy. This situation could however change, if the hard cross-sections $\sigma_{h}$ rise much stronger with energy as the ones given in Fig. 3 which are used here. It was already discussed in Section 2, that a stronger rise of $\sigma_{h}$ cannot be excluded from our present experimental and theoretical understanding of the hadron structure functions in the region $x \rightarrow 0$. 
Table 1

Energy fractions carried by the partons or strings corresponding to three different mechanisms: soft sea chains $(s s)$, soft valence chains $(s v)$ and hard chains $(h)$. The energy fractions were obtained by sampling between 200 and 2000 events at each energy.

\begin{tabular}{clll}
\hline \hline $\begin{array}{c}\sqrt{s} \\
(\mathrm{GeV})\end{array}$ & $E_{s s} / E_{0}$ & $E_{\text {sv }} / E_{0}$ & $E_{h} / E_{0}$ \\
\hline 200 & 0.020 & 0.944 & 0.036 \\
540 & 0.036 & 0.925 & 0.039 \\
900 & 0.043 & 0.920 & 0.037 \\
2000 & 0.055 & 0.905 & 0.040 \\
16000 & 0.071 & 0.883 & 0.046 \\
40000 & 0.070 & 0.886 & 0.044 \\
\hline \hline
\end{tabular}

\section{The Properties of the Model After the Fragmentation of All Strings into Hadrons}

After having selected the $x$-values, hard and primordial transverse momenta and flavors of all quarks, antiquarks and diquarks at the ends of the strings, the remaining task is the fragmentation of the strings into hadrons and the decay of all hadron resonances into stable hadrons.

There are more than one independent fragmentation and string fragmentation models available, which could be used. For the results reported here, we use the independent fragmentation chain decay code BAMJET [20] and the resonance decay code DECAY [21]. Figure 5 gives examples for the string structure in simple events with one hard and one soft Pomeron. In Table 2 we present average total and charged multiplicities for each of the three mechanisms as well as for the complete events. These multiplicities correspond to nondiffractive inelastic events. Diffraction is not included in the present version of the model.

In Fig. 6 we present pseudorapidity distributions at 6 energies of past (CERNISR) present (CERN-SPS and Fermilab collider) and future (SSC) hadron colliders. At ISR and SPS energies we compare with data from non single-diffractive events from the UA-5 collaboration [22] and find good agreement.

In Fig. 8 we present transverse momentum distributions integrated over the total longitudinal momentum or rapidity region at three energies. Again, we do not see significant changes with energy in the transverse momentum region below $5 \mathrm{GeV} / \mathrm{c}$. The onset of hard scattering is visible in the change of the slope of the distributions with increasing transverse momentum.

We find also good agreement of the model with semi-inclusive pseudorapidity 
Table 2

Model results for average charged and total multiplicities in nondiffractive inelastic collisions. The charged and total multiplicities include all charged and neutral hadrons from the pseudoscalar meson octet and the baryon octet and some photons resulting from the decay of hadron resonances.

\begin{tabular}{rcccccccc}
\hline \hline$\sqrt{s}$ & \multicolumn{2}{c}{ Total Events } & \multicolumn{2}{c}{ Soft Sea } & \multicolumn{2}{c}{ Soft Valence } & \multicolumn{2}{c}{ Hard } \\
$(\mathrm{GeV})$ & \multicolumn{3}{c}{} & \multicolumn{4}{c}{$\begin{array}{c}\text { Strings } \\
\text { Strings }\end{array}$} \\
& $n_{\text {tot }}$ & $n_{\text {ch }}$ & $n_{\text {tot }}$ & $n_{\text {ch }}$ & $n_{\text {tot }}$ & $n_{\text {ch }}$ & $n_{\text {tot }}$ & $n_{\text {ch }}$ \\
\hline 53 & 24.4 & 14.1 & 0.36 & 0.21 & 22.7 & 13.11 & 1.28 & 0.74 \\
200 & 41.0 & 23.8 & 2.72 & 1.58 & 34.3 & 19.94 & 4.03 & 2.33 \\
540 & 58.9 & 34.1 & 7.55 & 4.40 & 43.1 & 25.1 & 8.26 & 4.83 \\
900 & 69.8 & 40.5 & 11.9 & 6.93 & 47.7 & 27.7 & 10.6 & 6.20 \\
2000 & 88.8 & 51.7 & 18.4 & 10.8 & 54.6 & 31.8 & 15.2 & 8.8 \\
40000 & 185.3 & 107.6 & 57.0 & 33.1 & 83.2 & 48.3 & 45.2 & 26.0 \\
\hline \hline
\end{tabular}

distributions as measured by the UA-5 collaboration[22]. The model shows the correlations between the average $p_{\perp}$ and the multiplicity per rapidity interval as found experimentally by the UA-1 collaboration[23]. The model also violates KNO scaling of the multiplicity distributions in such a way as found experimentally by the UA-5 collaboration[24]. A comparison with these features of the model with data will be presented elsewhere.

The changes of the event structure predicted by the model when selecting subclasses of events with and without hard jets [minijets] follow from the changes of the Pomeron distribution (see Fig. 4) between the regions with $n_{h}=0$ and $n_{h} \neq 0$. In Fig. 9 we present pseudorapidity distributions at $540 \mathrm{GeV}$ obtained by selecting normal average events, events without hard jets and events with jets of a given minimum transverse momentum. In these distributions the jets are always the ones known to the model, not jets found by a jet finding mechanism. We find a dramatic rise of the plateau of the rapidity and pseudorapidity distributions when selecting events with jets. In Fig. 10 the same features of the model are shown at the energy of $40 \mathrm{TeV}$. These features of the model make it likely, that the model reproduces the rise of the plateau under the jets as determined by the jet finding algorithm of the UA-1 collaboration [8]. 

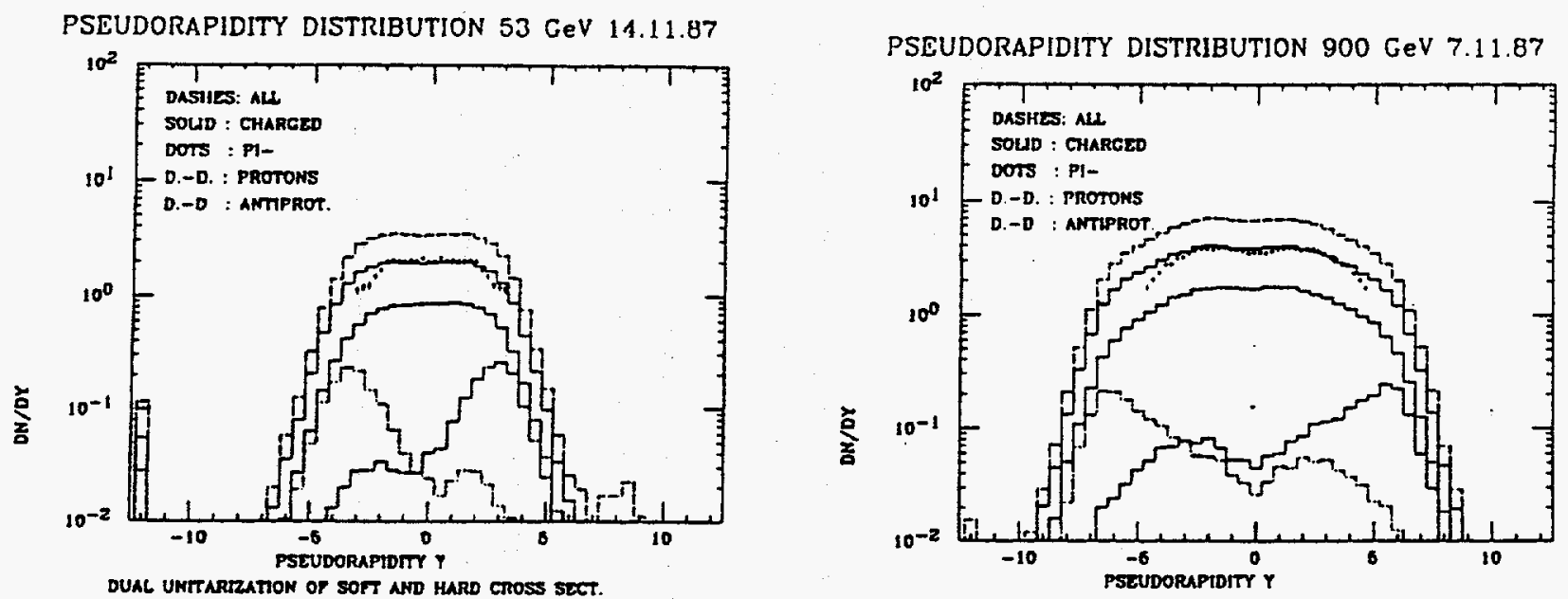

DUAL UNTTARZZATION OF SORT AND HARD CROSS SECT.

DUAL UNTTARIZATION OF SOTT AND HARO CROSS SECT.

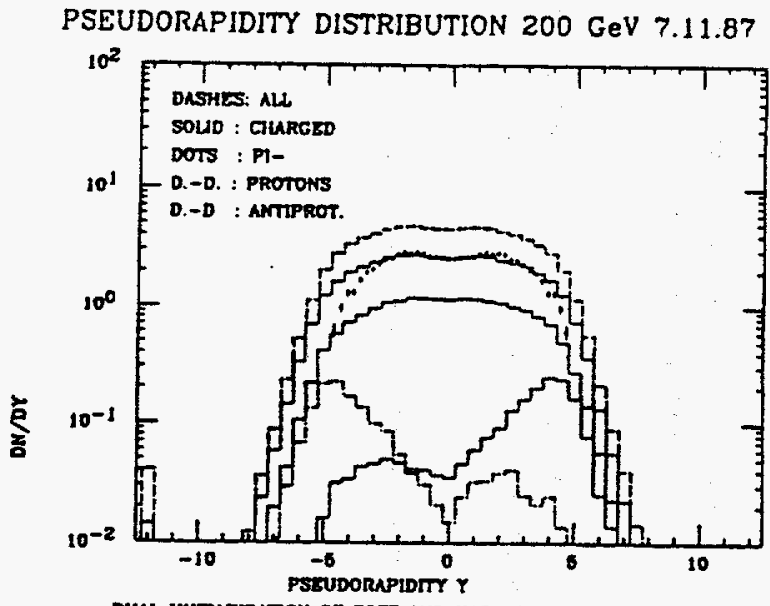

DUAL UATTARZZATON OF SOTT AND HARD CROSS SECT.

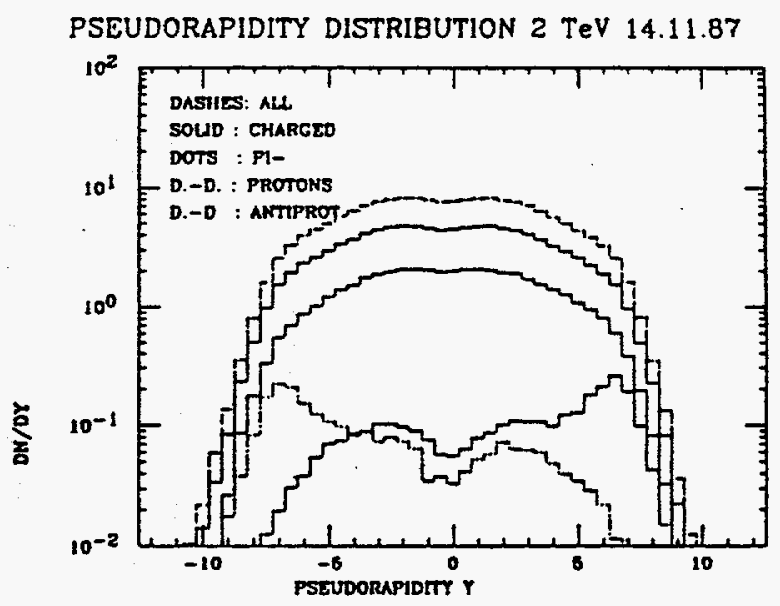

DUAL UNTTARTZATION OF SOTT AND KARO CROSS STET.

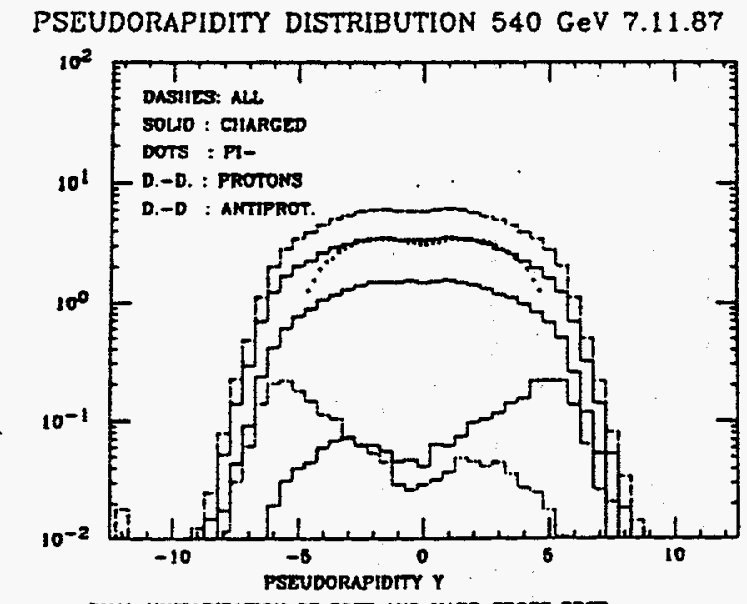

DUAL UNTTARIZATIOE OF SOTT AHD HAFD GROSS SECT.

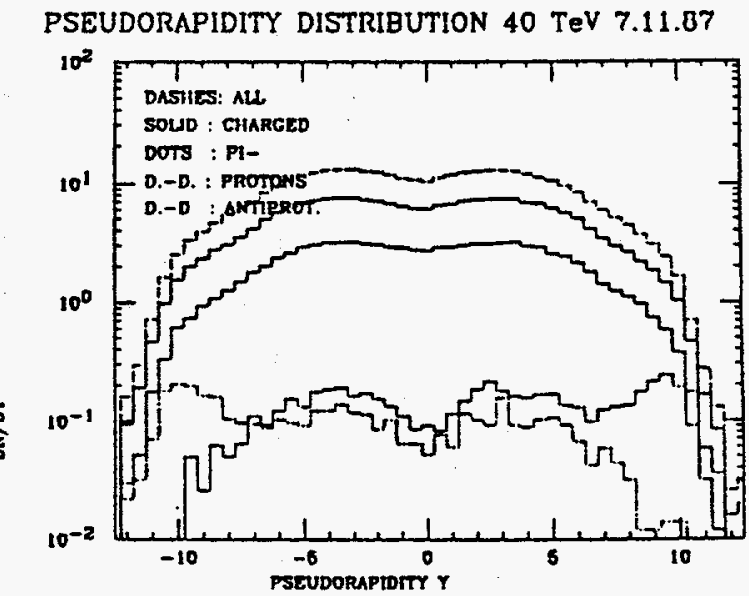

DUAL UNTARIZATION OF SOT AND HARD CROSS SECT.

FIG 6. Pseudorapidity distributions $d N / d \eta$ calculated at ISR to SSC energies in $p \bar{p}$ collisions. The data points at energies up to $\sqrt{s}=900 \mathrm{GeV}$ are from the UA-5 Collaboration [22]. The calculated distributions correspond to nondiffractive inelastic events. 
FEYNMAN X-DISTRIBUTION $40 \mathrm{TeV} 5.11 .87$

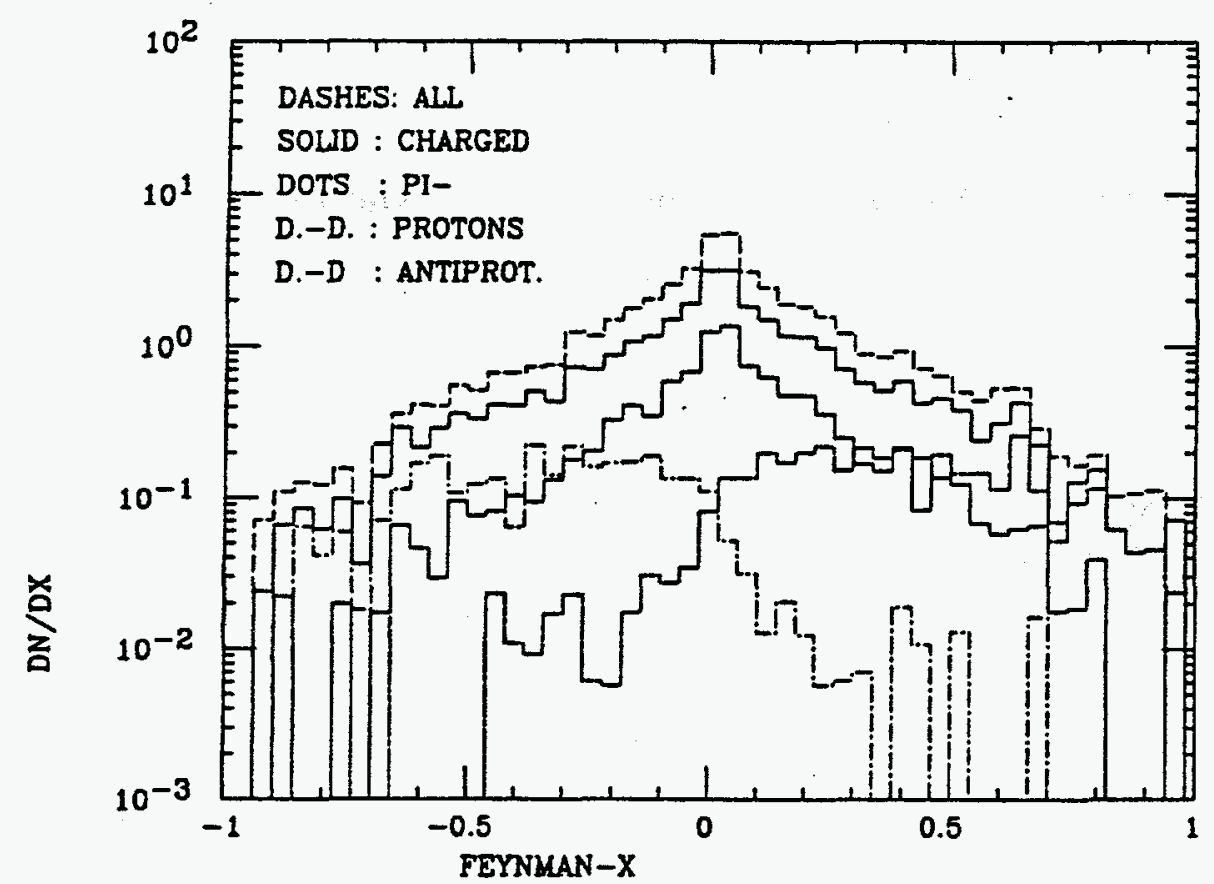

DUAL UNITARIZATION OF SOFT AND HARD CROSS SECT.

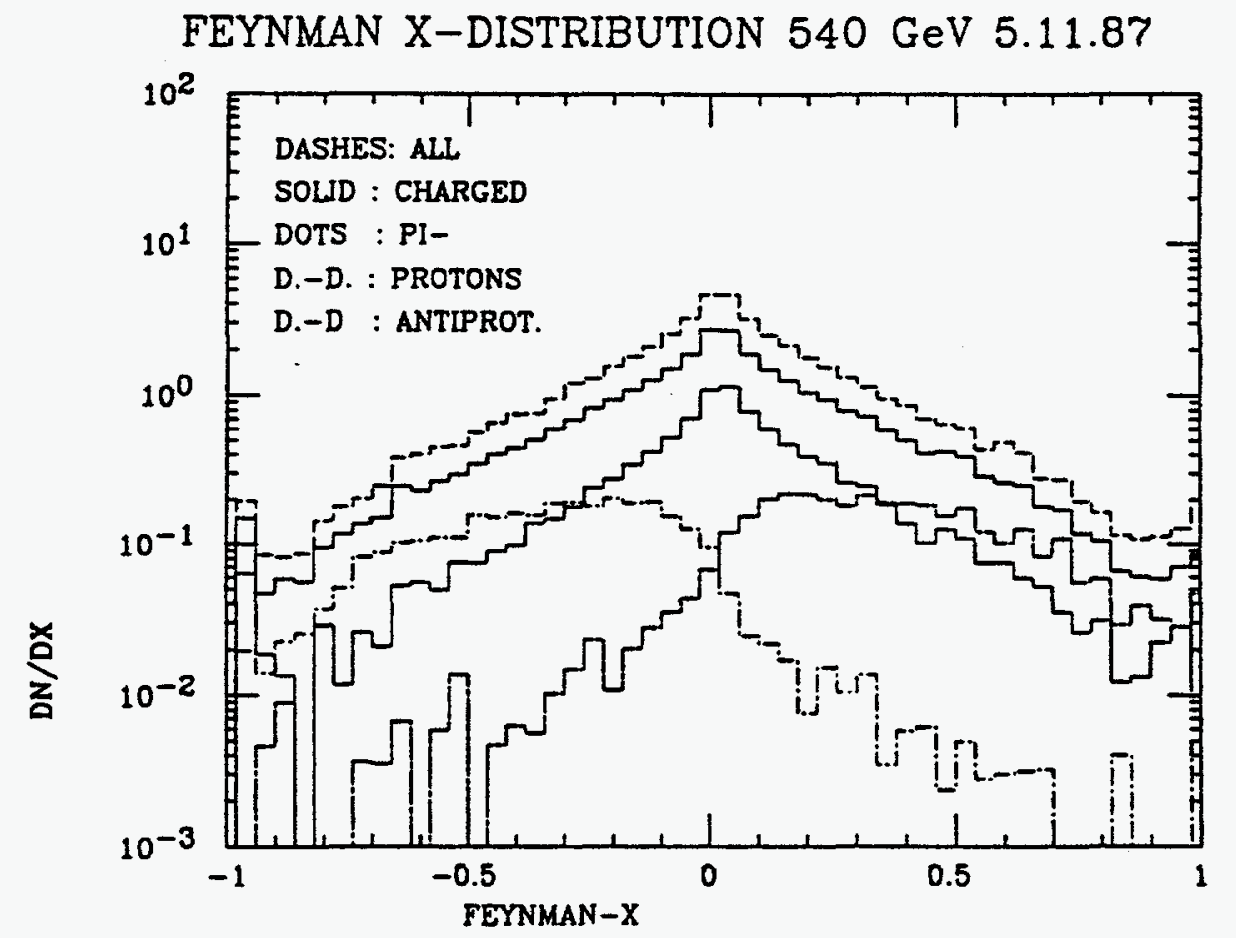

DUAL UNITARIZATION OF SOFT AND HARD CROSS SECT.

FIG. 7. Feynman- $x$ distributions calculated for $p \bar{p}$ collisions at $540 \mathrm{GeV}$ and $40 \mathrm{TeV}$. The calculated distributions correspond to nondiffractive inelastic events. 


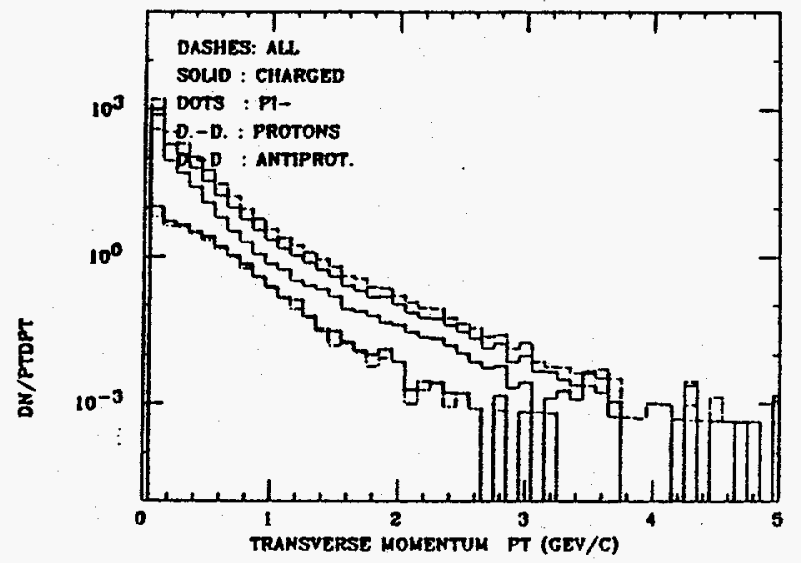

DUAL UNTARTZATION OF SOTT AND MARD CROSS SECT.

TRANSVERSE MOMENTUM DISTRIBUTION $540 \mathrm{GeV} 7.11 .87$

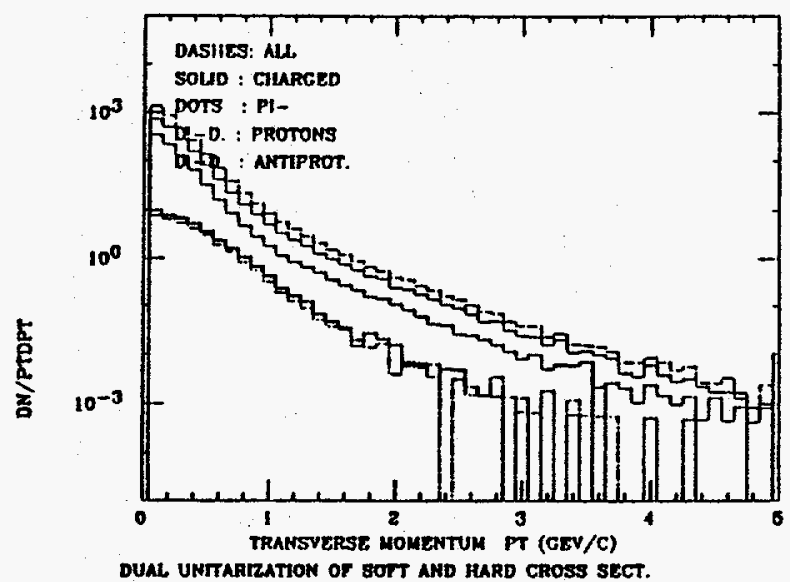

TRANSVERSE MOMENTUM DISTRIBUTION $40 \mathrm{TeV} 7.11 .87$

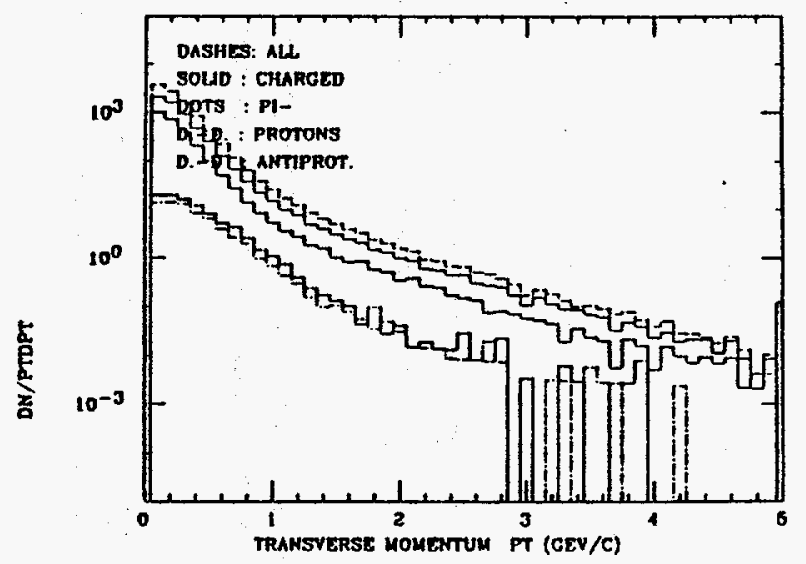

DUAL UATTARIZATION OF SOFT AHD HARD CROSS SECT.

FIG. 8. Transverse momentum distributions for minimum bins events at ISR to SSC energies. The calculated distributions correspond to nondiffractive inelastic events. 


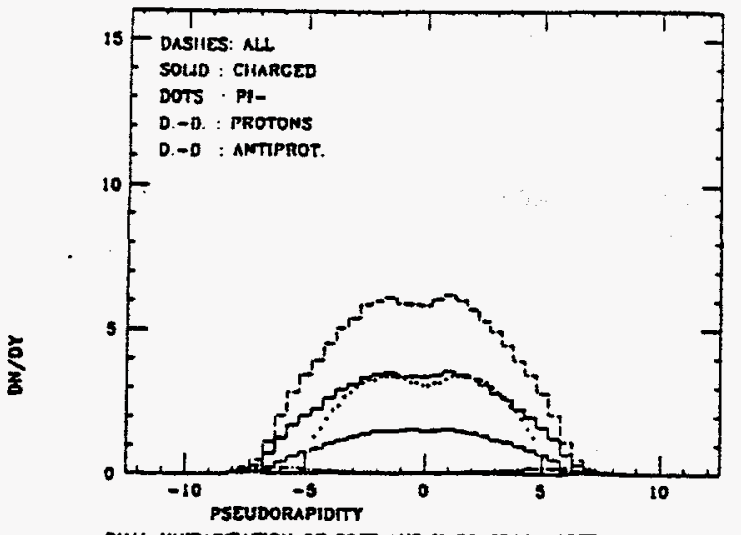

DUAL UNTARUZATION OF SOTT AND HARE CROSS SECT.

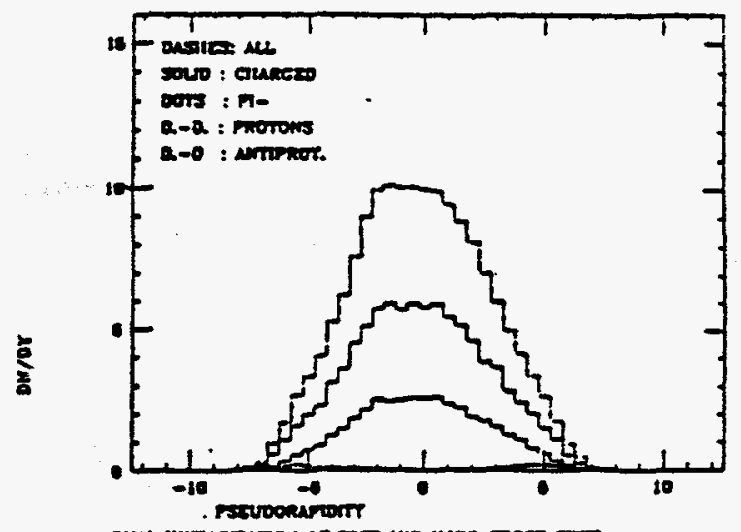

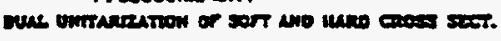

PSEUDORAPIDITY DISTRIBUTION $540 \mathrm{CEV}$ pL>7

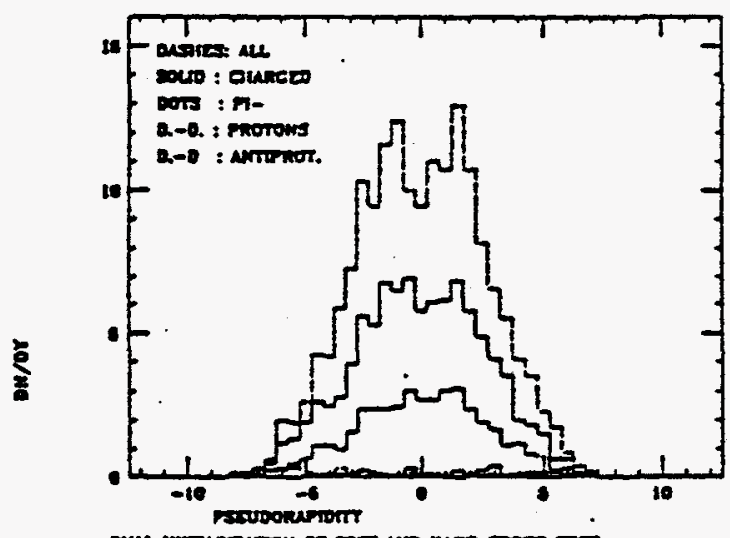

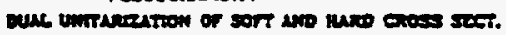

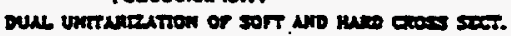

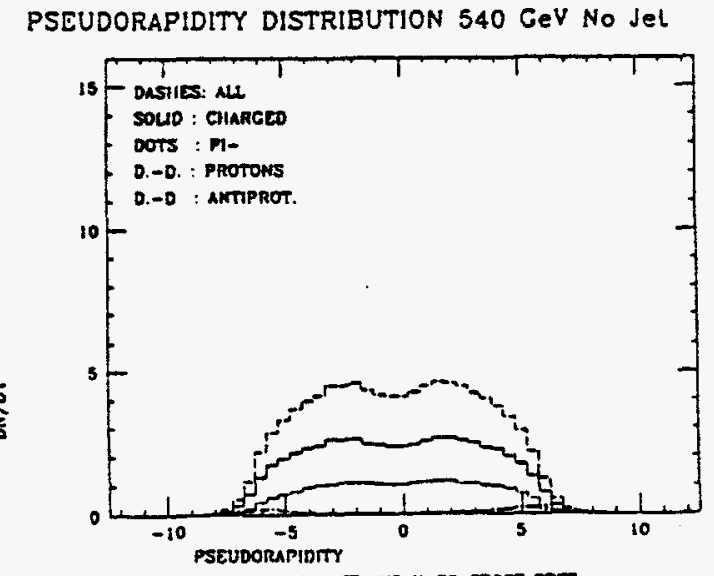

DUAL UNTTARIZATION OF SOTT AND UARD CROSS SECT.

FIG. 9. Pseudo rapidity distributions calculated for $p \bar{p}$ interactions at $540 \mathrm{GeV}$ for minimum bias events, events with hard scattered jets with transverse momenta above a given $p_{\perp}$ in $\mathrm{GeV}$ and for events without hard jets $\left(n_{h}=0\right)$. Hard "jets" are always the jets used in the construction of the model, not jets as determined by a jet finding algorithm from the events. 


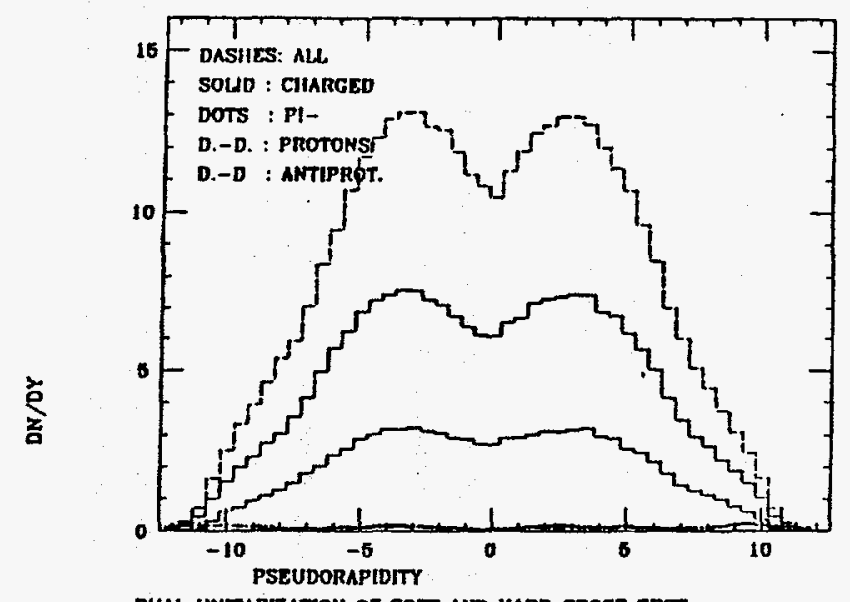

DUAL UNTARIZATION OF SOIT AND MARD CROSS SECT.

PSEUDORAPIDITY DISTRIBUTION $40 \mathrm{TeV}$ No jel

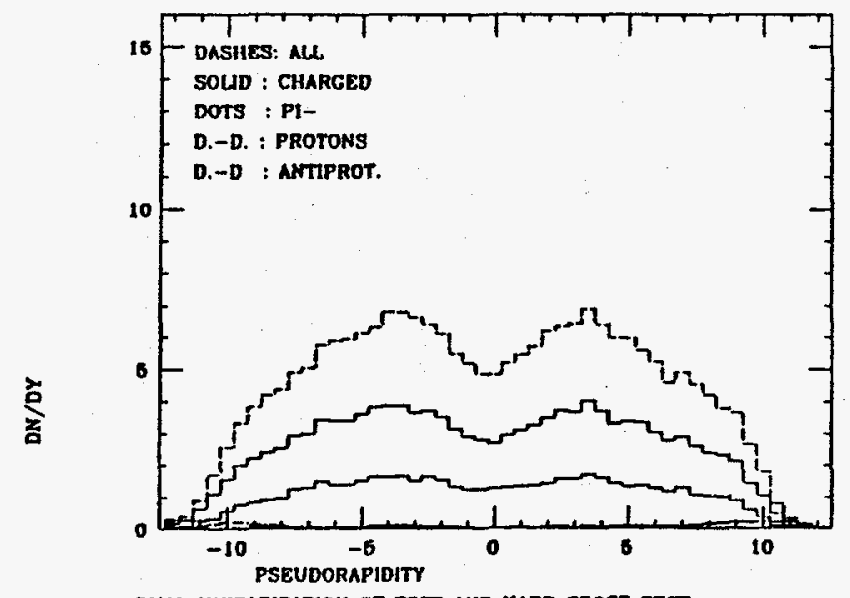

DUAL UNTTARIZATION OF SOTT AND BARD CRDSS SECT.

PSEUDORAPIDITY DISTRIBUTION $40 \mathrm{TeV}$ jet 3

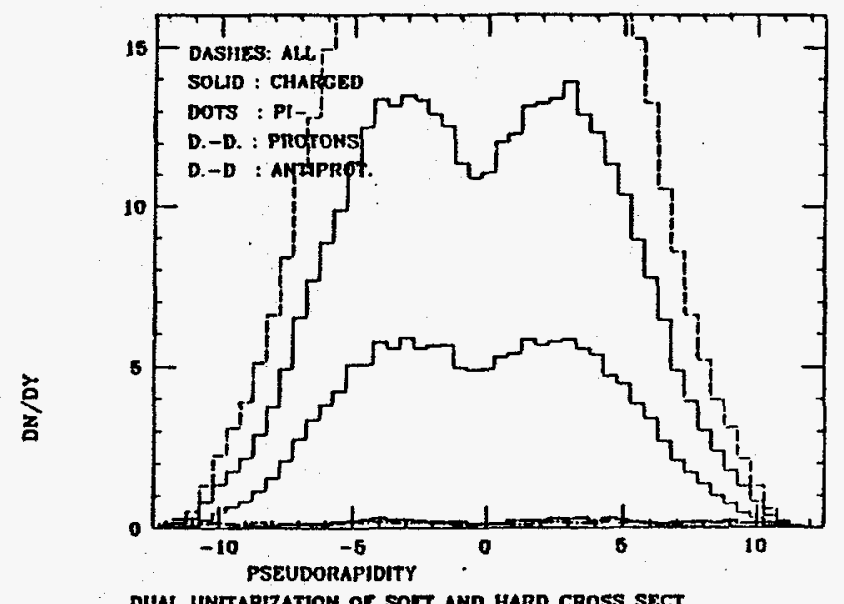

FIG. 10. Pseudorapidity distributions as given in Fig. 9 at $\sqrt{s}=40 \mathrm{TeV}$. 


\section{Hadron Production in $40 \mathrm{TeV}$ Collisions at the SSC}

In Table 3 we present multiplicities of different kinds of hadrons and energy fractions carried by these kinds of hadrons calculated by the DTUJET code. Some explanations to this table: The photons result from decay channels of hadron resonances. There are neutral kaons $K_{L}^{0}, K_{S}^{0}, K^{0}$ and $\bar{K}^{0}$. The $K_{L}^{0}$ and $K_{S}^{0}$ follow from some decay channels as given in the particle data tables. The $K^{0}$ and $\bar{K}^{0}$ are produced in BAMJET. The user should decide in which form he needs the neutral $K$-mesons. The table as given is for $p \bar{p}$ collisions, the only differences seen in $p \bar{p}$-collisions are obvious changes in the baryon and antibaryon multiplicities.

Table 3

Particle composition at $40 \mathrm{TeV} p \bar{p}$ collisions.

\begin{tabular}{lcc}
\hline \hline Particle & Multiplicity & Energy Fraction \\
\hline $\mathrm{p}$ & 2.02 & 0.0600 \\
$\overline{\mathrm{p}}$ & 2.01 & 0.0612 \\
$\gamma$ & 7.20 & 0.0191 \\
$n$ & 1.78 & 0.0379 \\
$\bar{n}$ & 1.81 & 0.0381 \\
$\mathrm{~K}_{L}^{0}$ & 0.25 & 0.0010 \\
$\pi^{+}$ & 45.7 & 0.2059 \\
$\pi^{-}$ & 45.7 & 0.2101 \\
$\mathrm{~K}^{+}$ & 5.7 & 0.0296 \\
$\mathrm{~K}^{-}$ & 5.7 & 0.0315 \\
$\Lambda$ & 0.84 & 0.0120 \\
$\bar{\Lambda}$ & 0.83 & 0.0100 \\
$\mathrm{~K}_{s}^{0}$ & 0.25 & 0.0010 \\
$\Sigma^{-}$ & 0.17 & 0.0010 \\
$\Sigma^{+}$ & 0.17 & 0.0023 \\
$\Sigma^{0}$ & 0.14 & 0.0016 \\
$\pi^{0}$ & 53.6 & 0.2114 \\
$\mathrm{~K}^{0}$ & 5.37 & 0.0288 \\
$\mathrm{~K}^{-0}$ & 5.34 & 0.0299 \\
\hline \hline
\end{tabular}

In Figs. 11-13 we give pseudorapidity distributions for all particles, all hadrons, particles leading to electromagnetic cascades $\left(\pi^{0}\right.$ and $\left.\gamma\right)$, all charged 
particles and all neutral particles. Figure 11 gives the multiplicities per pseudorapidity unit $d N / d \eta$, Fig. 12 gives the energy per pseudorapidity unit $d E / d \eta$ and Fig. 13 gives the total energy within the bounds $-\eta \ldots \eta$.

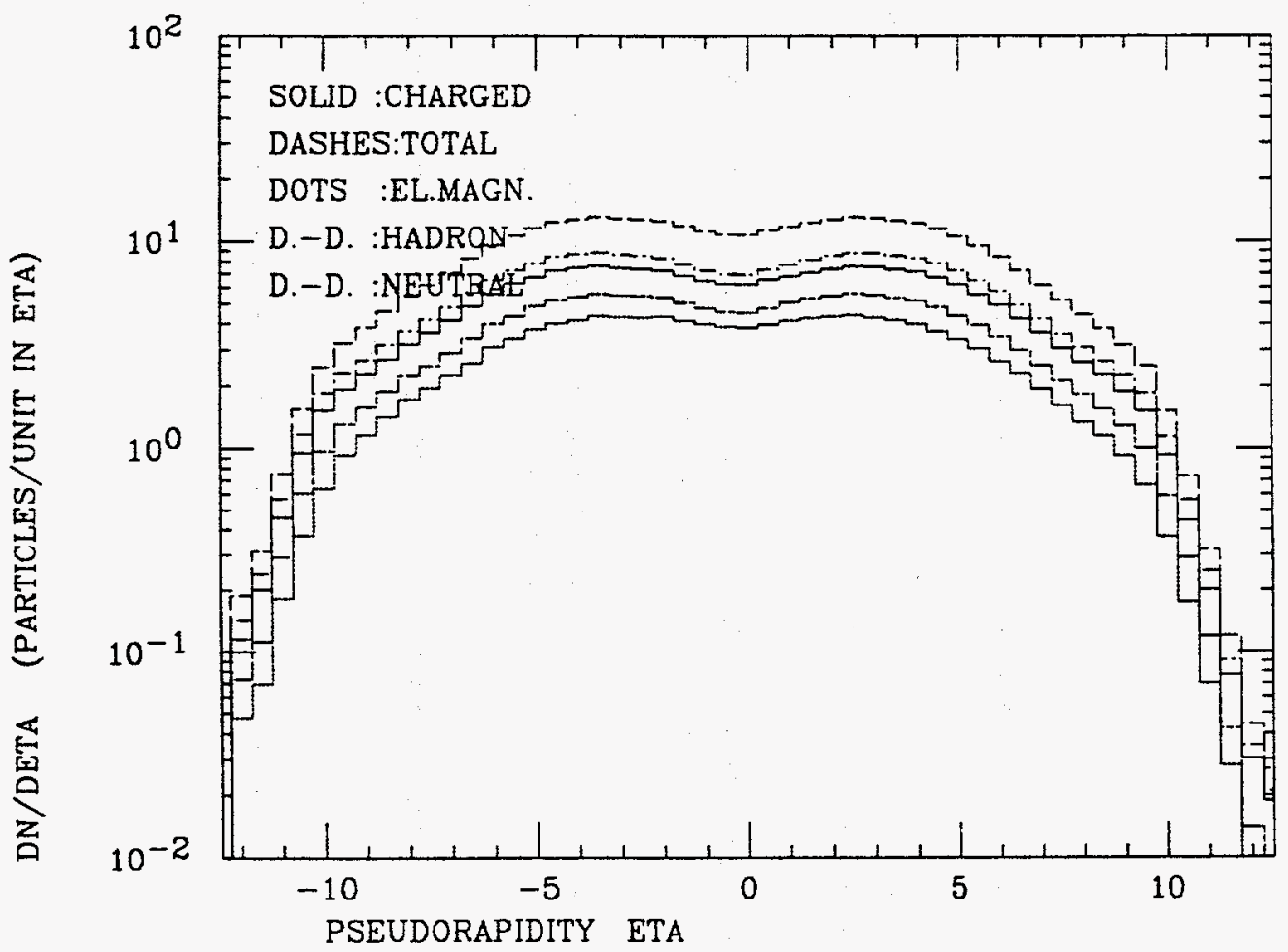

FIG. 11. Pseudorapidity distributions $d N / d \eta$ in $p \bar{p}$ collisions at $40 \mathrm{TeV}$. Plotted are the distributions for all particles, charged particles, all hadrons, particles leading to electromagnetic cascades $\left(\pi^{0}\right.$ and $\left.\gamma\right)$ and neutral particles.

In Figs. 14-16 we give similar histograms, this time as function of the polar angle $\theta$. Figure 14 gives $d N / d \Omega$ in particles per steradian, Fig. 15 gives $d E / d \Omega$ in $\mathrm{GeV} / \mathrm{sr}$ and Fig. 16 gives $d E / d \theta$ in $\mathrm{GeV} /$ radian.

In Figs. 17 and 18 we present two dimensional histograms representing $\mathrm{d}^{2} \mathrm{~N} / \mathrm{dpd} \eta$ for 50 momentum bins and 20 pseudorapidity bins of width $\Delta \eta=0.5$. Figure 17 gives $\mathrm{d}^{2} \mathrm{~N} / \mathrm{dpd} \eta$ for 10 pseudorapidity bins $0-0.5,1-1.5$, etc. as function of the momentum p. In each plot the histograms are given for all particles, all hadrons and all particles leading to e.m. cascades $\left(\pi^{0}\right.$ and $\gamma$ ). In Fig. 18 we present these histograms in the form $p d^{2} N / d p d \eta$ plotted over a logarithmic momentum scale. The 20 histograms starting from the leftmost histogram are for the pseudorapidity bins $0 .-0.5,0.5-1 ., 1 .-1.5, \ldots, 9.5-10$. 


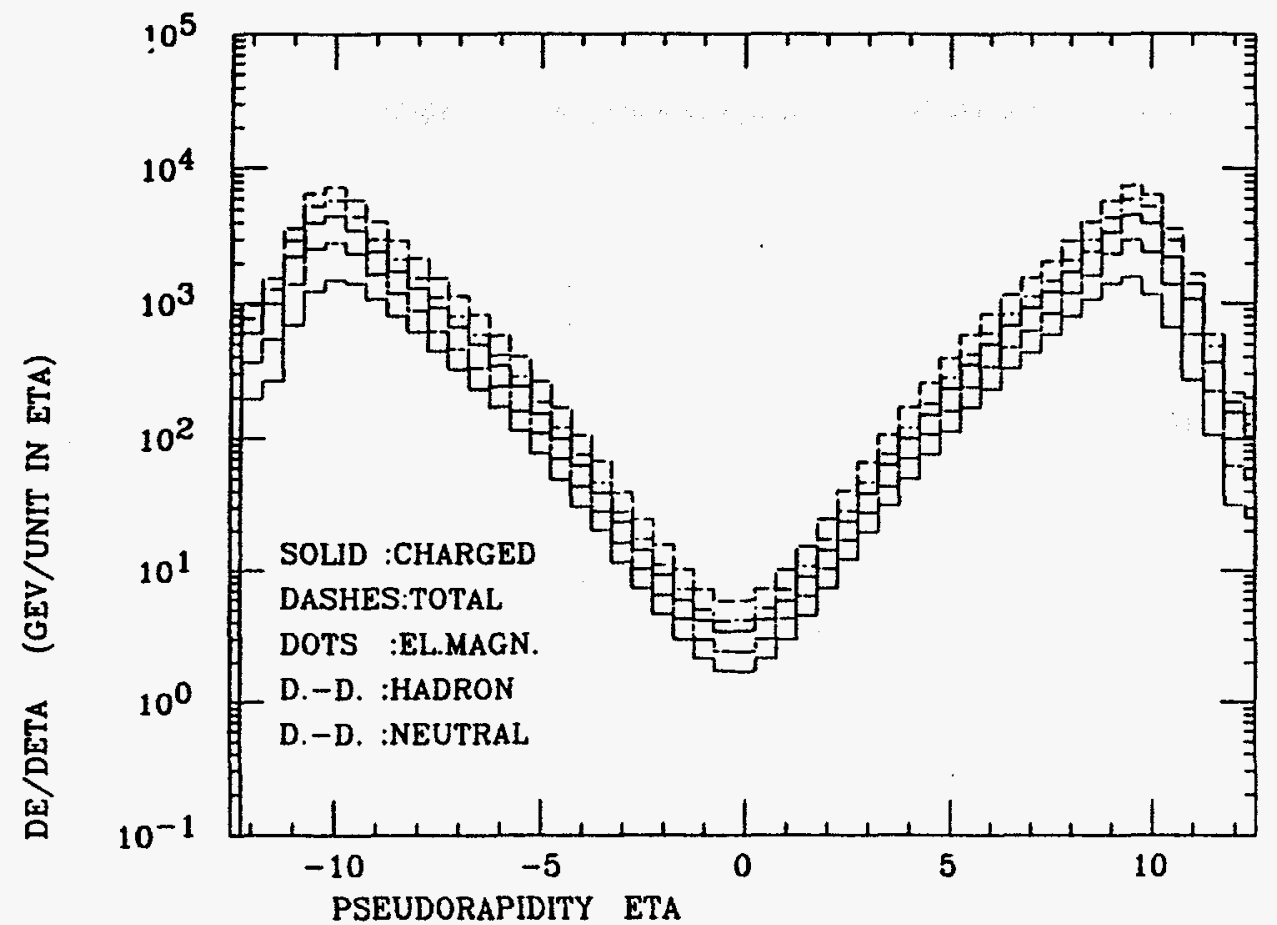

FIG. 12. Distributions $d E / d \eta$ in $p \bar{p}$ collisions at $40 \mathrm{TeV}$. Plotted for the same combinations of secondary particles as in Fig. 11.

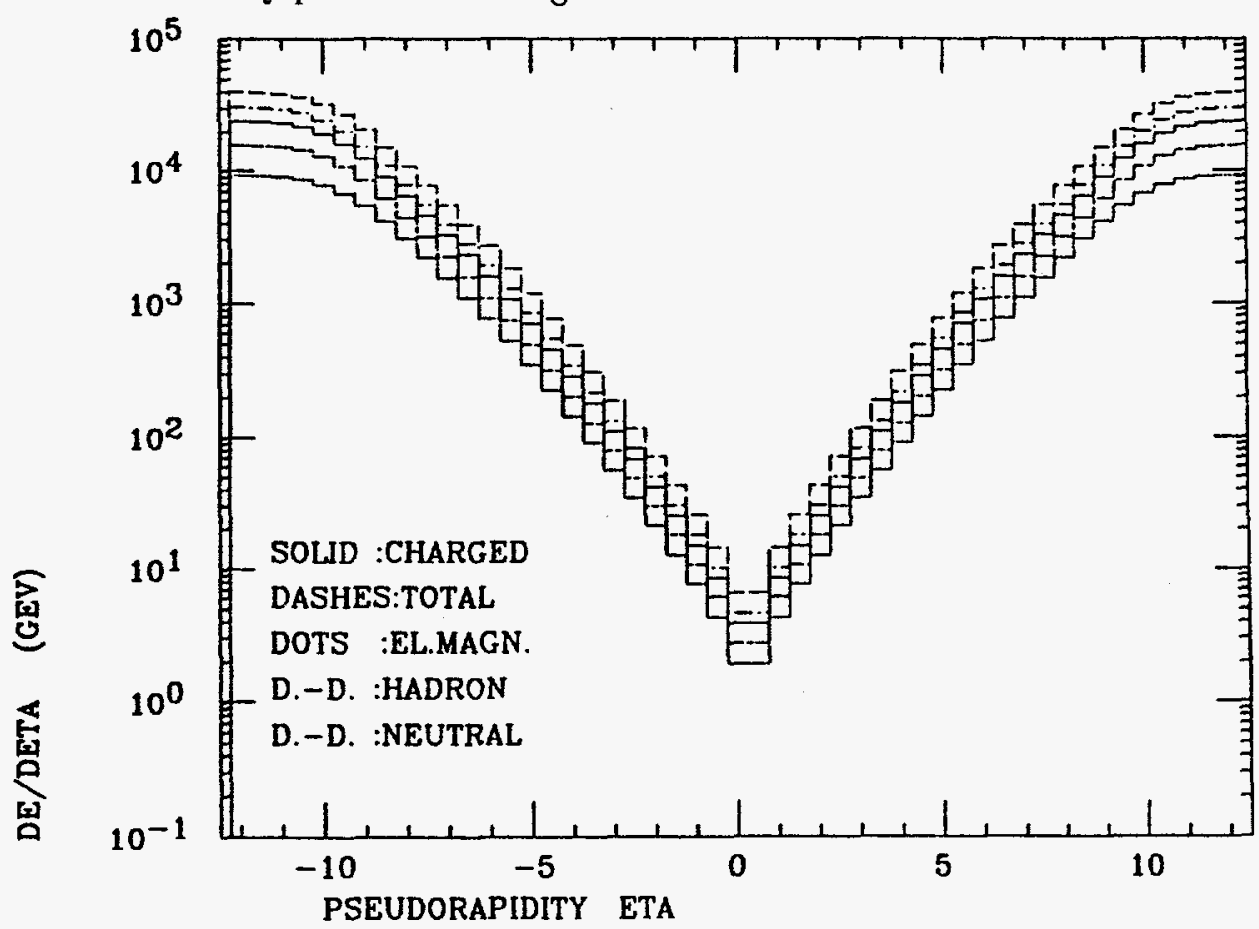

FIG. 13. Total energy within the pseudorapidity range $-\eta \leq \eta^{\prime} \leq \eta$ in $p \bar{p}$ collisions at $40 \mathrm{TeV}$, platted for the same combinations of secondaries as in Fig. 11. 
We would like to point out that the code DTUJET is available and a writeup will be available soon [12]. DTUJET-87 can be used to calculate histograms like the ones presented here or to write events to a computer file to be used by other codes.

\section{Summary}

We have presented results from the first version of a new model for multiparticle production in hadron-hadron collision. This model treats the soft and hard component of hadron production in a unified way. The basis for the model is the unitarization of the soft and hard cross sections.

The first results of the model presented here indicate that the model has the potential to describe all features of hadron production known at present, and the model makes detailed predictions at the energies of future hadron colliders.

The model as presented here is only the first version of a model, which will be completed in many respects, we mention only: diffractive events, initial and final state parton evolution, use of alternative fragmentation codes, working out the predictions of the model beyond the leading log approximation and applying the model to study the correlation between the soft hadron production and other types of hard collisions.

\section{Acknowledgements}

One of the authors (J. R.) would like to thank Prof. M. Tigner for an invitation to the SSC-Central Design Group, where the code DTUJET-87 was written. He thanks especially D.E. Groom for discussions and a fruitful collaboration at the SSC-CDG, and Dr. W. R. Nelson for continuous help in using the computer facilities at SLAC. He also thanks Dr. T. Sjöstrand for a copy of the PYTHIA code and the permission to use it within DTUJET. 


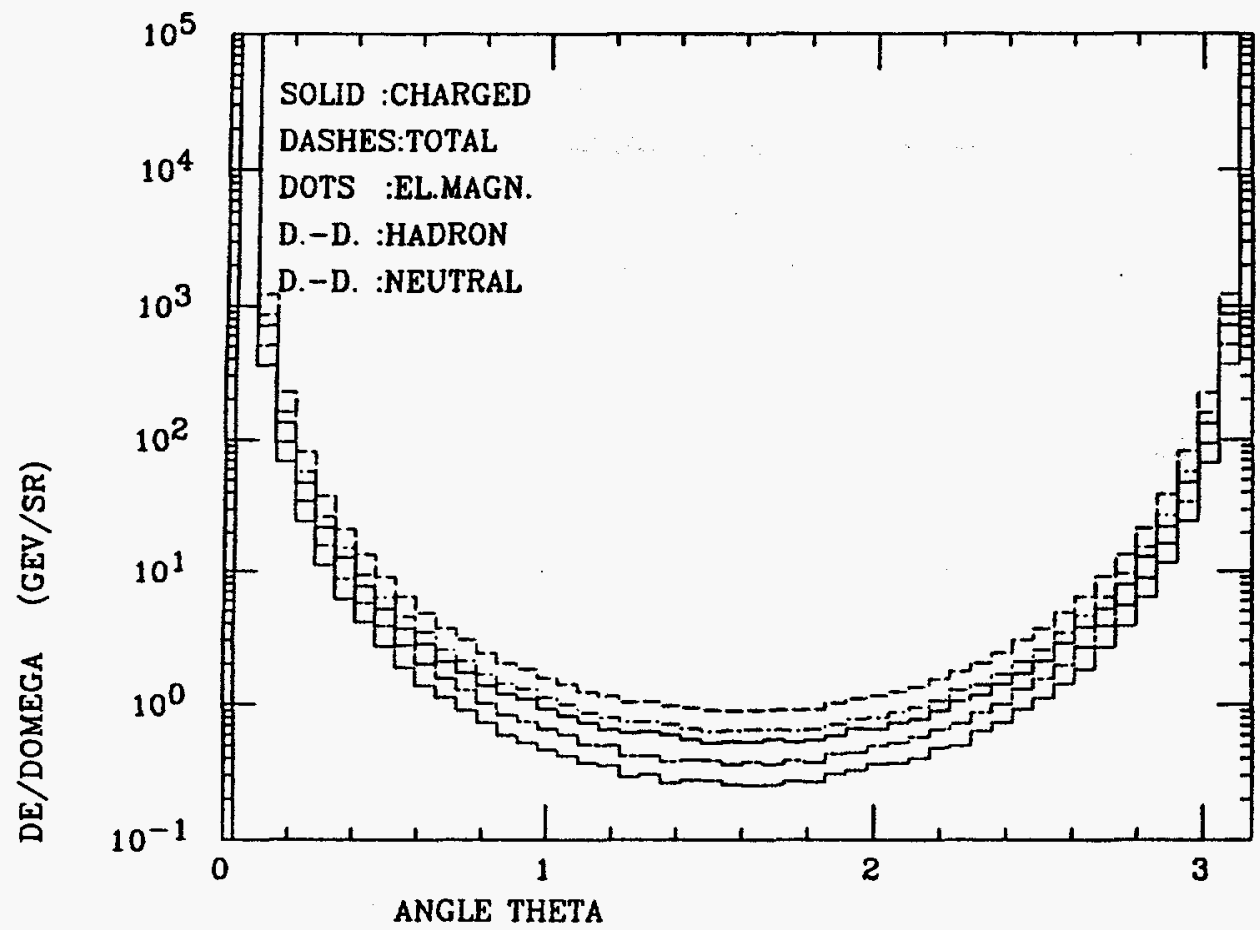

FIG. 14. The angular distribution $d N / d \Omega$ (particles per steradian) of secondaries produced in $p \bar{p}$ collisions at $40 \mathrm{TeV}$.

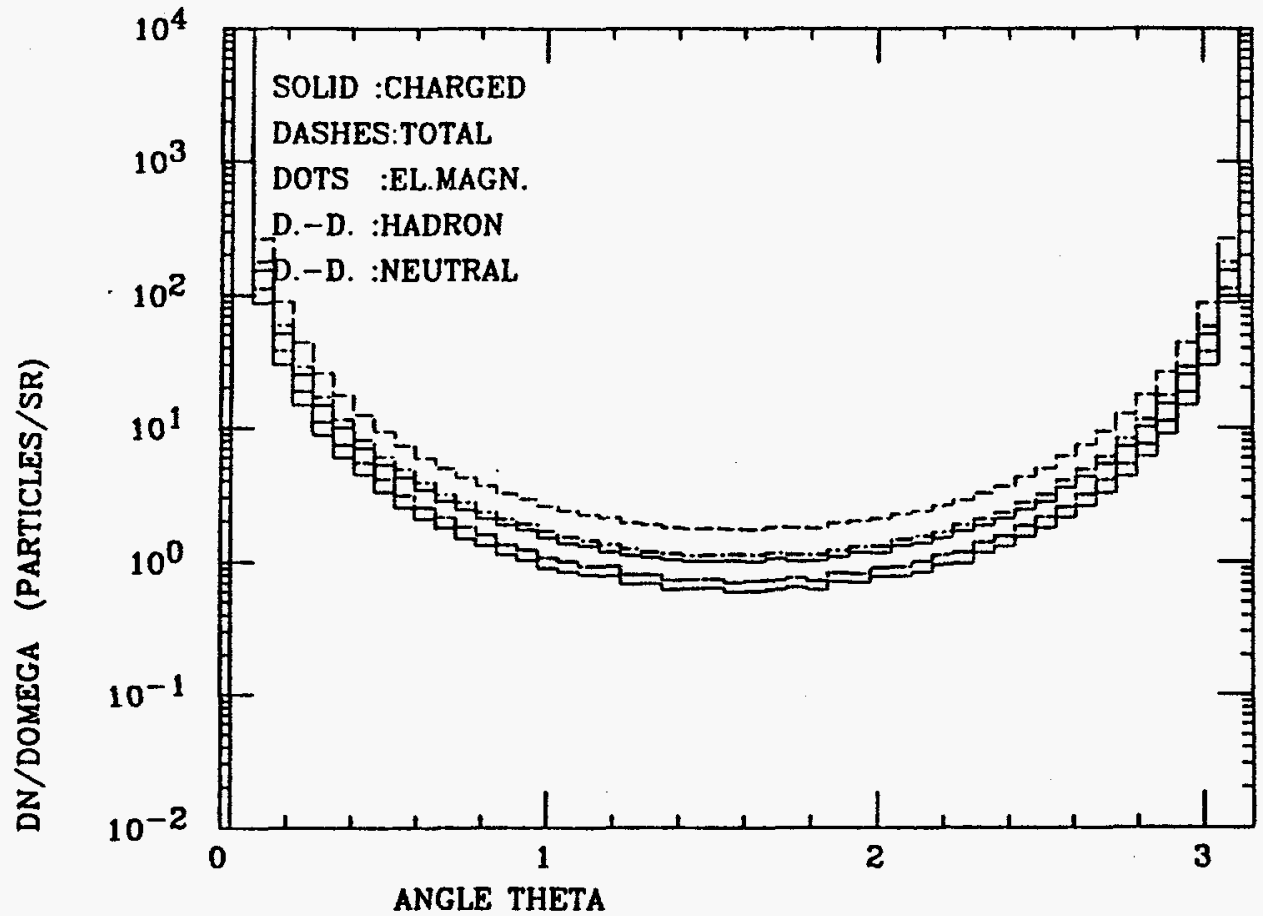

FIG. 15. The energy distribution $d E / d \Omega$ [GeV per steradian] of secondaries produced in $p \bar{p}$ collisions at $40 \mathrm{TeV}$. 


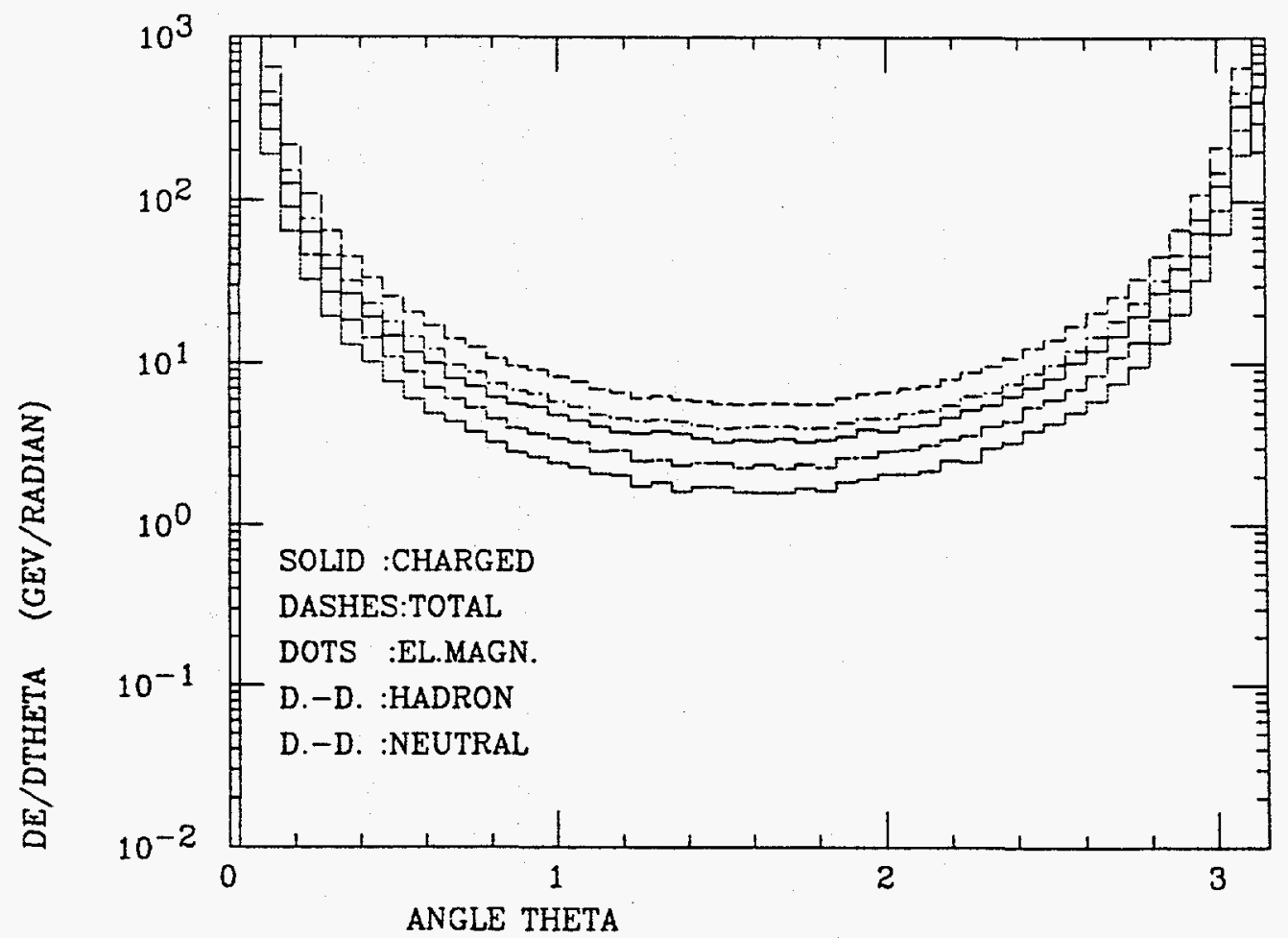

FIG. 16. The energy distribution $d E / d \theta$ [GeV per radian] of secondaries produced in $p \bar{p}$ collisions at $40 \mathrm{TeV}$. 

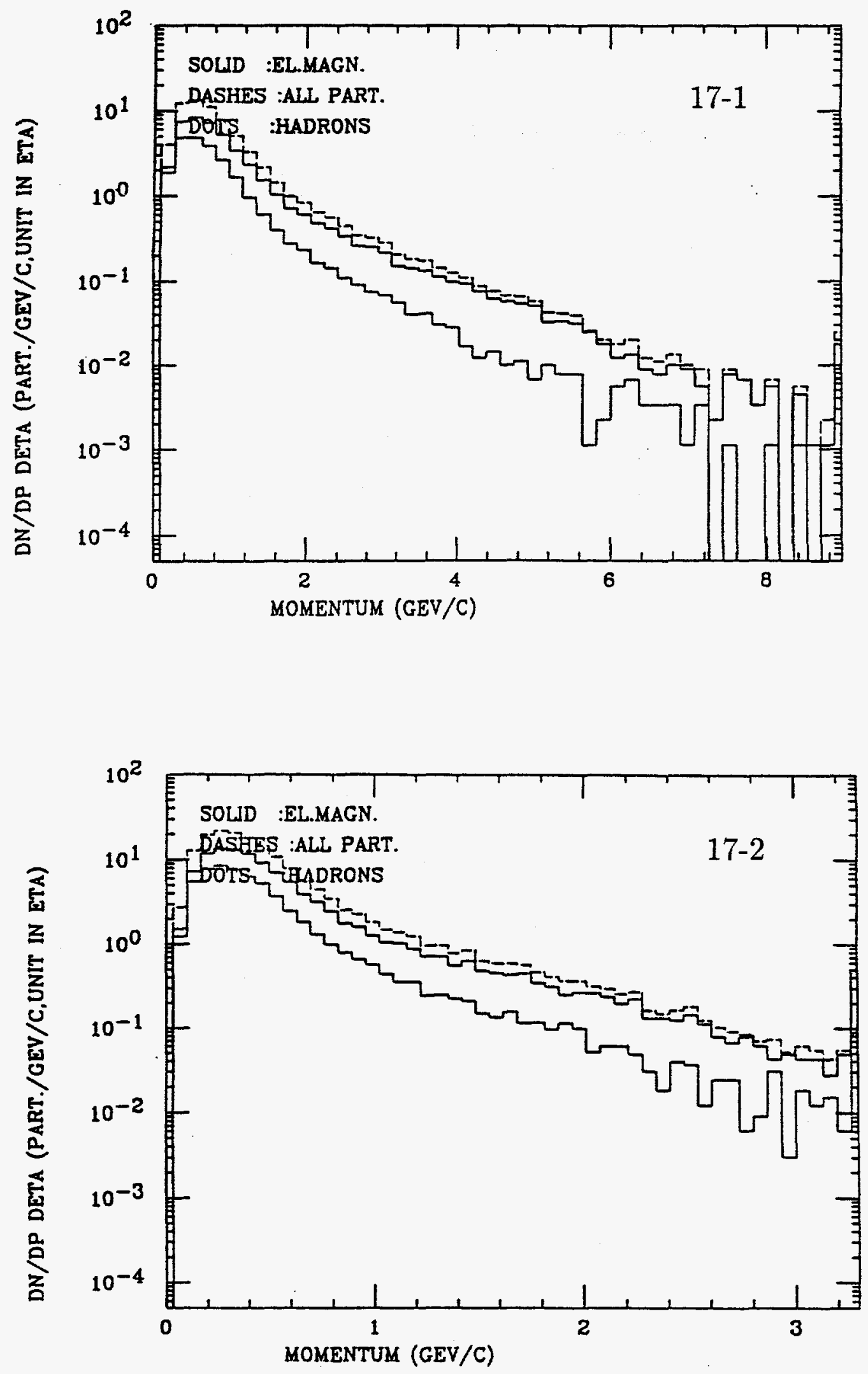

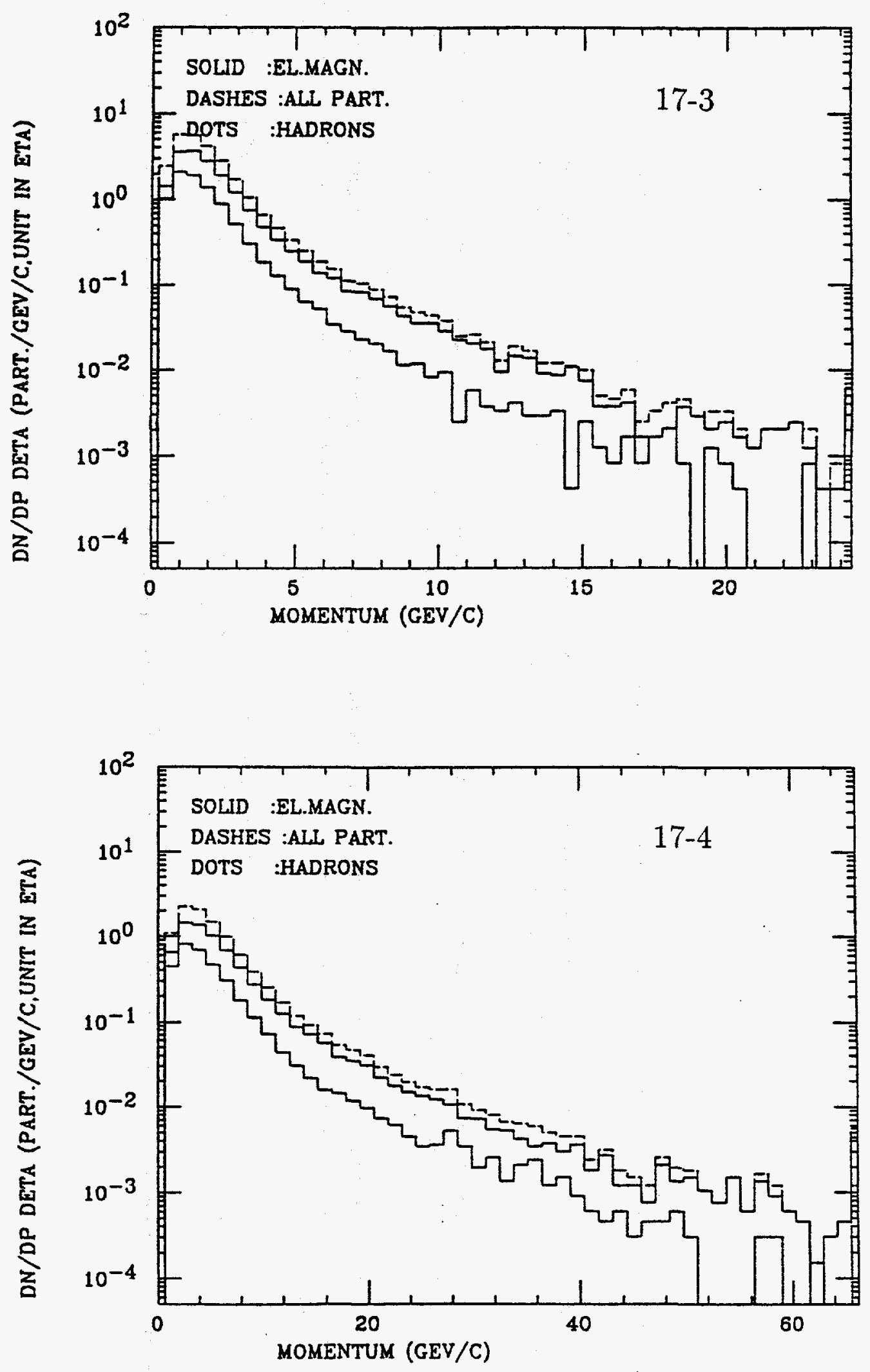

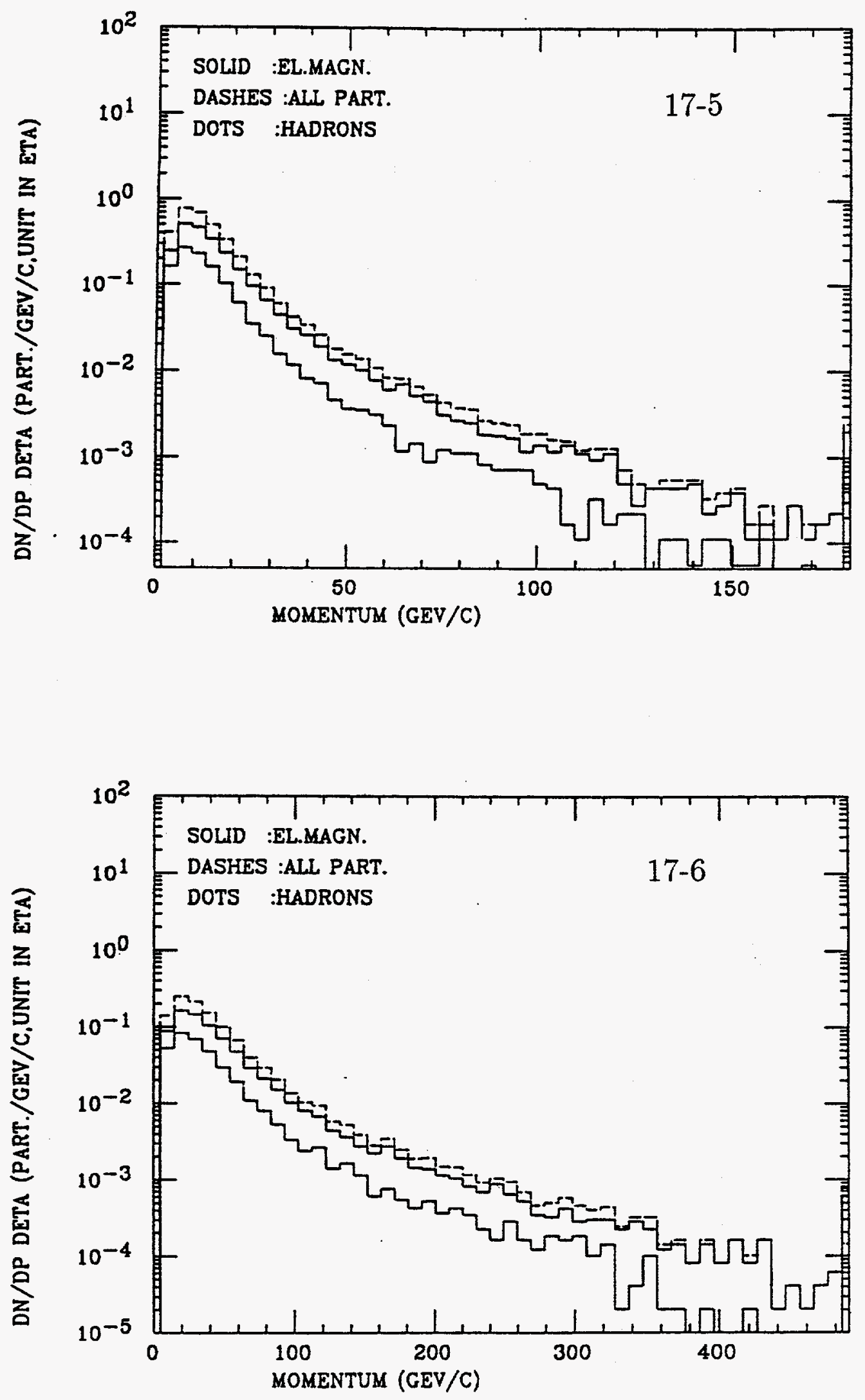

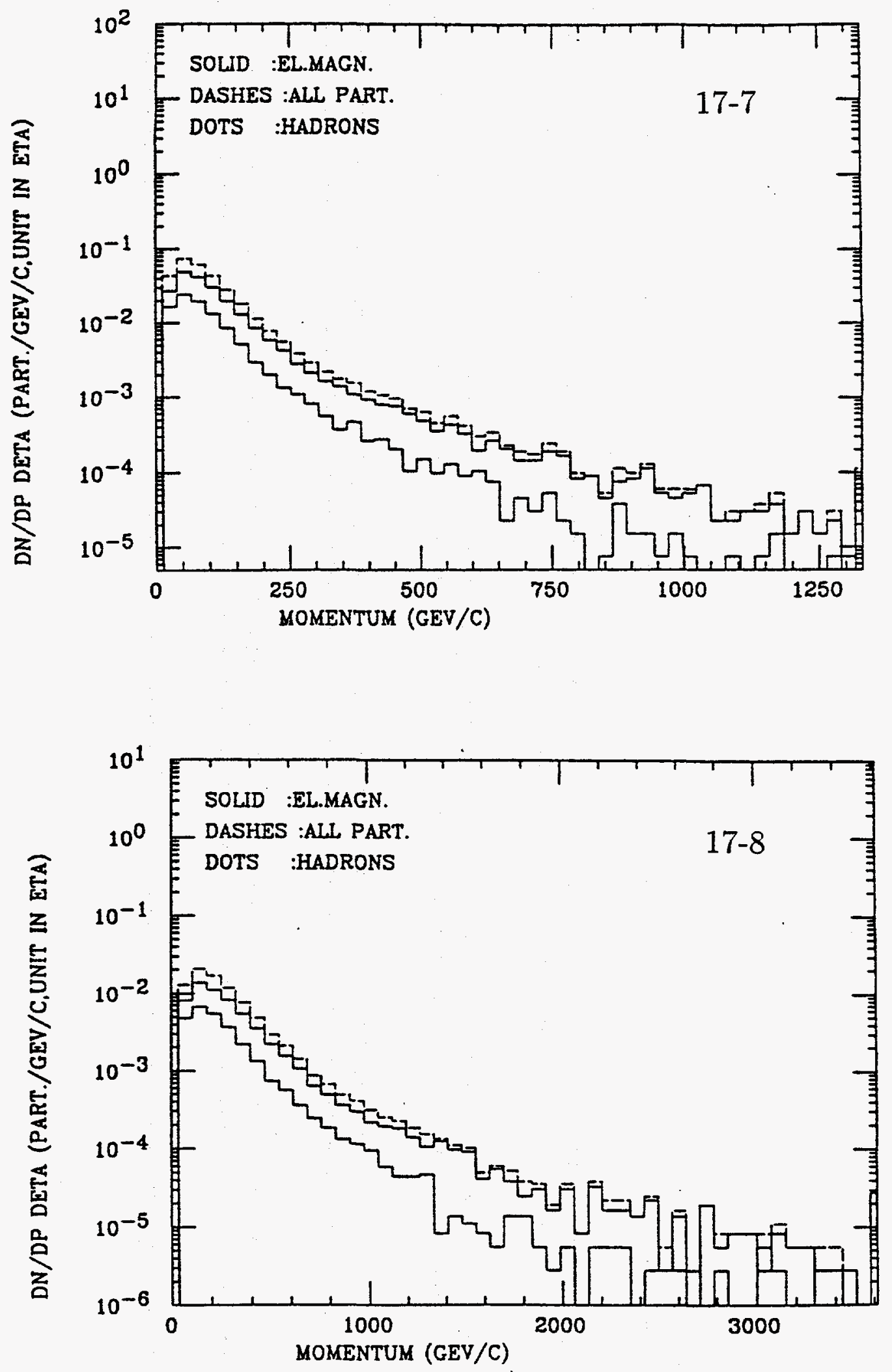

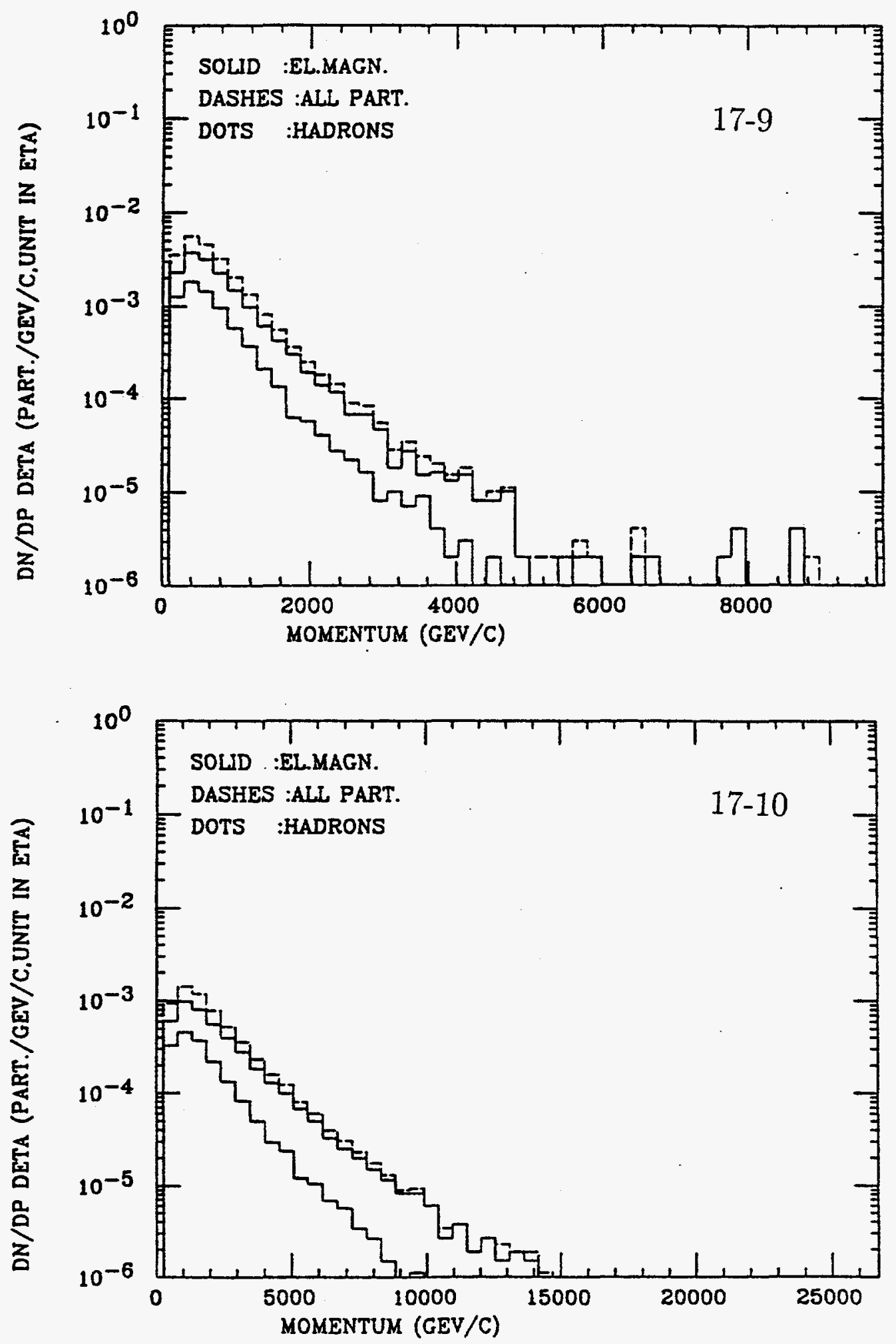

FIG. 17. Distributions $d^{2} N / d p d \eta$ [particles per $\mathrm{GeV} / \mathrm{c}$ and unit of pseudorapidity]. The Figures 17.1-17.10 give the distributions for the rapidity bins $0 .-0.5,1.0-1.5, \ldots, 9.0-9.5$. 
DTUJET P*DN/DP DETA ALL PARTICLES
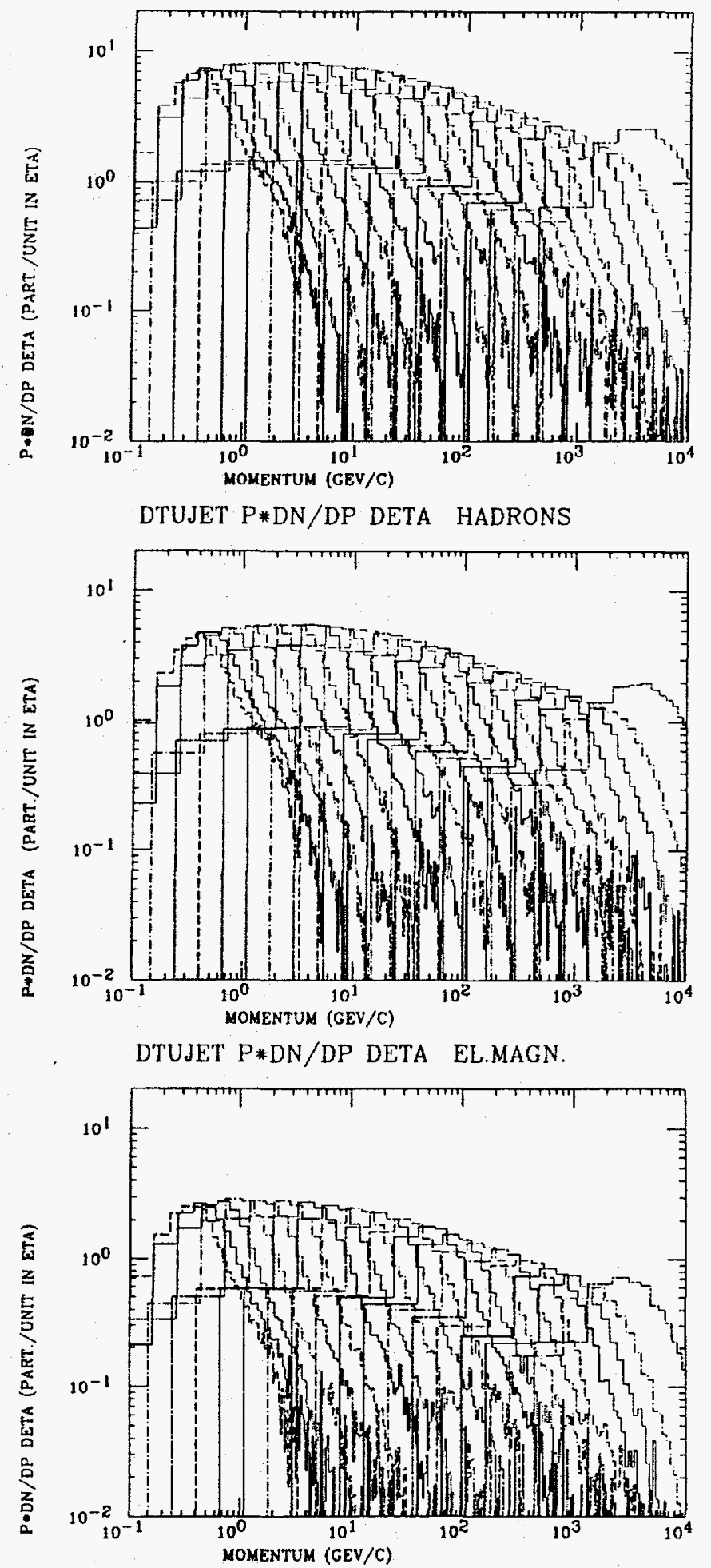

FIG. 18. Distributions $p d N / d p d \eta$ [particles per unit of pseudorapidity]. The 20 histograms on each of the three plots give the distributions for the pseudorapidity bias $0 .-0.5,0.5-1.0, \ldots, 9.5-10.0$. The curves in Fig. 18.1 to Fig. 18.3 are for all particles, hadrons, and particles leading to electromagnetic showers $\left(\pi^{0}\right.$ and $\left.\gamma\right)$. 


\section{APPENDIX A}

Expressions for the total and inelastic hadronic cross section as obtained by Capella et al. [10] from the cross sections $\sigma_{s}$ (bare soft Pomeron) and $\sigma_{h}$ (bare hard Pomeron) and a triple Pomeron contribution $\sigma_{T P}$ to take diffractive events into account.

$$
\begin{array}{rrr}
\sigma_{\text {tot }} & =\sum_{\ell+m+n \geq 1} I_{\ell, m, n} & (A-1) \\
\sigma_{\text {inel }} & =\sum_{\ell+m+n \geq 1} 2^{\ell+m+n-1} I_{\ell, m, n} & (A-2) \\
\sigma_{D} & =\sum_{\ell \geq 0, m \geq 0, n \geq 1} C_{n} 2^{\ell+m+n-1} I_{\ell, m, n} & (A-3) \\
\sigma_{h, \text { inel }} & =\sum_{\ell=0, m \geq 1, n=0} 2^{\ell+m+n-1} I_{\ell, m, n} & (A-4) \\
I_{\ell, m, n}=-\frac{8 \pi}{\ell ! m ! n !}\left[-\frac{\sigma_{s}}{8 \pi b_{s}}\right]^{\ell}\left[-\frac{\sigma_{h}}{8 \pi b_{h}}\right]^{m}\left[+\frac{\sigma_{T P}}{8 \pi b_{T P}}\right] & \\
\times\left[\frac{\ell}{b_{s}}+\frac{m}{b_{h}}+\frac{n}{b_{T P}}\right]^{-1} & (A-5)
\end{array}
$$




\section{APPENDIX B}

The cross sections for inelastic collisions with $i$ soft Pomerons ( $2 i$ soft strings) and $j$ hard Pomerons (2j hard strings).

Via a resummation of the expression given in (A-2) one obtains the inelastic cross section for $i$ soft and $j$ hard Pomerons in the form

$$
\begin{aligned}
\sigma(i, j)= & -\sum_{\substack{\ell^{\prime} \geq 0 \\
\left(j+\ell^{\prime}+n^{\prime} \geq 1\right)}} \sum_{\substack{n^{\prime} \geq 0 \\
n^{\prime}}} \sum_{m>j} \sum_{n \geq n^{\prime}} \sum_{a=0}^{n^{\prime}} \sum_{b=0}^{n^{\prime}-a} \\
& \left(\begin{array}{c}
n^{\prime} \\
a
\end{array}\right)\left(\begin{array}{c}
n^{\prime}-a \\
b
\end{array}\right)(-2)^{a} 4^{b} 2^{\ell+m+n-1}(-1)^{\ell^{\prime}+j-a-b} \\
& \times\left(\begin{array}{c}
\ell \\
\ell^{\prime}
\end{array}\right)\left(\begin{array}{c}
m \\
j
\end{array}\right)\left(\begin{array}{c}
n \\
n^{\prime}
\end{array}\right) I_{\ell, m, n} \delta_{\left(i-\ell^{\prime}-n^{\prime}-a\right)}
\end{aligned}
$$

where $I_{\ell, m, n}$ is given by (A-5).

The formula (B-1) (in the approximation with vanishing $\sigma_{T P}$ discussed in Section 4) originates from the simple formula in the impact parameter representation, which is manifestly positive and has an explicit probabilistic interpretation:

$$
\sigma(i, j, B)=\frac{\left(2 p_{s}(B)\right)^{i}}{i !} \times \frac{\left(2 p_{h}(B)\right)^{j}}{j !} \exp \left(-2 p_{s}(B)-2 p_{h}(B) \quad(B-2)\right.
$$

where $2 p_{s}(B)$ and $2 p_{h}(B)$ are the corresponding bare cross-sections in the impact parameter representation normalized as

$$
\begin{aligned}
& \int 2 p_{s}(B) d^{2} \vec{B}=\sigma_{s} \\
& \int 2 p_{h}(B) d^{2} \vec{B}=\sigma_{h}
\end{aligned}
$$

The formula (B-1) follows from Eq. (B-2) assuming a Gaussian shapes for $p_{s}(B)$ and $p_{h}(B)$. 


\section{References}

1. J. Ranft, P. Aurenche, F. Bopp, A. Capella, K. Hahn, J. Kwiecinski, P. Maire and J. Tran Thanh Van, SSC Report SSC-149 (1987).

2. A. Capella and J. Tran Thanh Van, Z. Phys. C10, 249 (1981); Phys. Lett. 114B, 450 (1982).

3. P. Aurenche and F. W. Bopp, Z. Phys. C13, 205 (1982); Phys. Lett. 114B, 363 (1982).

4. A. B. Kaidalov, Phys. Lett. 116B, 459 (1982), A. B. Kaidalov and K. A. Ter-Martirosyan, Phys. Lett. 117B, 247 (1982).

5. J. Ranft, P. Aurenche and F. W. Bopp, Z. Phys. C26, 279 (1984); P. Aurenche, F. W. Bopp and J. Ranft, Z. Phys. C23, 67 (1984); P. Aurenche, F. W. Bopp and J. Ranft, Phys. Lett. 147B, 212 (1984).

6. P. Aurenche, F. W. Bopp and J. Ranft, Phys. Rev. D33, 1867 (1986).

7. G. Arnison et al., UA1 Collaboration, Phys. Lett. 118B, 167 (1982).

8. UA1 Collaboration, presented by F. Ceradini at the Europhysics Conference on High Energy Physics, Bari 1985.

9. P. Aurenche, F. W. Bopp and J. Ranft, Contribution to the Workshop on Physics Simulations at High Energy, Madison, Wisconsin 1986, Annecy preprint LAPP-TH-161 (1986).

10. A. Capella, J. Tran Thanh Van and J. Kwiecinski, Phys. Rev. Lett. 58, 2015 (1987).

11. J. Durand and H. Pi, Phys. Rev. Lett. 58, 303 (1987).

12. J. Ranft, SSC-Report SSC-150, 1987.

13. V. A. Abramovski, V. N. Gribov and O. V. Kancheli, Yad. Phys. 18, 595 (1971).

14. B. L. Combridge, J. Kripfgaus and J. Ranft, Phys. Lett. 70B, 234 (1977).

15. E. Eichten, I. Hinchliffe, K. Lane and C. Quigg, Rev. Mod. Phys. 56, 579 (1984); Rev. Mod. Phys. 58, 1065 (1986).

16. J. C. Collins, Illinois Tech. preprint 86-0298 (1986).

17. M. M. Block and R. N. Cahn, Rev. Mod. Phys. 57, 563 (1985).

18. H. U. Bengtson and G. Ingelman, Comp. Phys. Commun. 34, 251 (1985); T. Sjöstrand and M. van Zijl, Phys. Rev. D36, 2019 (1987).

19. A. Capella, U. Sukhatme and J. Tran Thanh Van, Z. Phys. C3, 329 (1980); A. B. Kaidalov, Pis'ma Zh. Esp. Teuv. Fiz. 32, 494 (1980); Yad. Fiz. 33, 1369 (1981).

20. S. Ritter and J. Ranft, Acta Phys. Polonica B11, 259 (1980); S. Ritter, Z. Phys. C6, 27 (1982); Comp. Phys. Commun. 31, 393 (1984).

21. K. Hänßgen and S. Ritter, Comp. Phys. Commun. 31, 411 (1984).

22. G. J. Alner et al., UA-5 Collaboration, Z. Phys. C33, 1 (1986).

23. G. Arnison et al.,UA-1 Collaboration, Phys. Lett. 118B, 167 (1982).

24. G. J. Alner et al., UA-5 Collaboration, Phys. Lett. 138B, 304 (1984).

25. D. E. Groom, SSC-Report SSC-N-154 (1986). 



\title{
APPENDIX 4 \\ SIMULATION OF MINI-JETS IN MINIMUM-BIAS EVENTS
}

\author{
T. K. Gaisser and Todor Stanev \\ Bartol Research Institute \\ University of Delaware, Newark DE 19716
}

We are constructing a Monte Carlo algorithm for calculations of minimum bias events that takes account explicitly of production of multiple soft jets as calculated in perturbative QCD. A primary goal is the application to calculation of cosmic ray cascades in the atmosphere generated by cosmic rays with energies of order $1 \mathrm{PeV}\left(=10^{6} \mathrm{GeV}\right)$ to $1 \mathrm{EeV}\left(=10^{9} \mathrm{GeV}\right)$ (respectively corresponding to $\sqrt{s} \simeq 1 \mathrm{TeV}$ to $40 \mathrm{TeV}$ ). Examples of applications include the interpretation of coincident multiple muons observed with large, deep underground detectors (PeV range) and calculation of giant air showers as observed by the Fly's Eye and related detectors (EeV range). In both cases a primary goal of the experiments is to determine the gross features of the chemical composition of cosmic rays in a region where the flux is too low to be accessible to direct observation of the primary particles. In the higher energy range it is also possible to measure some gross features of hadronic cross sections in regions not yet accessible to accelerators.

We focus especially on the (forward) fragmentation region and require an interaction program that in a single run can easily handle a wide variety of projectile particles and energies. Although our intended applications are different from accelerator oriented programs such as PYTHIA, ISAJET and DTUJET, the physics is the same in both cases and it is useful to compare the results of the two kinds of simulations. Moreover, the emphasis here on inelasticities and the fragmentation region may also lead to some results of interest for studies of minimum bias events at colliders.

Several aspects are of particular interest for us in designing this algorithm.

- We need to get the transverse structure of interactions correct in detail (including correlations between multiplicity and $p_{\perp}$ and between longitudinal and transverse momentum).

The transverse structure is particularly important for calculation of multiple, coincident muons from cosmic ray air showers as seen in underground detectors. The detectors, though large, are nevertheless finite. Therefore, separation of the muons is crucial for understanding the relation between the number of muons detected and the total number in the shower. We 
are talking here about muons from decay of pions in the atmosphere that typically have energies of 1 to $10 \mathrm{TeV}$ or more at production.

- We need to be able to extrapolate the behavior of the fragmentation region beyond energies of present accelerators.

The basic idea here is to assume an underlying scaling form for hadronization of low $p_{\perp}$ jets, including beam jets, but to account correctly for the increase in the number of mini-jets with energy. This will explain the rise in the central rapidity distribution and (by energy conservation) will lead to a corresponding softening of the distribution in the fragmentation region.

- The algorithm has to be arranged so that it can readily be extended to calculated interactions on nuclear targets.

The idea of explaining the rise in the central region in terms of soft jets (the low $p_{\perp}$ tail of hard scattering) was suggested several years ago $[1,2]$. The cross section was written as the sum of two terms

$$
\sigma(s)=\sigma_{0}+\sigma_{\mathrm{jet}}\left(s, p_{\perp}^{\mathrm{min}}\right)
$$

The energy dependent, $\sigma_{\text {jet }}$ is the convolution of the structure functions for partons in the incident protons with the elementary cross section for hard scattering of the partons, subject to the condition

$$
x_{1} x_{2} s>4\left(p_{\perp}^{\min }\right)^{2}
$$

As usual, $x_{1}$ and $x_{2}$ are the fractions of the incident momentum carried by the partons that undergo hard scattering. Because of the rapid growth of the structure functions at small $x$, the constraint (2) causes $\sigma_{\text {jet }}$ to grow rapidly with energy - to the point where $\sigma_{\text {jet }} / \sigma$ exceeds unity at high enough energy.

In the past year several authors (e.g. Durand and Pi[3], Ametller and Treleani [4], and Dias de Deus and Kwiecinski [5]), have pointed out how to understand this. The answer is that $\sigma_{\text {jet }}$ calculated as described above is an inclusive cross section, so the ratio of the jet to inelastic cross sections is

$$
\frac{\sigma_{\text {jet }}}{\sigma}=\left\langle n_{\text {jet }}\left(p_{\perp}^{\min }\right)\right\rangle
$$

where $\left\langle n_{\text {jet }}\left(p_{\perp}^{\min }\right)\right\rangle$ is the mean number of jet pairs with transverse momentum above the cutoff. The whole calculation can be unitarized in an eikonal formalism, and the total cross section calculated in a consistent way and in agreement with the data. 
The formalism can also be used as the basis of a Monte Carlo interaction program in which $n_{\text {jet }}$ is chosen randomly from the model distribution. This is followed by a choice of $n\left\{x_{1}, x_{2}\right\}$ pairs, a scattering angle for each pair of jets in its $\mathrm{CM}$ frame and then hadronization of each jet. Finally, beam and target jets with momenta $\left(1-\sum x_{1}\right) \sqrt{s} / 2$ and $\left(1-\sum x_{2}\right) \sqrt{s} / 2$, respectively are hadronized. The attached figures give the results of a very preliminary version of this simulation program. The same choice of $p_{\perp}^{\min }=\sqrt{2} \mathrm{GeV}$ that gives a good account of the cross section [3] also gives a very reasonable prediction for the rise in the central rapidity density (Fig. 1). The corresponding (relatively small) scaling violation in the fragmentation region is shown in Fig. 2.

A reassuring feature of the model is that it is now relatively insensitive to the choice of $p_{\perp}^{\mathrm{min}}$. This is because of the unitarization provided by the eikonal formalism. The choice of $\sqrt{2} \mathrm{GeV}$ for $p_{\perp}^{\min }$ is made to reproduce the rise in the total cross section without introducing any energy dependence into $\sigma_{0}[3]$. If a different $p_{\perp}^{\min }$ is chosen some energy-dependence would have to be introduced, but it would be relatively small. For example, increasing the minimum transverse momentum by as much as a factor of two (without any other change of parameters) only leads to a $20 \%$ decrease in $d n / d y$. As $p_{\perp}^{\min }$ increases, the average number of jets above threshold decreases, but at the same time the multiplicity per jet increases. It is this compensation that leads to the relative stability of the result, and gives us some confidence in using the model for the extrapolation to $\sqrt{s} \simeq 40 \mathrm{TeV}$.

The pseudorapidity and angular distributions of the number and energy carried by charged particles in inelastic non-diffractive pp interactions at $\sqrt{s}=40$ $\mathrm{TeV}$ are shown on Figs. 3-6. The histograms are based on a run of 2000 events. The average charged multiplicity $\left\langle n_{\mathrm{ch}}\right\rangle=100 \pm 2$ particles.

ACKNOWLEDGMENT. We are grateful for a helpful conversation with L. Durand. One of us (T. S.) enjoyed the hospitality of D. E. Groom and the SSC Central Design Group. This work is supported by the National Science Foundation and by the U. S. Department of Energy.

\section{References}

1. T. K. Gaisser and F. Halzen, Phys. Rev. Lett. 541754 (1987).

2. G. Pancheri and Y. Srivastava, Proc. Santa Fe DPF Meeting, T. Goldman and M. M. Nieto, Eds., World Scientific, Singapore, p. 29 (1984).

3. Loyal Durand and Hong Pi, Phys. Rev. Lett. 58, 303 (1987).

4. Ll. Ametller and D. Treleani, Proc. La Thuile Workshop (1987).

5. J. Dias de Deus and J. Kwiecinski, CERN-TH.4759/87. 


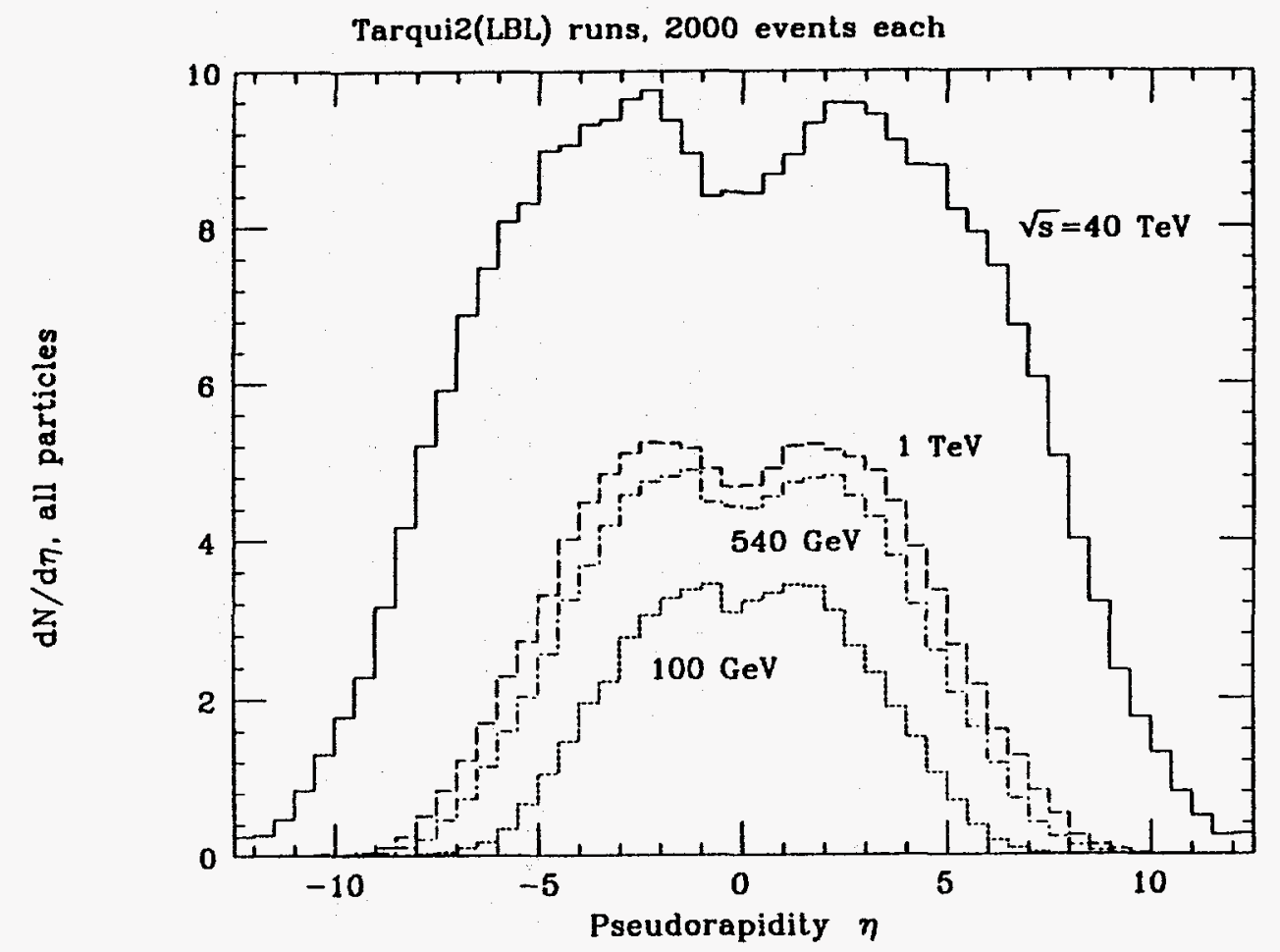

FIG. 1. Pseudorapidity distributions of all secondary particles at different energies.

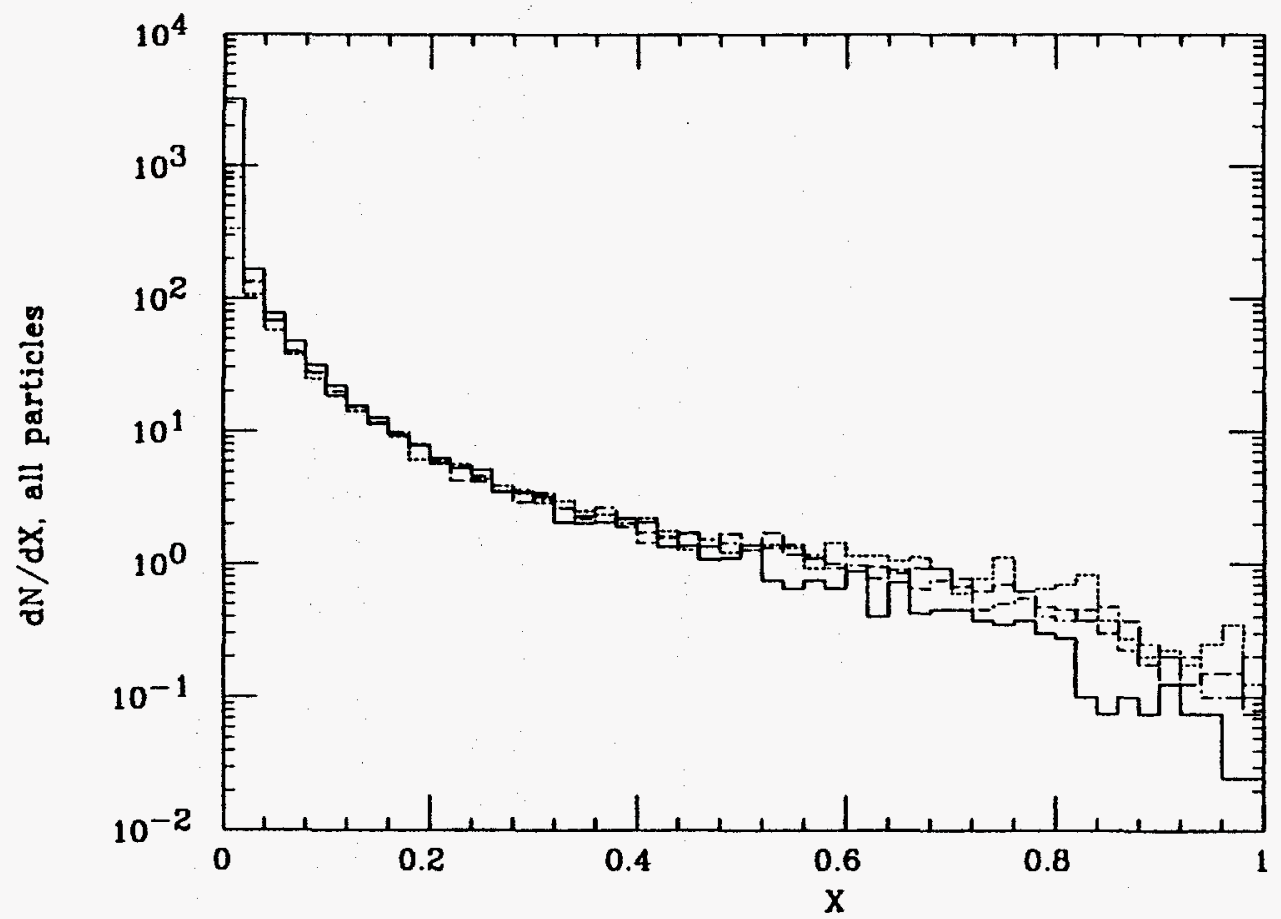

FIG. 2. $d N / d x$ distributions of forward secondary particles. The lines are coded as in Fig. 1. 


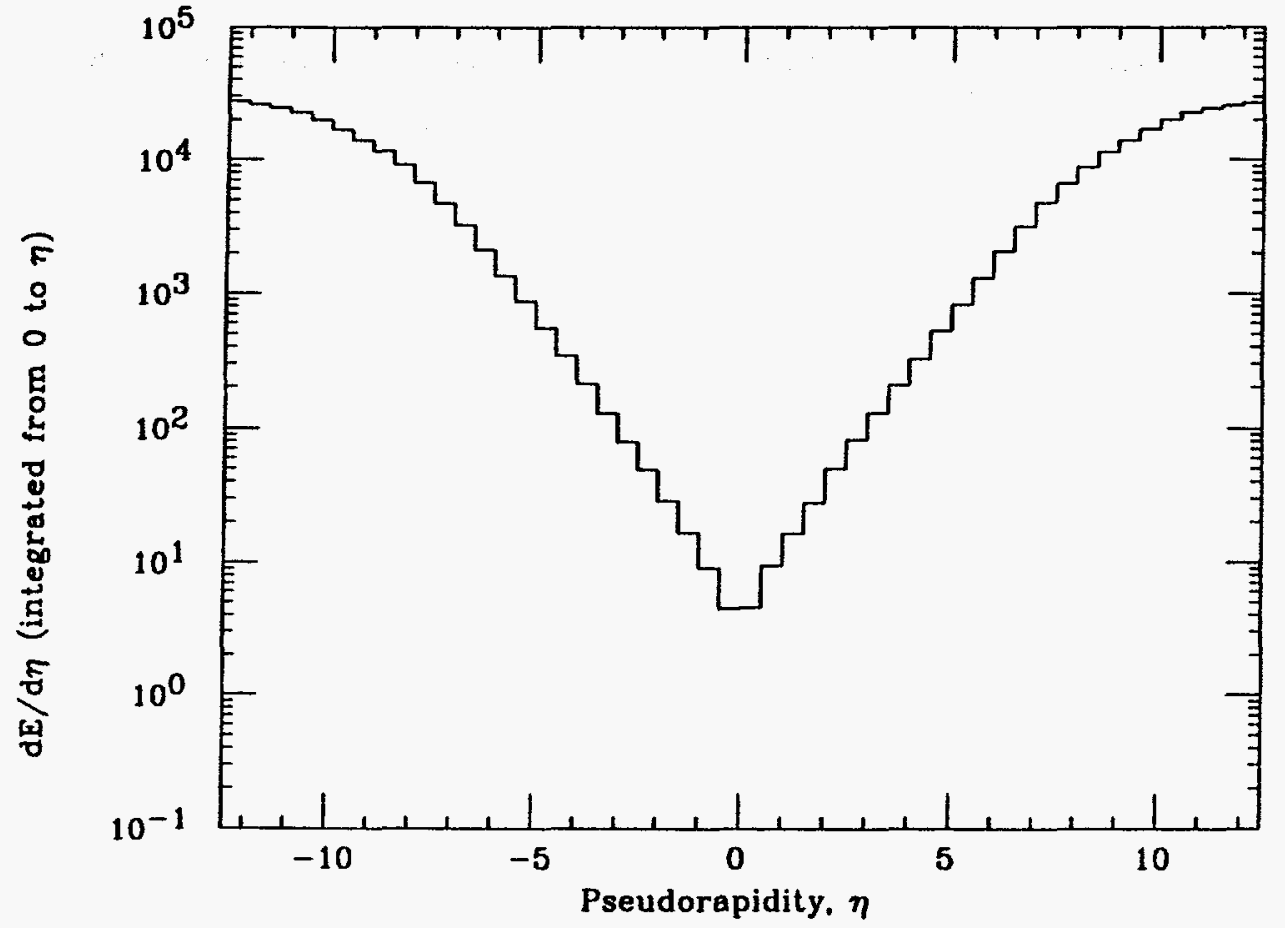

FIG. 3. Energy carried by charged particles with pseudorapidity $<\eta$ at $\sqrt{s}=40 \mathrm{TeV}$. The value at the kinematic limit shows the total energy carried by charged particles in each hemisphere.

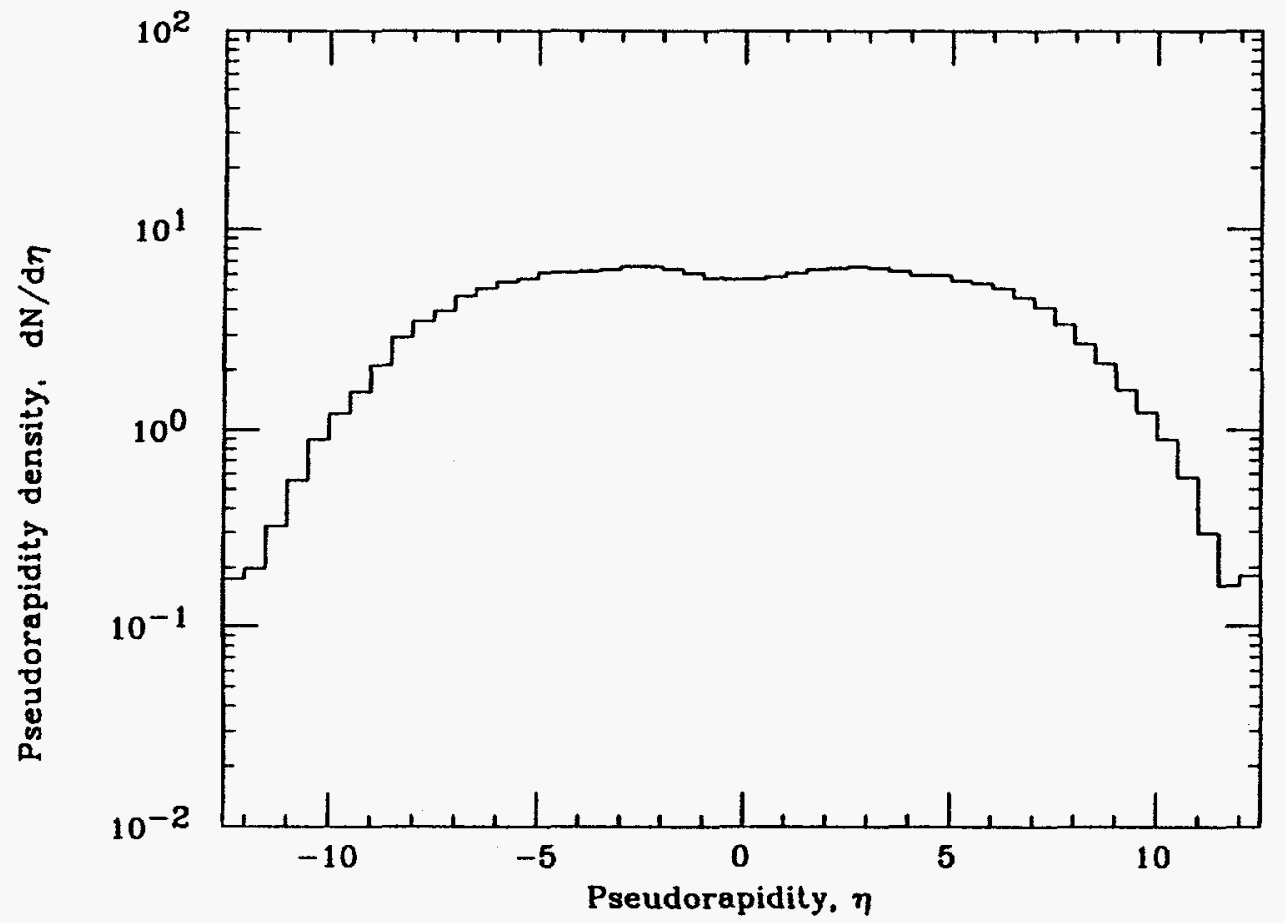

FIG. 4. Pseudorapidity distribution of charged particles at $\sqrt{s}=40 \mathrm{TeV}$. 


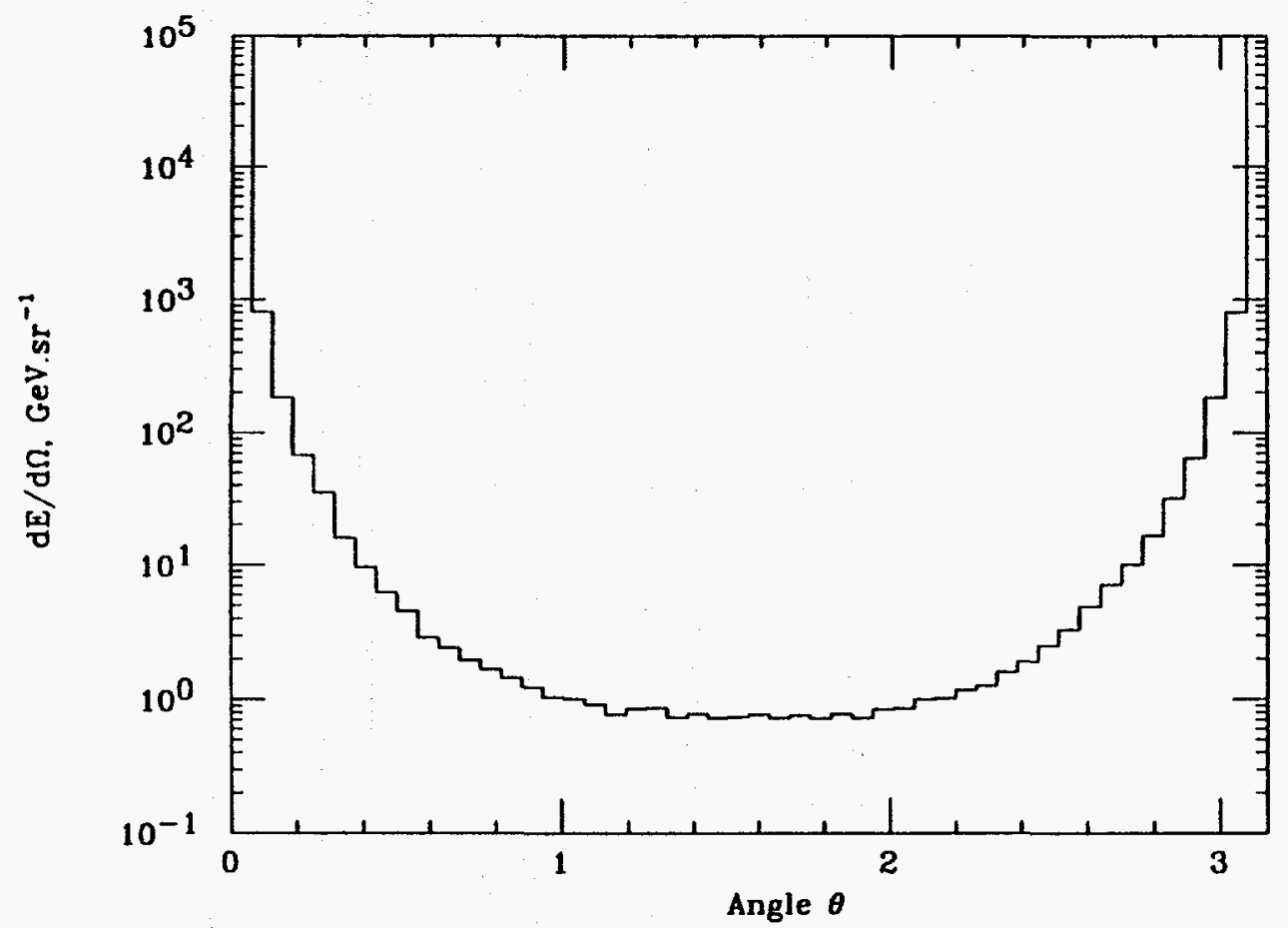

FIG. 5. Angular distribution of the energy carried by charged particles at $\sqrt{s}=40 \mathrm{TeV}$.

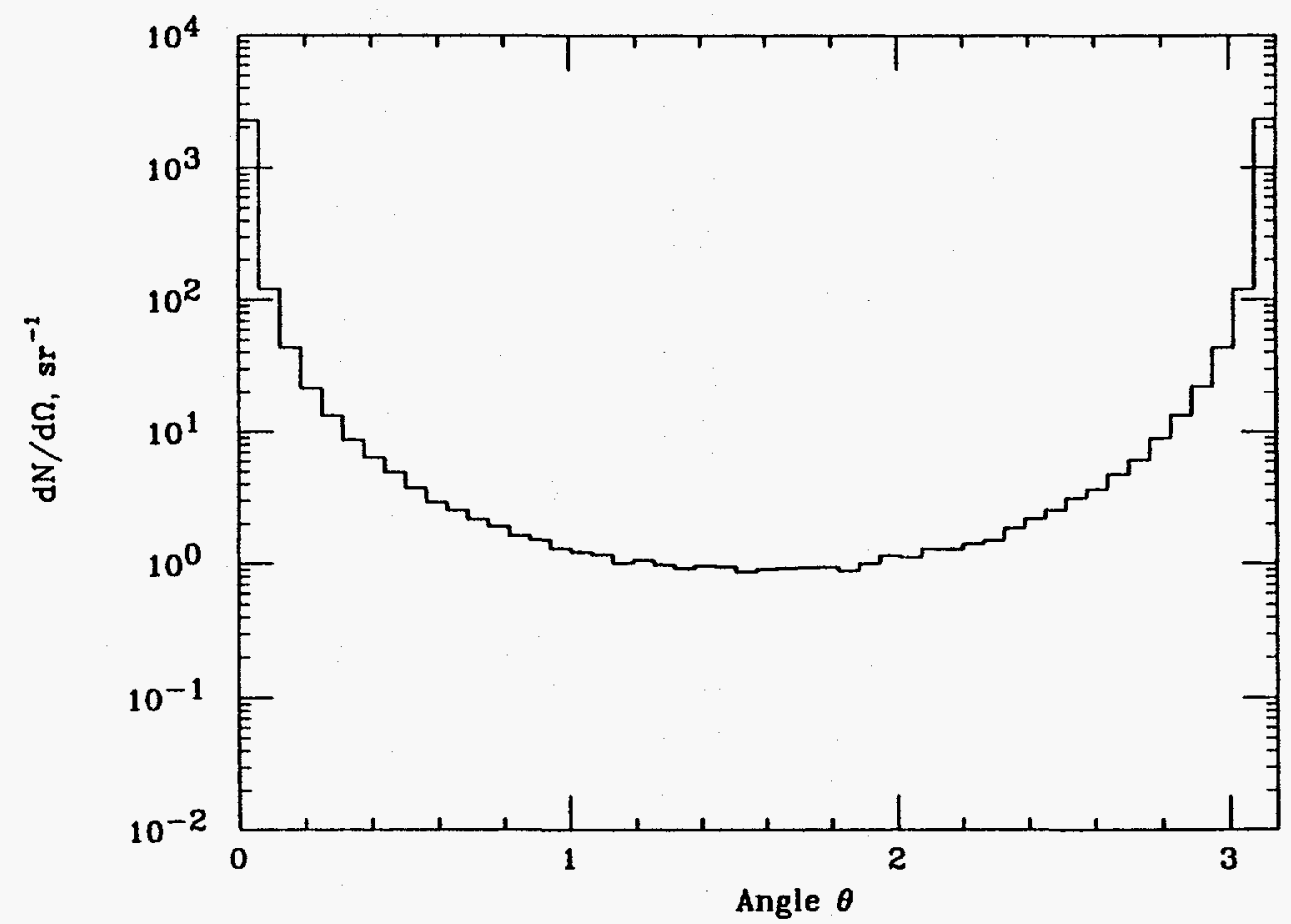

FIG. 6. Angular distribution of charged particles at $\sqrt{s}=40 \mathrm{TeV}$. 


\title{
APPENDIX 5 \\ CROSS SECTIONS, MULTIPLICITIES AND ENERGY FLOW AS PREDICTED BY ISAJET AND PYTHIA
}

\author{
Edward M. Wang \\ Lawrence Berkeley Laboratory \\ University of California \\ Berkeley, California 94720
}

The present state of the art description of low $p_{\perp}$ physics is described elsewhere in this Report. Only the predictions of ISAJET 5.34 and PYTHIA 4.9 physics Monte Carlo generators are presented here. The inelastic low $p_{\perp}$ events at $\sqrt{s}=40 \mathrm{TeV}$ are simulated by generating a mixture of QCD hard scatters (with a lower cutoff on the outgoing parton $p_{\perp}$ in the hard scatter frame) and soft scatters (different prescriptions implemented in ISAJET and PYTHIA) $[1,2]$.

\section{Cross Sections}

The behavior of low $p_{\perp}$ QCD hard scatters as a function of $p_{\perp}$ cutoff is plotted in Fig. 1. Thus at around $p_{\perp}=3 \mathrm{GeV}$, the QCD cross section about $200 \mathrm{mb}$. Default $\mathrm{Q}^{2}$ scale definitions used in the structure functions in ISAJET and PYTHIA are different, thus giving rise to a systematic difference in the estimate for QCD hard scatter cross sections.

The cross section for soft scattering events is $137 \mathrm{mb}$ (ISAJET) and $96 \mathrm{mb}$ (PYTHIA). The number from PYTHIA is essentially a subtraction of the diffractive plus elastic cross section from the total cross section (all of which are fits to data) [2].

\section{Multiplicities and Momentum Spectra}

Comparison between ISAJET and PYTHIA cannot be made in a completely consistent manner due to significant differences in the implementation of soft scatters. ISAJET uses Pomerons and (modified) independent fragmentation [1]. PYTHIA uses multiple QCD interactions and string fragmentation [2]. In ISAJET, one needs to generate QCD hard scatter and Minbias soft scatter events separately and relevant distributions are obtained by mixing. In PYTHIA the mixing of QCD and soft scatters (which are not necessarily Minbias events) are done automatically. Furthermore, multiple interactions are included in PYTHIA. Thus the QCD hard scattering distributions are well defined and can be compared while the soft scattering distributions cannot be compared easily. The net distributions, however, can be compared (and are the ones of interest anyway).

Figure 2 shows the overall distribution in $\eta$ (pseudorapidity) vs. $\log _{10} p$. Figure 3 shows the transverse momentum distribution for ISAJET Minbias and QCD 
events. Figures $4 \mathrm{a}$ and $4 \mathrm{~b}$ show momentum distributions given by ISAJET and PYTHIA for $\eta$ slices $=0,3,6$.

Figures $5 \mathrm{a}$ and $5 \mathrm{~b}$ give the $\eta$ distribution for the electromagnetic (EM) and hadronic components of QCD hard scatter $\left(p_{\perp}>3 \mathrm{GeV}\right)$ events. Similarly, Figs. $6 \mathrm{a}$ and $6 \mathrm{~b}$ give the $\eta$ distributions for the EM and hadronic components of soft scatter events. (Note: for PYTHIA, the distribution for events with "2-string scatters" [1] are shown. These are not directly comparable to ISAJET Minbias events).

Figures $7 \mathrm{a}$ and $7 \mathrm{~b}$ give the best estimate of the $\eta$ distributions for the appropriate mixture of hard and soft scatters (50\% each for ISAJET and complicated for PYTHIA).

The average total charged particle multiplicity per event is 110 for ISAJET (50\% mix of Minbias and QCD hard scatters) and 120 for PYTHIA.

\section{Energy Flow}

Finally, the average total EM and hadronic energy flow are given in Figs. 8a and $8 \mathrm{~b}$ in terms of the polar angle. Figures $9 \mathrm{a}$ and $9 \mathrm{~b}$ give the average total EM and hadronic energy flow in terms of cosine of the polar angle. Outgoing particles with polar angle less than $1 \mathrm{mR}$ are excluded.

\section{Conclusion}

The bottom line is that ISAJET and PYTHIA give nearly identical estimates for multiplicity and energy flow distributions though differing on how the hard and soft scattering components are mixed.

\section{References}

1. F. E. Paige and S. D. Protopopescu, "ISAJET 5.35, A Monte Carlo Event Generator for p-p and p-p Reactions," ISAJET PAM file.

2. H.-U. Bengtsson, Torbjorn Sjostrand, "The Lund Monte Carlo for Hadronic ProcessesPYTHIA version 4.8," LU TP 87-3 (Jan 1987). 


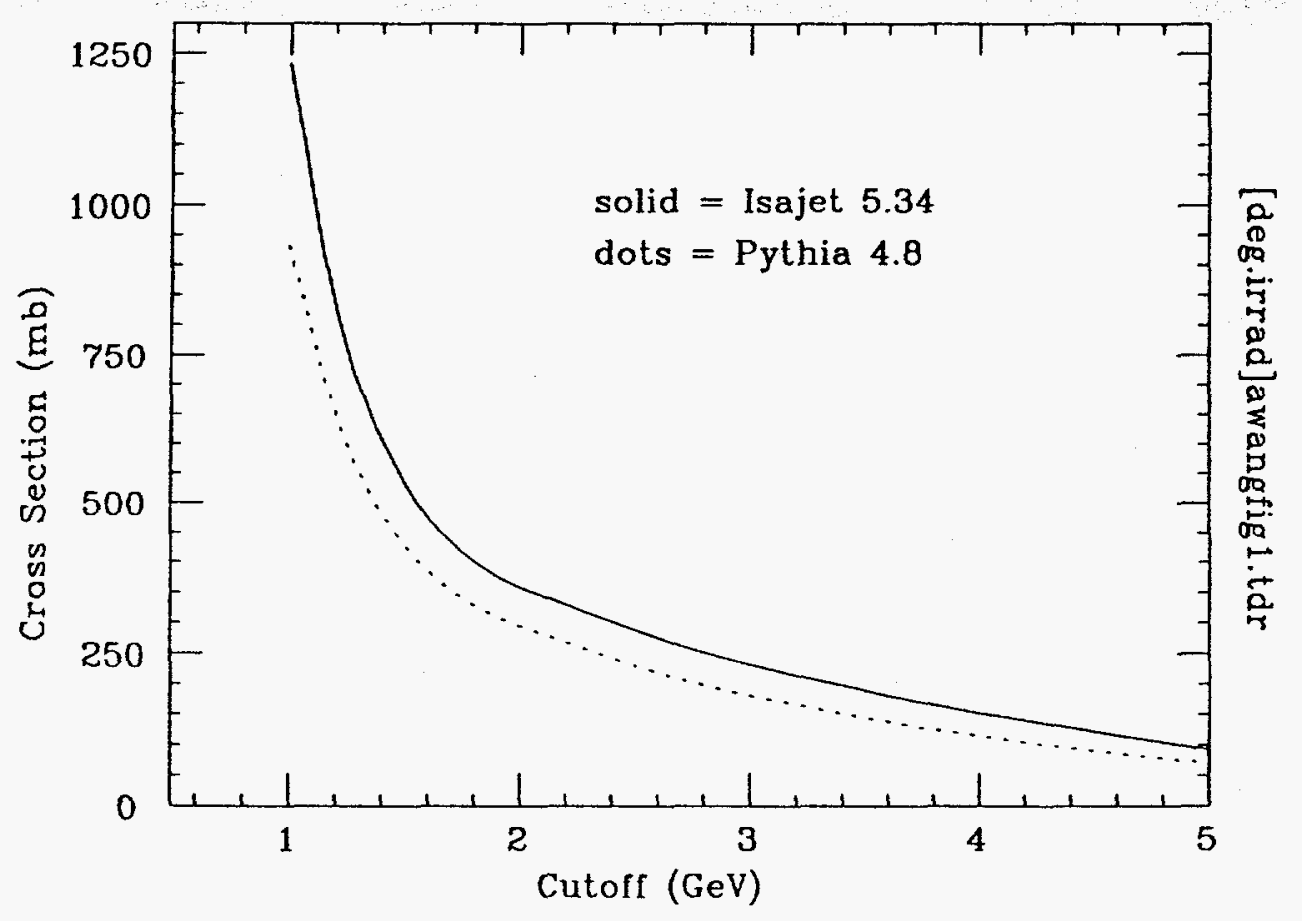

FIG. 1. Low $p_{\perp}$ QCD hard scattering behavior as a function of cutoff $p_{\perp}$.

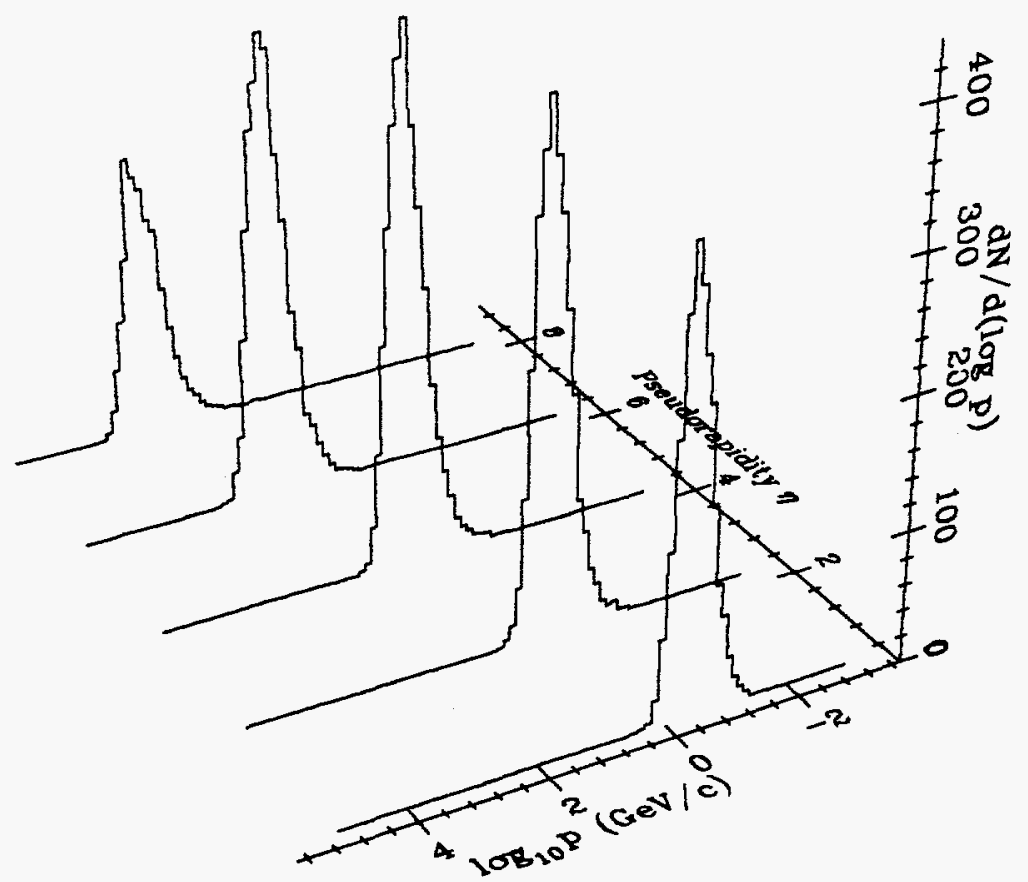

FIG. 2. Momentum distributions at constant $\eta$ for ISAJET events at $\sqrt{s}=40 \mathrm{TeV}$. 


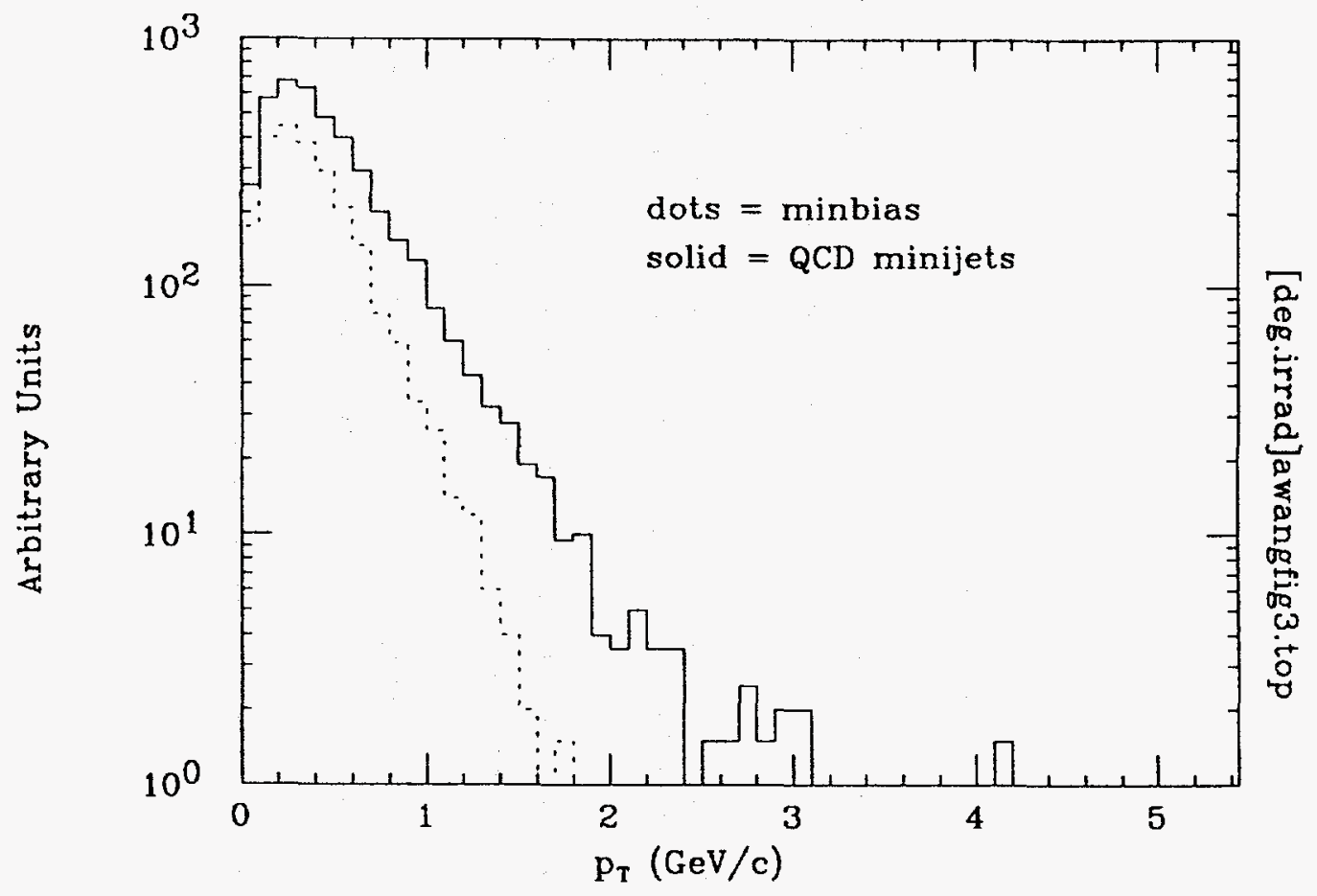

FIG. 3. Transverse momentum disstribution for ISAJET Minbias and QCD events. 

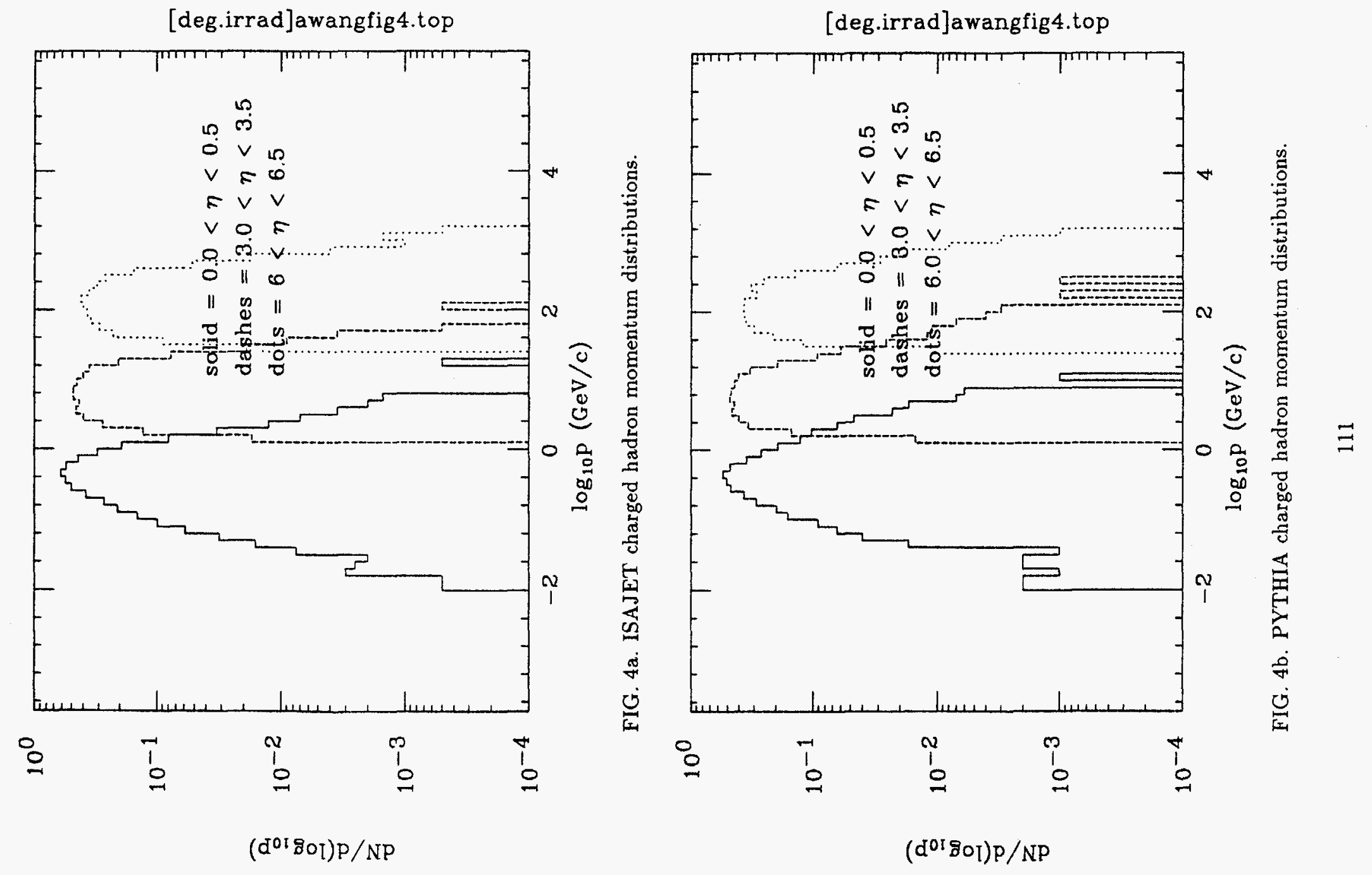


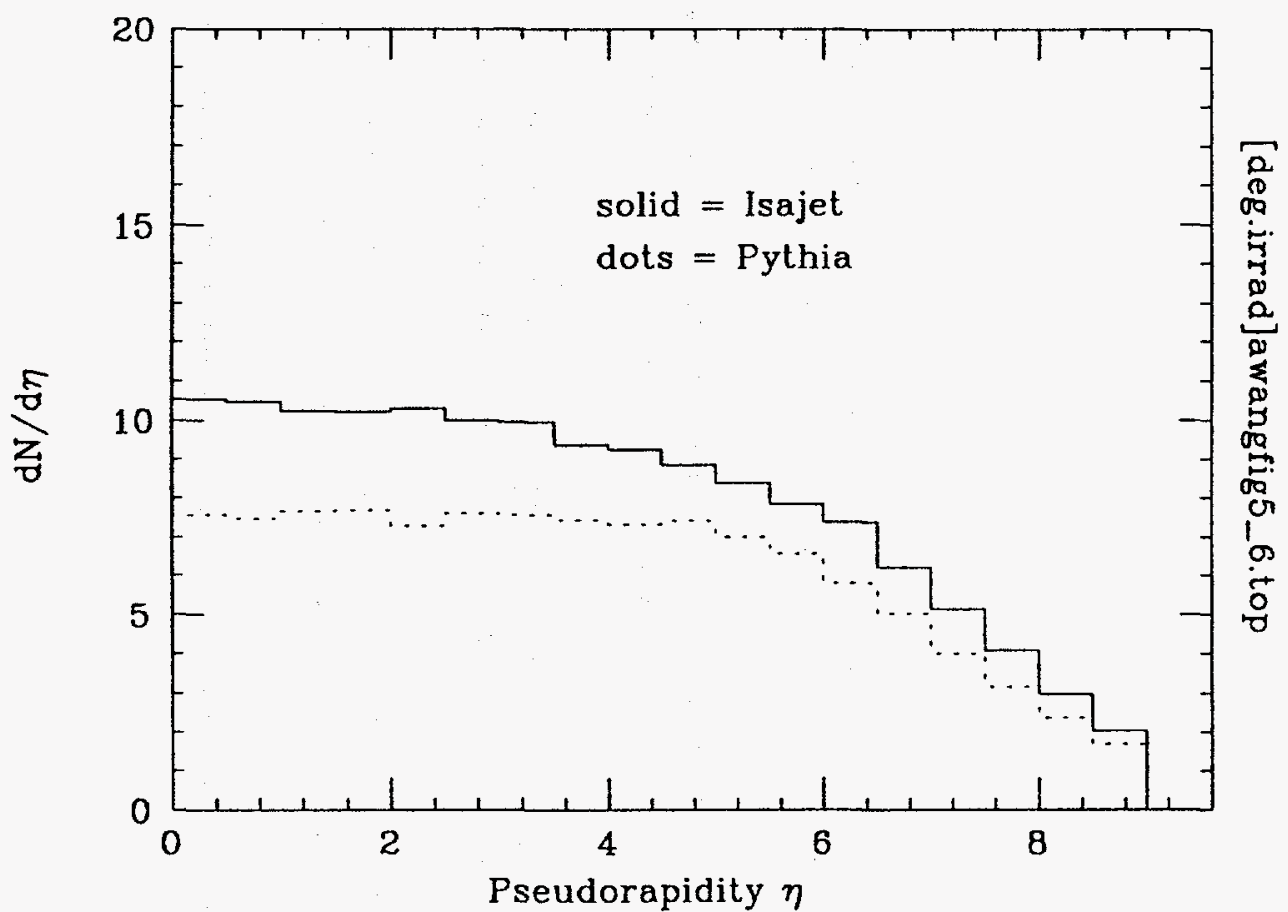

FIG. 5a. $\eta$ distribution for the electromagnetic components of QCD hard scatter $\left(p_{\perp}>3\right.$ $\mathrm{GeV}$ ) events.

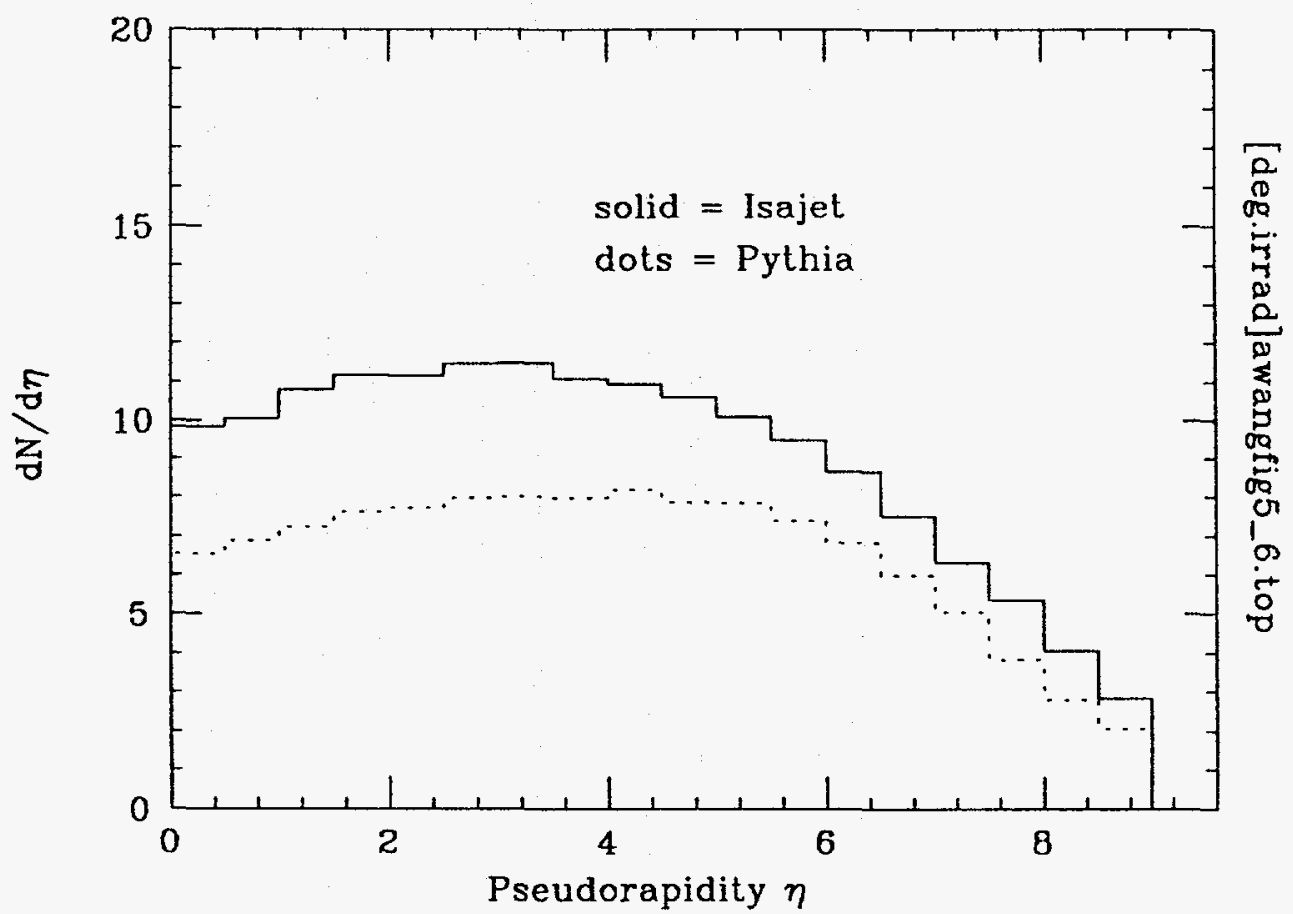

FIG. 5b. $\eta$ distribution for the charged hadronic components of QCD hard scatter $\left(p_{\perp}>3 \mathrm{GeV}\right)$ events. 


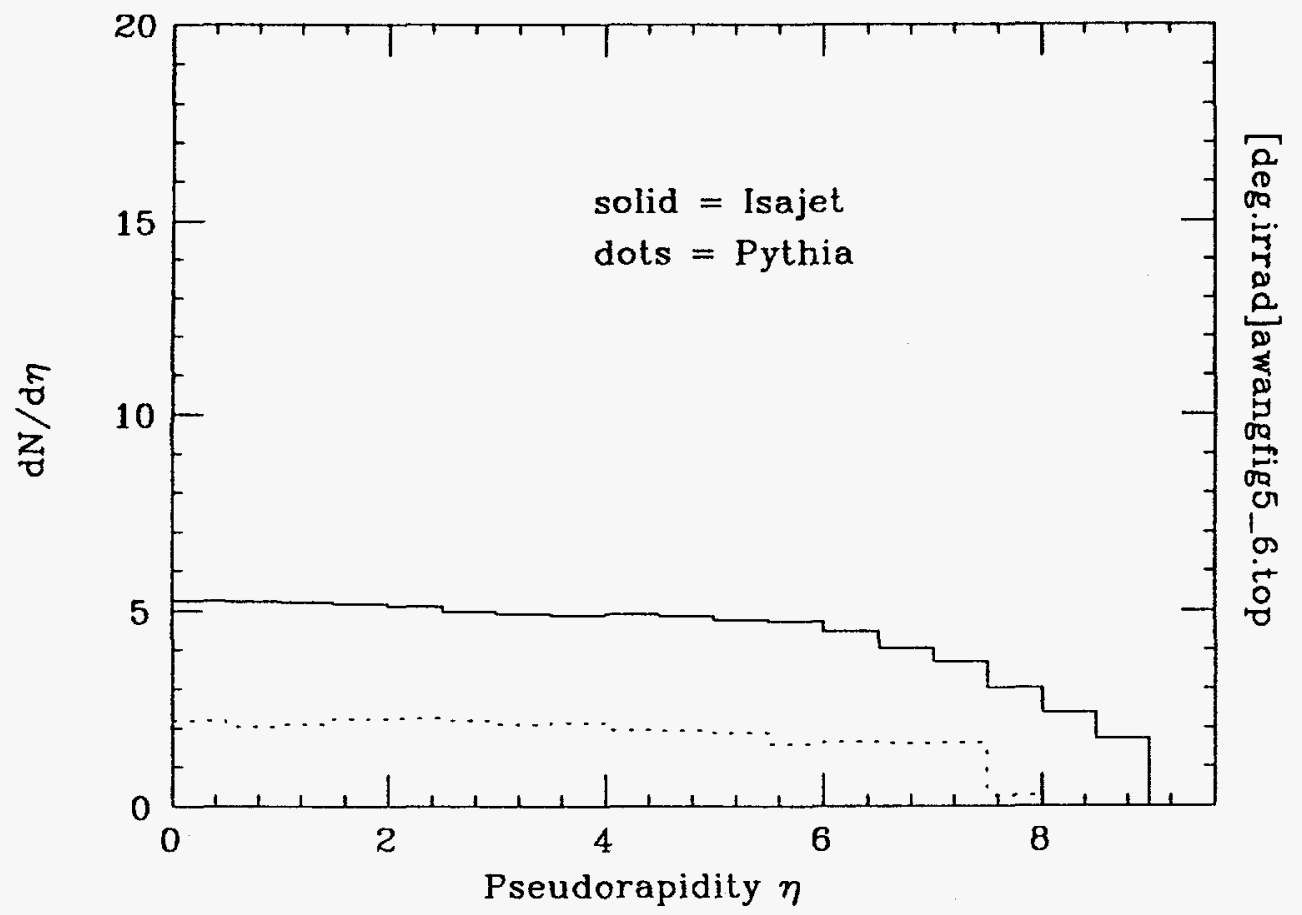

FIG. 6a. $\eta$ distribution for the electromagnetic components of QCD soft scatter events.

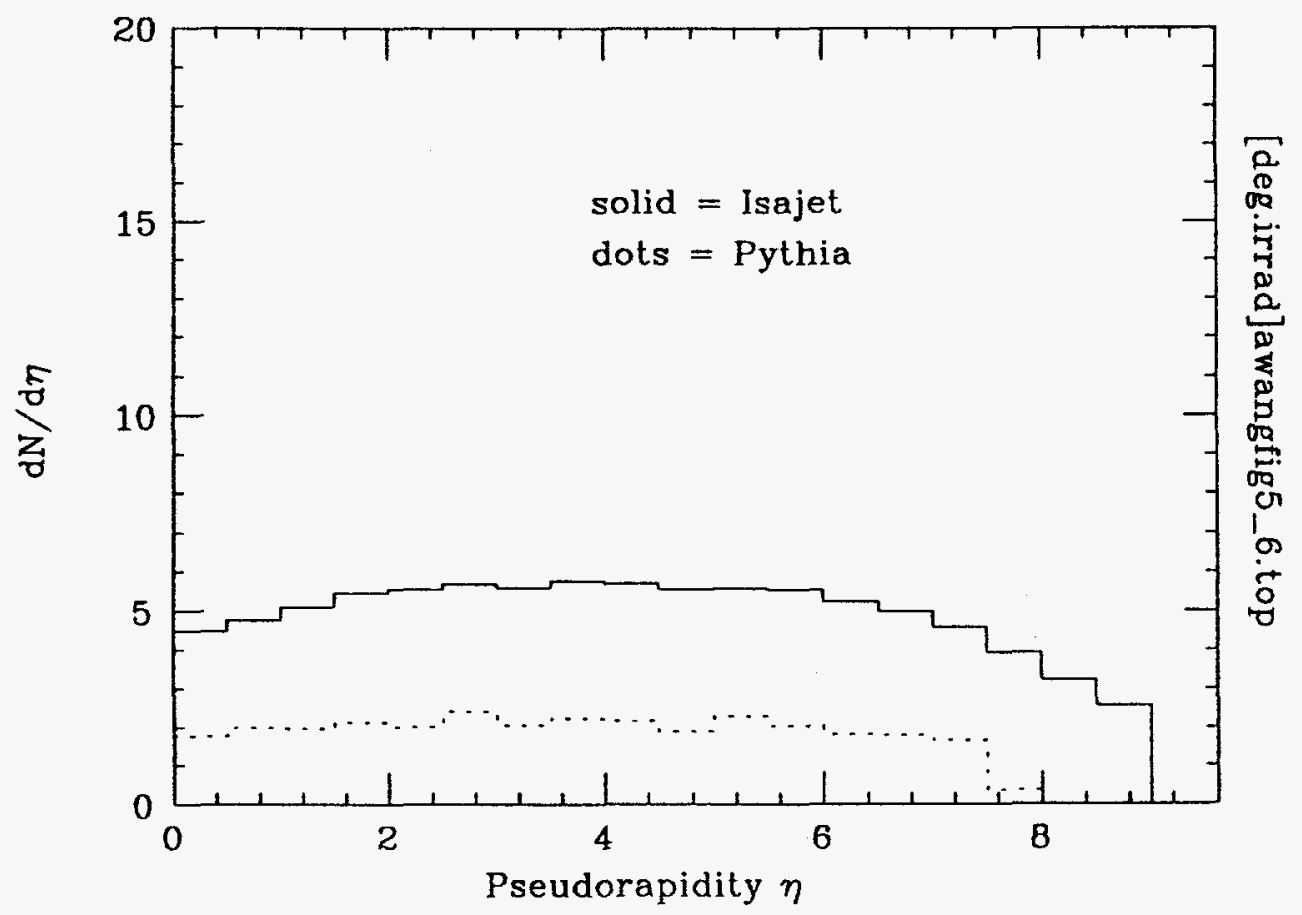

FIG. 6b. $\eta$ distribution for the charged hadronic components of QCD soft scatter events. 


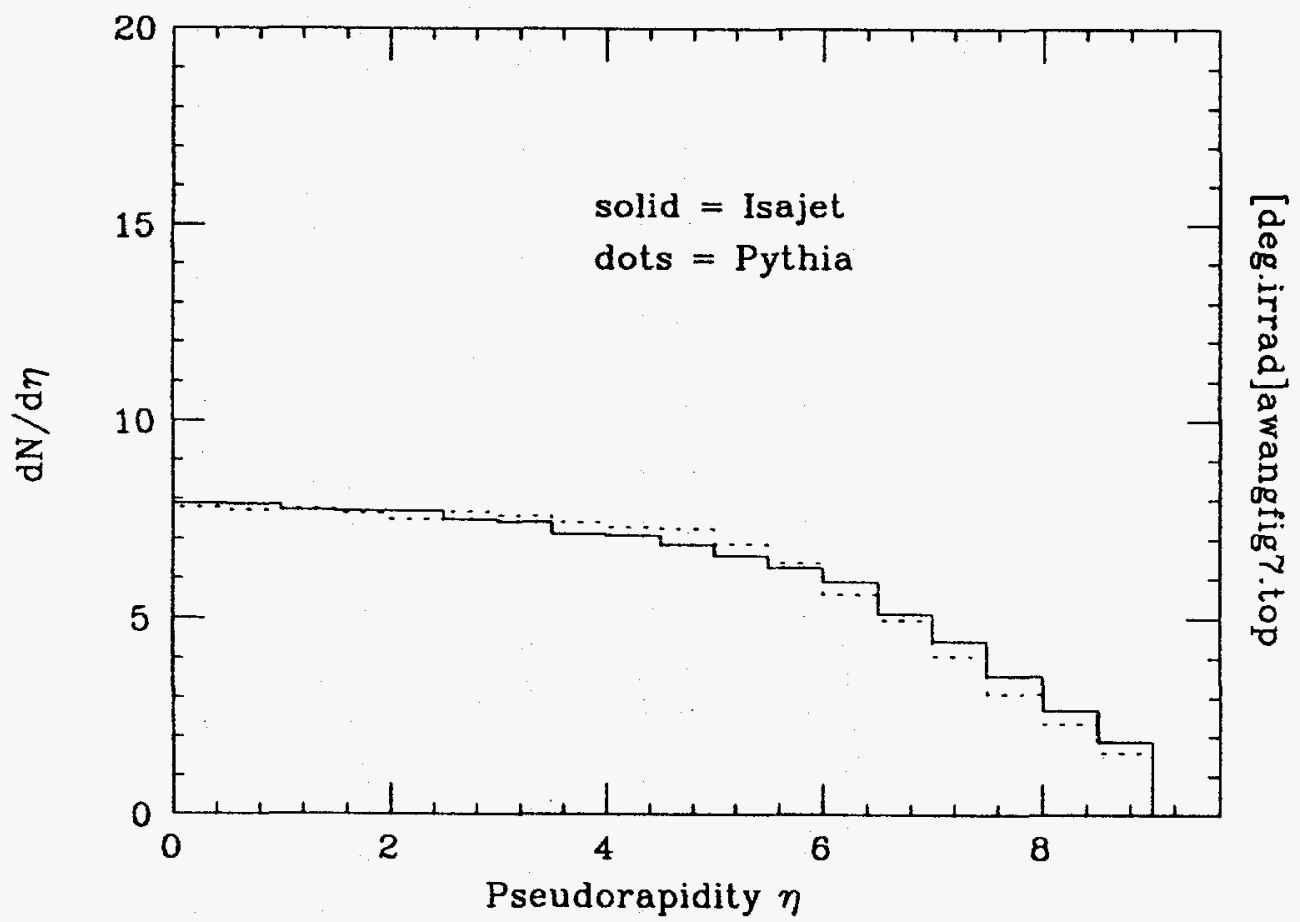

FIG. 7a. $\eta$ distributions for the electromagnetic components of an appropriate mixture of QCD hard and soft scatter events.

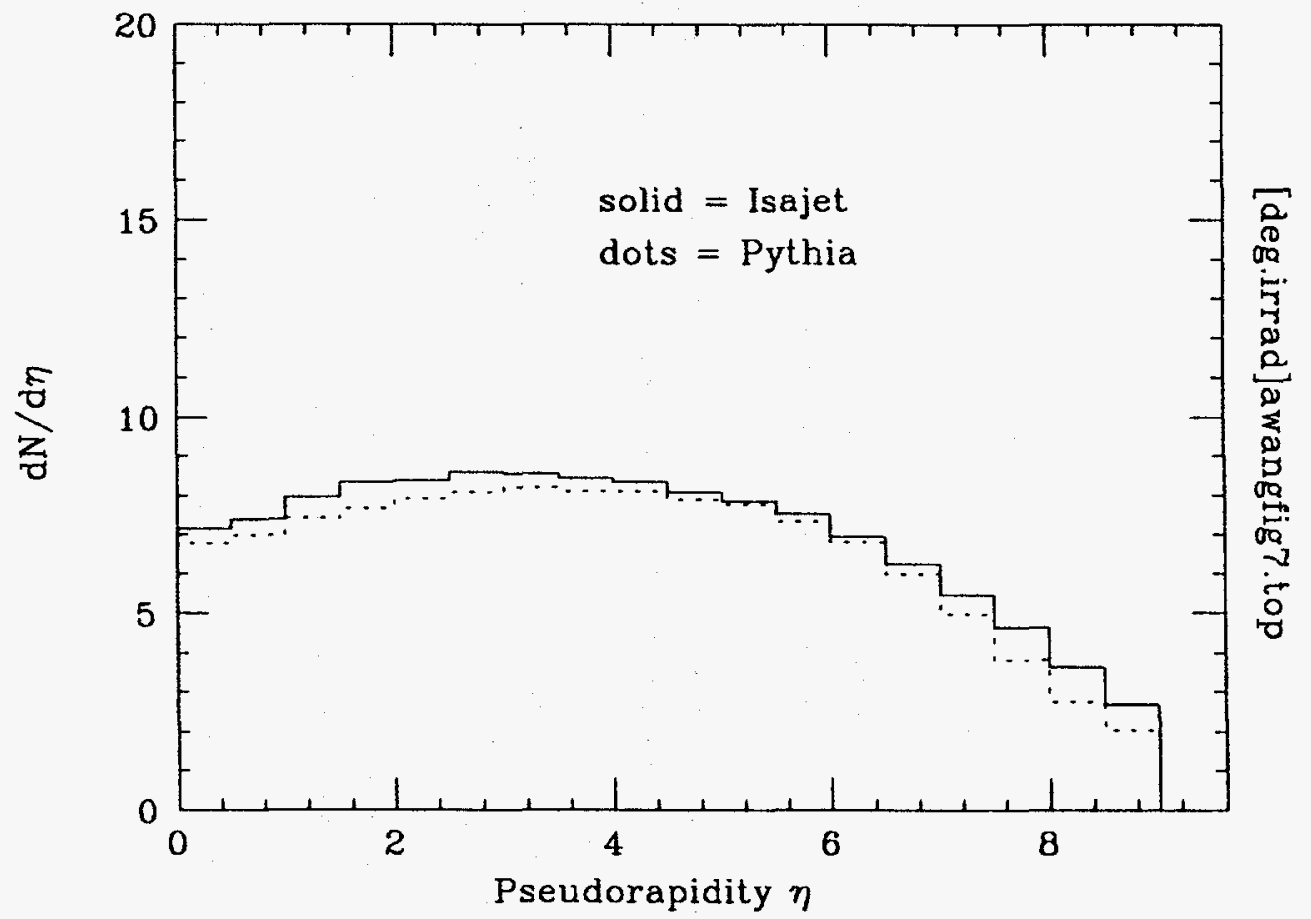

FIG. 7b. $\eta$ distributions for the charged hadronic components of an appropriate mixture of QCD hard and soft scatter events. 


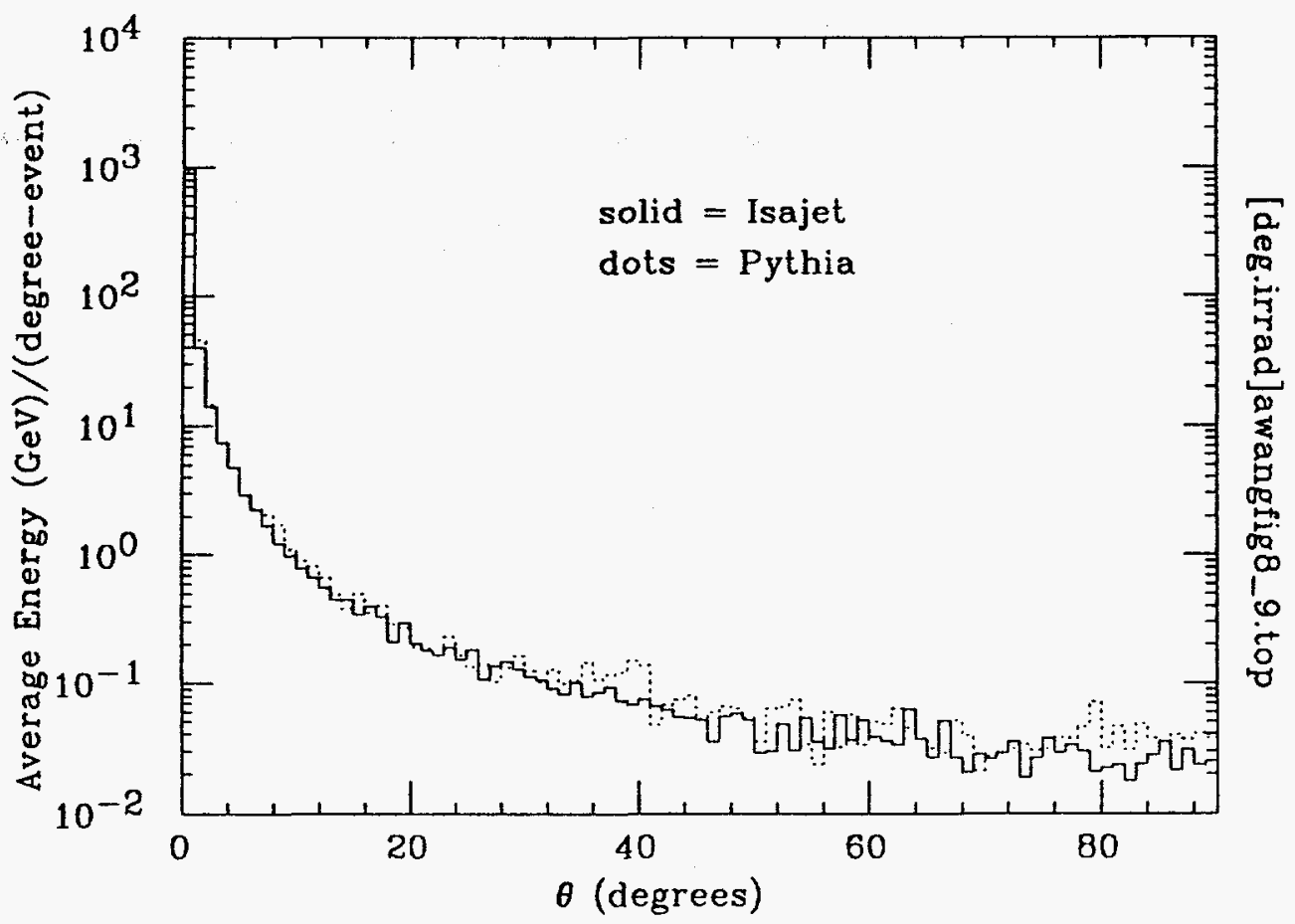

FIG. 8a. The average electromagnetic energy flow for an appropriate mixture of QCD hard and soft scattering events as a function of the polar angle. Particles with $\theta<1 \mathrm{mr}$ are excluded.

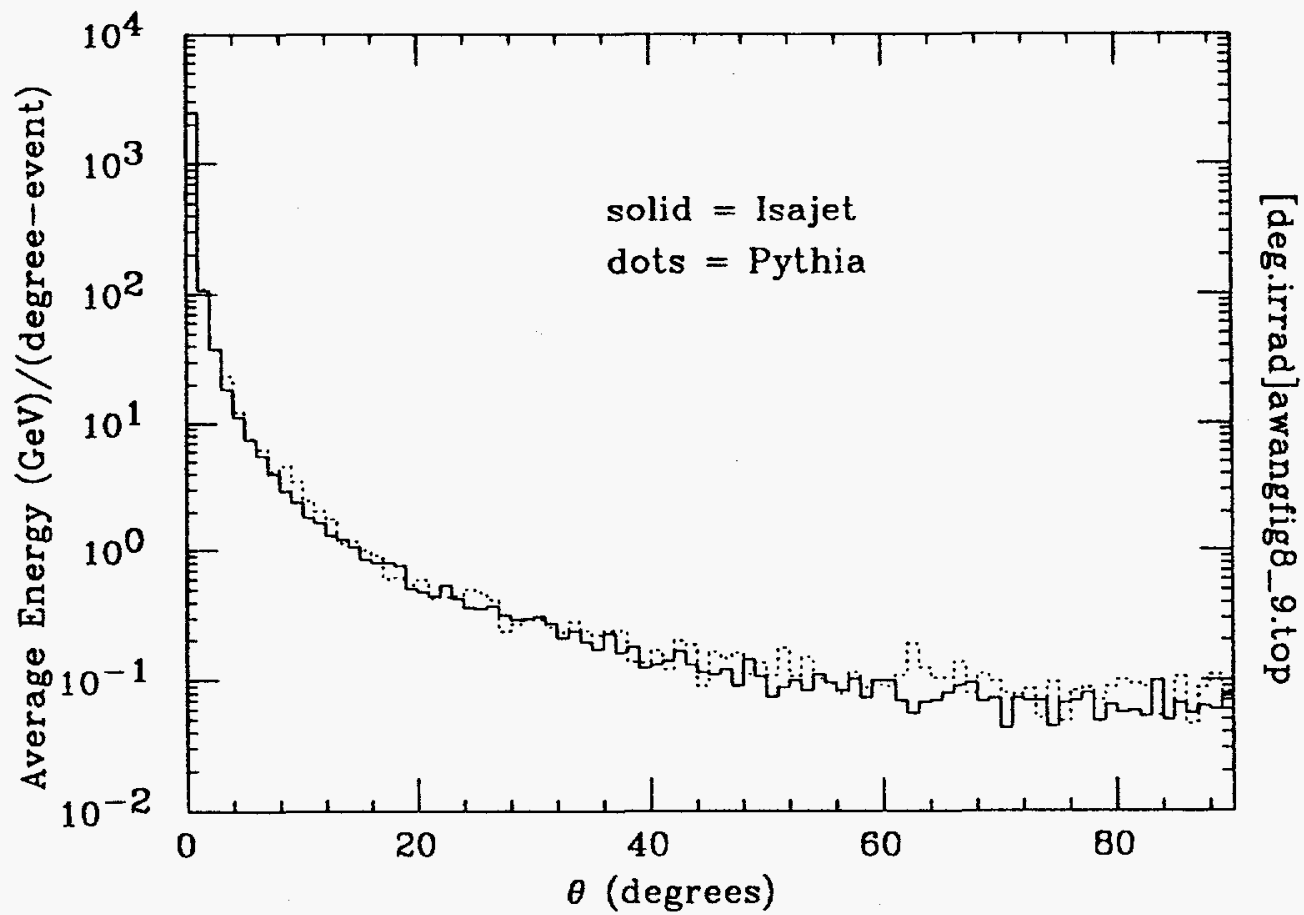

FIG. 8b. The average charged hadronic energy flow for an appropriate mixture of QCD hard and soft scattering events as a function of the polar angle. Particles with $\theta<1 \mathrm{mr}$ are excluded. 


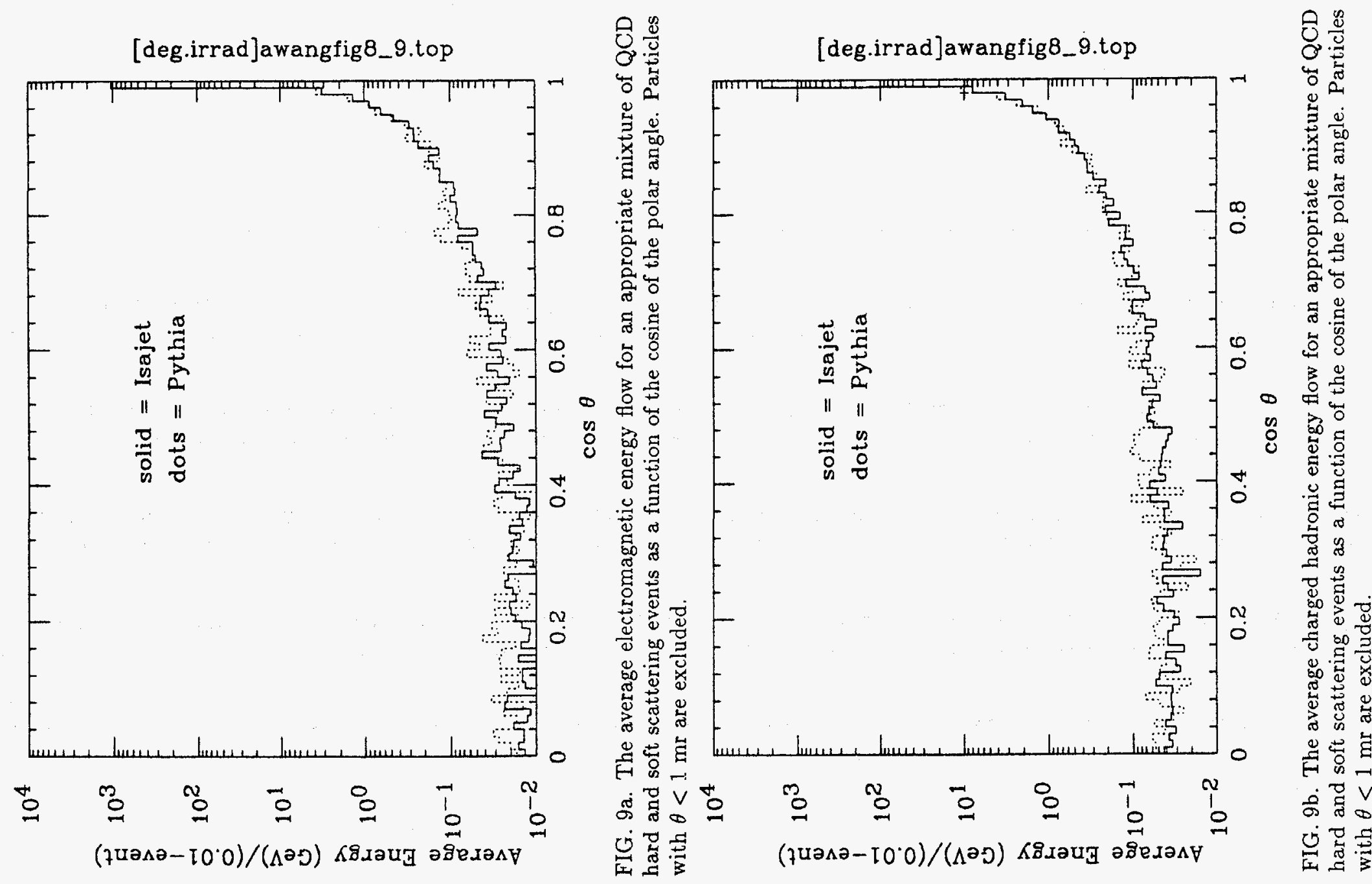




\title{
APPENDIX 6 APPLICABILITY OF EGS AT SSC ENERGIES
}

\author{
Alberto Fassò \\ European Organization for Nuclear Research \\ CERN, Geneva, Switzerland
}

Electromagnetic shower calculations are performed with great accuracy by EGS4 [1], which has been called "a recognized standard by the high-energy physics community" [2]. Physical processes are simulated in detail, and only a few approximations are made. Most of these are not especially related to high energy effects. In some cases the accuracy of the approximation even increases with energy, for instance the assumptions that electrons are not deflected by bremsstrahlung and that the polar angle of a radiated photon is uniquely determined by its energy. The approximations made and the extent to which they can affect results under various circumstances are clearly stated in the EGS4 manual, but since they are mentioned in the context of a very detailed description of the code they might escape attention of a casual user. Important examples are the treatment of triplet production in materials of low atomic number and of the bremsstrahlung "tip" differential cross section, i.e. i.e. for secondary photons or electrons having energies very close to that of the primary electron. Triplet production relies on the classical treatment by Bethe and Ashkin[3], with the more modern calculations of Tsai [4] only partially taken into account. The "tip" cross section is assumed equal to zero. New bremsstrahlung cross sections have since been published by Berger and Seltzer in 1985 [5]. These take into account several correction factors not present in EGS4, including the finite value at the high energy limit.

In general, then, EGS4 uses the best available cross sections and experimental data. The EGS4 manual, although describing the code as having a "dynamic range from a few tens of $\mathrm{keV}$ up to a few thousands GeV", warns that " 100 $\mathrm{GeV}$ is a safe upper limit to the present EGS version". In some cases, new and more accurate data have been published since the release of the present version. One may expect that some of them will be eventually incorporated in a possible future version of the code. The most important of these are the bremsstrahlung cross sections mentioned above and new data concerning the density effect in stopping-power calculations [6].

EGS4 has now been coupled to the FLUKA hadronic cascade simulation program [7]. The combined program simulates hadron photoproduction in electromagnetic showers and transports the photons from the decay of $\pi^{0}$ 's produced in hadronic showers. However, there are things EGS (in common with other sim- 
ulation codes) does not do. In particular, it does not simulate low-energy neutron production in electromagnetic cascades. Production of giant-resonance neutrons can be estimated, as done by Hirayama in Appendix 7, by folding EGS4 photon spectra with experimental photoneutron excitation functions. In other cases [8], a Lorentz theoretical curve has been used to describe the giant resonance cross section. The accuracy of such procedures, however, is not comparable with that attained in the description of other effects. The two main weak points concern the angular distribution and the spectrum. The angular distribution is in general far from isotropic (as it is generally assumed). Neutron emission is often enhanced at right angles to the photon direction. Recent experimental results from the Orsay group [9] show that the cross section weighted with neutron multiplicity stays nearly constant with energy at least up to a few hundred MeV. An additional uncertainty exists about the exact energy distribution of the photoneutrons. Only in a very crude approximation can it be assumed to be described by a fission spectrum, especially in materials of low atomic number and at energies above the resonance. The problem arises from the fact that each nucleus, even for a same atomic number, has different photoneutron emission properties depending in a complicated way from its shape, quantum numbers and so on. It is therefore difficult to see how a simple model could be incorporated into existing programs. The situation here is similar to that faced in writing MORSE and other Monte Carlo programs for low-energy neutron transport, where extensive evaluated cross-section data sets must be incorporated. Unfortunately, the photoneutron component could give an important contribution to the albedo fluence. ${ }^{\star}$ At lower energies, evaporation neutrons from the hadronic cascade certainly predominate, but at SSC energies, where electromagnetic showers play a major role, photoneutron production might become more significant.

Muon photoproduction presents quite a different problem. Since it is a purely QED process, it should not be difficult to incorporate into EGS if there is a need to do so. According to results obtained by Van Ginneken using a modified version of CASIM[10], muon pairs from the photons from $\pi^{0}$ 's produced in hadronic cascades are important contributors to the low-energy muon flux at the SSC. Direct muon production (actually from heavy-flavor decay) and charged meson decay also contribute, dominating at higher energies. The important problem of muon production at the SSC has not yet been fully addressed, but full inclusion of muons in EGS will contribute only a partial solution.

Collective QED effects at very high energies were addressed in the course of the workshop. Several such effects have been predicted theoretically, especially

\footnotetext{
* In Appendix 7, Hirayama and Nelson estimate the contribution as about $10 \%$.
} 
by the Soviet school. A very complete treatment can be found in a book by Ter-Mikaelyan [11]. Experimental evidence for most of these effects is still very poor and comes mostly from cosmic ray observations. The so-called Chudakov effect has been reported in several papers and is described in the well-known book on cosmic rays by Hayakawa[12]. An electron-positron pair is produced by a photon of very high energy at such a small angle that the two opposite charges partially cancel and stopping power is reduced. I am unfamiliar with any Monte Carlo program where which takes this into account (note that it would require simultaneous treatment of the two particles), but it would only affect the shower at its very beginning. It almost certainly can be ignored in treatments of radiation effects at the SSC, but might be important for detectors. The Landau-Pomeranchuk-Migdal (LPM) suppression effect is more widely known and experimental evidence from accelerators exists for it [13]. The effect arises from the interference of bremsstrahlung radiation emitted in the simultaneous interaction of an electron with several neighboring nuclei. Like similar interference effects, the crystal lattice configuration plays an important role and is difficult to include in a Monte Carlo code. The LPM effect was taken into account in the Monte Carlo program of Messel and Crawford [14], from which many of the EGS formulae and algorithms have been taken, but it is not implemented in EGS. Because the reduced bremsstrahlung cross section would give on average a deeper first interaction, and because it would result in a decreased number of low energy photons, the albedo photon flux might be reduced. The problem has been studied in the context of this workshop by $\mathrm{T}$. Stanev, whose results are reported in Appendix 8. His conclusion is that this interesting effect will be observable at the SSC but has little to do with radiation effects, where most of the contribution comes from fairly low-energy particles.

There is still a problem concerning computer speed, since at very high energy a full EGS simulation takes a prohibitively long time. For this reason, coupled Fluka-EGS calculations are normally run using the leading-particle option in EGS. Further reduction in computer time can be achieved by selecting higher energy cutoffs. However, it is not always clear to which extent the results can be affected by such approximations, which might engender more serious errors than most of the approximations and effects discussed above. It is perhaps worth mentioning that the GEANT tracking package[15], which used to refer to GHEISHA for hadron physics and to EGS for the electromagnetic part, has now been provided with a new electromagnetic shower package. The latter contains practically the same physics as EGS, but with a different coding. In some cases new data have been included, for instance the new bremsstrahlung cross sections of Berger and Seltzer. The authors claim a considerable gain in speed. 


\section{References}

1. W. R. Nelson, H. Hirayama, and D. W. O. Rogers, "The EGS4 Code System," Stanford Linear Accelerator Center Report SLAC-265 (1985).

2. C. W. Fabjan, "Calorimetry in High-Energy Physics," in Techniques and Concepts of High-Energy Physics, Vol. III, Ed. by T. Ferbel, Plenum (1985).

3. H. A. Bethe and J. Ashkin, "Passage of Radiation through Matter," in Experimental Nuclear Physics, Vol. I, Part II, E. Segre (Editor) (Wiley \& Sons, New York, 1953).

4. Tsai.

5. Berger and Selzer.

6. Sternheimer, Berger and Seltzer (1984).

7. P. A. Aarnio, A. Fassò, H-J. Möhring, J. Ranft and G. R. Stevenson, CERN Report TIS-RP/168 (1986).

8. Nelson, in a study for LEP.

9. Orsay? One of the authors is Veyssiere.

10. A. Van Ginneken, private communication.

11. Book by Ter-Mikaelyan.

12. Well-known book by Hayakawa.

13. Serpukhov.

14. H. Messel and D. F. Crawford, "Electron-Photon Shower Distribution Functions: Tables for Lead, Copper and Air Absorbers," Pergamon Press, Oxford (1970).

15. Reference to GEANT. 


\title{
APPENDIX 7 \\ RADIATION LEVELS IN ELECTROMAGNETIC CALORIMETER
}

\author{
Hideo Hirayama \\ KEK, National Laboratory for High Energy Physics \\ Oho-machi, Tsukuba-gun, Ibaraki, 305 Japan \\ and \\ Walter R. Nelson \\ Stanford Linear Accelerator Center \\ Stanford University, Stanford, California 94305
}

\section{Introduction}

Radiation levels in electromagnetic calorimeters are mainly determined by charged particles, which mainly distributed along the beam axis, and photons, which distributed widely inside the calorimeter. Photoneutrons produced by photons via photonuclear reaction also contributed but radiation levels due to these neutrons are small compared with charged particles and photons.

To understand radiation levels inside and outside the calorimeter, the energy depositions inside the calorimeter, the number of particles or its spectrum reflected from the calorimeter and the photoneutrons produced inside the converter are calculated by using Monte Carlo Code, EGS4 [1], for the combination of the two converter materials, $U$ and Lead, and the two active materials, plastic scintillator and liquid Ar. The incident electron energy varies from $1 \mathrm{GeV}$ to $1 \mathrm{TeV}$ to investigate the energy dependence.

\section{Methodology}

For the simulation of cascade showers in the calorimeter, a general electromagnetic radiation transport code called EGS4 (Electron-Gamma Shower version 4) [1] was used. EGS4 is capable of treating electrons, positrons, and photons with kinetic energies as high as a few thousand $\mathrm{GeV}$ and as low as $1 \mathrm{keV}$ (photons) and a few tens $\mathrm{keV}$ (electrons and positrons). The transport can take place in any of 100 different element, or in any mixture or compound of these elements. The computational portion of EGS4 system is divided into two parts. First, a preprocessor code (PEGS4) uses theoretical (and sometimes empirical) formulas to compute the various physical quantities needed (e.g., cross sections, branching ratios, etc.) and prepare them in a form suitable for fast numerical evaluation. Then the EGS4 code itself uses this data, along with user supplied data and routines, to perform the actual simulation. 
The calorimeter was supposed to be semi-infinite alternate slab of the converter ( $3 \mathrm{~mm} U$ or Lead) and the active material ( $3 \mathrm{~mm}$ plastic scintillator or liquid $\mathrm{Ar}$ ) having the total thickness of about $20 \mathrm{X}_{0}$. The cut-off energy of each materials is shown in Table 1. Incident particles are electrons having the energy of 1-, 10-, 100-, or 1000-GeV. The number of incident particles simulated at each energy is shown in Table 2 together with the typical computer time in HITAC $\mathrm{M} 680 \mathrm{H}$.

Table 1

Cut-off energy used in EGS4 calculation.

\begin{tabular}{lcc}
\hline \hline Materials & $\begin{array}{c}\text { Cut-off } \\
\text { Photon }\end{array}$ & $\begin{array}{c}\text { Kinetic } \\
\text { Electron and Positron }\end{array}$ \\
\hline Uranium & 0.1 & 1.0 \\
Lead & 0.1 & 1.0 \\
Polystyrene & 0.01 & 0.1 \\
Liquid Argon & 0.01 & 0.1 \\
\hline \hline
\end{tabular}

Table 2

Number of showers simulated and typical computing time in HITAC M680H.

\begin{tabular}{ccc}
\hline \hline $\begin{array}{c}\text { Incident Energy } \\
(\mathrm{GeV})\end{array}$ & $\begin{array}{c}\text { Number of } \\
\text { Showers }\end{array}$ & $\begin{array}{c}\text { Computing time } \\
(\mathrm{sec})\end{array}$ \\
\hline 1 & 3000 & 803 \\
10 & 300 & 775 \\
100 & 30 & 770 \\
1000 & 4 & 1054 \\
\hline \hline
\end{tabular}

\section{Radiation Level with Calorimeter}

\subsection{General Feature of Electromagnetic Cascade}

Figure 1 shows tracks of charged particles (solid lines) and photons (dots) for a cascade initiated by a single $10 \mathrm{GeV}$ electron normally incident on the calorimeter of the $U$ and the polystyrene scintillator. The same shower is given in Fig. 2, but this time only charged particles in the scintillator are shown. From these two 
figures, it is clearly shown that charged particles mainly distributed along the beam axis of the incident electrons and the lateral spread of the cascade shower caused by photons. It is also shown that energy was carried out back mostly by photons.

\subsection{Energy Deposition Inside the Calorimeter}

To see the differences of the energy deposition in the active material for the combination of the converter material and the active material, the maximum absorbed energy and the maximum absorbed dose for the normal incident parallel beam in the active material slab is plotted as the function of the incident electron energy for the various combinations and shown in Fig. 3. The energy dependence of the absorbed energy is similar between each combination. Therefore, the following detail investigations were done for the combination of the $U$ converter and the polystyrene scintillator. Figure 4 shows the distribution of absorbed energy and the absorbed dose for the normal incident parallel beam in the scintillator for the different incident energy as the function of the scintillator slab number. This figure shows the longitudinal cascade development inside the calorimeter. The energy deposition distribution is strongly affected by the incident angle. Figure 5 shows the tracks produced by a single $10 \mathrm{GeV}$ electron incident at $45^{\circ}$ to the normal. The effect of the incident angle to the energy deposition distribution is shown in Fig. 6 in the case of $10 \mathrm{GeV}$ electron incident. The peak position moves to forward and the peak value increases as the increase of the incident angle. But the total absorbed energy inside the scintillator is not affected by the incident angle. The existence of the magnetic field also affects the energy deposition distribution inside the scintillator shown in the tracks in Fig. 7 but its effect is far small compared with that of the slant incident shown in Fig. 6.

The absorbed dose inside the scintillator caused by the pencil beam are plotted in Figs. 8 and 9. Figure 8 shows the longitudinal dose distributions along the beam axis within $3-\mathrm{mm}$ radius. The general shape is similar to that of total energy deposition curve shown in Fig. 4. Figure 9 shows the radial dose distribution at the scintillator corresponding the peak values in Fig. 8. The absorbed energy dropped rapidly with increasing the radius in all energy. This figure also shows that the energy deposition mainly occurred along the beam axis.

\subsection{Albedo Radiations from the Scintillator}

The number of albedo electrons and positrons having the kinetic energy above $1 \mathrm{MeV}$ is shown in Fig. 10 for the combination of the converter and the active material as the function of the incident energy. The flux of albedo photons is shown in Fig. 11. In Fig. 11, albedo photon fluxes are plotted for the different cut-off energy. The number of albedo electrons and positrons is far small 
compared with that of photons as supposed from the general feature of the cascade development. The differences due to the combination of the converter and the active materials is very small for charged particles. In the case of photons, the flux from $\mathrm{Pb}$ converter are slightly smaller than from $\mathrm{U}$ one especially for higher cut-off energy. The absorbed dose of Si due to albedo photons from the normal incident parallel beam is shown in $\mathrm{Fi}_{5} .12$ as the function of the incident energy. The detailed investigation below is performed for the combination of the U-converter and the polystyrene scintillator. The albedo photon spectra for different incident energy are shown in Fig. 13. The shape of the spectrum can be expressed $\exp \left(-a E_{p}\right)$ above $1 \mathrm{MeV}$ and does not depend on the incident energy. The flux of albedo photons are also strongly affected by the incident angle supposed from Fig. 5. The variation of the flux of backscattered photons is shown in Fig. 14 as the function of the incident angle for $1-$ and $10-\mathrm{GeV}$ incident. The flux of photons increases with increasing the angle, about two times at $45^{\circ}$ and about 4 times at $60^{\circ}$. The incident angle affects slightly to the spectrum of albedo photons. Figure 14 shows the albedo photon spectra for $10 \mathrm{GeV}$ electrons having the different incident angle. The increase of the incident angle increases the higher energy parts. The effects of the magnetic field to the number of albedo photons are also shown in Fig. 14. The existence of the 2 Tesla magnetic field increases the albedo photons but its effects are very small compared with the increase with the incident angle.

\subsection{Photoneutron Production Inside the Calorimeter}

Inside the converter, many photoneutrons are produced by the photonuclear reaction. The cross sections at the giant resonance region are shown in Fig. 16 [2]. Number of produced photoneutrons is obtained by multiplying the differential photon track length with the reaction cross section. Figure 17 shows the total number of produced neutrons inside the converter for the combination of the converter and the active material as the function of the incident energy. The number is proportional with the incident energy and is about 0.35 neutrons/GeV for $U$ and about 0.2 neutrons for $\mathrm{Pb}$. The difference between $\mathrm{U}$ and $\mathrm{Pb}$ is due to the differences of the $(\gamma, 2 \mathrm{n})$ reaction cross section. These values are about $1 / 10$ of the low-energy neutron below $15 \mathrm{MeV}$ produced by high-energy pion. The number of photoneutrons in the $\mathrm{U}$ convertor is shown in Fig. 18 as the function of the incident energy. The distribution is very similar to that of the energy deposition in the scintillator shown in Fig. 4 but the peak position is shifted slightly deeper position. The photoneutrons have the spectrum very similar with that of fission neutrons and are emitted nearly isotropically. Therefore, it is possible to calculate the albedo neutrons by combining with the low energy neutron transport code like MORSE or ANISN. 


\section{References}

1. W. R. Nelson, H. Hirayama and D. W. O. Rogers, "The EGS4 Code System," SLAC-265, Stanford Linear Accelerator Center (Dec. 1985).

2. "Atlas of Photoneutron Cross Sections obtained with Monoenergetic Photons," Atomic Data and Nuclear Data Tables, 15, 319 (1975). 


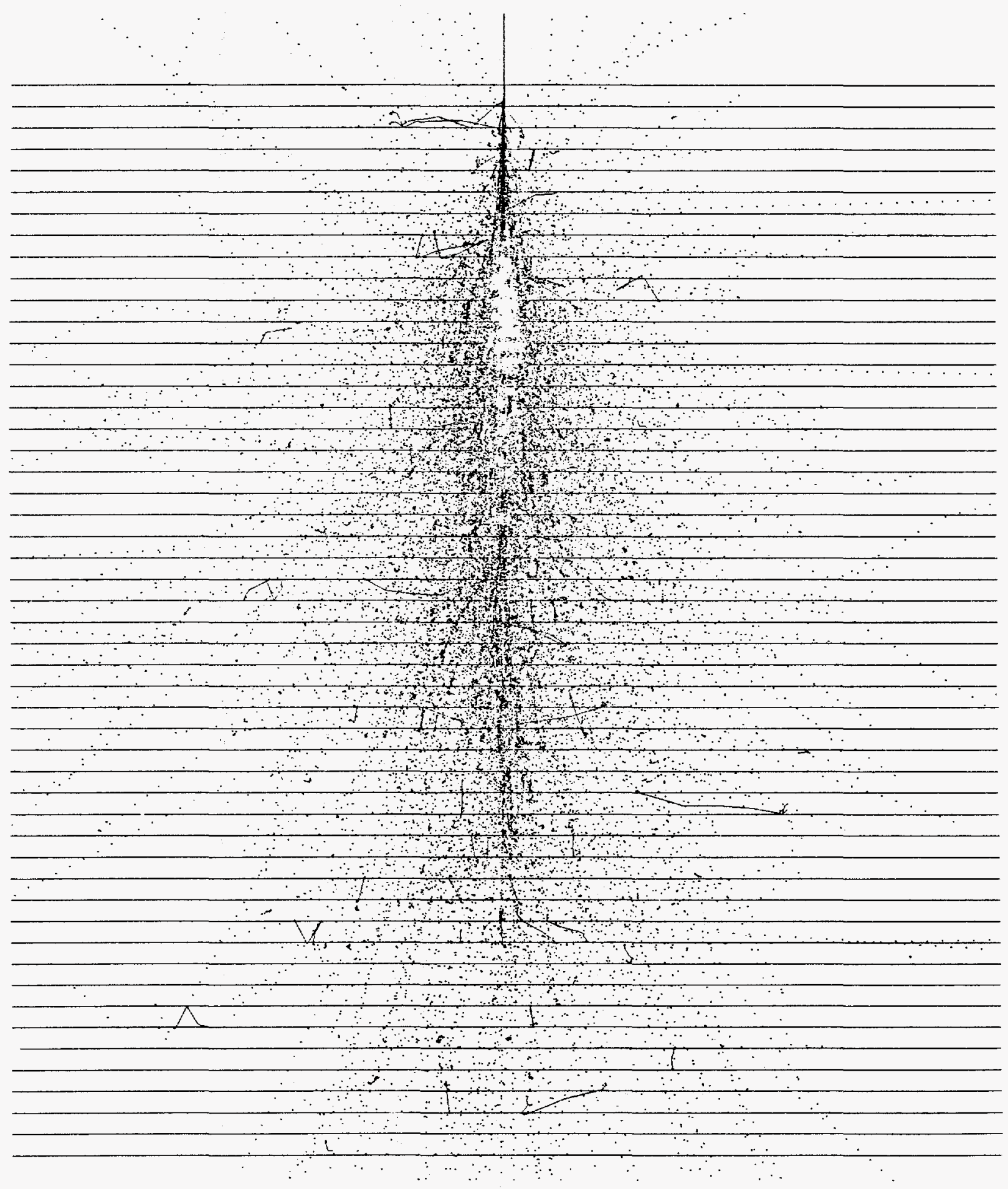

FIG. 1. EGS4 generated shower event in calorimeter of alternative slab $3 \mathrm{~mm} \mathrm{U}$ and $3 \mathrm{~mm}$ polystyrene scintillator by a single $10 \mathrm{GeV}$ electron incident normally on the calorimeter. Charged particle and photon tracks are depicted as slid and dotted lines, respectively. 


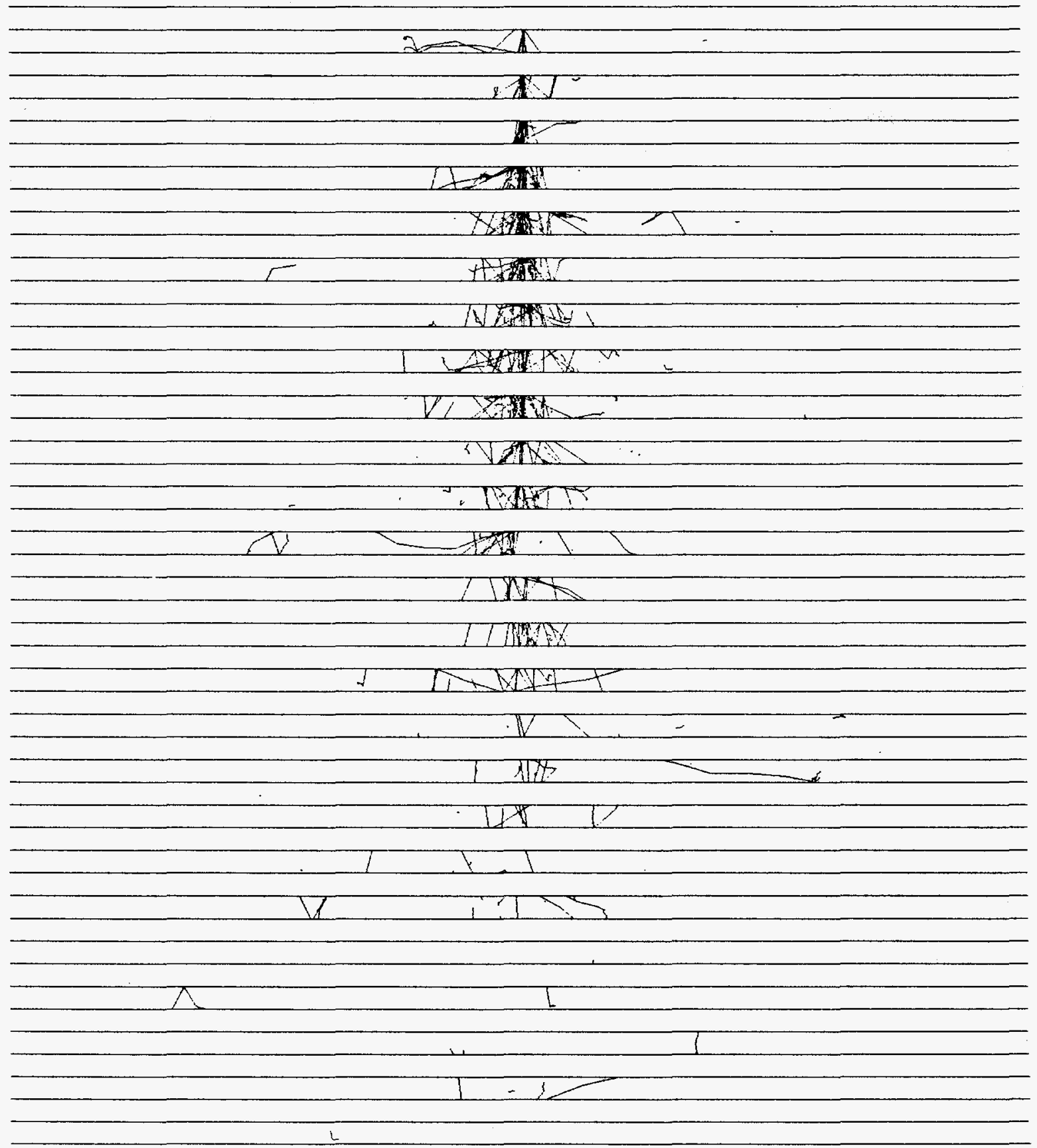

FIG. 2. Same as Fig. 1, but only charged particles in scintillator regions are shown. 


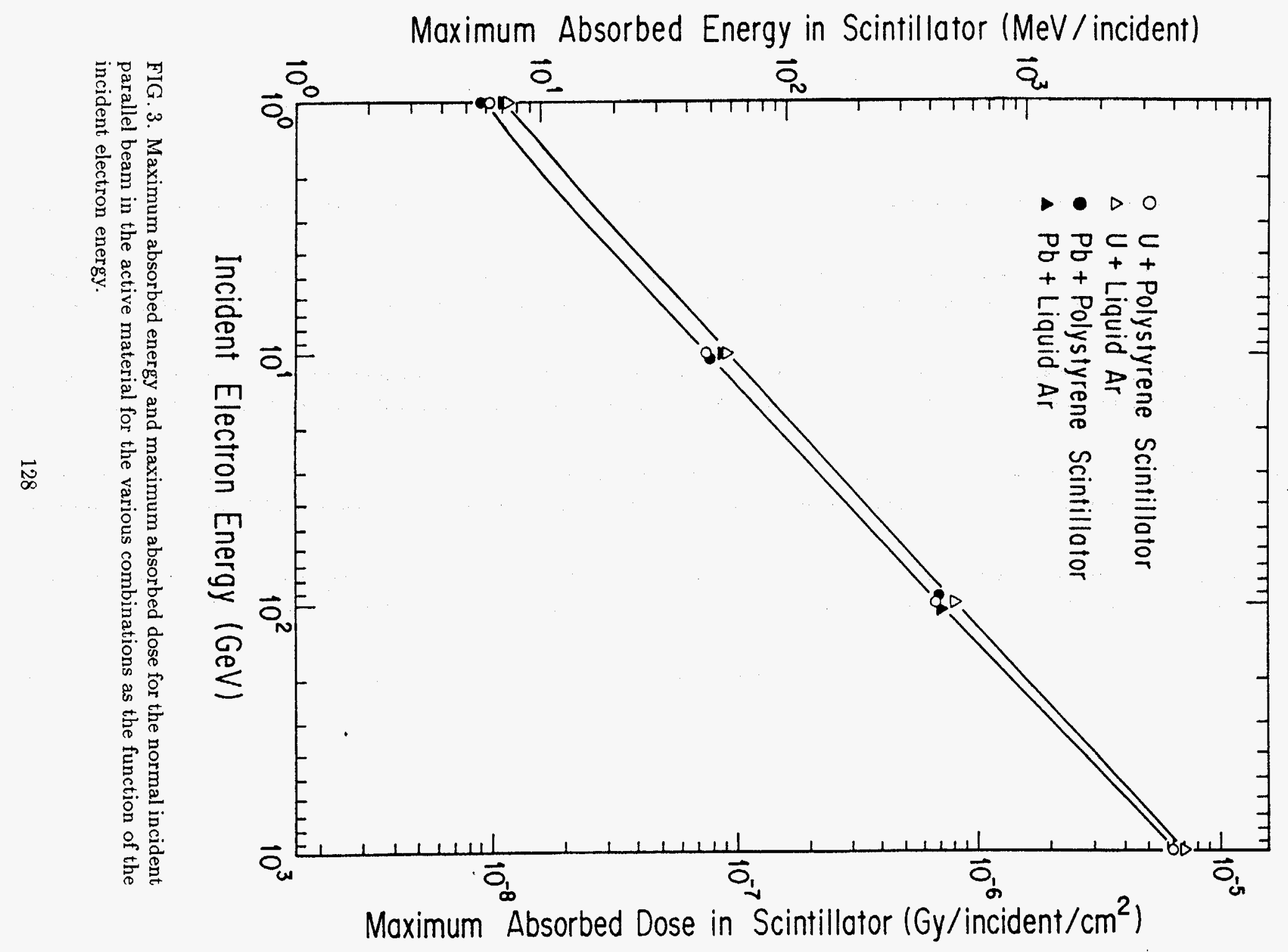




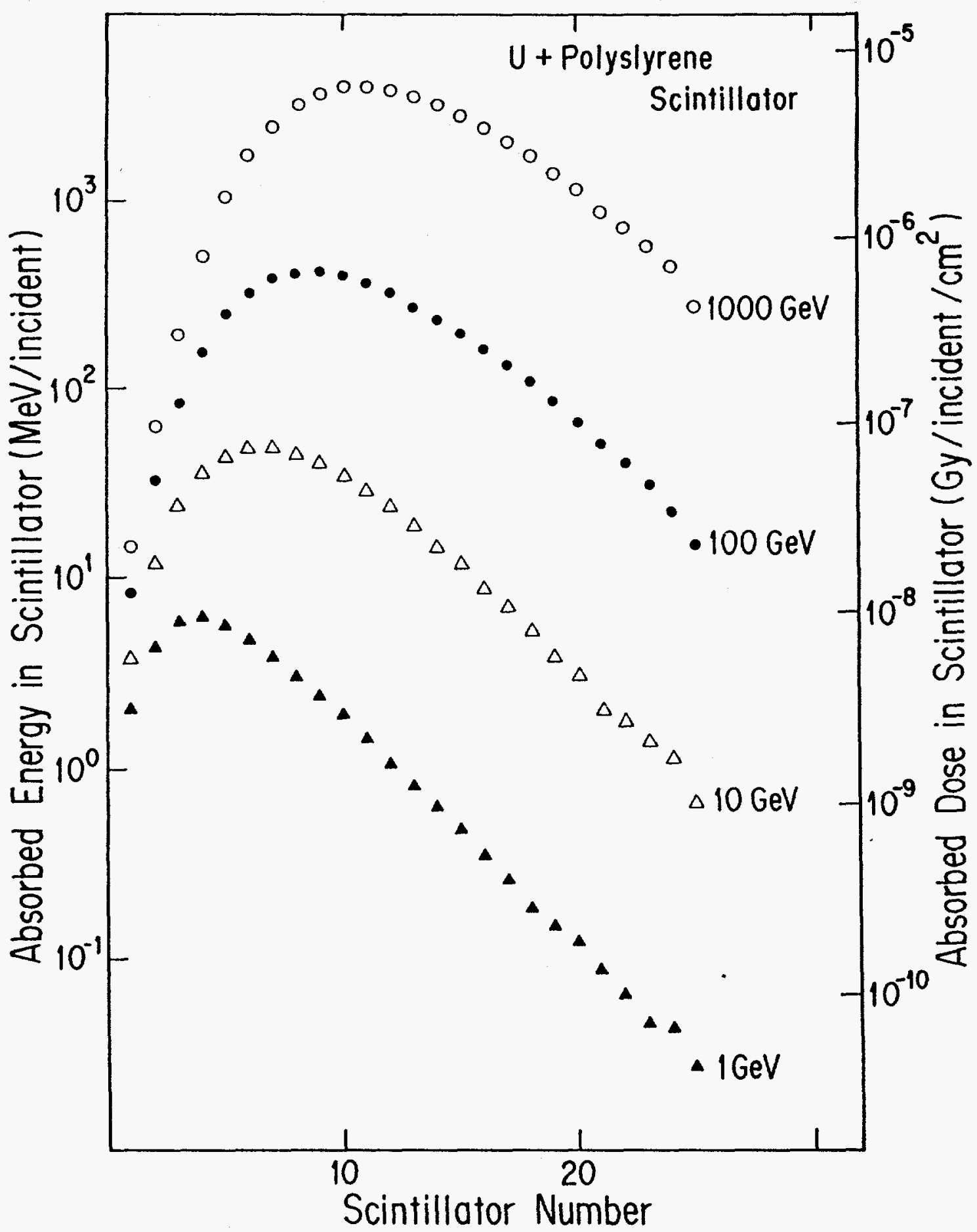

FIG. 4. Distribution of absorbed energy and absorbed dose for the normal incident parallel beam in the scintillator for the different incident energy. 


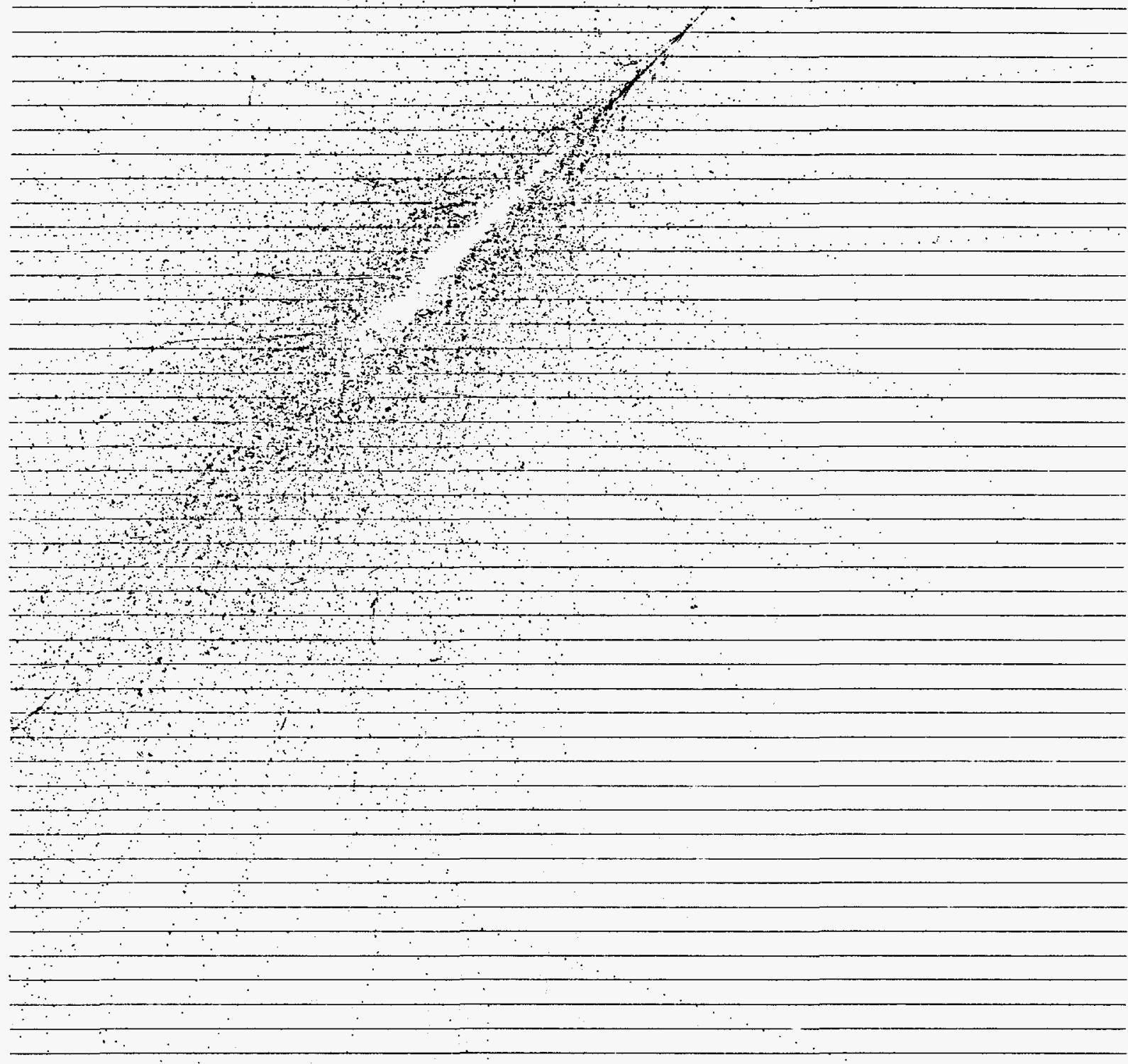




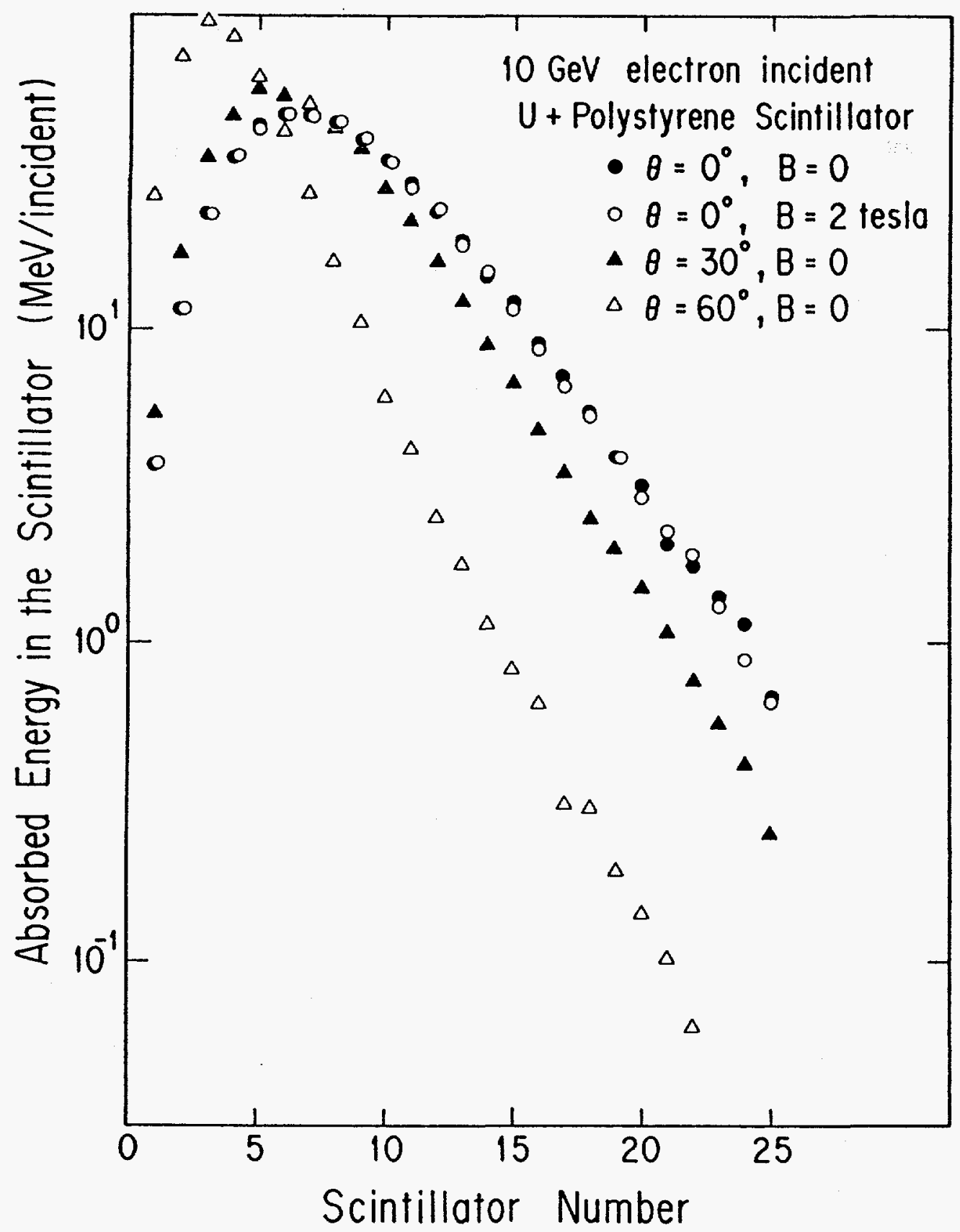

FIG. 6. Distribution of absorbed energy in the scintillator for $10 \mathrm{GeV}$ electrons having the different incident angle. 


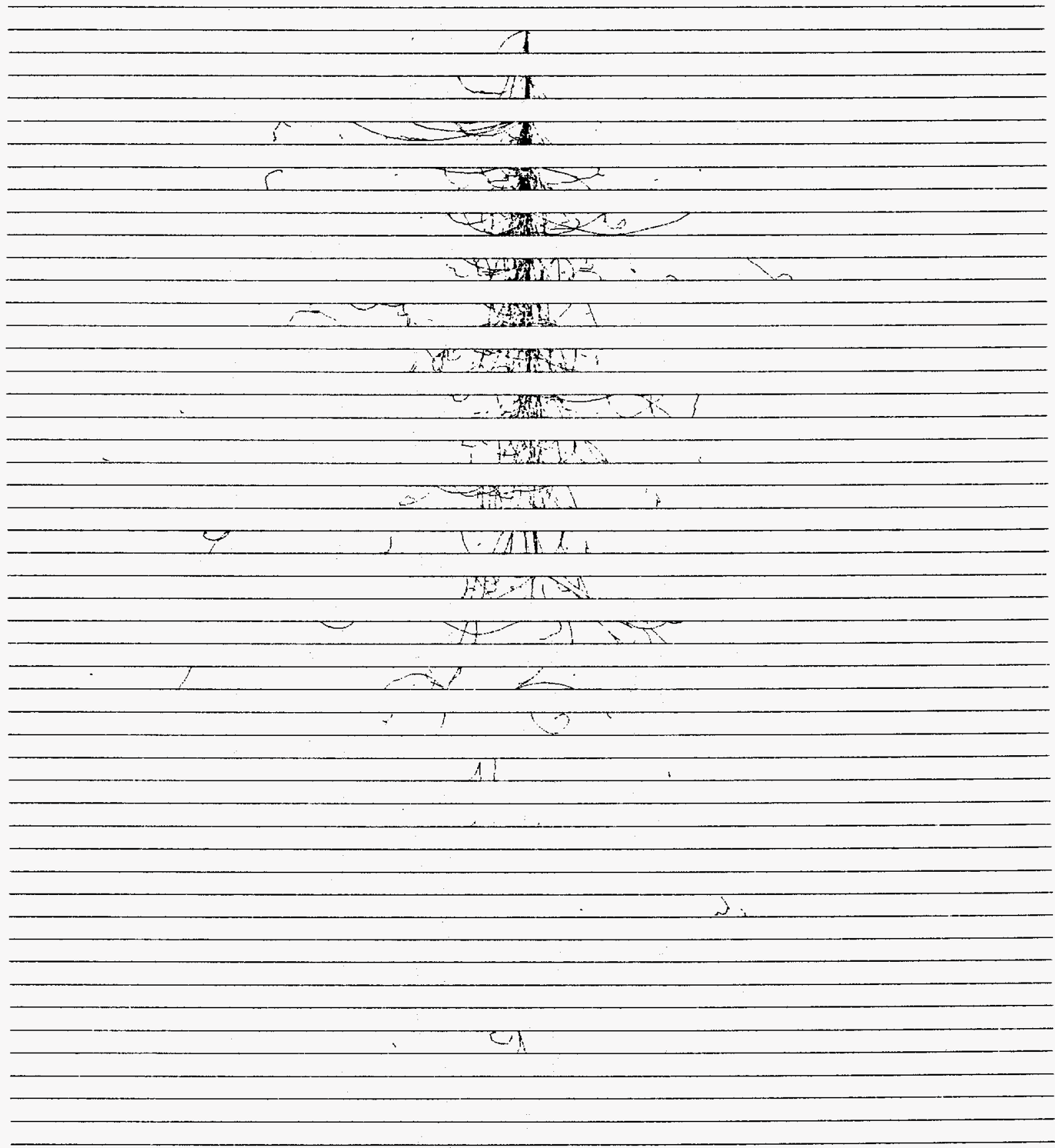

FIG. 7. EGS4 generate shower event in calorimeter of alternate slab of $3 \mathrm{~mm} \mathrm{U}$ and $3 \mathrm{~mm}$ polystyrene scintillator by a single $10 \mathrm{GeV}$ electron incident normally on the calorimeter with the 2 Tesla magnetic fields parallel to the $\mathrm{x}$-axis. 


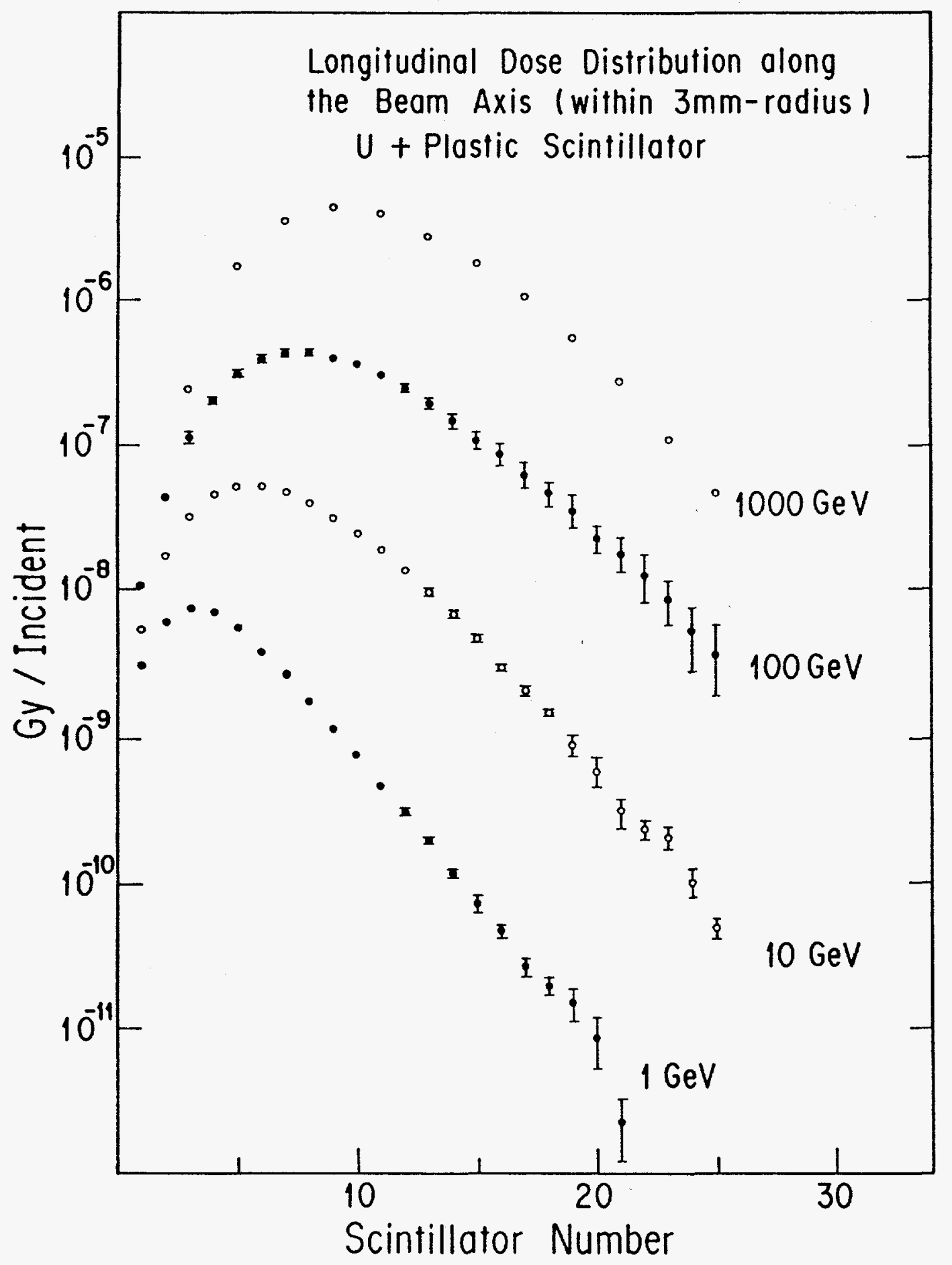

FIG. 8. Longitudinal absorbed dose distribution inside the scintillator along the beam axis within $3 \mathrm{~mm}$ radius. 


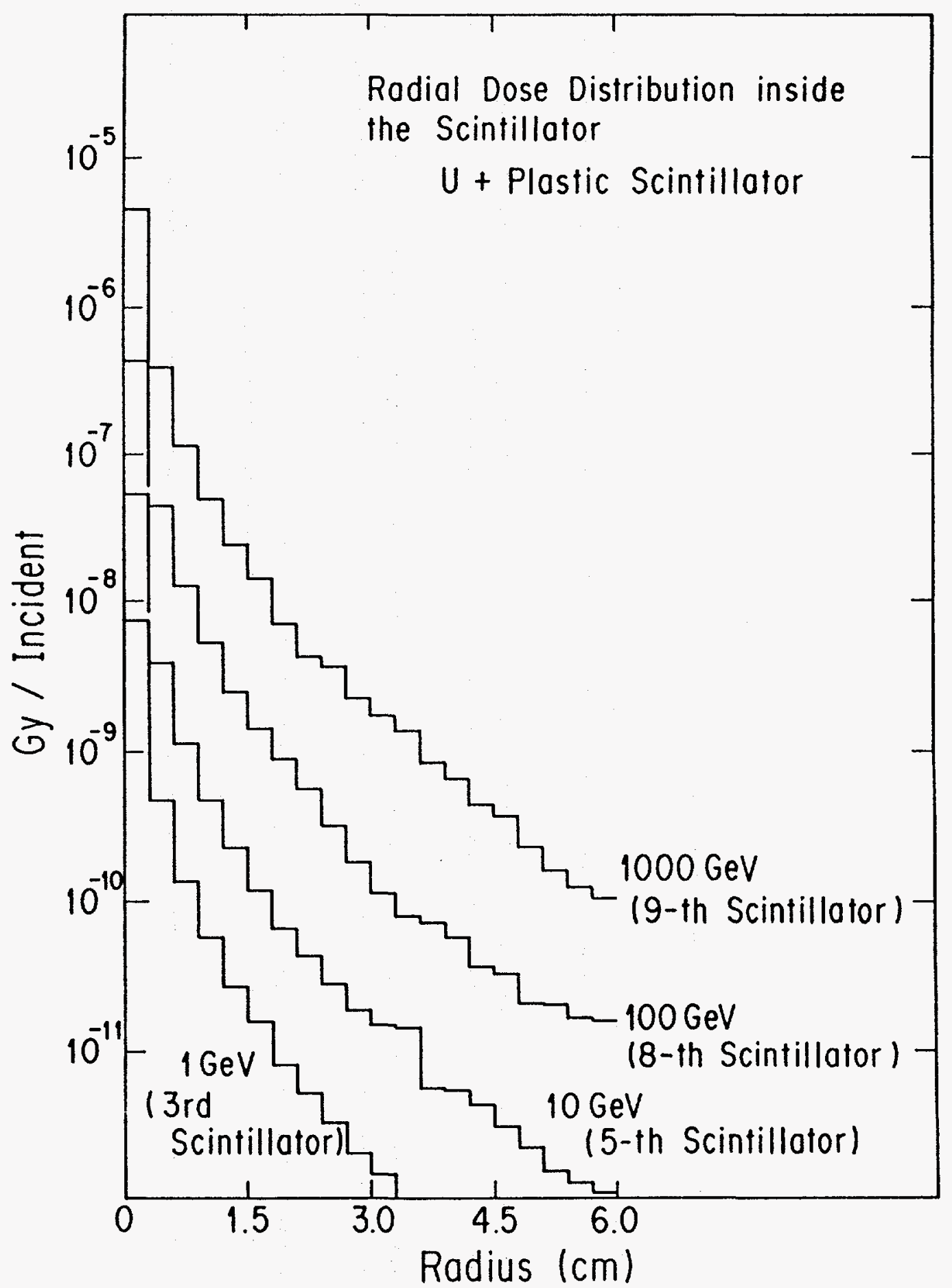

FIG. 9. Radial absorbed dose distribution at the scintillator corresponding the peak values in Fig. 8. 


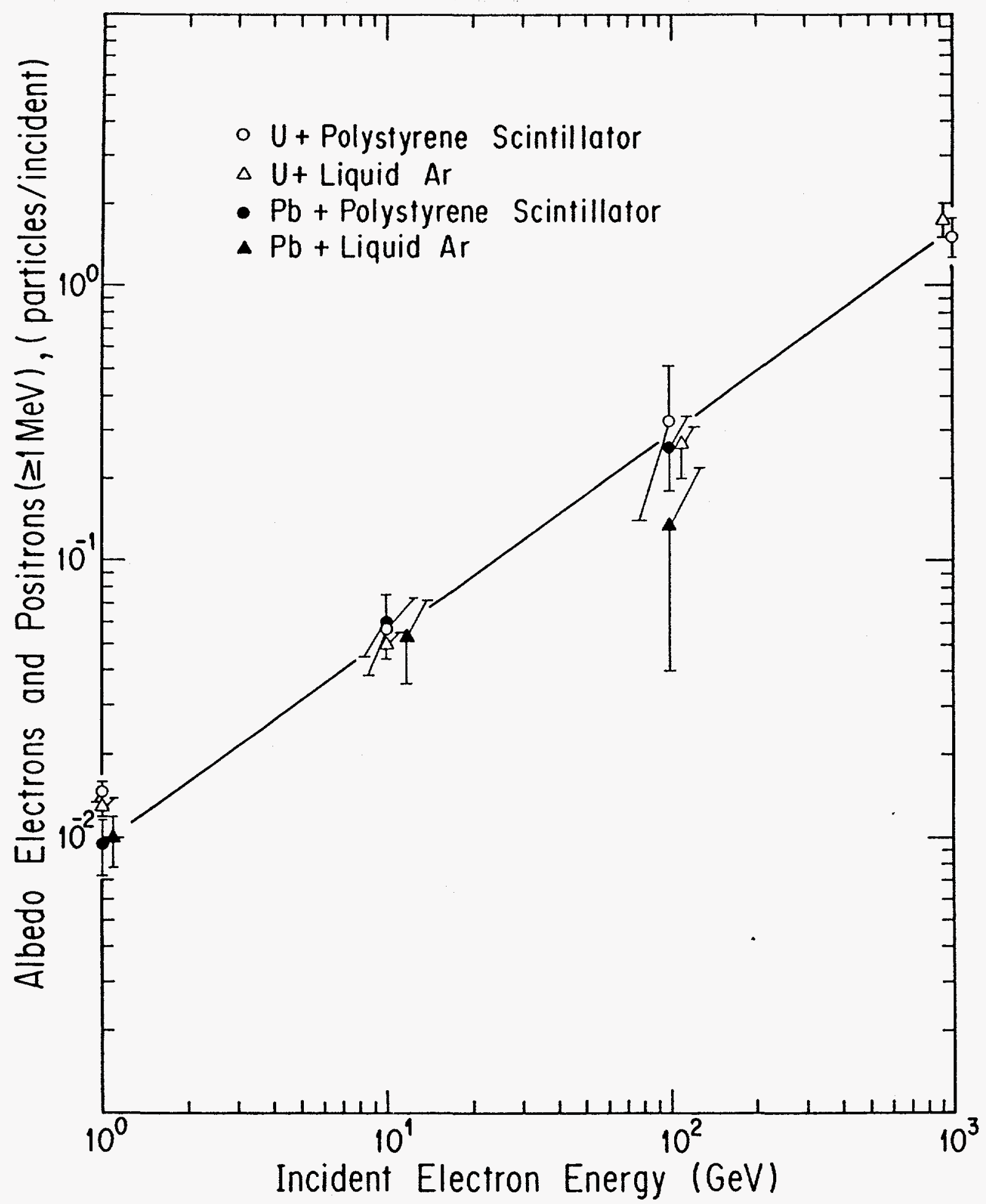

FIG. 10. Number of albedo electrons and positrons having the kinetic energy above $1 \mathrm{MeV}$ for the various combination of the converter and the active materials. 


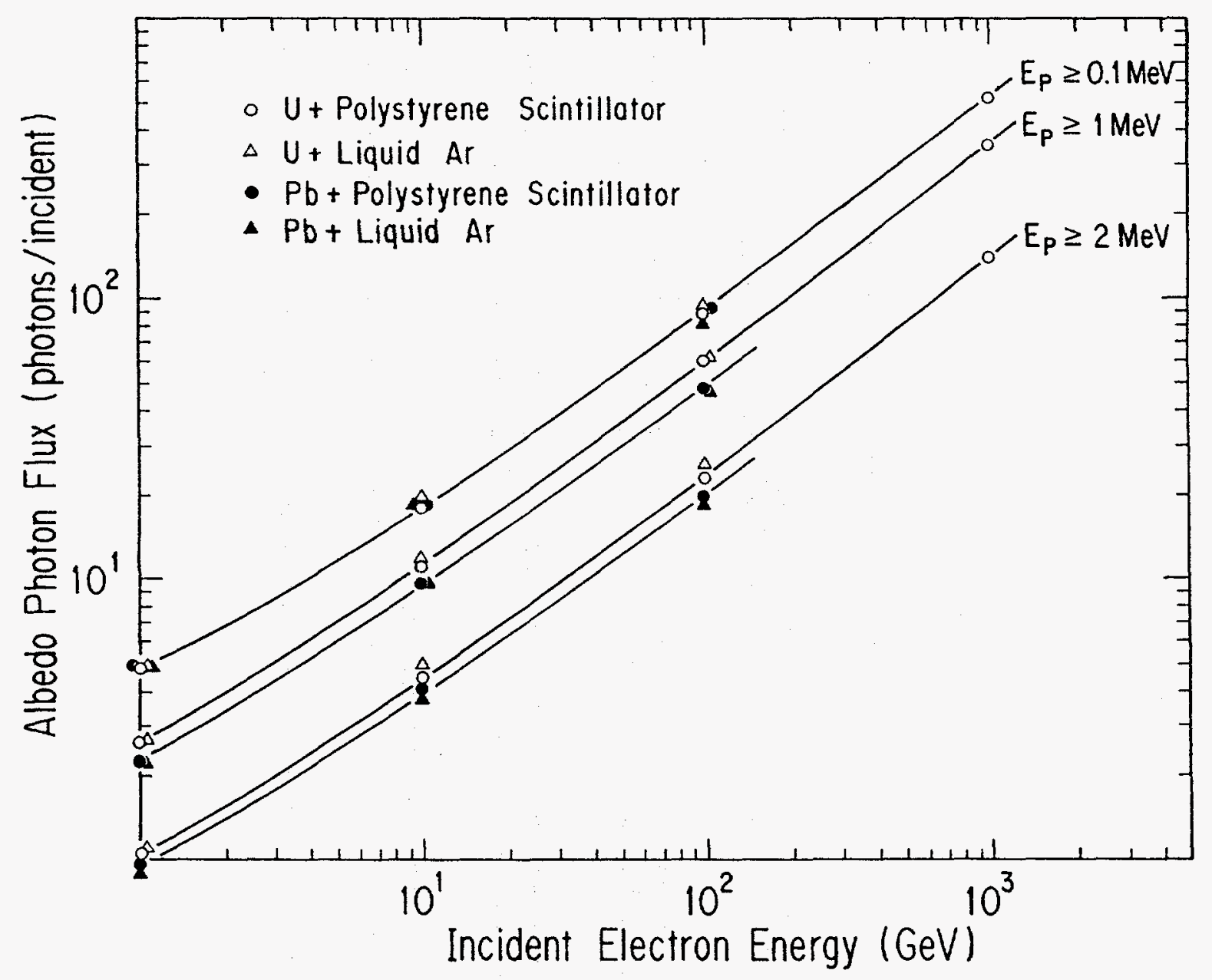

FIG. 11. Flux of albedo photons having the different cut-off energy for the various combination of the converter and active materials. 


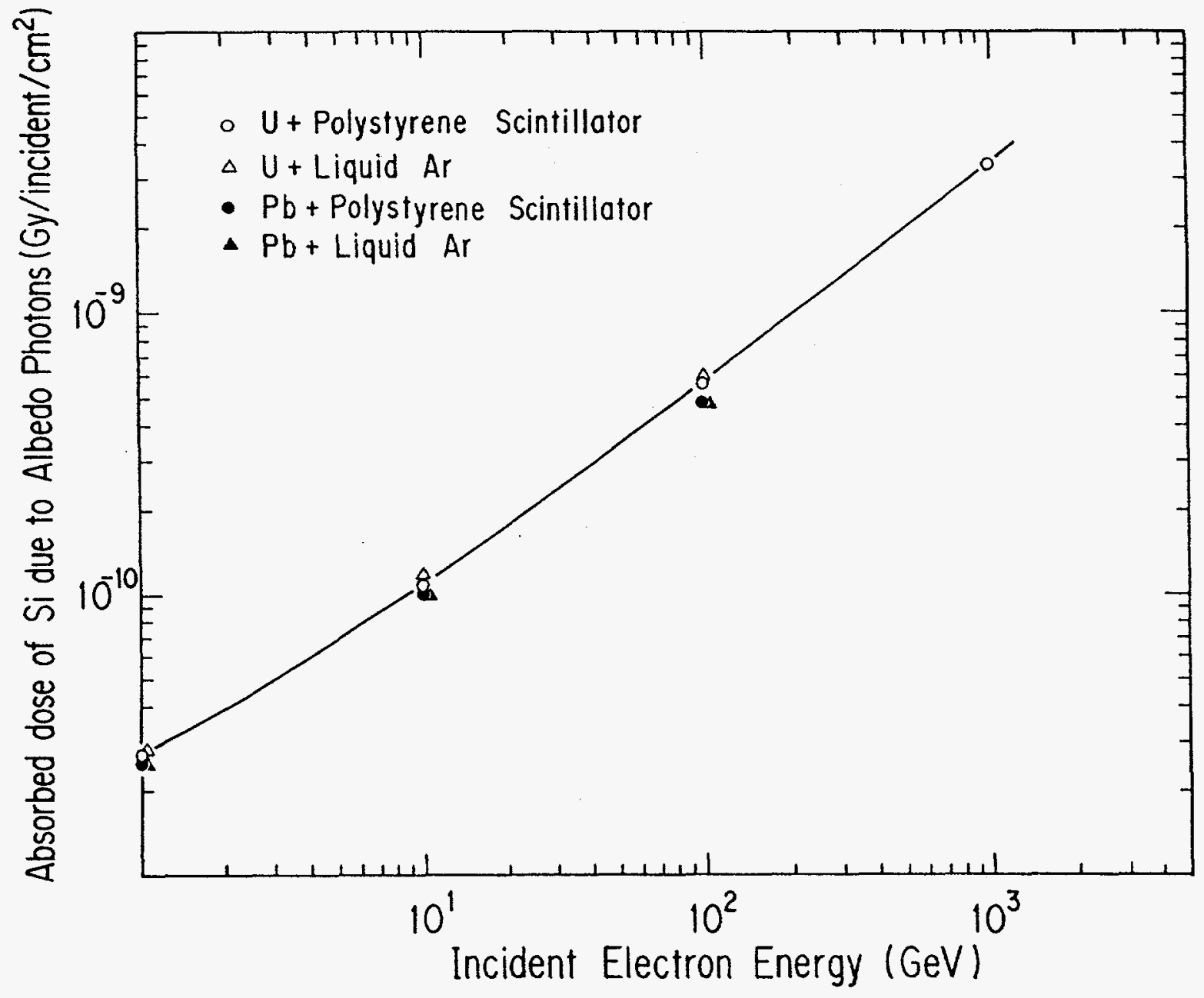

FIG. 12. Absorbed dose of Si due to albedo photons for the normal incident parallel beam for the various combination of the converter and the active materials. 


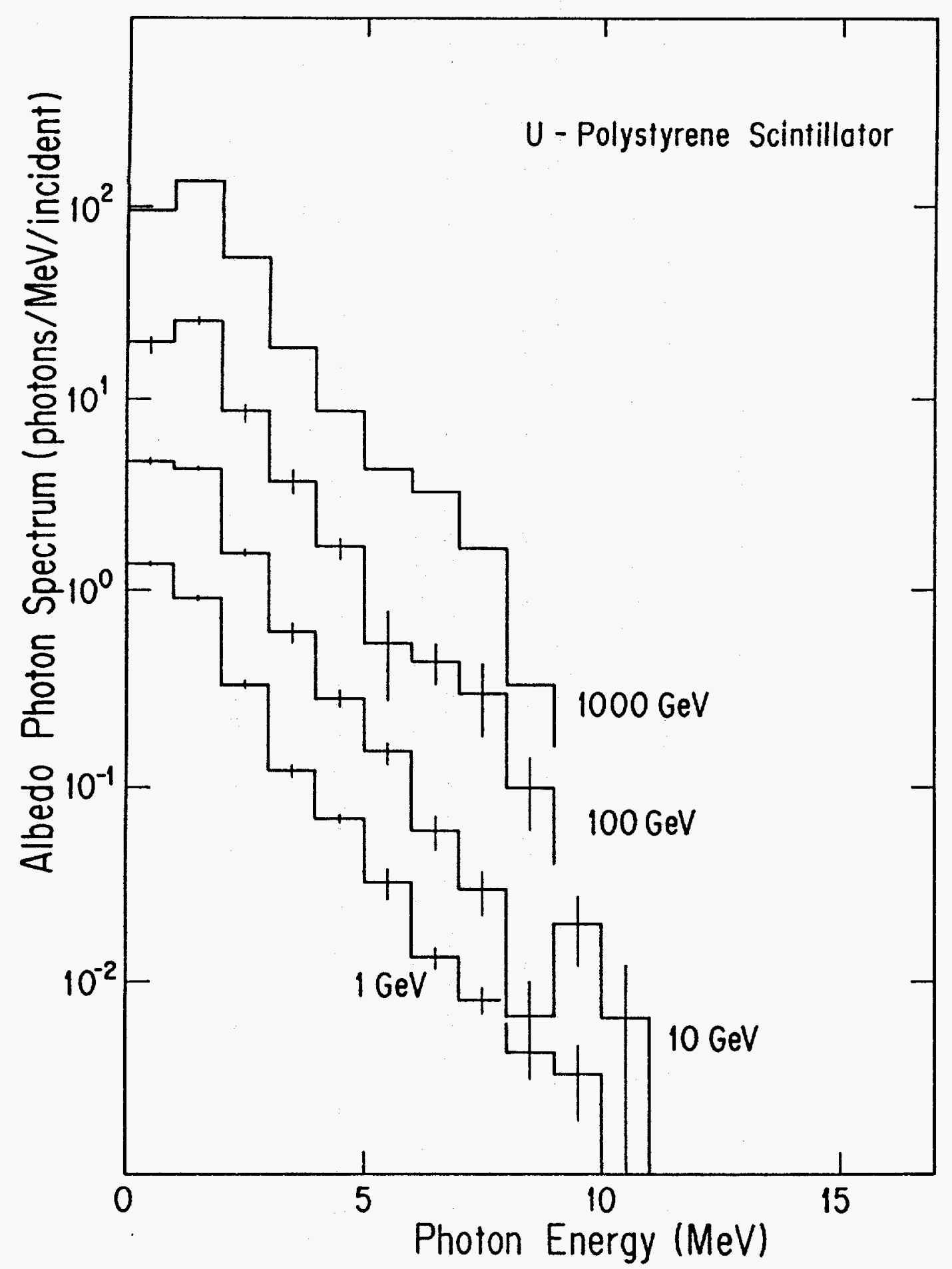

FIG. 13. Albedo photon spectra from the alternate slab of $3 \mathrm{~mm} \mathrm{U}$ and $3 \mathrm{~mm}$ scintillator for different incident energy. 


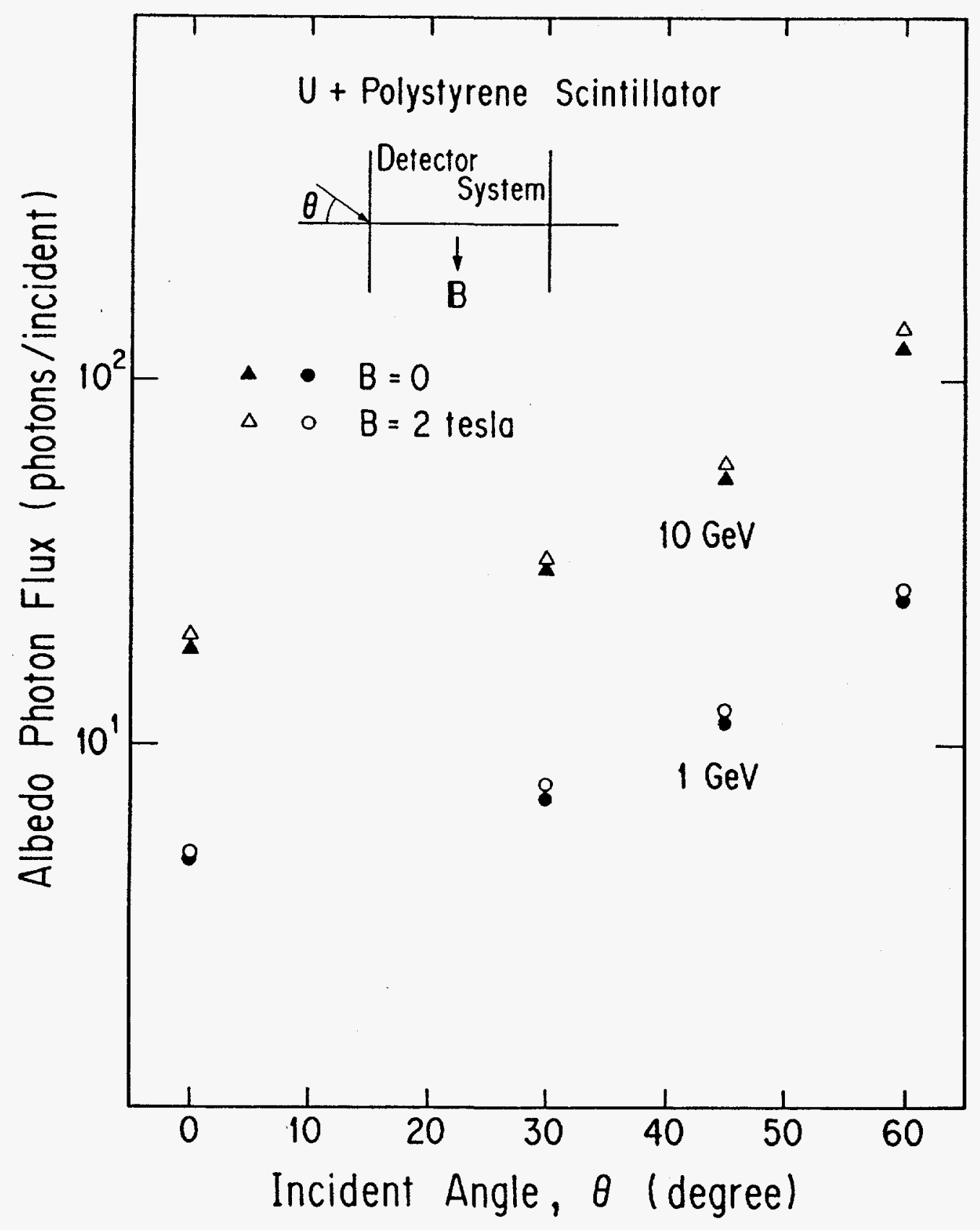

FIG. 14. Variation of the albedo photons for the different incident angle in the case of 1- or $10-\mathrm{GeV}$ electron with and without magnetic field. 


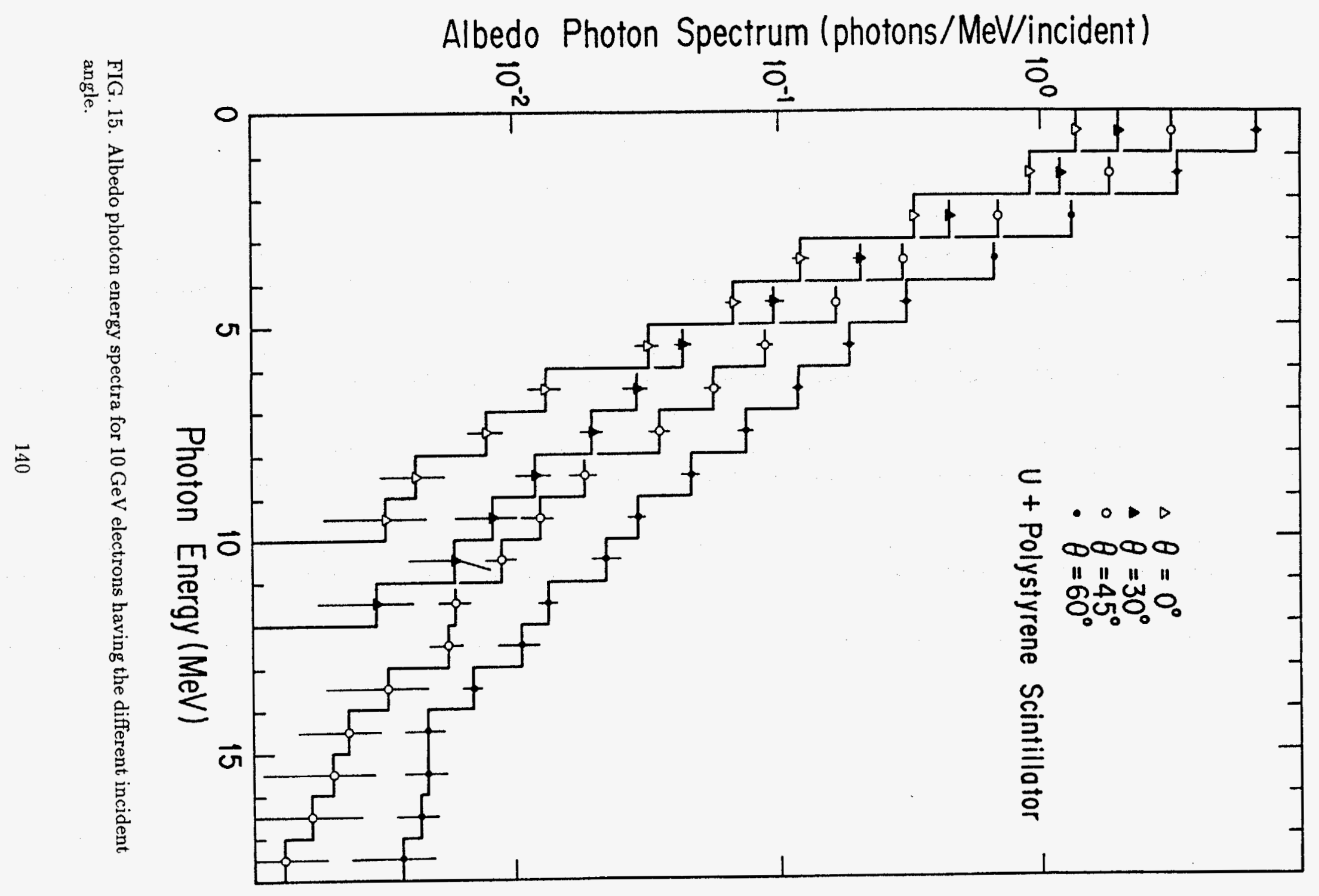



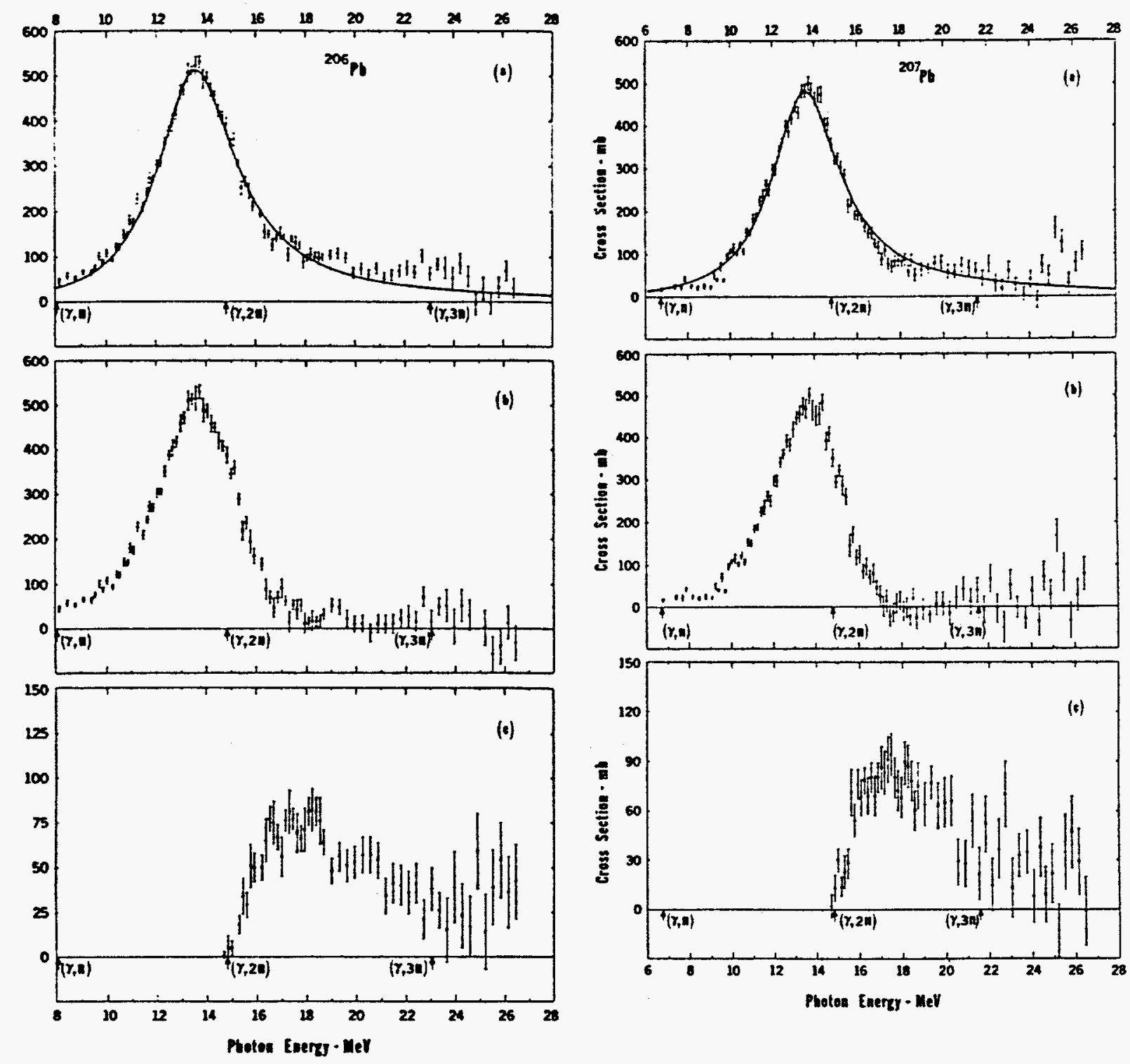

FIG. 16a. Photoneutron cross section of ${ }^{208} \mathrm{~Pb}$ and ${ }^{207} \mathrm{~Pb}$. 

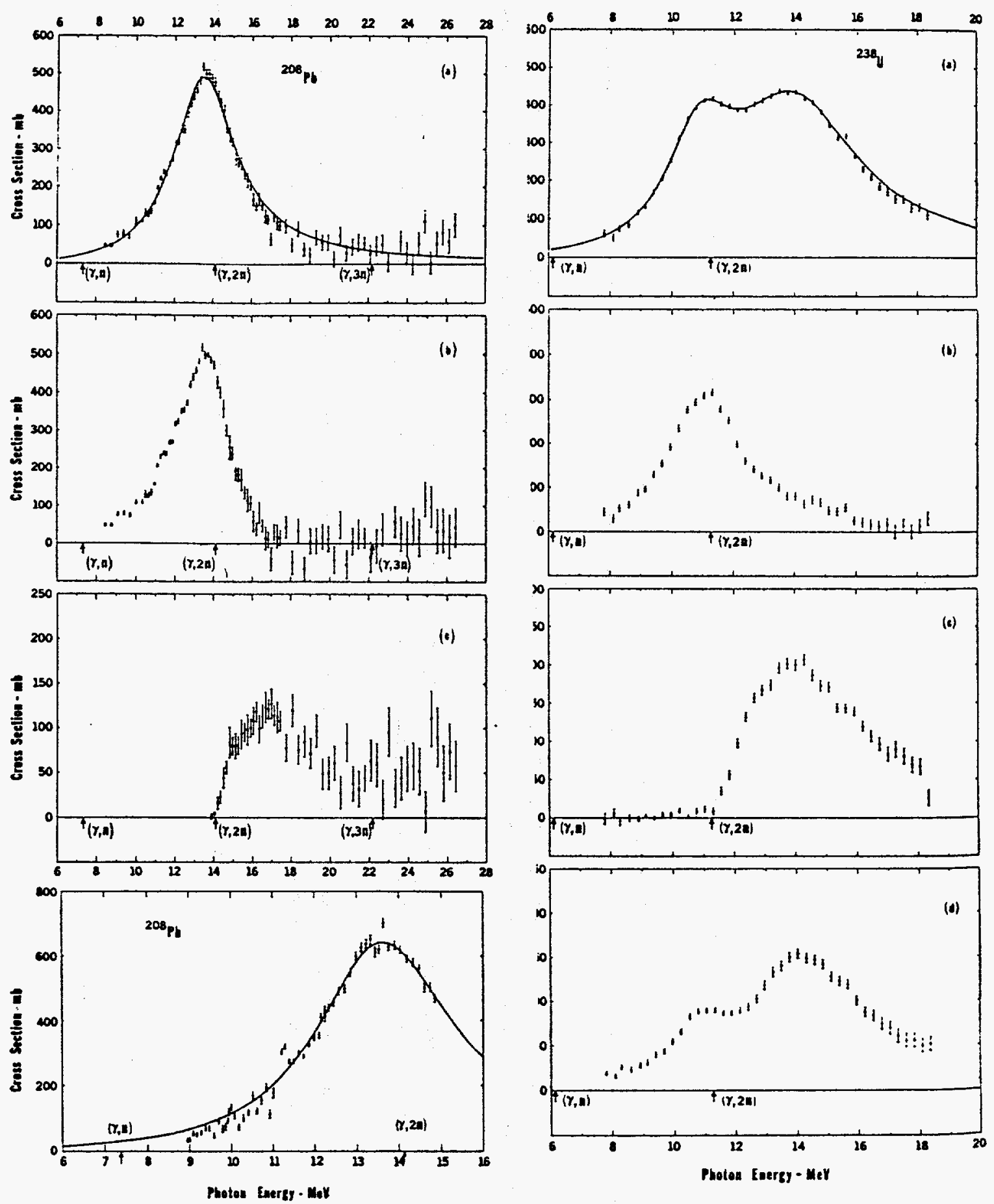

FIG. 16b. Photoneutron cross section of ${ }^{208} \mathrm{~Pb}$ and ${ }^{238} \mathrm{U}$. 


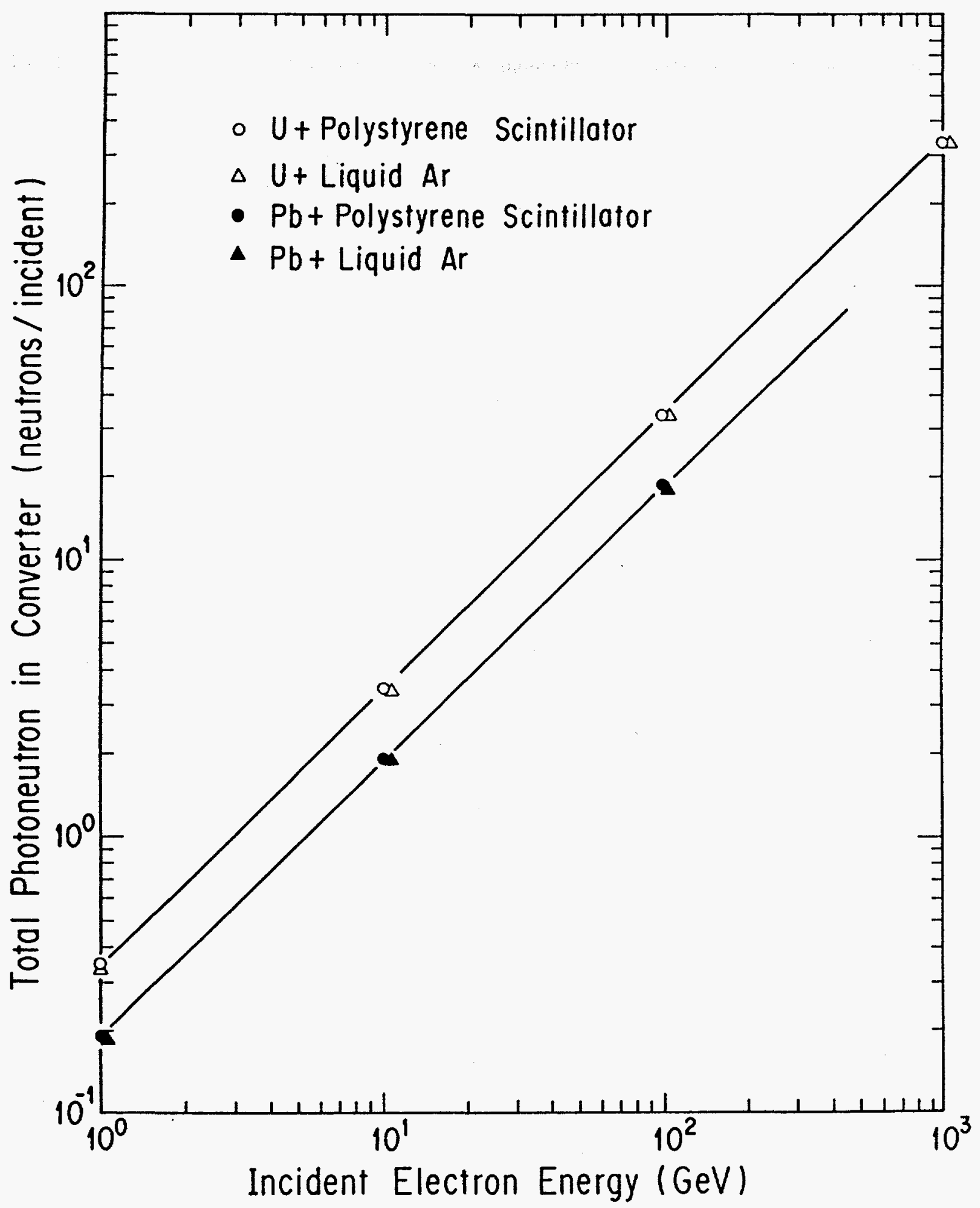

FIG. 17. Total number of photoneutrons produced in the converter for the various combination of the converter and the active materials. 


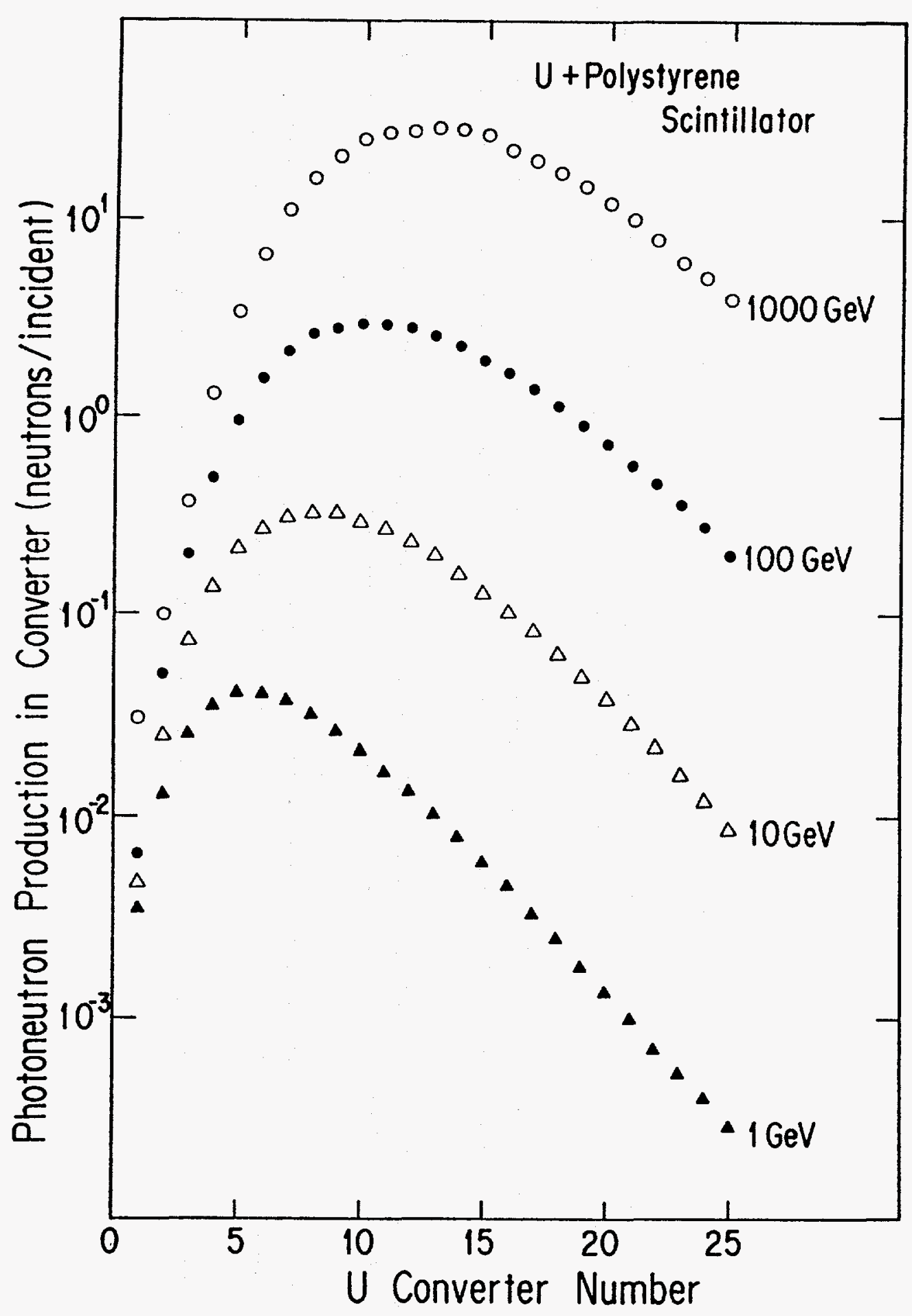

FIG. 18. Distribution of produced photoneutrons in U converter for different incident energy. 


\title{
APPENDIX 8 \\ LPM EFFECT IN THE SSC DETECTORS*
}

\author{
Todor Stanev \\ Bartol Research Institute \\ University of Delaware, Newark DE 19716
}

\section{Introduction}

The LPM effect $[1,2]$ decreases the bremsstrahlung and pair-production crosssection in dense materials at high energy and modifies the secondary production spectra. As a results the development of electromagnetic cascades is slowed down and the cascades penetrate deeper. Although it has been estimated that the effect affects cascade development significantly only at energies $>61.5 L_{\mathrm{cm}} \mathrm{TeV}$ (where $L_{\mathrm{cm}}$ is the value of the radiation length of the material in $\mathrm{cm}$ ) [3] the need to use heavy materials in the SSC calorimeters calls for a new and more detailed estimate. Another manifestation of the LPM effect is that with the decreased bremsstrahlung cross-section the electron energy loss becomes so small that at $\mathrm{TeV}$ energies some electrons might be misidentified as muons.

The LPM effect is due to the interference between multiple scattering and radiation when the distance between neighbouring nuclei is comparable to the radiated photon wavelength. When the two electron momenta (initial and final electron momenta for radiation processes or $e^{+}, e^{-}$momenta for pair production) become ultrarelativistic, the mass of the system at the vertex is negligible, so that the longitudinal momentum transfer $q_{\|}$can be very small. Conversely the distance $l$ along which the radiation occurs becomes very long. We have

$$
l \sim \frac{\hbar}{q_{\|}} \sim \frac{2 E(E-c k)}{\left(m c^{2}\right)^{2}} \frac{\hbar}{k}
$$

where $E$ is the initial electron energy, $k$ is the photon momentum, and $m$ is the electron mass. In media with sufficient density more that one atom is encountered on the distance $l$. These additional atoms cause multiple Coulomb scattering of the two electron waves introducing decoherence between the two states which reduces the result of the integration to obtain the transition matrix element.

The suppression of the radiation matrix element becomes important when when the rms multiple-Coulomb-scattering angle $\left\langle\theta_{s}^{2}\right\rangle^{1 / 2}$ becomes larger than the

* Also appears as SSC Central Design Group Report SSC-N-415 (1987). 
scattering angle $\theta_{r}$ due to the radiation process. A parameter $s$ is defined as

$$
s[\xi(s)]^{1 / 2} \equiv \theta_{r} / 2\left\langle\theta_{s}^{2}\right\rangle^{1 / 2} \sim \frac{u}{1-u}
$$

for the case of bremsstrahlung, where $\xi(s)$ is a logarithmic factor $O(1)$ and $u$ is the fractional energy of the radiated photon. The effect must be considered for $s \leq 1$. For pair production $s \sim 1 /\left(v-v^{2}\right)$, where $v$ is the fractional energy of the electron in the created pair, and since $1 /\left(v-v^{2}\right) \geq 4$ (while $u /(1-u$ ) can be arbitrarily small), the LPM effect in pair production becomes important at energies approximately two order magnitudes higher then for radiation.

Experimentally the LPM effect has been studied in cosmic rays, where it has been only qualitatively confirmed. A quantitative result comes from a comparison of the intensity ratios of 20 to $80 \mathrm{MeV}$ photons from $\mathrm{Pb}$ relative to $\mathrm{Al}$ targets and from $\mathrm{W}$ relative to $\mathrm{C}$ in experiments with a $40 \mathrm{GeV}$ electron beam in Serpukhov [4].

Since the LPM effect is much stronger for electrons and in heavy materials we have calculated the bremsstrahlung cross-section and the electron energy loss in uranium. These results give an upper limit of the influence of the LPM effect in the SSC energy range.

\section{Bremsstrahlung cross-section and energy loss in uranium}

Fig. 1 shows the photon production spectrum in uranium as a function of the fractional photon energy. The full line represents the Bethe-Heitler spectrum, while the dash, dash-dot and dot lines show the decrease of the probability for radiation of low energy photons with the energy. At fractional photon energies of $10^{-8}$ the suppression is significant ( $\sim$ factor of 100) even at electron energies of $1 \mathrm{GeV}$. For $10 \mathrm{TeV}$ electrons the suppression is up to four orders of magnitude.

This graph is, however, somewhat misleading, since from experimental point of view the interesting parameter is the probability for radiation of photons above certain energy threshold. Such a result is shown on Fig. 2, where the average number of photons radiated with energy above $1 \mathrm{MeV}$ and $1 \mathrm{GeV}$ is plotted versus the electron energy. Without the account for the LPM effect $\left\langle n_{\gamma}\left(E>E_{\gamma}\right)\right\rangle$ would continue to grow logarithmicaly with the electron energy. Because of the LPM effect the production of $>1 \mathrm{MeV}$ photons reaches a maximum at $\sim 10 \mathrm{GeV}$ and significantly declines in the $\mathrm{TeV}$ region. At $10 \mathrm{TeV}$ the production of $>1 \mathrm{MeV}$ photons is lower than the Bethe-Heitler spectrum by a factor of 7 .

The decrease of the bremsstrahlung cross-section leads, of course, to a corresponding decrease of the electron energy loss. The energy loss is not affected as strongly as the cross-section because the suppression is stronger at low photon 


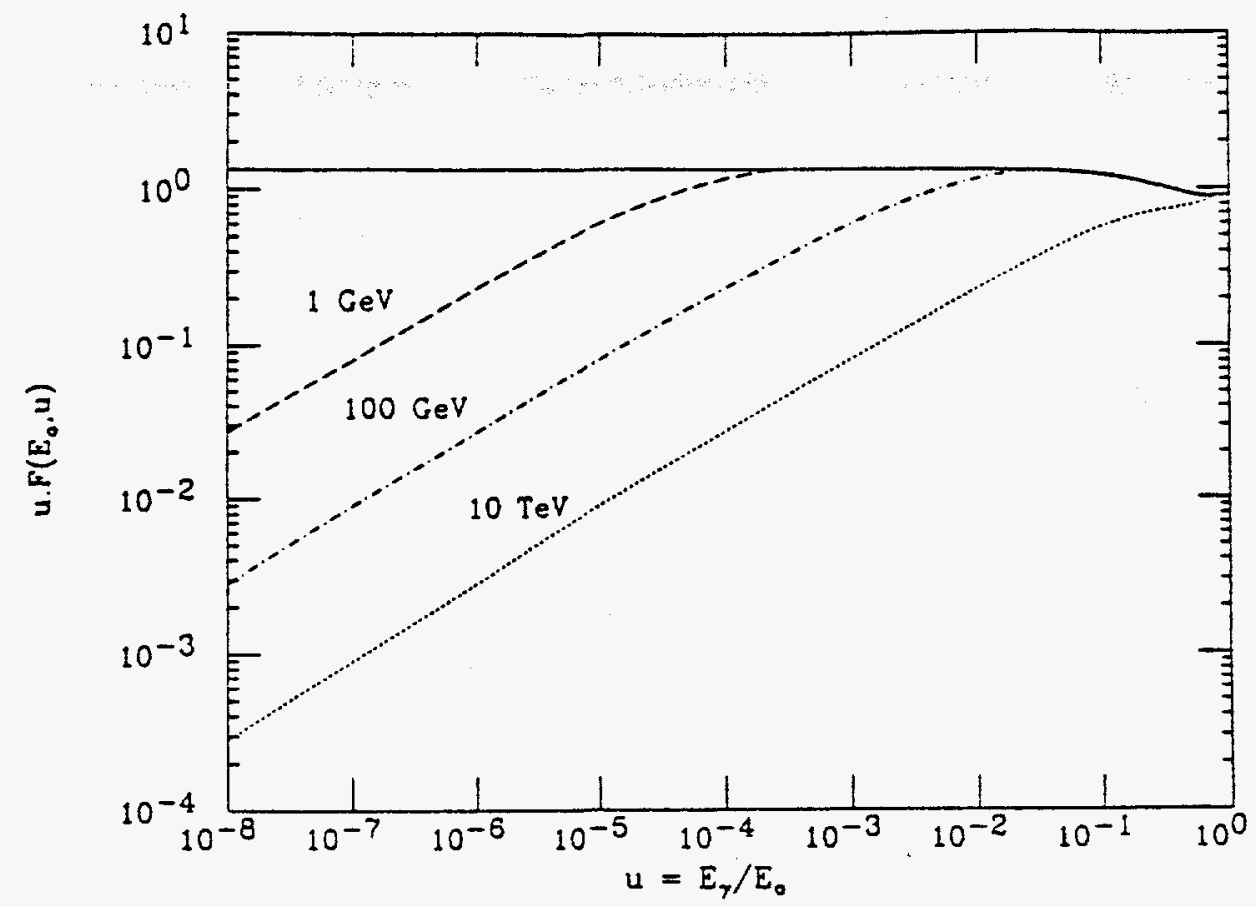

Fig. 1. Differential bremsstrahlung intensities per radiation length in uranium. The solid line is for the limiting Bethe-Heitler cross-section. The energy of the incoming electron is indicated by each curve.

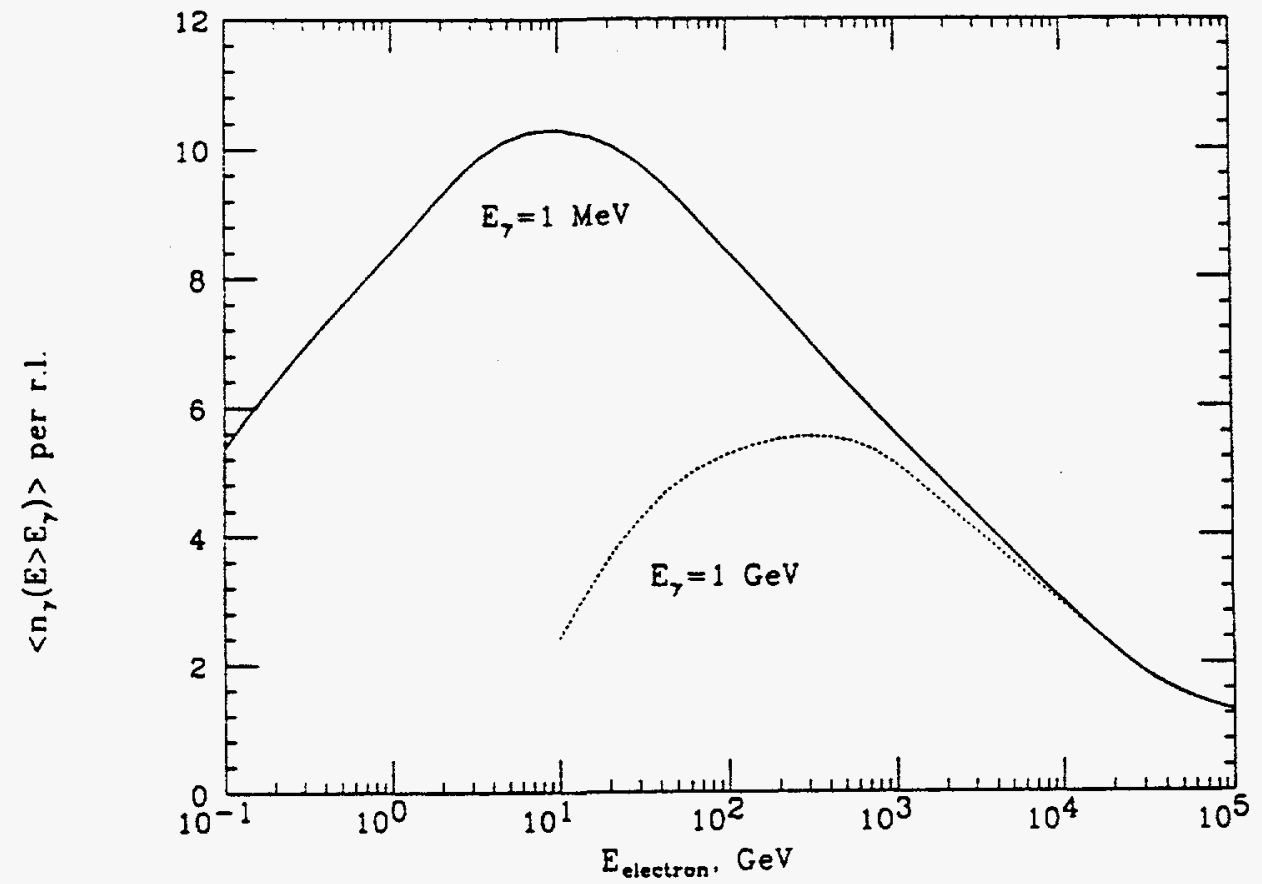

FIG. 2. Average number of photons with energy $>1 \mathrm{MeV}$ (colid curve) and $>1 \mathrm{GeV}$ (dotted curve) radiated in one radiation length of uranium as a function of the electron energy. 
energies (note the $u /(1-u)$ factor in Eq. 2). The fractional electron energy loss per $\mathrm{g} \mathrm{cm}^{-2}$ is shown in Fig. 3. The decline in the $\mathrm{MeV}$ region is due to the decreasing contribution of the ionization loss to the fractional energy loss. The influence of LPM effect can be detected at $\sim 100 \mathrm{GeV}$ but it only makes a difference of less than $30 \%$ even at $10 \mathrm{TeV}$.

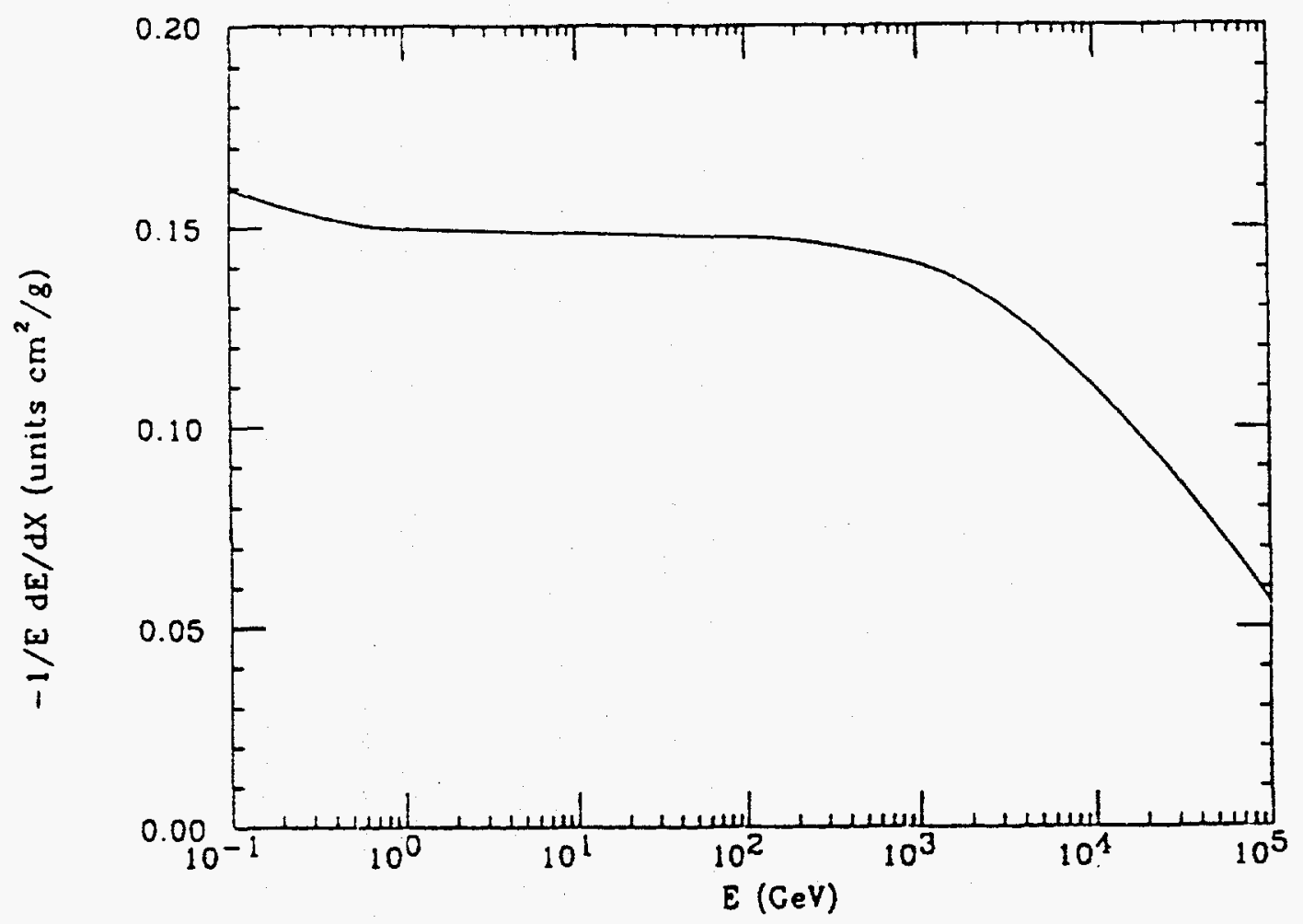

FIG. 3. Fractional electron energy loss in uranium.

\section{Conclusions}

The LPM effect will be present at the SSC energies, but it can hardly change the present estimates of the energy flow in the planned detectors. Past calculations of the development of electromagnetic showers with account of the LPM effect (see[3] for other references) show that the cascade development is noticeably affected only when the cross-section for $u \sim 1 / 2$ is decreased, i.e. at $20 \mathrm{TeV}$ in uranium. Some more subtle manifestations of the effect are possible. The angular and lateral distributions of the cascade particles in LPM cascades become narrower. The combination of the narrow angular spread and the larger depth of the first interaction will decrease the electromagnetic albedo from the detectors. Even this effect is not likely to be large, though, because the interaction products are dominated by photons, not electrons. 
The decrease of the electron energy loss is not significant enough to cause misidentification of electrons as muons, unless the ratio of electrons to muons is of the order of $10^{4}$. If a muon signal, however, has an electronic background of this order of magnitude, the LPM decrease of the electron energy loss must be accounted for in calculating the expected noise.

\section{Acknowledgments}

This work is supported in part by the National Science Foundation. The author is grateful to D. E. Groom and the SSC Central Design Group for their hospitality.

\section{References}

1. L. Landau and I. Pomeranchuk, Dok. Akad. Nauk SSSR 92, 535 (1953); 92, 735 (1953).

2. A. B. Migdal, Phys. Rev. 103, 1811 (1956); Sov. Phys.-JETP 5, 527 (1957).

3. T. Stanev, C. Vankov, R. E. Streitmatter, R. W. Ellsworth, and T. Bowen, Phys. Rev. D25, 1291 (1982). See also T. Bowen, R. W. Ellsworth, T. Stanev, R. E. Streitmatter, and C. Vankov, At. Data Nucl. Data Tables 24, 495 (1979).

4. A. A. Varfolomeev, V. I. Glebov, E. I. Denisov, A. M. Frolov, and A. S. Khlebnikov, Sov. Phys.-JETP 42, 218 (1976). 



\section{APPENDIX 9 \\ FLUX, CURRENT, AND LAMBERT'S LAW \\ D. E. Groom \\ SSC Central Design Group, LBL90-4040, Berkeley CA 94720}

\section{Flux}

To most physicists, a flux $F$ means the number of something (neutrons, ergs, millijanskys, ...) arriving in a particular solid angle interval and crossing unit area in unit time; the units might be $\mathrm{cm}^{-2} \mathrm{sr}^{-1} \mathrm{~s}^{-1}$. For example, con-

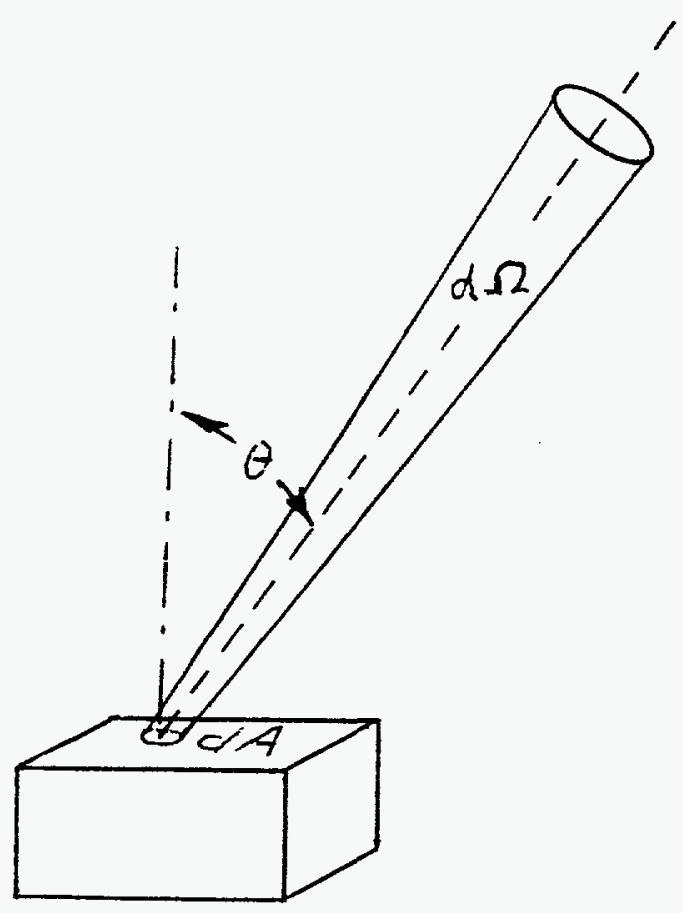

FIG. A9-1. Particle detector exposed to a flux of cosmic rays or other particles. sider the detector shown in Fig. A91. The counting rate in a small area $d A$ is $F d A \cos \theta d \Omega$, where $\theta$ is the angle between the normal to $d A$ and the direction of interest. The total counting rate is

$$
\text { Rate }=\int_{\text {Area }} \int_{\Omega} F d A \cos \theta d \Omega
$$

If the flux from all directions is the same, $F$ can be taken out of the integral, which is then purely geometrical and may be defined as the aperture, $\langle A \Omega\rangle$. For a plane detector with area $A,\langle A \Omega\rangle=2 \pi A$. For a sphere with $\pi r^{2}=A,\langle A \Omega\rangle=4 \pi A$.

In radiation problems the target (detector) is invariably a nucleus with no preferred orientation, so in an isotropic flux $F$ the interaction rate is $4 \pi F \sigma$, where $\sigma$ is the interaction cross section. It is therefore usual and convenient to define the flux $\&$ as $4 \pi F$, measured e.g. in $\mathrm{cm}^{-2} \mathrm{~s}^{-1}$, or in $\mathrm{cm}^{-2}$ per interacting primary particle. $\varphi$ is the flux $F$ integrated over a spherical detector with unit cross sectional area. It is the time derivative of the fluence as defined by the ICRU[1]. *

* "A.3a. The (particle) fluence, $\Phi$, is the quotient of $d N$ by $d a$, where $d N$ is the number of particles incident on a sphere of cross-sectional area da." - Ref. 1, p. 7. 
Even though $\varphi$ is simply the solid-angle integral of $F$ over a special geometry, there remains a basic conceptual difference. In Fig. A9-1, a count occurs when a particle crosses the surface of the detector. Aperture matters, not volume. Interactions with the material inside occur at a rate $\varphi N \sigma$ per unit volume, where $N$ is the number density of target nuclei. The total interaction rate is proportional to volume, not to the area presented by the detector: The size of a monopole detector is given in $\mathrm{cm}^{2} \mathrm{sr}$, while the size of a neutrino detector is given in tons.

A program such as MORSE "scores" flux in several different ways, among them by recording the total track length of the particles of interest in the scoring volume. Then

$$
\varphi=\frac{\text { Total track length }}{\text { Scoring volume }} .
$$

The correctness of this expression follows from the observation that flux defined this way times the interaction cross section is the interaction probability, as required. However, a more detailed look is useful for the following discussion of cur-

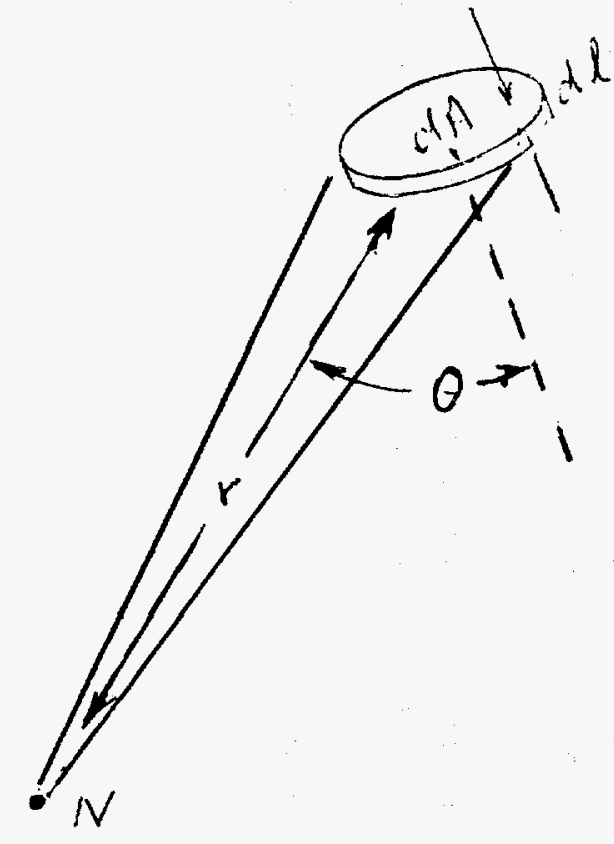

FIG. A9-2. A thin-disk scoring volume exposed to a point source of particles. rent. Consider a point source of radiation a distance $r$ from a thin scoring volume with area $d A$ and thickness $d \ell$, as shown in Fig. A9-2. If $N$ particles per unit time are emitted by the source, then the flux at a distance $r$ is just $N / 4 \pi r^{2}$. The number of particles crossing the scoring volume per unit time is $N d A \cos \theta / 4 \pi r^{2}$. The track length of each is $d \ell / \cos \theta$, for a total track length $N(d A d \ell) / 4 \pi r^{2}$. The flux is obtained by dividing by the volume $d A d \ell$.

\section{Current}

In this case, only the normal component is considered, which is equivalent to weighting each track segment by the cosine of the angle it makes with the normal to the entry surface of the scoring volume. In Fig. A9-

2 the current is $N \cos \theta / 4 \pi r^{2}$-few particles cross the flat detector if is nearly edge-on to the source. For an isotropic flux arriving on one side of a surface, the current is smaller than the flux by the average of $\cos \theta$ over the solid angle, or $\frac{1}{2}$. This same factor of $\frac{1}{2}$ showed up above when we compared the aperture of a 
plane detector with that of a spherical detector, where in both cases the sense of the crossing was ignored. The plane detector was measuring what we are now calling "current."

It is convenient to imagine "current" as the number of holes (per unit time and unit area) punched by the particles in a plane surface as they go through. Suppose particles traveling in one direction make green holes, and particles traveling in the opposite sense make red ones. Technically the total current is zero (red and green cancel), but a detector is color blind.

A variety of experiments and simulations are described in this report concerning the escape of neutrons from the front face of a calorimeter and the flux inside. Some authors report flux and some report current, so appropriate care must be taken.

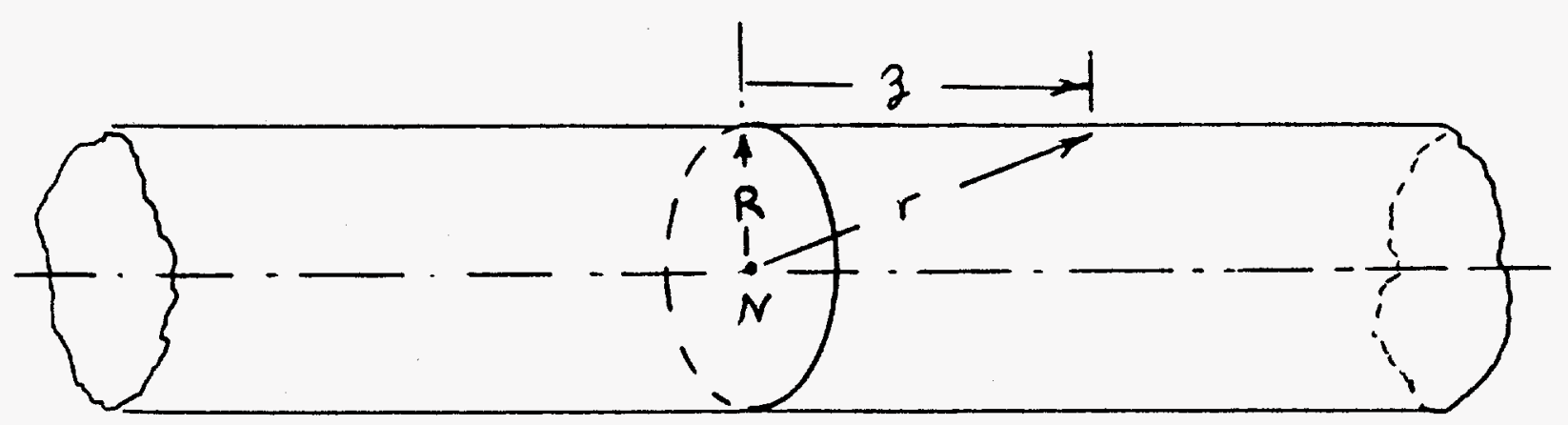

FIG. A9-3. Point particle source in the center of a long tunnel.

\section{Confusion}

Many of us find these ideas confusing because we are used to measuring flux by counting the number of surface crossings. As an example, consider a point source emitting $N$ particles per unit time, located on the axis of a cylindrical tunnel with radius $R$, as shown in Fig. A9-3. ${ }^{\star}$ The flux at the wall a distance $z$ downstream is $N / 4 \pi r^{2}$, where $r^{2}=z^{2}+R^{2}$. If we integrate this flux over the entire tunnel wall, the result is $\pi N / 2$. On the other hand, the current at the wall is $N \cos \theta / 4 \pi r^{2}$, and its integral over the cylindrical surface is $N$. This is reasonable, since we have interpreted the current as the number of surface crossings. If the scoring volume is a thin cylindrical shell on the tunnel wall, we see that the interaction probability of a particle striking far down the tunnel is much greater (by $1 / \cos \theta$ ) than one crossing near the source-hence the flux integral is greater than the current integral. Again: The current provides a count

\footnotetext{
^ I am indebted to W. P. Swanson for providing this example.
} 
of the number of particles entering the scoring volume, while the flux provides a measure of the havoc wreaked inside.

\section{Lambert's Law}

Let us consider an enclosure full of something in thermodynamic equilibrium, such as black body radiation or an ideal gas. We draw a plane with an arbitrary orientation inside the cavity, as shown in Fig. A9-4, and consider the flux crossing it in the positive sense. The isotropy of the situation implies that the flux into solid angle $d \Omega$ is independent of direction, in particular the direction the cone makes with the normal to the plane. It follows that the flux crossing (emitted from!) $d A$ of the surface is proportional to $\cos \theta$, where $\theta$ is the angle away from the normal. "Lambert's Law" is thus true for a mathematical surface anywhere in the enclosure, and is just another expression of the isotropy of the flux.

What about a physical enclosure wall, or a physical surface inside the enclosure? To fix ideas, consider a black disk inside a black thermal enclosure. Suppose Lambert's Law were not true, but that each surface element of the disk emitted the same amount of radiation into

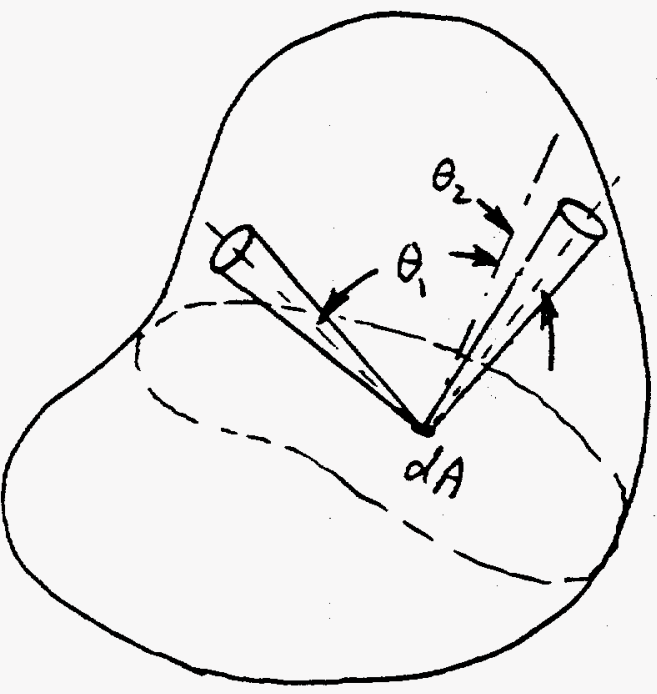

FIG. A9-4. Flux across a mathematical plane in a cavity in equilibrium. each solid angle cone. Then the $\cos \theta$ foreshortening of $d A$ would imply more radiation near the plane, and a bright area on the walls of the enclosure near the intersection of the plane with the wall. It would be hotter here, and heat would be transported from a cooler to a warmer place without any other work being done, in violation of the second law. For a system in thermodynamic equilibrium, Lambert's law is a necessary consequence of the second law of thermodynamics [2]. If we were considering an enclosure containing an ideal gas, then deviations from Lambert's Law would result in pressure inhomogeneities in the enclosure.

Lambert's Law has familiar consequences: a red hot ingot of iron appears as a uniformly luminous plane, and the sun and moon appear as uniformly illuminated discs. Exceptions are equally familiar: The $\mathrm{x}$-ray intensity from a tungsten anode peaks at $90^{\circ}$ rather than $0^{\circ}$ because of the radiation mechanism, and photographs of the sun show darkening near the limb, in contrast to our visual impression. The darkening is easily understood, since we see mostly light emitted by material 
about an optical absorption length into the photosphere along the line of sight. At the more nearly grazing incidence angles near the limb, this material is nearer the surface, and hence cooler and less luminous.

Now consider a block of material (e.g. uranium or lead) exposed to 200-300 $\mathrm{MeV}$ neutrons. Neutrons in this energy range have a reasonably small interaction probability and hence a large range. For our present purposes, we assume that the interactions are distributed uniformly throughout the volume. Secondary neutrons (e.g. $\sim 1 \mathrm{MeV}$ ) are produced isotropically in these interactions. As per our original argument, the "emission" from a surface drawn arbitrarily inside the volume obeys Lambert's Law. Now simply remove the material to one side of this surface; it is now a real boundary and the neutron flux from the material toward the outside is unchanged by the removal, at least if we ignore double scatterings. The "albedo" neutrons thus obey Lambert's Law to a good approximation.

In Appendix 13, Alsmiller et al. present simulation results for the entry-face neutron albedo for a calorimeter. In this case the progenitor flux is not really isotropic. The empirical ratio of flux to current is about 1.8, while complete compliance with Lambert's Law would yield two. We are therefore justified in assuming the near-validity of Lambert's Law for cases of interest in this report.

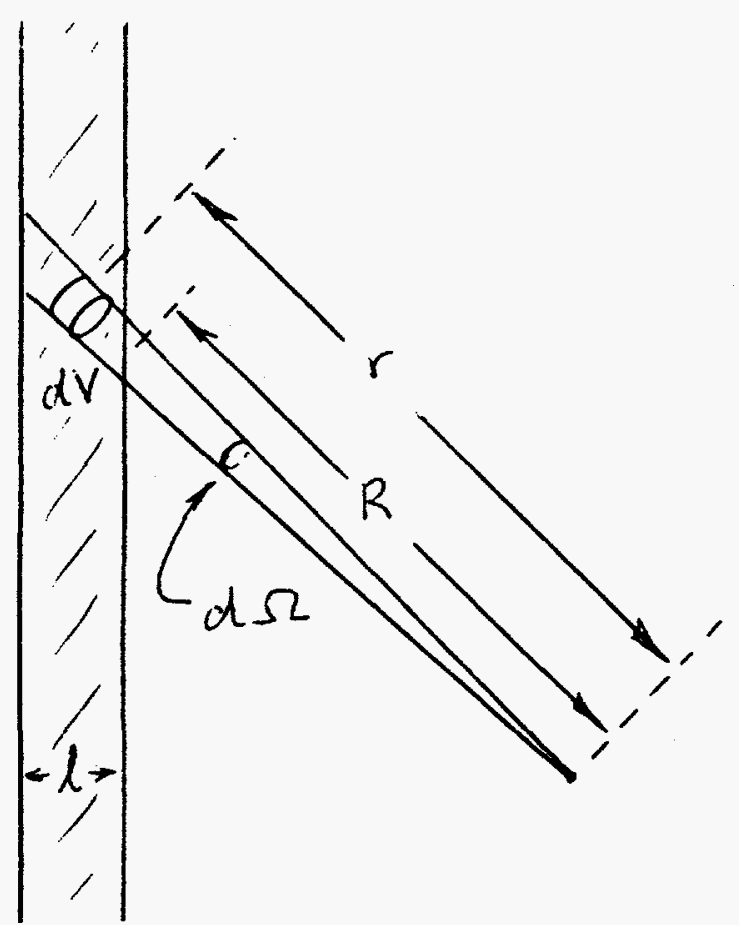

FIG. A9-5. Radiation from an activated slab.

A final example might help clarify the physical origin of Lambert's Law in a radiation situation. A slab of thickness $\ell$ is uniformly activated with a $\gamma$-ray emitting isotope such as ${ }^{137} \mathrm{Cs}$. A small detector outside the surface is sensitive only to full-energy $\gamma$-rays (so that we may ignore secondary scattering processes.) The $\gamma$ rays have an attenuation length $\lambda$ in the material and are not attenuated outside the slab. Using the geometry shown in Fig. A9-5, we see that the intensity of $\gamma$-rays produced in volume element $d V\left(=r^{2} d r d \Omega\right)$ at the detector is

$$
d^{2} I=\frac{A d V}{4 \pi r^{2}} \mathrm{e}^{-(r-R) / \lambda}
$$

where $A$ is the number of decays per 
unit volume per unit time. Integrating over the radial variable at fixed angle,

$$
d I=\frac{A d \Omega}{4 \pi} \lambda\left(1-\mathrm{e}^{-\ell / \lambda \cos \theta}\right)
$$

For $\ell>\lambda, d I / d \Omega$ is independent of $\theta$, as expected from Lambert's Law. This comes about because most of the radiation seen at the detector originates at a slant depth $\lambda$ or less into the slab, so that the amount of activity $(\alpha d \ell=\lambda \cos \theta)$ contributing to the intensity falls with increasing angle. The contribution to the intensity falls as $\cos \theta$, just compensating the $\cos \theta$ increase of the surface area intercepted by $d \Omega$.

If the slab is extremely thin, then

$$
\frac{d I}{d \Omega} \approx \frac{A}{4 \pi}\left(\frac{\ell}{\cos \theta}+\ldots\right)
$$

so that $d I / d \Omega$ varies as $\sec \theta$. The divergence as $\theta \rightarrow 90^{\circ}$ is prevented by higherorder term in the series, since for any small $\ell$ there is near-grazing angle of emission for which the maximum slant depth is no longer small compared with $\lambda$. An activated thin foil exhibits edge-brightening, but only up to a finite limit.

A final point must be raised. We have shown that inside a cavity in which something (radiation or gas) is in thermodynamic equilibrium, Lambert's Law is just another way of expressing isotropy. At a real boundary in the cavity it must also be exact to avoid violations of the second law. However, this is a physical surface, and there is no general reason why the $\cos \theta$ emission law should be exact. (It failed to be exact in the handful of non-equilibrium cases discussed above.) This point is uniformly glossed over in the literature. Joos, for example, who provides one of the rare discussions of the matter [2], merely says that it must be so.

\section{Neutrons inside a $4 \pi$ detector}

To some degree of approximation, a $4 \pi$ detector consists of a calorimeter outside a region which is essentially empty. This geometry is shown in Fig. A96. About 110 charged hadrons are produced in each $\mathrm{p}-\mathrm{p}$ collision at the center of the detector, with an angular distribution roughly constant as a function of $\eta \equiv-\ln \tan (\theta / 2)$. In Section 2 it is shown that the charged particle momentum distribution as a function of $\theta$ (or $\eta$ ) is adequately represented by

$$
\frac{d^{2} n_{\mathrm{ch}}}{d \eta d p}=\frac{1}{\cosh \eta} \frac{d^{2} n_{\mathrm{ch}}}{d \eta d p_{\perp}}=\frac{H}{\cosh \eta} f\left(p_{\perp}\right)
$$

for each collision at the interaction point, where we have used the identities $\sin \theta \cosh \eta=1$ and $p_{\perp}=p \sin \theta$. 


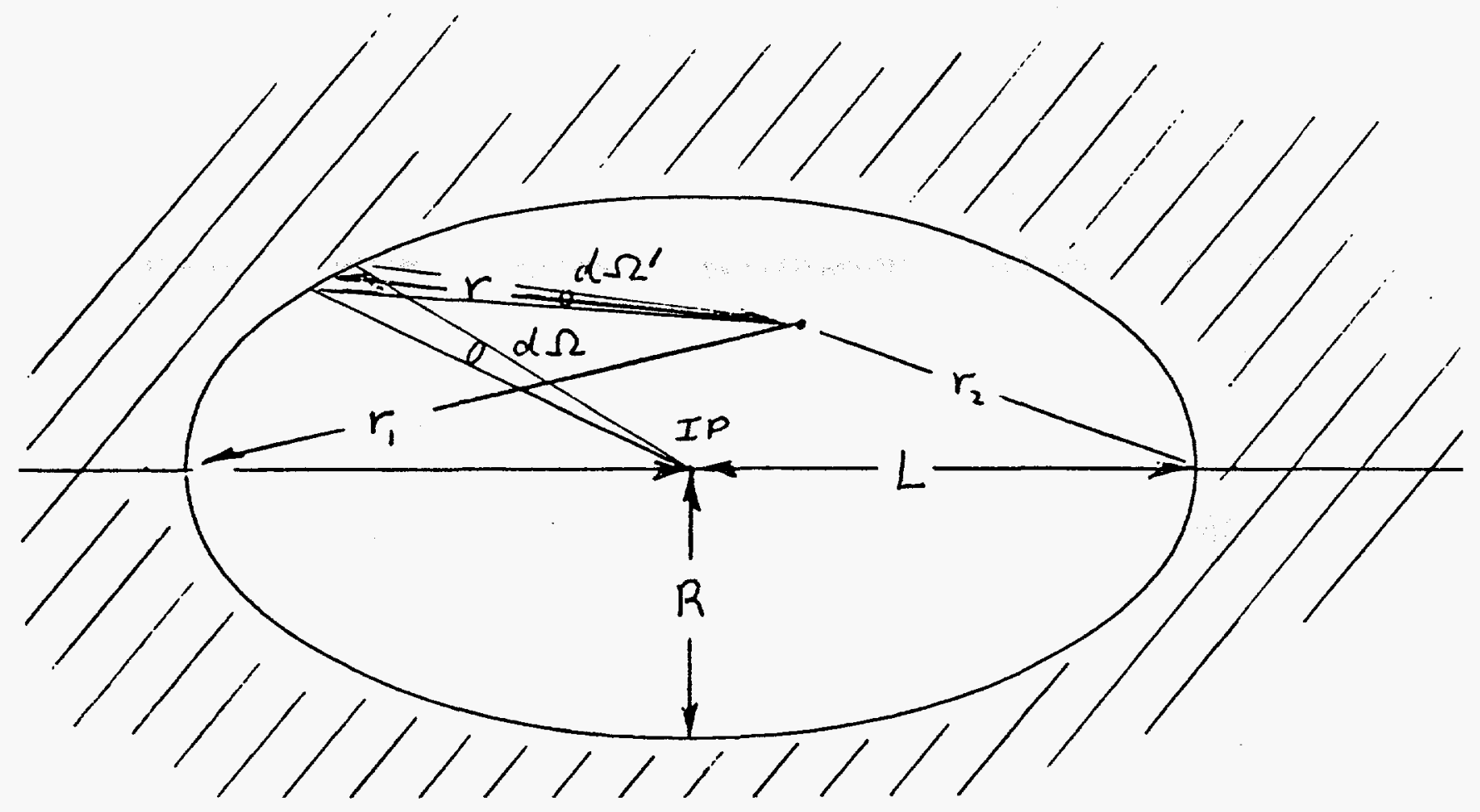

FIG. A9-6. An arbitrary $4 \pi$ detector.

When a hadron with momentum $p$ strikes the calorimeter, a substantial number of low-energy $(\approx 1 \mathrm{MeV})$ neutrons are backscattered into the central cavity. Since their distribution very nearly satisfies Lambert's law, the number from the area element involved which scatter into a solid angle $d \omega$ at an angle $\theta$ to the normal is

$$
d N_{n}=N(p) \cos \theta d \omega / \pi
$$

where $N_{n}(p)$ is the total number produced by a particle with this momentum. Since the contribution to the flux at an arbitrary point $r$ away is $N_{n}(p) \cos \theta / \pi r^{2}$, that of a surface element subtending $d \Omega^{\prime}$ as viewed from the point of interest is *

$$
\begin{aligned}
d \phi_{\text {direct }} & =\left(\int_{p} \frac{d^{2} n_{\mathrm{ch}}}{d \eta d p} \frac{d \eta}{d \Omega} \frac{d \Omega}{d \Omega^{\prime}} \frac{N_{n}(p)}{\pi r^{2}} d p\right) d \Omega^{\prime} \\
& =\left(\int_{p} \frac{d^{2} n_{\mathrm{ch}}}{d \eta d p} \frac{d \eta}{d \Omega} \frac{N_{n}(p)}{\pi r^{2}} d p\right) d \Omega
\end{aligned}
$$

As shown in Fig. A9-6, the surface element subtends $d \Omega$ as seen from the interaction point, and subtends $d \Omega^{\prime}$ from the observation point. The total flux is

* The area of the emitting surface defined by $d \Omega^{\prime}$ increases as the secant of the angle to the normal, so the $\cos \theta$ term does not appear in Eqns. (4) and (5). 
then

$$
\begin{aligned}
\phi_{\text {direct }} & =\int_{p_{\perp}, \Omega} \frac{d^{2} n_{\mathrm{ch}}}{d \eta d p_{\perp}} \frac{d \eta}{d \Omega} \frac{N_{n}\left(p_{\perp} / \sin \theta\right)}{\pi r^{2}} d p_{\perp} d \Omega \\
& =\int_{p_{\perp}, \eta} \frac{d^{2} n_{\mathrm{ch}}}{d \eta d p_{\perp}} \frac{N_{n}\left(p_{\perp} \cosh \eta\right)}{\pi r^{2}} d p_{\perp} d \eta .
\end{aligned}
$$

It is interesting to note that the total flux is obtained by integrating over the production angles rather than $d \Omega^{\prime}$, although the dependence of $r$ on these angles is generally quite complicated.

Before going on, we remark that

1. $N_{n}(p)$ is a slowly varying function of $p\left(\sim p^{0.5}\right.$, so low momenta are important. Note that $N_{n}(p)$ is the number of backscattered particles, or a current. If a flux is quoted, it must be divided by two.

2. $d \eta / d \Omega=1 / 2 \pi \sin ^{2} \theta$. The choice of $\eta$ as angular variable effectively moves the forward peaking of the inclusive cross section into this term.

3. Most of the flux comes from the two small-angle regions of the endcap calorimeters, so that we may write the integral of Eqn. (5) as the sum of a simpler integral with $r=r_{1}$ (the distance to one endcap) and an integral with $r=r_{2}$ (for the other endcap).

4. Because of the $1 / r^{2}$ dependence of the flux, it is advantageous if the endcaps are far from sensitive electronics or gas-filled volumes, or if such elements are the "shadow" of other parts of the calorimetry, as can be the case when the endcaps are physically separate from the central detector.

\section{Reflection}

Before the integral of Eqn. (5) is taken more seriously, it is worth considering another problem. So far, we have considered only the direct flux of albedo neutrons from the calorimeters. With a very high probability these neutrons again backscatter, particularly from atoms with high atomic number. In the accelerator tunnel situation discussed in Appendix 10, only about $20 \%$ of the flux at the wall comes directly from the source[3]. After several reflections their point of origin has been forgotten, and they appear as an isotropic neutron gas which fills the enclosure.

The injected flux is seldom isotropic. In the case of the main ring tunnel it comes from almost a line source, and in the case of a calorimeter most of it comes from the "hot spots" closest to the beam line. The reflected flux is more nearly isotropic. It is therefore convenient to treat the direct and reflected components 
separately, and to assume that the reflected flux is in fact isotropic. Let $a$ be the mean number of reflections from the walls; if $P$ is the probability of a reflection then $a=P /(1-P)$.

To do even this, one must make some assumptions about the energy spectrum of the direct flux and its degradation due to moderation and other processes upon reflection. The tunnel spectrum can be described by a broad gaussian in $\ln E_{k}$ (where $E_{k}$ is the neutron kinetic energy) with a peak at about $0.6 \mathrm{MeV}$. For albedo (backscattered) neutrons in a calorimeter cavity, the spectrum is similar but peaks at perhaps $1 \mathrm{MeV}$. In both cases most of the integral under the peak is above $0.1 \mathrm{MeV}$. In many problems the main issue is damage to silicon devices, and the effective threshold for such damage is about $0.16 \mathrm{MeV}$. Scattering cross sections increase as the energy decreases, ${ }^{\star}$ so reflection is more efficient at lower energies. For purposes of this note, we think of $\sim 1 \mathrm{MeV}$ as a typical energy, and drop a neutron from further consideration when its energy falls below $0.1 \mathrm{MeV}$.

Let $\mathcal{N}$ be the number of neutrons injected into a cavity per unit time. In the case of tunnel, let $\mathcal{K}$ be the number injected per unit length per unit time. These quantities play the same role in calculations, but are given separate names because of the difference in units.

For the moment suppose that the $\mathcal{N}$ neutrons per unit time injected into a cavity all have the same velocity $v$. Let $\ell$ be the mean total distance a neutron goes after the first reflection and before being absorbed. It survives for a mean time $\ell / v$, and so there are $\mathcal{N} \ell / v$ of these reflected neutrons "in the air" inside the cavity at any moment. Their volume density is $n=\mathcal{N} \ell / v V$, where $V$ is the volume of the cavity. The differential flux $F$ (the number of particles per unit solid angle crossing a normal unit area element in unit time) is given by $n v / 4 \pi$ and the isotropic flux $\varphi$ (the number crossing a sphere with unit cross sectional area in unit time) by $n v=\mathcal{N} \ell / V$. The velocity no longer appears.

In general, $\ell$ will scale as a characteristic linear dimension of an enclosure (call it $R$ ), and $V$ as the cube of this dimension. The scattered flux therefore scales as $1 / R^{2}$.

If the mean distance between reflections after the first bounce is $\lambda$ and there are $a$ such scatterings on the average before the neutron is absorbed or loses too much energy, then $\ell=a \lambda$. Given Lambert's Law, $\lambda$ is just the average chord length. For a solid which is everywhere convex and has surface area $S$ and volume $V$, the average chord length is $4 V / S[4,5]$. Thus for a cylinder of radius $R, \lambda=2 R$, and for a sphere, $\lambda=(4 / 3) R$.

* In the case of a uranium/scintillator calorimeter, the cross section is twice as large at 0.1 $\mathrm{MeV}$ as at $1.0 \mathrm{MeV}$. 
A detector cavity is intermediate between these cases. In general we may write $\lambda=b R$, where $b$ is slightly less than two. Then

$$
\begin{aligned}
\varphi_{\text {ref }} & =\mathcal{N} \frac{a \lambda}{V} \\
& =\mathcal{N} \frac{a b R}{V} \\
& =\mathcal{N} \frac{4 a}{S}
\end{aligned}
$$

where either the second or third form might be more useful in a given situation.

\section{Spheres}

For a sphere, the third form of Eq. (6) becomes

$$
\varphi_{\text {refl }}=\mathcal{N} \frac{a}{\pi R^{2}} .
$$

Suppose that every surface element on the inside of a sphere emits $\mathcal{N} d A / 4 \pi R^{2}$ neutrons per second, all with velocity $v$ and all of which are absorbed after crossing the cavity once. We assume that they are emitted according to Lambert's Law, so that $d A$ emits $\left[\mathcal{N} d A / 4 \pi R^{2}\right] \cos \theta d \Omega / \pi$ into a solid angle element $d \Omega$ at an angle $\theta$ with the normal. The contribution of $d A$ to the flux at the center of the sphere is then $\left(\mathcal{N} d A / 4 \pi R^{2}\right) / \pi R^{2}$. Integration over $d A$ yields $\mathcal{N} / \pi R^{2}$ for the flux at the center. This should be the same as the reflected flux with $a=1$, and we can use Eq. (7) to obtain the same result.

When reflection is added, we have

$$
\varphi_{\text {total }}=(1+a) \frac{\mathcal{N}}{\pi R^{2}}
$$

for the total flux inside. If the direct flux is not isotropic, then

$$
\varphi_{\text {total }} \approx \varphi_{\text {direct }}+a \frac{\mathcal{N}}{\pi R^{2}} .
$$

At the center of the cavity $\varphi_{\text {total }}=\mathcal{N} / \pi R^{2}$ no matter how the emission is distributed on the inside of the sphere, so long as it satisfies Lambert's Law.

Suppose holes are cut out of our spherical shell calorimeter, e.g. cones with half-angle $\theta_{0}$ along the beam direction, so that the remaining solid angle is $\Omega^{\prime}=$ $4 \pi \cos \theta_{0}$. In the case of isotropic flux, the reflection probability for a neutron inside is changed from $P$ to $P^{\prime}=P \Omega / 4 \pi$, or $P \cos \theta_{0}$. Since $a=P /(1-P)$, the new mean number of reflections $a^{\prime}=P^{\prime} /\left(1-P^{\prime}\right)$ can easily be calculated. A comparison of this calculation with Monte Carlo simulation results (Appendix 12) is made in Section 4. 


\section{Tunnels}

It is of interest compare results for a cylindrical tunnel geometry with those obtained above. We consider a cylindrical tunnel with radius $R$, e.g. in the SSC arcs. The source is the magnet string, which may be treated as a line source injecting $\mathcal{K}$ neutrons per unit length in unit time. At point of observation $r$ from the magnet string, $d z$ of the source a distance $z$ downstream contributes $\mathcal{K} d z / 4 \pi\left(r^{2}+z^{2}\right)$ to the direct flux. (We assume that the lateral size of the magnet is small compared with $r$.) The entire string thus contributes $\mathcal{K} / 4 r$.

For a length $L, \mathcal{N}=L \mathcal{K}$ and $S=2 \pi R L$, so $\mathcal{N} / S=\mathcal{K} / 2 \pi R$. The third form of Eq. (6) yields $2 a \mathcal{K} / \pi R$ for the reflection contribution to the flux. The total flux is then

$$
\varphi=\frac{\mathcal{K}}{4 r}\left(1+\frac{8 a r}{\pi R}\right) .
$$

The flux is enhanced through reflection by the factor $(1+8 \mathrm{ar} / \pi R)$ instead of the factor $(1+a)$ which was obtained for an isotropic source in a spherical cavity.

\section{Prolate spheroids}

A better approximation for a detector cavity might be a prolate spheroid with half-length $L$ and radius $R$, with its long axis along the beam line. Such a cavity has a volume $\frac{4}{3} \pi L R^{2}$. Its area is given by an elliptic integral, and decreases from $4 \pi R L$ at $R=L$ to $\pi / 4 \times 4 \pi R L$ for $L>>R$. For likely values of the aspect ratio ( $L / R=2$ or 3 ), $S \approx 0.85 \times 4 \pi L R$. Since most of the backscattered neutron flux comes from near the ends, the direct flux near the center of the detector is very nearly given by $\varphi_{\text {dir }}=\mathcal{N} / \pi L^{2}$. We then have

$$
\begin{aligned}
\varphi_{\text {total }} & =\varphi_{\text {dir }}+\varphi_{\text {refl }} \\
& =\frac{\mathcal{N}}{\pi L^{2}}+\mathcal{N} \frac{4 a}{S} \\
& \approx \frac{\mathcal{N}}{\pi L^{2}}\left(1+\frac{a}{0.85} \frac{L}{R}\right)
\end{aligned}
$$

The ratio $L / R$ might be two or three for a real detector. From Table 1 we see that $a \approx 0.7$ for a uranium/scintillator calorimeter. The factor in parentheses is thus about 3.1 for such a detector. The reflected contribution becomes relatively more important as the enclosure becomes longer, but only because the direct flux decreases as $1 / L^{2}$ while the reflected part goes as $1 / L$. If the structure is "open," with a large gap between the central section and the endcaps, then the analysis does not hold; in this case a different estimate of the direct flux must be made and the holes taken into account as above in estimating the reflection factor. 


\section{APPENDIX to Appendix 9: Summary of variable definitions}

a Mean number of reflections before a neutron is absorbed or loses enough energy to drop below a threshold.

$P \quad$ Probability of a reflection with acceptable energy. If $P$ is independent of the reflection number (not quite true because of the energy loss), then $1+a=1 /(1-P)$.

$E_{k} \quad$ Neutron kinetic energy.

$\mathcal{N}$ The number of neutrons injected into a cavity per unit time. Comment: Normalization of this and related quantities is often to one neutron injected into the cavity, the number of neutrons injected by one incident particle, etc.,rather than per unit time.

$\mathcal{K} \quad$ Neutrons per unit length injected into a tunnel per unit time.

$\ell \quad$ The mean distance traveled by a neutron before absorption or excessive energy degradation.

$\lambda \quad$ Mean distance between collisions; usually the average chord length in an enclosed volume.

$n \quad$ Number of neutrons per unit volume.

$\varphi \quad$ Isotropic neutron flux, i.e. the number crossing a sphere with unit cross sectional area in unit time.

$R$ Radius of a cylinder sphere, semiminor axis of prolate spheroid.

$L \quad$ Length of a tunnel segment or semimajor axis of prolate spheroid.

$V \quad$ Volume of the cavity.

$S \quad$ Surface area of the cavity.

$\theta_{0} \quad$ Half-angle of conical holes in opposite ends of a spherical-shell calorimeter.

\section{References}

1. "Radiation Units and Quantities," ICRU Report 33, International Commission on Radiation Units and Measurements (1980).

2. G. Joos, Theoretical Physics, 3rd. Ed., Hafner Pub. Co., New York, pp. 616-619 (1958).

3. T. A. Gabriel, F. S. Alsmiller, R. G. Alsmiller, Jr., B. L. Bishop, O. W. Hermann, and D. E. Groom, "Preliminary Simulations of the Neutron Flux Levels in the Fermilab Tunnel and Proposed SSC Tunnel," SSC Central Design Group Report SSC-110 (1987).

4. A. Cauchy, "Memoire sur la rectification des courbes et la quadrature des surfaces courbes" (1850); reprinted in Ouvres Completes, Vol. 2, Gauthier Villard, Paris (1908).

5. E. Czuber, "Zur Theorie der geometrischen Wahrscheinlichkeiten," Sitzungsber. Akad. Wiss. Wien Abt. 2, 90, 719-742 (1884). 
APPENDIX 10

\title{
MEASUREMENTS AND SIMULATIONS OF THE
} NEUTRON FLUX IN THE TEVATRON TUNNEL

\author{
D. E. Groom \\ SSC Central Design Group, LBL90-4040, Berkeley CA 94720
}

\section{Introduction}

In the fall of 1985, a FNAL/LBL group measured neutron spectra in the Tevatron tunnel[1]. These experiments were refined and extended [2] during the machine cycle which ended in the spring of 1987. Absolute magnitude and longitudinal distributions of the neutron flux were measured downstream (in the proton sense) from a warm section in the beam pipe. A controlled $\mathrm{N}_{2}$ gas leak was introduced near the center of the warm section, so that by measuring rates as a function of gas pressure, beam-gas rates could be separated from background rates. To help support this experimental effort, detailed simulations of particle cascades in the Fermilab tunnel initialed by hadron-nucleus collisions $\left(E_{p}=875 \mathrm{GeV}\right)$ in the center of the warm section were carried out at Oak Ridge National Laboratory (ORNL). A version of HETC [3] was used for high-energy particle transport, and the MORSE code [4] was used to transport the low-energy $(\leq 20 \mathrm{MeV})$ neutrons. The preliminary simulation results have been described in an SSC report [5], along with preliminary experimental results.

Many of the results are relevant to radiation in the collision halls and detectors. These include the neutron yield, scaling with energy, the neutron energy spectrum, and the role of neutron reflection from the tunnel walls. Accordingly, we present a short description of the work here, with emphasis upon these aspects.

\section{Motivation}

The study was motivated by concern about radiation damage to silicon semiconductors in the SSC tunnel, since about 400 racks of control circuitry are located at $200 \mathrm{~m}$ intervals around the ring. In addition, temperature sensors, beam pickups, and quench protection diodes are mounted in or on many of the 10,000 magnets. As can be seen from the first figure in Appendix 17, the effective threshold for silicon dislocation damage is about $160 \mathrm{keV}$. As will be seen, about half of the neutron flux in the tunnel is in a broad peak near $1 \mathrm{MeV}$, and most of the rest is thermal. Simulations of the hadron-induced flux in detector components yield similar spectra.

\section{Simulations}

A cross section of the "real" Tevatron tunnel[6] is shown in Fig. A10-1, and the cylindrical approximation used for the simulations is shown in Fig. A10-2. 


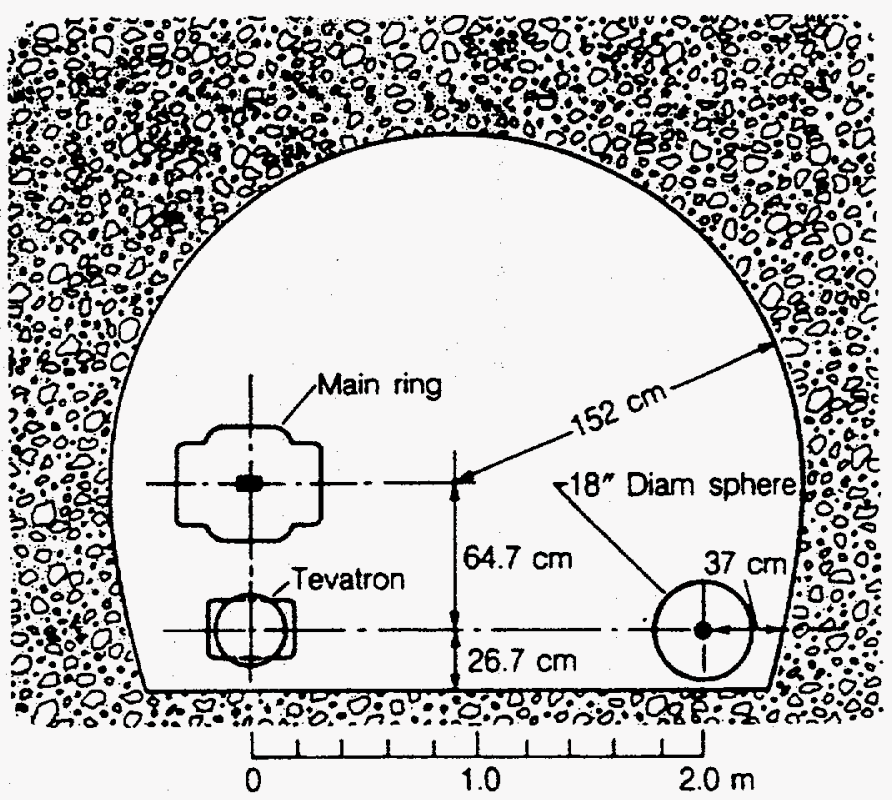

FIG. A10-1. Cross section of the Tevatron tunnel, showing relevant dimensions and the placement of the neutron flux measuring equipment.

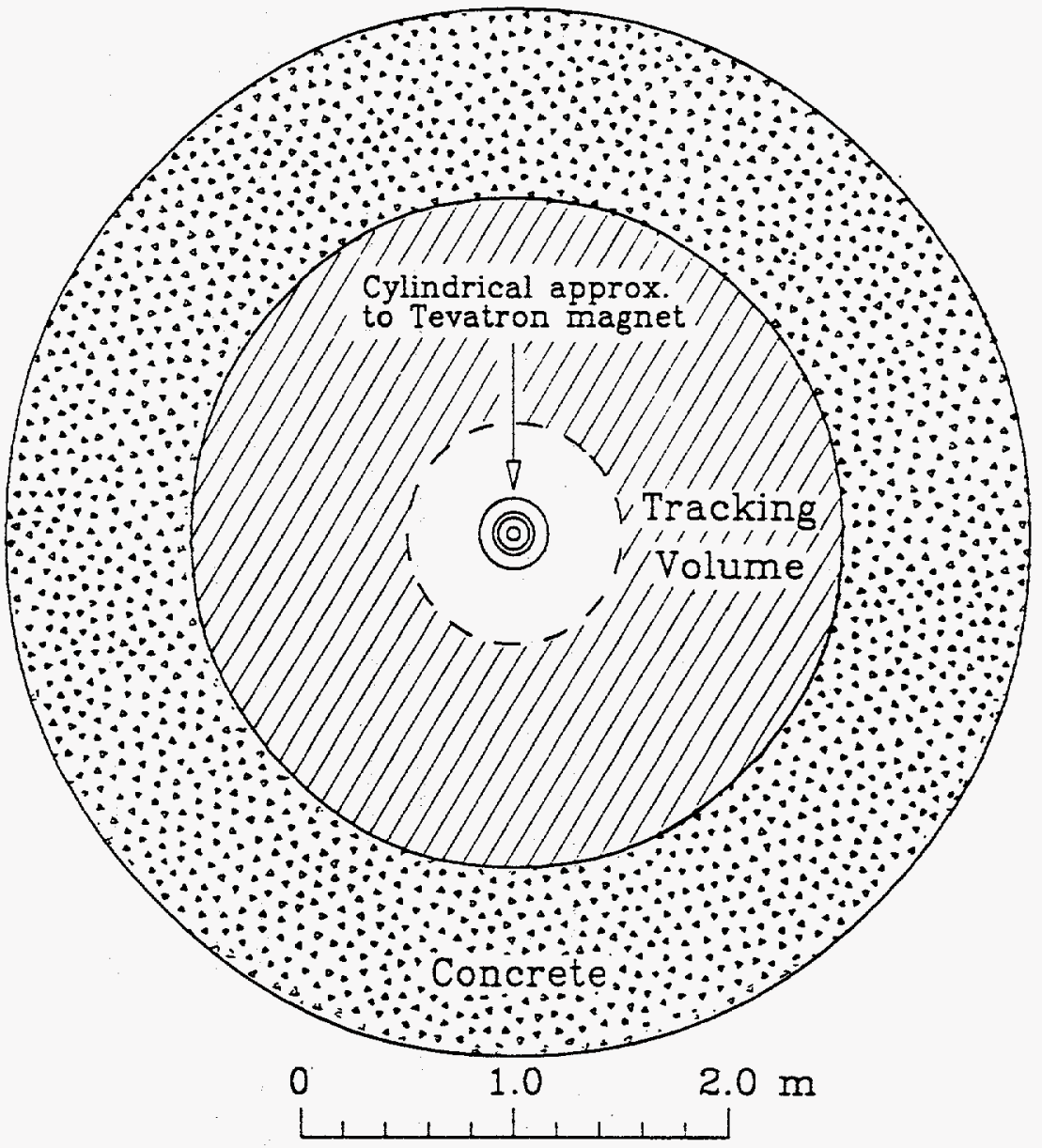

FIG. A10-2. Cross section of the ORNL model to the same scale as Fig. A10-1. Flux is obtained from neutron path lengths scored in the "tracking volume." 
The tunnel has the same radius, and the cylindrical approximation to the Tevatron magnet has the same bore area and yoke area as the real one. The correct Tevatron dipole field was used for the $875 \mathrm{GeV}$ simulations, and it was simply raised by $20 / 0.875$ for the $20 \mathrm{TeV}$ simulations. Sagitta was ignored. This approximation leads to problems with the longitudinal flux distribution, and it will be removed for the final version. Similarly, the large scoring volume precludes obtaining radial information about the flux, and finer radial segmentation is now being included.

(a)

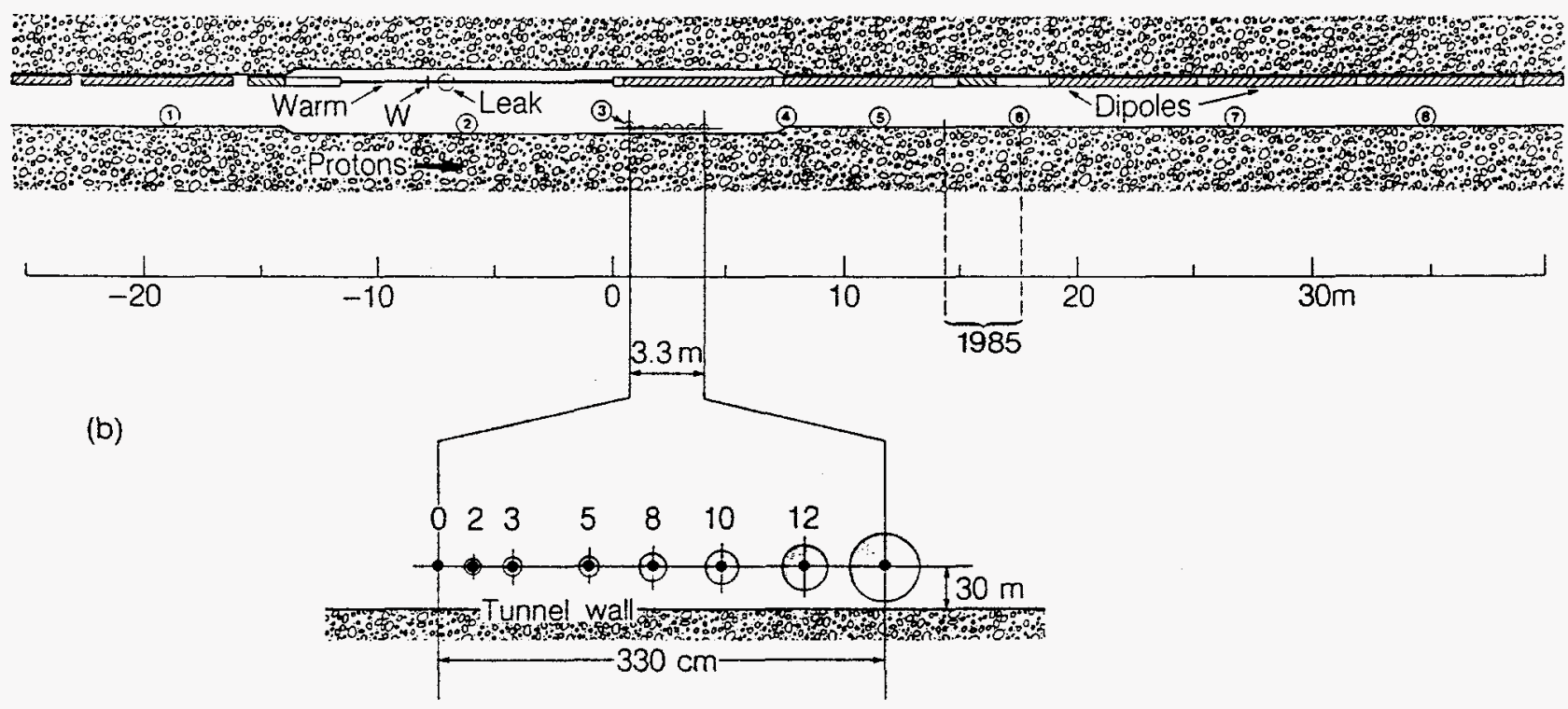

FIG. A10-3. Plan view of experimental setup in the Tevatron tunnel near the A17 straight section. Proton direction is left to right. Nitrogen was allowed to leak into the warm straight section at the point indicated, and the pressure was effectively zero at the cold dipole entrances on either end. Identical Bonner spheres at the numbered locations were used to measure the longitudinal distribution, and the full spectrometer (as shown in the enlargement) was used to obtain the spectrum shown in Fig. A10-4. The effective position of the spectrometer in the 1985 experiment (actually in A48) is marked by "1985."

A plan view of the Tevatron tunnel is shown in Fig. A10-3.

The actual density profile of the $\mathrm{N}_{2}$ target gas is triangular. Since experiments using a "flying wire" target were also planned, the target was modeled as a thin iron wire in the center of the warm section.

Approximately 3000 neutrons per $875 \mathrm{GeV}$ proton and 30,000 neutrons per $20 \mathrm{TeV}$ proton are produced in HETC for transport in the MORSE code. Fewer than $3 \%$ of these low-energy neutrons originate in collisions with hadrons of energy above $3 \mathrm{GeV}$. 


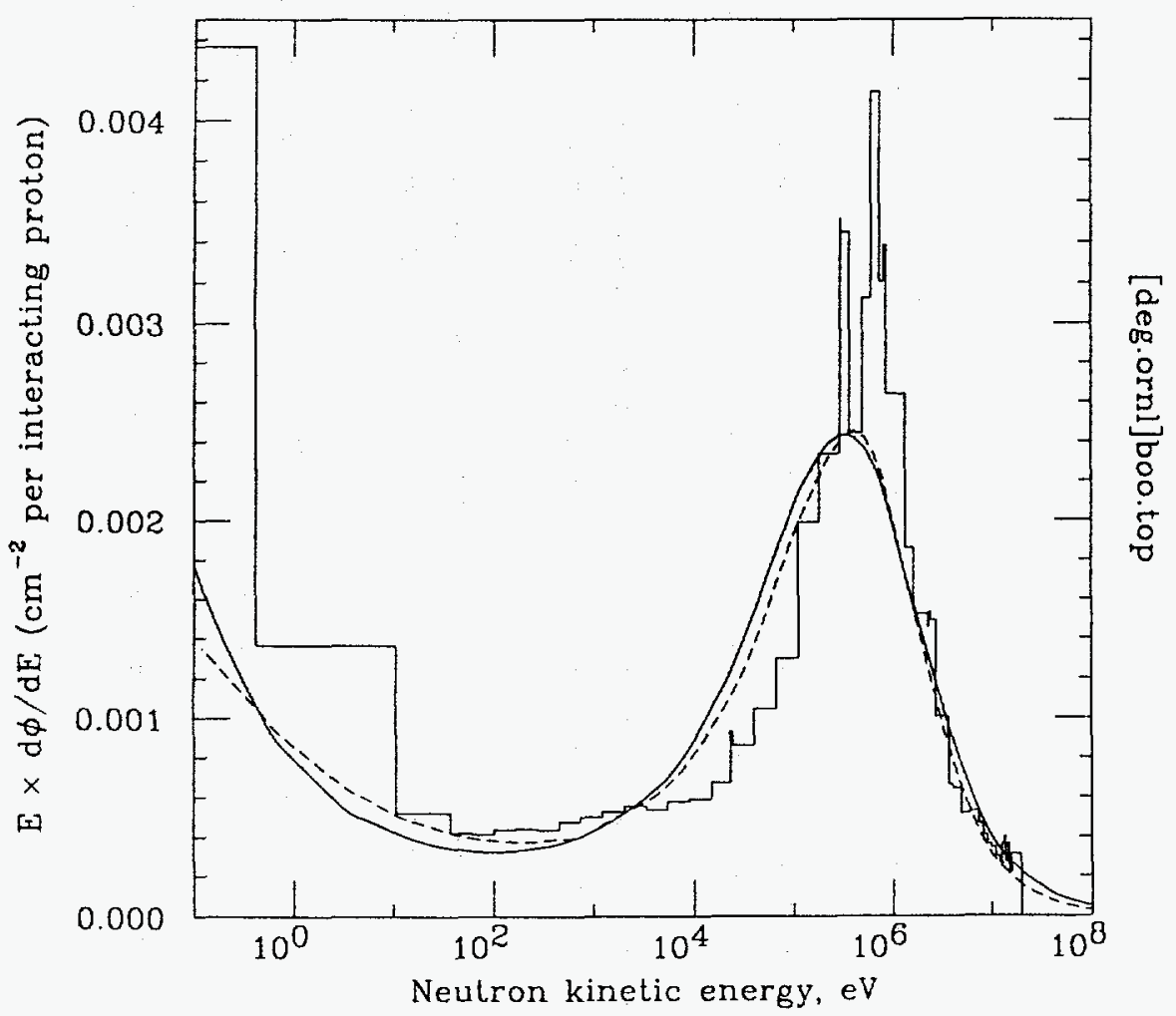

FIG. A10-4. The histogram shows the energy spectrum of the total neutron flux near the maximum of the longitudinal distribution in an $875 \mathrm{GeV}$ simulation. The dotted curve is the result of folding these data with the Bonner sphere responses and then unfolding using the program LOUHI[7]. The solid curve is obtained when the normalized rates from the $\mathrm{N}_{2}$ gas (slope data) are unfolded with the same program.

It was of interest to understand the role of neutron scattering from the tunnel walls. Accordingly, runs were made with the profile shown in Fig. A10-2 and with the same geometry with the concrete replaced by vacuum ("no tunnel walls"). The "no walls" case yielded the direct flux, and the difference of the two cases yielded the scattered or "albedo" flux. Runs for both cases were made at 875 $\mathrm{GeV}$ and $20 \mathrm{TeV}$.

A typical spectrum is shown by the histogram in Fig. A10-4. When plotted in this way $(d \varphi / d \ln E$ as a function of $\ln E$, where $E$ is the neutron's kinetic energy), about half the flux is in a broad gaussian peak centered at about 600 $\mathrm{keV}$. Since most of the computer time was speat transporting neutrons in the thermal region, most subsequent runs were made with the lower threshold in MORSE set at $40 \mathrm{keV}$. As can be seen from the figure, a cut at this energy yields about the same integral as does a cut at $160 \mathrm{keV}$ (our effective threshold for silicon damage).

Longitudinal distributions at $875 \mathrm{GeV}$ are shown by the histograms in Fig. A10-5. The total and direct fluxes are obtained directly from the simulations, while the albedo contribution is obtained by subtraction, as discussed above. 


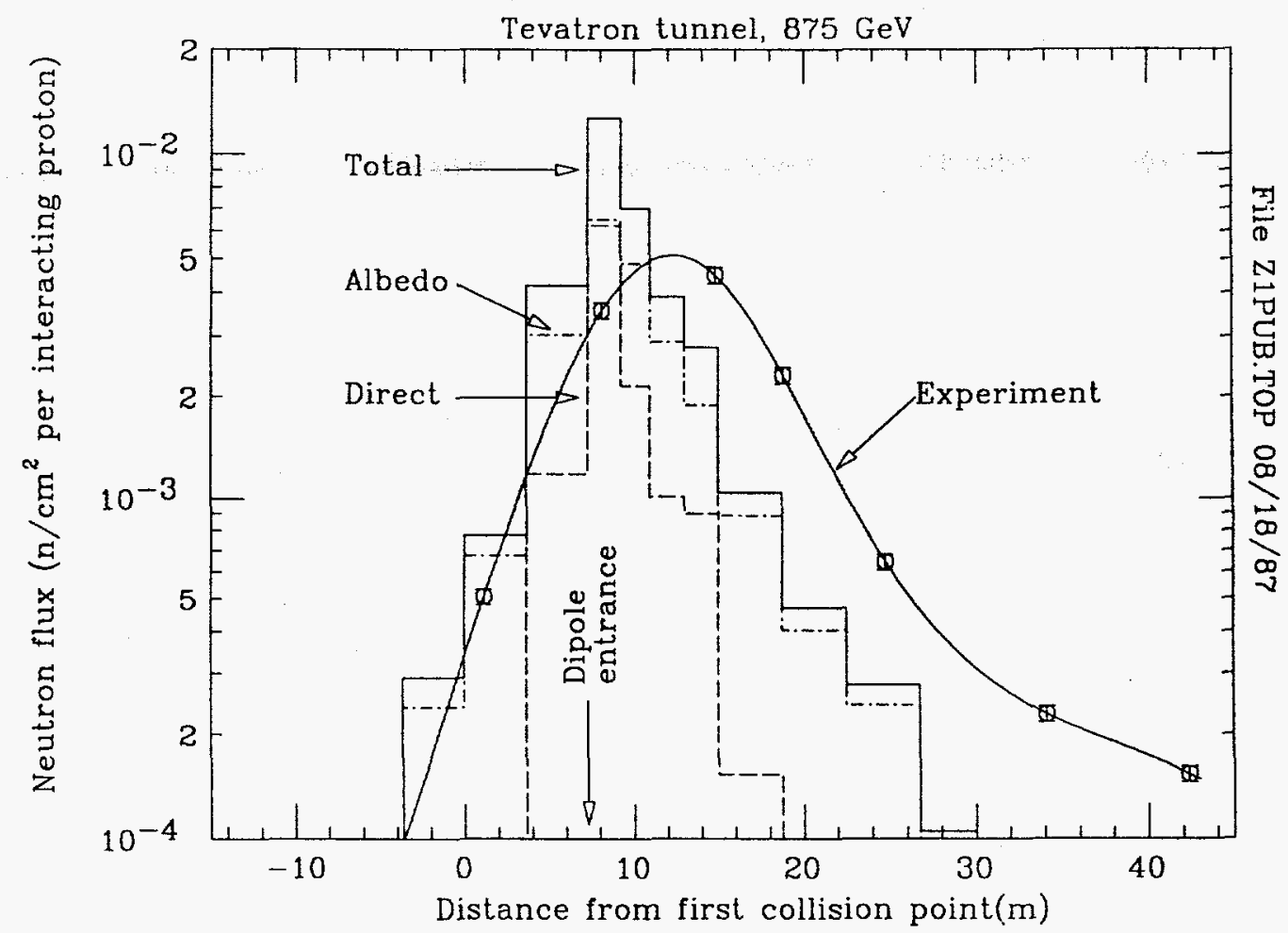

FIG. A10-5. Calculated longitudinal distributions of neutron flux $(E>40 \mathrm{keV})$ in the Tevatron tunnel for $875 \mathrm{GeV}$ incident proton energy (histograms), and measured distribution (symbols connected with spline).

The direct flux is more sharply peaked, since the albedo neutrons have had more opportunity to diffuse along the tunnel.

In addition to a more extended longitudinal distribution, the $20 \mathrm{TeV}$ results show a long, flat tail composed almost entirely of albedo particles.

Suppose that a proton interacts at a point $z^{\prime}$ with probability $d z^{\prime} / C$ in a continuous magnet string, as is the case for beam-gas collisions in the SSC. A detector at a point $z$, at a given distance from the magnet, measures a flux $f\left(z-z^{\prime}\right) d z^{\prime} / C$. The total flux in the detector from uniformly distributed sources of this kind will then be

$$
\begin{aligned}
\phi & =\frac{1}{C} \int f\left(z-z^{\prime}\right) d z^{\prime} \\
& =\frac{1}{C} \int f\left(z^{\prime}\right) d z^{\prime}
\end{aligned}
$$

when the integral is carried out over all $z^{\prime}$ for which $f$ is non-zero. In making the connection between the continuously distributed case and the localized distribution, it is thus the integral of the distribution which is relevant. The function $f(z)$ is somewhat different than the longitudinal distribution measured in the Tevatron experiment or calculated in the present simulation, where the source is 
Table A10-1

$z$-integrals of the total neutron flux above $40 \mathrm{keV}$

as obtained from the December 1986 simulations.

\begin{tabular}{|c|c|c|c|}
\hline $\begin{array}{r}8 \\
\text { ((neutrc } \\
\text { per inte }\end{array}$ & $\begin{array}{l}875 \mathrm{GeV} \\
\left.\text { ons } \mathrm{cm}^{-2}\right) \times \mathrm{cm} \\
\text { eracting proton) }\end{array}$ & $\begin{array}{c}20 \mathrm{TeV} \\
\left(\left(\text { neutrons } \mathrm{cm}^{-2}\right) \times \mathrm{cm}\right. \\
\text { per interacting proton) }\end{array}$ & Ratio \\
\hline Total & 7.70 & 105.6 & 13.7 \\
\hline Direct & 2.54 & 35.5 & 14.0 \\
\hline Albedo* & 5.15 & 70.1 & 13.6 \\
\hline Albedo + 0.66 Dir. & 6.83 & 93.5 & 13.7 \\
\hline
\end{tabular}

* Obtained by subtracting the direct flux from the total flux.

in a long field-free region. However, the integral is virtually the same, and it is the $z$-integrals of the functions shown in Fig. 10-4 which is needed.

The $z$-integrals of the flux above $40 \mathrm{keV}$ are given in Table A10-1. An upper limit to the ratio of the integrals (the scaling factor with energy) is given by the ratio $(20.0 \mathrm{TeV}) /(0.875 \mathrm{TeV})=22.9$. The expected value for the scale factor is somewhat lower than this limit; at the higher energies a larger fraction of the cascade is "bled off" into electromagnetic showers because there are more generations of $\pi^{0}$ production. Lindenbaum has suggested that the scaling with energy should be approximately a power law $E^{m}$, and the present best value for the exponent is $m=0.80 \pm 0.10$ [8]. This scaling would predict a ratio of $12.2_{-3.3}^{+4.5}$, in good agreement with the present result.

Let $a$ be the mean number of times a neutron is scattered from the tunnel walls before it is absorbed or loses too much energy to be counted. It is shown in Appendix 9 that the total flux is then enhanced by a factor $(1+8 a r / \pi R)$, where the point of observation is $r$ from the dipole string and the radius of the tunnel is $R$. The Tevatron tunnel has a radius of $152 \mathrm{~cm}$. The scoring volume in the simulation is such that $r$ should be replaced by a mean radius $\langle r\rangle=101 \mathrm{~cm}$, so that $(1+8 a r / \pi R)=(1+1.69 a)$. This quantity should be equal to the ratio of the total to direct flux, from which we obtain $a=1.18$ from the data in the table. At the tunnel wall, $(1+8 a r / \pi R)=(1+2.54 a)$. Comparing the two expressions, we see that only 0.66 of the direct flux scored in the simulation would be measured near the tunnel wall. The last row in the table thus contains the Monte Carlo prediction for the longitudinal flux integral.

Simulation spectra near the maximum of the longitudinal distribution are shown in Fig. A9-6. One might have expected the albedo contribution to be considerably softer than the direct part, because of moderation in the hydroge- 


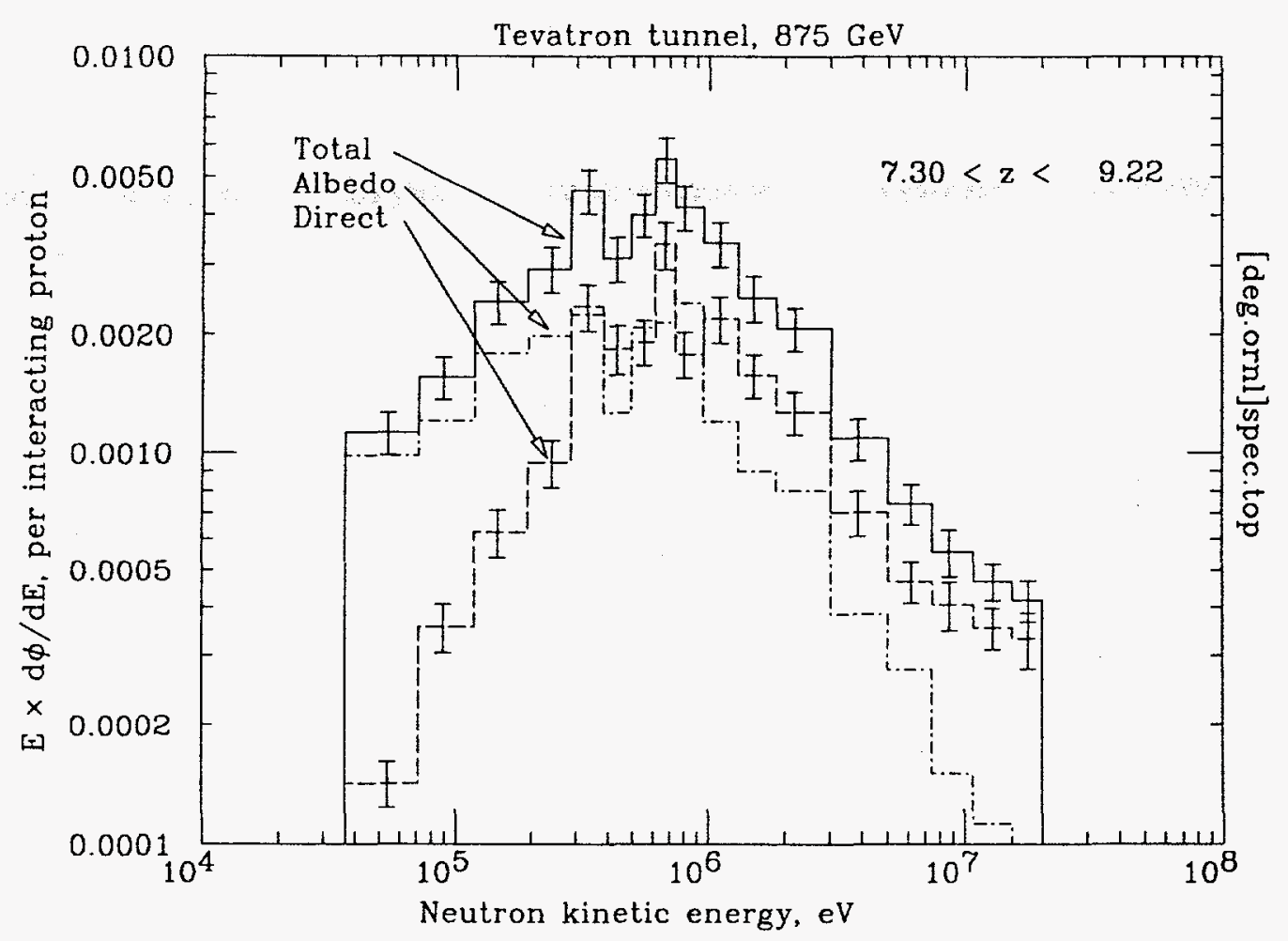

FIG. A10-6. Neutron spectra obtained in the $875 \mathrm{GeV}$ Tevatron tunnel simulations at a longitudinal position near the maximum.

nous walls. This is only slightly true. The main effect is a "fill-in" of the valley between the thermal and $1 \mathrm{MeV}$ peaks.

\section{Experiment}

Neutrons were counted with "Bonner spheres" $[9,10]$. Each consisted of a small ${ }^{6} \mathrm{LiI}$ crystal viewed by a photomultiplier, surrounded by a polyethylene sphere. LiI has about the same properties of $\mathrm{NaI}$, except that it is sensitive to thermal neutrons via the reaction ${ }^{6} \mathrm{Li}+\mathrm{n} \rightarrow{ }^{3} \mathrm{H}+\alpha$. The $4.8 \mathrm{MeV}$ recoil energy is deposited in the crystal, producing a sharp spectral peak whose area can be measured accurately even in the presence of a large background. A "naked" ${ }^{6} \mathrm{LiI}$ crystal is only sensitive to thermal neutrons. The surrounding polyethylene sphere moderates higher-energy neutrons, so the combination has an energy response dependent upon the size of the sphere. For example, in the Tevatron tunnel environment about $85 \%$ of the counts observed with 5 -inch diameter spheres were from neutrons with energies in excess of $100 \mathrm{keV}$-neutrons which could damage silicon. From the relative counting rates obtained using the full compliment of 8 detectors, the incident neutron spectrum could be unfolded in a relatively unambiguous fashion.

The setup is shown in Fig. A10-3. The pressure profile of the "target" gas was triangular, with its peak at the place marked "leak" and zeros at the entrances 


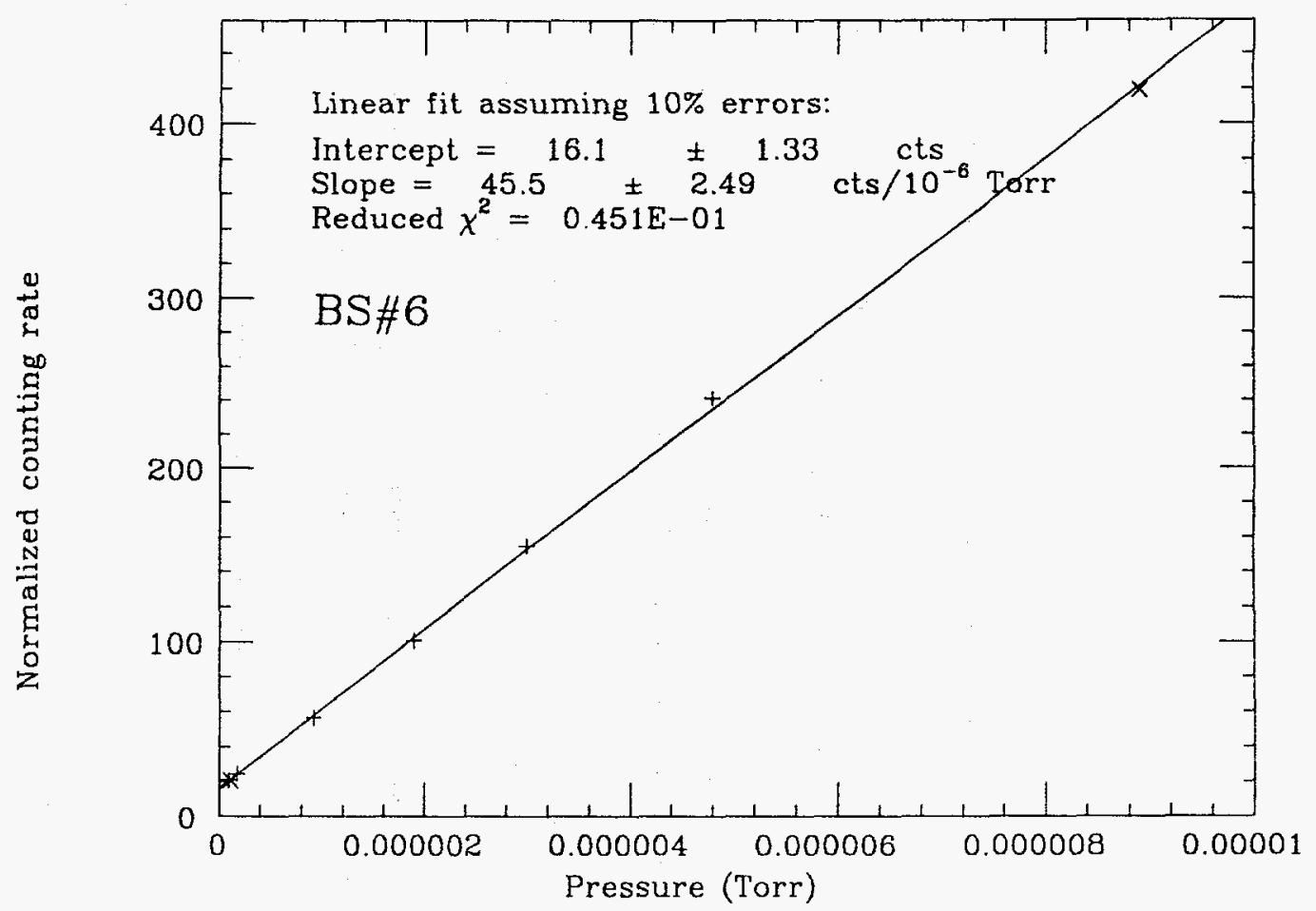

FIG. A10-7. Counting rate versus gauge pressure for a typical Bonner sphere. From the slope the rate from a background-free gas target may be extracted, and from the intercept the rate from beam loss and other sources not associated with beam-gas interactions in the straight section may be obtained.

to the cold magnets. The longitudinal distribution was measured using identical 5 -inch spheres for the reasons stated above. The spectrum was measured with the spheres close together near the flux maximum, as shown in the enlargement. The equivalent position of the spectrometer in 1985 is also shown.

Nitrogen gas leaked into the two-dipole long A17 straight section provided a target of known thickness. Since counting rates scaled linearly with the pressure in the middle of the section, the slope of the pressure-rate relationship yielded the rate change for a know pressure change, and from the intercept the beamwall and "other" contribution could be extracted. The pressure dependence on gauge pressure for a typical counter is shown in Fig. A10-7. The slopes times some reference pressure were then unfolded using a program such as LOUHI [7] to obtain experimental estimates of the spectra. Such a result is shown by the solid curve in Fig. A10-4. The Monte Carlo and experimental spectra are in rather fortuitous agreement.

The dominant experimental error arises because of the lack of an adequate pressure gauge calibration, but within this error the experimental and simulation rates agreed.

The measured longitudinal distribution of the flux is considerably broader 
than that obtained in the simulation. This might be expected for a longitudinally distributed source, but on the other hand most of the collision products should remain within the beam tube until the dipole field is encountered. Further simulations are being made with an extended source and a correctly curving beam tube in an attempt to resolve this question.

Analysis of the experiment is still not complete, but the tentative result is that we should expect an annual neutron fluence of $2 \times 10^{10}$ neutrons $\mathrm{cm}^{-2}$ at the $\mathrm{SSC}$, assuming a $300 \mathrm{hr}$ beam lifetime contribution for distributed losses around the ring [12] and $10^{14}$ protons in each ring for $10^{7}$ seconds per year. Neutron damage to semiconductors becomes a concern for fluences above $10^{12} \mathrm{~cm}^{-2}$, although carefully chosen components can survive another one or two orders of magnitude more exposure. Given uncertainties about beam loss during injection, the actual beam-gas lifetime, and possible future increases in the proton current, we conclude that the control electronics at alternate spool pieces (every $200 \mathrm{~m}$ ) should be shielded, either by using the ceiling recesses discussed in the Conceptual Design Report [11] or by placing the shielded electronics racks in niches the side of the main tunnel. Pending the results of further simulations now in progress, we also tentatively conclude that cold diodes inside the cryostats will survive for the life of the machine.

\section{References}

1. J. B. McCaslin, R-K. S. Sun, and W. P. Swanson; J. J. Elwyn, W. S. Freeman, and P. M. Yurista, SSC Central Design Group Report No. SSC-58 (1986).

2. J. D. Cossairt, A. J. Elwyn, W. S. Freeman, H. Jöstlein, C. D. Moore, and P. M. Yurista; J. B. McCaslin, R-K. S. Sun, and W. P. Swanson; and D. E. Groom, SSC Central Design Group Report, in preparation (1987).

3. R. G. Alsmiller, Jr. et al., "Modifications of the High Energy Transport Code (HETC) and Comparisons with Experimental Results," presented at the ANS Topical Conference on Theory and Practices in Radiation Protection and Shielding, 22-24 April 1987, Knoxville, Tennessee; K. C. Chandler and T. W. Armstrong, "Operating Instructions for the HighEnergy Nucleon-Meson Transport Code HETC," Oak Ridge National Laboratory Report ORNL-4744 (1972)

4. M. B. Emmett, "The MORSE Monte Carlo Radiation Transport Code System," Oak Ridge National Laboratory Report ORNL-4972 (February 1975).

5. T. A. Gabriel, F. S. Alsmiller, R. G. Alsmiller, Jr., B. L. Bishop, O. W. Hermann, and D. E. Groom, "Preliminary Simulations of the Neutron Flux Levels in the Fermilab Tunnel and Proposed SSC Tunnel," SSC Central Design Group Report SSC-110 (1987).

6. H. T. Edwards, Ann. Rev. Nucl. Part. Sci. 35, 605-660 (1985), and F. T. Cole, M. R. Donaldson, D. A. Edwards, H. T. Edwards, and P. F. M. Koehler, editors, "A Report on the Design of the Fermi National Accelerator Laboratory Superconducting Accelerator," Fermilab (1979).

7. J. T. Routti and J. V. Sandberg, "General purpose unfolding program LOUHI 78 with linear and nonlinear regularisations," Compt. Phys. Commun. 21, 119 (1980). 
8. R. H. Thomas and G. R. Stevenson, Radiological Safety Aspects of the Operation of Proton Accelerators, IAEA Technical Report Series (1987) (to be published).

9. R. L. Bramblett, R. L. Ewing and T. W. Bonner, Nucl. Instrum. Meth. 9, 1 (1960).

10. M. Awschalom and R. Sanna, "Applications of Bonner sphere detectors in neutron fields," Rad. Prot. and Dosimetry 10, 89 (1985).

11. "Superconducting Super Collider Conceptual Design," SSC Central Design Group Report SSC-SR-2020 (1986).

12. D. Bintinger, P. Limon, H. Jöstlein, and D. Trbojevic, "Status of the SSC Photodesorption Experiment," SSC Central Design Group Report SSC-102(1986). 


\section{APPENDIX 11 \\ NEUTRON REFLECTION IN SPHERICAL CALORIMETEPS \\ R. A. Lillie, R. G. Alsmiller, Jr., and T. A. Gabriel, Oak Ridge National Laboratory, Oak Ridge TN 37830}

\section{Introduction}

Substantial numbers of low-energy neutrons are backscattered from the surface of a calorimeter as the result of cascades generated by incident hadrons. In the case of an SSC detector, the calorimeter almost completely surrounds a central cavity filled with very low-density tracking devices. The neutrons pass through this volume, strike the calorimeter elsewhere, and may again backscatter. It is essential to obtain a reliable estimate of the the flux enhancement in the cavity due to secondary reflections. To this end we have modeled this problem for four simple calorimeters, and simulated the fluxes inside with the aid of the discrete ordinates code ANISN [1].

\section{Model}

All of the calorimeters consisted of thick spherical shells with inside radii of $2 \mathrm{~m}$. The neutron source was uniformly distributed over the inside surface, and emitted uniformly into all solid angles. A $\delta$-function energy distribution at $1 \mathrm{MeV}$ was desired; because of the structure of the code it was convenient to approximate this distribution by equal numbers of neutrons from the group with energies between $0.55 \mathrm{MeV}$ and $1.10 \mathrm{MeV}$ and from the group between $1.10 \mathrm{MeV}$ to $1.80 \mathrm{MeV}$.

Closed spheres of vacuum, concrete, uranium/scintillator (equal parts by volume), and uranium were chosen. The first of these was for normalization and checking purposes, since the expected flux $\left(0.796 \times 10^{-5} \mathrm{~cm}^{-2}\right.$ per source neutron) can easily be calculated analytically (see Appendix 9). The concrete calorimeter provided results which could be compared with earlier simulations for the SSC arcs (see Ref. 2 and Appendix 10). The uranium/scintillator calorimeter is taken as a standard reference case throughout this report, and the solid uranium reflector is relevant for any device without a hydrogenous moderator (e.g. a calorimeter with silicon or liquid argon readout.)

\section{Results}

Neutron and photon spectra were calculated for the four cases. The total fluxes are given in Table A11-1. The column labeled " $(1+a)$ " is the reflection enhancement factor, which is unity in the case of no reflection. The small deviation from unity in the vacuum case is the result of statistical fluctuations in the simulation. The input neutron spectrum is shown in Fig. A11-1; note that in the 
Table A11-4

Total neutron and photon flux inside a closed spherical shell calorimeter with a $200 \mathrm{~cm}$ inside radius. Results are for an isotropic source from two energy groups bracketing $1 \mathrm{MeV}$ (normalized to one source neutron) on the inside of the shell. $f_{>}$ is the fraction of the flux with energy in excess of $0.1 \mathrm{MeV} .(1+a)$ is the enhancement factor due to neutron reflection, and is equal to the flux above $0.1 \mathrm{MeV}$ divided by $\pi \times(200 \mathrm{~cm})^{2}$, the expected flux in the absence of reflection.

\begin{tabular}{lcccc}
\hline Material & $\begin{array}{c}\text { Neutron } \\
\text { flux }\left(\mathrm{cm}^{-2}\right)\end{array}$ & $f_{>}$ & $(1+a)$ & $\begin{array}{c}\text { Photon } \\
\text { flux }\left(\mathrm{cm}^{-2}\right)\end{array}$ \\
\hline Void & $0.786 \times 10^{-5}$ & 1.00 & 0.99 & 0.00 \\
Concrete & $2.83 \times 10^{-5}$ & 0.49 & 1.77 & $5.04 \times 10^{-6}$ \\
U/scint. & $1.32 \times 10^{-5}$ & 0.89 & 2.47 & $1.76 \times 10^{-6}$ \\
Uranium & $2.23 \times 10^{-5}$ & 0.81 & 1.35 & $0.87 \times 10^{-6}$ \\
\hline \hline
\end{tabular}

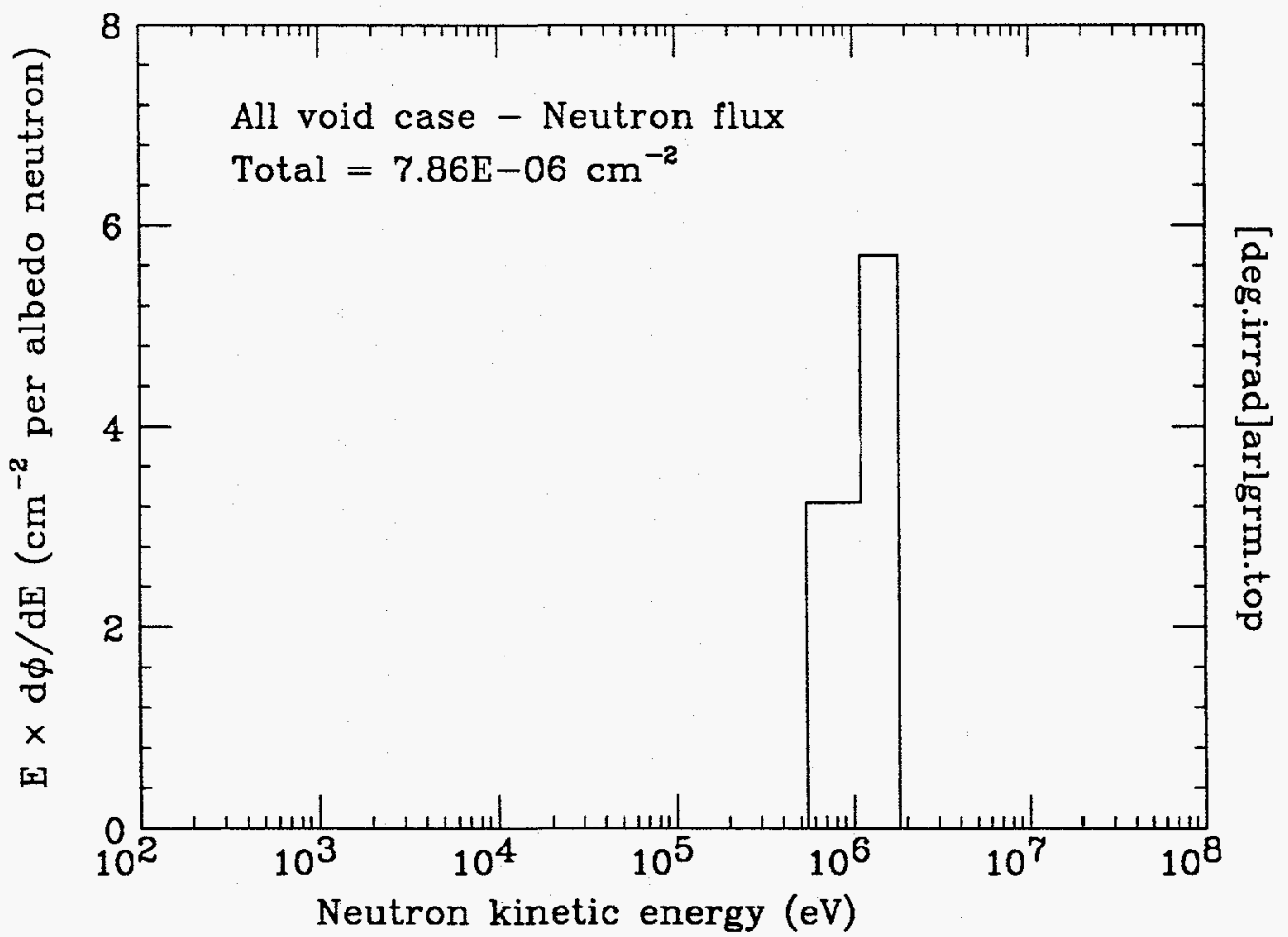

FIG. A11-1. Neutron spectrum at any point inside $2 \mathrm{~m}$ sphere in the absence of reflection. The area in each of the two source groups is the same.

plot of $d \varphi / d(\log E)$ the equal number of neutrons in the two groups is skewed by the $E$ factor. Differential and integral spectra for the three non-trivial cases are shown in Figs. A11-2 through A11-7. 


\section{Discussion}

After the above results were obtained, comparisions were made with those reported by a Lawrence Livermore Laboratory group (Appendix 12). Further calculations were then made in an attempt to understand some of the differences. One difference in the input was the assumed concrete composition. Another had to with source energy spectra, which for Livermore was a $\delta$-function at $1 \mathrm{MeV}$ and for Oak Ridge was two energy groups spanning the interval $0.55 \mathrm{MeV}<E<$ $1.80 \mathrm{MeV}$. Accordingly, we (a) adopted the Livermore concrete composition and (b) made runs with separate energy groups $(0.55 \mathrm{MeV}$ to $1.10 \mathrm{MeV}$ and $1.10 \mathrm{MeV}$ to $1.80 \mathrm{MeV}$ ) as well as with the combined groups. These results are summarized in Table A11-2.

The variation with energy is consistent with that reported in Table A124 (Appendix 12). The concrete composition change results in some neutron flux increase; that it is still $6 \%$ below the Livermore result is not regarded as significant. A problem remains with the uranium/scintillator case, where we find an enhancement factor of 1.35 using a $0.1 \mathrm{MeV}$ threshold, while the Livermore result with the same threshold is 1.87 . This means that the mean number of reflections is 0.35 in the Oak Ridge case and 0.87 in the Livermore case. The discrepancy is viewed as serious, and it has not yet been resolved. One might have expected a result between those for the concrete and solid uranium cases.

\section{Table A11-2}

Neutron flux $(\varphi)$ inside a closed spherical shell calorimeter with a $200 \mathrm{~cm}$ inside radius. The Livermore concrete composition was used, which differs slightly from that used for the results reported in Table A11-1. The first three columns were obtained using source energies from the two groups used above, while for the second and third sets of columns the lower and upper groups were used. $f_{>}$means the fraction of the neutrons in the cavity with energies in excess of $0.1 \mathrm{MeV}$, and $(1+a)=10^{5} \varphi / \varphi_{0}$ is the factor by which the flux is enhanced when reflection is included.

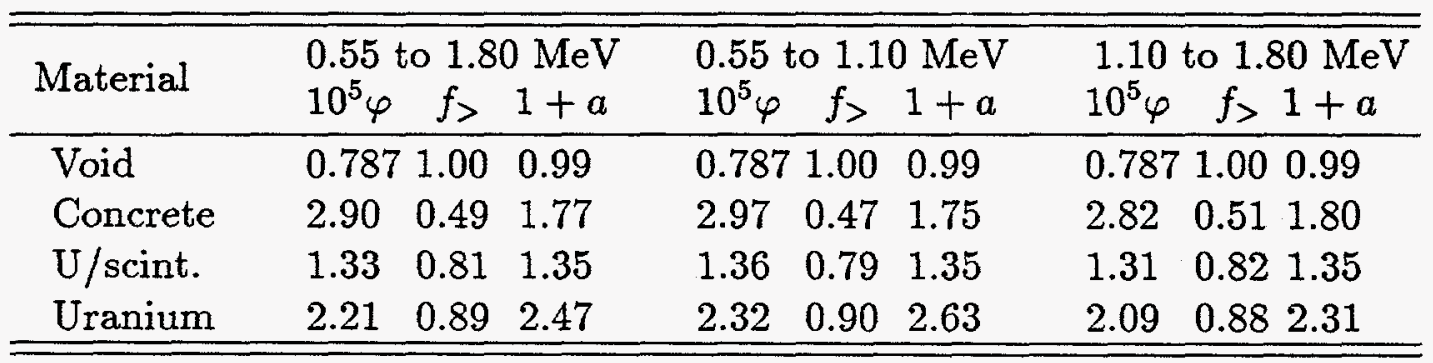




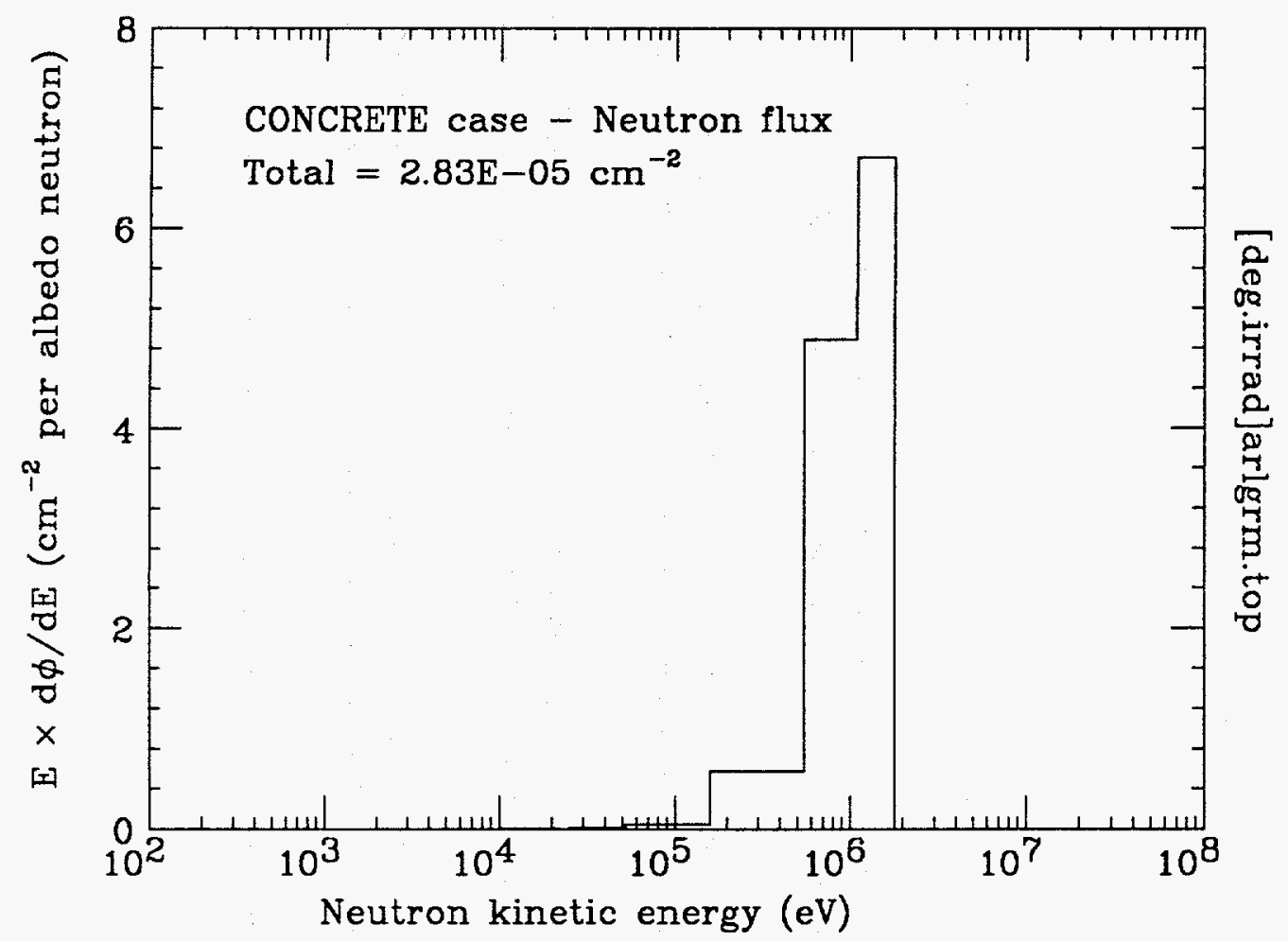

FIG. A11-2. Neutron spectrum at any point inside a $2 \mathrm{~m}$ concrete sphere.

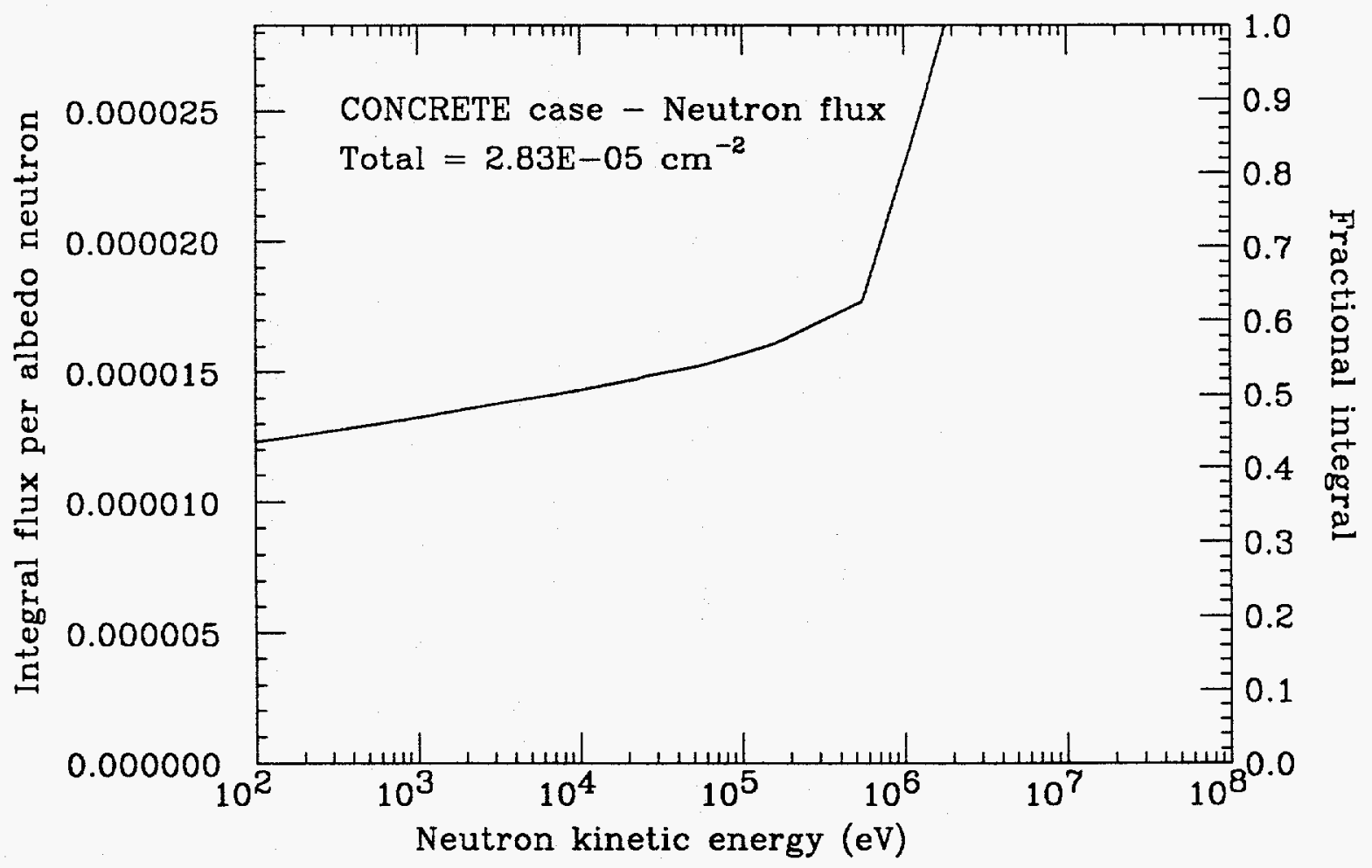

FIG. A11-3. Integral neutron spectrum at any point inside a $2 \mathrm{~m}$ concrete sphere. 


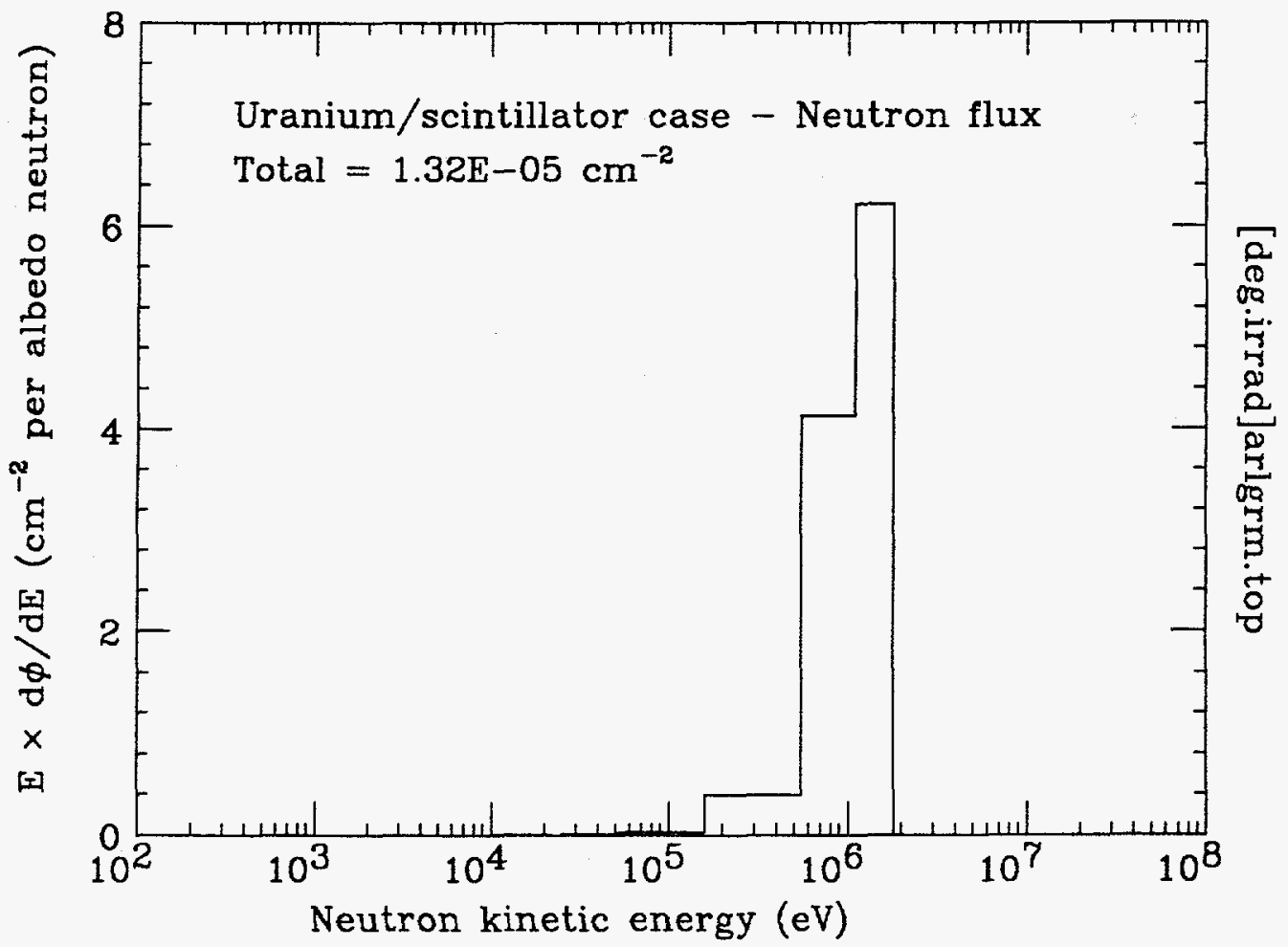

FIG. A11-4. Neutron spectrum at any point inside a $2 \mathrm{~m}$ uranium/scintillator sphere.

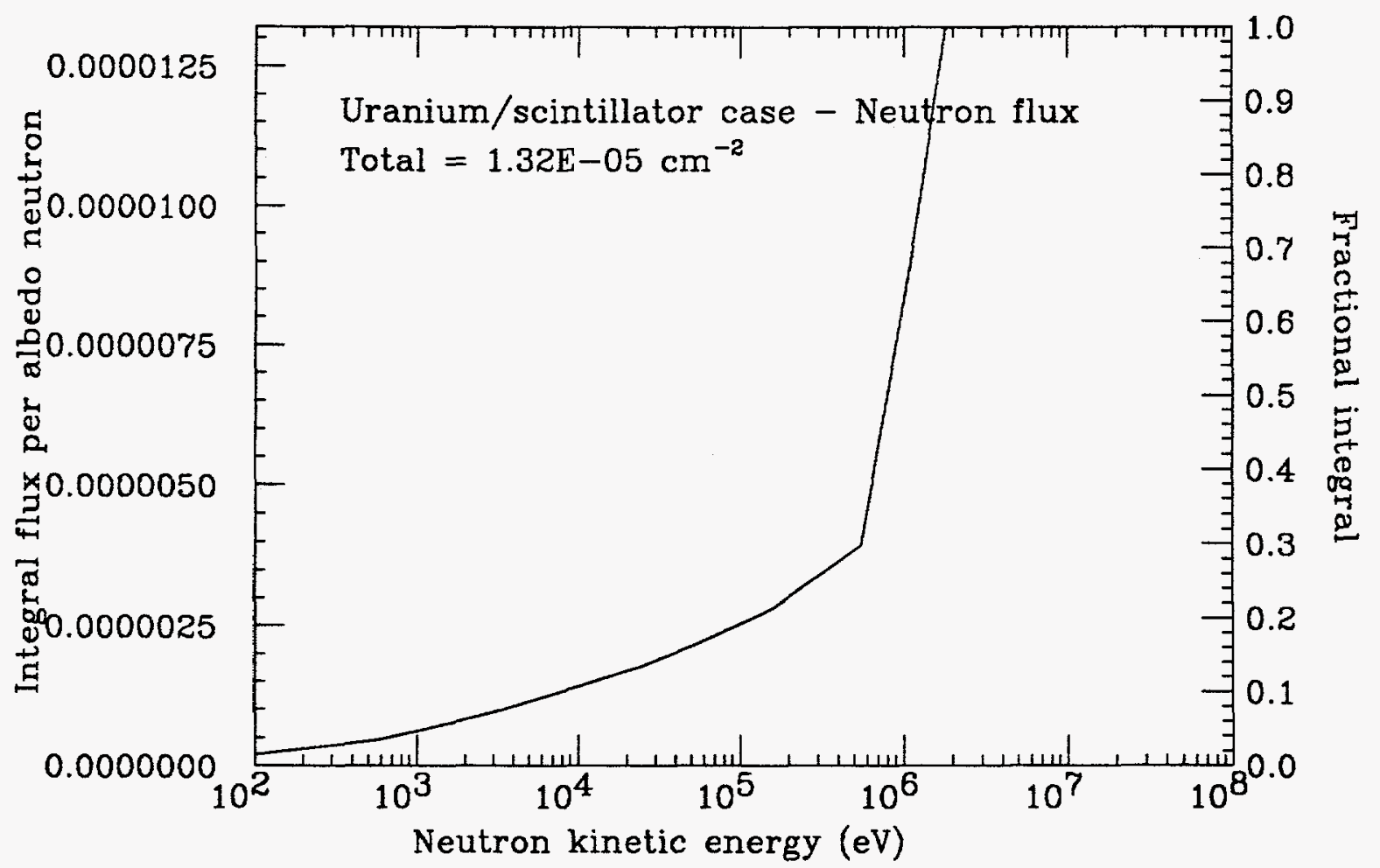

FIG. A11-5. Integral neutron spectrum at any point inside a $2 \mathrm{~m}$ uranium/scintillator sphere. 


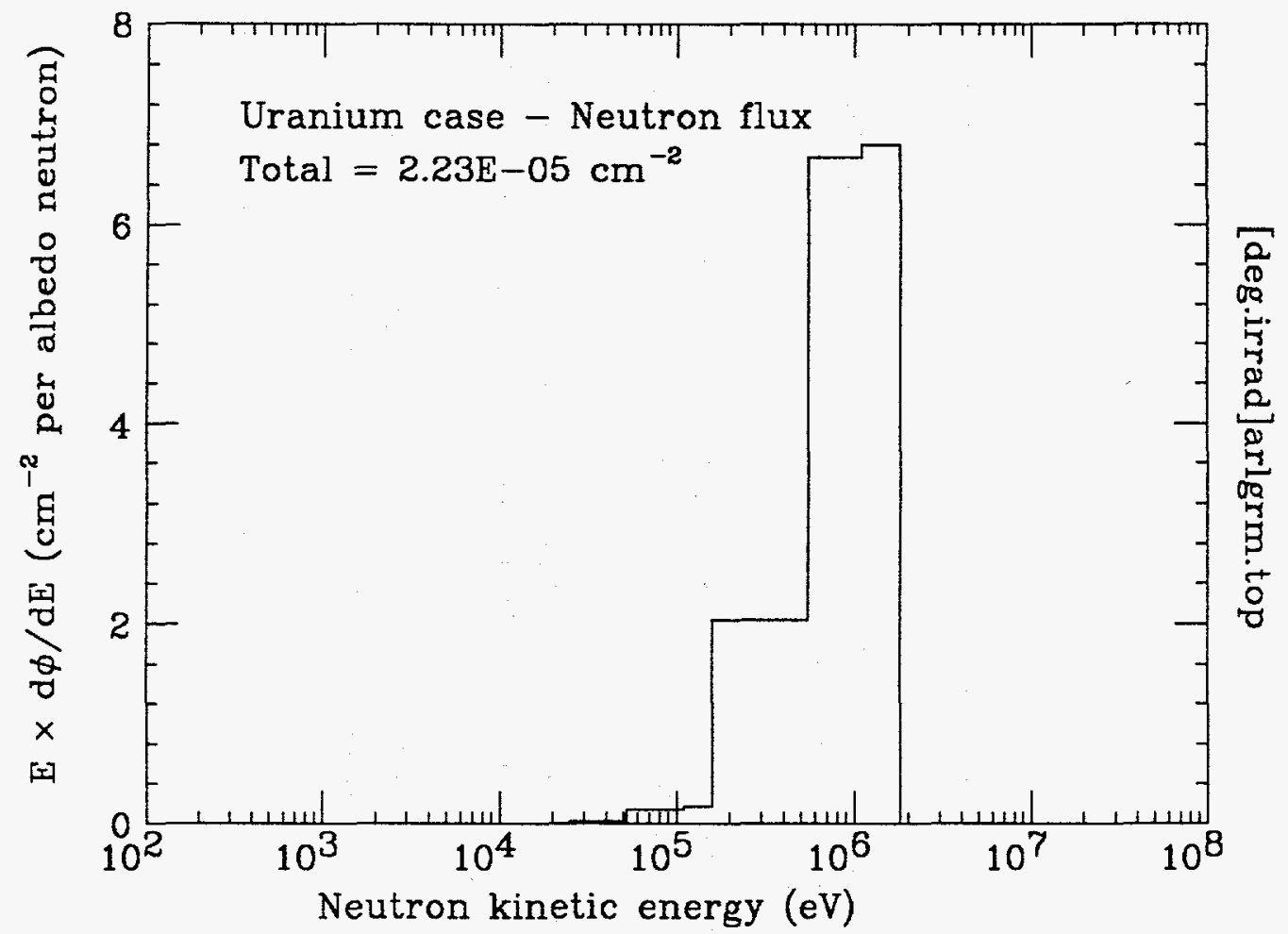

FIG. A11-6. Neutron spectrum at any point inside a $2 \mathrm{~m}$ uranium sphere.

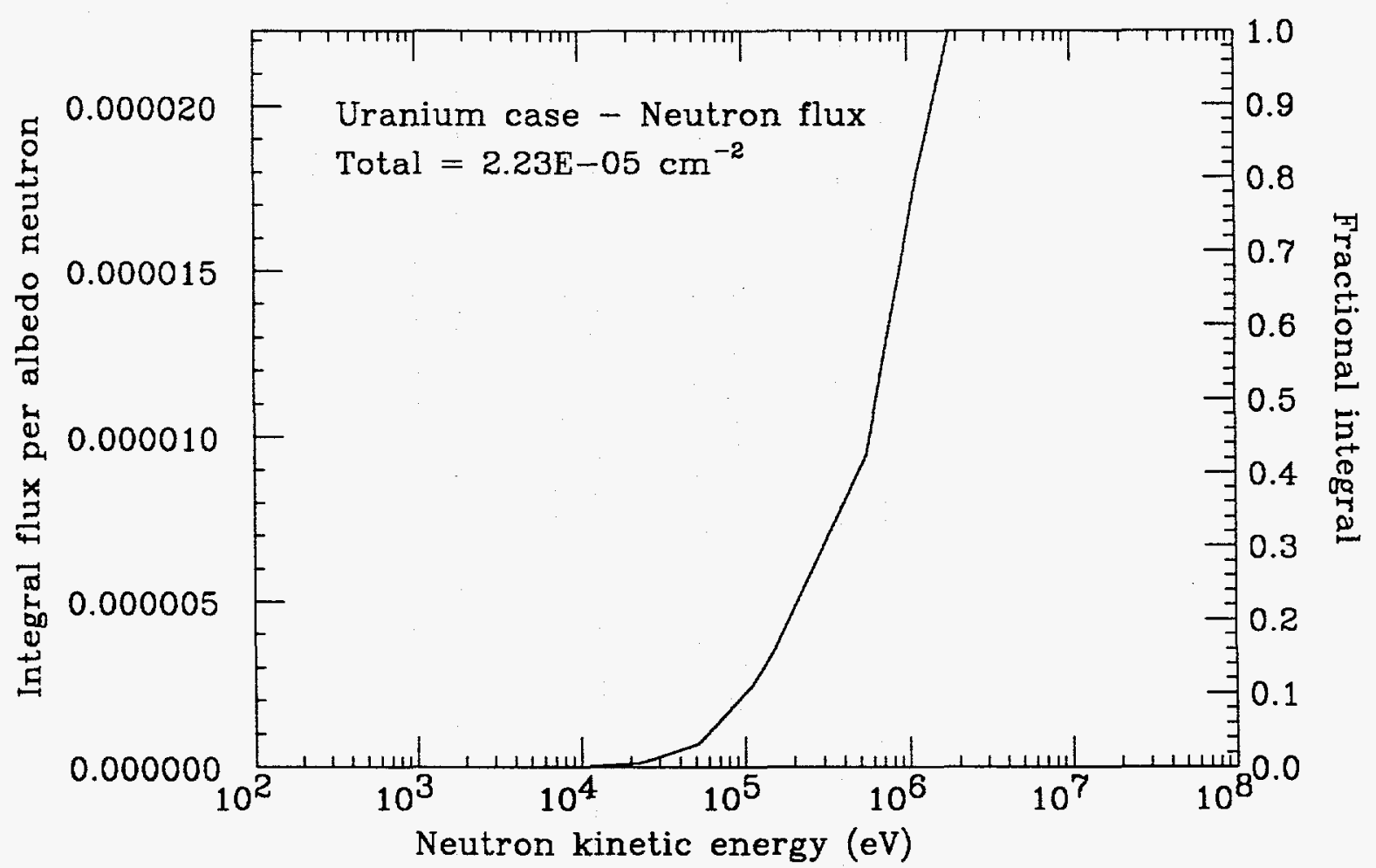

FIG. A11-7. Integral neutron spectrum at any point inside a $2 \mathrm{~m}$ uranium sphere. 


\section{References}

1. W. W. Engle, Jr., “A USER'S MANUAL FOR ANISN, A One-Dimensional Discrete Ordinates Transport Code with Anisotropic Scattering," K-1693, Union Carbide Corporation, Oak Ridge, TN (1967).

2. T. A. Gabriel, F. S. Alsmiller, R. G. Alsmiller, Jr., B. L Bishop, O. W. Hermann, and D. E. Groom, "Preliminary Simulation of the Neutron Flux Levels in the Fermilab Tunnel and Proposed SSC Tunnel," SSC Central Design Group Report SSC-110 (1987). 



\section{APPENDIX 12 \\ NEUTRON REFLECTION IN SPHERICAL CALORIMETERS \\ Thomas P. Wilcox, Jr. \\ Lawrence Livermore Laboratory, Livermore CA 94550}

\section{Introduction}

The following report summarizes the calculations performed to assist the members of the Superconducting Super Collider Central Design Group, LBL, in understanding the effects of "low" energy neutrons. These results are for a selection of very simple cases and are meant to assist in the development of ballpark numbers. Future work will undoubtedly require more concentrated efforts.

\section{Physical geometry}

It was assumed that the geometry of the system could be represented by a hollow spherical ball. The inside radius of this ball was $200 \mathrm{~cm}$ while the outside radius was $300 \mathrm{~cm}$. The innermost region was filled with air at $1.0 \times 10^{-10}$ of its normal density. The ball itself, which represents a calorimeter, was, in various problems, made of one of the following materials:

1) Vacuum

2) Concrete -

$\begin{array}{ll}\mathrm{H} & 0.013 \mathrm{gm} / \mathrm{cc} \\ \mathrm{O} & 1.091 \\ \mathrm{Na} & 0.046 \\ \mathrm{Mg} & 0.034 \\ \mathrm{Al} & 0.016 \\ \mathrm{Si} & 0.756 \\ \mathrm{Ca} & 0.477 \\ \mathrm{Fe} & 0.077 \\ \text { Total } & 2.510 \mathrm{gm} / \mathrm{cc}\end{array}$

3) U238 with a density of $18.9 \mathrm{gm} / \mathrm{cc}$

4) Depleated uranium - U235 $\begin{aligned} & \text { U.132 } \mathrm{gm} / \mathrm{cc} \\ & \text { U238 } 18.768\end{aligned}$

Total $\quad 18.900 \mathrm{gm} / \mathrm{cc}$

5) U/scintillator - $\quad \mathrm{H} \quad 0.044 \mathrm{gm} / \mathrm{cc}$

C $\quad 0.528$

$\mathrm{U} 235 \quad 0.066$

$\mathrm{U} 238 \quad 9.384$

Total $\quad 9.962 \mathrm{gm} / \mathrm{cc}$ 
Any particle exiting from this sphere enters into an infinite void and does not return to the defined geometry.

Some problems were run with a conical hole cut through the solid sphere. The center of the cone was at the sphere's center and both pieces of the spherical shell were removed. The measure of the cone's size is its half angle; i.e., the angle from the cone's axis to the side of the cone.

\section{Fixed Source}

The fixed source of neutrons in the calculation had the following characteristics:

\section{Position:}

Most cases were run with a uniformly distributed source strength per unit area located on the inside of the spherical shell. For cases with holes in the shell, that part of the source occurring on the missing part of the shell was not used but the normalization was kept at a level as if a full shell source had been employed.

Two cases were run where the source strength varied as $1.0 \sin ^{2.5} \theta$ where the angle $\theta$ is measured from the center of the sphere and starting from some reference direction. Theta values from $5.7^{\circ}$ to $90^{\circ}$ degrees were used.

\section{Initial Direction:}

The probability of a particle being initially directed at an angle $\phi$, measured from the inward directed normal to the spherical shell, was assumed to be proportional to $\cos \phi$. No outward directed source particles were generated.

\section{Initial Energy:}

Most of the problems were run with the initial energy set to one specific value. The generally used value was $1 \mathrm{MeV}$ but a series of problems were run with values running from $20 \mathrm{MeV}$ to $100 \mathrm{keV}$. One problem was run using the albedo neutron spectrum as described by:

$$
\frac{d \Phi}{d E}=\frac{1}{E \sigma \sqrt{2 \pi}} \exp \left\{-\frac{\left(\ln \left(E / E_{o}\right)\right)^{2}}{2 \sigma^{2}}\right\}
$$

where $E_{o}$ is $1.3 \mathrm{MeV}$ and $\sigma$ is 1.4 . This was simulated by a tabulated point-wise input.

\section{Problem Results - Overview}

With the exception of the cases with a conical hole, all problems were normalized to one source neutron. Since no time dependence was considered, this may also be taken to be one source neutron per unit time if the stated results are altered to have the specified units per unit time. 
COG [1] problems were run so as to determine the average neutron and photon fluxes in the center "void" region. These values are integrated over all energies down to zero. An energy dependent response function was also added to this calculation so as to determine the energy absorption in $\mathrm{SiO}_{2}$. This would again be the average value in the "void" region assuming that so little of this material would be added so as to not perturb the flux values. The code calculated results in $\mathrm{MeV} / \mathrm{cc}$ for $2.64 \mathrm{gm} / \mathrm{cc}$ dense $\mathrm{SiO}_{2}$ have been multiplied by $6.07 \times 10^{-11}$ to obtain results in Grays. This energy deposition includes not only the neutron and photon interactions but also all energy carried by produced charged particles not followed by COG.

One problem was calculated with point detectors in order to determine the energy deposition and fluxes at various points within the central region.

Partial results are presented here. More complete results may be obtained from the full computer output.

\section{Problem Set "A" - Material Variations}

This set of five problems investigates the effect of changing the calorimeter materials. Each has a closed geometry and a $1 \mathrm{MeV}$ source energy uniformly distributed on the inner surface of the calorimeter. The results are summarized in Table A12-1.

Table A12-1

\begin{tabular}{lclcc}
\hline \hline Material & Prob. & Neutrons $/ \mathrm{cm}^{2}$ & Photons $/ \mathrm{cm}^{2}$ & Grays \\
\hline Void & 1A & $7.95 \times 10^{-6}[.002]^{*}$ & 0 & $2.64 \times 10^{-17}[.002]$ \\
Concrete & 1B & $3.08 \times 10^{-5}[.01]$ & $4.39 \times 10^{-6}[.02]$ & $5.58 \times 10^{-17}[.01]$ \\
D38 & 1C & $2.36 \times 10^{-5}[.02]$ & $9.47 \times 10^{-7}[.07]$ & $4.37 \times 10^{-17}[.01]$ \\
D38/scint. & 1D & $2.38 \times 10^{-5}[.07]$ & $4.39 \times 10^{-6}[.16]$ & $5.59 \times 10^{-17}[.04]$ \\
U238 & 1E & $2.23 \times 10^{-5}[.01]$ & $8.49 \times 10^{-7}[.06]$ & $4.21 \times 10^{-17}[.01]$ \\
\hline \hline
\end{tabular}

* Numbers in brackets are the fractional standard deviation-one standard deviation divided by the calculated quantity.

For the uranium calorimeters, approximately $10 \%$ of the deposited energy comes from the secondary gammas. For the calorimeters which have hydrogen in them, this rises to approximately $40 \%$. For the latter case, this implies that the use of a shielding material, like lead, could drop the energy deposition in materials placed in the "void" region by as much as a factor of two.

Figs. A12-1 through A12-6 show details of spectra and energy deposition for problems 13 (concrete). Figs. A12-7 through A12-10 show differential and integral neutron spectra for problems $1 \mathrm{C}$ and $1 \mathrm{D}$. 


\section{Problem Set "B" - Variations in Spatial and Energy Distributions}

Two problems were run in order to see the variations when going to the $1 / \sin ^{2.5} \theta$ spatial distribution and, then, to a full source energy distribution. Each of the problems had the D38/scintillator material. These results are shown in Table A12-2, along with those for a reference problem (a $1 \mathrm{MeV}$ source with a uniform distribution on the inside of the sphere).

Table A12-2

\begin{tabular}{lclll}
\hline \hline & Prob. & Neutrons $/ \mathrm{cm}^{2}$ & Photons $/ \mathrm{cm}^{2}$ & Grays \\
\hline Unif. $1 \mathrm{MeV}$ & $1 \mathrm{D}$ & $2.38 \times 10^{-5}[.07]$ & $4.39 \times 10^{-6}[.16]$ & $5.59 \times 10^{-17}[.04]$ \\
sin, dist. & 2 & $2.48 \times 10^{-5}[.03]$ & $4.60 \times 10^{-6}[.06]$ & $5.26 \times 10^{-17}[.03]$ \\
sin, $1 \mathrm{MeV}$ & 3 & $2.14 \times 10^{-5}[.02]$ & $3.68 \times 10^{-6}[.06]$ & $5.41 \times 10^{-17}[.03]$ \\
\hline \hline
\end{tabular}

Point calculations were conducted in a re-run of problem number 2. The results are shown in Fig. A12-11 for the two cases that can be formulated from this run-the first with the spatial distribution from 5.7 to 90 degrees and the second from 5.7 to 174.3 degrees. For both cases the numbers indicate the energy deposition divided by the average value given in Table A12-2. above.

\section{Problem Set " $C$ " - Variations in Conical Holes}

A series of problems were run with conical holes cut through the solid calorimeter ball. All problems had a uniform source spatial distribution, $1 \mathrm{MeV}$ initial energy, and the D38/scintillator material. Results are given in Table A12-3.

Table A12-3

\begin{tabular}{lclll}
\hline \hline & Prob. & Neutrons $/ \mathrm{cm}^{2}$ & Photons $/ \mathrm{cm}^{2}$ & Grays \\
\hline Closed & $1 \mathrm{D}$ & $2.38 \times 10^{-5}[.07]$ & $4.39 \times 10^{-6}[.16]$ & $5.59 \times 10^{-17}[.04]$ \\
$5.7 \mathrm{deg}$ & $4 \mathrm{~A}$ & $2.12 \times 10^{-5}[.02]$ & $3.46 \times 10^{-6}[.07]$ & $5.26 \times 10^{-17}[.03]$ \\
$10 \mathrm{deg}$ & $4 \mathrm{~B}$ & $2.20 \times 10^{-5}[.05]$ & $5.74 \times 10^{-6}[.42]$ & $6.49 \times 10^{-17}[.22]$ \\
$20 \mathrm{deg}$ & $4 \mathrm{C}$ & $1.86 \times 10^{-5}[.01]$ & $3.00 \times 10^{-6}[.08]$ & $4.64 \times 10^{-17}[.02]$ \\
$30 \mathrm{deg}$ & $4 \mathrm{D}$ & $1.57 \times 10^{-5}[.02]$ & $2.10 \times 10^{-6}[.07]$ & $3.93 \times 10^{-17}[.02]$ \\
$40 \mathrm{deg}$ & $4 \mathrm{E}$ & $1.17 \times 10^{-5}[.01]$ & $1.62 \times 10^{-6}[.23]$ & $3.54 \times 10^{-17}[.13]$ \\
\hline \hline
\end{tabular}




\section{Problem Set "D" - Variations in Initial Source Energy}

In the final set of problems the initial neutron energy is varied. All cases have a closed geometry, uniform spatial distribution, and are for the D38/scintillator material. The results are given in Table A12-4.

Table A12-4

\begin{tabular}{lclll}
\hline \hline & Prob. & Neutrons $/ \mathrm{cm}^{2}$ & Photons $/ \mathrm{cm}^{2}$ & Grays \\
\hline $20 \mathrm{MeV}$ & $5 \mathrm{~A}$ & $5.19 \times 10^{-5}[.07]$ & $1.26 \times 10^{-5}[.16]$ & $2.19 \times 10^{-16}[.03]$ \\
$10 \mathrm{MeV}$ & $5 \mathrm{~B}$ & $5.58 \times 10^{-5}[.39]$ & $1.11 \times 10^{-5}[.28]$ & $1.65 \times 10^{-16}[.24]$ \\
$5 \mathrm{MeV}$ & $5 \mathrm{C}$ & $2.72 \times 10^{-5}[.09]$ & $5.44 \times 10^{-6}[.05]$ & $7.34 \times 10^{-17}[.06]$ \\
$1 \mathrm{MeV}$ & $1 \mathrm{D}$ & $2.38 \times 10^{-5}[.07]$ & $4.39 \times 10^{-6}[.16]$ & $5.59 \times 10^{-17}[.04]$ \\
$0.5 \mathrm{MeV}$ & $5 \mathrm{D}$ & $2.30 \times 10^{-5}[.02]$ & $4.11 \times 10^{-6}[.06]$ & $3.49 \times 10^{-17}[.04]$ \\
$0.1 \mathrm{MeV}$ & $5 \mathrm{E}$ & $2.37 \times 10^{-5}[.01]$ & $5.39 \times 10^{-6}[.10]$ & $3.67 \times 10^{-17}[.08]$ \\
\hline \hline
\end{tabular}

\section{References}

1. T. P. Wilcox, Jr., E. M. Lent, "COG: A Particle Transport Code Designed to Solve the Boltzman Equation for Deep-Penetration (Shielding) Problems-Volume I, Users Manual," Lawrence Livermore National Laboratory Report M-221-1. 


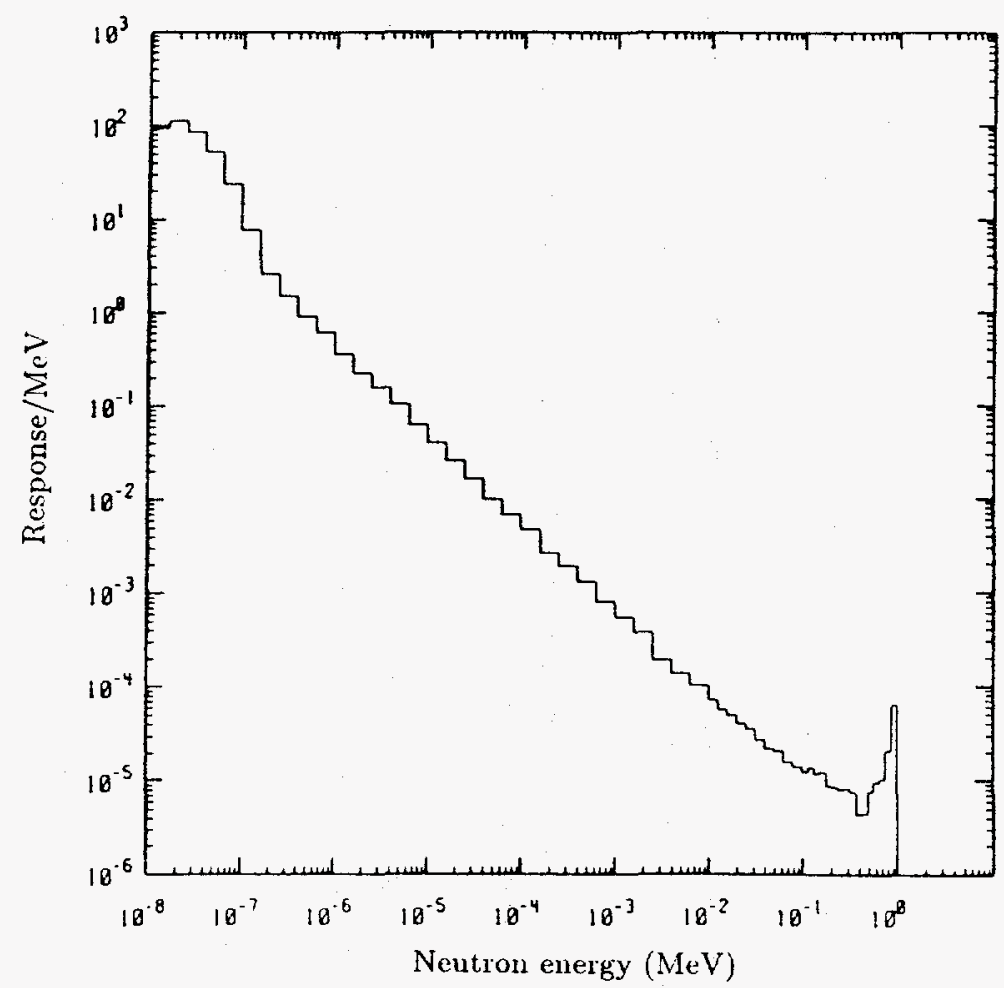

FIG. A12-1. Differential neutron spectra for problem 1B-Concrete. Response is in neutrons/square centimeter.

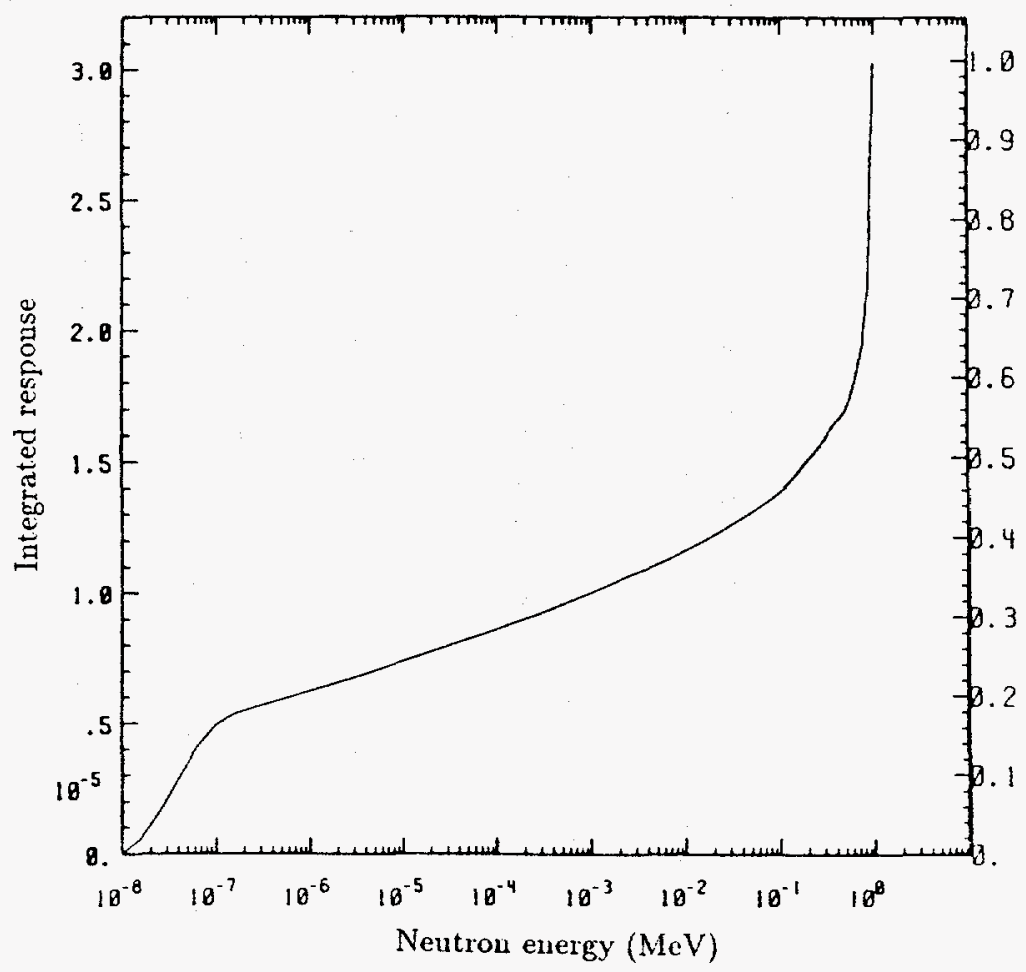

FIG. A12-2. Integrated neutron spectra for problem 1B-Concrete. Left side represents neutrons/sq. $\mathrm{cm}$ below given energy. 


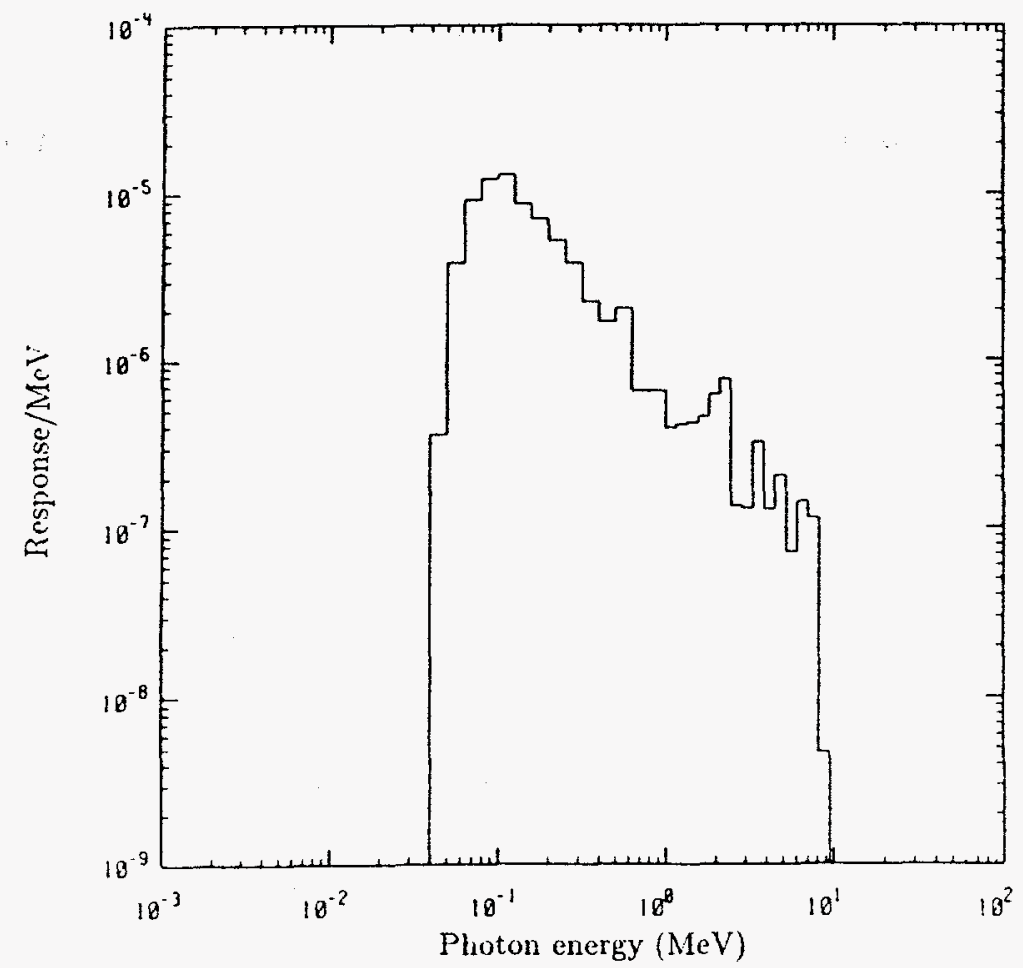

FIG. A12-3. Differential photon spectra from problem 1B-Concrete. Response is in photons/square centimeter.

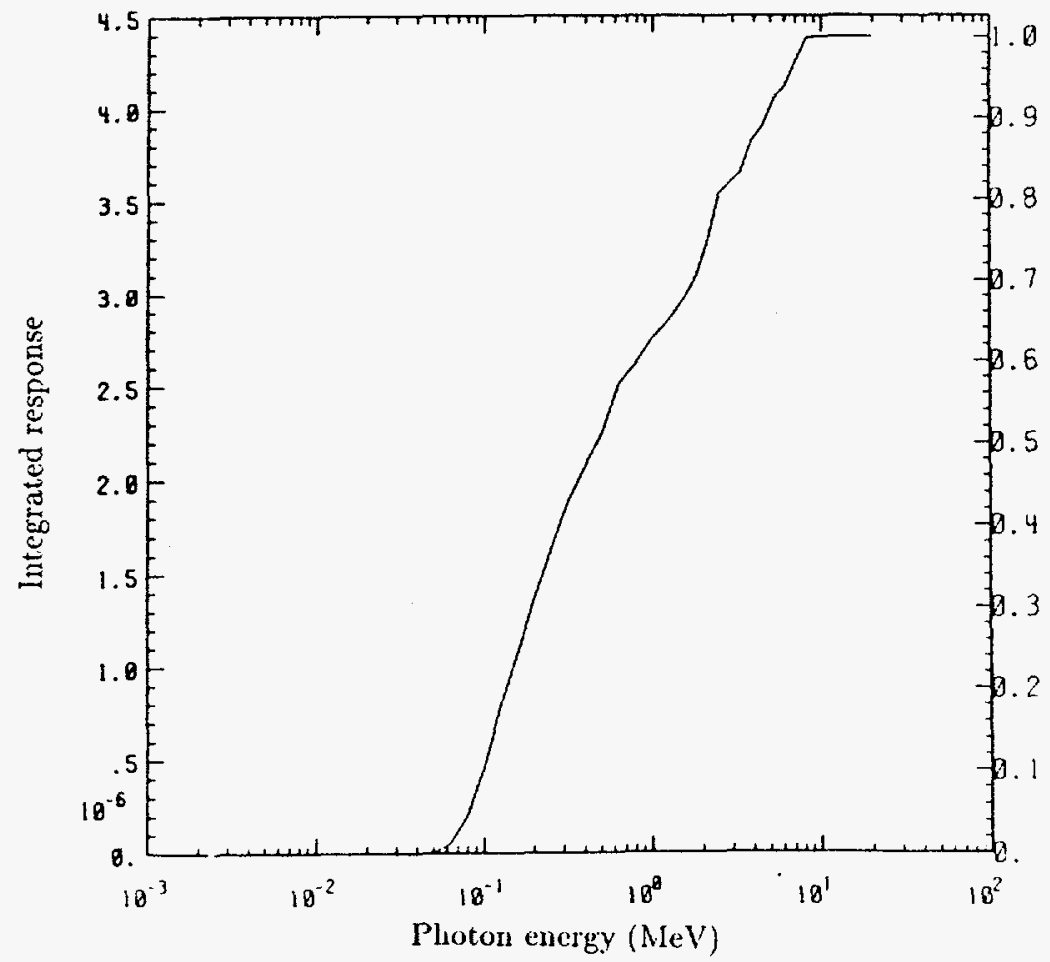

FIG. A12-4. Integrated photon spectra from problem 1B-Concrete. Left side represents photons/sq. $\mathrm{cm}$ below given energy. 


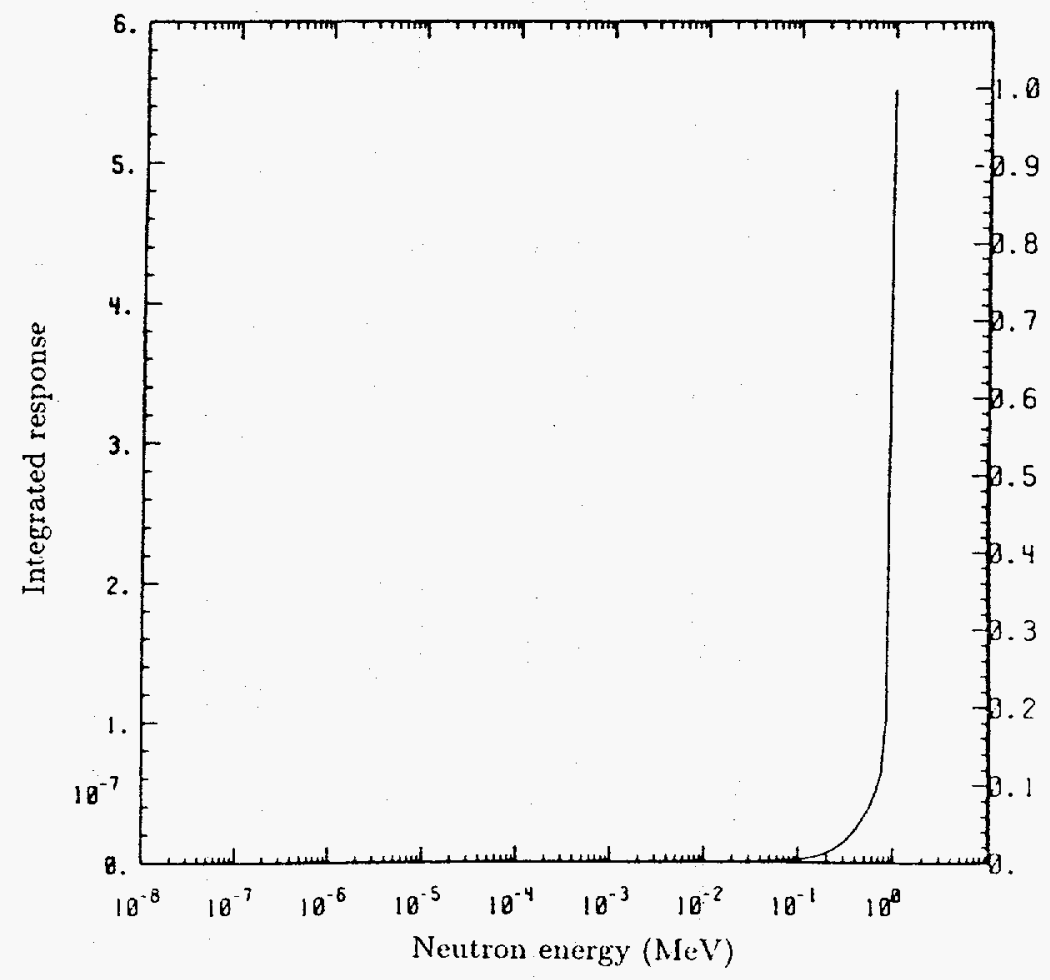

FIG. A12-5. Energy deposition for neutrons below a given energy for problem 1BConcrete. Left side is in $\mathrm{MeV} / \mathrm{cc}$ in $\mathrm{SiO}_{2}$.

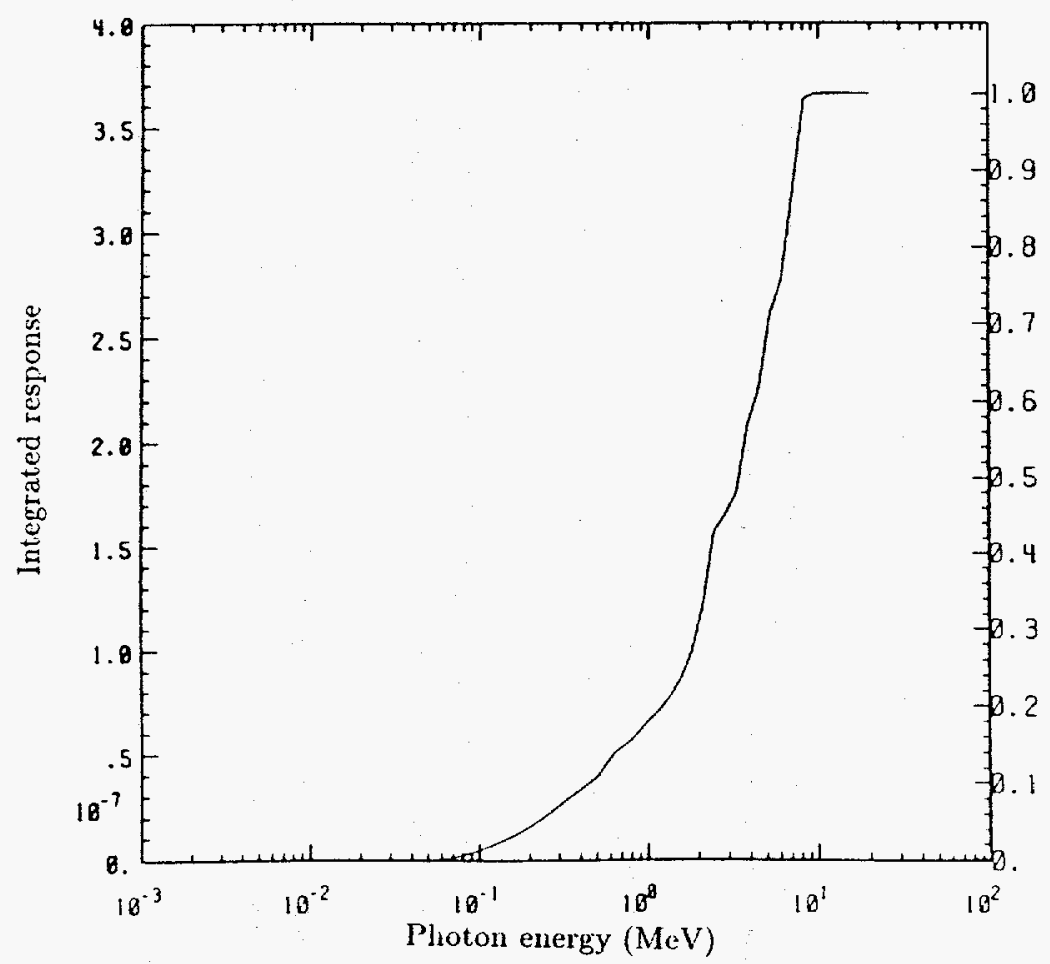

FIG. A12-6. Energy deposition for photons below a given energy for problem 1BConcrete. Left side is in $\mathrm{MeV} / \mathrm{cc}$ in $\mathrm{SiO}_{2}$. 


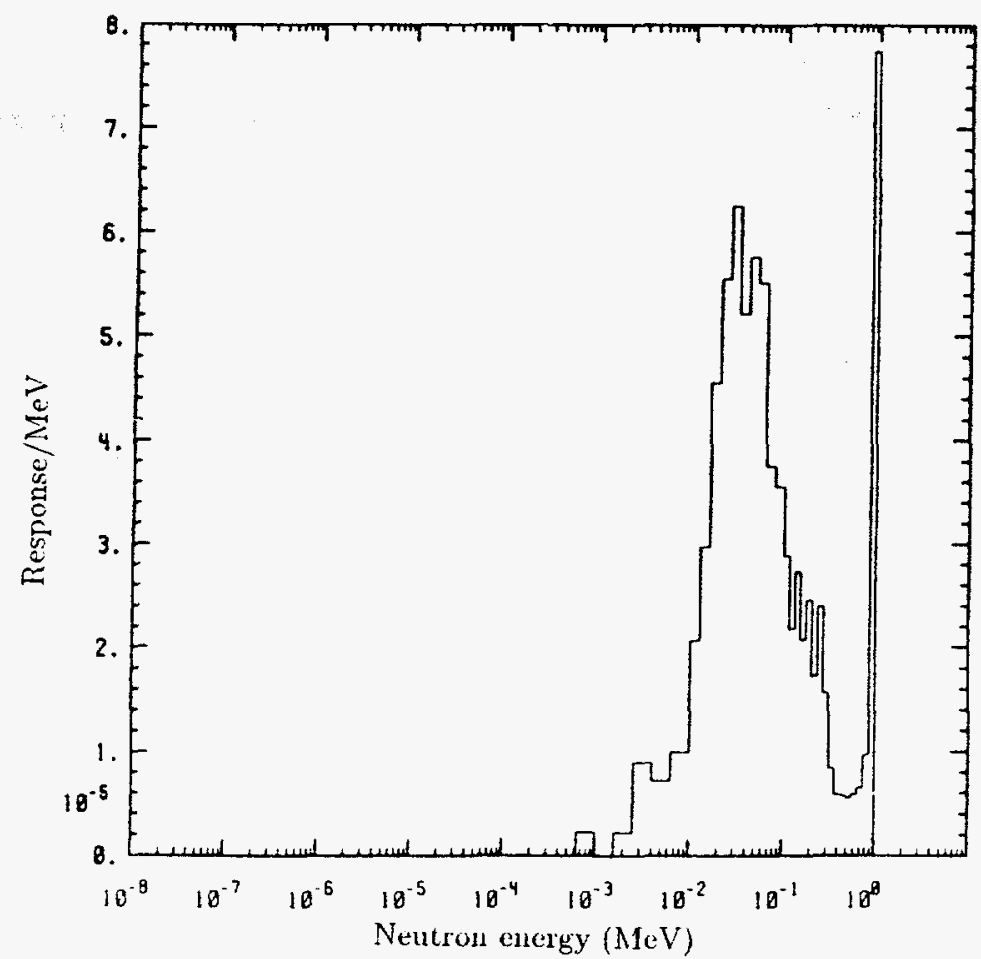

FIG. A12-7. Differential neutron spectra for problem 1C-D38. Response is in neutrons/sq. centimeter.

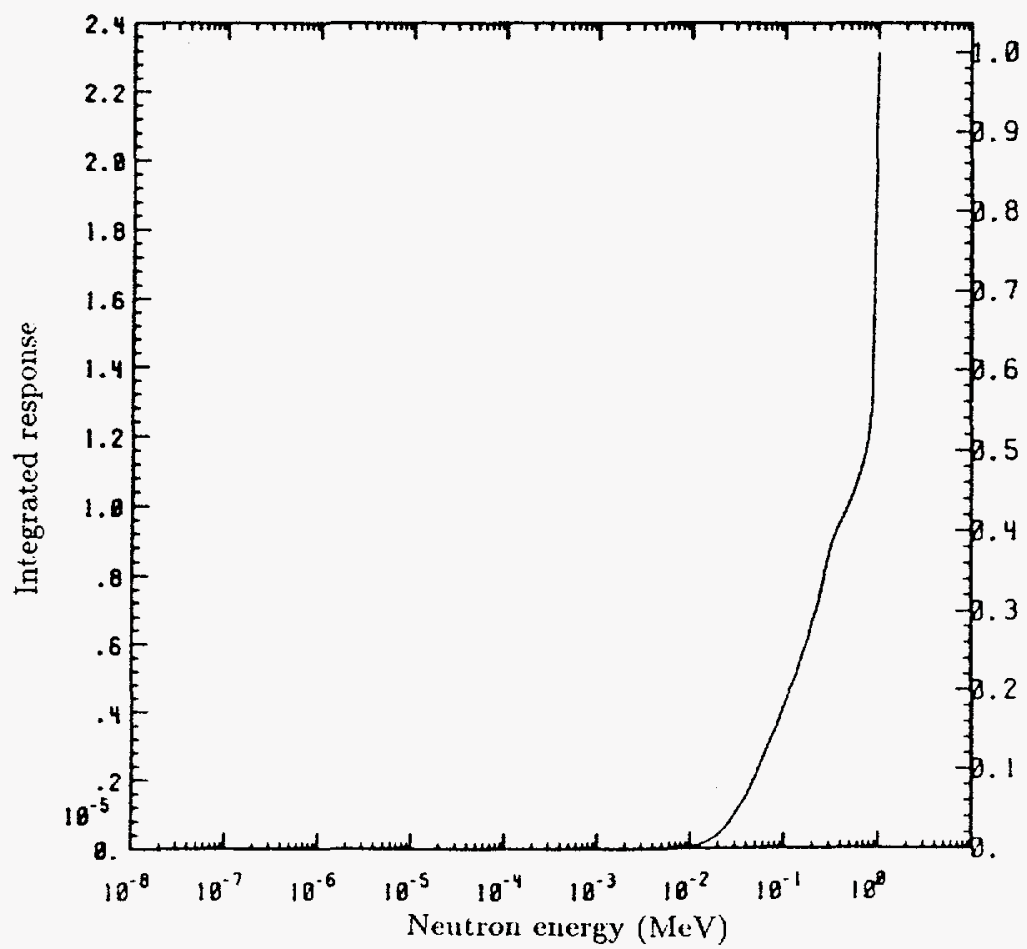

FIG. A12-8. Integrated neutron spectra for problem 1C-D38. Left side represents neutrons/sq. $\mathrm{cm}$ below given energy. 


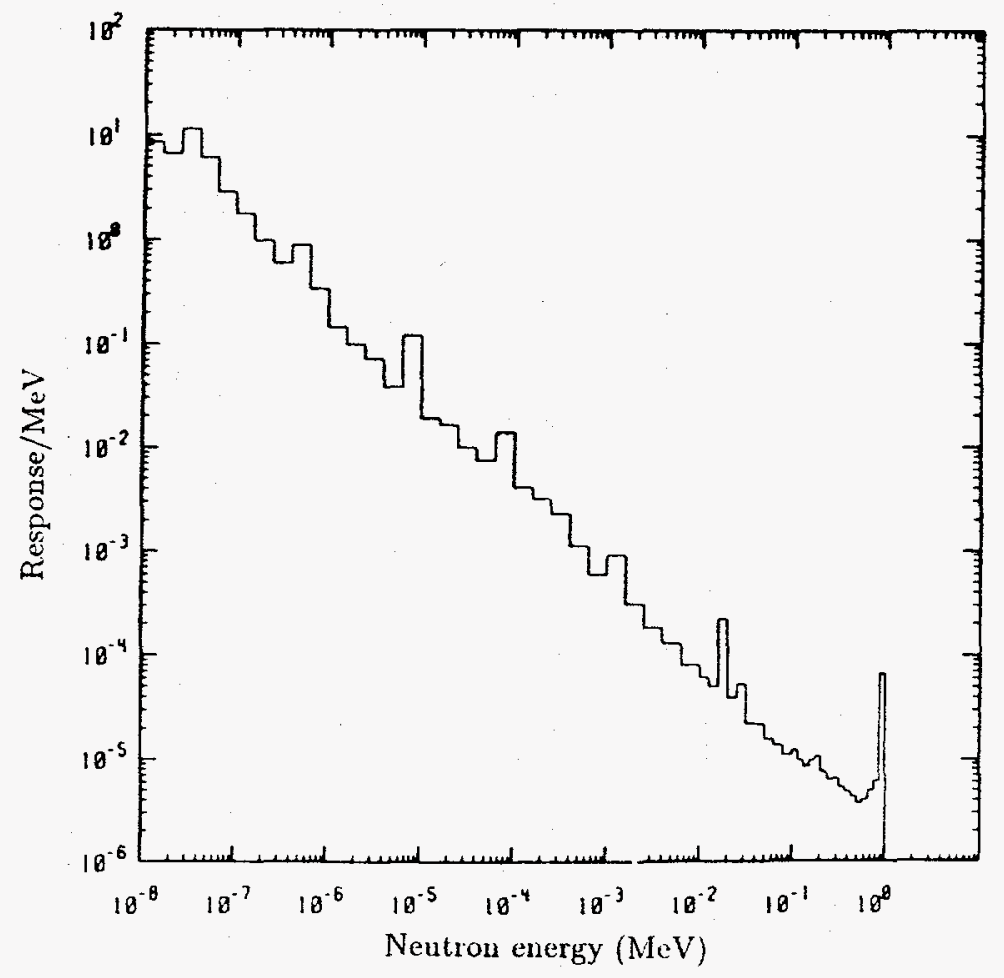

FIG. A12-9. Differential neutron spectra for problem 1D-D38/scintillator. Response is in neutrons/square centimeter.

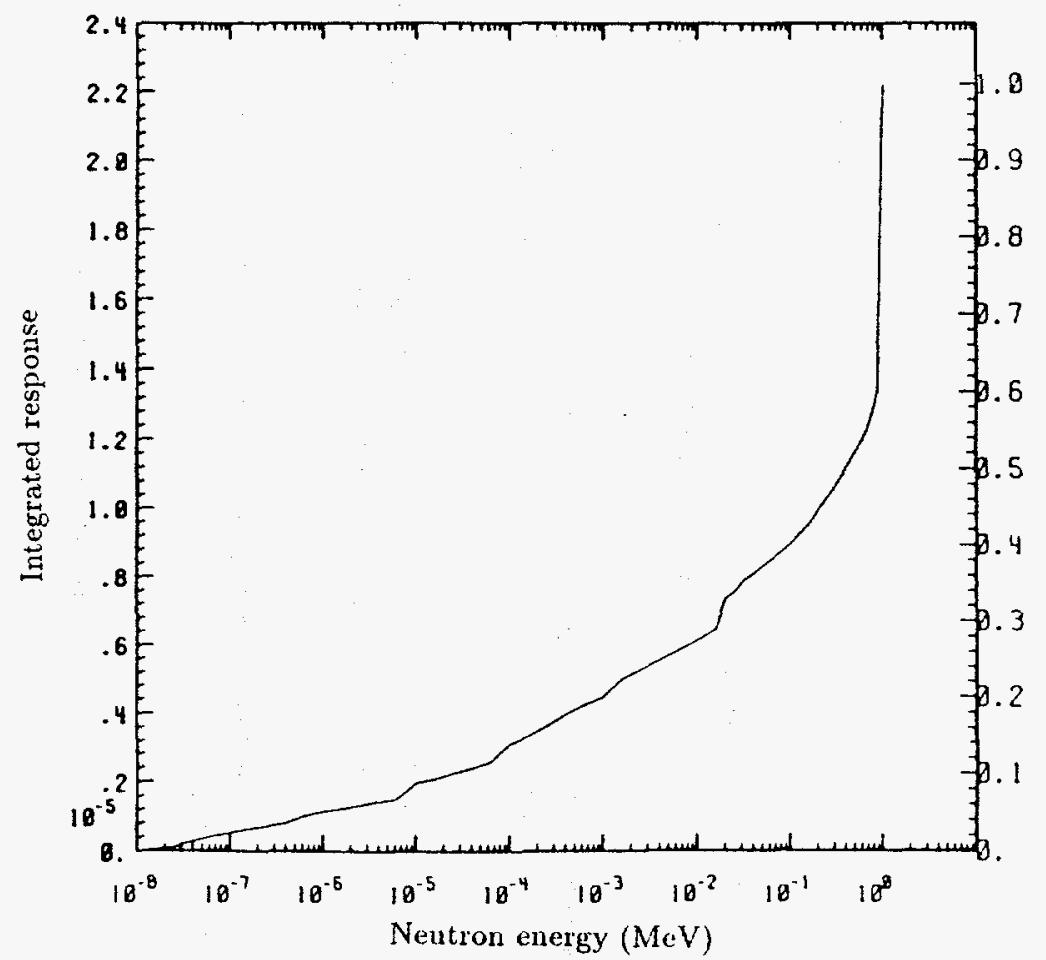

FIG. A12-10. Integrated neutron spectra for problem 1D-D38/scintillator. Left side represents neutrons/sq. $\mathrm{cm}$ below given energy. 

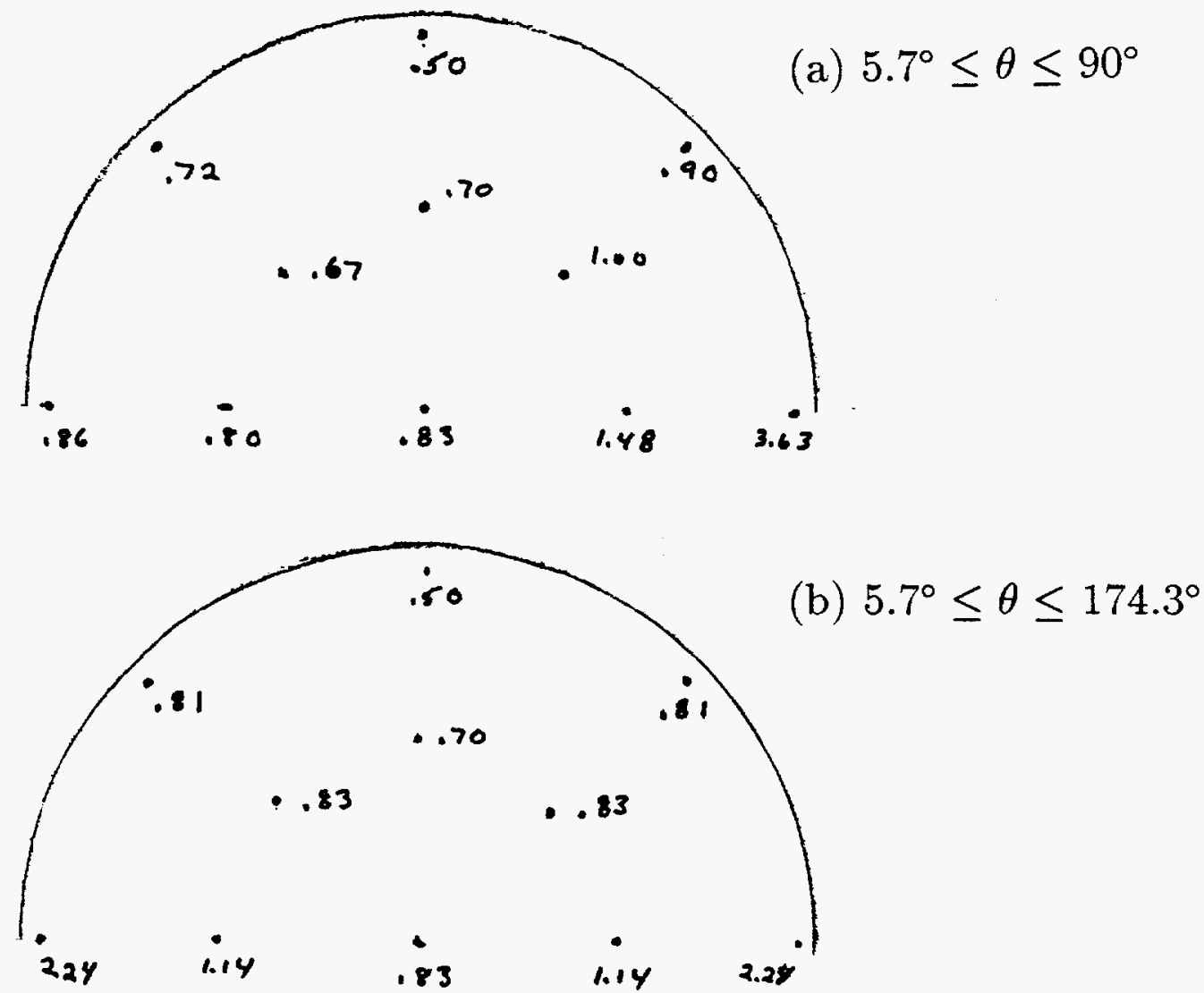

FIG. A12-11. Neutron distributions in a $2 \mathrm{~m}$ radius cavity for a $1 / \sin ^{2.5} \theta$ source distribution over the angular range indicated. A closed uranium/scintillator calorimeter surrounds the cavity. 

APPENDIX 13

\title{
CALCULATED RESULTS FOR MONOENERGETIC PROTONS INCIDENT ON A URANIUM-PLASTIC CALORIMETER
}

\author{
R. G. Alsmiller, Jr., F. S. Alsmiller, T. A. Gabriel, \\ B. L. Bishop and O. W. Hermann \\ Oak Ridge National Laboratory, Oak Ridge TN 37830
}

Calculations have been carried out for protons with energies between $1 \mathrm{GeV}$ and $100 \mathrm{GeV}$ normally incident on a uranium-plastic calorimeter. The geometry used in the calculations is shown in Fig. 1. Cylindrical symmetry is used and a proton beam with zero width is incident along the axis of the cylinder. The calorimeter is composed of homogeneous regions of depleted uranium and plastic (50\% by volume of depleted uranium and $50 \%$ by volume of plastic) separated by thin layers of plastic. The depleted uranium has $0.2 \% \mathrm{U}^{235}$ and a density of $18.9 \mathrm{~g} \mathrm{~cm}^{-3}$. The plastic is composed of carbon and hydrogen in the ratio of 1.0 to 1.2 and has a density of $1 \mathrm{~g} \mathrm{~cm}^{-3}$.

The calculations were carried out using the modified version of the HighEnergy Transport Code (HETC) described in Refs. 1 and 2 and used in Ref. 3. This version of the code will hereinafter be referred to as HETC(82). As explained in Ref. 1, this version of the code differs from that used previously (see Refs. 4 and 5 and the references given therein) in that the model for differential particle production from hadron-nucleus nucleus at energies $>3 \mathrm{GeV}$ has been revised.

Calculations have been carried out for incident proton energies of $1,5,10$, 20,50 , and $100 \mathrm{GeV}$. In Fig. 2 the calculated results for the number of albedo neutrons (that is, the number of neutrons emerging from the front face of the calorimeter) with energies between 0.11 and $20 \mathrm{MeV}$ are shown as a function of incident proton energy. Also shown in the Figure for incident proton energies of $1 \mathrm{GeV}$ and $5 \mathrm{GeV}$ are the results that are given by the older version of HETC described in Refs. 4 and 5. The error bars where shown are statistical only and represent one standard deviation; where not shown they are smaller than the size of the plotted points.

For $1 \mathrm{GeV}$ incident proton energies the two versions of the code are in agreement, as they must be, since the two version of the code are the same at energies below $3 \mathrm{GeV}$. For an incident energy of $5 \mathrm{GeV}$ there is however a significant difference between results using the two versions of the code. Since the two versions of the code yield the same results at energies less than $3 \mathrm{GeV}$, this discrepancy is due to the difference in the particle production models between 3 and $5 \mathrm{GeV}$. 
In Fig. 3 the energy distributions of the albedo neutrons given by the two models are compared and are not significantly different. At present it is not known which of the two calculations is the more trustworthy, so for practical purposes an average of the two should be used with perhaps slightly more weighing given to the higher value, that is the value given by the earlier version of the model. At the higher incident energies $(\$ 20 \mathrm{GeV})$ the older code is thought to be unreliable and should not be used. *

In Fig. 4 the flux of neutrons with energies from $0.11 \mathrm{MeV}$ to $20 \mathrm{MeV}$ is shown as a function of depth in the calorimeter for incident proton energies of 1 , 5 , and $20 \mathrm{GeV}$.

In Fig. 5 the average and approximate maximum energy deposition in the plastic layers is shown as a function of incident proton energy. Actually shown is a straight line drawn by eye through the calculated values rather than the calculated points.

In Fig. 6 the fraction of the total energy deposited in the plastic strips is shown as a function of incident proton energy. The source of the various contributions shown in Fig. 6 are described on the plot. The sum of the contributions at a given incident energy is unity. As before, a smooth curve drawn by eye through the calculated points is shown rather than the points themselves.

\section{References}

1. R. G. Alsmiller, Jr., F. S. Alsmiller, T. A. Gabriel, and O.W. Hermann, "Modification of the High-Energy Transport Code (HETC) and Comparisons with Experimental Results," Proc. of the ANS Topical Conference on the Theory and Practices in Radiation Protection and Shielding, April 22-24, 1987, Knoxville, TN.

2. F. S. Alsmiller, R. G. Alsmiller, Jr., and O. W. Hermann, "Low-Energy Particle Production and Residual Nuclei Production in High-Energy Hadron-Nucleus Collisions," Proc. of the ANS Topical Conference on the Theory and Practice in Radiation Protection and Shielding, April 22-24, 1987, Knoxville, TN.

3. T. A. Gabriel, F. S. Alsmiller, R. G. Alsmiller, Jr., B. L Bishop, O. W. Hermann, and D. E. Groom, "Preliminary Simulation of the Neutron Flux Levels in the Fermilab Tunnel and Proposed SSC Tunnel," SSC Central Design Group Report SSC-110 (1987).

4. T. N. Armstrong et al., "Monte Carlo Calculations of High-Energy Nucleon-Meson Cascades and Comparisons with Experiments," Nucl. Sci. and Eng. 49, 82 (1972).

* The version of the particle production model used in HETC 82 has since been updated by its authors (J. Ranft et al.; see Ref. 6 and the Refs. given therein), and therefore a code HETC87 is in preparation. Only a very few calculations have been performed with "HETC87," but for an incident energy of $10 \mathrm{GeV}$ the number of albedo neutrons from HETC87 is 20.6 while the value from HETC82 (see Fig. 2) is 15.7. 
5. R. G. Alsmiller, Jr. "Nucleon-Meson Transport Calculations," in Spallation Nuclear Reactions and Their Applications, Ed. by B. S. P. Shen and M. Mirkner, Vol. 59, 1976, D. Reidell Publishing Co., Dodrecht-Holland/Boston, U.S.A.

6. J. Ranft and S. Ritter, "Rapidity Ratios, Feynman- $x$ Distributions and Forward-Backward Correlations in Hadron-Nucleus Collisions in a Dual Monte-Carlo Multi-Chain Fragmentation Model," Z. Phys. C27, 569 (1985).

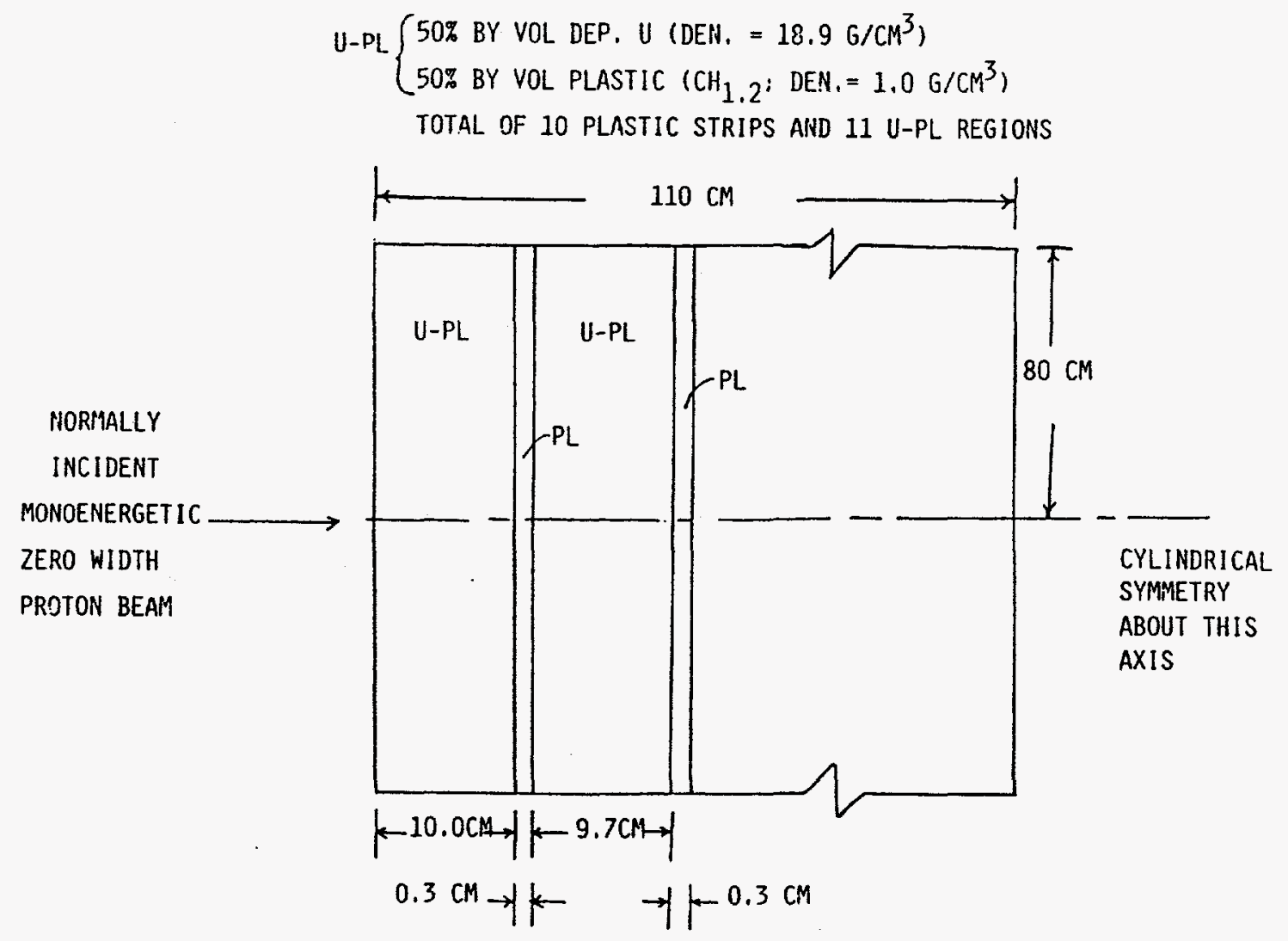

FIG. 1. Schematic of the uranium-plastic calorimeter used in the calculations. 


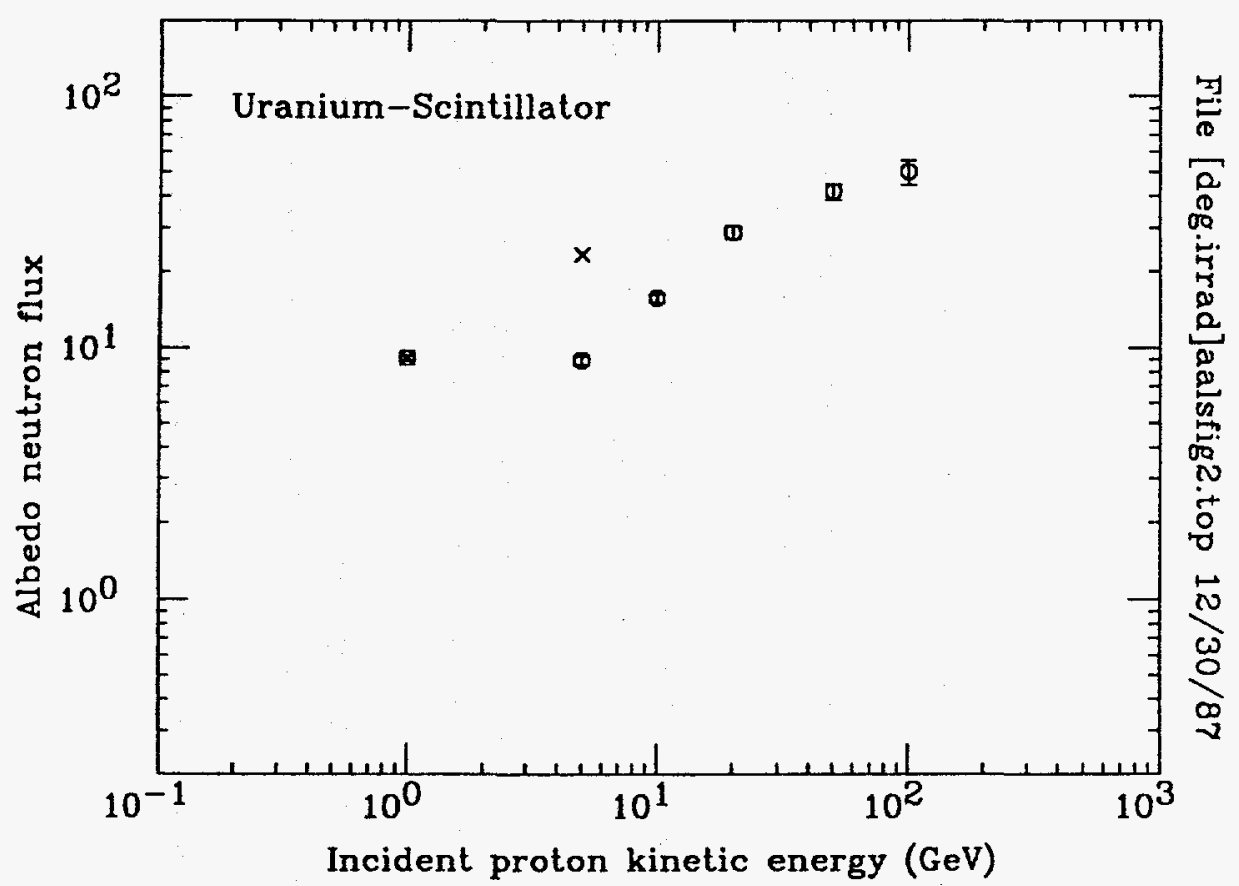

FIG. 2. Number of albedo neutrons with energies between $0.11 \mathrm{MeV}$ and $20 \mathrm{MeV}$ vs. incident proton kinetic energy. The circles are for results obtained with HETC82, and the $x$ 's (at $1 \mathrm{GeV}$ and $5 \mathrm{GeV}$ ) are for results obtained with the old version.

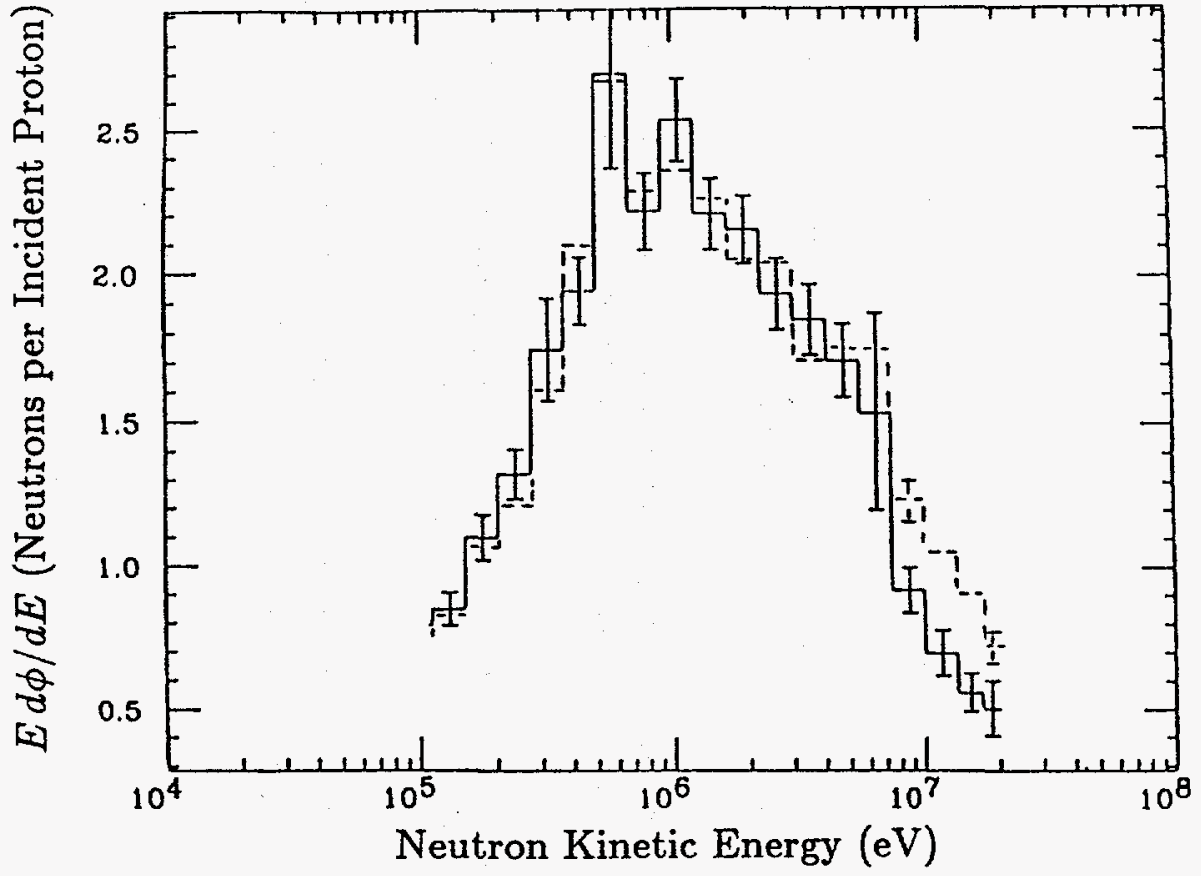

FIG. 3. Albedo neutron spectra for $5 \mathrm{GeV}$ incident protons. The solid histogram was obtained using HETC82, and the dotted using HETC82(OLD). Normalization of both is to an integral of 9.0 neutrons. 


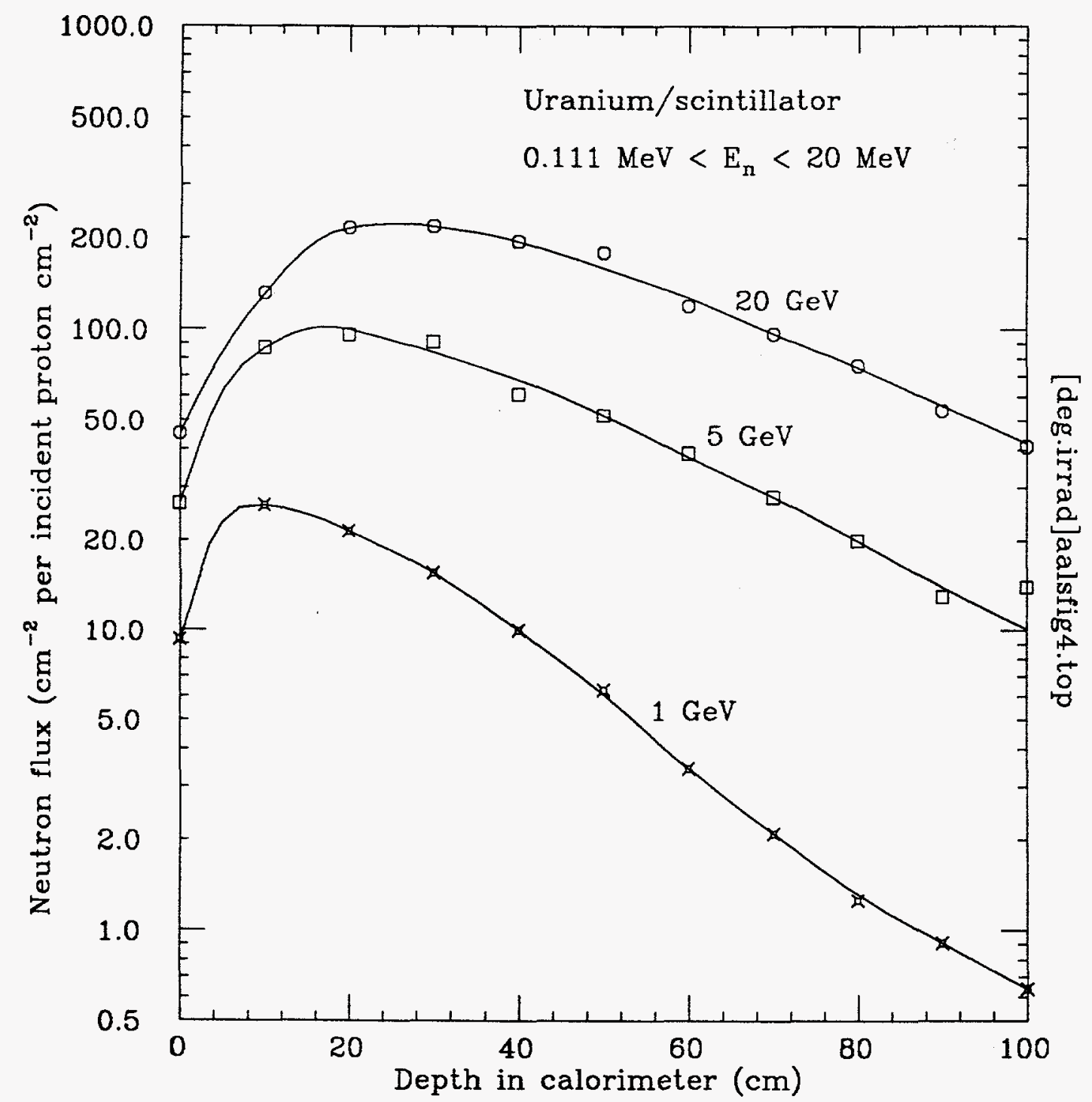

FIG. 4. Fast neutron flux $(0.11 \mathrm{MeV} \leq E \leq 20 \mathrm{MeV})$ vs. depth for 1,5 , and $20 \mathrm{GeV}$ incident protons. 


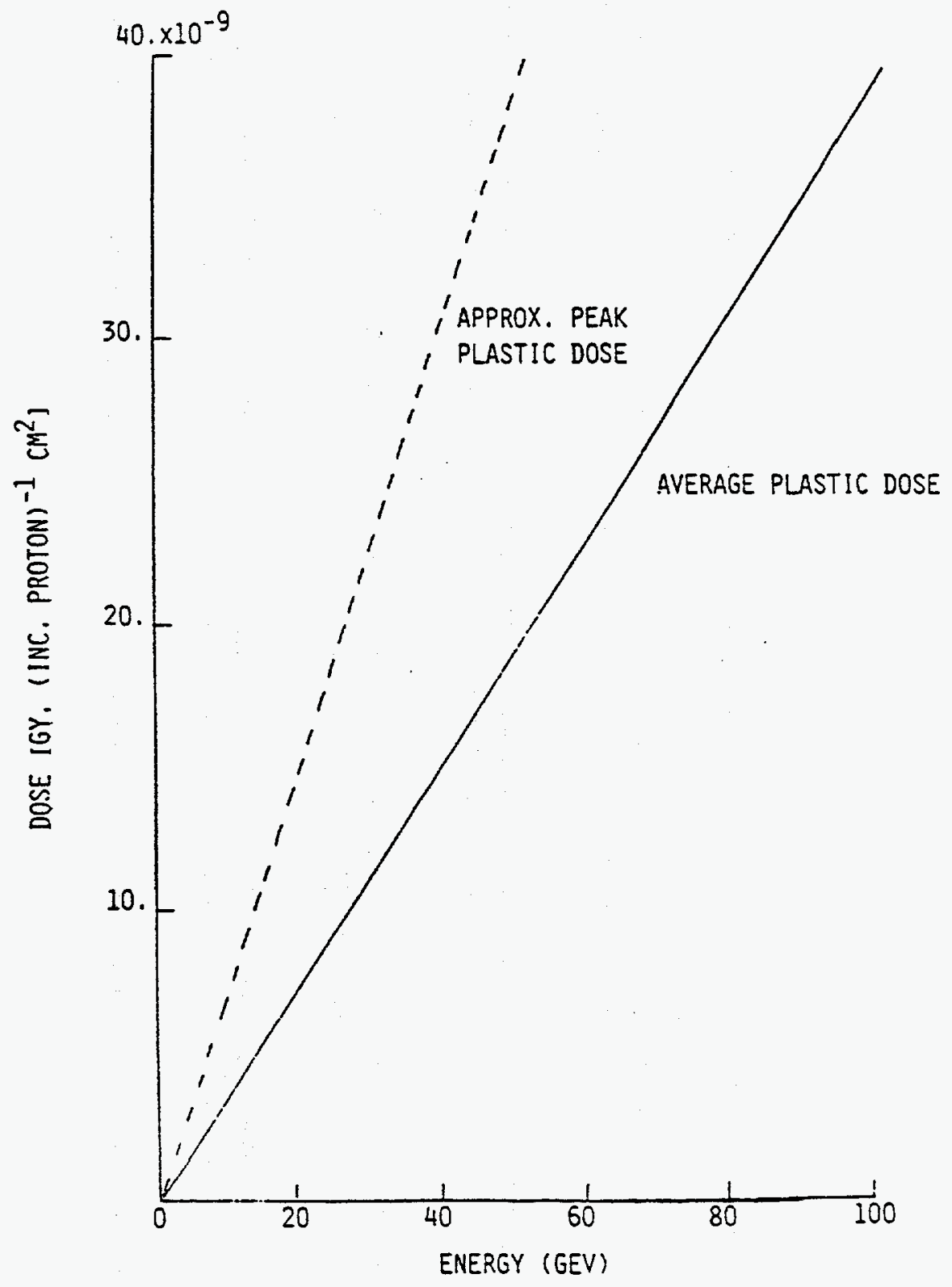

FIG. 5. Average and peak dose in the plastic from incident protons as a function of incident kinetic energy. 


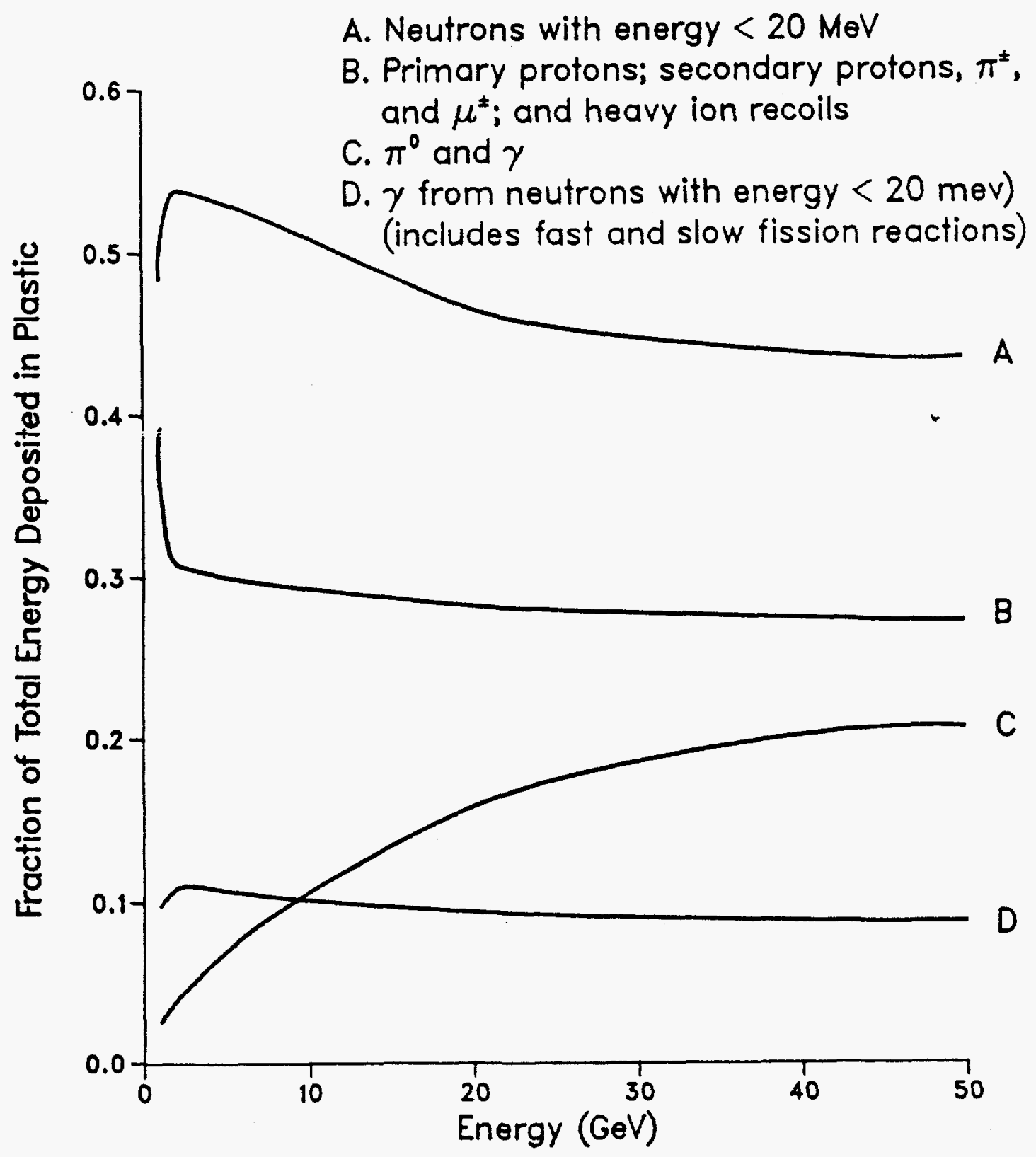

FIG. 6. The fraction of the total energy deposited in the plastic scintillator as a function of incident proton kinetic energy. 



\title{
APPENDIX 14 \\ PREDICTIONS OF NEUTRON YIELDS FROM GHEISHA + ANISN
}

\author{
Syuichi Ban, Takahiko Kondo, and Makoto Asai ${ }^{\star}$ \\ KEK, National Laboratory for High Energy Physics, Japan
}

\section{Introduction}

In the calorimeter, neutron fluxes are dominant in the energy region between 0.1 and $15 \mathrm{MeV}$ because of evaporation and fission neutrons. It is therefore important to calculate the transport of low energy neutrons. However, it is not easy to simulate high energy showers.

To simulate the shower development in the calorimeter, Monte Carlo code GHEISHA (Version 7.03) [1] was used. Two kinds of calculations were done. In one case, the calculation was done using GHEISHA only down to $0.1 \mathrm{MeV}$. In another case, simulation was done using GHEISHA except for neutrons below $15 \mathrm{MeV}$. Low-energy neutron transportation was calculated separately using Discrete Ordinate code ANISN [2]. These conditions are shown in Tables 1 and 2.

Table 1

Cut-off kinetic energy in GHEISHA-7.

\begin{tabular}{lcl}
\hline \hline & Particle & Energy \\
\hline Case 1: & $\gamma$ & $1 \mathrm{MeV}$ \\
& $e^{ \pm}$ & $1 \mathrm{MeV}$ \\
& Charged hadrons & $0.1 \mathrm{MeV}$ \\
& Neutral hadrons (not $\mathrm{n}$ ) & $15 \mathrm{MeV}$ \\
& $\mu^{ \pm}$ & $1 \mathrm{MeV}$ \\
\hline Case 2: & $\gamma$ & $1 \mathrm{MeV}$ \\
& $e^{ \pm}$ & $1 \mathrm{MeV}$ \\
& Charged hadrons & $0.1 \mathrm{MeV}$ \\
& Neutral hadrons & $0.1 \mathrm{MeV}$ \\
\hline \hline
\end{tabular}

\footnotetext{
* Hiroshima Inst. Tech.
} 
Table 2

\begin{tabular}{cl}
\hline \hline GHEISHA - 7.03 & $\begin{array}{l}\text { simulates the transport of particles } \\
\text { down to } 0.1 \mathrm{MeV} \\
\text { Low energy neutrons below } 15 \mathrm{MeV} \text { were } \\
\text { dumped on disk files }\end{array}$ \\
neutrons below & $\begin{array}{l}\text { sorted into } 13 \text { energy groups, } 16 \text { angle } \\
\text { groups and } 400 \text { space intervals. } \\
15 \mathrm{MeV}\end{array}$ \\
ANISN & $\begin{array}{l}\text { simulates the transport of neutrons } \\
\text { from } 15 \mathrm{MeV} \text { to } 0.1 \mathrm{MeV}\end{array}$ \\
\hline
\end{tabular}

\section{Calculations}

In the calculations, the calorimeters are $120 \mathrm{~cm}$ long and of infinite lateral extent. They consist of absorbers (uranium or lead) and detectors (scintillators or liquid argon) both $3 \mathrm{~mm}$ thick, as shown in Table 3 . The densities of the materials are shown in Table 4. A $\pi^{+}$or proton was normally incident on the calorimeter. The numbers of source particles processed in GHEISHA are shown in Table 5.

Table 3

Model calorimeters studied.

\begin{tabular}{|c|c|c|c|c|c|}
\hline Calorimeter & $\begin{array}{l}\text { Total } \\
\text { Length }\end{array}$ & Layer & Thickness & Layer & Thickness \\
\hline 1 & $120 \mathrm{~cm}$ & Uranium & $3 \mathrm{~mm}$ & Scint. & $3 \mathrm{~mm}$ \\
\hline 2 & $120 \mathrm{~cm}$ & Uranium & $3 \mathrm{~mm}$ & Liq. Ar. & $3 \mathrm{~mm}$ \\
\hline 3 & $120 \mathrm{~cm}$ & Lead & $3 \mathrm{~mm}$ & Scint. & $3 \mathrm{~mm}$ \\
\hline 4 & $120 \mathrm{~cm}$ & Lead & $3 \mathrm{~mm}$ & Liq. Ar. & $3 \mathrm{~mm}$ \\
\hline
\end{tabular}

In the low-energy neutron transport calculation using ANISN, DLC-37 [3] were used. There are 100 groups neutron cross section data from $15 \mathrm{MeV}$ to thermal energy. In this case, their energy group structure is too fine, so they were collapsed to 13 groups as shown in Table 6 . To calculate the angular distribution of the neutron flux in ANISN, 16 discrete directions were used as shown in Table 7. Calorimeters (120 cm long) were divided into 400 space mesh. 
Table 4

Density of materials used in model calorimeters.

\begin{tabular}{lcc}
\hline \hline Material & Density & \\
\hline Uranium & 18.95 & \\
Lead & 11.35 & \\
Scintillator & 1.032 & $\mathrm{C}: \mathrm{H}=1: 1.1$ \\
Liquid argon & 1.38 & \\
\hline \hline
\end{tabular}

Table 5

Number of events processed in GHEISHA-7.

\begin{tabular}{lcccc}
\hline \hline & \multicolumn{5}{c}{ Momentum (GeV/c) } \\
Calorimeter & 1 & 3 & 10 & 100 \\
\hline & \multicolumn{5}{c}{ Number of incident $\pi^{+}$'s } \\
$\mathrm{U}$ + Scint. & 200 & 200 & 100 & 10 \\
$\mathrm{~Pb}$ + Scint. & 200 & 200 & 200 & 20 \\
$\mathrm{U}$ + Liq. Ar. & 200 & 200 & 100 & 10 \\
$\mathrm{~Pb}$ + Liq. Ar. & 200 & 200 & 100 & 10 \\
\hline \hline $\mathrm{U}+$ Scint. & 200 & 100 & 100 & 10 \\
$\mathrm{~Pb}$ + Scint. & 200 & 100 & 200 & 20 \\
\hline \hline
\end{tabular}

\section{Results}

The number of neutrons (whose kinetic energy is between 0.1 and $15 \mathrm{MeV}$ ) produced in GHEISHA (see Table 2) in case of incident $\pi^{+}$are shown in Fig. 1. This is the number (not a "flux") of neutrons produced in whole regions of the calorimeter. The difference between uranium and lead is large but small between scintillator and argon. Energy spectrum of these produced neutrons is shown in Fig. 2. In the case of uranium, the lower energy part is dominant due to fission neutrons.

Neutron fluxes $\left(0.1<E_{k}<15 \mathrm{MeV}\right)$ in the calorimeter as calculated using ANISN are shown in Fig. 3. Energy spectra of neutron fluxes calculated using ANISN are shown in Fig. 4. They are shown at the depth of one-inelasticinteraction length and three-interaction lengths. Energy spectra of albedo neutron current (not a flux) are shown in Fig. 5, which were also calculated using ANISN. 
Table 6

Energy group structure of neutron cross sections.

\begin{tabular}{ccc}
\hline \hline Group & $\begin{array}{c}\text { Upper Group } \\
\text { Energy (MeV) }\end{array}$ & $\begin{array}{c}\text { Lower Group } \\
\text { Energy (MeV) }\end{array}$ \\
\hline 1 & 14.918 & 12.214 \\
2 & 12.214 & 10.000 \\
3 & 10.000 & 8.1873 \\
4 & 8.1873 & 6.0653 \\
5 & 6.0653 & 4.4933 \\
6 & 4.4933 & 3.0119 \\
7 & 3.0119 & 2.0190 \\
8 & 2.0190 & 1.4957 \\
9 & 1.4957 & 1.0026 \\
10 & 1.0026 & 0.74274 \\
11 & 0.74274 & 0.49787 \\
12 & 0.49787 & 0.20242 \\
13 & 0.20242 & 0.11109 \\
\hline \hline
\end{tabular}

The number of albedo neutrons $\left(0.1<E_{k}<15 \mathrm{MeV}\right)$ is shown in Table 8 for both in Case-1 (GHEISHA + ANISN) and Case-2 (GHEISHA only) as seen in Table 1. The statistics are not good, especially for the $100 \mathrm{GeV} / \mathrm{c}$ case, because the number of source particles is not enough, as seen in Table 5. These results are also plotted in Fig. 6 (Case-2) and Fig. 7 (Case-1). The agreement between case-1 and case- 2 is good for the lead calorimeter but bad for the uranium calorimeter.

For the case of protons incident on the calorimeters, the number of albedo neutron is shown in Table 9 . The energy spectrum of albedo neutron current is shown in Fig. 8 in the case of $10-\mathrm{GeV}$ incident protons.

\section{Neutrons Below $0.1 \mathrm{MeV}$}

To estimate the contribution of neutrons below $0.1 \mathrm{MeV}$, neutron transport down to thermal energy was calculated for only one case, that is, $10 \mathrm{GeV} / \mathrm{c} \pi^{+}$ incident on uranium/scintillator. 
Table 7

Symmetric quadrature sets used in ANISN.

\begin{tabular}{cc}
\hline \hline Direction Cosines & Weights \\
\hline-0.9805009 & 0.0244936 \\
-0.9092855 & 0.0413296 \\
-0.8319966 & 0.0392569 \\
-0.7467506 & 0.0400796 \\
-0.6504264 & 0.0643754 \\
-0.5370966 & 0.0442097 \\
-0.3922893 & 0.1090850 \\
-0.1389568 & 0.1371702 \\
+0.1389568 & 0.1371702 \\
+0.3922893 & 0.1090850 \\
+0.5370966 & 0.0442097 \\
+0.6504264 & 0.0643754 \\
+0.7467504 & 0.0400796 \\
+0.8319966 & 0.0392569 \\
+0.9092855 & 0.0413296 \\
+0.9805009 & 0.0244936 \\
\hline \hline
\end{tabular}

Twenty-three groups of neutron cross section data were made from DLC-37. The energy group structure of the upper 13 groups is the same as the previous ones (shown in Table 6). Data from $0.1 \mathrm{MeV}$ to the thermal energy group were divided into 10 groups. However, DLC-37 was originally prepared for reactors at high temperatures, so its thermal neutron cross sections were weighted with a Maxwellian peaked at $800 \mathrm{~K}$. To correct for this the absorption cross section for the thermal energy group was multiplied by $\sqrt{800 / 293}$, so that it approximately corresponds to the cross section at $293 \mathrm{~K}$.

The calculation using GHEISHA and ANISN was done as shown in Table 2, but in this case 23 groups of cross section data were used for ANISN. Energy spectra of neutron fluxes at the depth of one and three interaction-lengths are shown in Fig. 9. The energy spectrum of albedo neutron current is shown in Fig. 10. The albedo neutron flux is 1.8 times larger than the albedo current. The ratio of low-energy neutrons below $0.1 \mathrm{MeV}$ to total neutrons is shown in Table 10. 
Table 8

Number of Albedo Neutrons $\left(0.1<E_{k}<15 \mathrm{MeV}\right)$.

\begin{tabular}{cccc}
\hline \hline Material & $\begin{array}{c}\pi^{+} \text {Momentum } \\
(\mathrm{GeV} / \mathrm{c})\end{array}$ & $\begin{array}{c}\text { GHEISHA-7 } \\
\text { (case-2) }\end{array}$ & $\begin{array}{c}\text { GHEISHA-7 \& ANISN } \\
\text { (case-1) }\end{array}$ \\
\hline U/Scint. & 1 & $0.78 \pm 0.06^{*}$ & 0.37 \\
$(3 \mathrm{~mm} / 3 \mathrm{~mm})$ & 3 & $4.4 \pm 0.2$ & 1.9 \\
& 10 & $12.2 \pm 0.4$ & 4.3 \\
& 100 & $27.0 \pm 2.0$ & 18.0 \\
\hline U/Liq. Ar. & 1 & $0.86 \pm 0.07$ & \\
& 3 & $6.47 \pm 0.2$ & \\
& 10 & $17.8 \pm 0.4$ & \\
\hline $\mathrm{Pb} /$ Scint. & 100 & $113.0 \pm 3.0$ & 0.075 \\
& 3 & $0.06 \pm 0.02$ & 0.56 \\
& 10 & $0.66 \pm 0.06$ & 2.2 \\
& 100 & $3.4 \pm 0.2$ & 7.9 \\
\hline $\mathrm{Pb} /$ Liq. Ar. & 1 & $9.0 \pm 0.7$ & \\
& 3 & $0.08 \pm 0.02$ & \\
& 10 & $0.80 \pm 0.06$ & \\
& 100 & $21.0 \pm 0.2$ & \\
\hline \hline
\end{tabular}

${ }^{*}$ Square-root (number of neutrons)/ number of incident pion

\section{References}

1. H. Fesefeldt, "The Simulation of Hadronic Shower-Physics and Applications," RWTH Aachen PITHA 85/02 (1985).

2. W. W. Engle, Jr., "A USER'S MANUAL FOR ANISN, A One-Dimensional Discrete Ordinates Transport Code with Anisotropic Scattering," K-1693, Union Carbide Corporation, Oak Ridge, TN (1967).

3. "EPR, Coupled 100-Group Neutron 21-Group Gamma-Ray Cross Sections for EPR Neutronics," Oak Ridge, DLC-37. 
Table 9

Number of albedo neutrons $\left(0.1<E_{k}<15 \mathrm{MeV}\right)$.

\begin{tabular}{cccc}
\hline Material & $\begin{array}{c}\text { Proton Energy } \\
(\mathrm{GeV} / \mathrm{c})\end{array}$ & $\begin{array}{c}\text { GHEISHA-7 } \\
\text { (case-2) }\end{array}$ & $\begin{array}{c}\text { GHEISHA-7 \& ANISN } \\
\text { (case-1) }\end{array}$ \\
\hline U/Scint. & 1 & $0.92 \pm 0.07^{*}$ & 0.39 \\
$(3 \mathrm{~mm} / 3 \mathrm{~mm})$ & 3 & $5.0 \pm 0.2$ & 2.1 \\
& 10 & $10.6 \pm 0.4$ & 6.0 \\
& 100 & $68.0 \pm 3.0$ & 15.0 \\
\hline $\mathrm{Pb} /$ Scint. & 1 & $0.05 \pm 0.02$ & 0.045 \\
& 3 & $0.77 \pm 0.09$ & 2.8 \\
& 10 & $3.4 \pm 0.2$ & \\
\hline \hline
\end{tabular}

*Square-root (number of neutrons)/ number of incident protons.

Table 10

Ratio of each neutron group to total neutron (from thermal energy to $15 \mathrm{MeV}$ ) in case $10 \mathrm{GeV} / \mathrm{c} \pi^{+}$incident on Uranium/Scintillator calorimeter.

\begin{tabular}{lccc}
\hline \hline & $\begin{array}{l}E_{k}<0.4 \mathrm{eV} \\
\text { (thermal) }\end{array}$ & $0.4 \mathrm{eV}<E_{k}<0.1 \mathrm{MeV} \quad 0.1 \mathrm{MeV} E_{k}<15 \mathrm{MeV}$ \\
\hline $\begin{array}{l}\text { Number of albedo } \\
\begin{array}{l}\text { Flux at one } \\
\text { interaction length }\end{array}\end{array}$ & 0.011 & 0.23 & 0.75 \\
$\begin{array}{l}\text { Flux at three } \\
\text { interaction length } \\
\text { Total flux in } \\
\text { the calorimeter }\end{array}$ & 0.025 & 0.40 & 0.58 \\
\hline \hline
\end{tabular}




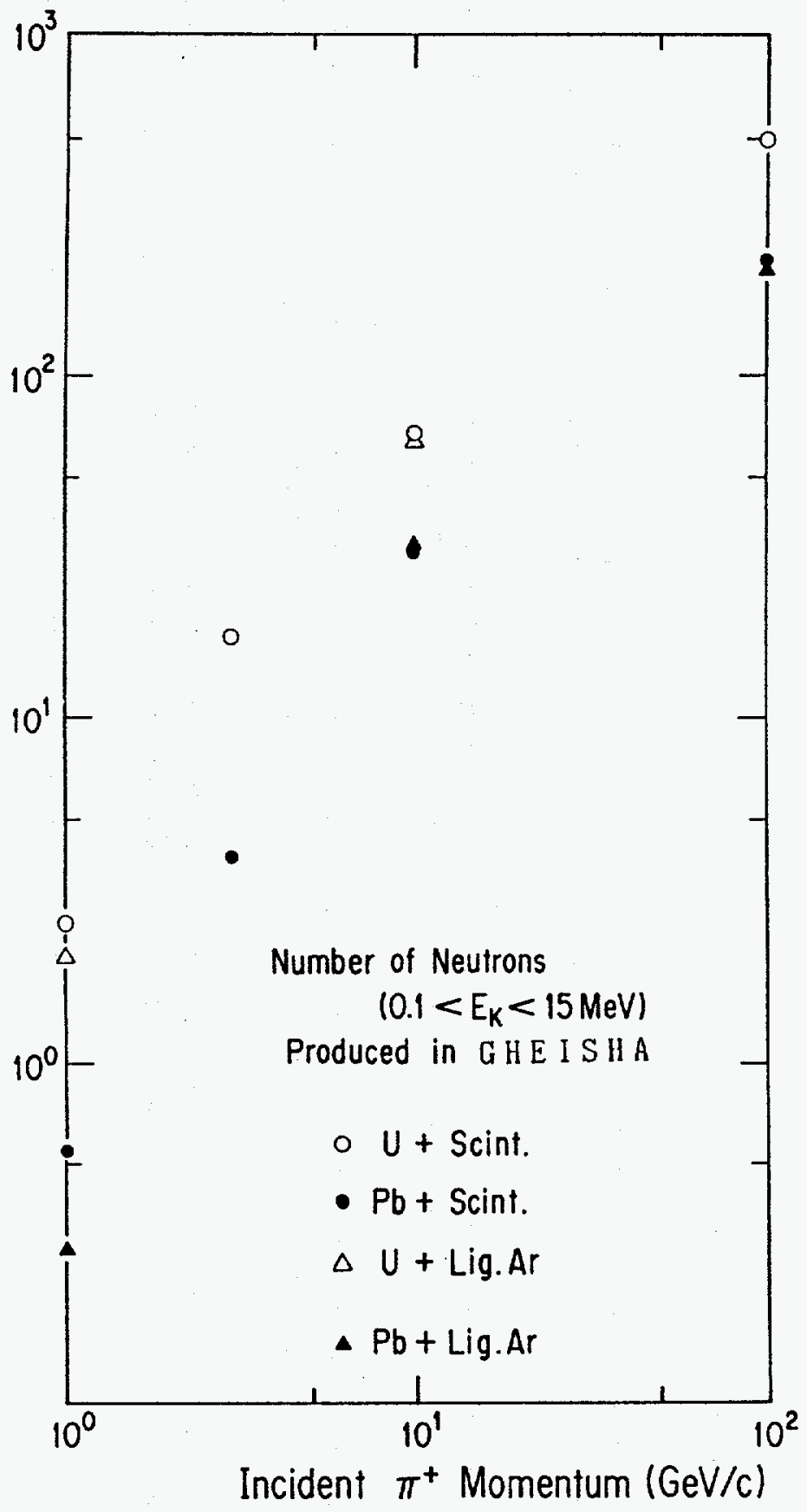

FIG. 1. Number of neutrons from 0.1 to $15 \mathrm{MeV}$ produced in calorimeters. Neutron production due to higher energy particles was calculated using GHEISHA-7 (see Table 2). 


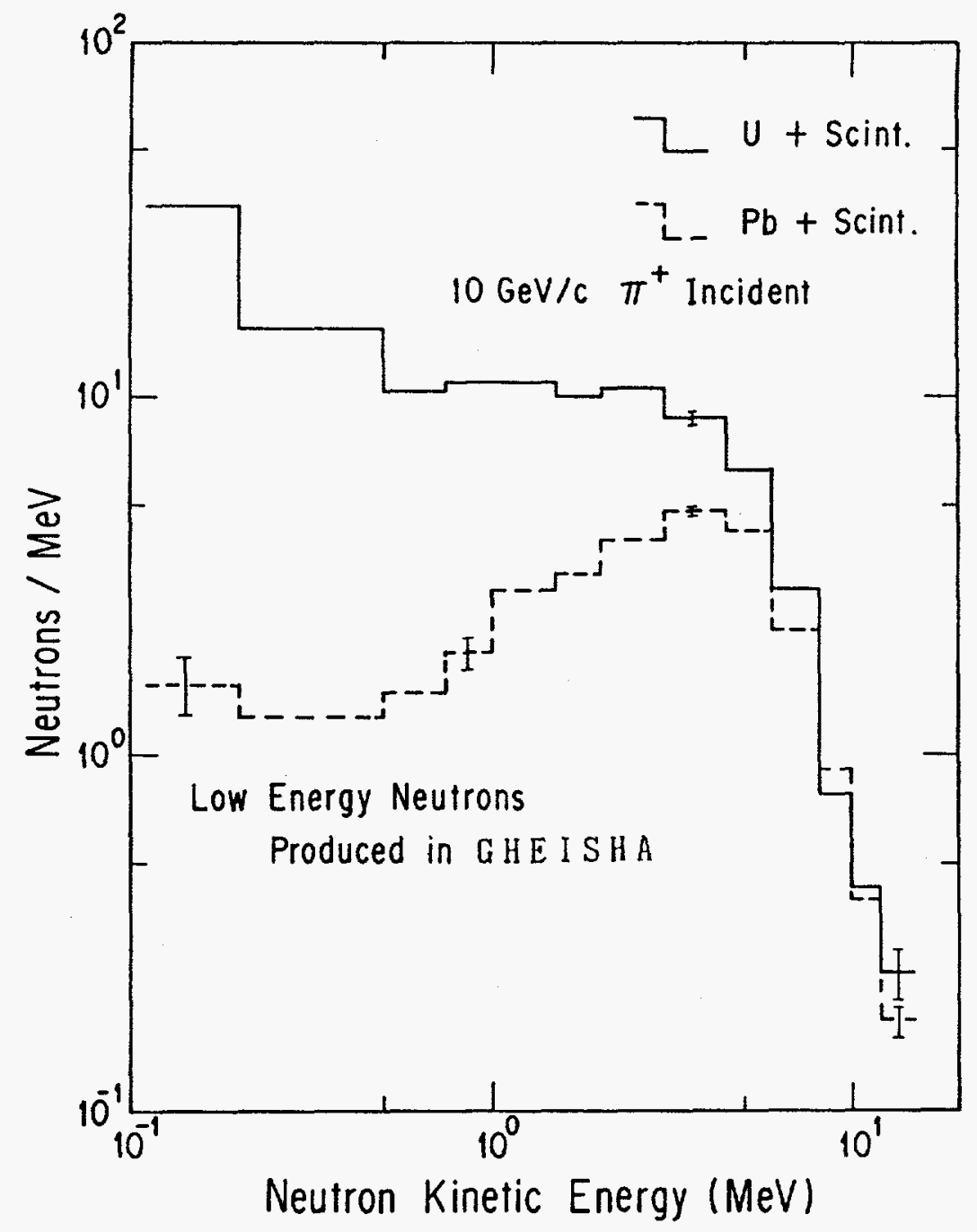

FIG. 2. Energy spectrum of produced low-energy neutrons. They were calculated using GHEISHA-7 (see Table 2). $10 \mathrm{GeV} / \mathrm{c} \pi^{+}$incident on U/Scint. and $\mathrm{Pb} /$ Scint. calorimeters. 


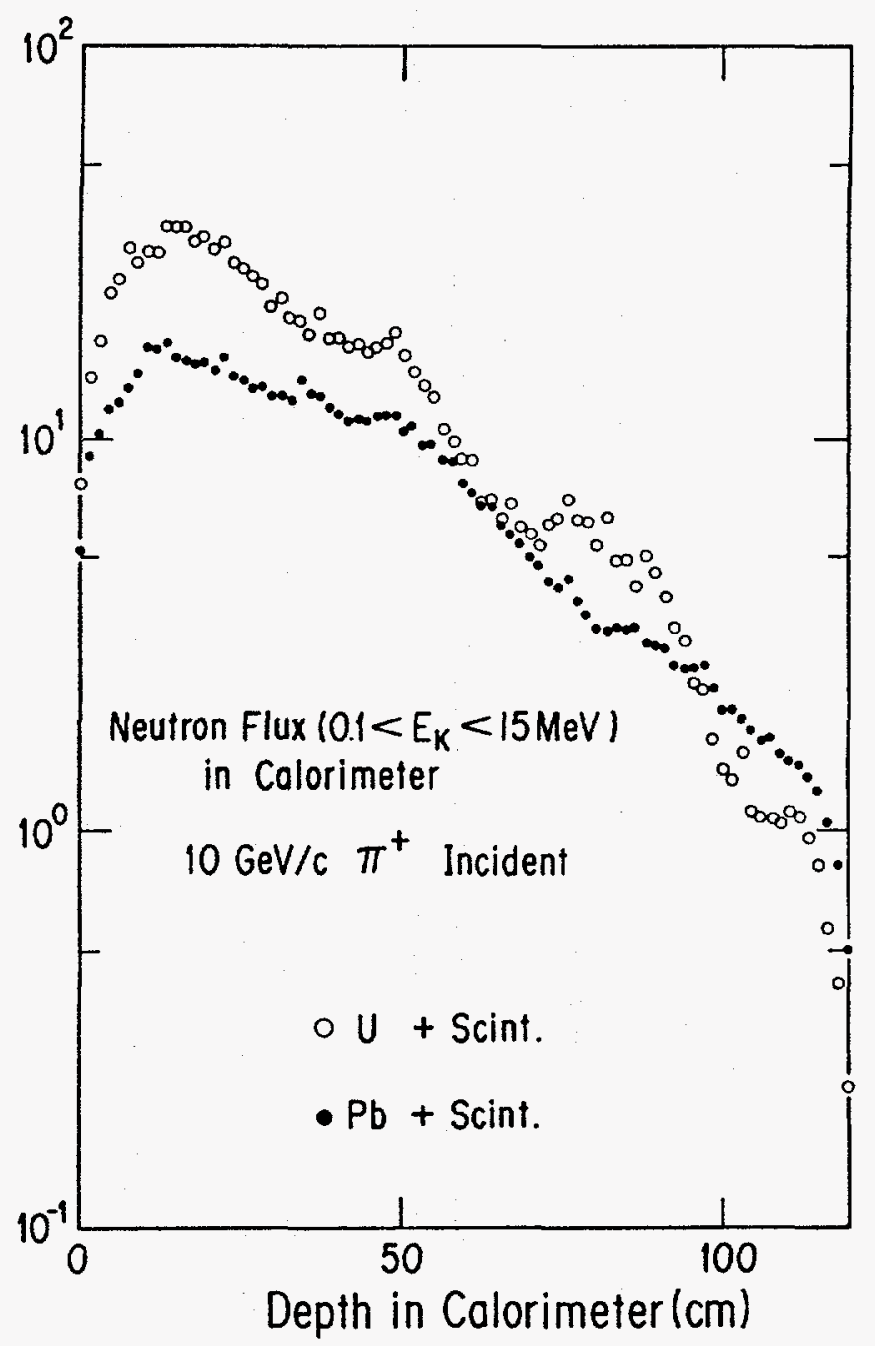

FIG. 3. Neutron fluxes distribution in the calorimeter calculated using ANISN in case of $10 \mathrm{GeV} / \mathrm{c} \pi^{+}$incident on $\mathrm{U} / \mathrm{scint}$. and $\mathrm{Pb} / \mathrm{Scint}$. These neutrons have energy between 0.1 and $15 \mathrm{MeV}$. 


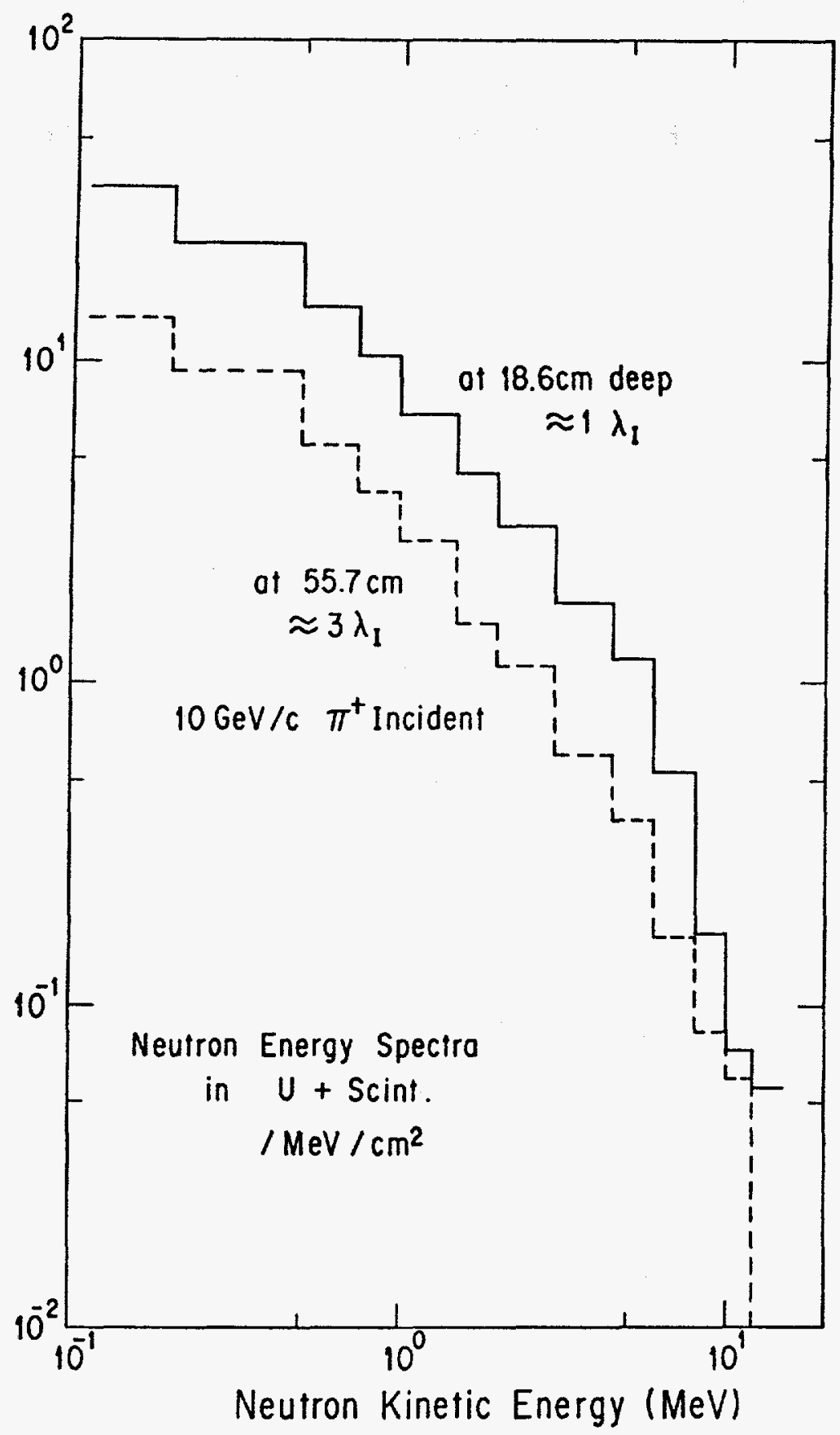

FIG. 4. Energy spectra of neutron fluxes in the calorimeter calculated using ANISN in case $10 \mathrm{GeV} / \mathrm{c} \pi^{+}$incident on U/scint. calorimeter. They are shown at the depth of one and three inelastic-interaction length. 


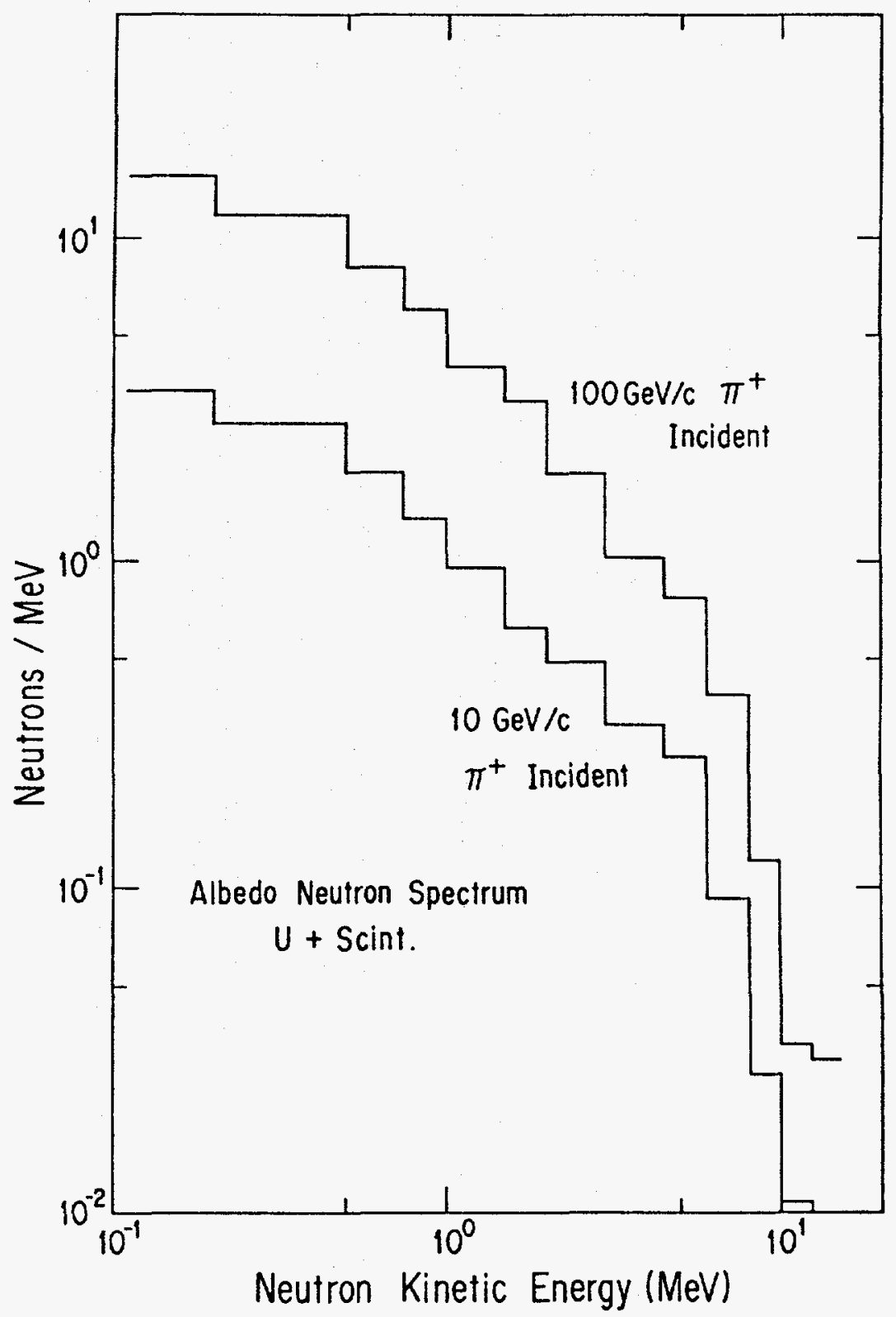

FIG. 5. Energy spectra of albedo neutron current calculated using ANISN in case of 10 and $100 \mathrm{GeV} / \mathrm{c} \pi^{+}$incident on $\mathrm{U} /$ scint. calorimeter. 


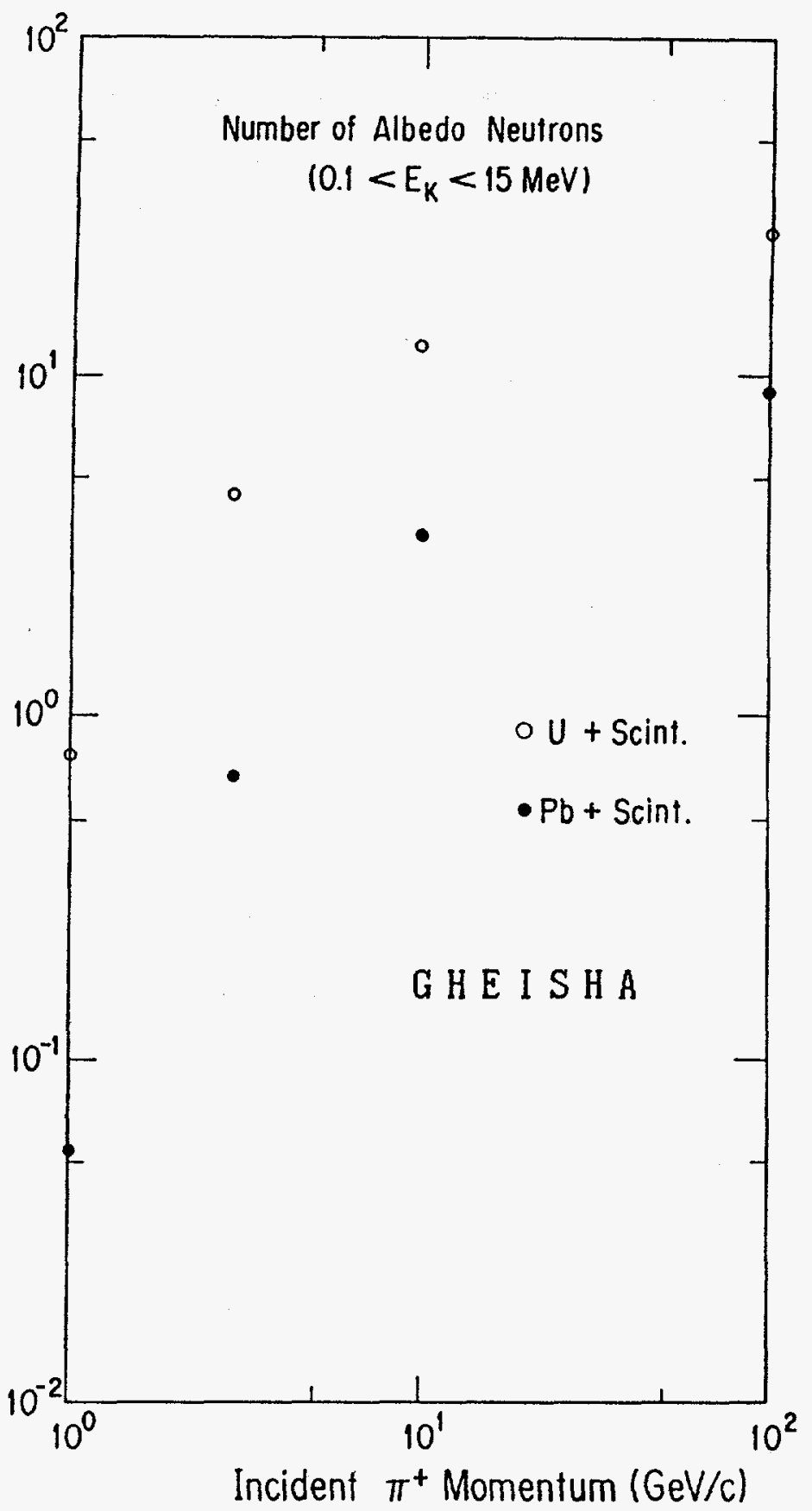

FIG. 6. Number of albedo neutrons in case of $\pi^{+}$incident. They were calculated using GHEISHA only (see case- 2 in Table 1 ). 


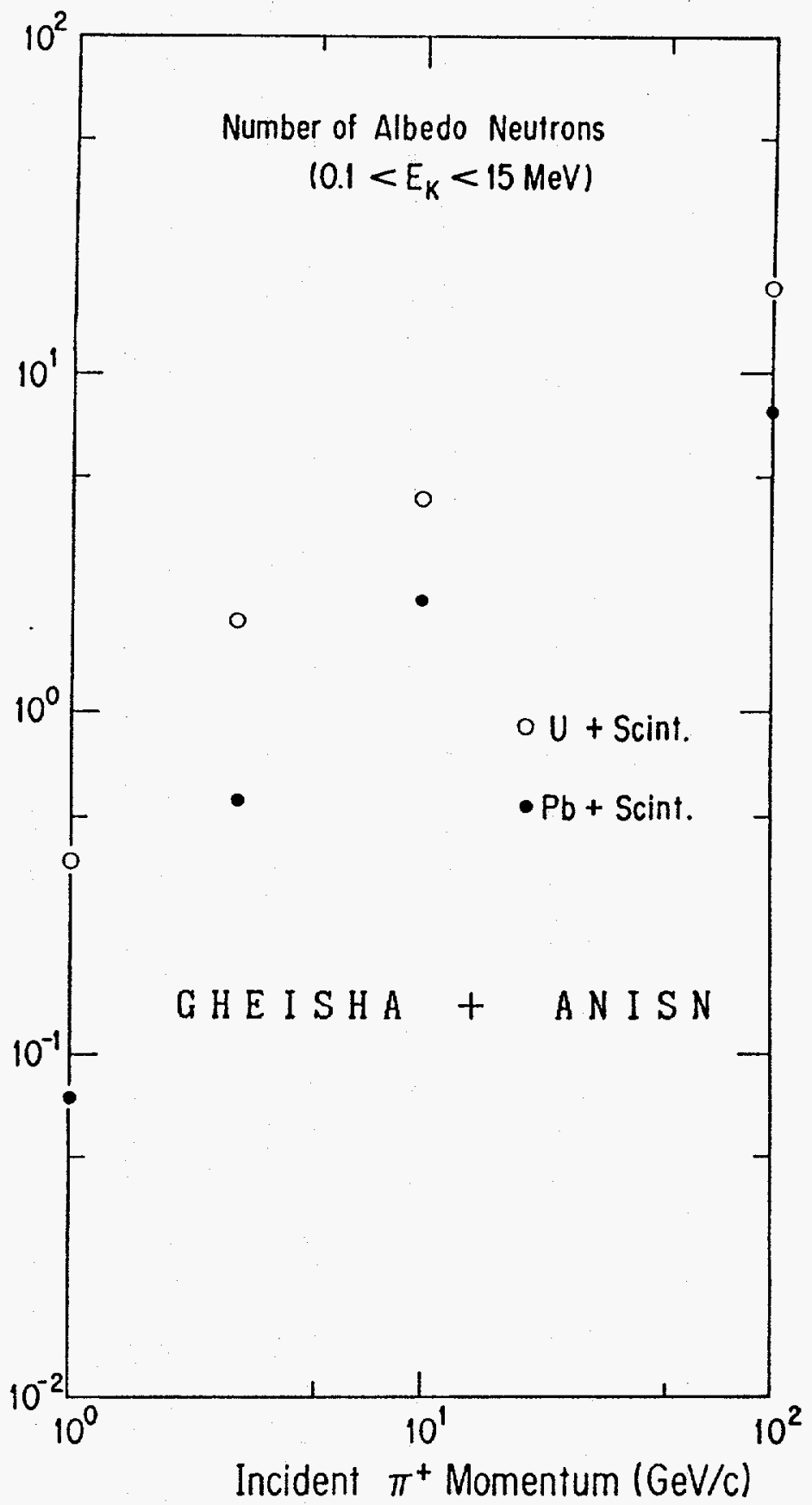

FIG. 7. Number of albedo neutrons in case of $10 \mathrm{GeV}$ proton incident on $\mathrm{U} / \mathrm{scint}$. calorimeter. 


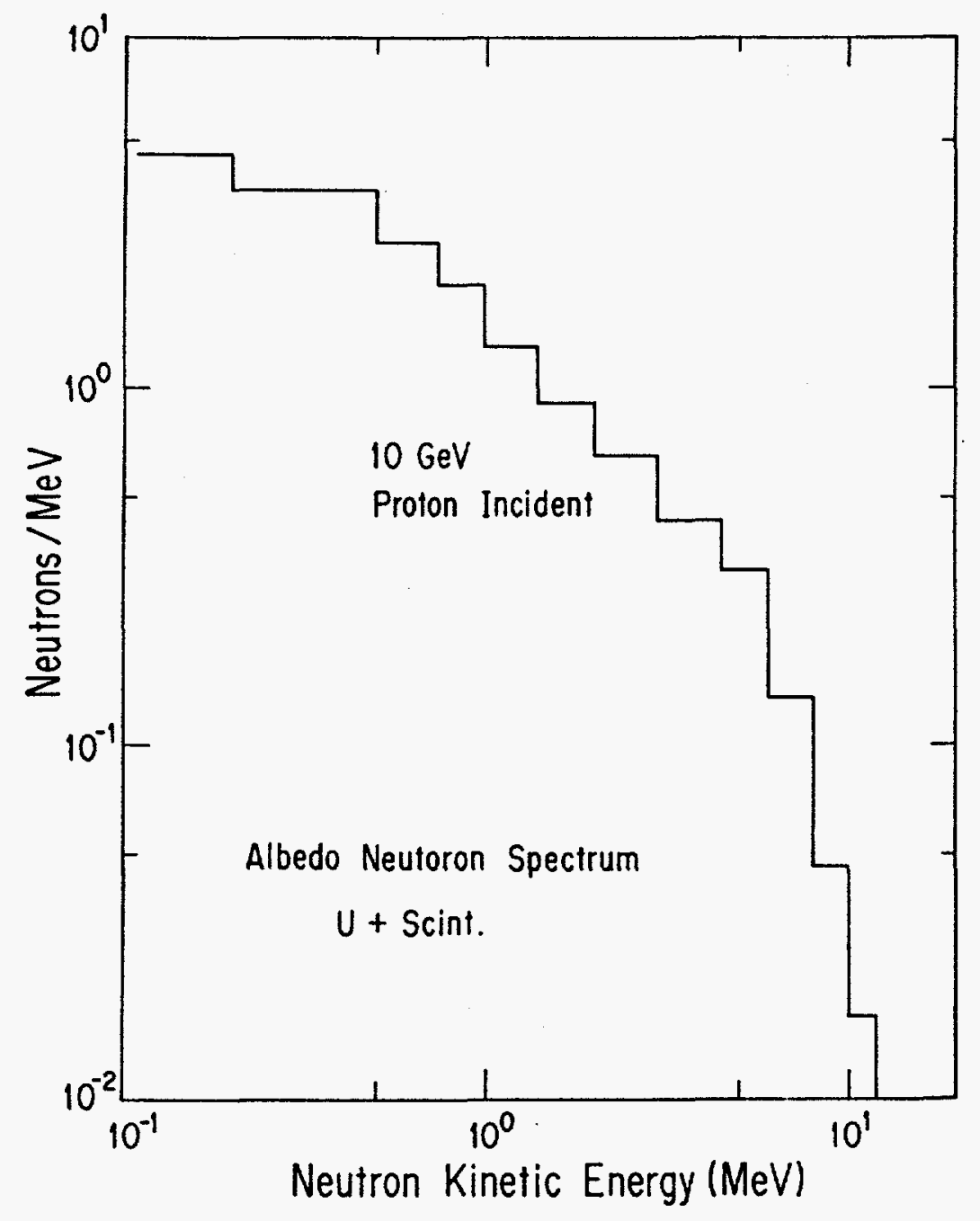

FIG. 8. Energy spectrum of albedo neutron current in case of $10 \mathrm{GeV}$ proton incident on $\mathrm{U} / \mathrm{scint}$. calorimeter. 


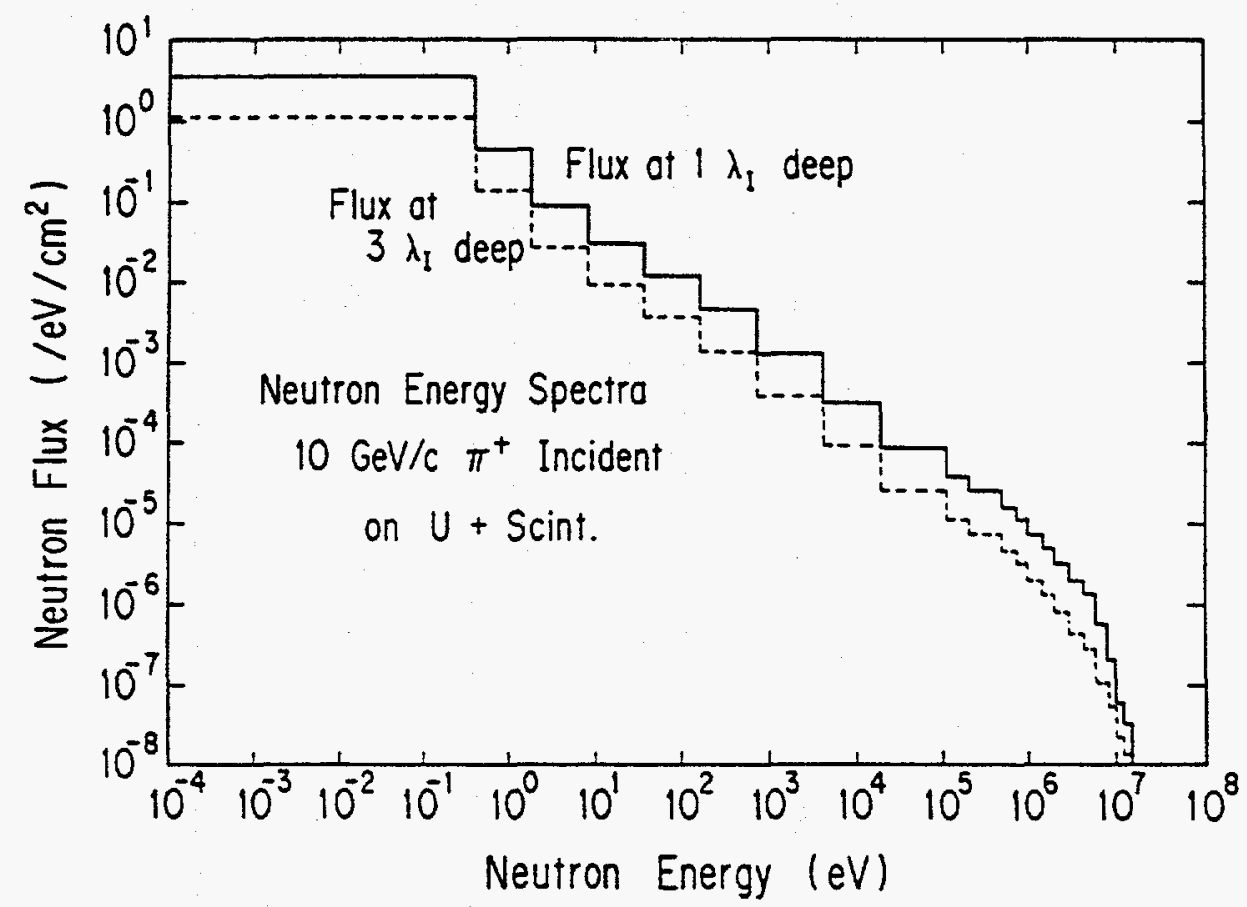

FIG. 9. Energy spectra of neutron fluxes down to thermal energy in the calorimeter calculated using anisin in case of $10 \mathrm{GeV} / \mathrm{c} \pi^{+}$incident on $\mathrm{U} / \mathrm{scint}$. calorimeter. They are shown at depths of one and three inelastic-interaction lengths.

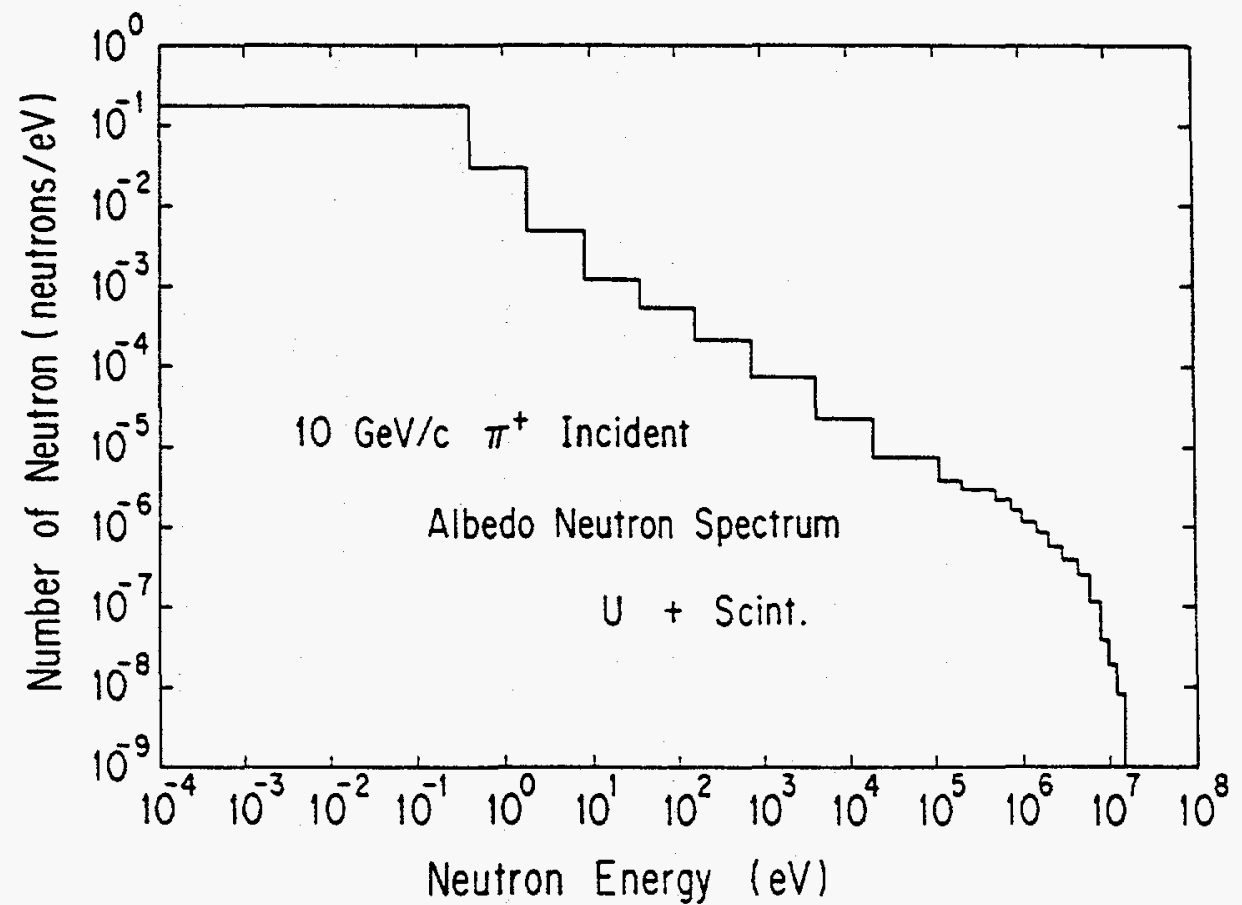

FIG. 10. Energy spectra of albedo neutron current down to thermal energy calculated using ANISN in case of 10 and $100 \mathrm{GeV} / \mathrm{c} \pi^{+}$incident on $\mathrm{U} /$ scint. calorimeter. 


\title{
APPENDIX 15 \\ CALCULATIONS OF THE NEUTRON FLUX IN IRON AND URANIUM CALORIMETERS
}

\author{
James E. Brau \\ University of Tennessee, Knoxville TN 37996 \\ T. A. Gabriel \\ Oak Ridge National Laboratory, Oak Ridge TN 37830
}

\section{Introduction}

A series of calculations have been performed to determine the expected neutron rates from iron and uranium calorimeters. The sampling media studied are silicon and scintillator. The Oak Ridge Monte Carlo, CALOR, has been used in this study. Details of the calculations are contained in Ref. 1. The detailed description of the hadronic showers comes from the high energy transport code, HETC. This code employs the MORSE neutron transport code to follow the details of the neutron propagation below $20 \mathrm{MeV}$. For these studies the neutrons were cut off at $25 \mathrm{keV}$.

\section{Comparison with Experimental Measurements}

Leroy, Sirois, and Wigmans [2] have measured the concentration of fission products within various calorimeter stacks. From these concentrations they deduce the fission density per hadronic shower. Two of the stacks studied were a massive depleted uranium stack and a uranium/scintillator stack consisting of 3 $\mathrm{mm}$ uranium sheets interleaved with $2.5 \mathrm{~mm}$ scintillator sheets. These stacks had a cross section of $20 \mathrm{~cm} \times 120 \mathrm{~cm}$ in each case. Table 1 gives the reported fissions/ $591 \mathrm{MeV}$ incident proton for these two configurations. We have simulated nearly these stacks by modeling the $20 \mathrm{~cm} \times 120 \mathrm{~cm}$ massive uranium stack and a $20 \mathrm{~cm} \times 120 \mathrm{~cm}$ stack of $3 \mathrm{~mm}$ depleted uranium and $3 \mathrm{~mm}$ scintillator. 1000 incident protons have been generated for each calorimeter. Transport of neutrons has been stopped at $25 \mathrm{keV}$. We have calculated with CALOR the expected longitudinal distribution of fissions by folding the calculated neutron flux spectrum from $1 \mathrm{MeV}$ to $20 \mathrm{MeV}$ with the ${ }^{238} \mathrm{U}$ fission cross section at several depths in the calorimeter. Fig. 1 shows the longitudinal distribution of the fissions. The fissions per $\lambda_{\text {int }}$ are more than a factor of two fewer in the uranium/scintillator stack. This results from the neutron flux reduction from the large energy loss of neutrons in their collisions with hydrogen in the scintillator. Fig. 2 shows the result of extending the calculations for calorimeters with unlimited tranverse extent. The fission densities increase slightly with the largest increases appearing at the greater depths as expected. 
Table 1

Neutron induced fissions and albedo fluxes. The numbers are per incident $591 \mathrm{MeV}$ proton. Neutrons with energies greater than 20 $\mathrm{MeV}$ are not included in the albedo case.

\begin{tabular}{|c|c|c|}
\hline & Massive uranium & $\mathrm{U} /$ scintillator \\
\hline $\begin{array}{l}\text { Experimentally } \\
\text { deduced fissions [2] }\end{array}$ & $3.8 \pm 0.4$ & $3.0 \pm 0.4$ \\
\hline Monte Carlo fissions & $4.1 \pm 0.3$ & $2.3 \pm 0.2$ \\
\hline $\begin{array}{l}\text { Monte Carlo fissions } \\
\qquad\left(E_{n}<20 \mathrm{MeV}\right)\end{array}$ & $3.3 \pm 0.2$ & $1.4 \pm 0.1$ \\
\hline $\begin{array}{c}\text { Monte Carlo albedo } \\
20 \mathrm{~cm} \times 120 \mathrm{~cm} \\
\left(1 \mathrm{MeV}<E_{n}<20 \mathrm{MeV}\right)\end{array}$ & 5.6 & 3.1 \\
\hline $\begin{array}{c}\text { Monte Carlo albedo } \\
20 \mathrm{~cm} \times 120 \mathrm{~cm} \\
\left(E_{n}>25 \mathrm{keV}\right)\end{array}$ & 15.4 & 5.3 \\
\hline $\begin{array}{c}\text { Monte Carlo albedo } \\
\text { (no transverse restriction; } \\
E_{n}>25 \mathrm{keV} \text { ) }\end{array}$ & 22.5 & 6.1 \\
\hline
\end{tabular}

Table 1 compares the experimentally determined number of fissions with the fission sums computed directly by counting the number of fissions in MORSE (as opposed to the above fission density calculations). The MORSE sums agree with the integrals of the curves shown on Fig. 1. Neutron induced fissions for neutron energies greater than $20 \mathrm{MeV}$ are not included in the albedo case and are expected to contribute approximately ten percent, based on previous calculations [3] at this energy. The fission sums agree well in the case of massive uranium while the calculated uranium/scintillator results are somewhat lower than the experimental results. The difference in the sums for the simulations of the two stacks can be traced directly to the depletion of neutron energy by the hydrogen in the scintillator. Neutron fluxes simply are not as great when the hydrogenous medium is introduced due to the interaction of the neutrons with the medium.

Table 1 further tabulates the results of calculations of neutron albedos for these two stacks. First the albedo of neutrons with energy in excess of $1 \mathrm{MeV}$ are presented. These are the numbers which are most directly extracted from the experimental data. Then we present the neutron albedo flux for all neutrons 
between $25 \mathrm{keV}$ and $20 \mathrm{MeV}$. Finally we show the effect of extending the transverse dimension of the calorimeter since the stacks of $20 \mathrm{~cm} \times 120 \mathrm{~cm}$ represent a somewhat optimistic estimate of the total albedo flux.

\section{Calorimeters}

Calculations have been completed for $400 \mathrm{MeV}, 3 \mathrm{GeV}$, and $20 \mathrm{GeV}$ incident $\pi^{-}$'s on four calorimeter configurations. The calorimeters modeled have the following basic cell structures:

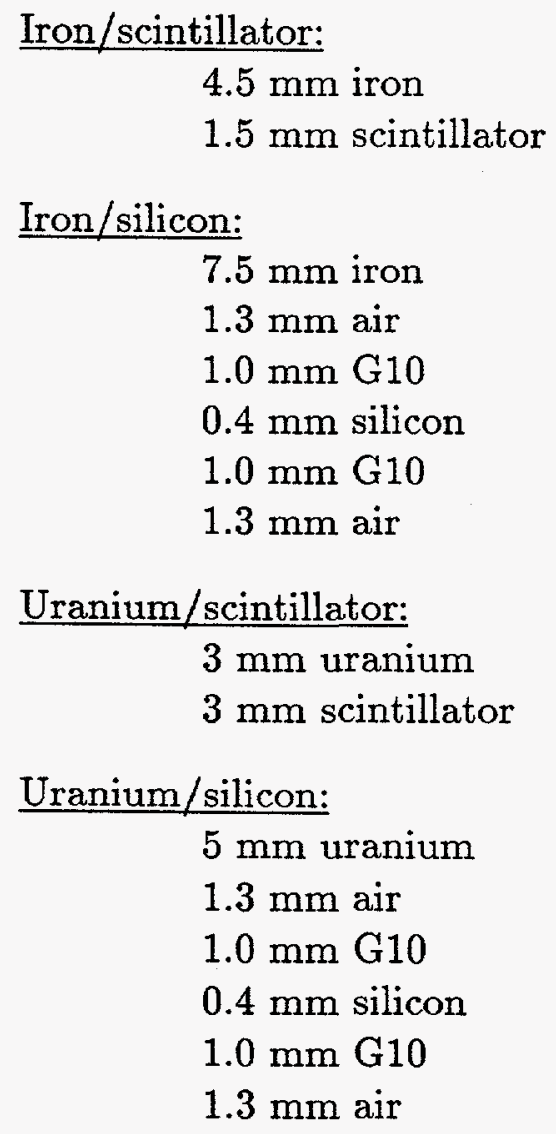

$7.5 \mathrm{~mm}$ iron

$1.3 \mathrm{~mm}$ air

$1.0 \mathrm{~mm} \mathrm{G10}$

$0.4 \mathrm{~mm}$ silicon

$1.0 \mathrm{~mm} \mathrm{G10}$

$1.3 \mathrm{~mm}$ air

Uranium/scintillator:

$3 \mathrm{~mm}$ uranium

$3 \mathrm{~mm}$ scintillator

Uranium/silicon:

$5 \mathrm{~mm}$ uranium

$1.3 \mathrm{~mm}$ air

$1.0 \mathrm{~mm} \mathrm{G10}$

$0.4 \mathrm{~mm}$ silicon

$1.0 \mathrm{~mm} \mathrm{G10}$

$1.3 \mathrm{~mm}$ air

\section{Results}

The number of incident $\pi^{-}$'s calculated for each case is shown in Table 2.

The results are summarized in the attached Figs. 3-12. As above, all neutrons have been cut off at $25 \mathrm{keV}$ and the fluxes presented are for neutrons in the range $25 \mathrm{keV}<E_{n}<20 \mathrm{MeV}$. All neutron fluxes presented have been obtained by using the boundary crossing estimator. This involves weighting each neutron boundary crossing by $1 /|\cos \theta|$. 
Table 2

Number of incident $\pi^{-}$'s.

\begin{tabular}{cccc}
\hline \hline & $400 \mathrm{MeV}$ & $3 \mathrm{GeV}$ & $20 \mathrm{GeV}$ \\
\hline $\mathrm{Fe} / \mathrm{scint}$ & 1000 & 1000 & 200 \\
$\mathrm{Fe} /$ silicon & 1000 & 750 & 150 \\
$\mathrm{U} / \mathrm{scint}$ & 1000 & 1000 & 200 \\
$\mathrm{U} /$ silicon & 1000 & 400 & 75 \\
\hline
\end{tabular}

Figs. 3 through 6 show the longitudinal neutron flux development for each of the calorimeters at each incident $\pi^{-}$energy.

Figs. 7 through 10 present the spectra for each case, showing the distributions off the front surface (albedo) and at the maximum of the neutron shower development.

Fig. 11 displays the maximum neutron flux for each case.

Fig. 12 shows the albedo flux for each case.

\section{References}

1. J. E. Brau and T. A. Gabriel, Nucl. Inst. and Meth. A238, 489 (1985).

2. C. Leroy, Y. Sirois, and R. Wigmans, Nucl. Inst. and Meth. A252, 4 (1986).

3. T. A. Gabriel, Nucl. Inst. and Meth. 150, 145 (1978). 


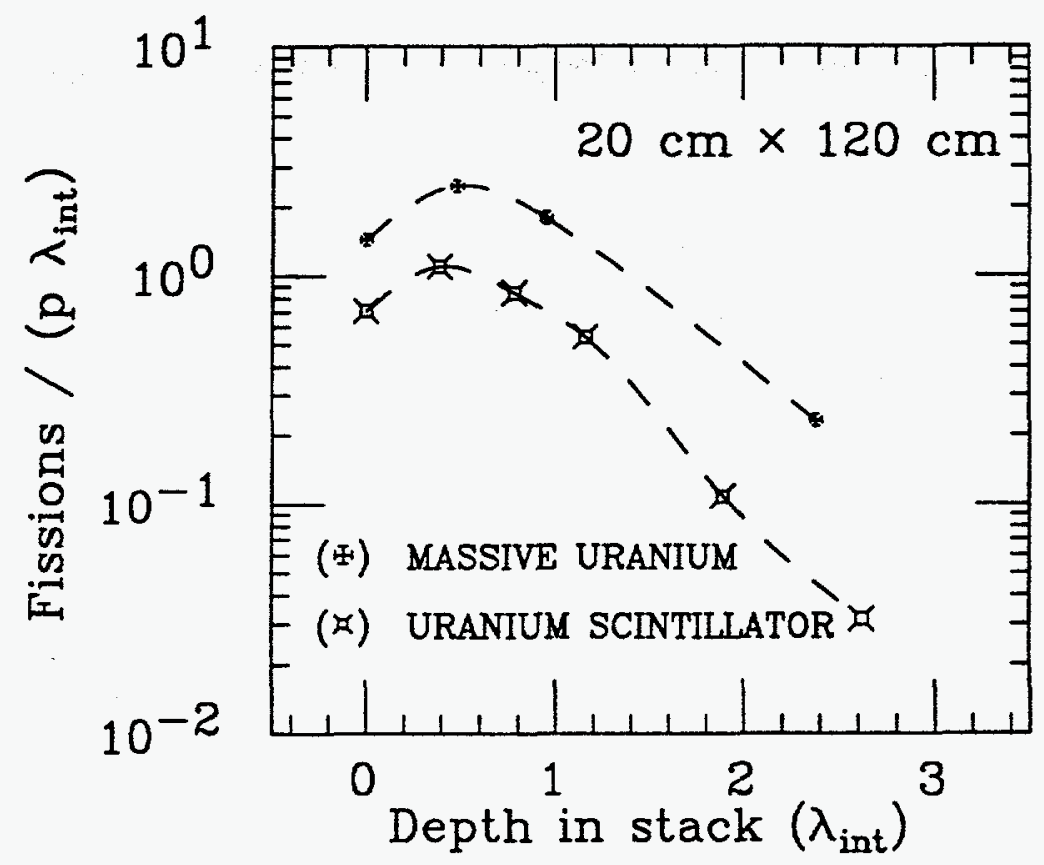

FIG. 1. Calculated longitudinal distribution of fissions in uranium and uranium/scintillator calorimeters for normally incident $591 \mathrm{MeV}$ protons. The calorimeter has a $20 \mathrm{~cm} \times 120 \mathrm{~cm}$ transverse section.

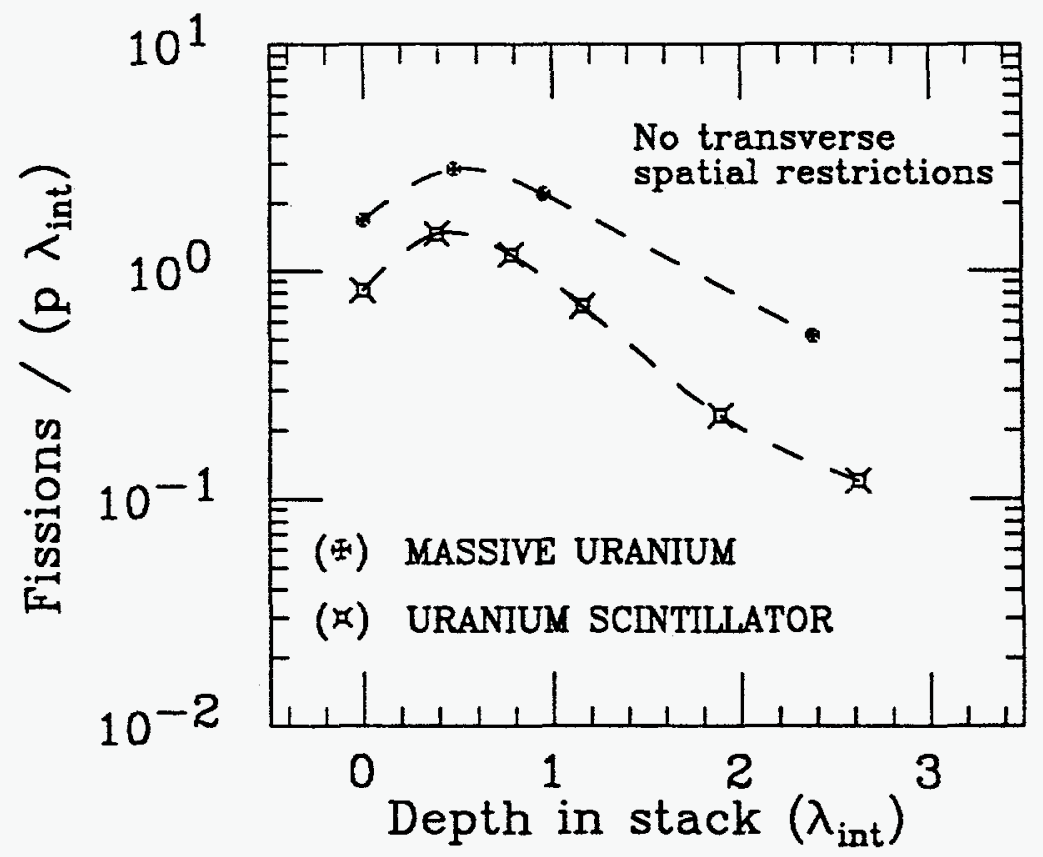

FIG. 2. Same as Fig. 1, except that the calorimeters have unlimited transverse extent. 


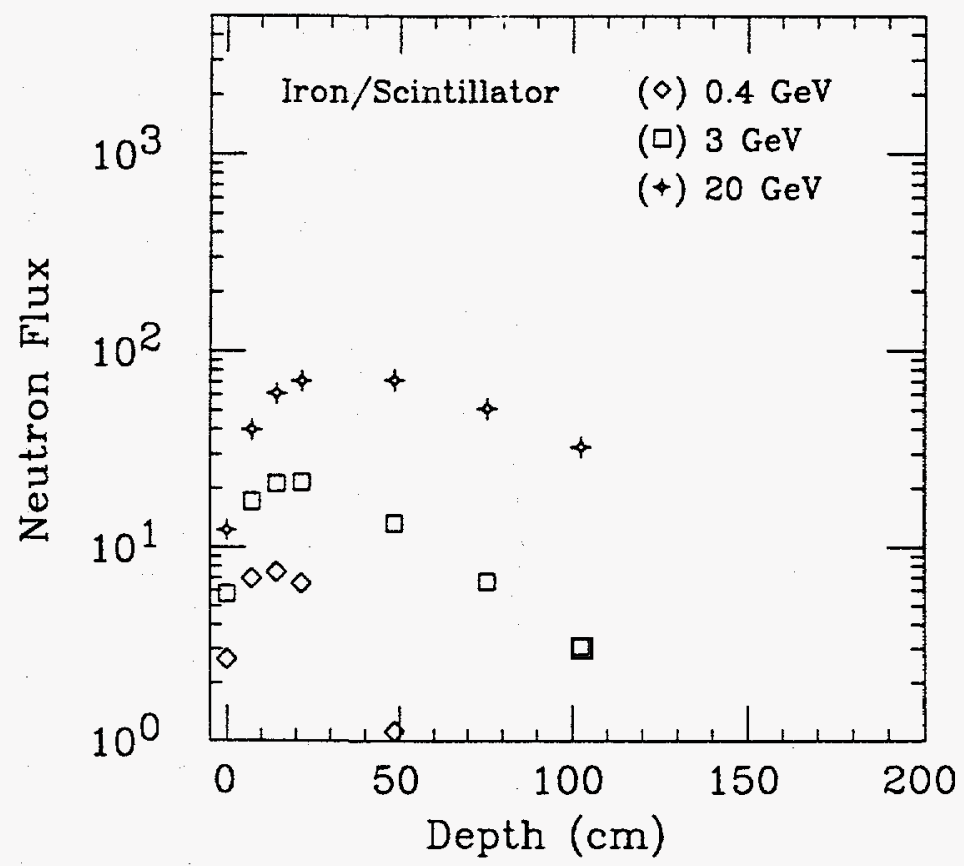

FIG. 3. Longitudinal neutron flux development in the iron/scintillator calorimeter at $0.4 \mathrm{GeV}, 3 \mathrm{GeV}$, and $20 \mathrm{GeV}$.

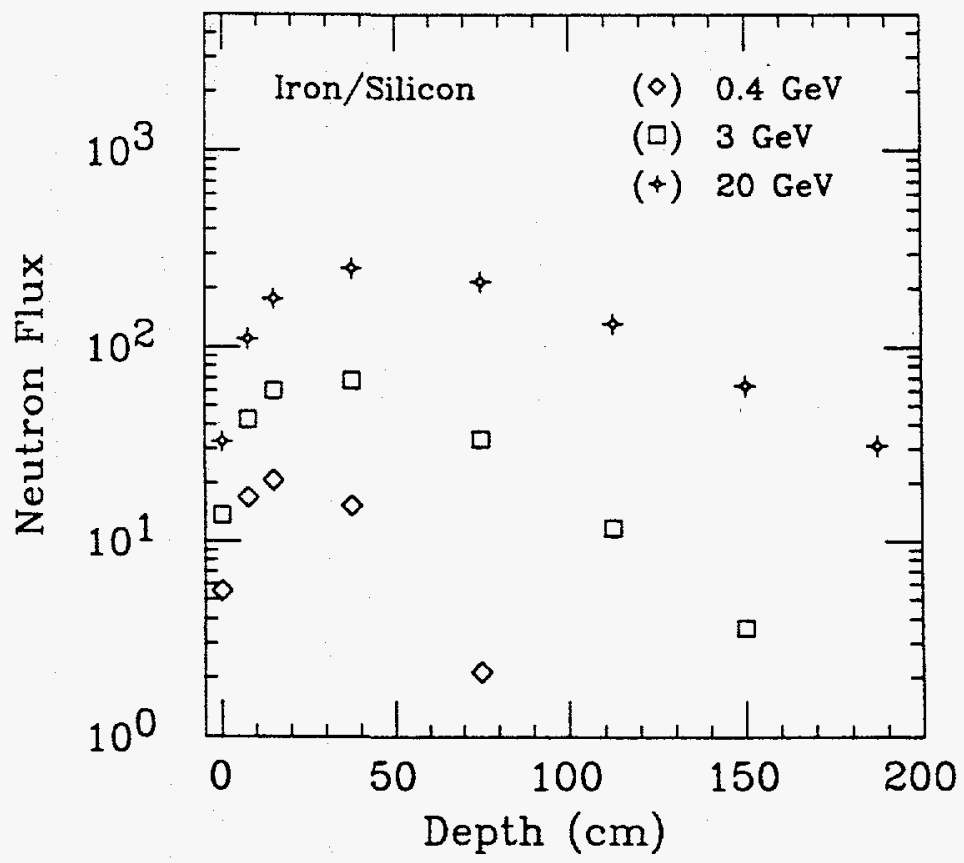

FIG. 4. Longitudinal neutron flux development in the iron/silicon calorimeter at 0.4 $\mathrm{GeV}, 3 \mathrm{GeV}$, and $20 \mathrm{GeV}$. 


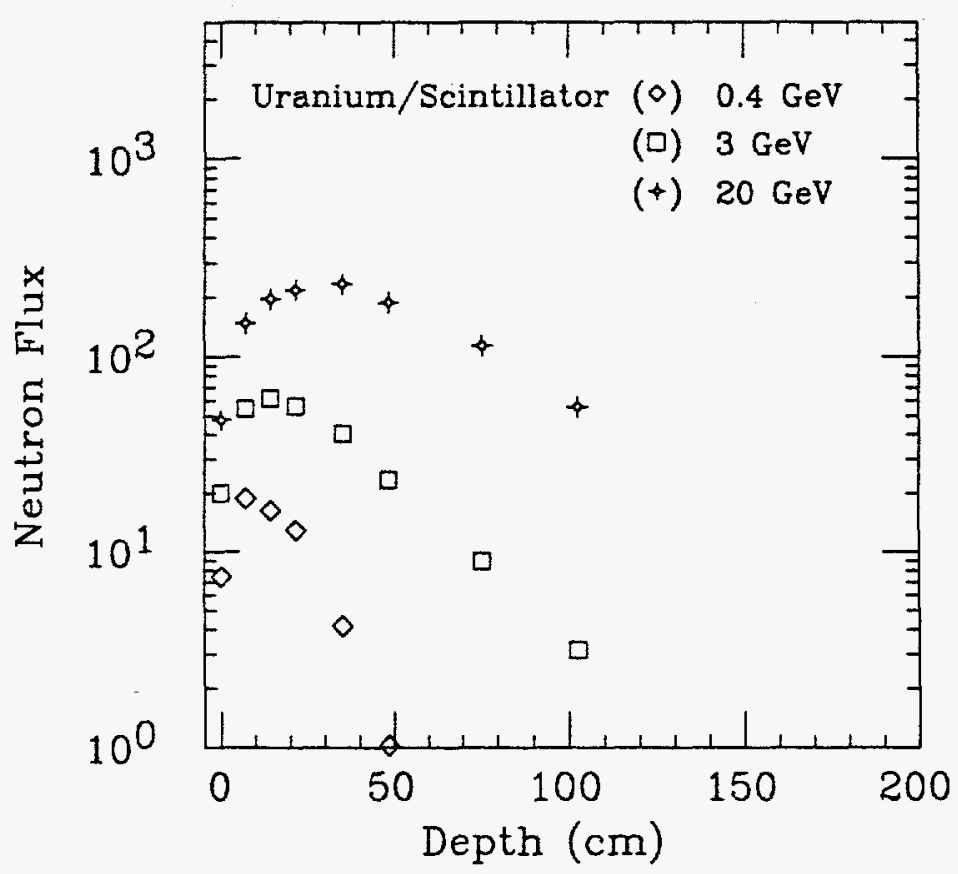

FIG. 5. Longitudinal neutron flux development in the uranium/scintillator calorimeter at $0.4 \mathrm{GeV}, 3 \mathrm{GeV}$, and $20 \mathrm{GeV}$.

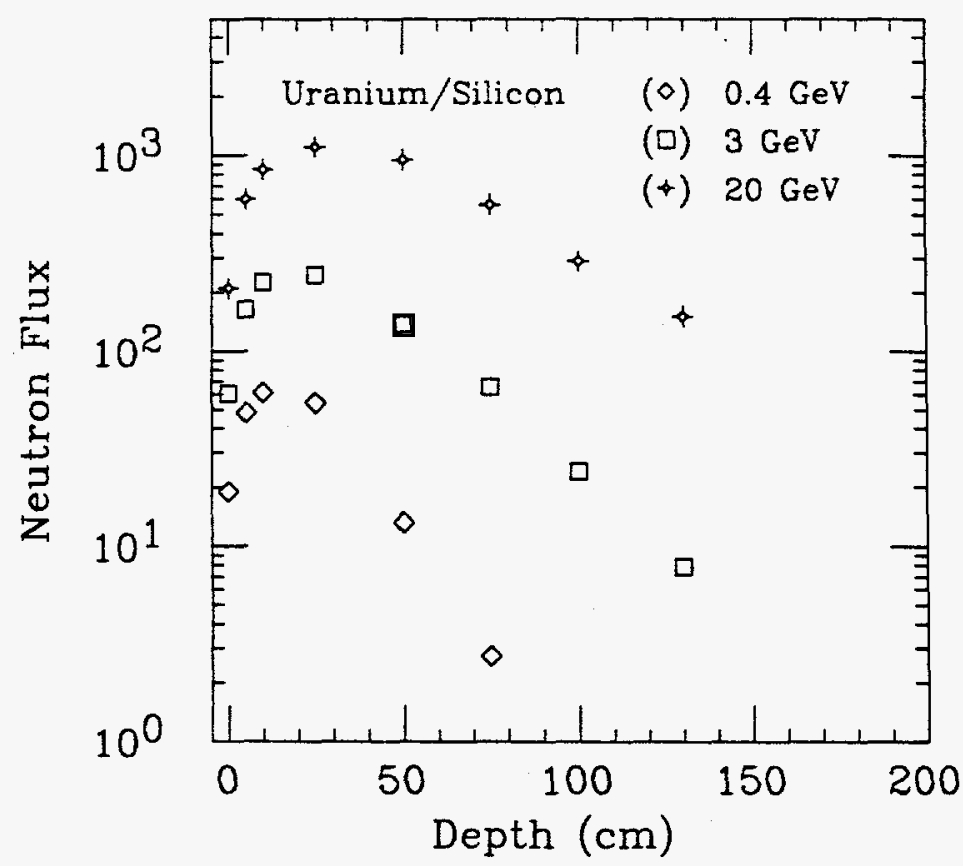

FIG. 6. Longitudinal neutron flux development in the uranium/silicon calorimeter at $0.4 \mathrm{GeV}, 3 \mathrm{GeV}$, and $20 \mathrm{GeV}$. 


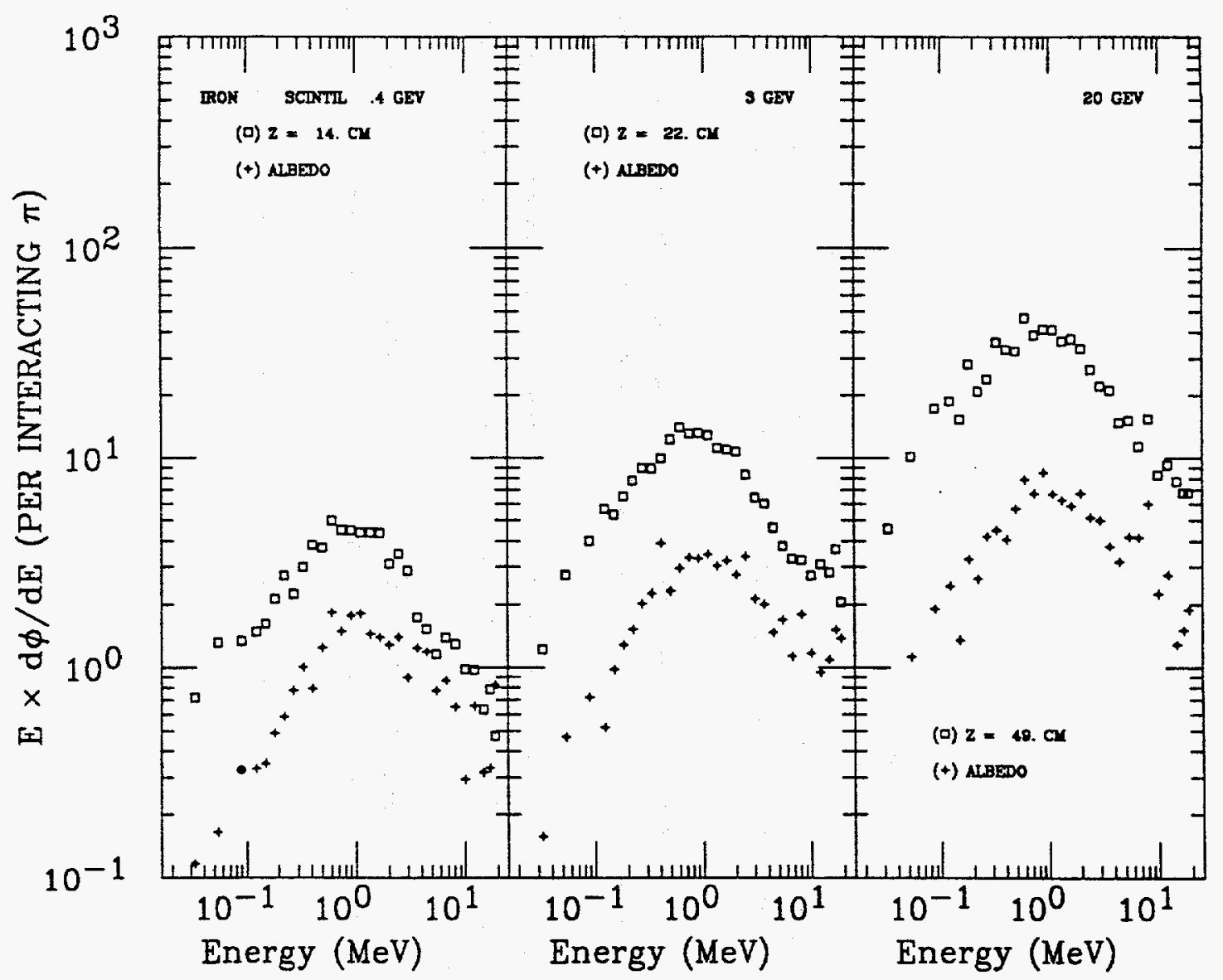

FIG. 7. Neutron spectra at the shower development peak and of albedo neutrons for the iron/scintillator calorimeter at $0.4 \mathrm{GeV}, 3 \mathrm{GeV}$, and $20 \mathrm{GeV}$. 


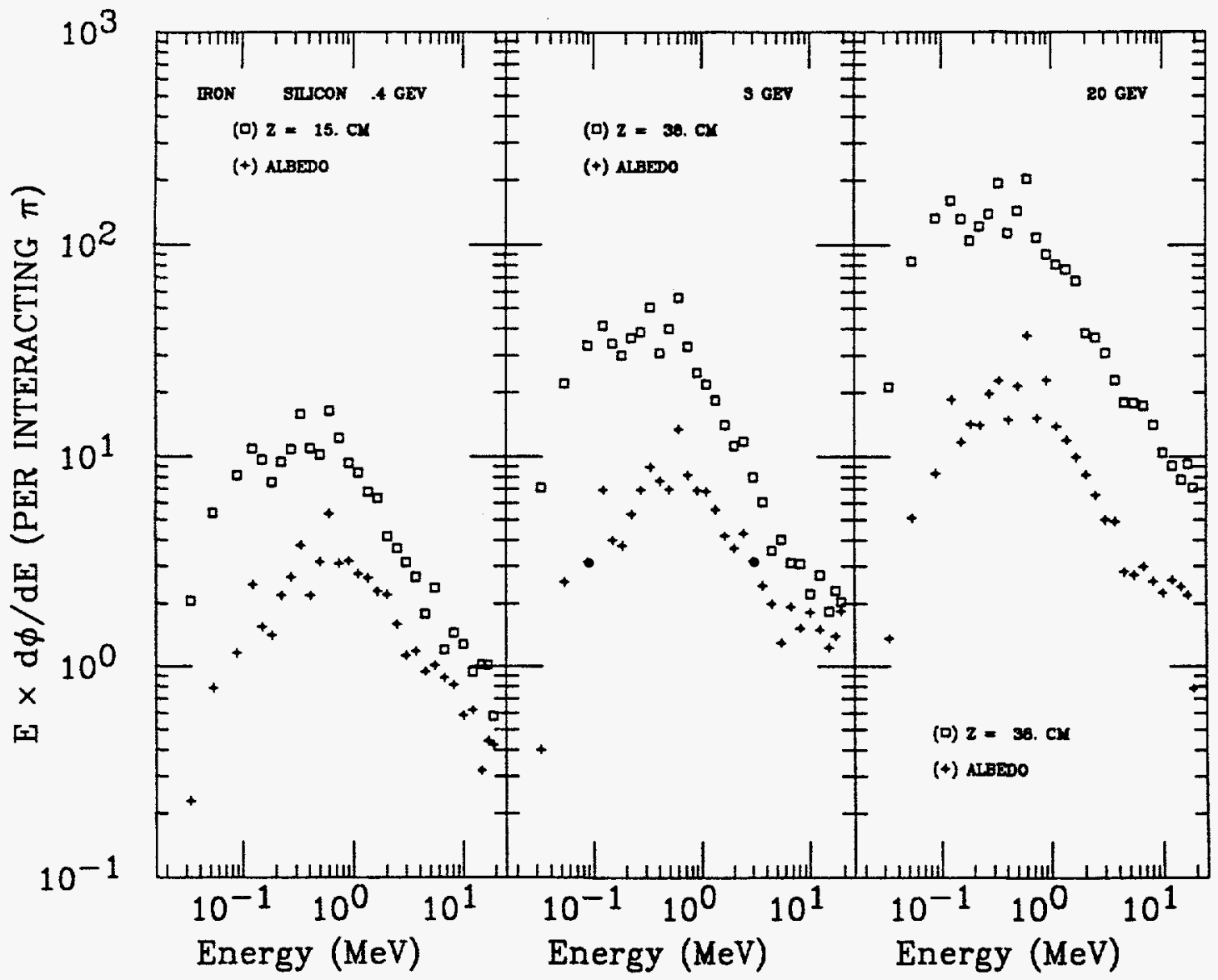

FIG. 8. Neutron spectra at the shower development peak and of albedo neutrons for the iron/silicon calorimeter at $0.4 \mathrm{GeV}, 3 \mathrm{GeV}$, and $20 \mathrm{GeV}$. 


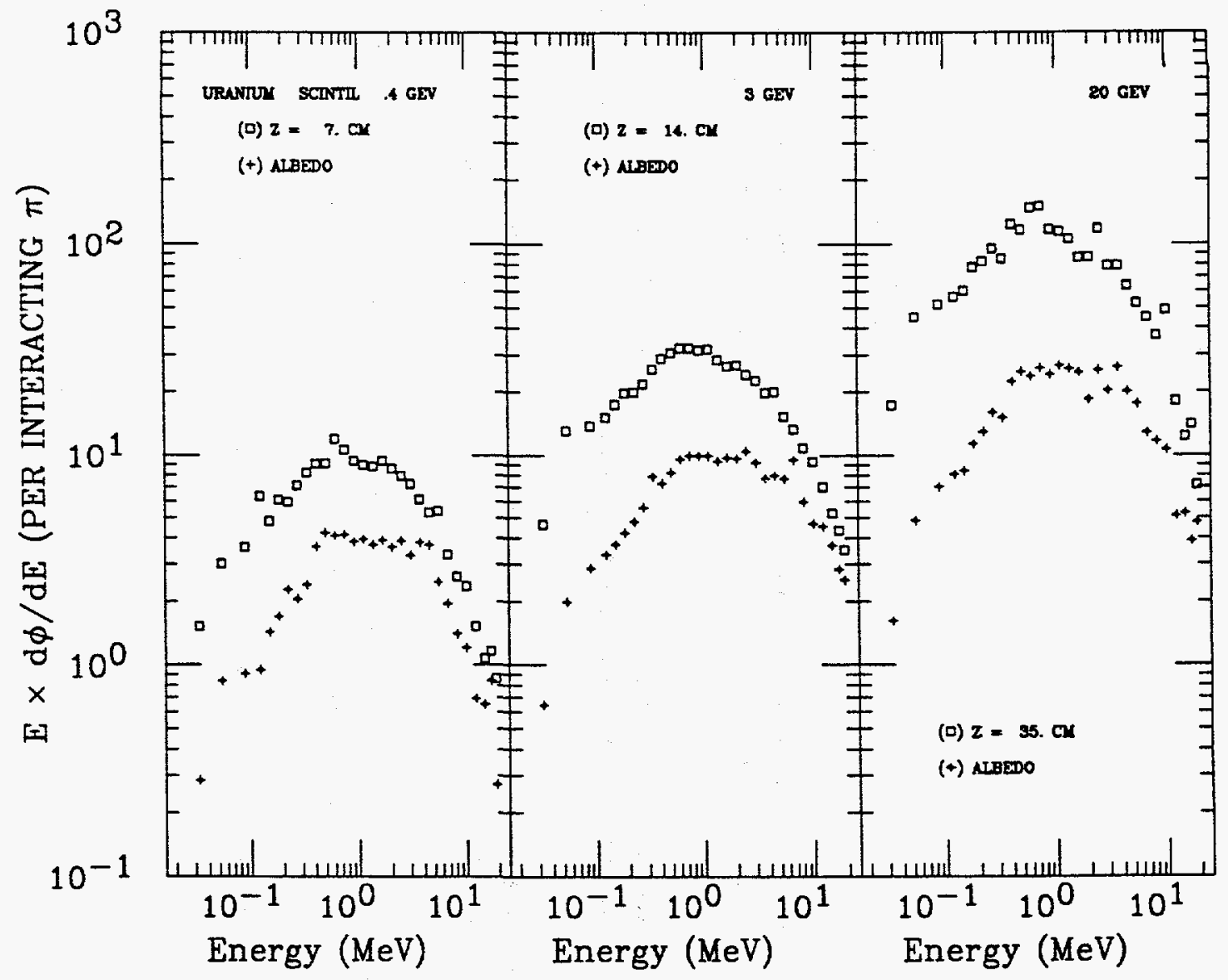

FIG. 9. Neutron spectra at the shower development peak and of albedo neutrons for the uranium/scintillator calorimeter at $0.4 \mathrm{GeV}, 3 \mathrm{GeV}$, and $20 \mathrm{GeV}$. 


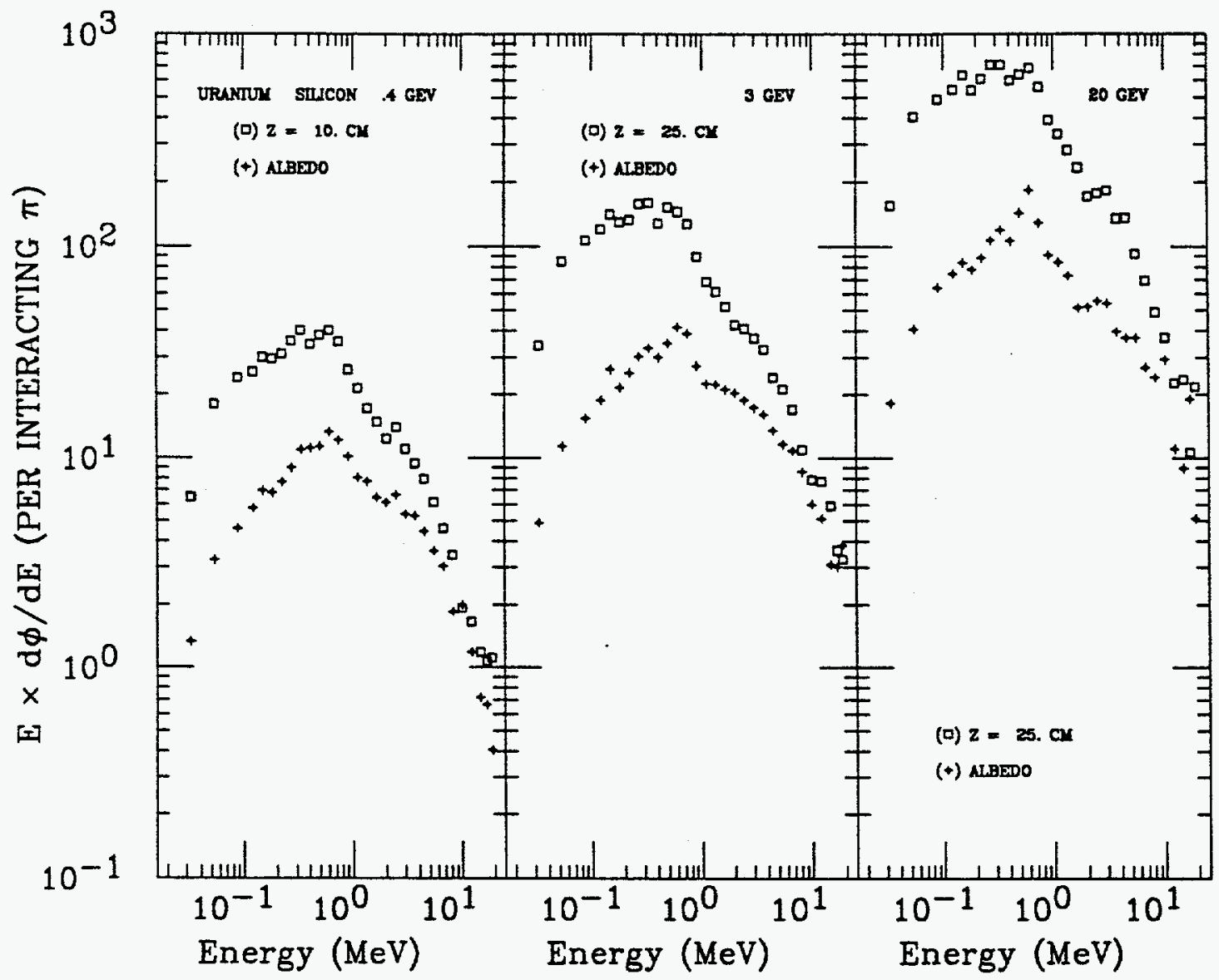

FIG. 10. Neutron spectra at the shower development peak and of albedo neutrons for the uranium/silicon calorimeter at $0.4 \mathrm{GeV}, 3 \mathrm{GeV}$, and $20 \mathrm{GeV}$. 


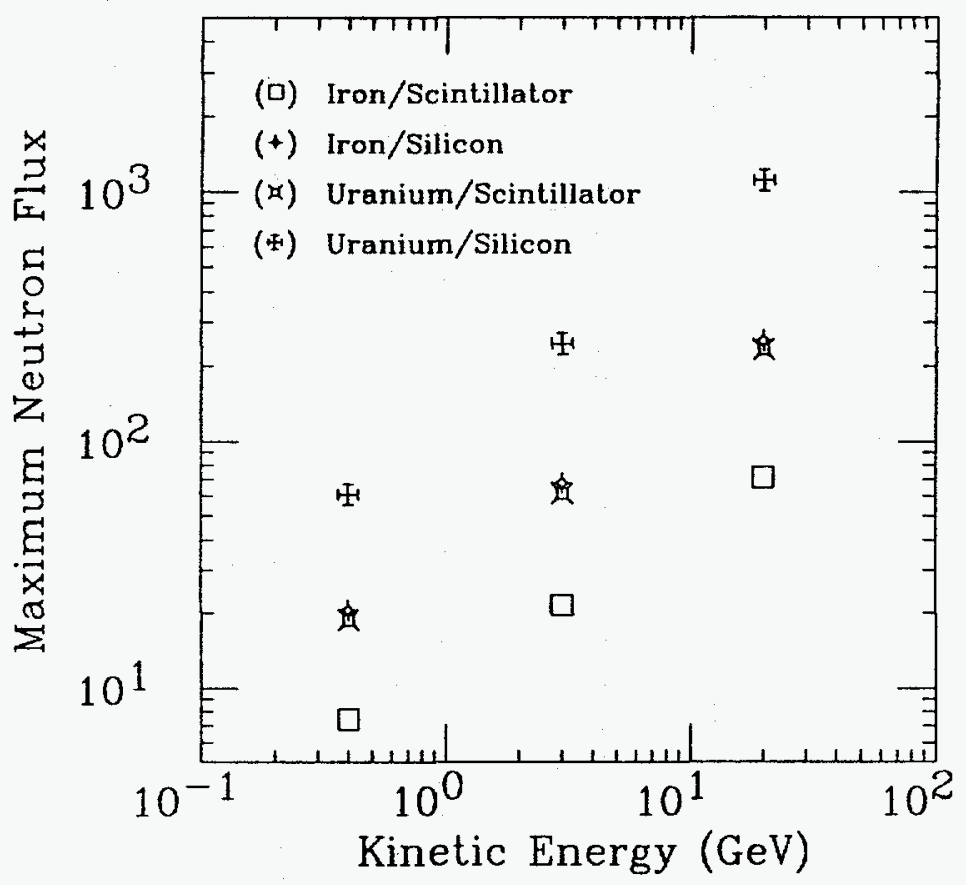

FIG. 11. Maximum neutron flux in the calorimeters as a function of energy.

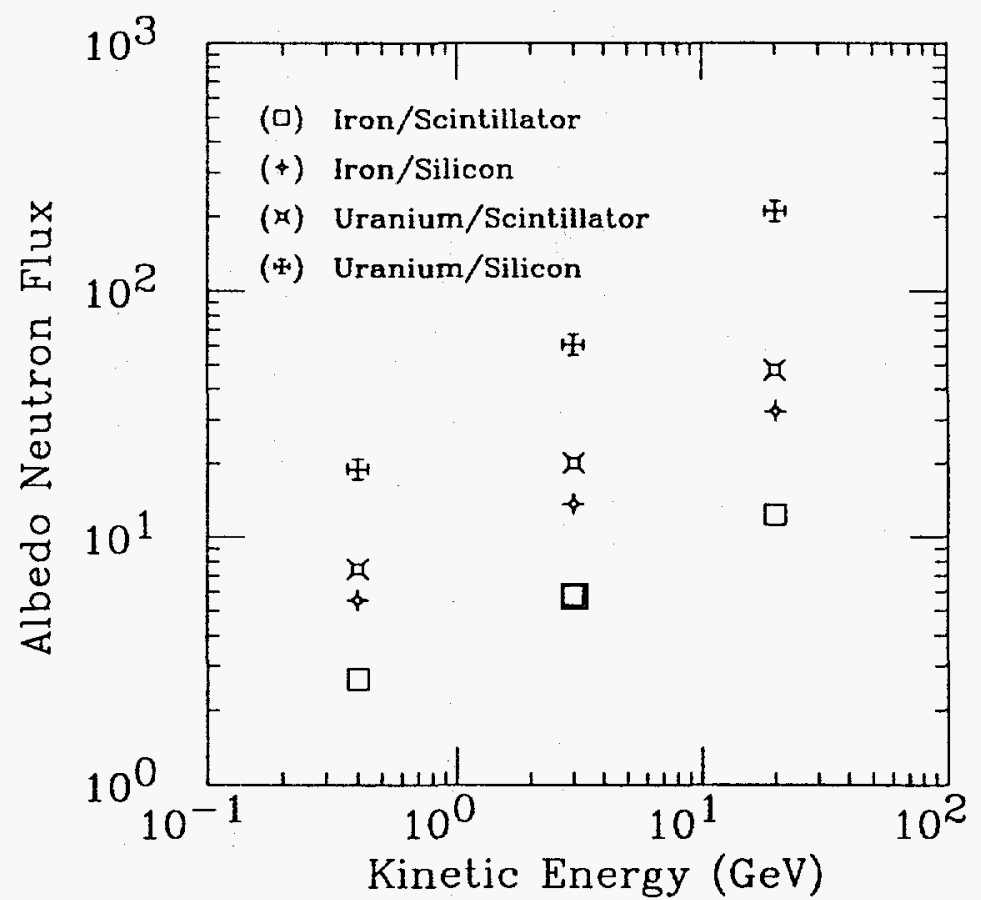

FIG. 12. Albedo neutron flux in the calorimeters as a function of energy. 


\title{
APPENDIX 16 \\ NEUTRON YIELDS IN HADRON CALORIMETERS \\ Harm Fesefeldt \\ III. Physikalisches Institut, Technische Hochschule Aachen, D5100 Aachen, Germany
}

\begin{abstract}
Neutron fluxes and neutron energy spectra in various hadron calorimeters are discussed. Other neutron correlated quantities, like average numbers of spallations, fissions and neutron captures as function of the longitudinal shower depth, are compared with the neutron currents.
\end{abstract}

\section{Introduction}

Hadron calorimeters will play a central role in all experiments at the future high energy super colliders. One of the main goals will be to reconstruct jets in order to calculate the invariant mass of two partons from new heavy particles. It is evident that this requires small calorimeter cells and good energy resolutions. However, these very clear physical requirements must be confronted with the limitations from the intensive radiative background, expected e.g. at the SSC. To give a rough estimate, we expect in uranium calorimeters, at an event rate of $10^{8} \mathrm{~s}^{-1}$ and 30 produced neutrons per $\mathrm{GeV}$ absorbed energy [1], approximately $10^{12}-10^{14}$ neutrons per second. Thus radiation damage of the readout materials and the local analog electronics will be a serious problem. Furthermore, radiation damage may also influences the decisions for the construction of other nearby detector parts. Albedo at the front of the hadron calorimeter and leakage at the end may destroy the performance of the vertex chamber and the muon chambers respectively. In this study we will present a quantitative analysis of the neutron flux and of some other related quantities for various types of calorimeters, for different kinds of particles with incident kinetic energies between 0.1 and 200 $\mathrm{GeV}$.

We begin in section 2 with a short summary of the used shower simulation program, the GHEISHA (version 8.02) code. In section 3 we summarize the model detectors, used in the present studies. We have concentrated on uranium calorimeters, but, since calorimeters with not fissionable materials as absorbers produce neutron fluxes one or two orders of magnitude smaller than uranium calorimeters, we will study also iron and lead calorimeters as alternatives. The neutron flux is naturally correlated with other quantities like the number of spallations, fissions and neutron captures per unit length. These quantities can be determined experimentally by measurements of the induced radioactivity [1]. The neutrons produce pulse heights by elastic and inelastic scattering with the atoms of the readout material. Thus there must be also a strong correlation between 
the neutron flux and the total pulse height. Neutron correlated quantities are discussed in section 4 . In section 5 a systematic study of the neutron flux at various locations inside and outside of the hadron calorimeters will be given. Whereas solid materials are extremely sensitive for high energetic neutrons $(\approx 1$ $\mathrm{MeV}$ ), wire chambers with hydrogenous gases may be damaged by neutrons with energies in the $\mathrm{keV}$ region. Consequently the energy spectrum of the neutrons must be considered too. A summary will be given in section 6 .

\section{The GHEISHA Shower Code}

In the GHEISHA shower code [2] three different types of inelastic hadron nucleus interactions are distinguished. Coherent and incoherent inelastic high energy interactions and low energy nuclear interactions of the types $A\left(N, N^{\prime}(\gamma)\right) A^{\prime}$, where $N, N^{\prime}$ are protons, neutrons or heavier particles. The coherent scattering is well understood in terms of the exchanged quanta. The incoherent reactions are separated into three phases, namely first a hadron interaction with one of the protons or neutrons of the nucleus, where the target nucleon is considered to be free, but smeared out in momentum space by Fermi motion, secondly a rapid development of an intranuclear cascade, where the final state particles of the first interaction may undergo further elastic and inelastic scattering with the downstream nucleons of the target nucleus, and finally the deexcitation of the thermally excited nucleus by a relatively slower process of evaporation. For fissionable elements nuclear fission must be considered as competing process to evaporation. The combined process of cascade and evaporation is sometimes called spallation. The evaporation is well separated in time from the cascading process, so one would not expect a strong correlation between them. Nevertheless the number of evaporated nucleons and nuclear fragments are measured to be proportional to the number of cascade particles, indicating that the temperature of the excited nucleus must be a function of the strength of the intranuclear cascade. The evaporation process is quite conventionally described by a thermodynamic model.

The first quasi free interaction and the following cascade scattering are simulated in a framework of a fragmentation model based on local quantum number conservation. For energies below $5 \mathrm{GeV}$ this model is partly replaced by a quasi two body resonance model, which represents a naive extrapolation of the reaction mechanisms known from hadron nucleon interactions to higher $A$ values. It is naive in the sense, that for such low energies the nucleons inside the nucleus can no more be considered as free. Nevertheless the model leads to a reliable description of final state multiplicities of pions and protons compared to experimental data.

Low energy nuclear interactions $A\left(N, N^{\prime}(\gamma)\right) A^{\prime}$ dominate the energy region 
from $\approx 10$ to $100 \mathrm{MeV}$, for higher energies they are continuously replaced by high energy spallation processes. The total inelastic cross sections at low energies have been determined by optical model calculations, the partial cross sections for different reaction channels with specific $N$ and $N^{\prime}$ are calculated by a simple model based on the $Q$ value of the respective reaction. Charged pions and all kaons have been assumed to interact always by spallation type processes. The history of the neutrons within a hadronic shower may be divided into three stages, first the production of neutrons, secondly the moderation and finally neutron capture. A short description of the neutron slowing down simulation inside the GHEISHA (version 8) code is given in Ref. 3.

\section{The Model Calorimeters}

The calorimeters used for this study consist of heavy absorber plates interleaved by readout layers. The transverse dimensions are $500 \times 500 \mathrm{~cm} 2$, the longitudinal depths are between 12 and 15 absorption lengths, so that leakage at the transverse sides and at the end of the calorimeters may be neglected. We will report only on studies with iron, lead and uranium as absorber materials. Aluminum, copper and tungsten have been used in some cases in order to investigate the A dependence. We have restricted ourselves to TMS, organic scintillators, liquid xenon and proportional counters as readout techniques. This covers the proposed applications at the SSC in the sense, that TMS and organic scintillators work as strong moderators for the neutrons produced in the absorber plates, for liquid xenon and proportional counters, on the other hand, one does not expect a sizable difference compared to a massive uranium block.

If not stated differently, the hadron calorimeters are not surrounded by other detectors, the incident particles from the primary interaction enter directly the calorimeters. This assumption does of course not resemble exactly the situation proposed for the experiments in situ. The influence of an electromagnetic calorimeter $\left(\approx 1 \lambda_{0}\right)$ in front of an uranium calorimeter (U/TMS) has thus been studied in some detail. The differences to the stand-alone uranium calorimeter are threefold. First of all the incident particles must pass one absorption length of a not fissionable material, secondly there will be backscattering not only from the uranium into the liquid xenon, but also from the xenon into the uranium. Consequently the neutron flux in the air gap between the electromagnetic and the hadron calorimeter, which is often used for fixing local analog electronics, may be an order of magnitude higher compared to the albedo of a stand-alone hadron calorimeter. Third, the interaction region, where the vertex detection device has to be installed, is protected against the uranium calorimeter by one absorption length. This model detector is similar to the $\mathrm{senon}$ Olive Detector[4]. In contrast to all other model calorimeters, a $4 \pi$ geometry has been assumed. 
An important parameter in studies of the neutron flux in sampling calorimeters is the sampling fraction, which is defined as ratio of the readout material content to the total mass of the calorimeter. To be more precise, if $\Delta z_{\text {readout }}$ and $\Delta z_{a b s o r b e r}$ are the layer thicknesses of the readout and the absorber plates respectively in longitudinal $\mathrm{Z}$ direction along the incident particle direction, then we define as sampling fraction:

$$
\lambda_{\text {readout }} / \lambda_{\text {tot }} \equiv \frac{\Delta z_{\text {readout }} / \lambda_{\text {readout }}}{\Delta z_{\text {readout }} / \lambda_{\text {readout }}+\Delta z_{\text {absorber }} / \lambda_{\text {absorber }}} .
$$

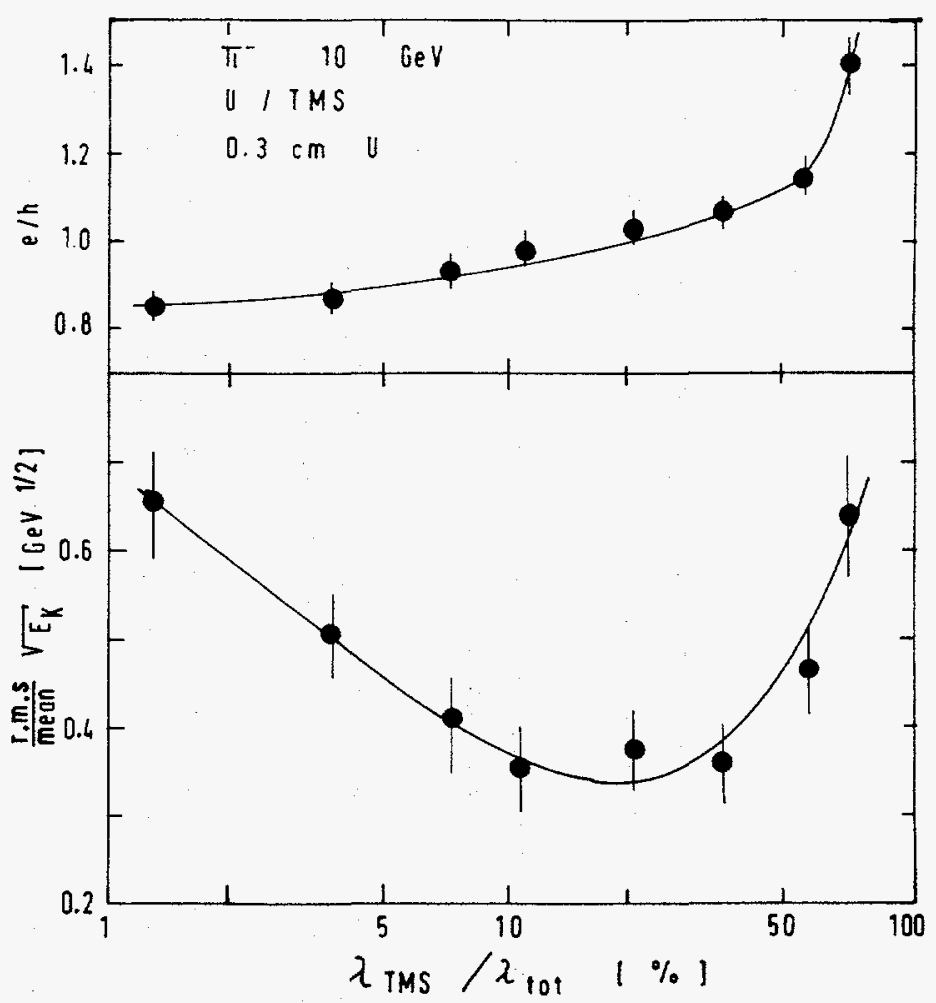

FIG. A16-1. e/h ratios and energy resolutions as function of the sampling fraction, for negative pions at $10 \mathrm{GeV}$, incident on $\mathrm{U} / \mathrm{TMS}$ calorimeters with $0.3 \mathrm{~cm}$ U layers.

In Fig. A16-1 we show the $e / h$ ratios and the energy resolutions as function of the sampling fraction, for an U/TMS calorimeter with $0.3 \mathrm{~cm}$ uranium plates and variable thickness of the TMS layers. In contrast to all other calculations in this study we used a time gate of $100 \mathrm{nsec}$. This reduces the number of neutrons counted by nearly a factor 1.5 . The $e / h$ ratio is defined as ratio of the signals 


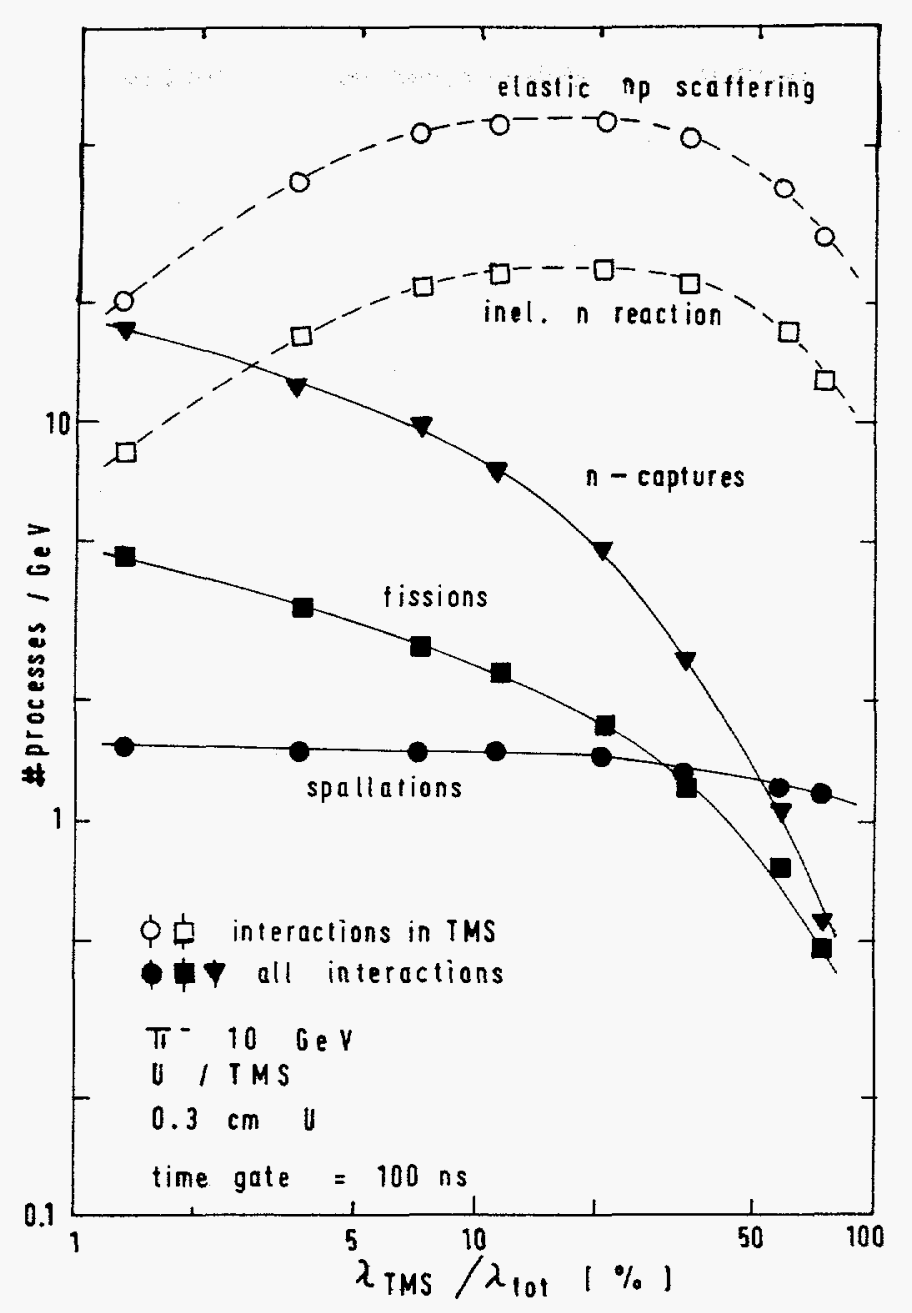

FIG. A16-2. Average numbers of various processes in hadronic showers as function of the sampling fraction. Results are given for U/TMS calorimeters with $0.3 \mathrm{~cm} \mathrm{U}$ layers.

from incident electrons and hadrons (here negative pions) at the same kinetic energy. The optimal energy resolution of $\frac{\text { r.m.s. }}{\text { mean }} \sqrt{E_{k}} \approx 35 \% \mathrm{GeV}^{1 / 2}$ is obtained for a sampling fraction of $10-30 \%$, which corresponds to $0.5-1.5 \mathrm{~cm}$ TMS layers for our fixed choice of $0.3 \mathrm{~cm}$ uranium plates. The $e / h$ ratio is close to 1 , however a clear increase with increasing sampling fraction is observed. The underlying physics behind this behaviour is shown in Fig. A16-2 (see also [3], [5] and [6]). Plotted are the average numbers of processes, per $\mathrm{GeV}$ incident energy, namely for spallations, fissions and neutron captures. It is seen that an increasing amount of TMS leads to a decrease of fissions and thus also to a strong decrease of neutrons produced in the shower, whereas the number of spallations is nearly not effected. The neutrons produced are sampled in the TMS layers by elastic neutron proton scattering and some inelastic reactions. On the one 
hand we expect a higher rate of scattering with increasing amount of TMS in the calorimeter, on the other hand the TMS works as a moderator, thus preventing the multiplication of neutrons by fission. The combined effect leads to a maximum of elastic and inelastic scattering in the TMS at $\approx 10-20 \%$ sampling fraction. This is shown also in Fig. A16-2. It is seen, by comparison of Fig. A16-1 and Fig. A16-2, that the best energy resolution corresponds just to this maximum of reactions. However, $0.5-1.5 \mathrm{~cm}$ TMS layers would lead to an unacceptable large depth of the total calorimeter. So a compromise between energy resolution and available space is necessary. We chose model calorimeters with U/TMS layers of $0.3 / 0.2(1.2 / 0.8)$ and $0.3 / 0.3 \mathrm{~cm}$, which corresponds to $5-7 \%$ sampling fraction. For these calorimeters the energy resolution is expected to be in the order of $\approx 40 \% \mathrm{GeV}^{1 / 2}$.

Organic scintillator readout will be studied with layer thicknesses of $0.3 / 0.3$ $\mathrm{cm}(\mathrm{U} / \mathrm{Sci}), 0.5 / 0.1 \mathrm{~cm}(\mathrm{~Pb} / \mathrm{Sci})$ and $0.45 / 0.15$ (Fe/Sci), which correspond to $11.6 \%, 5.0 \%$ and $4.0 \%$ respectively. For any other choice of the sampling fractions the total number of neutrons produced and thus also the neutron fluxes are different from those given in the present study. However, rough estimates of the differences may be performed using the curves for U/TMS in Fig. A16-2.

\section{Neutron Correlated Quantities}

Neutrons are produced by high energy spallation reactions and, in case of fissionable elements, by nuclear fission. They are moderated by a number of elastic and inelastic collisions and finally captured by a nucleus with the following emission of one or two photons or an electron. In Monte Carlo simulations the moderation must stop at a lower cutoff energy $E_{C}$. For the present study we used $E_{C}=1 \mathrm{keV}$. A neutron below this energy $E_{C}$ is definitely captured. The delay time of the capture is calculated according to a simple solution of the transport equation. The cutoff energy of $1 \mathrm{keV}$ is well below the minimum of the ionization energy of charged particles in gases. Thus $1 \mathrm{keV}$ is an adequate choice for the simulations of gaseous readout of hadron calorimeters. For liquids and solid readout materials a much higher value may be chosen. For all calculations, except of Fig. A16-1 and Fig. A16-2, we used a time gate of $1 \mu \mathrm{s}$.

According to this simple picture we expect a strong relationship between the neutron flux and the spatial distributions of spallations, nuclear fissions and neutron captures. The latter ones can be accurately measured by the method of induced radioactivity [1]. An approximate estimate can be made by the relation

$$
\left\langle n_{\text {capt }}\right\rangle=\langle\text { spall }\rangle\left\langle n_{\text {spall }}\right\rangle\langle\text { fiss }\rangle_{n}\left(\left\langle n_{\text {fiss }}\right\rangle-1\right),
$$

where $\langle$ spall $\rangle$ is the average number of spallation reactions $\left(\approx 1+E_{k}[\mathrm{GeV}]\right.$ $([3])),\left\langle n_{\text {spall }}\right\rangle$ is the number of neutrons produced in one spallation $(\approx 8.5)$, 
$\langle\text { fiss }\rangle_{n}$ the average number of fissions for one of the produced neutrons $(\approx 1.2)$ and $\left\langle n_{f i s s}\right\rangle$ the number of neutrons produced in one fission $(\approx 3.2)$. One neutron must be subtracted from the number of fission neutrons, since just the neutrons themselves do produce the fissions. The product of these four quantities gives a rough estimate for the total number of neutrons produced and thus also for the total number of neutron captures. We get

$$
\left\langle n_{\text {capt }}\right\rangle \approx 22\left(1+E_{k}\right)
$$

where $E_{k}$ must be inserted in $\mathrm{GeV}$. It is evidently clear that the number of neutron captures per absorption length is proportional to the number of neutrons $I_{N}(z)$ passing through this distance,

$$
\frac{d\left\langle n_{\text {capt }}\right\rangle}{d z} \sim I_{N}(z)
$$

where $\mathrm{z}$ is expressed in absorption lengths. Later we will show that the proportionality can be replaced by an equal sign. Deviations from this simple picture will be discussed in the rest of this section.

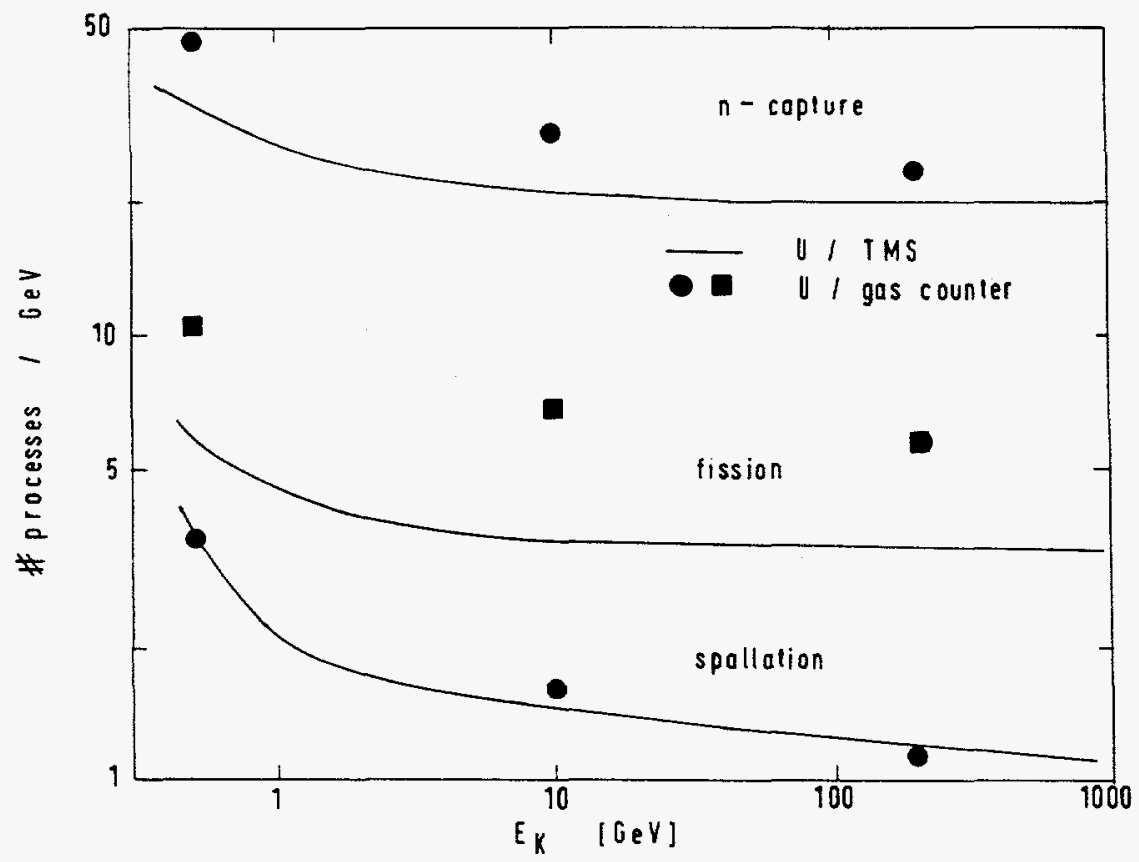

FIG. A16-3. Number of spallations, fissions and neutron captures in uranium calorimeters with gas counter and TMS readout respectively, as function of the incident pion energy. 


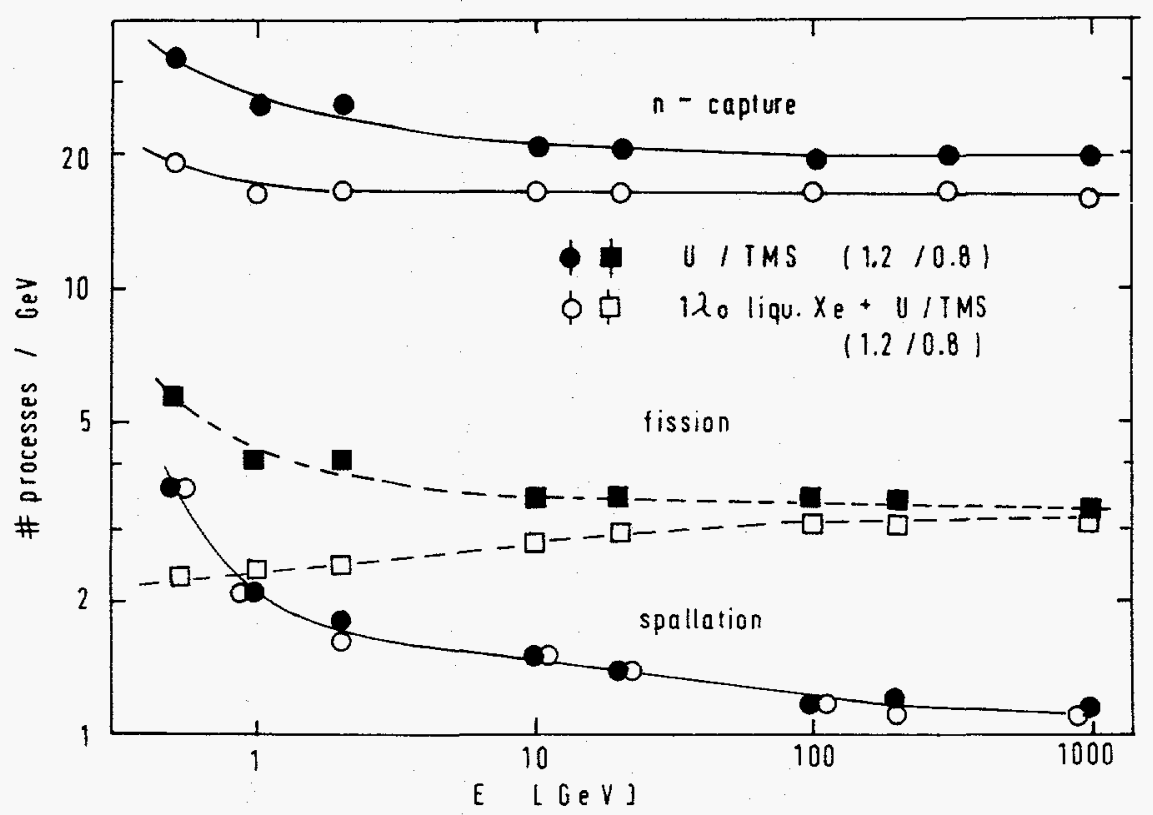

FIG. A16-4. Number of spallations, fissions and neutron captures for an U/TMS calorimeter with and without an electromagnetic liquid xenon calorimeter in front. Results are given for incident negative pions as function of the energy.

In Fig. A16-3 we show results for two extreme cases, an uranium calorimeter with gas counter and TMS respectively as readout technique. A sampling fraction of $5 \%$ (U/TMS $1.2 / 0.8 \mathrm{~cm}$ ) has been chosen. Incident particles are negative pions. TMS acts as a strong moderator, due to elastic scattering with the free protons of the methyl radicals of the $\mathrm{C}_{4} \mathrm{H}_{12} \mathrm{Si}$ molecules. Thus the neutrons are rapidly slowed down below the fission threshold $(\approx 2 \mathrm{MeV})$ and the number of produced neutrons is expected to be much smaller compared to gas counter readout or any other readout material without free protons. In gas counters one may use, of course, vapour fillings with hydrogen mixtures. However the cross sections are so small that the moderation effect is negligible [7]. We observe a $50 \%$ decrease for fissions and $30 \%$ for neutron captures. The number of spallations, on the other hand, is the same for both readouts.

If the incident pions do not start at the first plane of the hadron calorimeter, as has been assumed for the results of Fig. A16-3, but at the front face of a $1 \lambda_{0}$ liquid xenon e.m. calorimeter, placed in front of the hadron calorimeter, we expect a further decrease of the number of fissions and neutron captures. This is shown in Fig. A16-4. Especially at low energies around $1 \mathrm{GeV}$ only a small part of the hadronic shower is able to reach the uranium calorimeter, so the number of fissions is considerably reduced. The total number of neutrons produced is reduced by a factor 1.5 compared to the stand-alone U/ I'MS calorimeter and by a factor 2 compared to the uranium calorimeter with gas counter readout. 


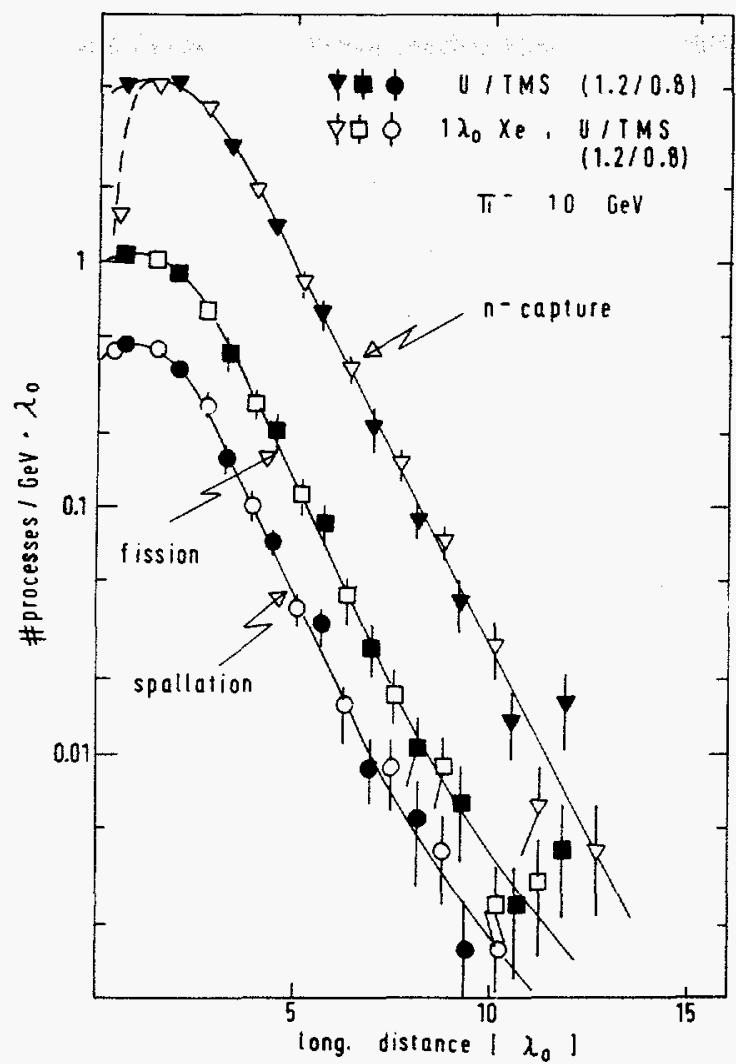

FIG. A16-5. Longitudinal distributions of spallation, fission and neutron capture in the calorimeters from Fig. A16-4. The results are for $10 \mathrm{GeV}$ incident pions.

Some more insight into the shower dynamic is obtained by inspection to Fig. A16-5. Plotted are the longitudinal distributions of the number of processes, for the two calorimeters from Fig. A16-4. In case of the $1 \lambda_{0} \mathrm{Xe}+\mathrm{U} / \mathrm{TMS}$ calorimeter the first absorption length is covered by a not fissionable element. Thus the number of fissions is exactly zero and the number of neutrons is small. For $z \geq 1 \lambda_{0}$, however, the number of fissions and neutron captures is precisely the same for both calorimeters. This may be simply explained by the intuitive model, that the longitudinal propagation of the shower is dominated by high energy particles, whereas the neutrons behave as a short range component. The neutrons themselves travel long distances, but, due to the large amount of elastic scattering, the net propagation is small $\left(\approx 1 \lambda_{0}\right)$. This leads directly to the idea that the number of fissions and neutron captures is simply proportional to the total amount of absorbed ionization energy, independent on the history of the shower. This is shown in Fig. A16-6. $E_{a b s}$ is the ionization energy absorbed by both, the TMS and the uranium. A perfect agreement between the results with and without the liquid xenon detector in front of the uranium calorimeter can be 
observed. This conclusion is still true, if the incident single particle is replaced by all secondary particles of pp collisions. For the results in Fig. A16-6 we used a $4 \pi$ geometry for the calorimeters, but still with a beam pipe of $5 \mathrm{~cm}$ radius. So a big part of particles from high energy pp collisions do not enter the calorimeters or touch upon the endcaps only. The decrease in the results for $\mathrm{pp}$ collisions compared to single particles is due to the $\pi^{0}$ conponent $(\approx 30 \%)$.

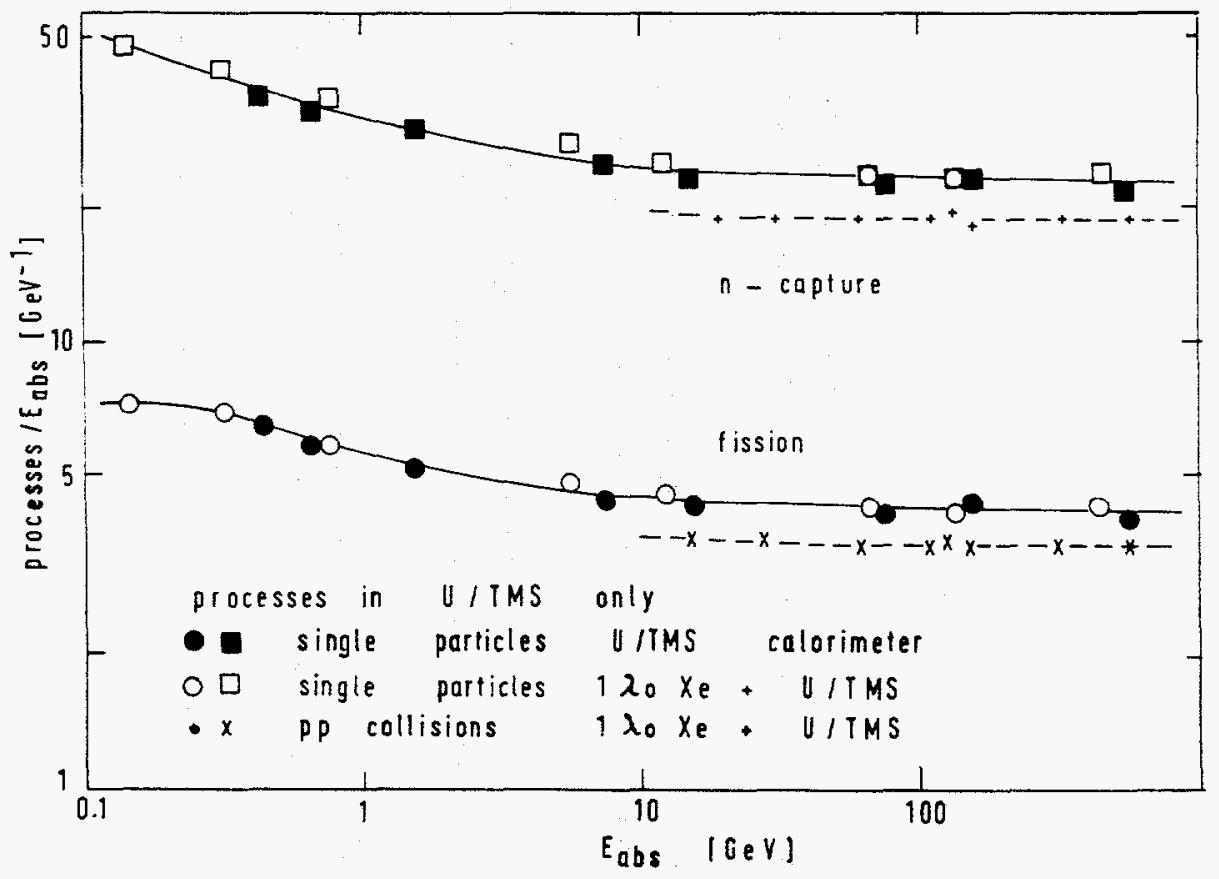

FIG. A16-6. Number of fissions and neutron captures as function of the total absorbed ionization energy in the uranium calorimeter. Given are results for single negative pions and for particles from complete pp collisions.

The total number of neutron captures may be taken safely as an upper limit of the neutron current. For uranium we found $I_{N} / E_{k} \leq 22 \mathrm{GeV}^{-1}$. As can be seen from Fig. A16-7, for $\mathrm{Pb}$ and $\mathrm{Fe}$ as absorber material the neutron current is expected to be an order of magnitude smaller, namely $I_{N} / E_{k} \leq 4 \mathrm{GeV}^{-1}$ and $I_{N} / E_{k} \leq 2 \mathrm{GeV}^{-1}$ respectively.

If not stated differently we used negative pions as incident particles. In Fig. A16-8 we compare the total number of neutron captures for incident pions, kaons, antiprotons and protons, for an uranium scintillator calorimeter with $12 \%$ $(\mathrm{U} / \mathrm{Sci} 0.3 / 0.3 \mathrm{~cm})$ sampling fraction. For all particles we get $\left\langle n_{\text {capt }}\right\rangle / E_{k} \approx$ $19 \mathrm{GeV}^{-1}$ in the limit $E_{k} \geq 100 \mathrm{GeV}$. For lower energies we observe strong differences. Showers from negative pions produce the lowest number of neutrons, proton showers show a strong decrease for $E_{k} \rightarrow 0$, showers with antiprotons 


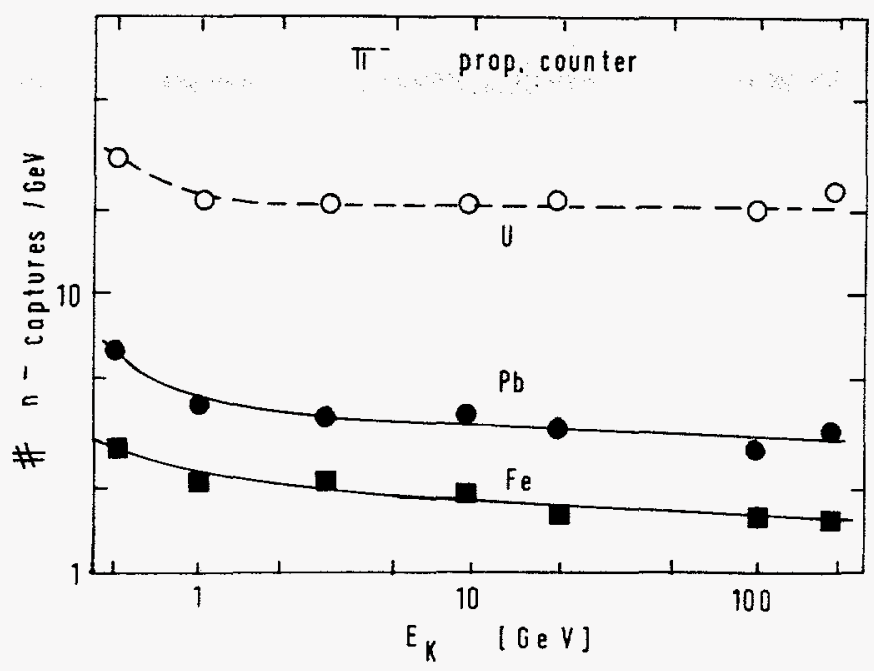

FIG. A16-7. Number of neutron captures for various absorber materials in sampling calorimeters with gas counter readout.

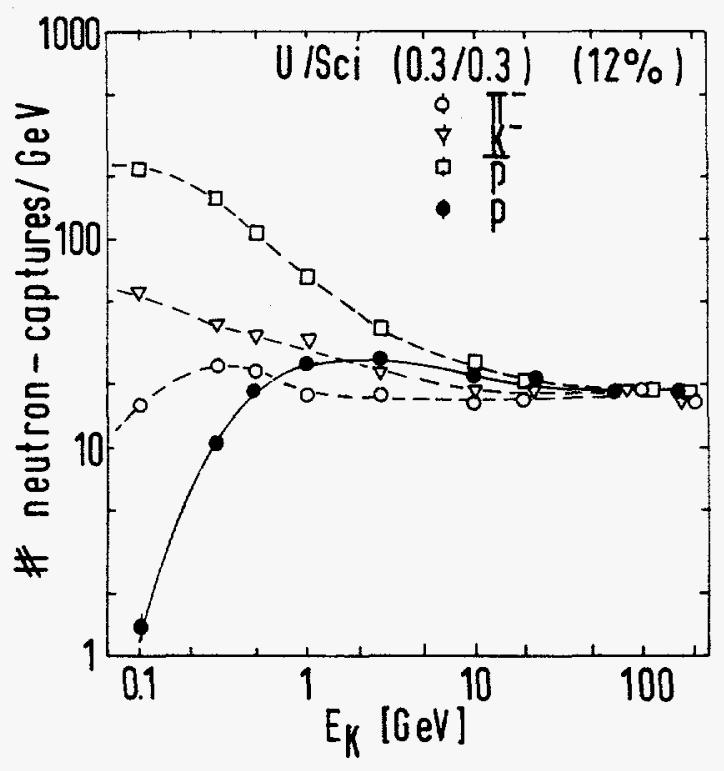

FIG. A16-8. Number of neutron captures as function of incident energy for different particles. All results are for uranium scintillator sampling calorimeters.

as incident particles seem to saturate with $\left\langle n_{\text {capt }}\right\rangle / E_{k} \approx 220 \mathrm{GeV}^{-1}$ for $E_{k} \leq$ $0.1 \mathrm{GeV}$. The finite value for $E_{k} \rightarrow 0$ for negative pions and kaons may be explained by pion and kaon capture at rest with the emission of some neutrons in turn. A safer upper limit is thus obtained as $I_{N} / E_{k} \leq 50 \mathrm{GeV}^{-1}$ for $E_{k} \geq$ $0.5 \mathrm{GeV}$. This number includes the results for pions, kaons and protons and has been scaled up from the uranium scintillator calorimeter to massive uranium. 


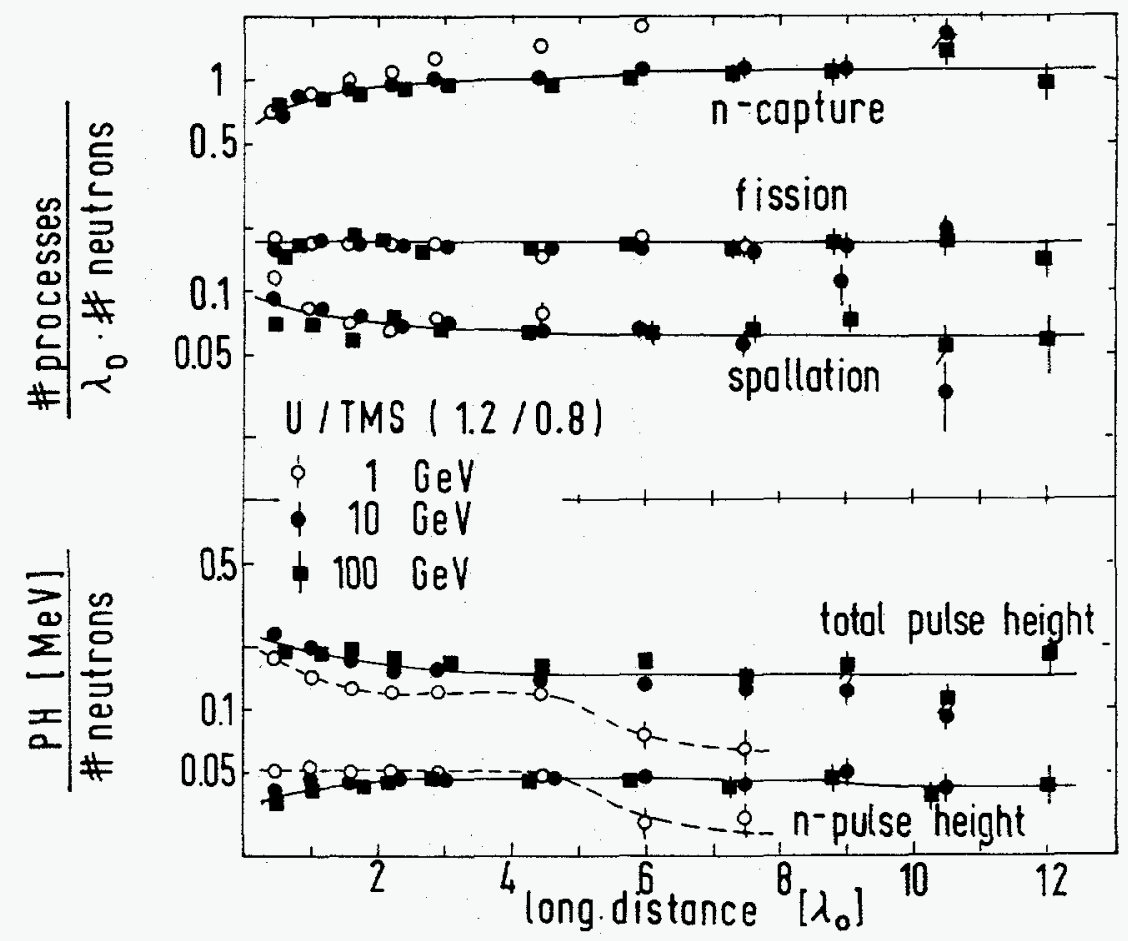

FIG. A16-9. Number of spallations, fissions and neutron captures per absorption length as function of the longitudinal depth, normalized to the number of neutrons (upper part) and neutron pulse heights and total pulse heights, normalized to the number of neutrons (lower part).

The approximate proportionality of the neutron correlated quantities, namely the number of spallations, fissions and neutron captures as well as the pulse height caused by neutrons and the total pulse height, all as function of the longitudinal depth in the calorimeter, is shown in Fig. A16-9. Deviations are observed for the $1 \mathrm{GeV}$ low energy results and for small longitudinal depths in case of all energies. The longitudinal distribution for the number of neutrons, normalized to the incident particle energy, is plotted in Fig. A16-10. It is clear that we expect, apart from a scale factor, similar distributions for all other neutron correlated quantities.

\section{Neutron Fluxes and Energy Spectra}

In the previous section we used simply the number of neutrons, crossing the boundary of the calorimeter plates, as measure for the neutron current. For studies of radiation damage it is useful to introduce the neutron flux, defined in this study as normalized tracklength in the readout material. Thus every neutron has been weighted by $1 /|\cos \theta|$, where $\theta$ is the angle of the momentum vector with respect to the axis perpendicular to the plane. Since the neutrons 


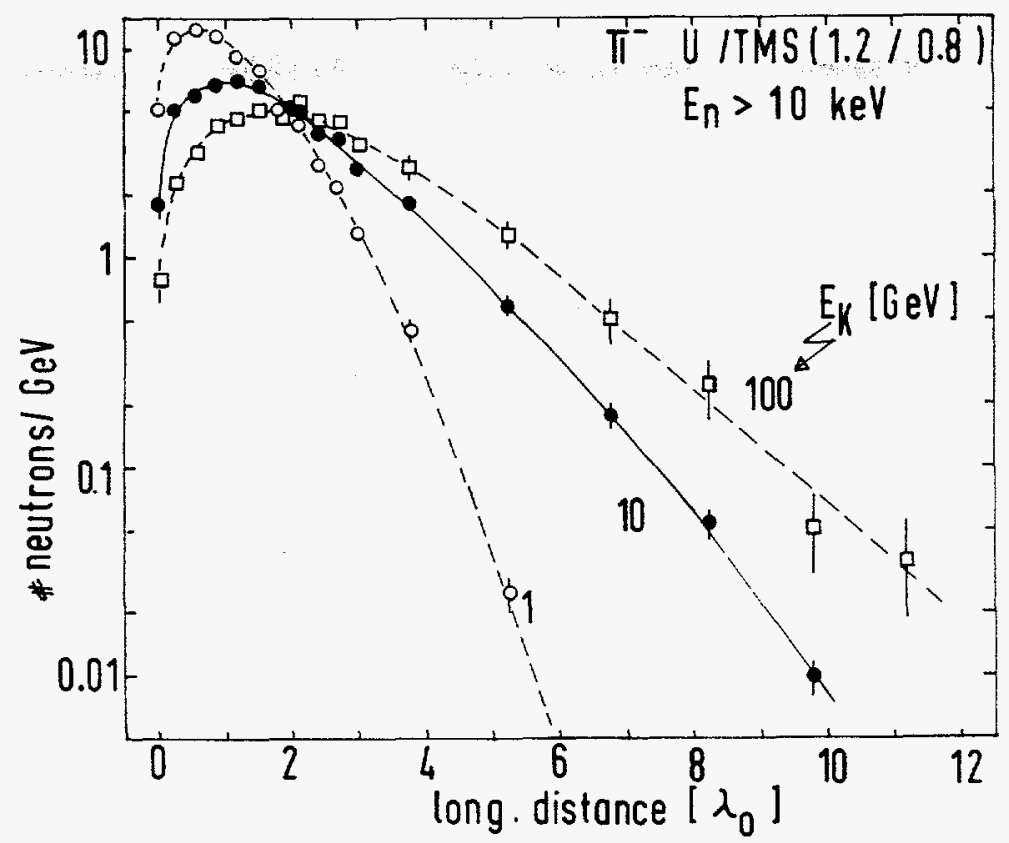

FIG. A16-10. Number of neutrons, normalized to the incident pion energy, as function of the longitudinal depth.

obey a distribution of the form

$$
\frac{d N}{d|\cos \theta|} \sim|\cos \theta|,
$$

we expect the relationship

$$
\Phi_{N} \approx 2 I_{N}
$$

between the flux and the current. The relation (6) is exact, at least in this Monte Carlo simulation, for homogeneous calorimeters only. For proportional counter readout or liquid xenon and liquid argon readout the two relations (5) and (6) are still well fulfilled. For scintillator and TMS readout, however, we found $\Phi_{N} / I_{N} \approx 2.0-2.5$, depending on the sampling fraction of the calorimeters.

Neutron fluxes at various locations inside and outside the hadron calorimeters are shown in Fig. A16-11, for all neutrons with energies above $10 \mathrm{keV}$. In Fig. A1611a we plotted for incident negative pions the neutron fluxes at the maximum of the longitudinal shower distribution and at the front plane (albedo). Two different U/TMS calorimeters are considered, with $5 \%(1.2 / 0.8 \mathrm{~cm})$ and $7 \%(0.3 / 0.3$ $\mathrm{cm}$ ) sampling fraction respectively. A $30 \%$ difference in the results is observed, indicating that the sampling fraction is an important parameter in studies of neutron fluxes. If we place a liquid xenon shower counter in front of the hadron 

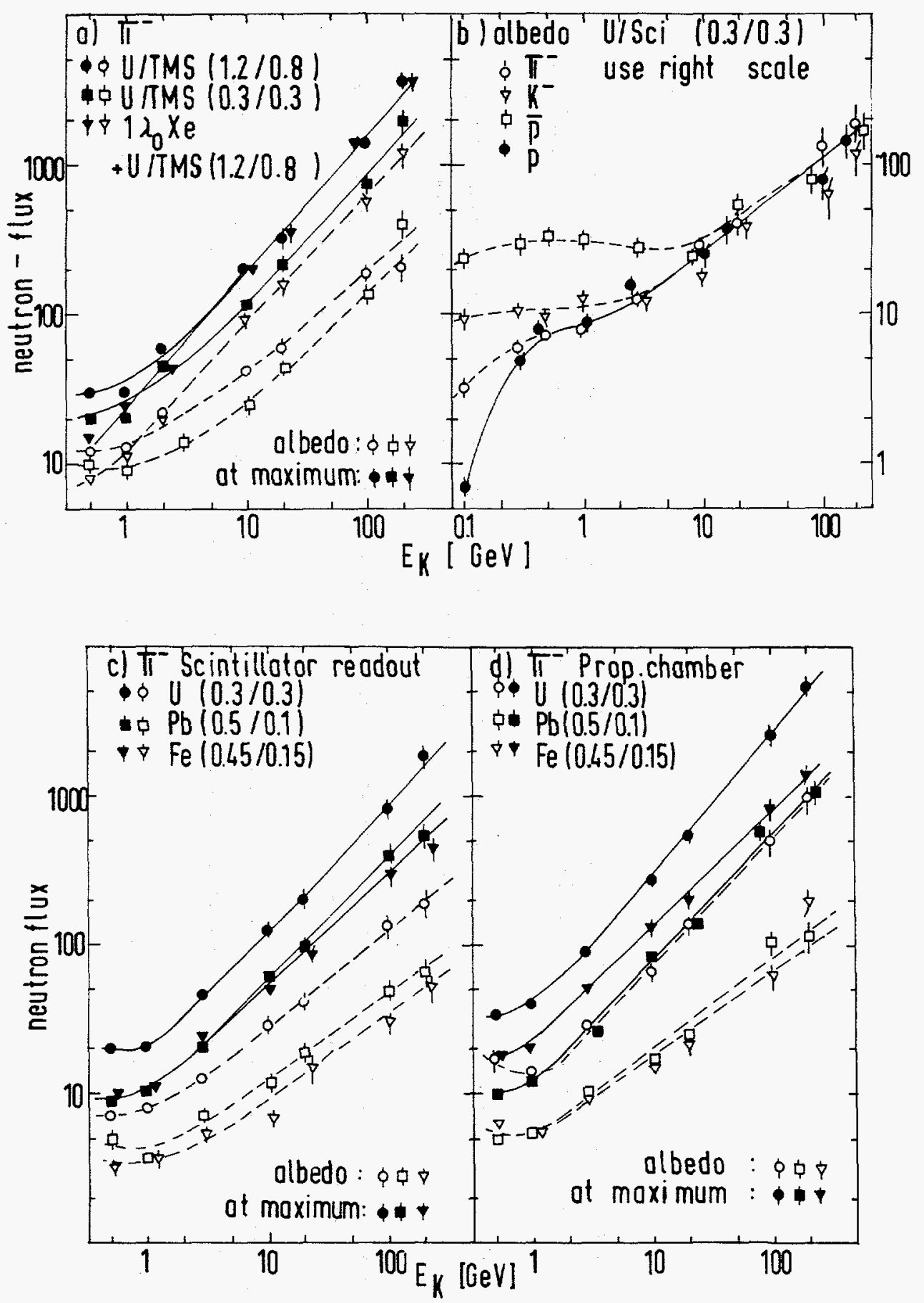

FIG. A16-11. Neutron fluxes at the shower maximum and at the front plane (albedo) for various calorimeters and various incident particles as function of energy. The neutron cutoff energy is $10 \mathrm{keV}$. 
calorimeter, we observe a strong increase of the albedo in the air gap between the shower counter and the hadron calorimeter. Albedo calculations for an uranium scintillator calorimeter with $12 \%(0.3 / 0.3 \mathrm{~cm})$ sampling fraction are shown in Fig. A16-11b, for various incident particles. For energies $E_{k} \geq 10 \mathrm{GeV}$ all results agree with each other, however for low energies differences up to factors of 10 can be observed. A similar observation has been made already in Fig. A16-8, where we studied the total number of produced neutrons. In Fig. A16-11c and 11d we compare the neutron fluxes for uranium, lead and iron calorimeters, for scintillator readout (Fig. A16-11c) and for proportional counter readout (Fig. A16-11d). All numbers given in Fig. A16-11 are collected in Table 1 to Table 4.

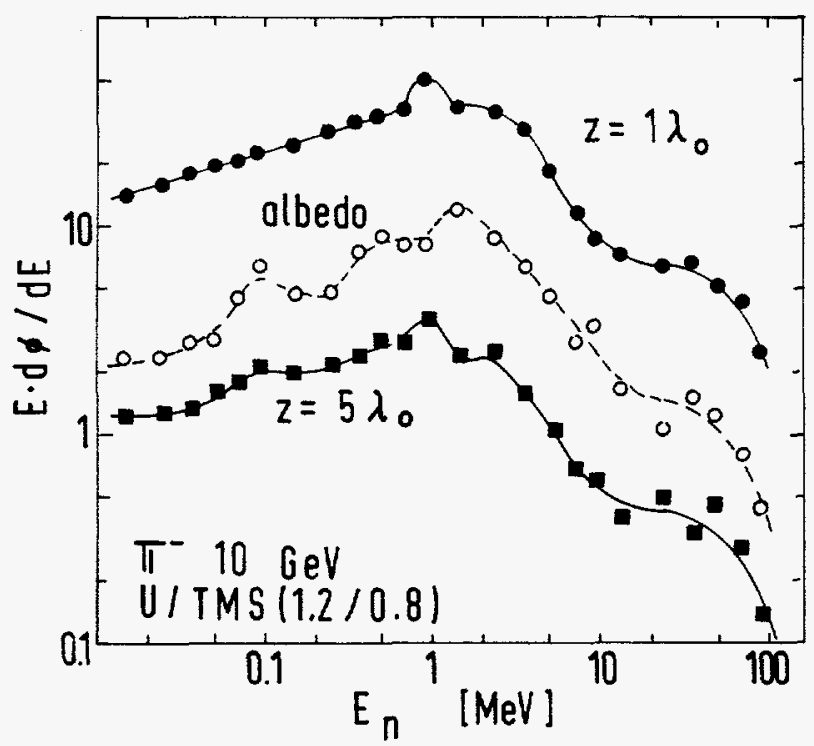

FIG. A16-12. Neutron energy spectra at various longitudinal shower depths for U/TMS calorimeter.

Neutron energy spectra have been studied using the quantity

$$
E_{N} \frac{d \Phi_{N}}{d E_{N}}
$$

where the differential flux is multiplied by the neutron energy. This takes into account that the strength of radiation damage is proportional to the energy. In Fig. A16-12 we show the neutron energy spectra for an U/TMS calorimeter at various longitudinal depths. We observe a pronounced maximum at $E_{N} \approx 1$ $\mathrm{MeV}$ and a long tail for lower energies. The distributions are nearly the same for all longitudinal depths. The energy spectra shown in Fig. A16-12, with TMS as readout material, are different from the energy spectra in Fig. A16-13. Here 
Table 1: Numerical values of the results shown in fig.11a.

\begin{tabular}{|c|c|c|c|c|c|c|}
\hline$E_{k}[G \in V]$ & $\begin{array}{l}\text { U/TMS } \\
\text { albedo }\end{array}$ & $\begin{array}{c}(1.2 / 0.8) \\
\Phi_{\max } \\
\end{array}$ & $\begin{array}{l}\text { U/TMS } \\
\text { albedo }\end{array}$ & $\begin{array}{c}(0.3 / 0.3) \\
\Phi_{\max } \\
\end{array}$ & $\begin{array}{c}1 \lambda_{0} \mathrm{Xe}+\mathrm{U} / \mathrm{TMS} \\
\text { albedo }\end{array}$ & $\begin{array}{c}(1.2 / 0.8) \\
\Phi_{\max }\end{array}$ \\
\hline 0.5 & $12.6 \pm 0.3$ & $30.9 \pm 0.7$ & $10.3 \pm 1.8$ & $21.0 \pm 2.0$ & & $14.9 \pm 0.4$ \\
\hline 1.0 & $13.1 \pm 0.5$ & $33.0 \pm 1.0$ & $0 \pm 1.2$ & 21. & & $23.0 \pm 0.7$ \\
\hline 3.0 & $23.0 \pm 1.0$ & $61.0 \pm 2.0$ & $14.4 \pm 1.2$ & 46.1 & & $42.0 \pm 1.5$ \\
\hline 10. & $44.0 \pm 2.0$ & $207 . \pm 5.0$ & $25.0 \pm 3.0$ & $115 . \pm 7.0$ & $92.0 \pm 5.0$ & $197 . \pm 11$ \\
\hline 20. & $60.0 \pm 7.0$ & 335. \pm 30 . & $45.0 \pm 6.0$ & $225 . \pm 17$ & $152 . \pm 13$ & $340 . \pm 25$ \\
\hline 100 & 185. \pm 20 . & $1430 \pm 50$ & $145 . \pm 20$ & $750 . \pm 100$ & $581 . \pm 80$ & $1400 \pm 150$ \\
\hline 200 & $205 . \pm 40$ & $3600 \pm 300$ & $400 . \pm 100$ & $2000 \pm 300$ & $1200 \pm 200$ & $3600 \pm 400$ \\
\hline
\end{tabular}

Table 2: Numerical values of the results shown in fig.11b.

\begin{tabular}{c|rrrr}
$E_{k}[\mathrm{GeV}]$ & \multicolumn{1}{|c}{$\pi^{-}$} & \multicolumn{1}{c}{$K^{-}$} & \multicolumn{1}{c}{$\bar{p}$} & \multicolumn{1}{c}{$p$} \\
\hline & & & & \\
0.1 & $3.3 \pm 0.3$ & $9.8 \pm 0.8$ & $24.0 \pm 2.0$ & $0.7 \pm 0.1$ \\
0.3 & $6.0 \pm 0.4$ & $10.8 \pm 1.0$ & $\mathbf{3 0 . 0} \pm 4.0$ & $5.0 \pm 0.6$ \\
0.5 & $7.4 \pm 0.6$ & $9.5 \pm 1.0$ & $33.0 \pm 3.0$ & $8.1 \pm 0.9$ \\
1.0 & $8.1 \pm 0.9$ & $12.5 \pm 2.0$ & $32.0 \pm 3.0$ & $9.0 \pm 0.7$ \\
3.0 & $12.9 \pm 1.1$ & $12.3 \pm 1.0$ & $29.0 \pm 2.0$ & $16.0 \pm 2.0$ \\
10. & $29.0 \pm 3.0$ & $17.3 \pm 1.7$ & $25.0 \pm 2.0$ & $26.0 \pm 4.0$ \\
20. & $41.0 \pm 7.0$ & $39.0 \pm 6.0$ & $55.0 \pm 9.0$ & $39.0 \pm 5.0$ \\
100 & $136 . \pm 40$. & $61.0 \pm 20$. & $80.0 \pm 15$. & $80.0 \pm 15$. \\
200 & $191 . \pm 50$. & $118 . \pm 40$. & $170 . \pm 40$. & $154 . \pm 30$ \\
\hline
\end{tabular}

Table 3: Numerical values of the results shown in fig.11c.

\begin{tabular}{|c|c|c|c|c|c|c|}
\hline$E_{k}[\mathrm{GeV}]$ & $\begin{array}{l}\text { U/Sci } \\
\text { albedo }\end{array}$ & $\begin{array}{c}(0.3 / 0.3) \\
\Phi_{\max }\end{array}$ & $\begin{array}{l}\mathrm{Pb} / \mathrm{Sci} \\
\text { albedo }\end{array}$ & $\begin{array}{c}(0.5 / 0.1) \\
\Phi_{\max } \\
\end{array}$ & $\begin{array}{l}\mathrm{Fe} / \mathrm{Sci} \\
\text { albedo }\end{array}$ & $\begin{array}{c}(0.45 / 0.15) \\
\Phi_{\max } \\
\end{array}$ \\
\hline & $7.4 \pm 0.6$ & $20.5 \pm 1.8$ & & & $3.2 \pm 0.3$ & \\
\hline 1.0 & \pm 0.9 & $21.0 \pm 1$ & $3.7 \pm 0.2$ & 10.5 & $3.7 \pm 0.4$ & \pm 0.6 \\
\hline 3.0 & $12.9 \pm 1.1$ & 47.0 & $7.1 \pm 0.4$ & 21.0 & $5.5 \pm 0.4$ & $0 \pm 1.7$ \\
\hline 10. & & 128. & $12.0 \pm 1.7$ & 60. & 0.8 & \pm 4.0 \\
\hline 20. & $41.0 \pm 7.0$ & $203 . \pm 20$ & $19.0 \pm 3.0$ & 100. \pm & $15.0 \pm 3.0$ & $90.0 \pm 10$ \\
\hline & $136 . \pm 40$ & & $48.0 \pm 10$ & 415. \pm & $31.0 \pm 5.0$ & $300 . \pm 50$ \\
\hline 200 & $191 . \pm 50$ & $1900 \pm 200$ & $66.0 \pm 15$ & $550 . \pm 100$ & $53.0 \pm 6.0$ & $450 . \pm 100$ \\
\hline
\end{tabular}

Table 4: Numerical values of the results shown in fig.11d.

\begin{tabular}{|c|c|c|c|c|c|c|}
\hline$E_{k}[G e V]$ & $\begin{array}{l}\text { U/p.c. } \\
\text { albedo }\end{array}$ & $\begin{array}{c}(0.3 / 0.3) \\
\Phi_{\max }\end{array}$ & $\begin{array}{l}\mathrm{Pb} / \mathrm{p} . \mathrm{c} . \\
\text { albedo }\end{array}$ & $\begin{array}{c}(0.5 / 0.1) \\
\Phi_{\max }\end{array}$ & $\begin{array}{l}\mathrm{Fe} / \mathrm{p} . \mathrm{c} . \\
\text { albedo }\end{array}$ & $\begin{array}{c}(0.45 / 0.15) \\
\Phi_{\max }\end{array}$ \\
\hline 0.5 & $17.4 \pm 3.0$ & $34.0 \pm 2.0$ & $5.1 \pm 0.5$ & $10.6 \pm 0.6$ & $6.3 \pm 0.9$ & $18.1 \pm 1.0$ \\
\hline 1.0 & $14.1 \pm 0.9$ & $40.0 \pm 2.0$ & $5.6 \pm 1.0$ & $12.4 \pm 0.7$ & $5.6 \pm 0.6$ & $21.0 \pm 1.0$ \\
\hline 3.0 & $29.0 \pm 2.0$ & $92.0 \pm 4.0$ & $10.7 \pm 1.7$ & $27.0 \pm 1.0$ & $9.4 \pm 1.0$ & $52.0 \pm 2.0$ \\
\hline 10. & $67.0 \pm 6.0$ & $280 . \pm 18$ & $17.4 \pm 1.2$ & $83.0 \pm 4.0$ & $15.0 \pm 1.3$ & $131 . \pm 7.0$ \\
\hline 20. & $142 . \pm 15$ & $570 . \pm 40$ & $25.0 \pm 2.0$ & $140 . \pm 8.0$ & $22.0 \pm 3.0$ & $203 . \pm 14$ \\
\hline 100 & $500 . \pm 100$ & $2700 \pm 300$ & $105 . \pm 25$. & $600 . \pm 50$ & $63.0 \pm 10$ & $850 . \pm 100$ \\
\hline 200 & $1000 \pm 200$ & $5700 \pm 900$ & 112. \pm 30 . & $1100 \pm 100$ & $200 . \pm 40$ & $1410 \pm 70$ \\
\hline
\end{tabular}




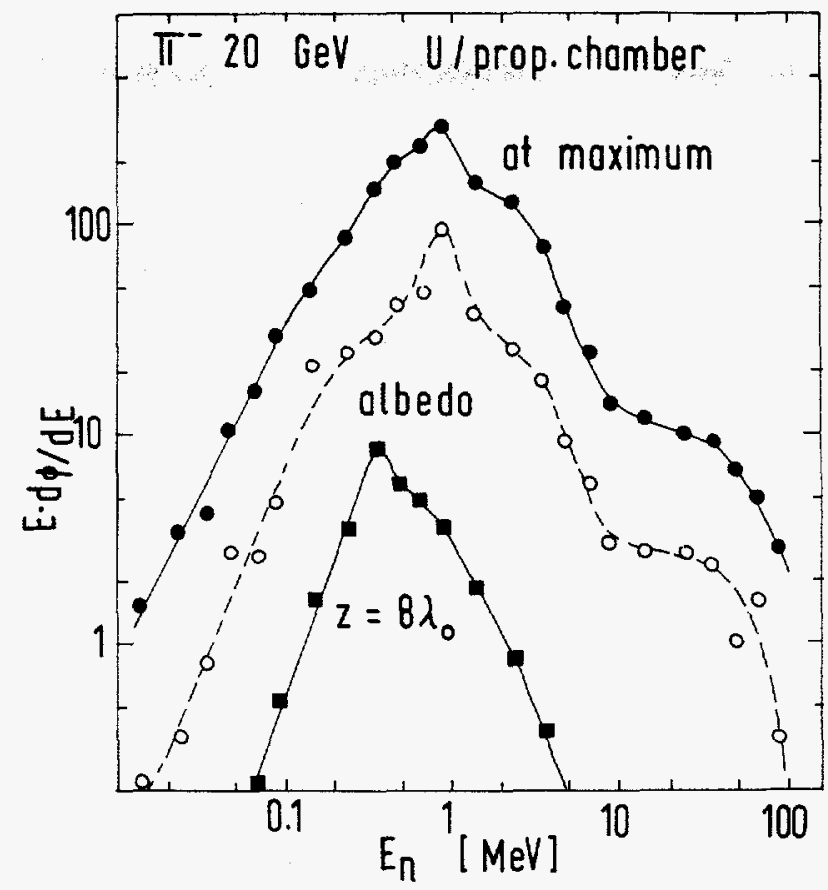

FIG. A16-13. Neutron energy spectra at various longitudinal shower depths for an uranium calorimeter with gas counter readout.

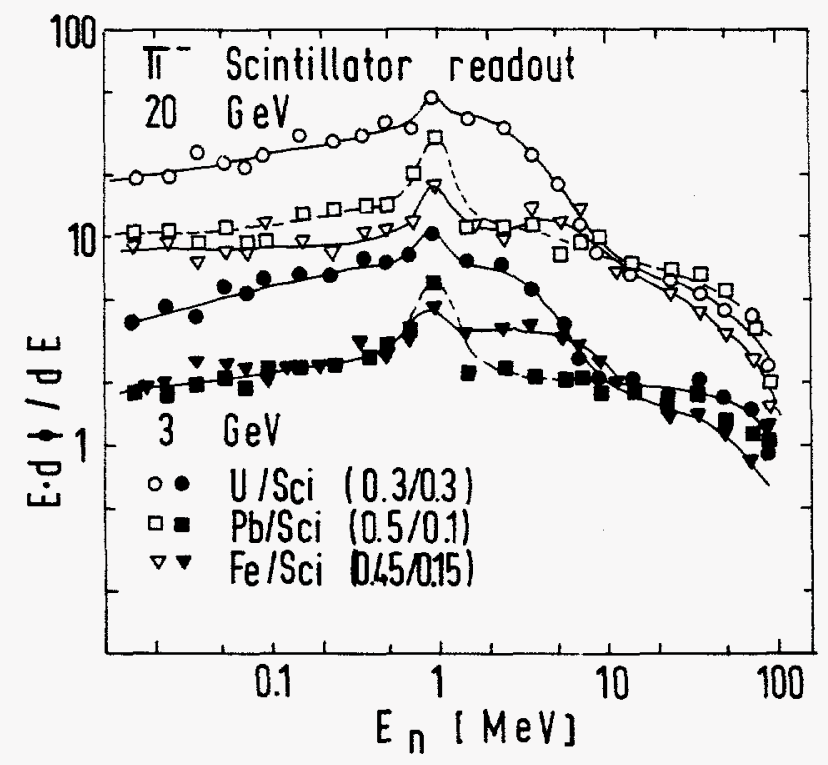

FIG. A16-14. Neutron energy spectra for uranium, lead and iron calorimeter with scintillator readout. 
we used proportional counter readout, thus avoiding the moderation by elastic neutron proton scattering. The distributions in Fig. A16-13 are sharply peaked around $1 \mathrm{MeV}$ for the albedo and the first few absorption lengths. For the longitudinal shower tail $\left(z=8 \lambda_{0}\right)$ however, we observe a shift of the distribution to $\approx 500 \mathrm{keV}$. A comparison for different absorber materials is made in Fig. A1614 , for scintillator readout and two energies of the incident pions. There seems to be no significant differences between various absorber materials and between the two energies. This is in contrast to proportional counter readout (Fig. A16-15), where the spectrum for iron absorbers show a pronounced tail at low energies. Uranium and lead, on the other hand, do not.

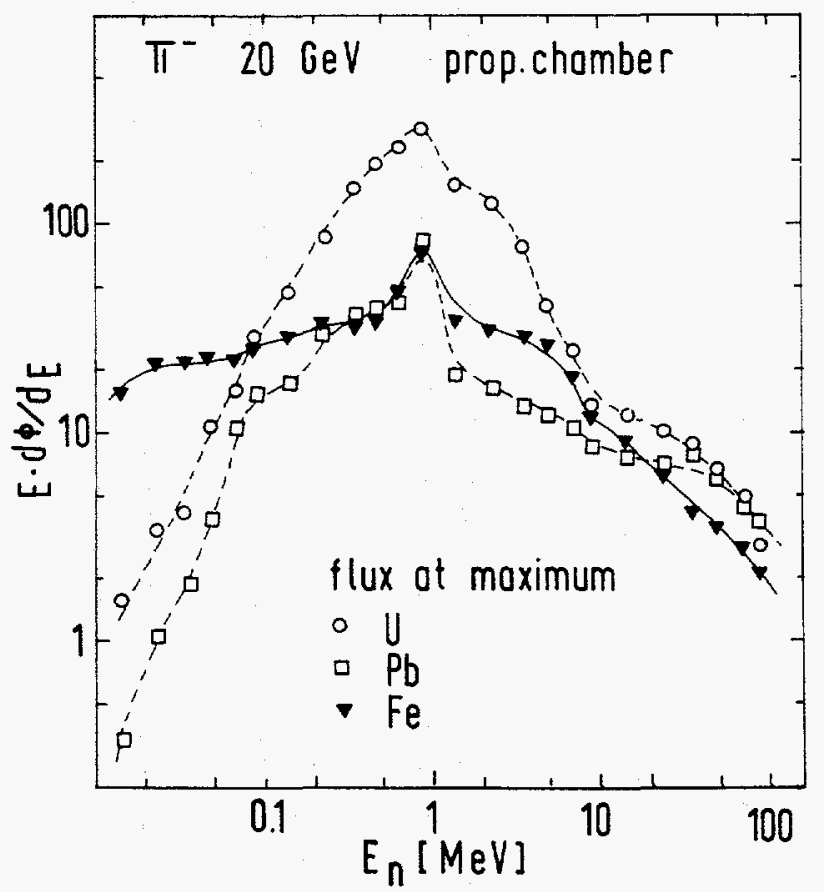

FIG. A16-15. Neutron energy spectra at the shower maximum for uranium, lead and iron with proportional counter readout.

It is of course not easy to explain or even verify the detailed structure of the plots in Fig. A16-12 to Fig. A16-15. In the GHEISHA code a relatively simple treatment is used for the neutron slowing down simulation (see [3] for details). We can not exclude systematic errors within a factor of about two in the energy spectra. We have thus omitted error bars.

\section{Summary}

A systematic study of the neutron currents and neutron fluxes in hadron calorimeters has been performed. The relationship between the neutron currents 
and the spatial distribution of processes, which produce, moderate and capture them, has been investigated in detail. Calculations for the albedo and the neutron flux at the maximum of the shower distribution have been presented, for various calorimeters and for various incident particles. The neutron energy spectra show a pronounced peak at $1 \mathrm{MeV}$, the low energy tail depends in general on the readout material.

This work has been funded by the German Federal Minister for Research and Technology (BMFT) under the contract number 054AC36P7.

\section{References}

1. C. Leroy, Y. Sirois and R. Wigmans, Nucl. Instrum. and Methods A252, 4 (1986); R. Wigmans, contribution to this report.

2. H. Fesefeldt, "The Physics of Hadronic Showers-Physics and Applications," RWTH Aachen PITHA 85/02 (1985).

3. H. Fesefeldt, "Low Energy Phenomena in Hadronic Showers," PITHA 87/20 and Proc. Workshop on Detector Simulation for the SSC, Argonne National Laboratory, Argonne IL, USA, August 24-28 (1987).

4. M. Chen et al., "The Xenon Olive Detector for the Next Generation Colliders," Proc. of the Workshop on Experiments, Detectors, and Experimental Areas for the Supercollider, ed. by R. Donaldson and M G. D. Gilchriese (Berkeley CA 1987).

5. R. Wigmans, Nucl. Instrum. and Meth. A259, 389 (1987).

6. H. Brückmann et al., "On the Theoretical Understanding and Calculation of Sampling Calorimeters," DESY 87-064 (1987) and Proc. of XVI Winter Meeting on Fundamental Physics, Sevilla, Spain, Feb.23-27 (1987).

7. H. Fesefeldt, \A Study of Proportional Counter Readout in Uranium Calorimeters," RWTH Aachen PITHA 87/21, to be published in Nucl. Instrum. and Methods A. 
APPENDIX 17

\title{
LOW-ENERGY NEUTRON MEASUREMENTS IN AN IRON CALORIMETER STRUCTURE IRRADIATED BY $200 \mathrm{GeV} / \mathrm{c}$ PIONS
}

\author{
James S. Russ \\ Carnegie-Mellon University, Pittsburgh, PA 15219 \\ and \\ Graham R. Stevenson and Alberto Fassò \\ European Organization for Nuclear Research \\ CERN, CH-1211 Genève 23, Switzerland
}

\section{Introduction}

In the design of detectors for the high-luminosity hadron-hadron colliders such as the Large Hadron Collider (LHC) in the LEP tunnel at CERN or the Superconducting Super Collider (SSC) at a site to be chosen in the USA, one must evaluate the damaging effects on detector devices from the thousands of secondary particles produced in each collision. These multi-TeV events deposit approximately 200 watts in the hadronic calorimeters surrounding each intersection region, discounting the fragments produced in the forward directions which stay in or close to the beam pipe. These fragments however will give rise to back-scattered radiation in the detector since they will interact in machine components. One of the serious questions raised at recent collider studies $[1,2]$ concerns the radiation-damage effects of low energy neutrons produced by spallation, evaporation or fission processes. Such neutrons will have a broad angular and energy distribution, and they may be particularly harmful to silicon-based electronics or organic materials. Damage factors for neutrons in silicon peak in the 0.1-10 MeV range, just where these hadron cascade neutrons are most copious. Figure 1 shows the neutron damage coefficient as a function of neutron energy for silicon [3]. At present collider luminosities $\left(10^{30} \mathrm{~cm}^{-2} \mathrm{~s}^{-1}\right)$ slow neutron effects are starting to be seen in gaseous detectors [4]. With the luminosity increases of $10^{3}-10^{5}$ discussed for future colliders, these effects may become devastating. One must clearly understand the behaviour of such potentially damaging particles in proposed detector systems.

At present there is a dearth of experimental information on the number of neutrons with energies between 0.1 and $10 \mathrm{MeV}$ in the cascades originating from 1 to several hundred $\mathrm{GeV}$ hadrons. Most data as do exist were obtained for shielding studies $[5,6,7]$. Except for the last-mentioned reference, only the highenergy cascade-propagating components of the cascade were studied. Even in the study of Volynchikov et al.[7] there are no data on the number of neutrons in the 
energy region of interest. Other experimental data were obtained for calorimeter studies $[8,9]$ where only radially integrated energy depostion as a function of depth in the cascade is of interest Some of the elaborate neutron transport codes originally developed for nuclear reactor studies have been coupled with hadron cascade programs to simulate these processes $[10,12]$ and they have been used in comparisons with data from calorimeter studies [11]. Other models incorporate parametrization of the low energy multiplication to account for the enhancement [13]. However, without experimental checks of the numbers of low-energy neutrons from cascades initiated by hadrons of known energy and in simplified geometries, one cannot decide if the predictions of the cascade programs in the complex geometries of collider detectors and in cascades initiated by the fragments of the hadron-hadron collisions are correct. Therefore, an experimental program was set out in order to measure neutron longitudinal and radial profiles and energy distributions within the volume of various calorimeter-type geometries. The results of an initial run with an iron dump exposed to a $200 \mathrm{GeV} / \mathrm{c}$ positive secondary beam at the CERN SpS are reported here.

The parameters of interest from detector considerations in such a study are the following:

a. Number and energy distribution of backscattered neutrons emerging from the front face of a calorimeter or a machine element as a function of bombarding particle energy (albedo).

b. Neutron fluence versus energy at various depths in the cascade, especially near shower maximum.

c. Radial spread of the hadron cascade, to define the radius around the entry point within which one must search to capture a certain percentage of the total energy.

d. Energy leakage after a given number of interaction lengths and the attenuation length after cascade maximum, to determine the optimal calorimeter thickness and tail catcher characteristics.

In order to measure these properties, one must design a measurement method which does not interfere with the cascade development and a dump module which simulates the actual calorimeter materials. It is especially important to include the correct mixture of hydrogenous materials with heavy nuclei, since the moderating effects of hydrogenous material on the observed neutron spectrum can be quite dramatic [14]. In this experiment inorganic neutron activation detectors were chosen as the detector elements. Each detector represented less than $0.4 \%$ of a nuclear interaction length, so its presence will not have perturbed the shower development at all to the accuracy of our measurements. These detectors were 
principally made of aluminium, which closely simulates the presence of the silicon in some proposed collider calorimeters.

\section{Description of the Experiment}

\subsection{Choice of Detectors}

In order to develop the information necessary to evaluate silicon damage potential, it is necessary to measure neutrons to less than $1 \mathrm{MeV}$ kinetic energy. In this experiment activation detectors were chosen which had energy thresholds of $0.5,2.0$, and $5.0 \mathrm{MeV}$, along with a high-energy spallation reaction which is generally sensitive to any high-energy hadron. This latter detector was included in order to give a direct comparison with simulations made using codes which are not able to predict the low-energy neutron component of the cascade. The activation reactions and their energy thresholds are summarized in Table 1. Further details are given in Chapter 3.

Table 1

Summary of Activation and Dosimetric Techniques

\begin{tabular}{lllll}
\hline Reaction & Sample size & $\begin{array}{l}\text { Measurement Assumed } \\
\text { Technique }\end{array}$ & $\begin{array}{l}\text { Nominal Energy } \\
\text { cross- } \\
\text { section }\end{array}$ & $\begin{array}{l}\text { Limits and } \\
\text { particles detected }\end{array}$ \\
\hline a) Activation Detectors & & & \\
${ }^{115} \mathrm{In}\left(\mathrm{n}, \mathrm{n}^{\prime}\right)^{115} \mathrm{In}$ & $0.3 \mathrm{~mm} \times 10 \mathrm{~mm} \phi$ & $\mathrm{GeLi}$ & $\mathrm{AFA}$ & $0.5-10 \mathrm{MeV}$ neutrons \\
${ }^{32} \mathrm{~S}(\mathrm{n}, \mathrm{p})^{32} \mathrm{P}$ & $6 \mathrm{~mm} \times 23 \mathrm{~mm} \phi$ & $\mathrm{GMT}$ & $300 \mathrm{mb}$ & $3-25 \mathrm{MeV}$ neutrons \\
${ }^{27} \mathrm{Al}(\mathrm{n}, \alpha)^{24} \mathrm{Na}$ & $4 \mathrm{~mm} \times 10-30 \mathrm{~mm} \phi \mathrm{GMT}$ & $300 \mathrm{mb}$ & $6-25 \mathrm{MeV}$ neutrons \\
${ }^{27} \mathrm{Al}(\mathrm{h}, \mathrm{x})^{18} \mathrm{~F}$ & $4 \mathrm{~mm} \times 10-30 \mathrm{~mm} \phi \mathrm{NaI}$ & $8 \mathrm{mb}$ & $>35 \mathrm{MeV}$ hadrons
\end{tabular}

b) Dosimeters

Radiophotoluminescent dosimeters Thermoluminescent dosimeters Kodak x-ray film
$6 \mathrm{~mm} \times 1 \mathrm{~mm} \phi \quad$ Schott-Jerner DOS2 $x \times y \times z \mathrm{~mm}^{3}{ }^{7} \mathrm{LiF}$

$\mathrm{X} \mathrm{mm} \times \mathrm{Y} \mathrm{mm}$ sheets

The dosimeter types used to measure absorbed dose are also summarized in Table 1. A range of dosimeter types were irradiated in order to cover as wide a range of dose as possible. After the irradiation it was found that the thermoluminescent dosimeters and $\mathrm{x}$-ray films had been irradiated to doses higher than their acceptable response range and so the results from these dosimeter types were abandoned. 
The activation detectors were mounted in holes, accurately punched out of $4 \mathrm{~mm}$ thick by $24 \mathrm{~cm} \times 30 \mathrm{~cm}$ aluminium plates. The radiophotoluminescent and thermoluminescent dosimeters were first placed in polyethylene capsules of $8 \mathrm{~mm}$ internal diameter before being mounted into the aluminium detector plates. In the case of the aluminium activation detectors, the samples were the same discs that has been punched from the holes. A sketch of a detector plate containing the aluminium samples is given in Fig. 2. It will be seen that the size of the detectors was made larger as the radial distance off axis increased. This was an attempt to keep the total activity in each sample approximately constant so as to ease the general problems in the assay of the samples, e.g., constant counting times, single source-detector distance etc. In addition this method of mounting the detectors provided a radial positional accuracy of better than $0.5 \mathrm{~mm}$ and considerably eased the problem of book-keeping.

\subsection{Description of the Dump}

The iron dump was made of twenty $5 \mathrm{~cm}$ thick iron plates, with the bottom and one side machined flat to ensure accurate alignment. Before assembly several of the plates were weighed and measured to determine the density of the iron. This was found to be $7.86 \pm 0.02 \mathrm{~g} \cdot \mathrm{cm}^{-3}$. The plates were welded to an iron framework with gaps of $7 \mathrm{~mm}$ between the plates. Extra detector slots were provided in front of the first and behind the last plates A sketch of the dump is given in Fig. 3.

Fiducial marks were engraved at the centre of the front and back faces and along the axis on the top surface of the dump. These marks served to survey the dump into its correct position in the beam line where it was placed on and adjustable iron table. They also allowed the accurate positioning of the detector plates within the dump.

\subsection{Arrangement of the Detector Plates}

Because of the limited space in the slots, it was not possible to expose each detector type in each position and so obtain as complete a picture of the cascade development as would have been desirable. The choice of detectors as a function of position is given in Table 2.

When the time came to load the dump with the plates after the initial settingup of the beam, it was found that Plate 9 would not fit into its correct slot because of welding irregularities. At the last minute it was decided to combine the x-ray film of Plate 2 with the other dosimeters of Plate 1 and move the other plates up to and including Plate 9 forward one slot. This was unfortunate in that Slot 9 was expected to be at the point of maximum developmnnt of the cascade, but the tight schedule in the dump manufacture (it was only finished several hours 
Table 2

Arrangement of Detector Plates

\begin{tabular}{rrl}
\hline \hline \multicolumn{1}{c}{ Plate } & Slot & Detectors \\
\hline 0 & 0 & Indium, Aluminum $\left({ }^{18} \mathrm{~F}\right.$ and $\left.{ }^{24} \mathrm{Na}\right)$ \\
1 & 1 & RPL + TLD + X-ray film \\
3 & 2 & Aluminum $\left({ }^{24} \mathrm{Na}\right.$ only $)$ \\
4 & 3 & Sulphur \\
5 & 4 & RPL + TLD + X-ray film \\
6 & 5 & Aluminum $\left({ }^{18} \mathrm{~F}\right.$ and $\left.{ }^{24} \mathrm{Na}\right)$ \\
7 & 6 & Sulphur \\
8 & 7 & Indium \\
9 & 8 & RPL + TLD \\
& 9 & Empty \\
10 & 10 & Aluminum $\left({ }^{24} \mathrm{Na}\right.$ only $)$ \\
11 & 11 & X-ray film \\
12 & 12 & Aluminum $\left({ }^{18} \mathrm{~F}\right.$ and $\left.{ }^{24} \mathrm{Na}\right)$ \\
13 & 13 & Sulphur \\
14 & 14 & Indium \\
15 & 15 & RPL + TLD \\
16 & 16 & Aluminum $\left({ }^{24} \mathrm{Na}\right.$ only $)$ \\
17 & 17 & X-ray film \\
18 & 18 & Aluminum $\left({ }^{18} \mathrm{~F}\right.$ and $\left.{ }^{24} \mathrm{Na}\right)$ \\
19 & 19 & RPL + TLD \\
20 & 20 & Indium, Aluminum $\left({ }^{24} \mathrm{Na}\right.$ only \\
\hline
\end{tabular}

before it was due to be put into the beam-line) did not allow all slot positions to be checked.

\subsection{Irradiation Profile}

The time profile of the irradiation was monitored in a number of ways. Three multi-wire proportional counters were installed in the $\mathrm{H} 6$ beam line. Two of these, TSCAL(37) and TSCAL(17), were positioned upstream of the experimental setup and one of them some XX metres downstream. At the intensities used for this experiment it was known that the first two of these would suffer somewhat from saturation effects. The third counter, TSCAL(19), being behind the dump, saw only those hadrons which had not interacted inelastically in the dump, and so did not suffer from saturation. However its response could not be determined 
in terms of the number of particles incident on the dump. The signal from each of these three counters was read out on a pulse-by-pulse basis.

A fourth time profile was obtained from on of the monitors of the stray radiation field for radiation protection purposes in the area, PAXN1261, placed about $2 \mathrm{~cm}$ laterally from the dump. The charge output from an $18 \mathrm{~atm}$ Argon chamber was digitized and the signals fed to a zatemeter with a time constant of approximately $100 \mathrm{~s}$. The output of this ratemeter was interrogated every 3 minutes.

The irradiation profiles are shown in Fig. $4 \mathrm{a}-\mathrm{d}$ for each of the four monitors. The nominal start and end of the irradiations were at 185 minutes and $1362 \mathrm{~min}-$ utes respectively on the time scale of the TSCAL counters and at 157 minutes and 1334 minutes respectively on the time scale of the PAXN1261 monitor. In real-time terms the nominal end of the irradiation was taken to be at $1437 \mathrm{~h}$ Central Europe Daylight-saving Time on Thursday 10th September, and the duration of the irradiation was taken to be 1177 minutes. Due to a computer failure, the last 30 minutes of the irradiation could not be monitored with the three TSCAL counters. The time profile for this last part of the irradiation for these three counters was therefore reconstructed from that of the PAXN1261 monitor.

\subsection{Beam Intensity and Profile}

There was no calibrated beam monitor available close to the experimental dump. The beam intensity was therefore measured from the production of ${ }^{24} \mathrm{Na}$ in a $0.5 \mathrm{~mm}$ thick aluminium sheet placed $60 \mathrm{~cm}$ upstream of the dump. A production cross-section of $8.1 \pm 0.6 \mathrm{mb}$ was assumed [15] for the $200 \mathrm{GeV}$ incident particles which at that energy are known to consist of at least two-thirds protons [16].

The beam intensity averaged over the nominal irradiation time was $1.32 \times$ $10^{7} \mathrm{~s}^{-1}$ with an error of $10 \%$ which includes systematic errors in the cross-section and the efficiency of the gamma spectrometer. The nominal beam intensity during the exposure was $2 \times 10^{8}$ particles per pulse with a pulse repetition period of 14.4 seconds.

The radial profile of the beam was measured using the radioactivity induced in the aluminium beam-monitoring plate. After the exposure an auto-radiograph of the plate was made using an x-ray film and the beam profile measured using a densitometer. The vertical and horizontal sigmas of the beam were $9 \mathrm{~mm}$ and $12 \mathrm{~mm}$ respectively. 


\section{Experimental Techniques}

\subsection{Introduction}

As explained in the previous sections, the fluence and dose measurements were all based on techniques which integrated the quantity to be measured over the time of the experiment. The radioactivation techniques were chosen because the isotopes to be measured had convenient half-lives, i.e., were neither too short so that variations of the fluence rate during exposure caused unacceptable errors in correcting for this variation, nor too long so that the low levels of radioactivity produced could not be measured without sophisticated assay techniques. Most of the isotopes produced could be measured with a simple sodium iodide gamma spectrometer or Geiger counter.

This chapter contains a brief description of the different activation and dosimetric techniques used in the experiment and a description of the corrections made to obtain the true average fluence rates.

\section{2 ${ }^{18} \mathrm{~F}$ from Aluminum}

The spallation reaction in aluminium, ${ }^{27} \mathrm{Al}(\mathrm{x}, \mathrm{spall}){ }^{18} \mathrm{~F}$ was used to estimate the flux-density of hadrons having energies above about $35 \mathrm{MeV} .{ }^{18} \mathrm{~F}$ has a halflife of $109.8 \mathrm{~min}$ and decays via positron emission $\left(96.9 \%, \mathrm{E}_{\max }=0.635 \mathrm{MeV}\right)$. Provided that the positrons are annihilated close to the source, which can be ensured by placing the source between two sheets of perspex, counting can be carried out by gamma spectrometry. The isotope was assayed by counting the $0.511 \mathrm{MeV}$ annihilation quanta coming from the samples when placed on a perspex cap over a 3 " $\times 3$ " sodium iodide crystal coupled to a standard amplifier + pulse height analyser chain.

The measurement of the annihilation photons was complicated by the contribution in the $0.511 \mathrm{MeV}$ peak window of the Compton photons coming from the 1.369 and $2.754 \mathrm{MeV}$ gamma rays of the ${ }^{24} \mathrm{Na}$, also present in the aluminium samples. The procedure adopted was to make at least three measurements of the activity of each sample at different times after the irradiation and to determine the annihilation and Compton photon contributions from a least squares analysis.

The cross section for the production of ${ }^{18} \mathrm{~F}$ from ${ }^{27} \mathrm{Al}$ as a function of hadron energy is given in Fig. 5. An effective cross-section of $11 \mathrm{mb}$ for the production of ${ }^{18} \mathrm{~F}$ from aluminium was chosen, mainly for historical reasons, in order to convert the measured activity into the flux density of hadrons above $35 \mathrm{MeV}$. Newer data, presented as a function of energy in Fig. 5, suggests that a better energy-averaged values would be $7 \mathrm{mb}$. The data in this report is based on the older value; it will be reassessed in the final publication. 


\section{$3.3^{24} \mathrm{Na}$ from Aluminum}

The production of ${ }^{24} \mathrm{Na}$ from ${ }^{27} \mathrm{Al}$ was used to estimate the fluence of neutrons in the energy range $6-25 \mathrm{MeV} .{ }^{24} \mathrm{Na}$ decays by $\beta^{-}$and gamma emission; the photons have energies of 1.369 and $2.754 \mathrm{MeV}(100 \%)$ and the half-life is $15.02 \mathrm{~h}$. The isotope was assayed by determining the counts in the window of the upper $2.754 \mathrm{MeV}$ peak when the sample was placed on the perspex cap covering the $3 " \times 3$ " sodium iodide crystal mentioned above.

The cross-section for the production of ${ }^{24} \mathrm{Na}$ as a function of energy is given in Fig. 6. An effective cross-section of $85 \mathrm{mb}$ can be used to derive a nominal flux density of neutrons between 6 and $25 \mathrm{MeV}$, even in the presence of high-energy hadrons.

\section{$3.4{ }^{32} \mathrm{P}$ from Sulphur}

Alberto Fassò to provide.

Figure 7 contains the xsec.

\section{$3.5^{115 \mathrm{~m}} \mathrm{In}$ from Indium}

Alberto Fassò to provide.

Figure 8 contains the xsec.

\subsection{Radiophotoluminescent Dosimeters}

The radiophotoluminescent dosimeters used in this experiment to measure the integrated absorbed dose were glass rods of $1 \mathrm{~mm}$ diameter and $6 \mathrm{~mm}$ length, made by Schott-Jenner Glaswerke, type DOS2. After irradiation, the luminescent emission from the glass when stimulated with UV irradiation was measured with a Toshiba FGD-6 reader. The useful dose range of these dosimeters is in the range 0.1 gray to 1 Mgray [17].

\subsection{Standard Calculations in Radioactivity Measurements}

Radioactive decay of the radioisotopes produced occurs during the irradiation, during the time that elapses before counting the sample and during the actual counting of the sample. The instantaneous count rate at the start of the counting period was determined from the measured average count rate by multiplying this latter by the factor:

$$
f_{\text {count }}=\frac{\lambda \cdot t_{c}}{1-\mathrm{e}^{\lambda t_{c}}},
$$

where $\lambda$ is the decay constant of the radionuclide and $t_{c}$ is the duration of the count. The activity at the nominal end of the irradiation was calculated by 
multiplying the instantaneous count rate at the start of the counting period by the factor:

$$
f_{\text {lapse }}=\mathrm{e}^{\lambda t_{1}},
$$

where $t_{1}$ is the time elapsed between the nominal end of the irradiation and the start of the count. The saturation activity, assuming a uniform irradiation, is determined by multiplying the activity at the end of the irradiation by the factor:

$$
f_{\text {irrad }}=\frac{1}{1-\mathrm{e}^{-\lambda t_{i}}},
$$

where $t_{i}$ is the irradiation time.

When the efficiency of the counter is known explicitly, as is the case in the radioassay by gamma spectrometry, the saturation count rates can be converted into disintegration rates (activity). From these and the masses of the samples the saturation specific activity in $\mathrm{Bq}$ per mole of parent atom could be calculated.

The saturation specific activity is to be regarded as the physical quantity actually measured in the experiment. To derive flux-densities from this quantity is a matter of educated guess-work since the activity is the integral from the threshold energy to the energy of the incident protons of the fluence spectrum multiplied by the cross-section, summed over all particle types in the cascade. When one particular process is dominant and the cross-section is approximately independent of energy, the activity can be expressed as the product of a nominal flux density and an effective cross-section.

When the efficiency of the counter is not known explicitly, as in the case of beta counting for ${ }^{32} \mathrm{P}$ with the Geiger counters, the nominal flux density is obtained by the application of a calibration factor derived from the irradiation of an identical sample in a field of known characteristics.

The authors approach the conversion of activity to flux density with some caution and do not wish to imply that the definitions of nominal flux density are necessarily correct. The conversion of activity to nominal flux density is made in the belief that the true flux densities correspond to the present definitions to within a factor of two. In quoting such definitions it is not implied that other types of particles or particles outside the specified energy range do not contribute to the activities measured.

\subsection{Correction for Non-Uniform Irradiation}

The assumption that the incident flux density is constant with time is seldom, if ever, valid, and in the case of isotopes with short half lives, the flux density calculated on this assumption can be significantly different from the true average 
flux density (total fluence divided by time of exposure). Correction for the nonuniform irradiation profile was made by calculating a Run-uniformity Factor for each isotope [18]. This procedure calculates the correction factor by which the flux density, calculated using the nominal irradiation times and the assumption of uniform irradiation, must be multiplied to give the average flux density, i.e., the time-integrated flux density divided by the nominal irradiation time.

The Run-uniformity Factors were calculated for each isotope measured on the basis of the four radiation profiles obtained, three from the TSCAL counters and one from the PAXN1261 monitor. These factors are given in Table 3. The final column of this table shows the values of the Run-uniformity factors used for correcting the data.

Table 3

Run-Uniformity Factors

\begin{tabular}{|c|c|c|c|c|c|c|}
\hline \multirow{2}{*}{$\begin{array}{l}\text { Isotope } \\
{ }^{18} \mathrm{~F}\end{array}$} & $\begin{array}{l}\text { Half-life } \\
\text { (minutes }\end{array}$ & \multicolumn{4}{|c|}{ TSCAL(37)TSCAL(17)TSCAL(19)PAXN1261 } & $\begin{array}{l}\text { Assumed } \\
\text { value }\end{array}$ \\
\hline & 109.8 & 1.523 & 1.527 & 1.537 & 1.519 & 1.527 \\
\hline${ }^{115 m}$ In & 269.2 & 1.187 & 1.187 & 1.191 & 1.176 & 1.185 \\
\hline${ }^{24} \mathrm{Na}$ & 900.0 & 1.047 & 1.046 & 1.048 & 1.042 & 1.046 \\
\hline${ }^{32} \mathrm{P}$ & 20578.0 & 1.002 & 1.002 & 1.002 & 1.002 & 1.002 \\
\hline
\end{tabular}

\section{Experimental Results}

\subsection{Radial Fluence and Dose Measurements}

After the irradiation, the various activation samples were dismantled from the detector plates. After a waiting period of 2-3 hours to allowed short-lived activities such as ${ }^{11} \mathrm{C}$ to decay, the assay of the aluminium for ${ }^{18} \mathrm{~F}$ and of the indium foils for ${ }^{115} \mathrm{~m}$ In was started. It took approximately 8 hours to process the 82 aluminium samples and 50 indium foils-about the maximum that could be achieved with single counting systems before the decay of the radioactivity to be measured. While counting the aluminium samples for ${ }^{18} \mathrm{~F}$, they were also assayed for ${ }^{24} \mathrm{Na}$. On the following day, the remaining aluminium samples were assayed for ${ }^{24} \mathrm{Na}$ (another 102 samples). The remaining sulphur pellets and the RPL dosimeters were not measured until several days after the irradiation.

The measurements of fluence and dose from the different detector systems are shown plotted as a function of the radial distance off-axis in Figs. 9-13. In these Figures the different azimuthal measurements have been plotted separately. It will be seen that the scatter in the points at different azimuths for a given 
radial distance is of the order of a few percent for the three low-energy neutron detectors. This can be assumed to be due to the statistical errors in the counting of the radioactivity which were maintained at the few-percent level. The ${ }^{18} \mathrm{~F}$ from aluminium and the dose measurements, however, show significant scatter (the former due to the fitting procedure needed to subtract the ${ }^{24} \mathrm{Na}$ activity from the measurements and the second due to the inherent inaccuracy of the dosimetry system), but none of this is correlated with the azimuthal direction. An exception to this azimuthal symmetry is to be found in the

In measurements in the downwards direction at the extreme radial position. On average this detector showed a flux density measurement some $20 \%$ higher than the equivalent position in the upwards direction. This is presumably an effect due to the $5 \mathrm{~cm}$ thick steel support plate on which the dump was installed during the irradiation. Nevertheless the cascade measurements were assumed to be azimuthally symmetric and the various measurements at a given radial position combined to give a mean value. These are shown plotted against radius in Figs. 14-18. The vertical error bar on these measurements is the standard error on the mean value; the error on the single measurement at zero radius was estimated from the scatter in the points at the smallest radial position. The error bar on the radial position indicates the radial extent of the circular samples. In the case of the RPL dosimeters this error is the size of the container in which the dosimeters were placed and so indicates a real uncertainty in the radial position.

These results show clearly the importance of the low-energy neutron component of the hadronic cascade. As can be seen from the comparative plots of fluxes measured by the different neutron detectors in Fig. 19a-d, the largest neutron flux is observed by the indium foil measurements: the neutron flux as measured by the ${ }^{27} \mathrm{Al}-{ }^{24} \mathrm{Na}$ reaction is about the same as that measured by the ${ }^{32} \mathrm{~S}-{ }^{32} \mathrm{P}$ reaction and is in general more than one order of magnitude less than that measured by the indium foils. Except on the axis of the cascade, the highenergy hadron flux is also of the same order of magnitude as the flux measured by either of the ${ }^{27} \mathrm{Al}-{ }^{24} \mathrm{Na}$ or ${ }^{32} \mathrm{~S}-{ }^{32} \mathrm{P}$ reactions. This means that the energy carried by neutrons in the $1 \mathrm{MeV}$ region is comparable to that carried by neutrons in the decade around $10 \mathrm{MeV}$ and is not much lower than that carried by the high-energy hadrons. This is consistent with the picture of hadronic cascades developed from interpretation of the results of simulation programs like HETC+MORSE [REF??].

The concept of an equilibrium cascade is illustrated in Fig. 20 where the fluence measurements from the ${ }^{27} \mathrm{Al}-{ }^{24} \mathrm{Na}$ reaction are shown on the same plot. The radial distribution becomes slowly less and less peaked as the depth increases, until, after cascade maximum in slot 9 , the radial shape remains constant with 
depth even though the absolute magnitude of the fluence changes.

Another interesting feature of the measurements is that the profile of the total energy deposition density measured by the RPL detectors is quite different from either the high-energy hadron or the neutron distributions. Figure 21a-d shows clearly that it is far more sharply peaked in radius. Furthermore, its on-axis value has its maximum at a shallower depth than the purely hadronic energy sampled by the activation detectors, as is seen in Fig. 22, The total dose includes both hadronic ( $\mathrm{dE} / \mathrm{dx}$ and nuclear excitation) and electromagnetic shower $\left(\pi^{0}\right.$ and $\eta$ meson) effects. The photons from the decays of these latter mesons have small opening angles at high energies: the average energy of these mesons will also be highest in the initial stages of the cascade. Since the electromagnetic showers will reach their maximum in about 5 radiation lengths ( 1.8 iron plates used for the dump), it is natural that the total energy density should reach its maximum after about 2 plates ( 0.6 interaction lengths) whereas the fluence of high-energy hadrons dose not reach a maximum until Slot 9 (2.4 interaction lengths). These different characteristics of the total energy deposition distribution indicate that it is sensitive to the early, high-energy, small-angle behaviour of the incident particle interactions and hence may well be a good measure of the interaction point and angle which is better than the hadronic-dominated distributions, as suggested by Akesson et al. [1].

In contrast, the radial dependence of the low-energy neutrons is much flatter. It can be represented by a negative exponential in radius which is approximately independent of depth. The low energy neutron flux is derived from the evaporation neutrons produced in the nuclear de-excitation process after a high-energy spallation reaction. These evaporation neutrons are essentially isotropically emitted from the struck nucleus. This hypothesis was roughly checked by using the high-energy fluence measurements using the ${ }^{27} \mathrm{Al}-{ }^{18} \mathrm{~F}$ reaction to calculate a "star density" distribution using an absorption mean free path of $16 \mathrm{~cm}$, assuming a low-energy neutron multiplicity of 10 and letting these neutrons be transported with a mean free path of $10 \mathrm{~cm}$. The resulting radial distributions are compared with the Indium fluence measurements in Fig. 23a-d.

No extra normalization has been included in these calculations. The agreement between the calculated and measured values is excellent given the simplicity of the calculation which does not take the transport in air into account on the front or back faces (Slots 0 and 20) nor in the gaps themselves (Slots 7 and 14). This supports the idea that the low-energy neutrons from the hadronic cascade form a "neutron gas" that percolates through the volume of a calorimeter. These data permit one to determine the fluence escaping from the detector and compare to detailed neutron transport models. These simulations are in progress and will 
be reported in another paper.

Other data of interest which can be obtained from Figs. 14-18 concern the variation of the radially integrated particle fluence (or dose) as a function of depth in the cascade. Simple fits by eye were made to the data in these figures and the radial fluence integrals calculated from these fits. The resulting curves as a function of radius at the different depths are given in Figs. 24-28. Features of the radial distributions mentioned in the above paragraphs are also exemplified in these integral plots. Those for the high-energy fluence and dose (Figs. 24 and 28) are much flatter and reach there plateau value at a smaller radius (more peaked radial fluence distributions). It is also encouraging that the integral of the highenergy hadron fluence per incident particle is close to unity (Fig. 14a), indicating a consistency in the cross-sections used. A comparison of the radial integrals for several different detectors is given in Fig. 29. The radial integrals are plotted as a function of depth in Fig. 30. However the low-energy neutron distributions, especially the indium measurements, indicate considerable flux-leakage from the sides of the dump. This leakage increases the observed low-energy neutron fluence by approximately a factor of 1.8 .

To summarize these data, for $200 \mathrm{GeV}$ hadron and at the maximum of the cascade, one produces approximately 3 neutrons per $\mathrm{GeV}$ of incident energy in an iron dump. The majority of these neutrons, some $70 \%$, are of low energy (0.1$5 \mathrm{MeV}$ ). The remaining $30 \%$ are fairly uniformly distributed in energy between 5 and several $100 \mathrm{MeV}$. The radially integrated albedo fluence is about $(60 \pm 30)$ neutrons $/ 200 \mathrm{GeV}$, of which some 45 have energies below $5 \mathrm{MeV}$. The number of neutrons leaving the stack after about 6 interaction lengths of iron is essentially identical to the albedo fluence leading to the picture that the low energy neutrons form a gas which permeates throughout the entire volume of a calorimeter. This is especially true for iron calorimeters, and those of some other heavy materials, because of the "hole" in the absorption cross-section of these neutron in the 0.01 $1 \mathrm{MeV}$ region. Thus neutrons in most non-hydrogenous calorimeters will survive many interaction lengths of material. This makes a proper understanding of the ultimate fate of these neutrons an important consideration in assessing damage. 


\section{References}

1. T. Akesson, M. Albrow, P. N. Burrows, T. Cox, J. P. deBrion, C. Fabjan, M. Holder, G. Ingelman, G. Jarlskog, K. Meier, P. G. Rancoita, J. Russ, J. Schukraft, G. Stevenson, A. Weidberg and R. Wigmans, "Detection of jets at future accelerators, in Proceedings of the Workshop on Physics at Future Accelerators," CERN 87-07, p. 182 (1987).

2. Proceedings of the LBL Workshop on Physics at the SSC, July, 1987 to be published (1987).

3. J. A. Harrell, P. L. Manke, and J. E. Gover, "The Application of Remote Electronics in a Nuclear Fuel Reprocessing Environment: Radiation Effects and Design Guidelines," SAND82-2151 (Albuquerque: Sandia National Laboratories, January 1983).

4. Report of the Fermilab Colliding Detector Facility to the Fermilab Program Advisory Committee, April 1987 and December, 1987.

5. A. Citron, L. Hoffmann, C. Passow, W. R. Nelson and M. Whitehead, "A study of the nuclear cascade in steel initiated by $19.2 \mathrm{GeV} / \mathrm{c}$ protons," Nucl. Instrum. and Meth. 32, 48 (1965).

6. G. W. Bennett, H. N. Brown, H. W. J. Foelsche, J. D. Fox, D. M. Lazarus, G. S. Levine, T. E. Toohig, R. H. Thomas and J. Kostoulas, "Particle distribution in a steel beam stop for $28 \mathrm{GeV}$ protons," Particle Accelerators 41, 229 (1973).

7. A. I. Volynchikov, V. B. Getmanov, A. A. Kuznetsov, G. I. Krupnyi, V. S. Lukanin, Ya. N. Rastsvetalov and V. H. Tatarenko, "Secondary radiation field measurements in a steel block absorbing $69 \mathrm{GeV} / \mathrm{c}$ proton beams," Serpukhov Inst. for High Energy Physics preprint (in Russian) IFVE 83-86; translated by I. Schulz-Dahlen in DESY Internal Report DESY L-Trans-284 (1983).

8. M. de Palma, C. Favuzzi, G. Maggi, E. Nappi, A. Ranieri and P. Spinelli, "Measurement, parametrization and fast simulation of hadronic showers in lead," Nucl. Instrum. and Meth. 219, 87 (1984).

9. K. Crase, W. E. Farley, H. Kruger, D. Selway and G.S. Sidhu. "Measurement of energy deposited by charged particle beams in composite targets," Lawrence Livermore Laboratory Report UCRL-52459 (1977).

10. T. A. Gabriel, "The high-energy transport code HETC," Oak Ridge National Laboratory Report ORNL/TM-9727 (1985); presented at the LEP Experimenters' Workshop on Shower Simulation, CERN, January 29-31 1985.

11. T. A. Gabriel, B. L. Bishop, A. I. Mincer, H. T. Freudenreich, J. A. Goodman, S. C. Tonwar, G. B. Yodh, D. Berley and R. W. Ellsworth, "A Monte-Carlo simulation of a hadronic calorimeter to protons of momentum 3.5 to $200 \mathrm{GeV} / \mathrm{c}$," Oak Ridge National Laboratory Report ORNL/TM-9270 (1985).

12. Examples of combined neutron transport and hadron cascade codes include HETC+MORSE, Oak Ridge National Laboratory. For more details of these and other similar programs, see the proceedings of the Argonne Workshop on Detector Simulation at the SSC, August, 1987. (To be published).

13. GEISHA, H. Fesefeldt, Aachen. For more details of these and other similar programs, see the proceedings of the Argonne Workshop on Detector Simulation at the SSC, August, 1987 (to be published).

14. C. Leroy, Y. Sirois, and R. Wigmans, "An experimental study of the contribution of nuclear fission to the signal of uranium hadron calorimeters," Nucl. Instrum. and Meth. A252, 4 (1986). 
15. S. B. Kaufman, M. W. Weisfield, E. P. Steinberg, B. D. Wilkins and D. Henderson, "Nuclear reactions of ${ }^{197} \mathrm{Au}$ with 11.5 and $300 \mathrm{GeV}$ protons," Phys. Rev. C14, 1121 (1976).

16. H. W. Atherton, C. Bovet, N. Doble, G. von Holtey, L. Piemontese, A. Placci, M. Placidi, D. E. Plane, M. Reinharz and E. Rossa, "Precise measurements of particle production by $400 \mathrm{GeV} / \mathrm{c}$ protons on beryllium targets," CERN Renort 80-07 (1980).

17. A. Izycka and H. Schonbacher, "High-level dosimetry for radiation damage studies at high-energy accelerators," CERN Divisional Report HS-RP.040/CF (1979), presented at the 3rd ASTM-Euratom Symposium on Reactor Dosimetry, Ispra 1-5 Oct. 1979.

18. G. R. Stevenson, "Normalization for non-uniform irradiation of activation detectors," Rutherford Laboratory Internal Report RHEL RP/PN 39 (1969). 


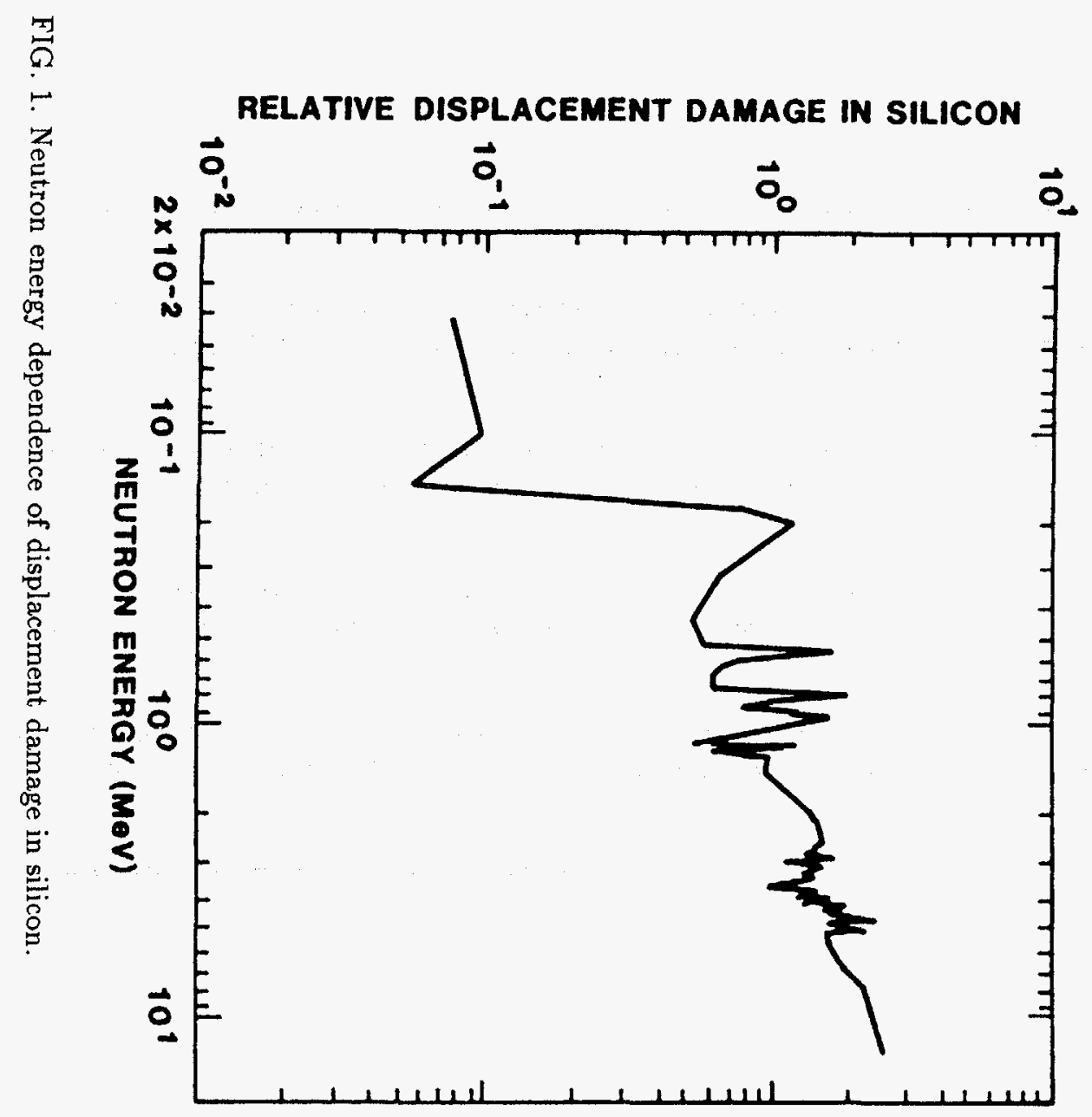




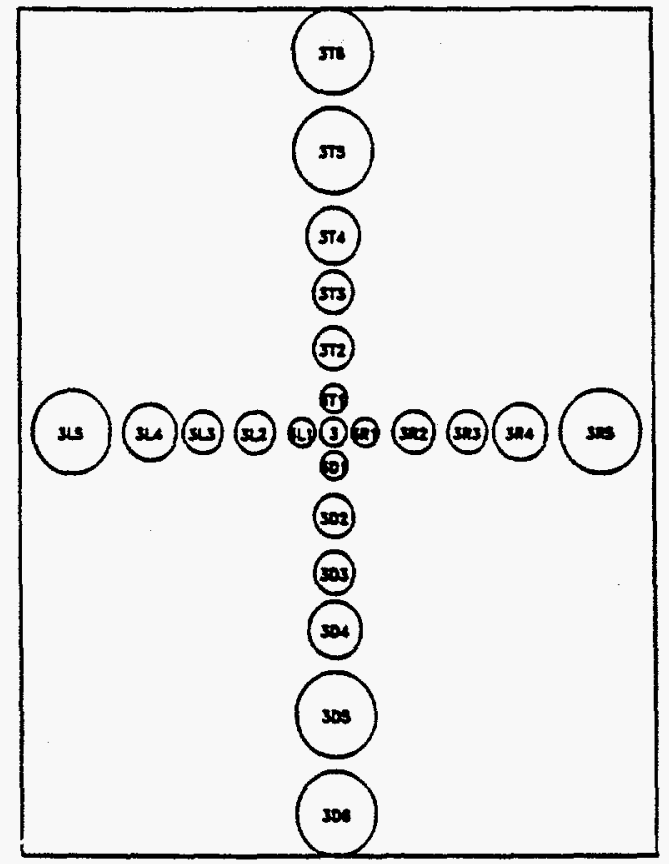

FIG. 2. Sketch of aluminium sample plate showing holes for the aluminium samples. The numbering scheme is indicated.

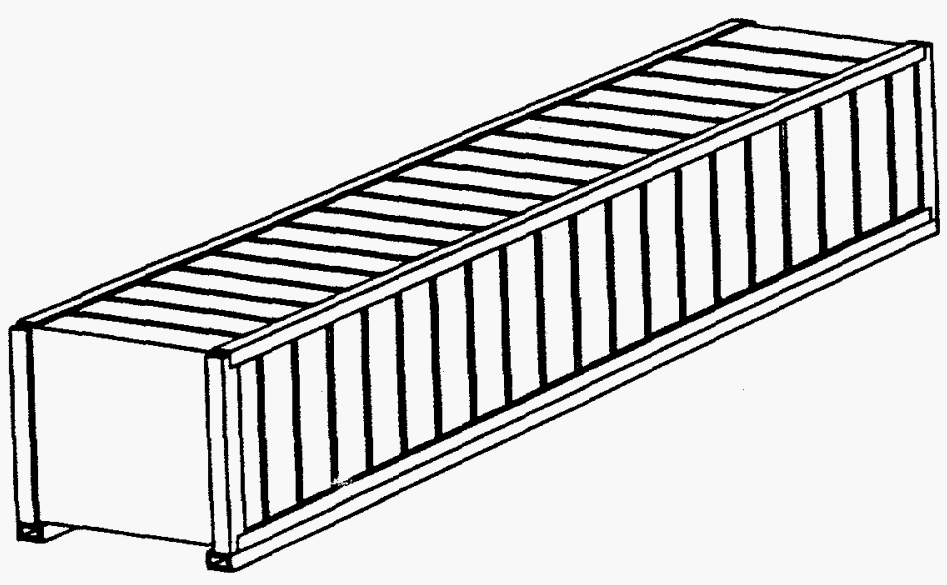

FIG. 3. Sketch of the dump assembly. 

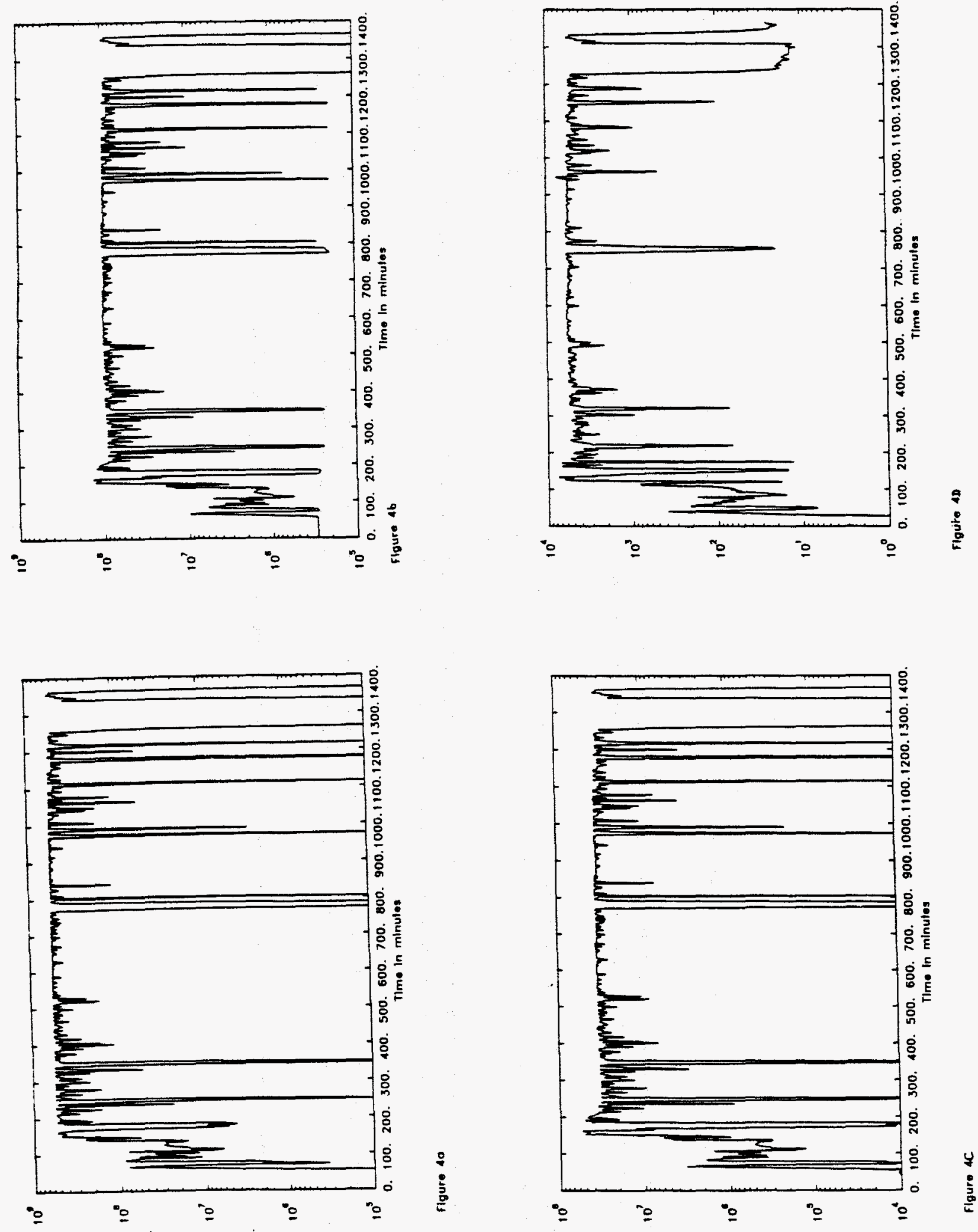

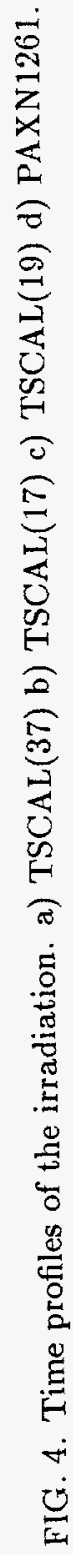




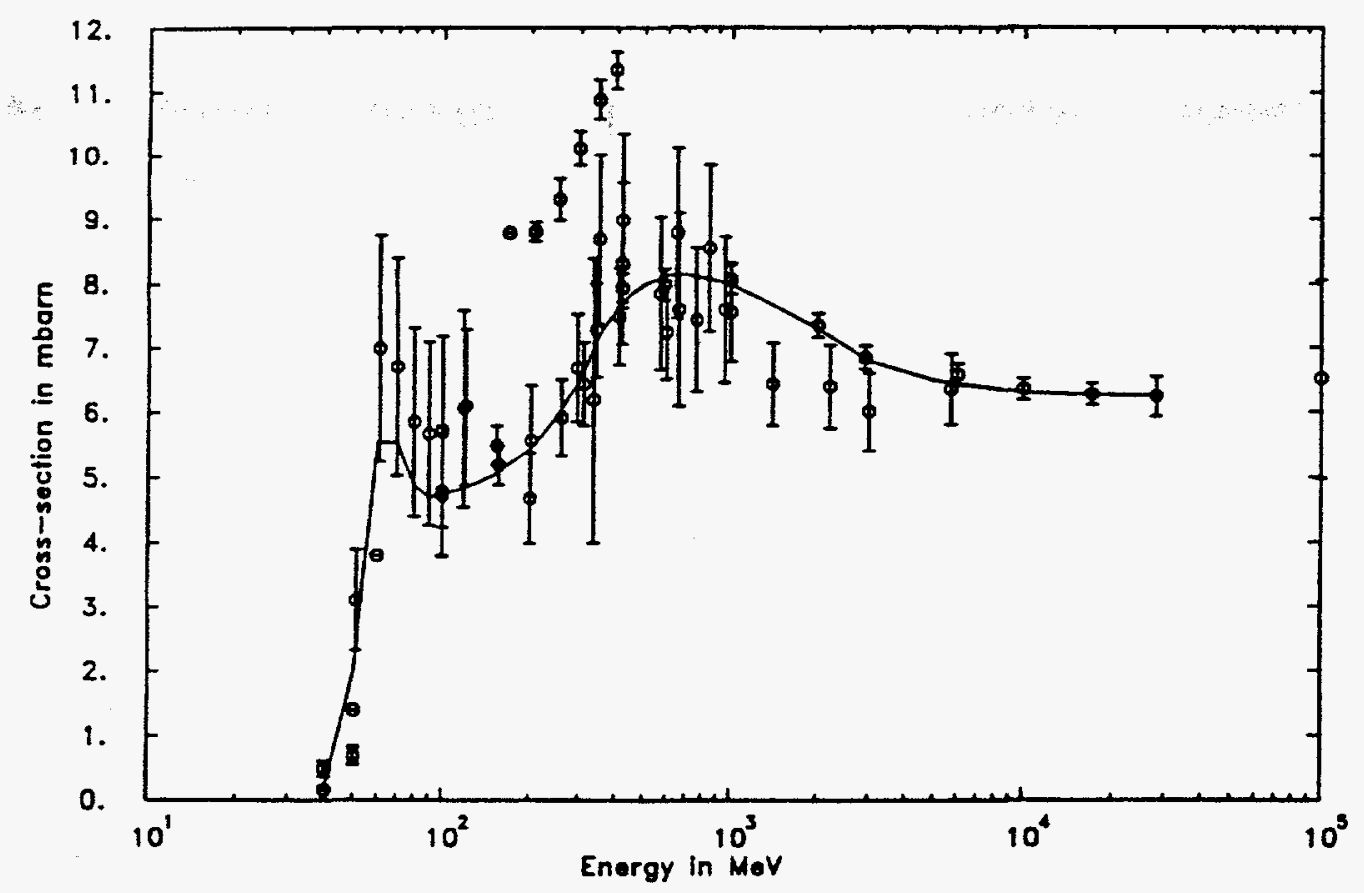

FIG. 5. Cross-section for the production of ${ }^{18} \mathrm{~F}$ from ${ }^{27} \mathrm{Al}$ as a function of hadron energy.

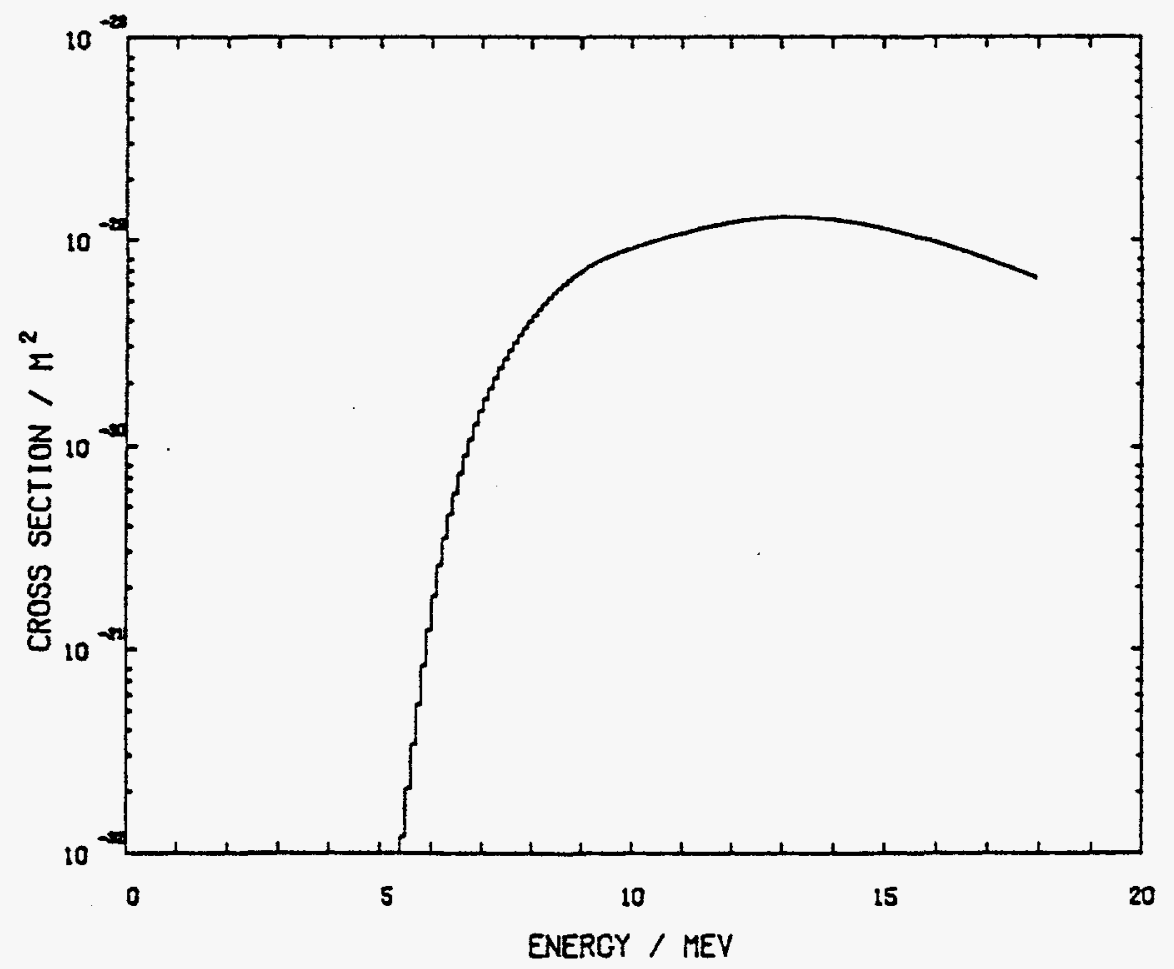

FIG. 6. Cross-section for the production of ${ }^{24} \mathrm{Na}$ from ${ }^{27} \mathrm{Al}$ as a function of hadron energy. 


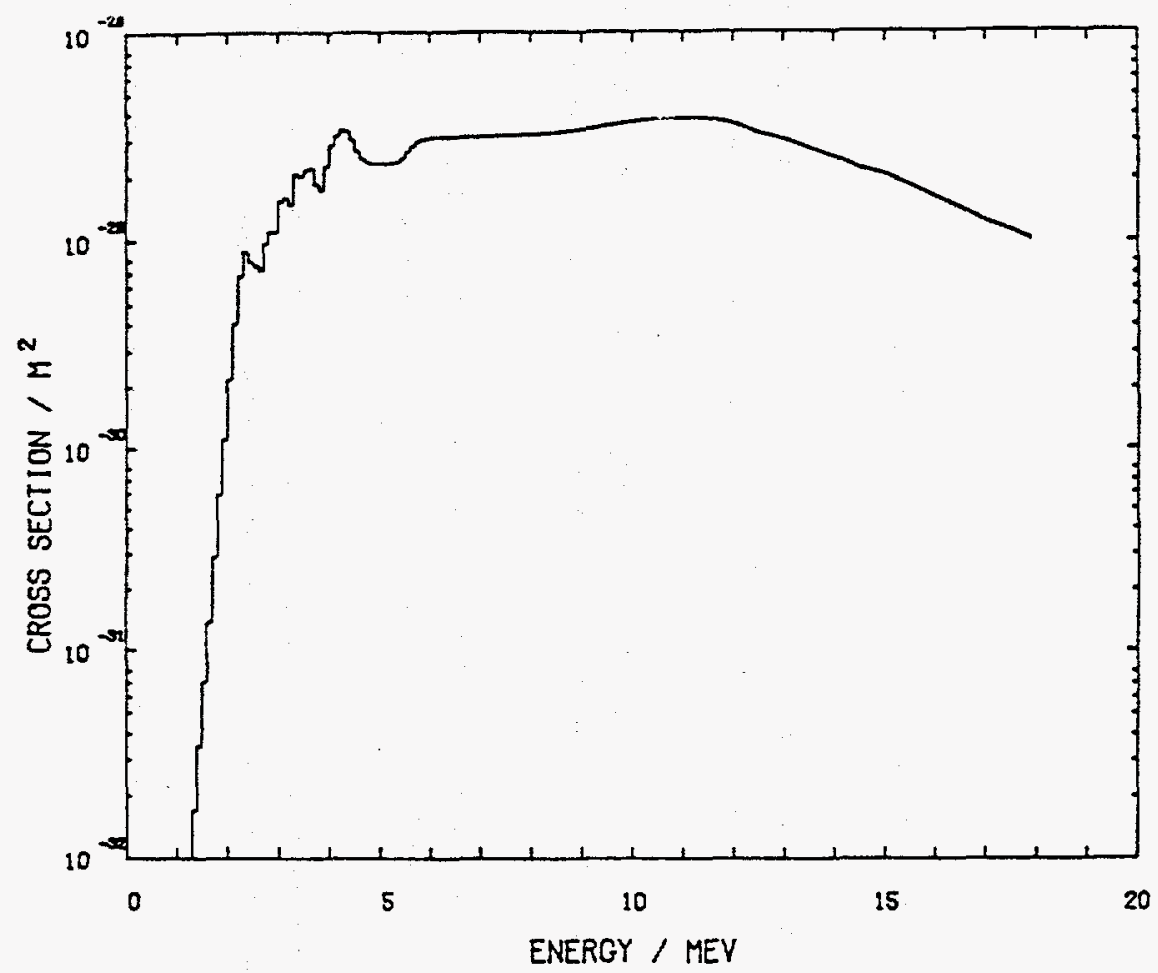

FIG. 7. Cross-section for the production of ${ }^{32} \mathrm{P}$ from ${ }^{32} \mathrm{~S}$ as a function of hadron energy.

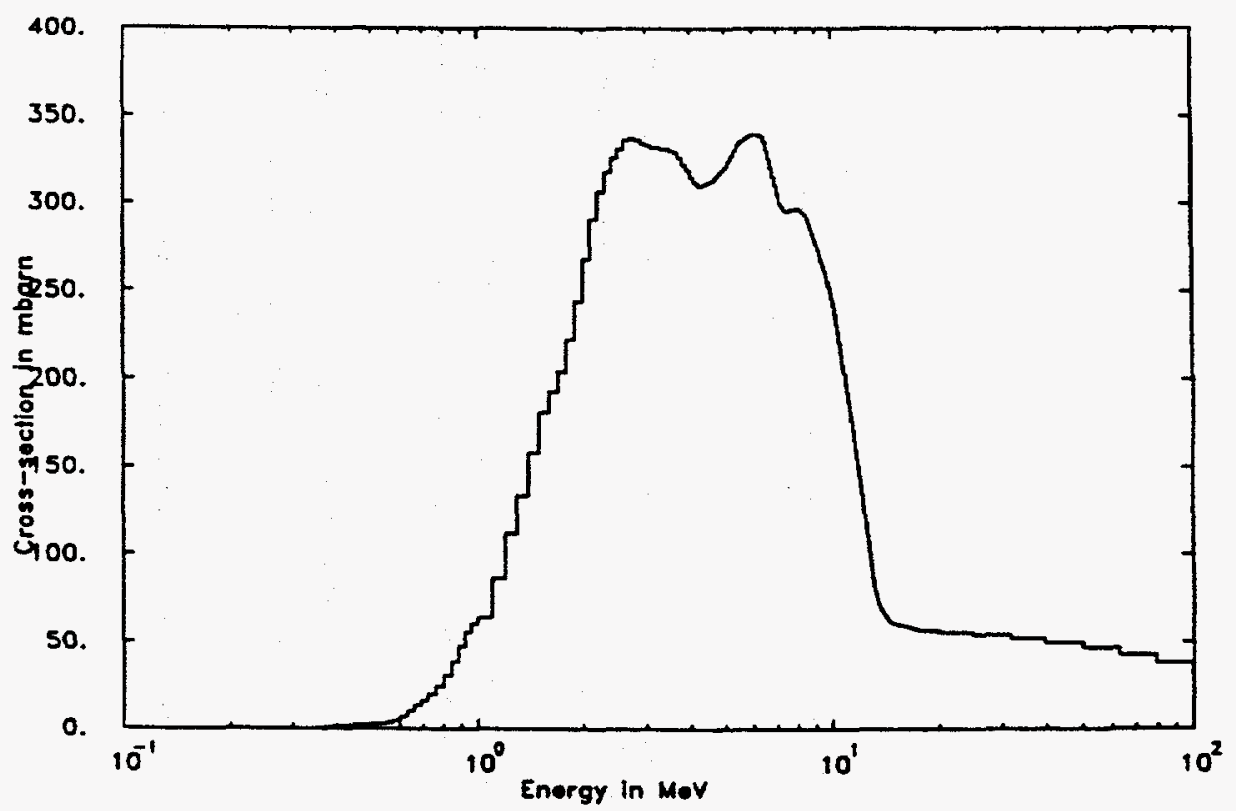

FIG. 8. Cross-section for the production of ${ }^{115} \mathrm{~m} I n$ from ${ }^{115} \mathrm{~m} I n$ as a function of neutron energy. 

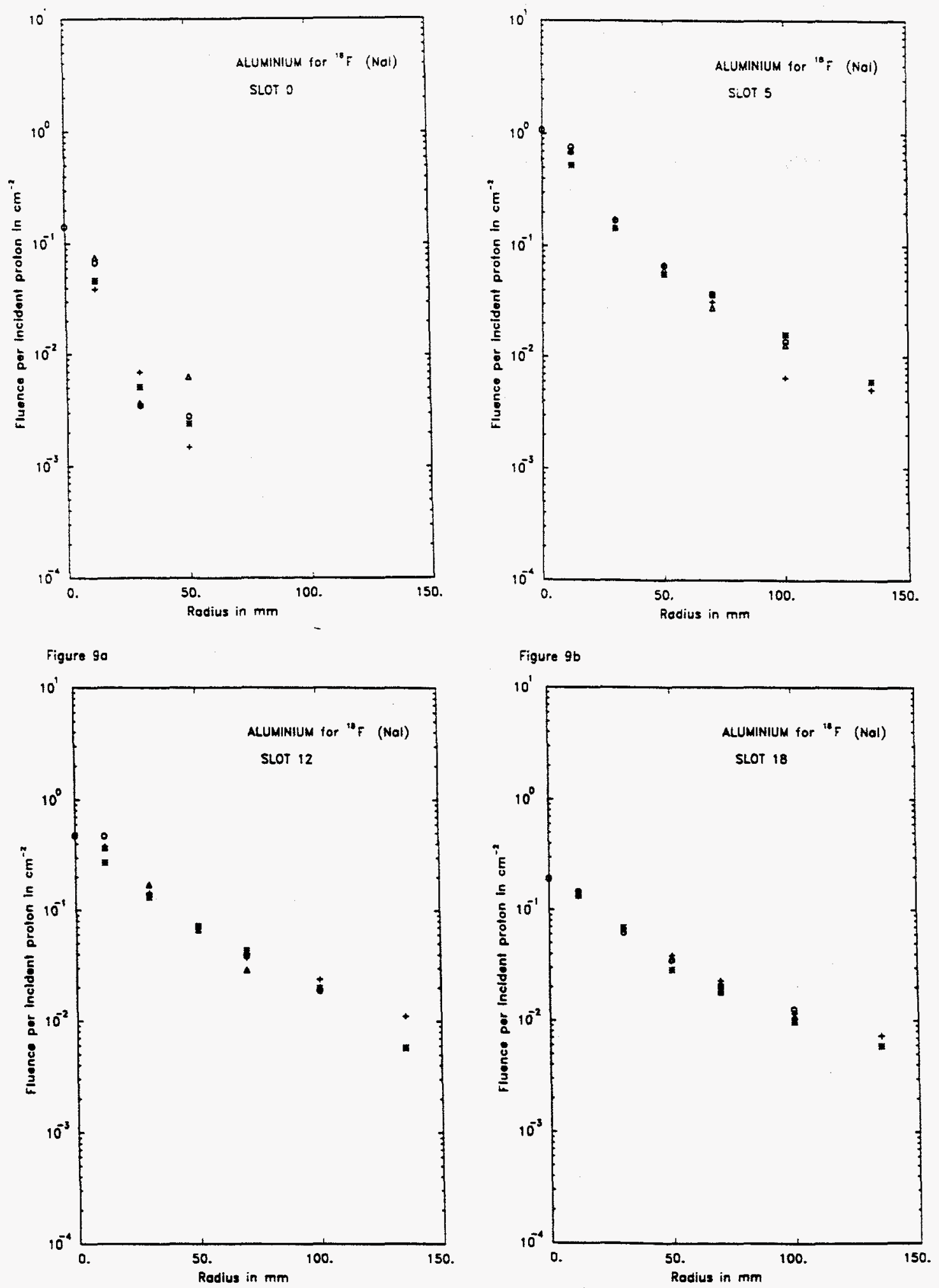

Figure 9c

Figure 9d

FIG. 9. Radial variation of hadron fluence as measured by ${ }^{18} \mathrm{~F}$ from ${ }^{27} \mathrm{Al}$. Meaning of symbols is: $\star \mathrm{D}$ (own), o L(eft), $\Delta \mathrm{R}$ (ight), $+\mathrm{T}(\mathrm{op})$. a) Slot 0, b) Slot 5 , c) Slot 12, d) Slot 18 . 

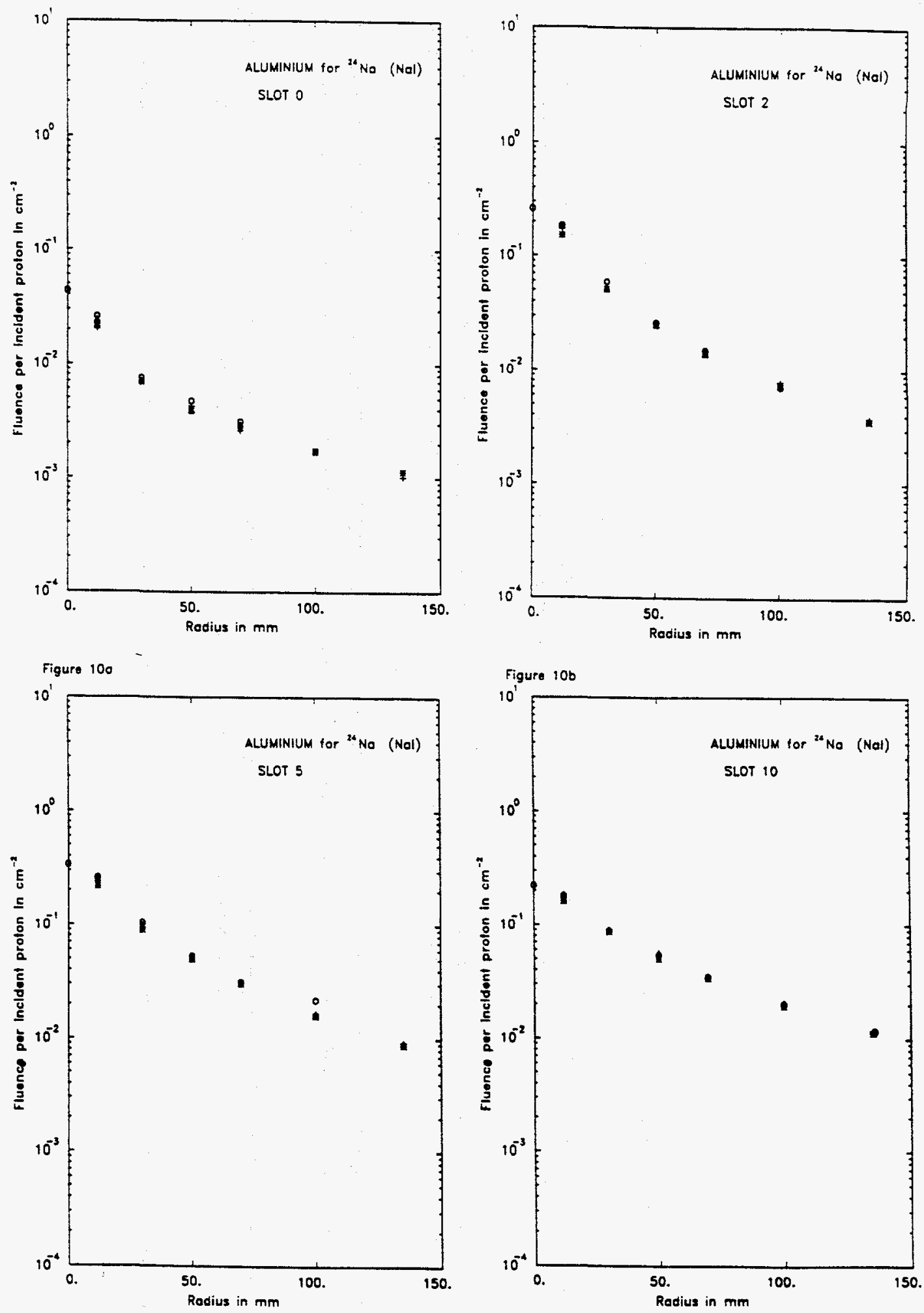

Figure $10 \mathrm{e}$

Figure $10 \mathrm{~d}$

FIG. 10. Radial variation of neutron fluence as measured by ${ }^{24} \mathrm{Na}$ from ${ }^{27} \mathrm{Al}$. Meaning of symbols is: $\star \mathrm{D}$ (own), o L(eft), $\Delta \mathrm{R}$ (ight), $+\mathrm{T}(\mathrm{op})$. a) Slot 0, b) Slot 2 , c) Slot 5 , d) Slot 10, 

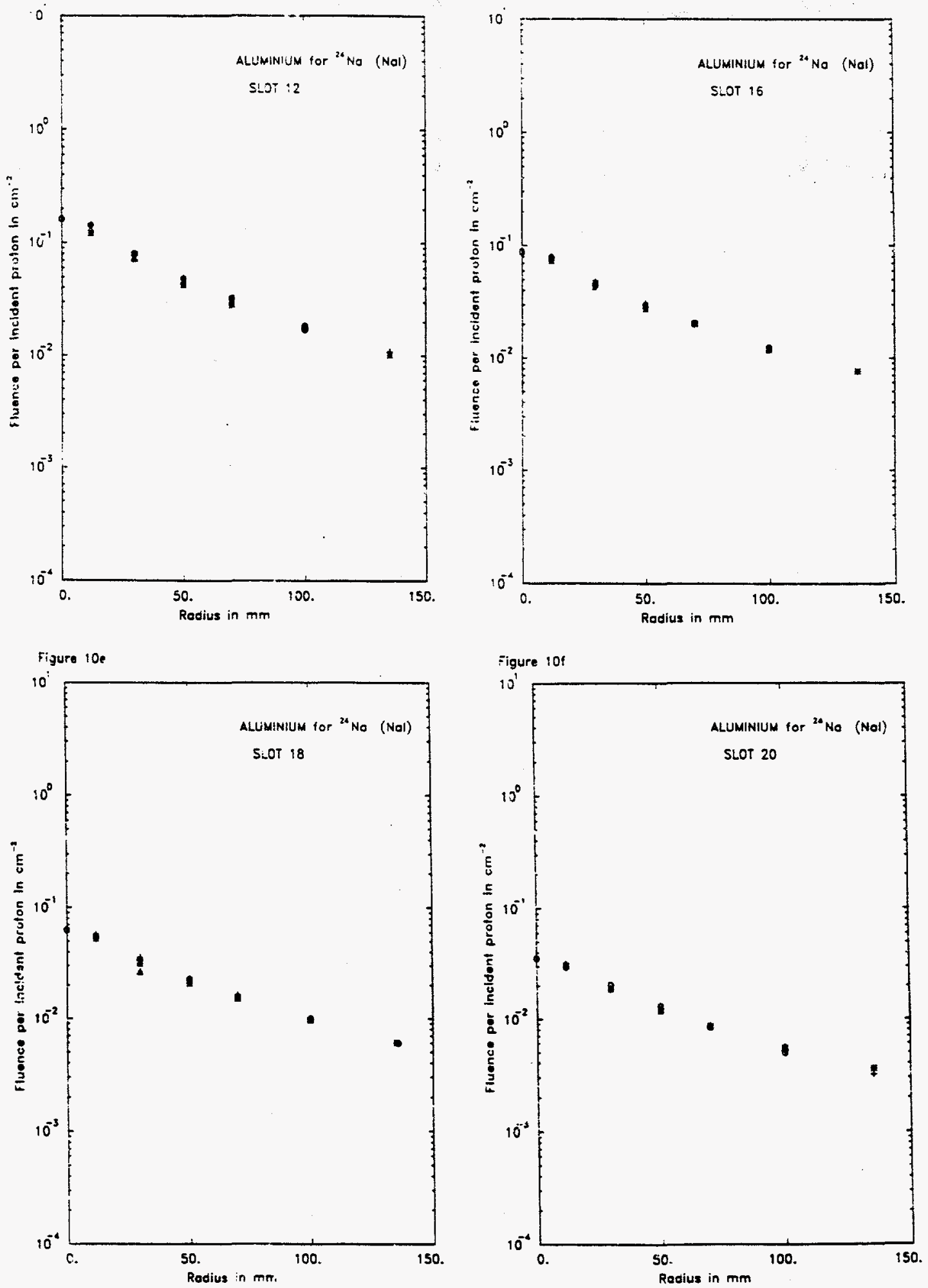

Figure 100

Figure $10 \mathrm{t}$

FIG. 10. Radial variation of neutron fluence as measured by ${ }^{24} \mathrm{Na}$ from ${ }^{27} \mathrm{Al}$. Meaning of symbols is: $\star D($ own $), \circ L(e f t), \Delta R($ ight $),+T(o p)$. e) Slot 12, f) Slot 16, g) Slot 18, h) Slot 20. 

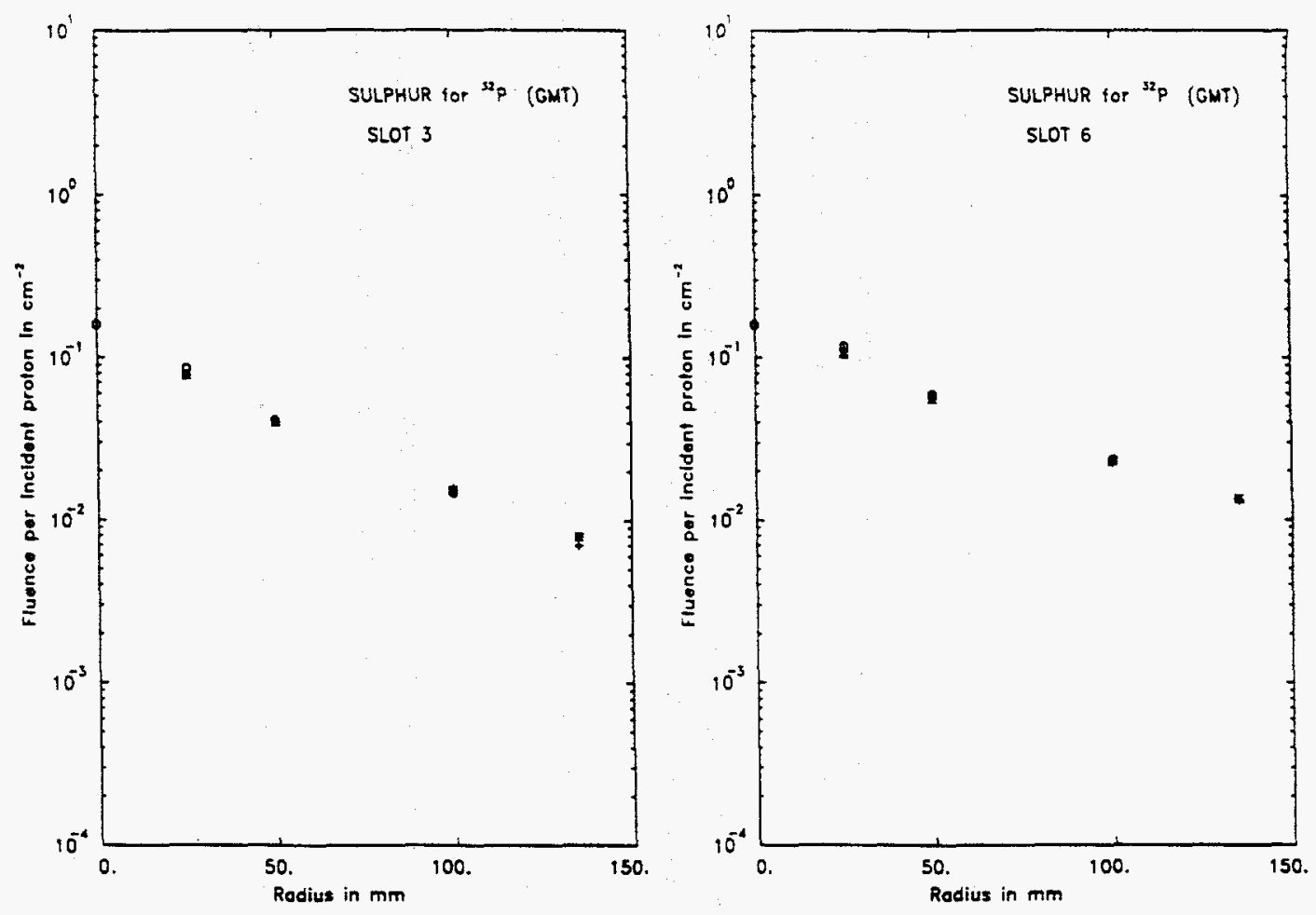

Figure 110

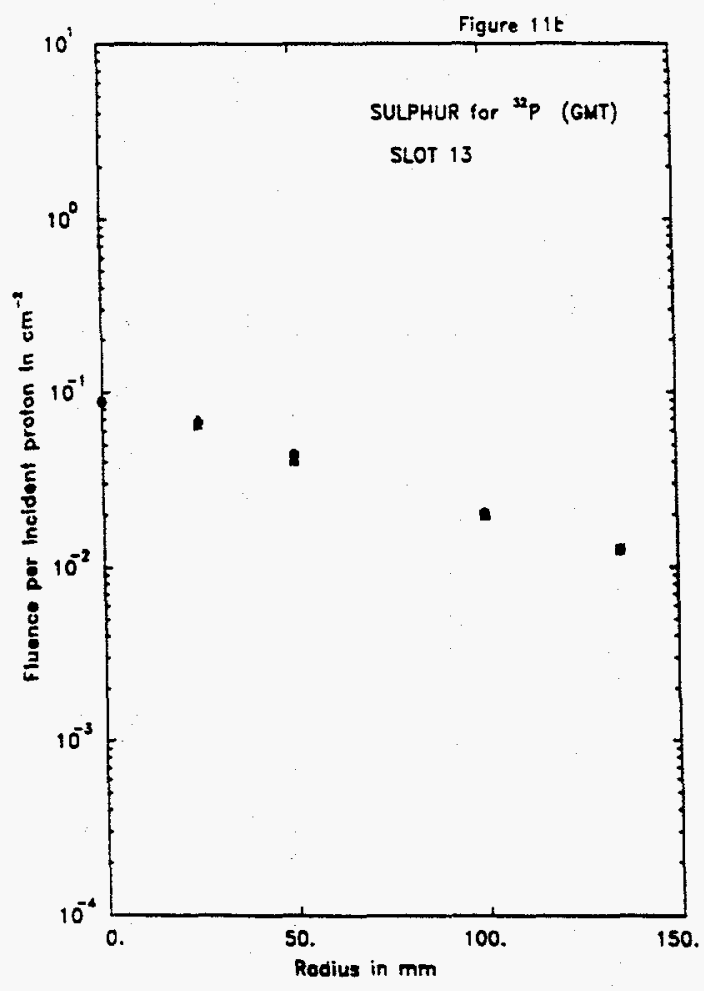

Figure $11 \mathrm{c}$

FIG. 11. Radial variation of neutron fluence as measured by ${ }^{32} \mathrm{P}$ from ${ }^{32} \mathrm{~S}$. Meaning of symbols is: $\star \mathrm{D}$ (own), oL(eft), $\Delta \mathrm{R}$ (ight),$+\mathrm{T}(\mathrm{op})$. a) Slot 3, b) Slot 6, c) Slot 13 . 

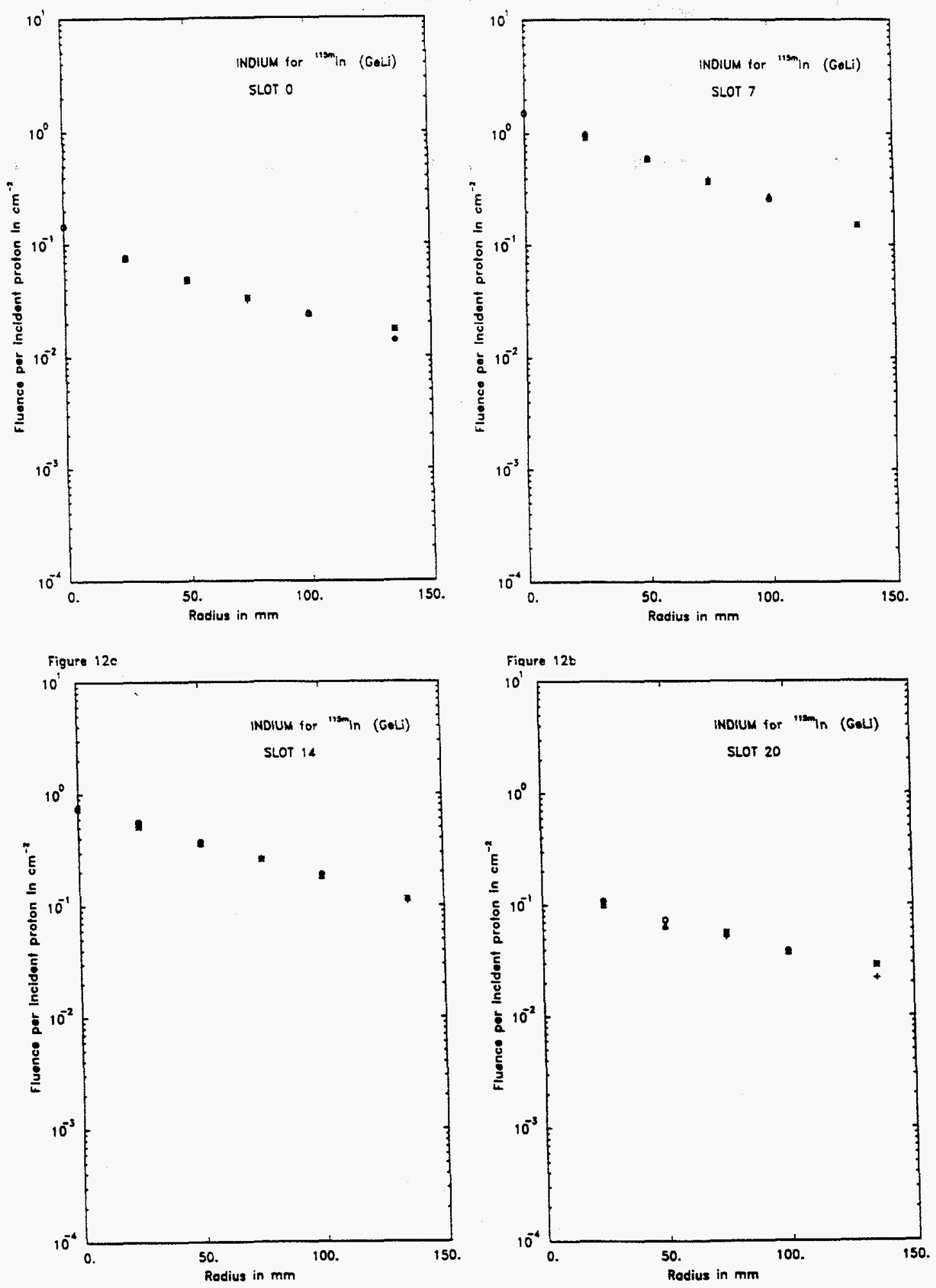

Figure $12 c$

Figure 12d

FIG. 12. Radial variation of neutron fluence as measured by ${ }^{115 \mathrm{~m}} \mathrm{In}$ from ${ }^{115 \mathrm{~m}} \mathrm{In}$. Meaning of symbols is: $\star D($ own $), \circ L($ eft $), \Delta R($ ight $),+T(o p)$ a) Slot $0, b)$ Slot 7, c) Slot 14 , d) Slot 20 . 

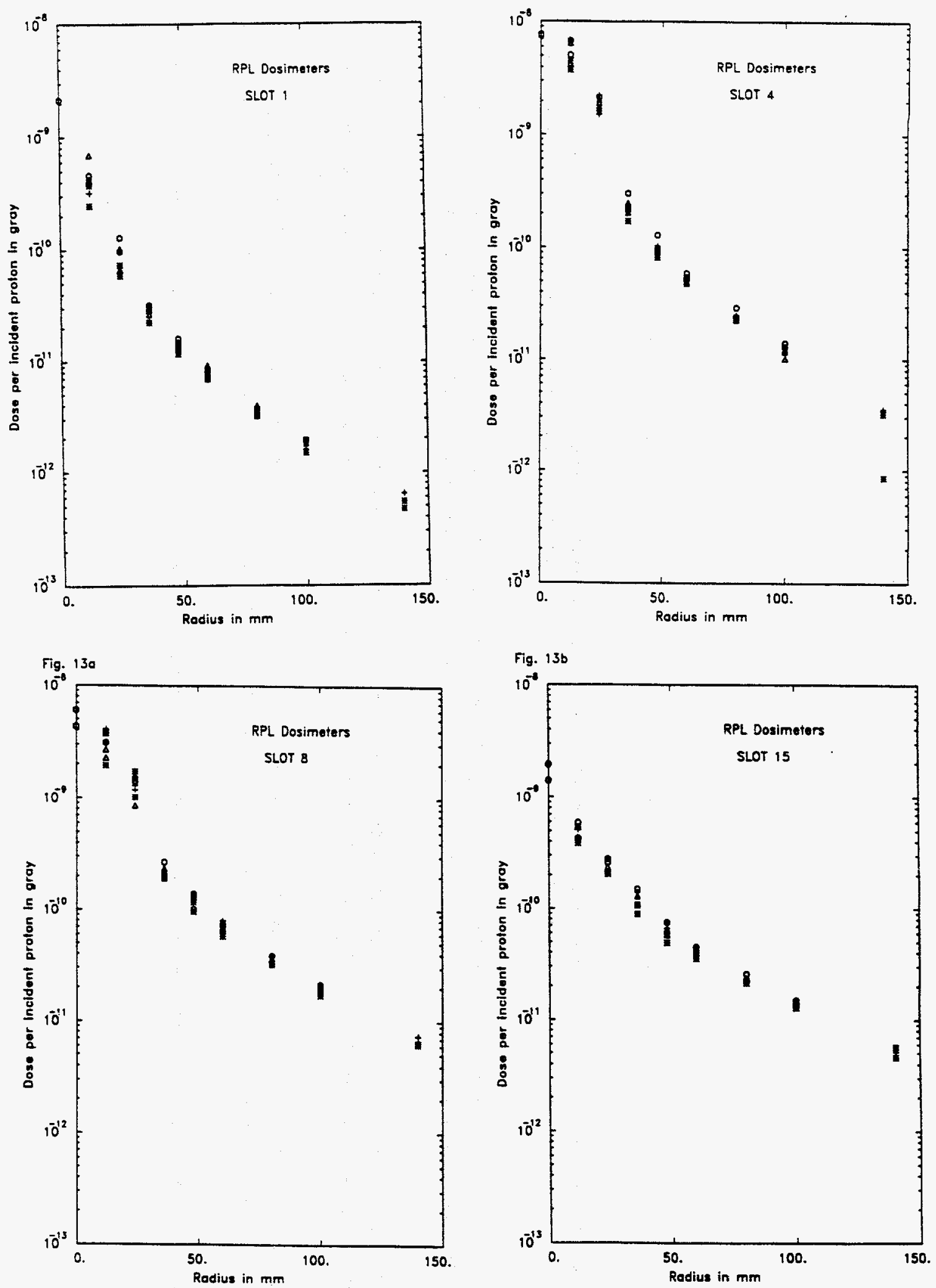

Fig. $13 \mathrm{c}$

Fig. 13d

FIG. 13. Radial variation of dose as measured by the RPL dosimeters. Meaning of symbols is: $\star D($ own $), \circ \mathrm{L}(\mathrm{eft}), \Delta \mathrm{R}(\mathrm{ight}),+\mathrm{T}(\mathrm{op})$. a) Slot 1, b) Slot 4, c) Slot $8, \mathrm{~d})$ Slot 15. 

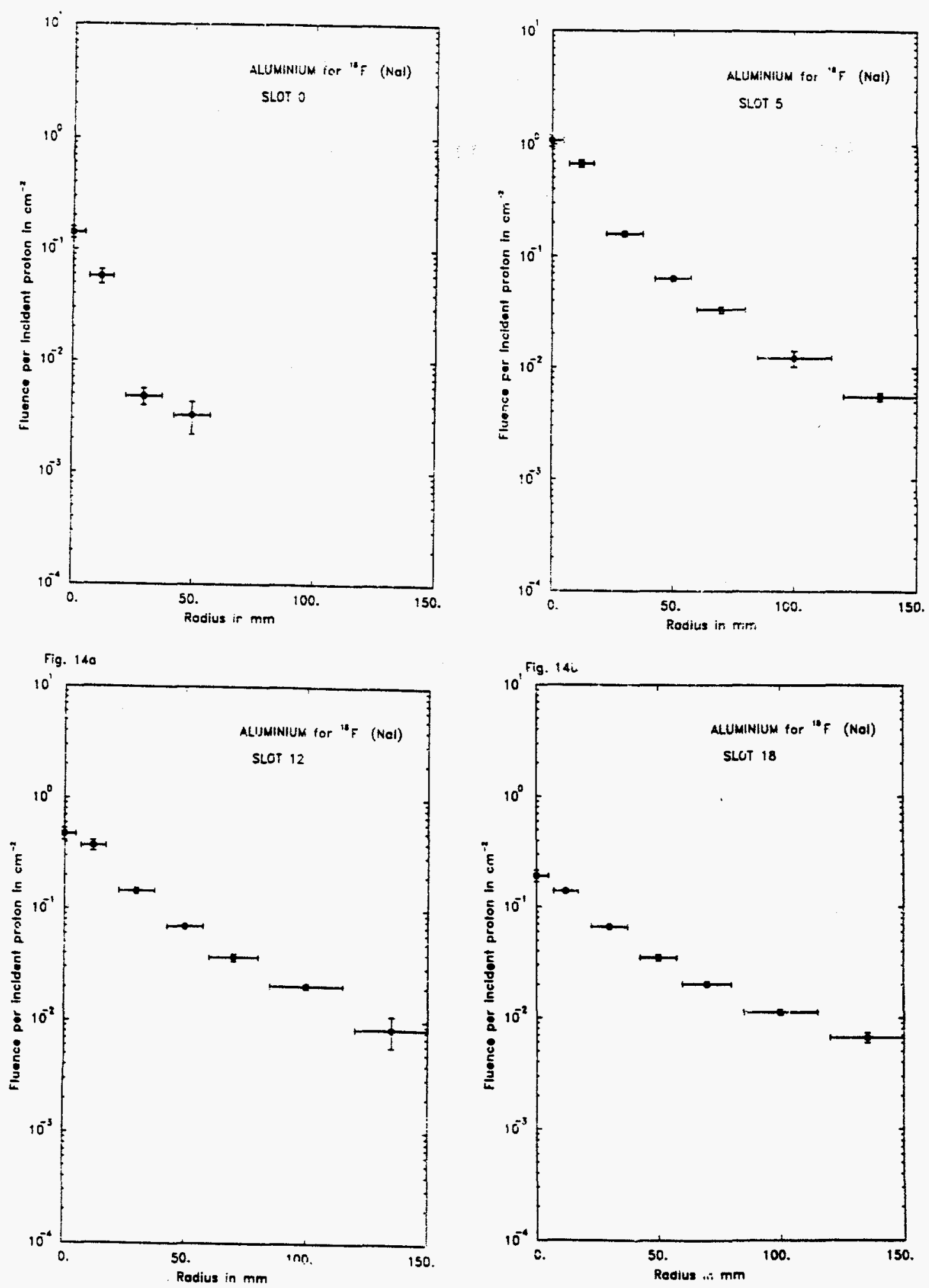

Fig. 14e

Fig. 14d

FIG. 14. Radial variation of hadron fluence as measured by ${ }^{18} \mathrm{~F}$ from ${ }^{27} \mathrm{Al}$. a) Slot 0 , b) Slot 5, c) Slot 12, d) Slot 18 . 

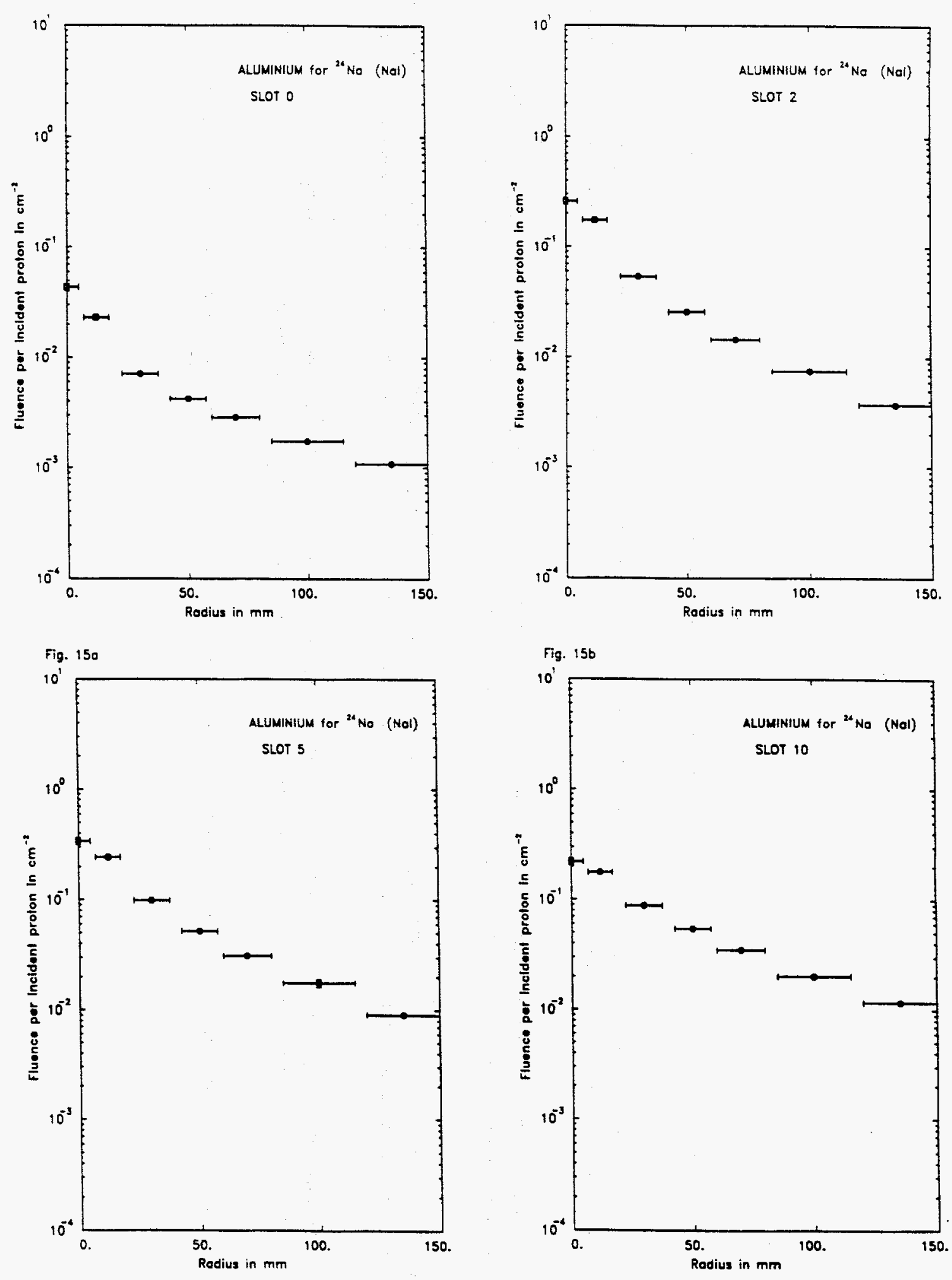

Fig. 15e

Fig. $15 d$

FIG. 15. Radial variation of neutron fluence as measured by ${ }^{24} \mathrm{Na}$ from ${ }^{27} \mathrm{Al}$. a) Slot 0 , b) Slot 2, c) Slot 5 , d) Slot 10. 

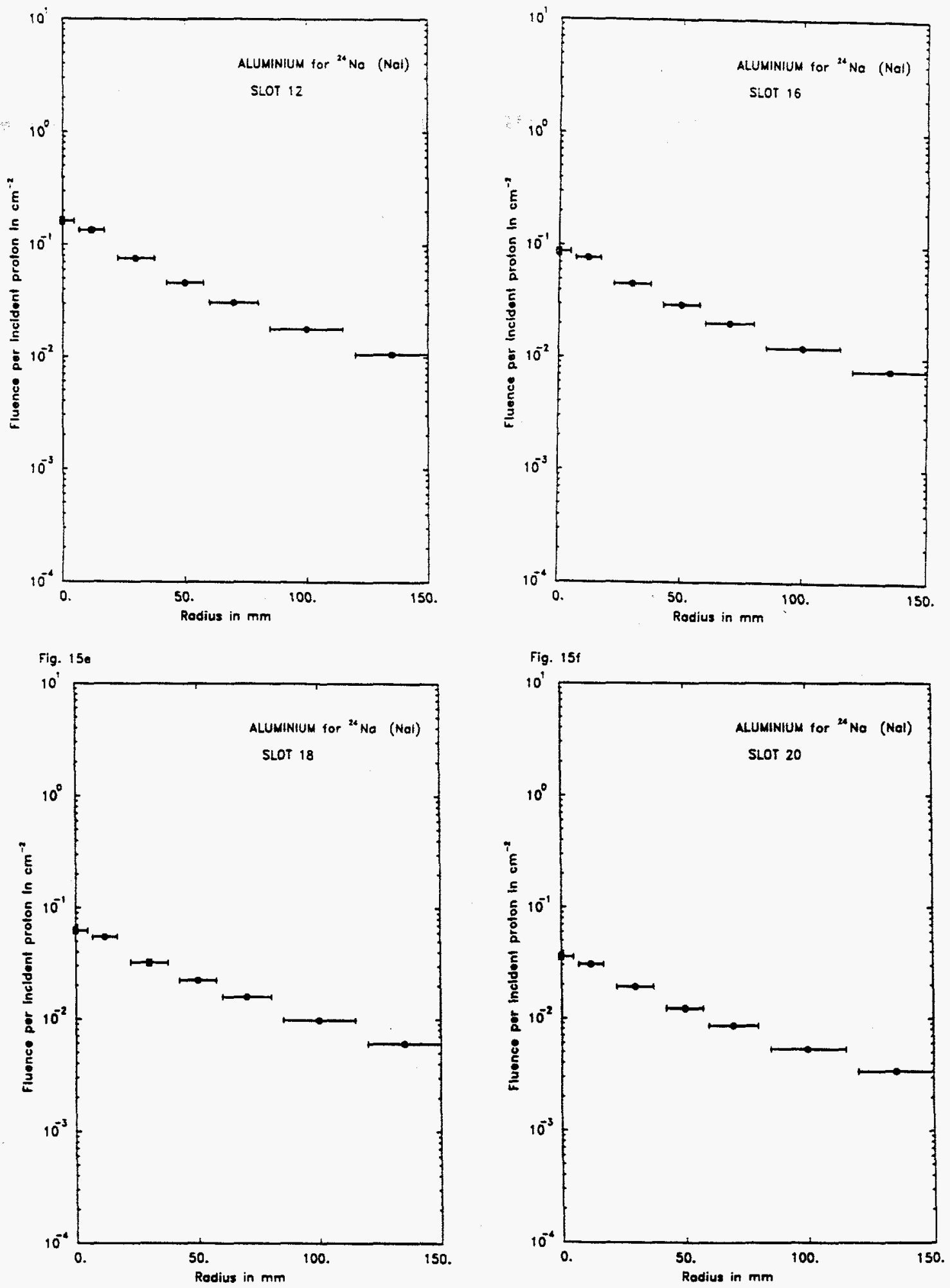

Fig. 159

Fig. $15 \mathrm{~h}$

FIG. 15. Radial variation of neutron fluence as measured by ${ }^{24} \mathrm{Na}$ from ${ }^{27} \mathrm{Al}$. e) Slot 12 , f) Slot 16 .

277 


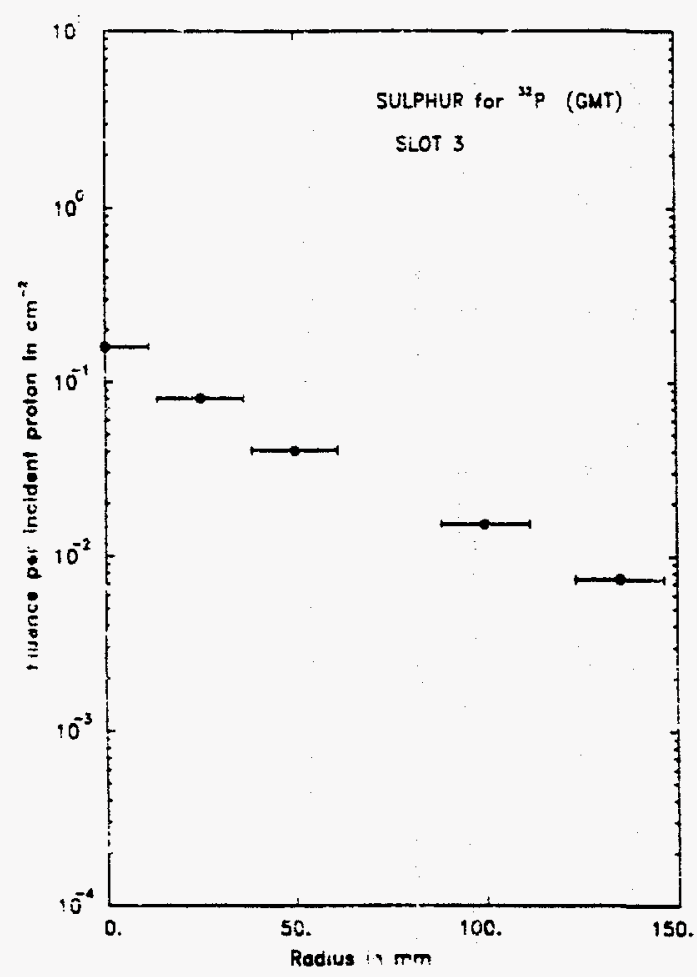

Figure 160

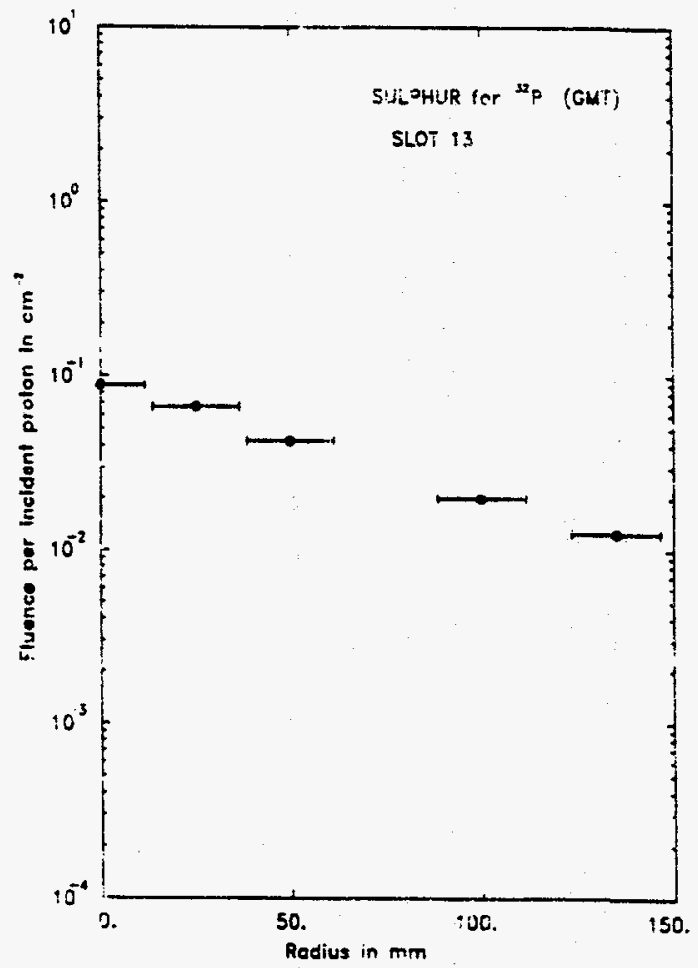

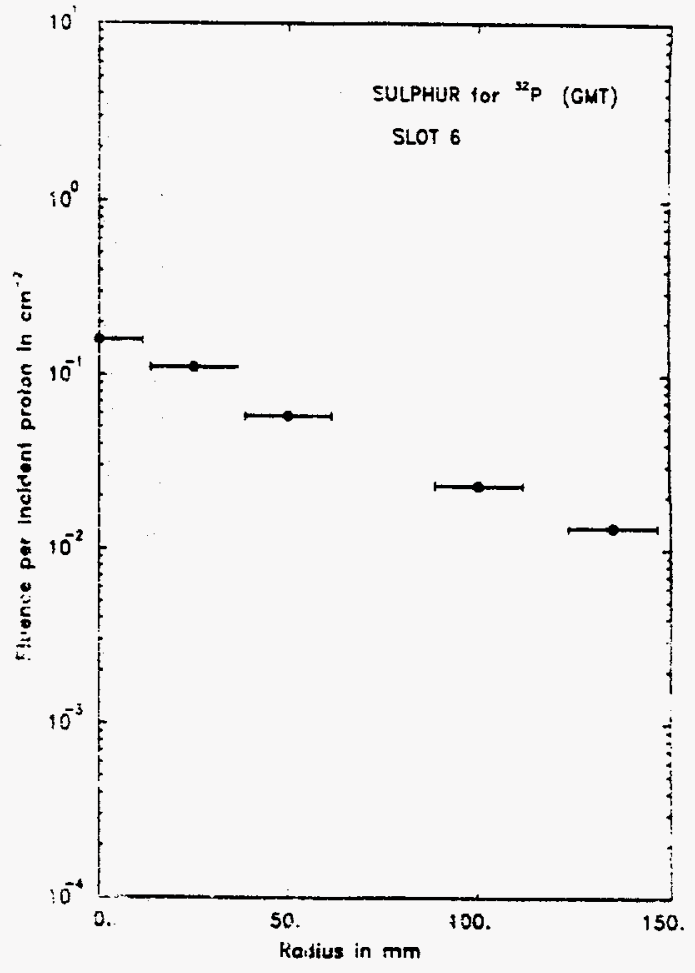

Fig. 160

FIG. 16. Radial variation of neutron fluence as measured by ${ }^{32} \mathrm{P}$ from ${ }^{32} \mathrm{~S}$. a) Slot 3 , b) Slot 6, c) Slot 13 . 

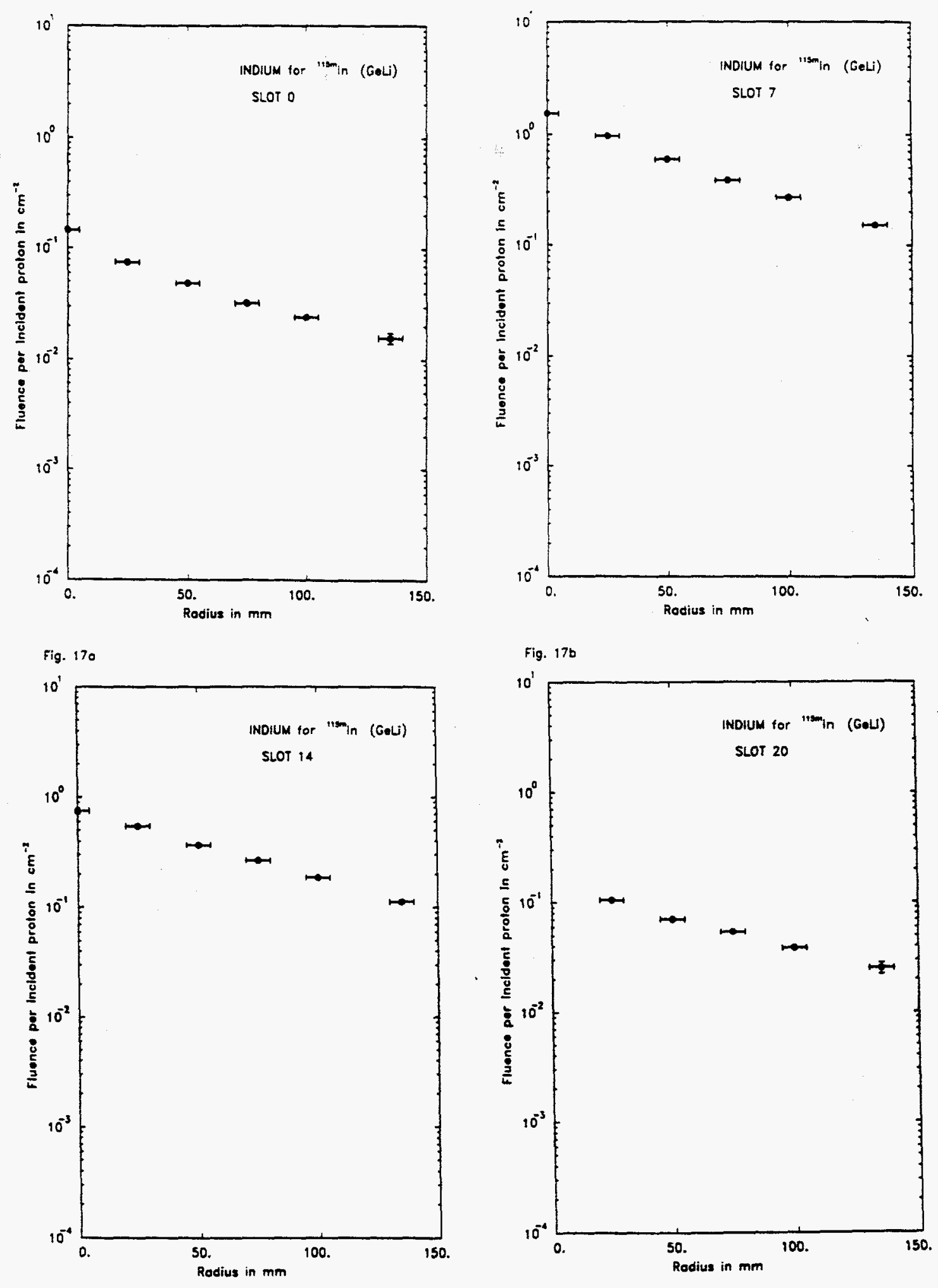

Fig. $17 e$

Fig. $17 d$

FIG. 17. Radial variation of neutron fluence as measured by $115 \mathrm{~m} I n$ from ${ }^{115} \mathrm{~m} I n$. a) Slot 0, b) Slot 7, c) Slot 14, d) Slot 20. 

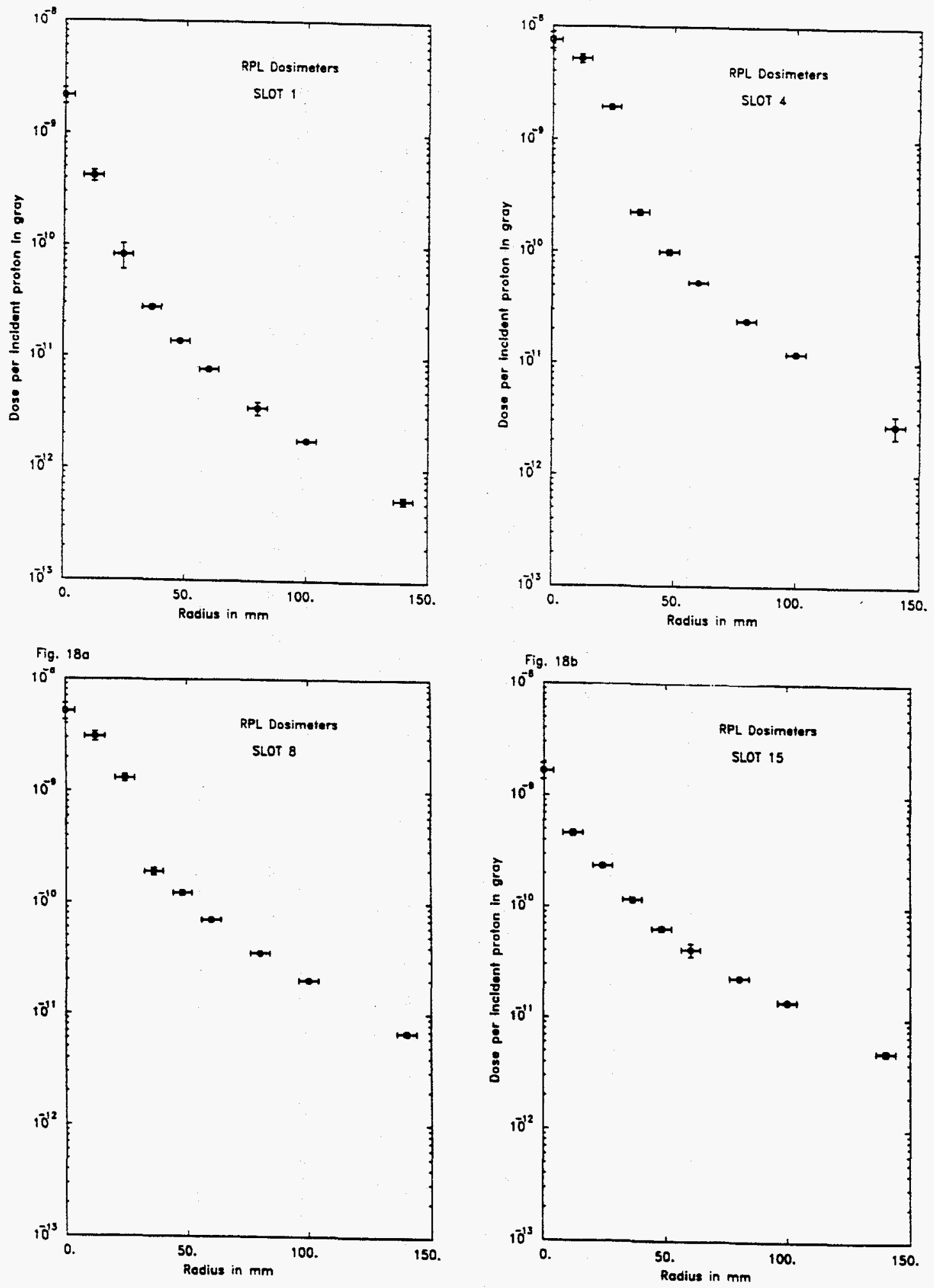

Fig. $18 \mathrm{e}$

Fig. 18d

FIG. 18. Radial variation of dose as measured by the RPL dosimeters. a) Slot 1 , b) Slot 4, c) Slot 8, d) Slot 15. 

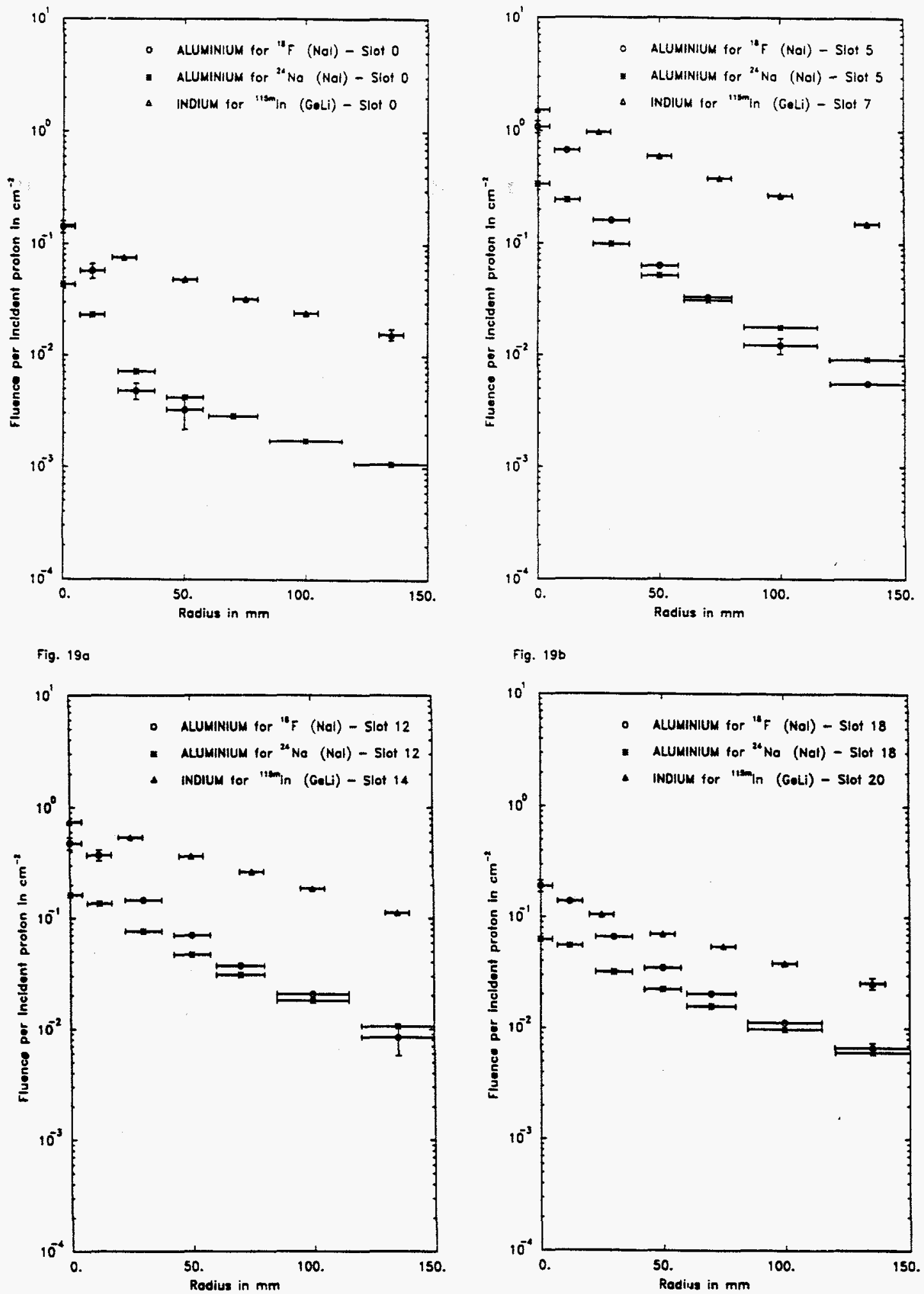

FIG. 19. Comparison of the radial distributions of hadron and neutron fluence as measured by the aluminium and indium detectors at similar depths in the cascade. 0 hadron fluence from ${ }^{27} \mathrm{Al}-{ }^{18} \mathrm{~F} ; \star-$ neutron fluence from ${ }^{27} \mathrm{Al}-{ }^{24} \mathrm{Na} ; \Delta$ - neutron fluence from ${ }^{115} \mathrm{In}-{ }^{115 \mathrm{~m}} \mathrm{In}$. a) at zero depth, b) at about $30 \mathrm{~cm}$ depth, c) at about $65 \mathrm{~cm}$ depth, d) at about $95 \mathrm{~cm}$ depth. 


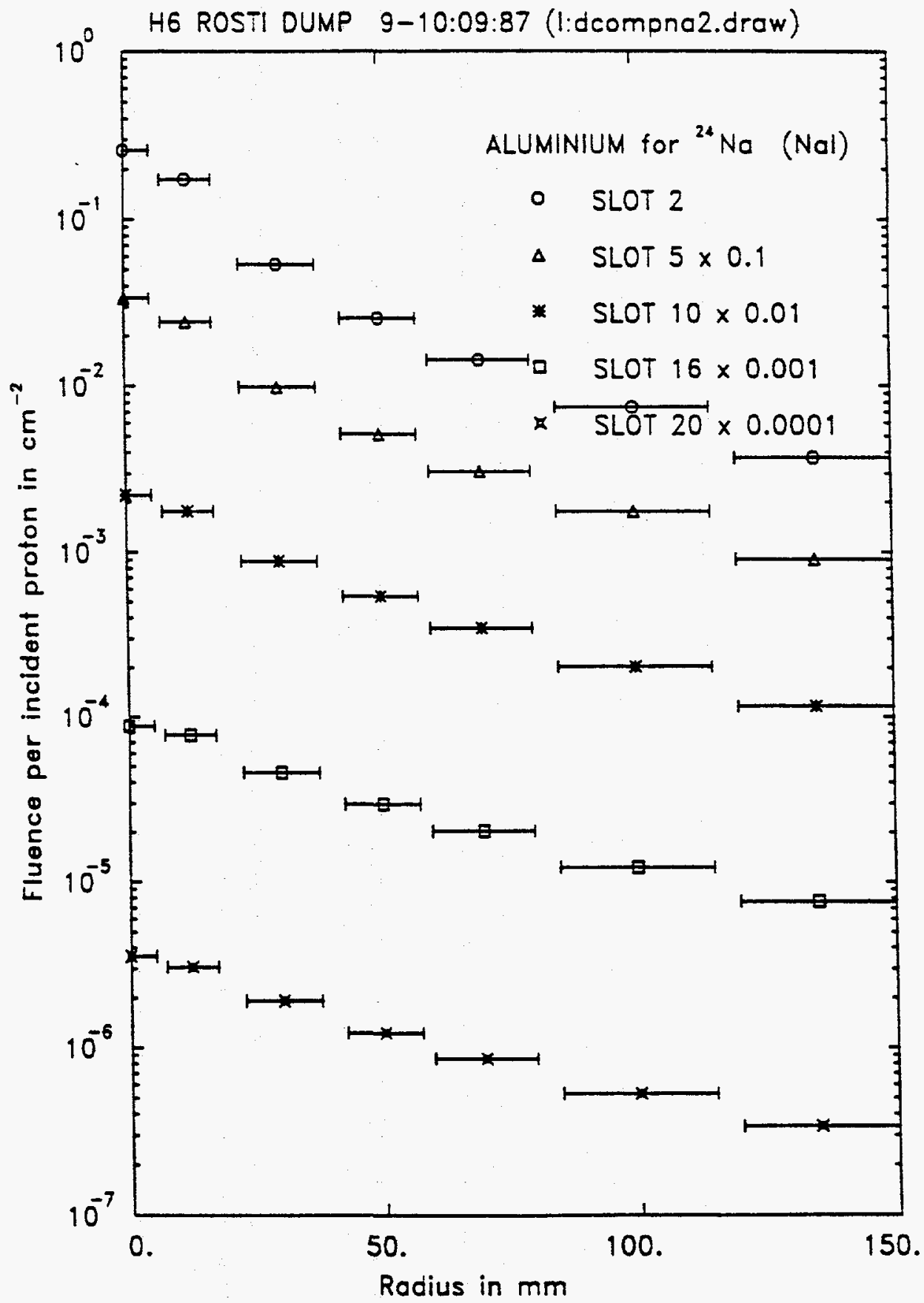

FIG. 20. Comparison of the radial distributions of fluence as measure by the ${ }^{27} \mathrm{Al}-{ }^{24} \mathrm{Na}$ reaction as a function of depth in the cascade. 

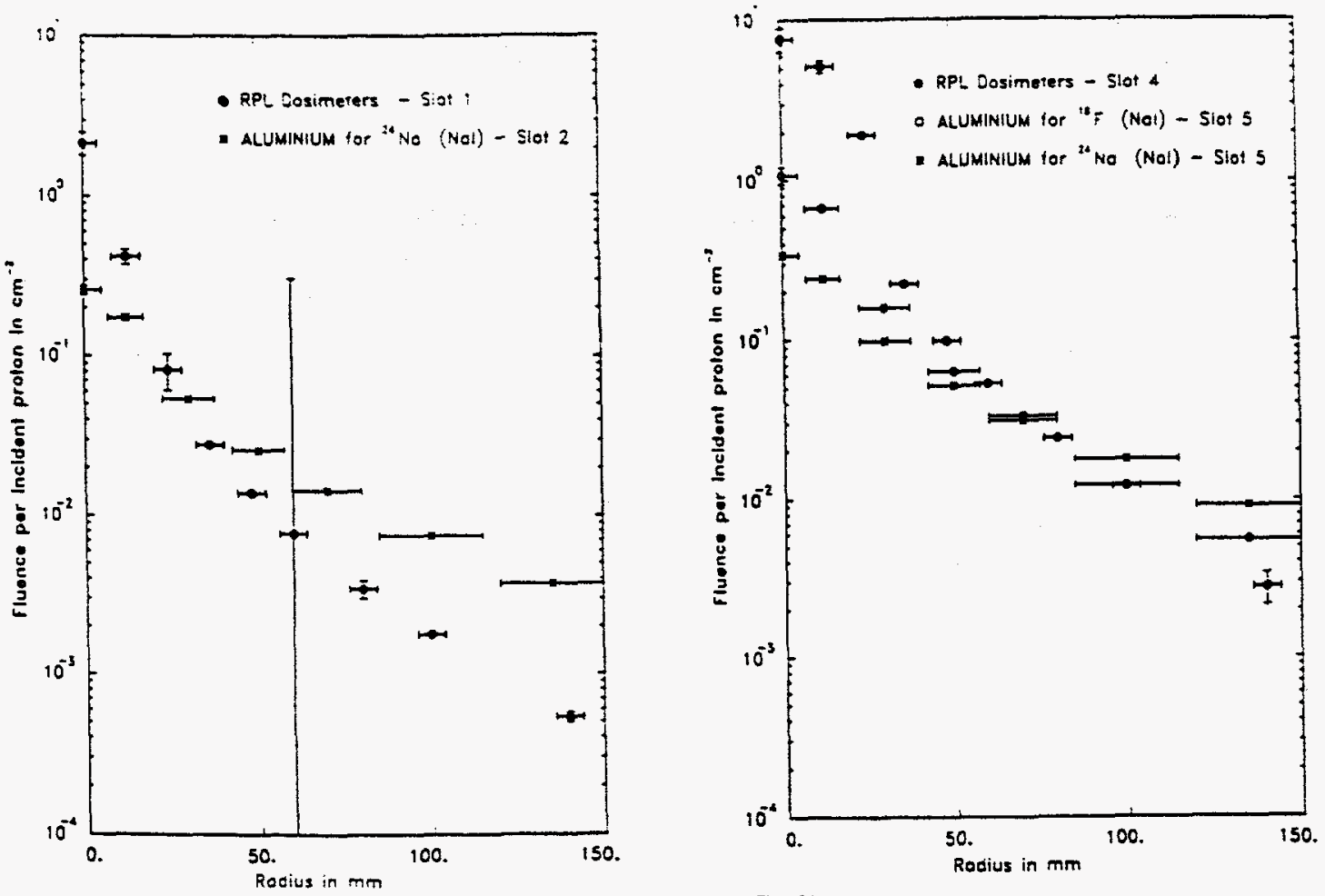

Fig. 210
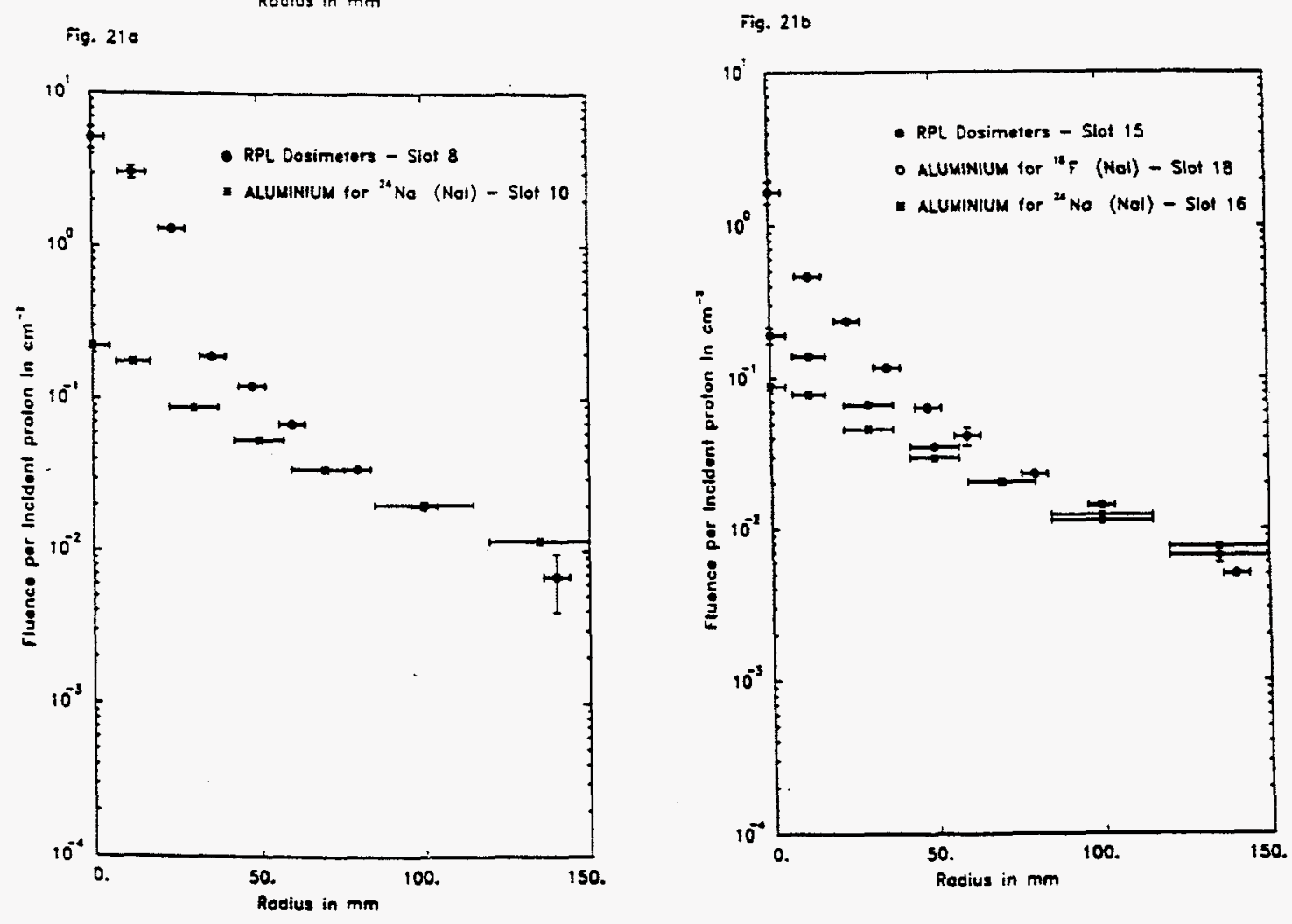

Fig. 2ic

Fig. $21 \mathrm{~d}$

FIG. 21. Comparison of the radial distributions of total energy deposition density, highenergy hadron fluence and low-energy neutron fluence at similar depths in the cascade. $\circ$ - dose from the RPL detectors (data multiplied by $10^{9} \mathrm{~cm}^{-2} \cdot \mathrm{Gy}^{-1}$ ), $\circ$ - hadron fluence from ${ }^{27} \mathrm{Al}-{ }^{18} \mathrm{~F}$, $\star$ - neutron fluence from ${ }^{27} \mathrm{Al}-{ }^{24} \mathrm{Na} ; \Delta$ - neutron fluence from ${ }^{115} \mathrm{In}-$ ${ }_{115 \mathrm{~m}} \mathrm{In}$. a) at about $5 \mathrm{~cm}$ depth, b) at about $20 \mathrm{~cm}$ depth, c) at about $45 \mathrm{~cm}$ depth, d) at about $65 \mathrm{~cm}$ depth. 


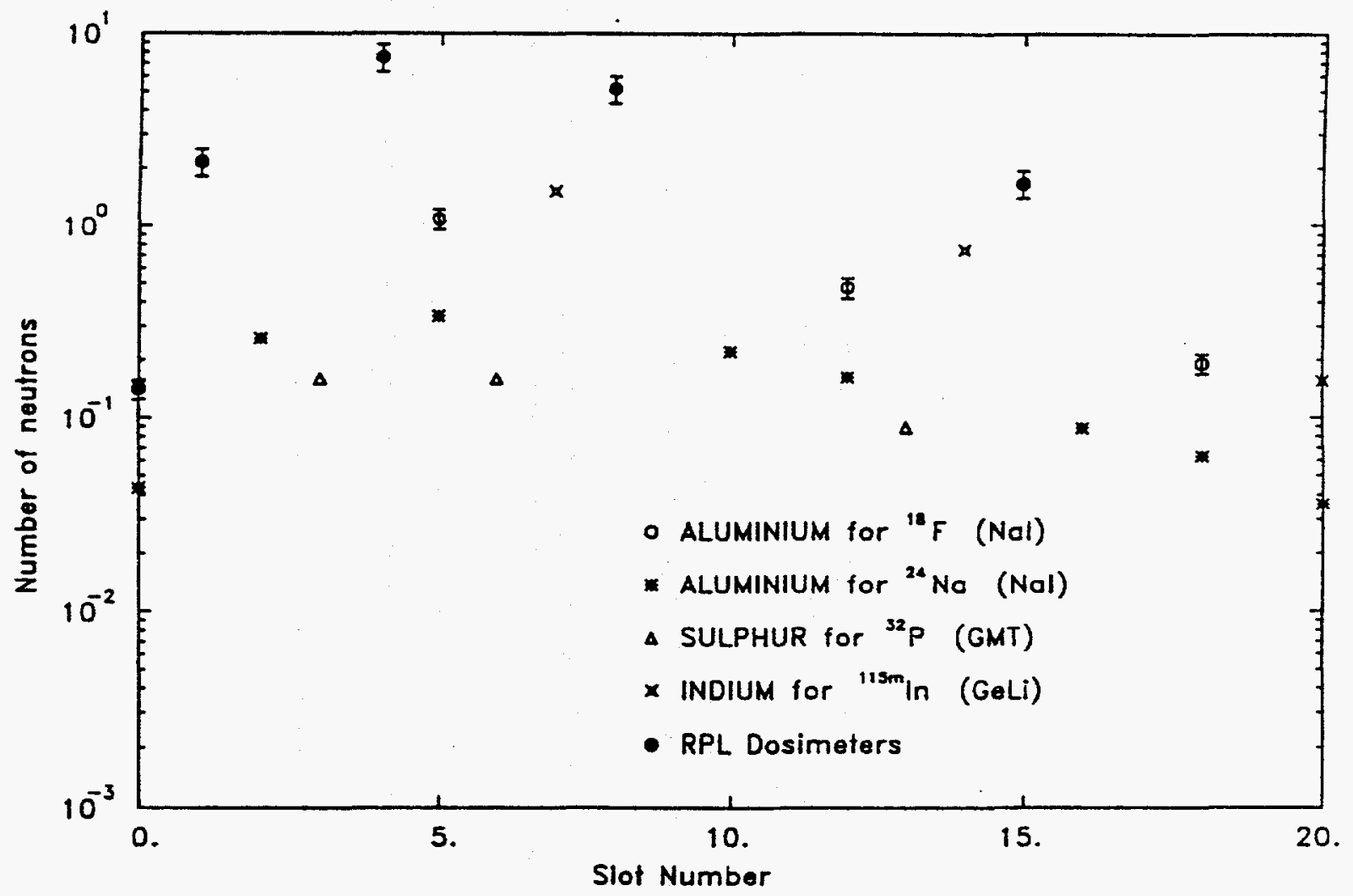

FIG. 22. Longitudinal variation of on-axis fluence and energy deposition. RPL data have been multiplied by $10^{9} \mathrm{~cm}^{-2} \cdot \mathrm{Gy}^{-1}$ before plotting. 

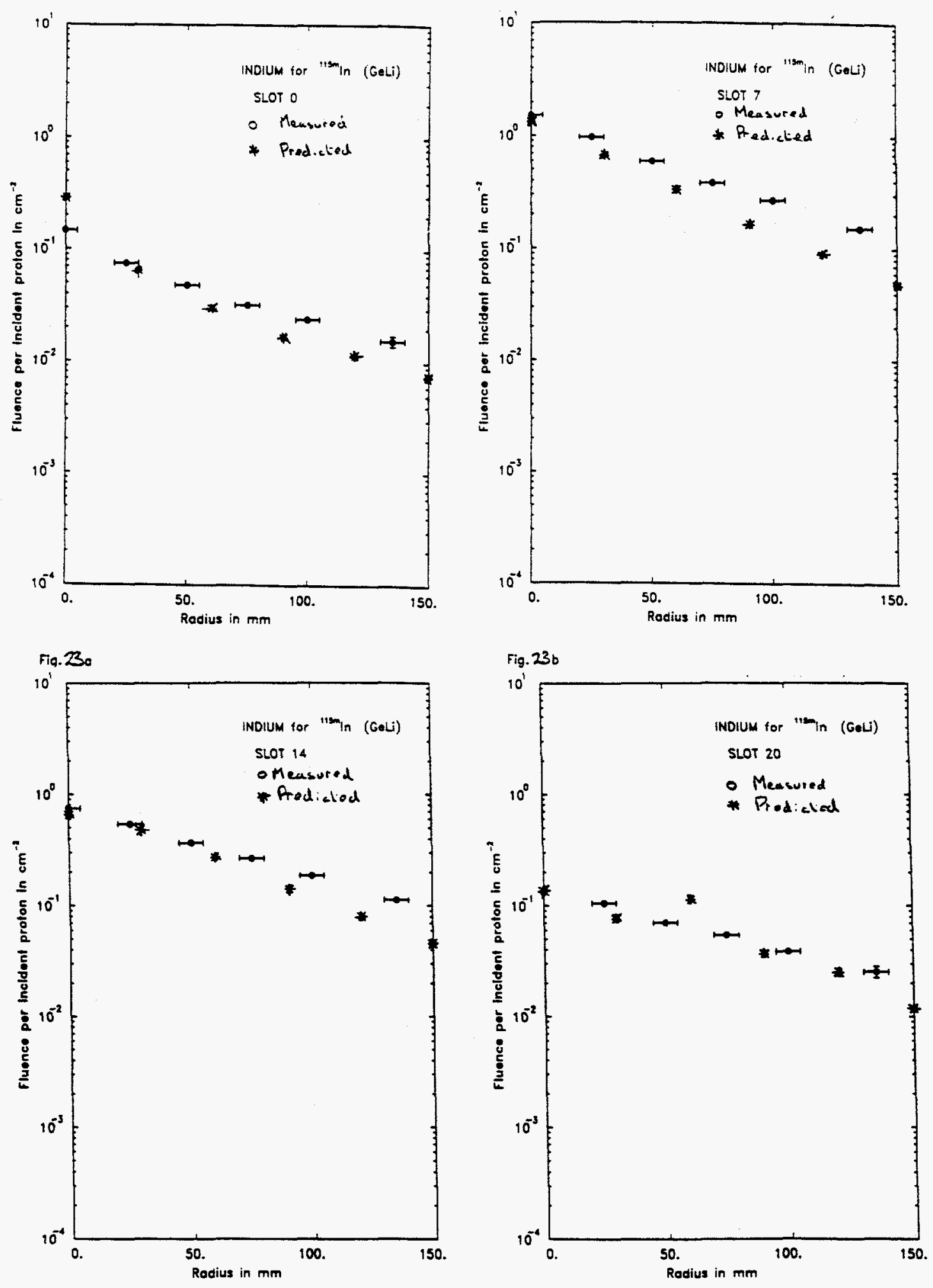

Fig. $23 \mathrm{e}$

Fig. 23d

FIG. 23. Comparison of the low-energy neutron fluence determined using the stars produced by the ${ }^{27} \mathrm{Al}-{ }^{18} \mathrm{~F}$ hadron fluence as the source and the fluence measured by the ${ }^{115} \mathrm{In}-{ }^{115 \mathrm{~m}}$ In reaction. a) Slot 0, b) Slot 7, c) Slot 14 , d) Slot 20. 

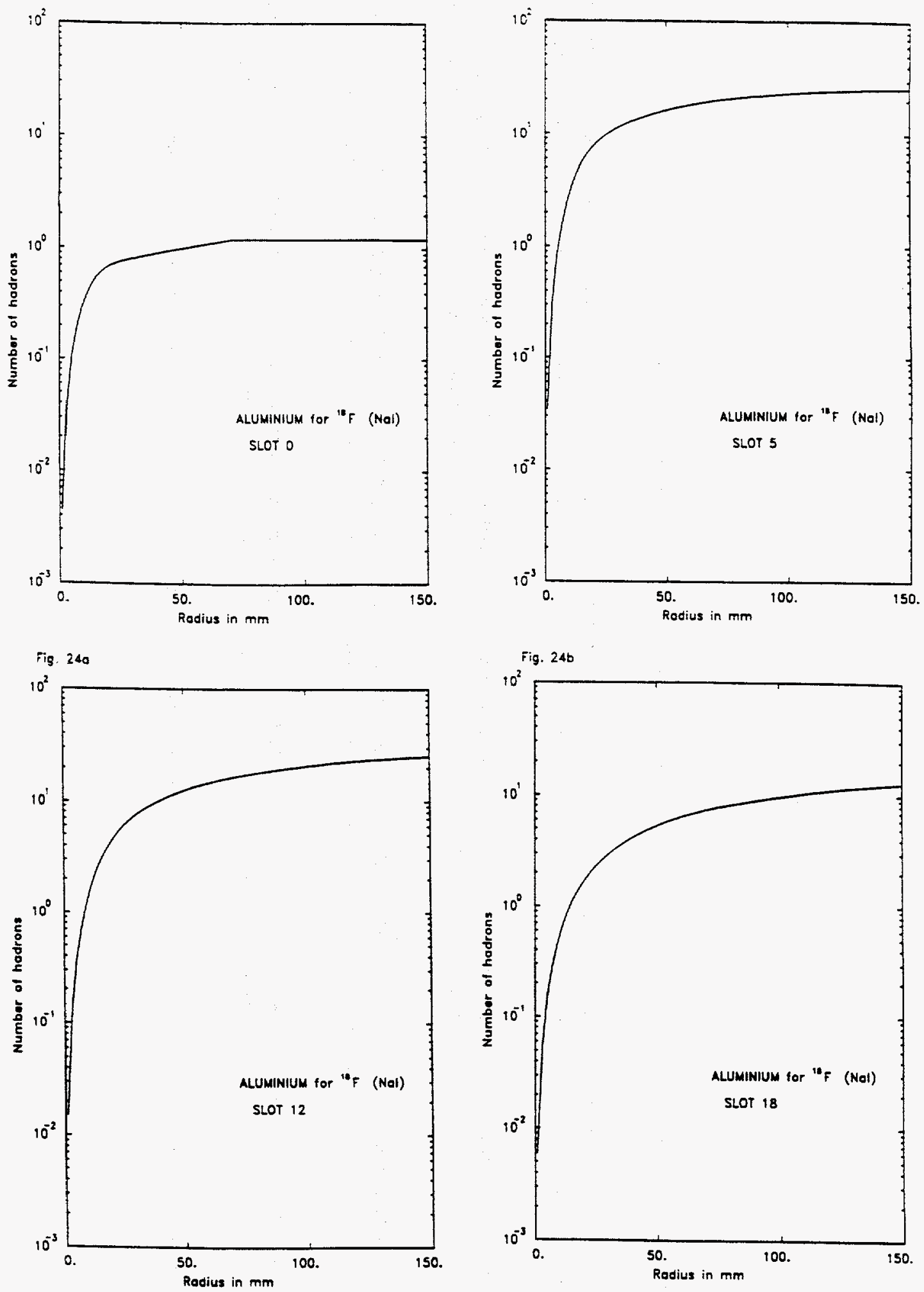

Fig. 24e

Fig. 24d

FIG. 24. Variation of the radially integrated hadron fluence as measured by ${ }^{18} \mathrm{~F}$ from ${ }^{27} \mathrm{Al}$ as a function of radius. a) Slot $0, \mathrm{~b}$ ) Slot 5, c) Slot 12 , d) Slot 18. 

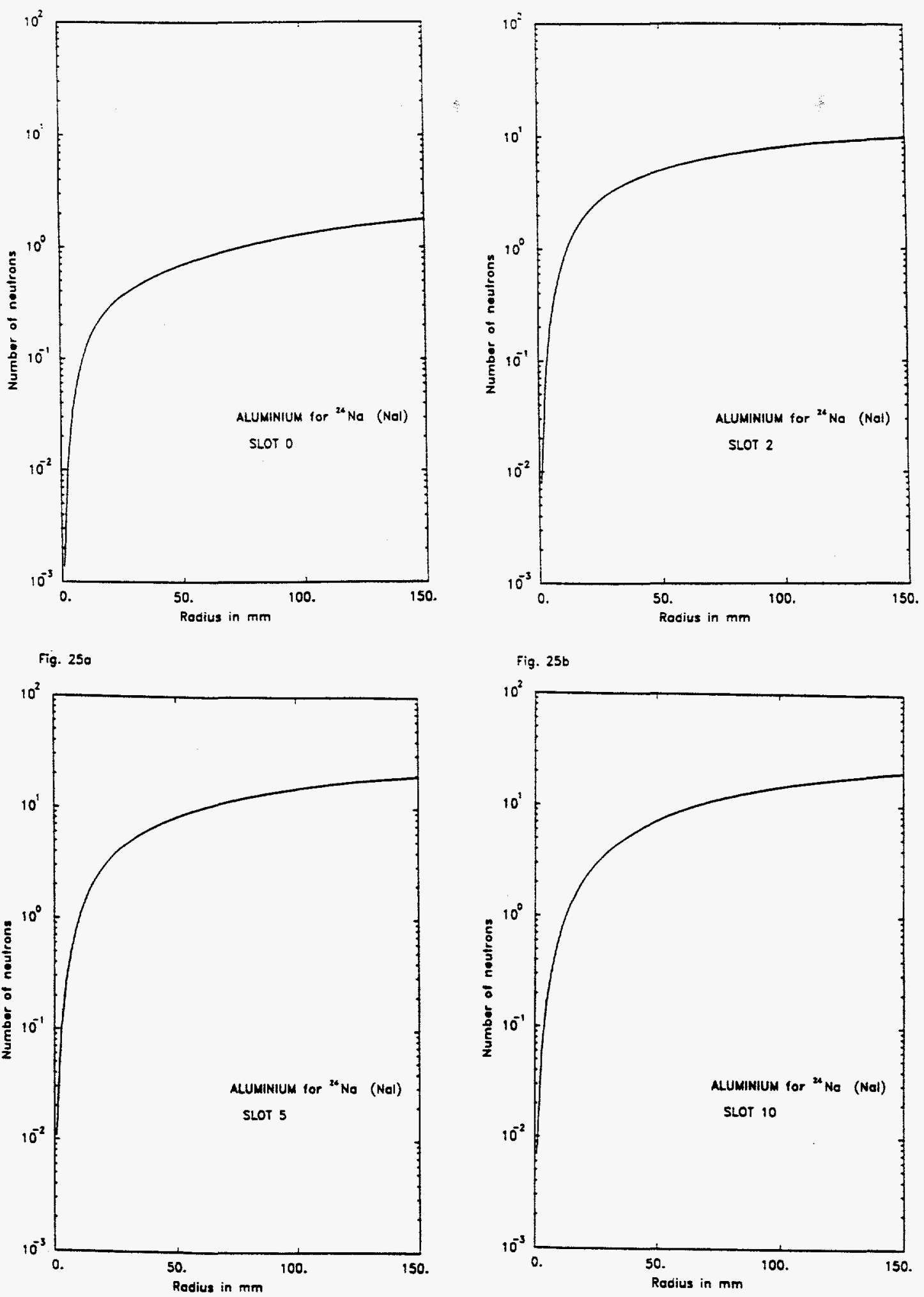

Fig. $25 \mathrm{c}$

Fig. 25d

FIG. 25. Variation of the radially integrated neutron fluence as measured by ${ }^{24} \mathrm{Na}$ from

${ }^{27} \mathrm{Al}$ as a function of radius. a) Slot $0, \mathrm{~b}$ ) Slot 2, c) Slot 5, d) Slot 10. 

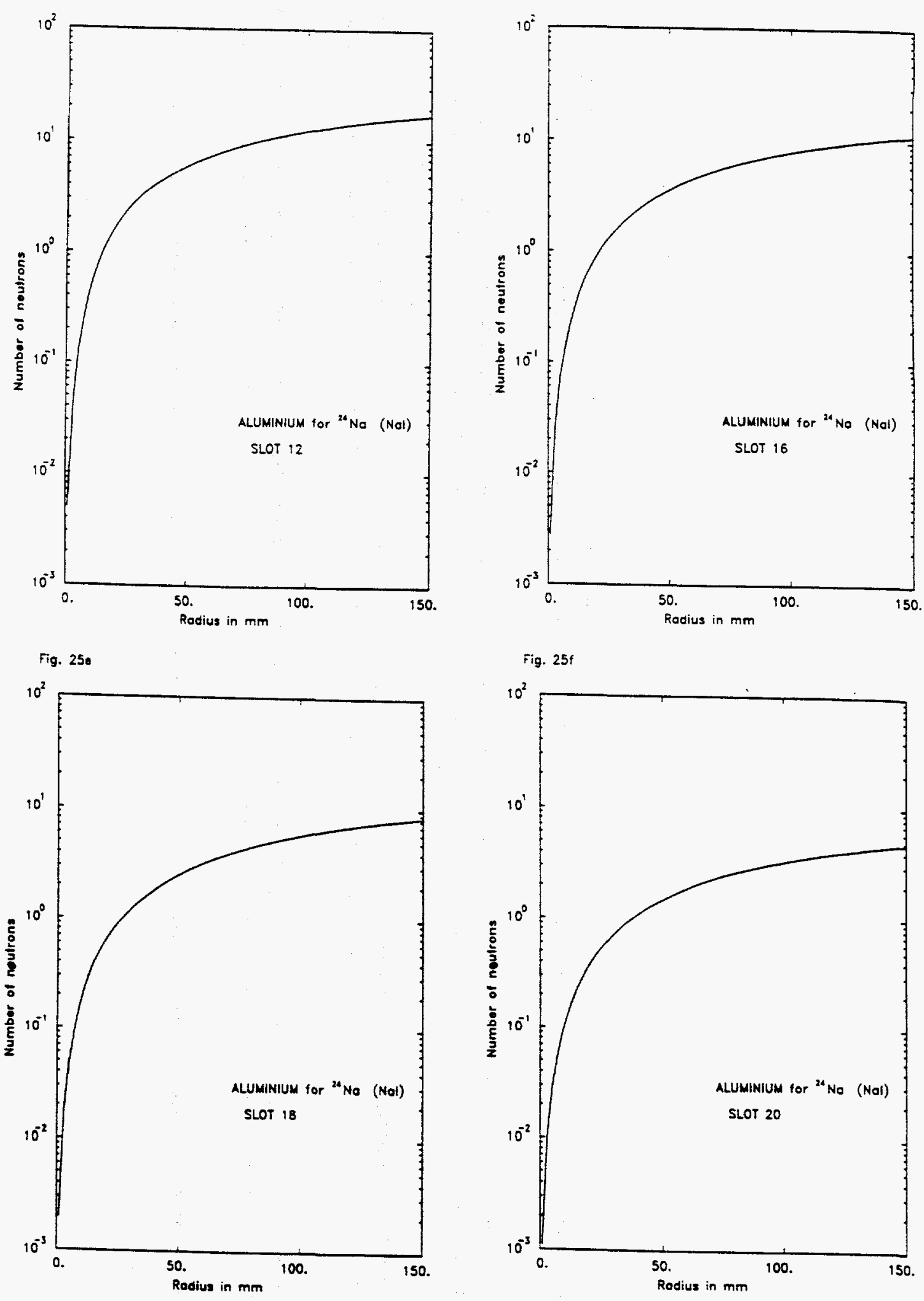

Fig. 25g

fig. $25 n$

FIG. 25. Variation of the radially integrated neutron fluence as measured by ${ }^{24} \mathrm{Na}$ from ${ }^{27} \mathrm{Al}$ as a function of radius. e) Slot $12, \mathrm{f}$ ) Slot $16, \mathrm{~g}$ ) Slot $18, \mathrm{~h}$ ) Slot 20. 

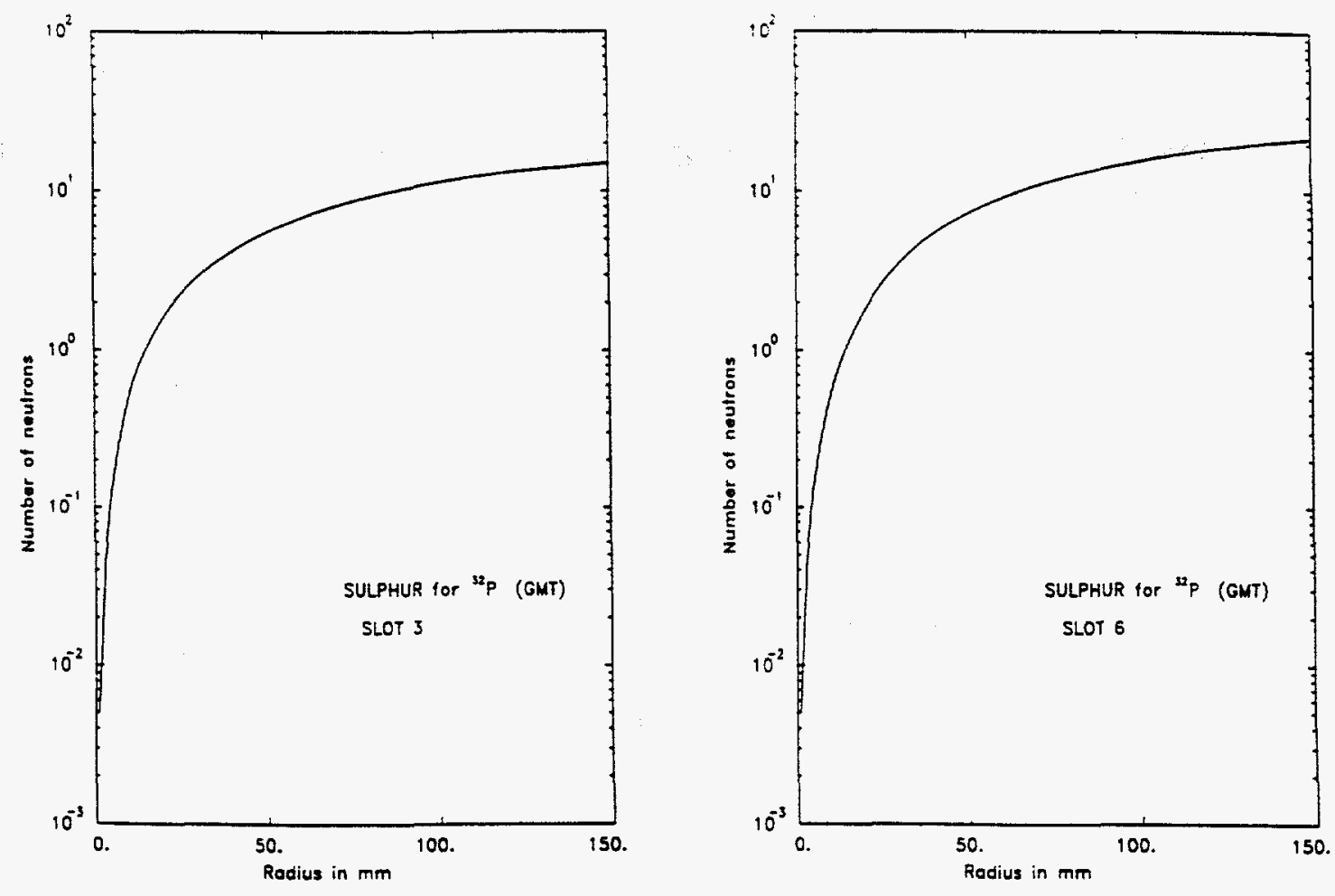

Fig. 260

Fig. 26b

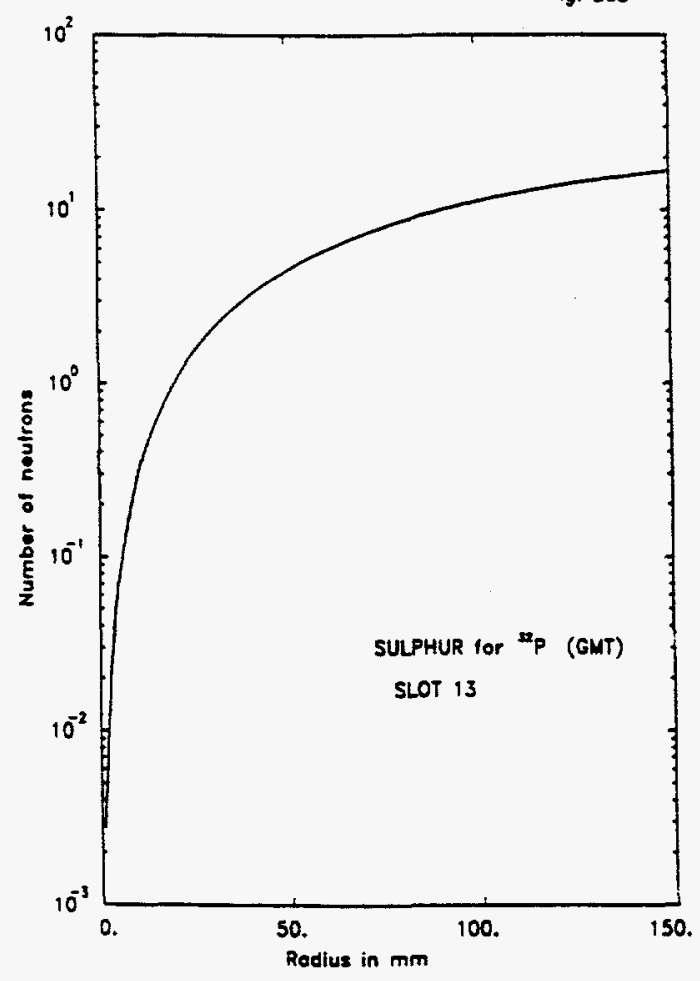

Fig. $26 e$

FIG. 26. Variation of the radially integrated neutron fluence as measured by ${ }^{32} \mathrm{P}$ from ${ }^{32} \mathrm{~S}$ as a function of radius. a) Slot $3, \mathrm{~b}$ ) Slot 6, c) Slot 13. 

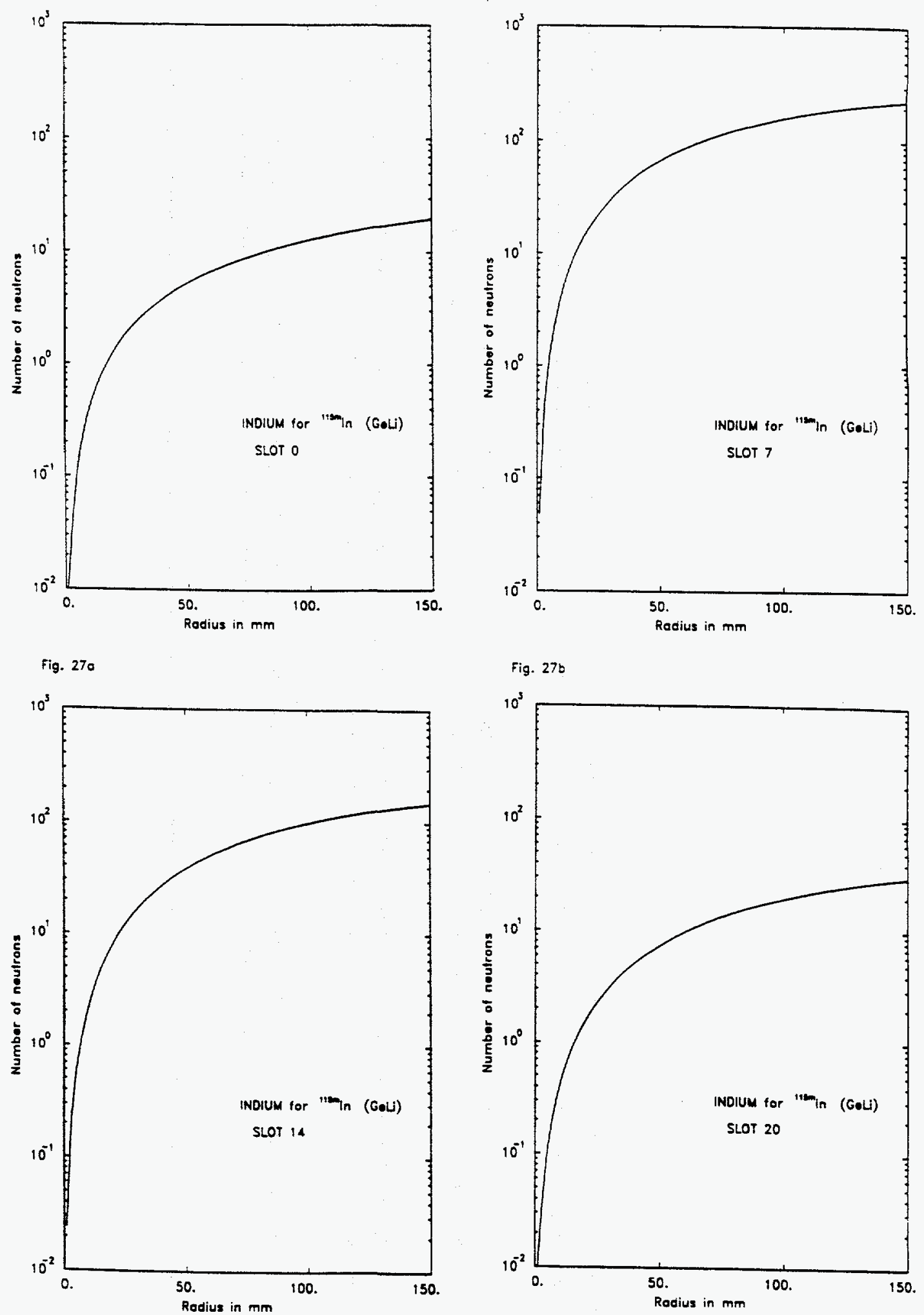

Fig. 27c

Fig. 27d

FIG. 27. Variation of the radially integrated neutron fluence as measured by $115 \mathrm{~m} I n$ from ${ }^{115}$ In as a function of radius. a) Slot 0, b) Slot 7, c) Slot 14, d) Slot 20. 

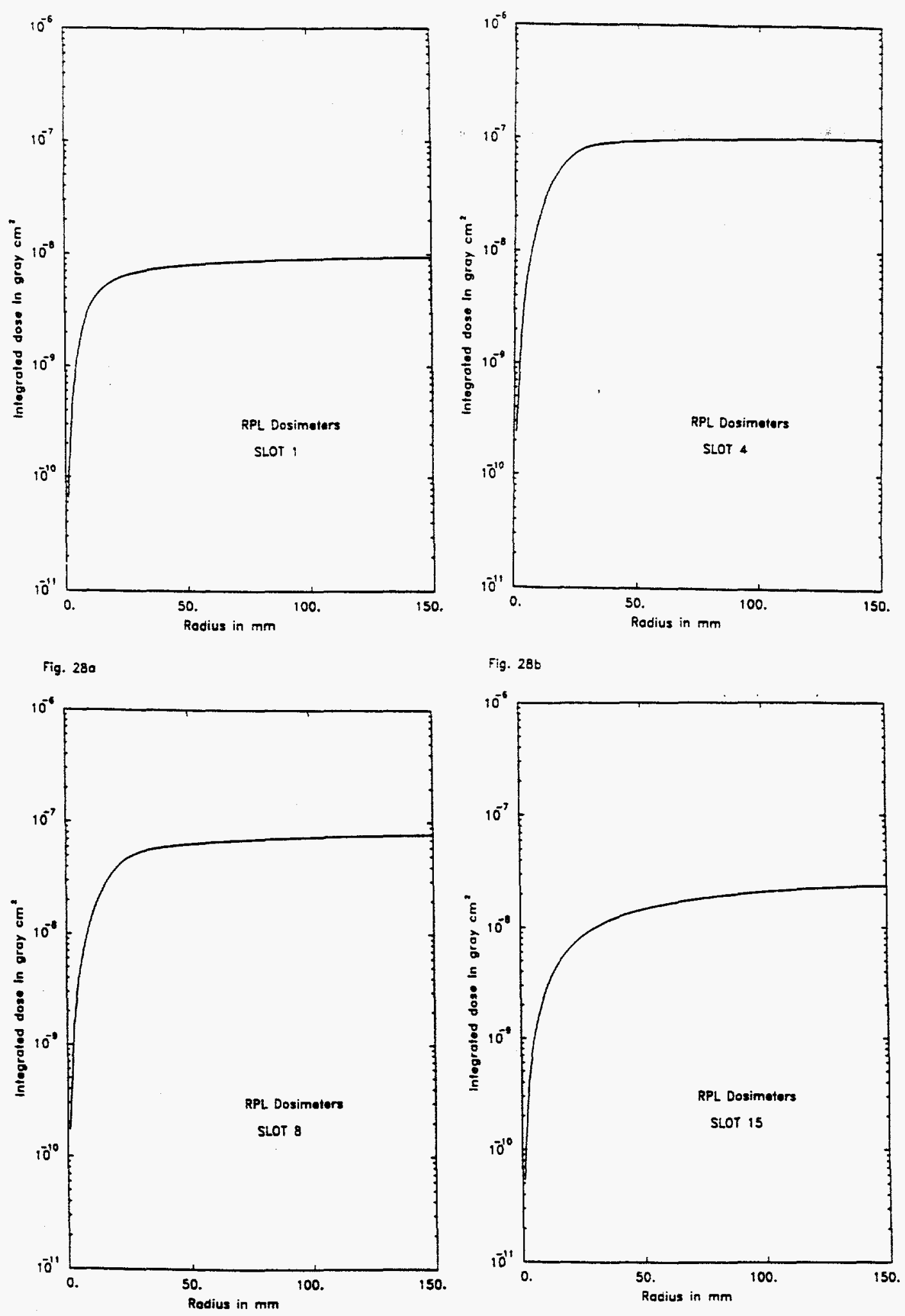

Fig. 28e

Fig. $28 d$

FIG. 28. Variation of the radially integrated dose as measured by the RPL dosimeters. as a function of radius. a) Slot 1, b) Slot 4, c) Slot 8, d) Slot 15. 


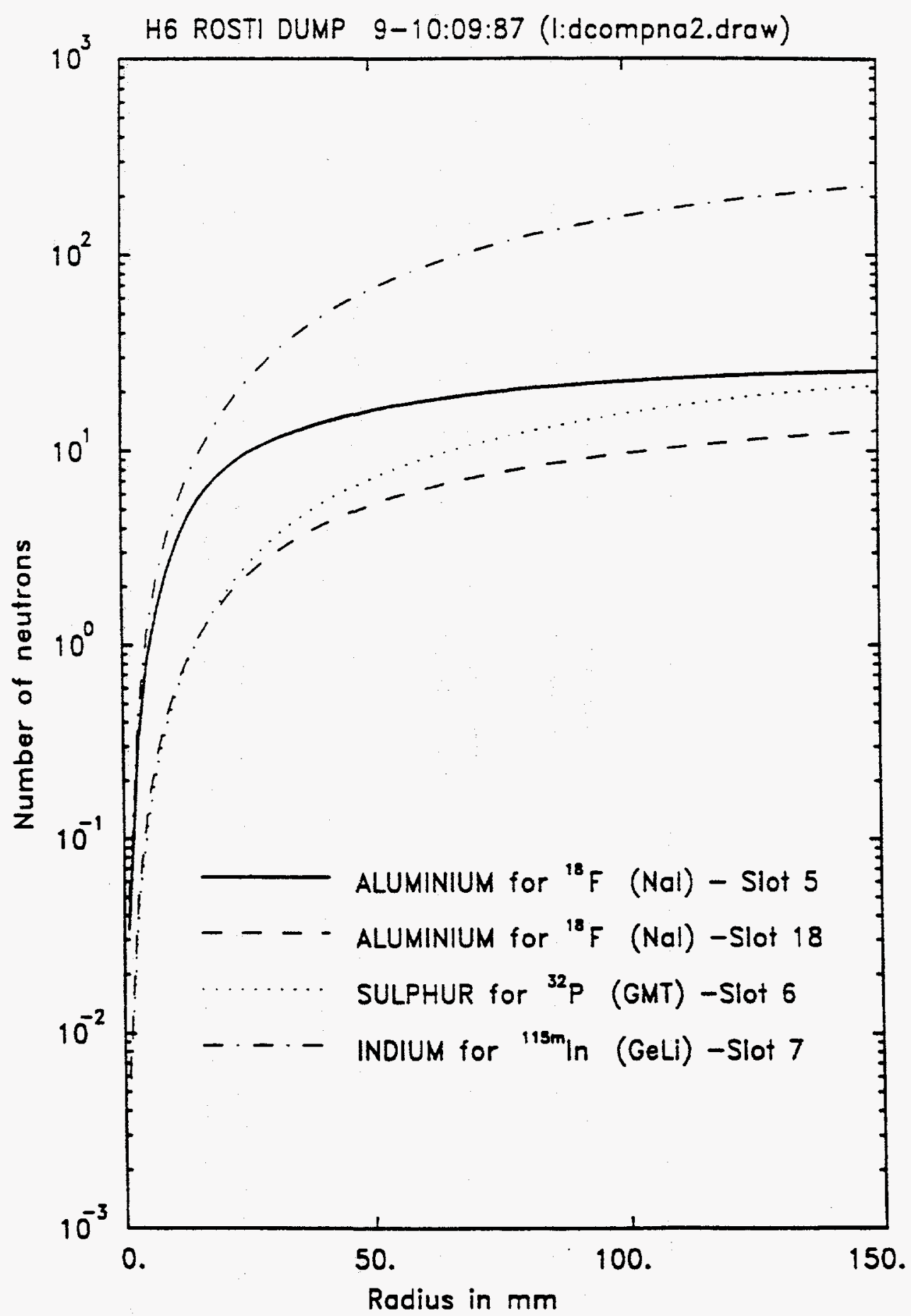

FIG. 29. Comparison of the radially integrated fluence as a function of radius for several detector systems. 


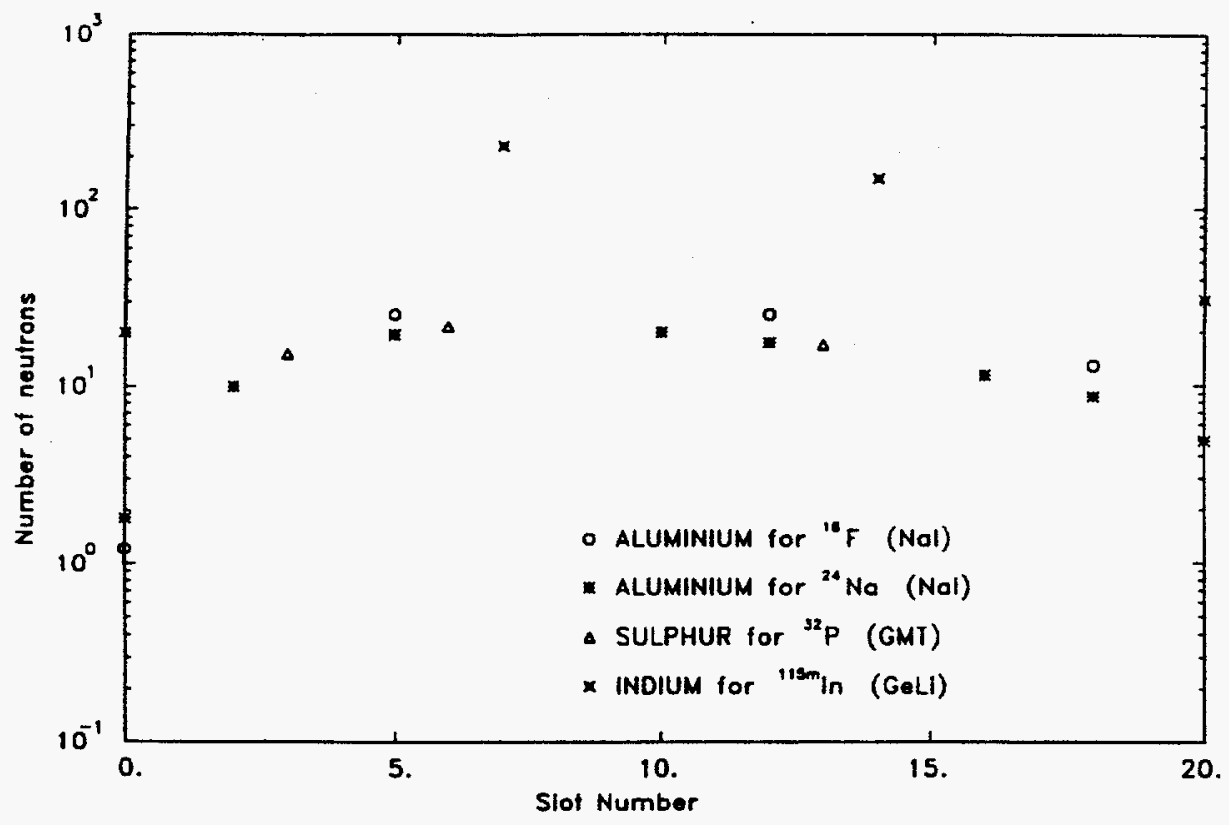

Fig. 300

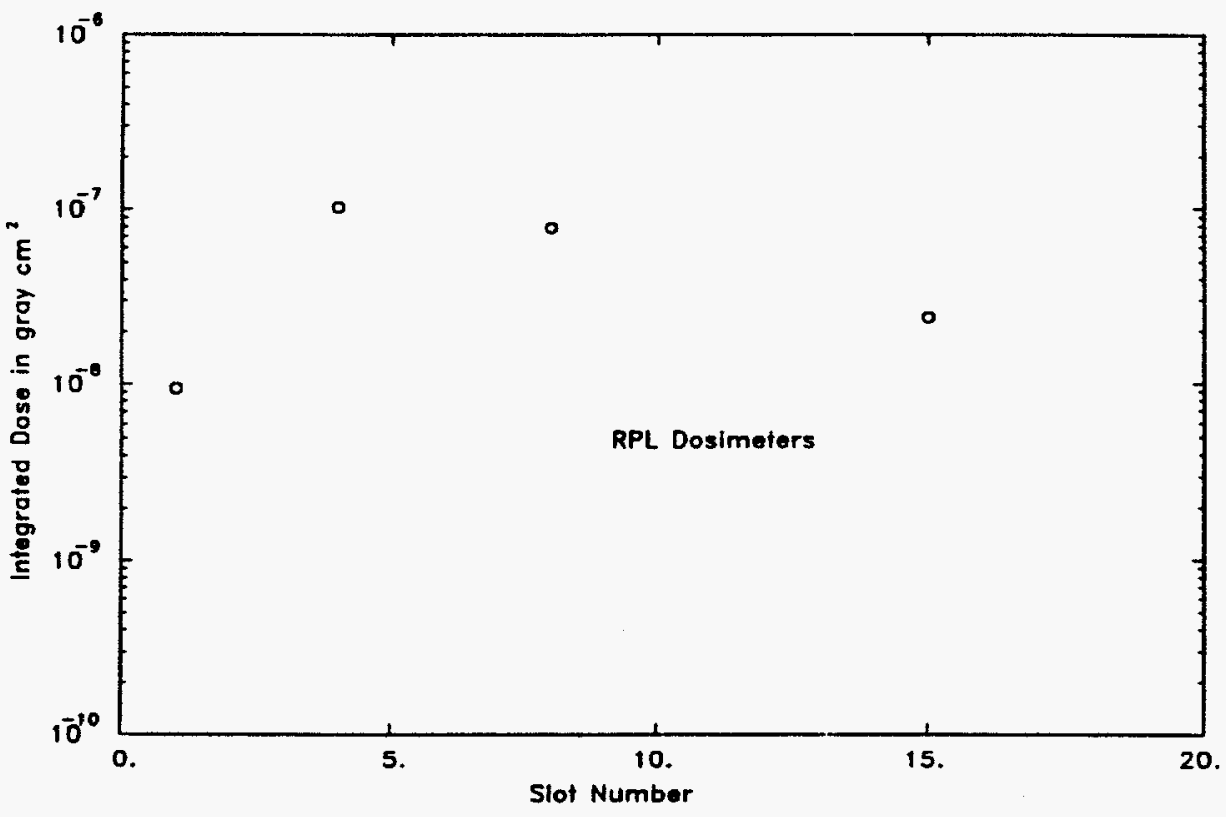

FIg. $30 b$

FIG. 30. Variation of the radial integral of the detector response as a function of depth in the cascade: a) hadron and neutron fluence, b) dose. 

APPENDIX 18

\title{
ESTIMATION OF NEUTRON FLUX AND ALBEDO FROM ACTIVATION ANALYSIS RESULTS
}

\author{
Richard Wigmans* \\ European Organization for Nuclear Research \\ CERN, Geneva, Switzerland
}

The analysis of induced radioactivity in blocks of matter, mainly uranium (-scintillator) structures, as described in Ref. 1, may provide information on the neutron flux and neutron albedo caused by showering hadrons. The longitudinal profiles of the induced activity shown in Figs. $1\left(300 \mathrm{GeV}^{-}\right)$and $2(591 \mathrm{MeV}$ p) give at the same time the flux of particles that created the measured nuclides. In the case of reactions induced by neutrons, the activity measured at the surface of the structure gives the neutron albedo.

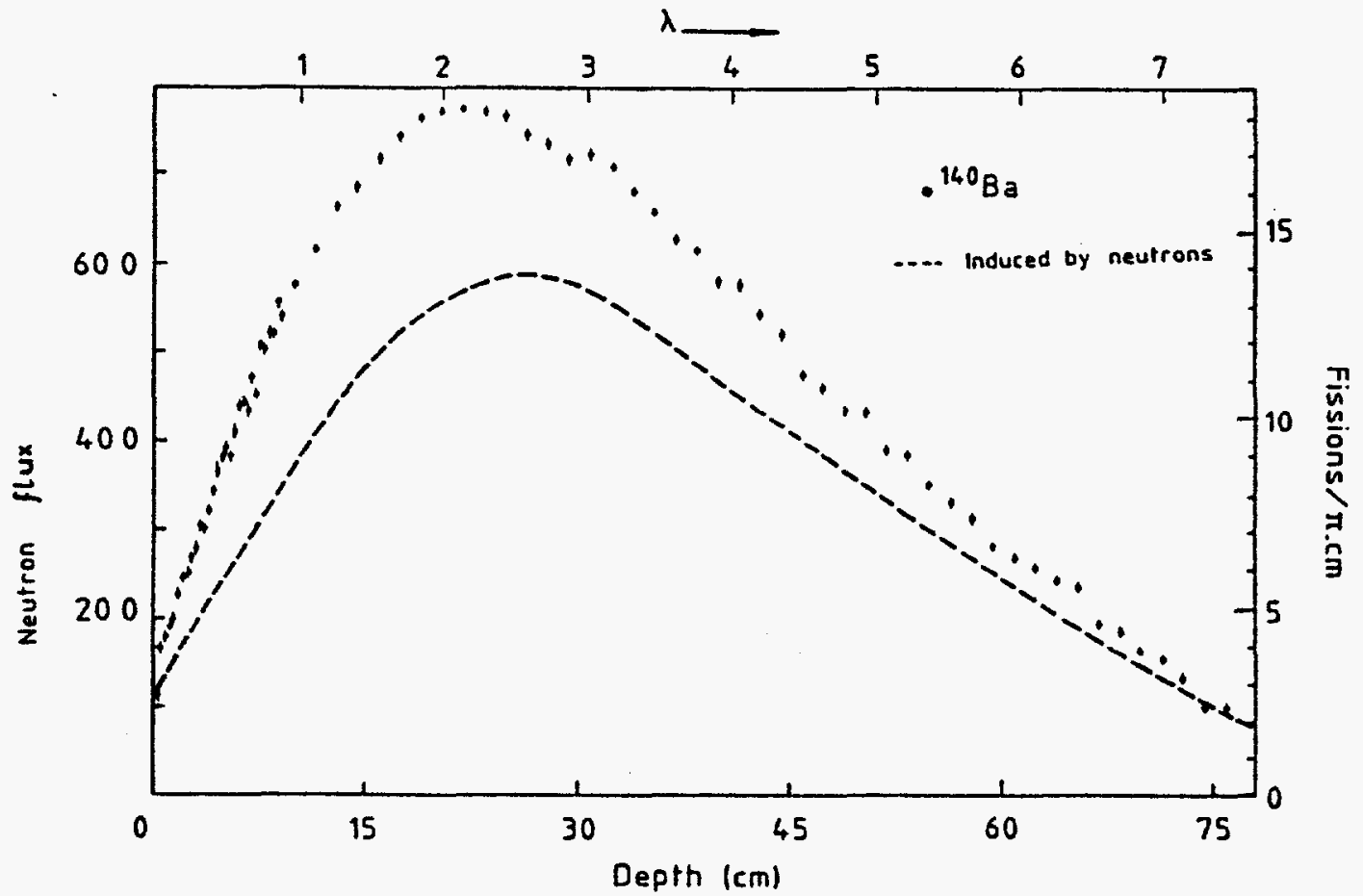

FIG. 1. Distribution of fission products $\left({ }^{140} \mathrm{Ba}\right)$ produced by $300 \mathrm{GeV} \pi^{-}$showers in a massive uranium stack, as a function of depth. The right hand scale gives the number of fissions per shower and per $\mathrm{cm}$ of uranium. The left hand scale gives the neutron flux $\left(E_{n}>1.5 \mathrm{MeV}\right)$ through a plane perpendicular to the beam direction and applies only to the $\mathrm{n}$-induced fission component (the dashed line).

* On leave of absence from NIKHEF, Amsterdam 


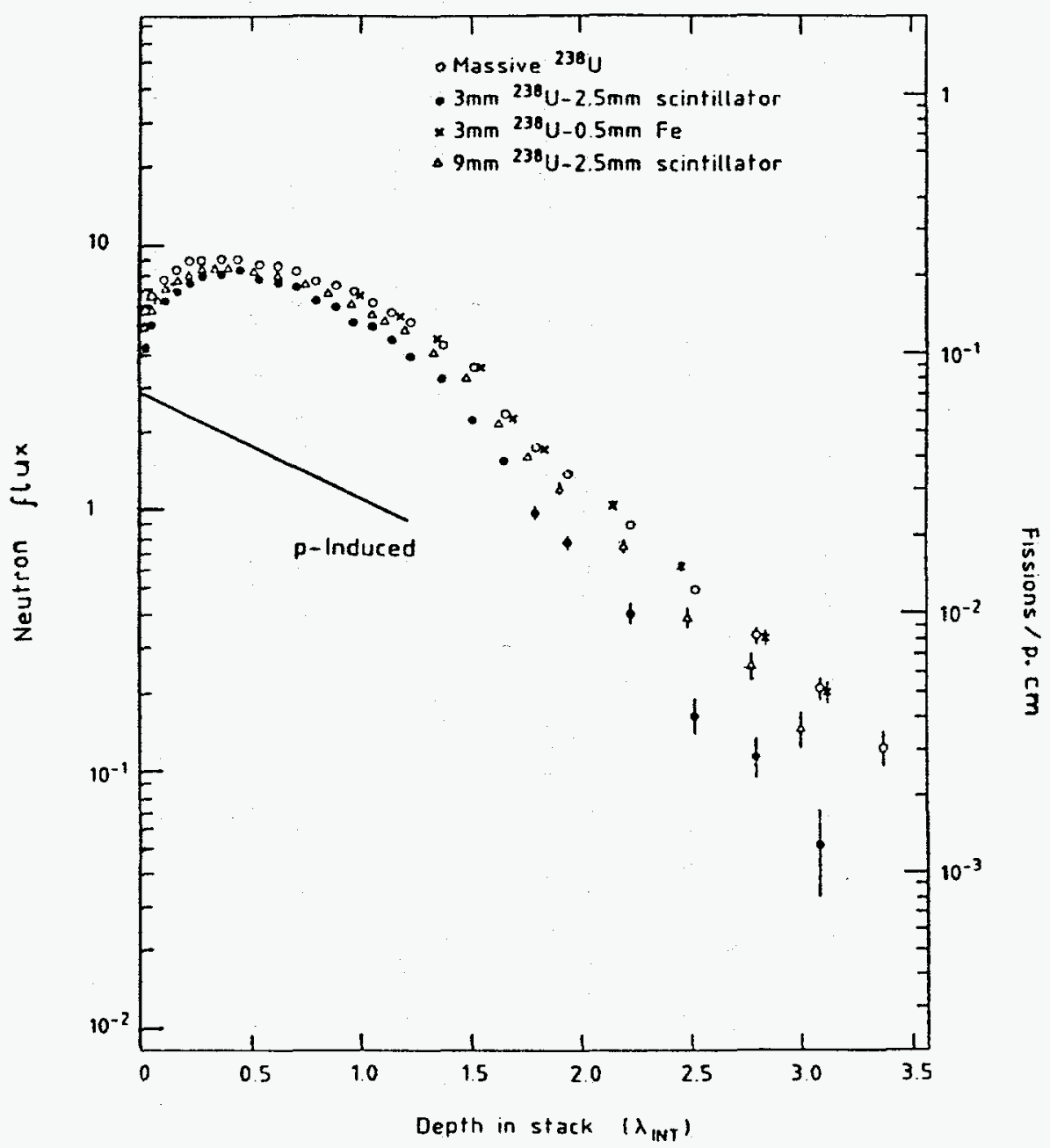

FIG. 2. Distribution of fission products $\left({ }^{140} \mathrm{Ba}\right)$ produced by $591 \mathrm{MeV}$ protons in various uranium stack configurations, as a function of depth. The right hand scale gives the number of fissions per incoming proton and per $\mathrm{cm}$ of uranium. The left hand scale gives the neutron flux $\left(E_{n}>1.5 \mathrm{MeV}\right)$ through a plane perpendicular to the beam direction and applies to the neutron-induced component, i.e., the experimental values minus the proton-induced fission component.

Most relevant for SSC radiation damage studies are the radioactive nuclides created by neutrons. First of all, there are the ${ }^{238} \mathrm{U}$ fission products, which allow extracting neutron fluxes for $E_{n}>1.5 \mathrm{MeV}$, the ${ }^{238} \mathrm{U}$ fission threshold. However, the fissions are not exclusively produced by neutrons and, therefore one has to be careful especially in evaluating the albedo. Figure 3 shows that $\sim 15 \%$ of the fissions induced by $591 \mathrm{MeV}$ proton showers are caused by the protons themselves and that at the surface, protons account for about half of the fissions (massive uranium). The number of $\mathrm{p}$-induced fissions per incoming particle does not depend on the presence or absence of scintillator in the stack, the number 


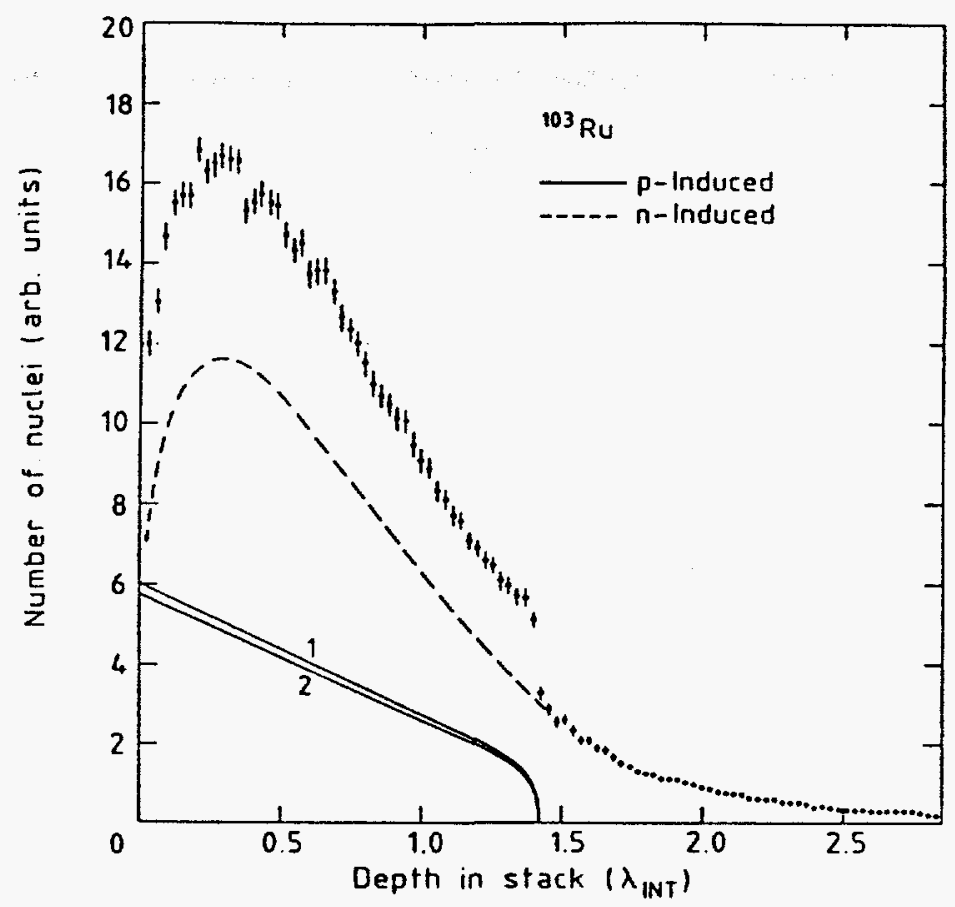

FIG. 3. Decomposition of the measured longitudinal distribution of fission products into a $591 \mathrm{MeV}$ proton-induced part and a neutron-induced part. See Ref. 1 for details.

of $\mathrm{n}$-induced fission does. Because of the fact that the neutron spectrum is softened by elastic collisions with hydrogen nuclei, less neutrons will cause fission in calorimeters with hydrogenous active material.

Unfolding of the $\mathrm{p}$ - and $\mathrm{n}$-induced fissions is straightforward in the case of $591 \mathrm{MeV}$ incident protons (Fig. 3). It is less trivial for the high-energy data. It seems reasonable to assume that also here something like $15 \%$ of the fissions are induced by pions and protons rather than by neutrons. As in the low-energy case, the longitudinal distributions of both components will be somewhat different. In particular, almost no contribution of $\pi^{-}$- or p-induced fission is expected near the calorimeter surface in this case. Each incoming $300 \mathrm{GeV} \pi^{-}$may induce at maximum 1 fission itself, which is negligible compared to the total number of 1230 fissions generated in the shower (the integral of the curve in Fig. 1), but substantial with respect to the 4 fissions created by $591 \mathrm{MeV}$ incoming protons. We may therefore safely assume that the fissions observed near the surface in the high-energy exposure are exclusively $n$-induced and therefore are a good measure for estimating the neutron albedo. Supporting evidence comes from Fig. 4, which shows that the ${ }^{140} \mathrm{Ba} /{ }^{131} \mathrm{I}$ production ratio near the surface is consistent with the asymmetric mass yield typical for soft $n$-induced fissions. Going inside the calorimeter the fissions become rapidly more symmetric, which indicates that faster particles contribute too. 


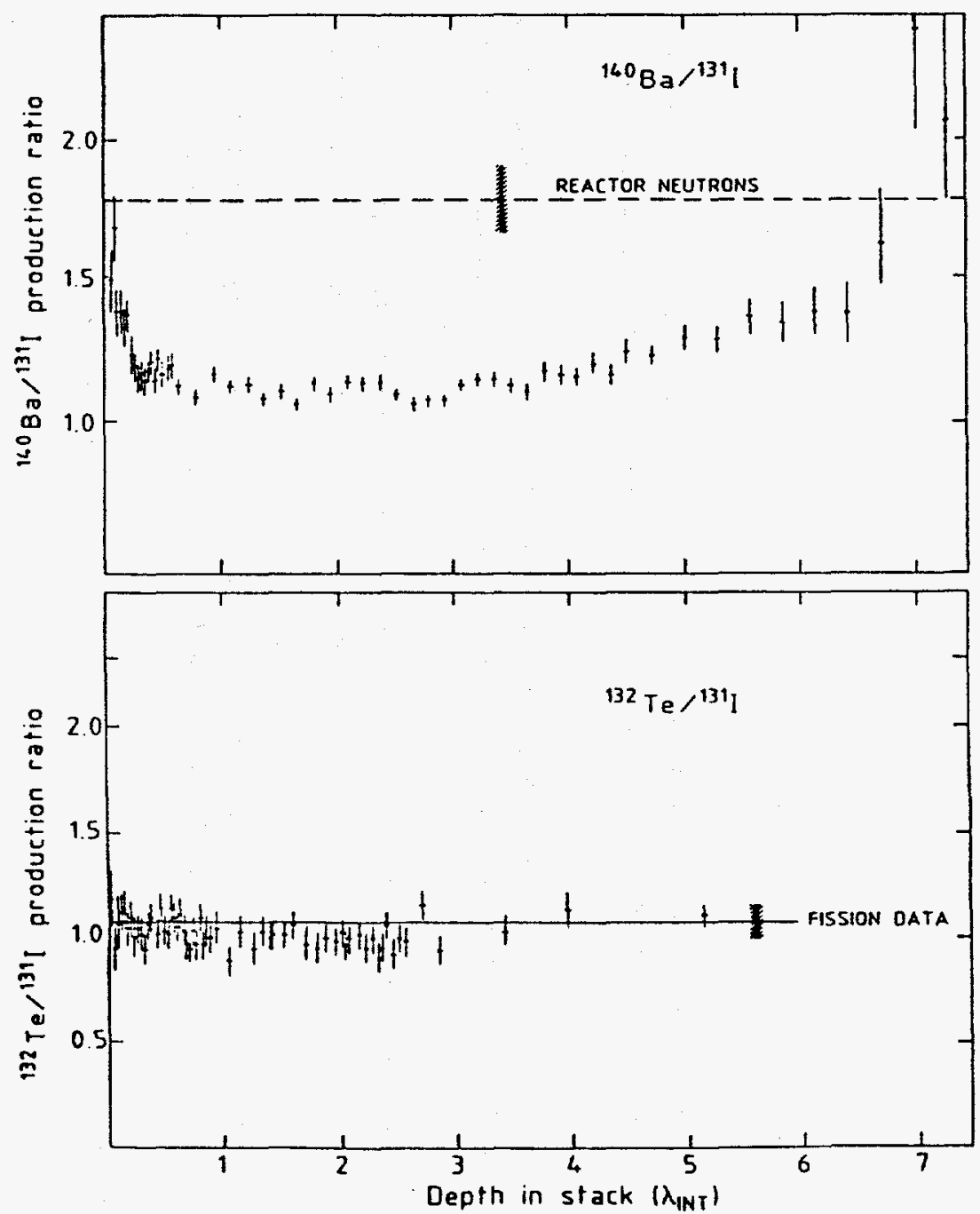

FIG. 4. The ratio of the yields of two fission products, ${ }^{140} \mathrm{Ba}$ and ${ }^{131} \mathrm{I}$, produced by $300 \mathrm{GeV} \pi^{-}$showers in massive ${ }^{238} \mathrm{U}$, as a function of depth (a). The same for two fission products with almost equal mass, ${ }^{131} \mathrm{I}$ and ${ }^{132} \mathrm{Te}$, to check for systematic effects (b).

These considerations leads to the dashed curve in Fig. 1, as an estimate of the $\mathrm{n}$-induced component of the fissions observed in $300 \mathrm{GeV}$ showers.

The data in Figs. 1 and 2 are presented as a number of fissions created per incoming particle in a plane of given thickness, perpendicular to the beam direction (right hand scale). In order to convert the vertical scale into a neutron flux, we will have to divide by the probability that a neutron crossing this plane will indeed cause fission to occur. For this we need the fission cross section. This amounts to 0.5 barns for neutrons between 1.5 and $6 \mathrm{MeV}, \sim 1$ barn for neutrons between 6 and $12 \mathrm{MeV}$, etc. The mass distribution of the $\mathrm{n}$-induced fission products indicates that neutrons below $6 \mathrm{MeV}$ largely dominate and therefore 
we will take $\sigma=0.5$ barn as the relevant cross section. This corresponds to a mean interaction length for $\mathrm{n}$-induced ${ }^{238} \mathrm{U}$ fission of $\sim 42 \mathrm{~cm}$. Therefore, the distributions in Figs. 1 and 2 can be converted into longitudinal neutron flux profiles by multiplying the scale by a factor $42 /($ thickness plane $(\mathrm{cm}))$.

\section{Conclusions}

For the $300 \mathrm{GeV}$ pions incident on massive uranium the maximum flux of neutrons crossing a given plane perpendicular to the beam direction is reached at $\sim 2 \lambda_{\text {int }}$ and amounts to 600 neutrons (faster than $1.5 \mathrm{MeV}$ ) per incoming beam particle. From the lateral activity profiles one can conclude that the flux per $\mathrm{cm}^{2}$ is at maximum $\sim 10 \%$ of this number. The flux of albedo neutrons is on average $\sim 100$ per incoming pion.

In the case of $591 \mathrm{MeV}$ incident protons on massive uranium the maximum flux occurs at $0.3 \lambda_{\text {int }}$ and amounts to about 7 neutrons $\left(E_{n}>1.5 \mathrm{MeV}\right)$. The flux of albedo neutrons is on average 3 per incoming proton. For a fine-sampling uranium-plastic scintillator calorimeter the maximum flux is about 5 neutrons crossing a plane, and 1.5 albedo neutrons, always with $E_{n}>1.5 \mathrm{MeV}$.

For calorimeters with lead absorber the fluxes of these "fast" neutrons were found to be a factor of 2 lower compared to uranium (see Ref. 1 for details).

\section{Slow neutrons}

The distributions of ${ }^{239} \mathrm{~Np}$, resulting from neutron capture by ${ }^{238} \mathrm{U}$, provide some handle on the neutrons softer than $1.5 \mathrm{MeV}$. The capture process predominately occurs at very low ("thermal") energies, i.e., below $\sim 10 \mathrm{keV}$. Complete profile measurements were only done for the $591 \mathrm{MeV}$ proton data. Interpretation of this data in terms of neutron fluxes is complicated by the fact that the cross section for capture is strongly energy dependent $(\sim 1$ barn at $10 \mathrm{keV}, \sim 2.5$ barns at $1 \mathrm{keV}, \sim 10$ barns at $100 \mathrm{eV}$, etc.). Measurements done in uraniumscintillator structures showed, however, that the ${ }^{239} \mathrm{~Np}$ concentration varied in a very systematic way in between any two subsequent scintillator plates (distance $9 \mathrm{~mm}$ ), suggesting that most of the captures occur at neutron energies where the cross sections amount to many barns (very short mean free neutron paths). See Fig. 15b from Ref. 1 for more details.

\section{Conclusions}

If we take the canonical integrated capture cross section of 2.7 barns as a basis for the flux calculations (mean free path $8 \mathrm{~cm}$ ) we find $1.5-2.5$ albedo neutrons and a maximum flux of $3-8$ neutrons, depending on the calorimeter configuration (Fig. 5). In any case, the maximum flux for these soft neutrons occurs at a depth significantly beyond the one for the neutrons capable of inducing nuclear 


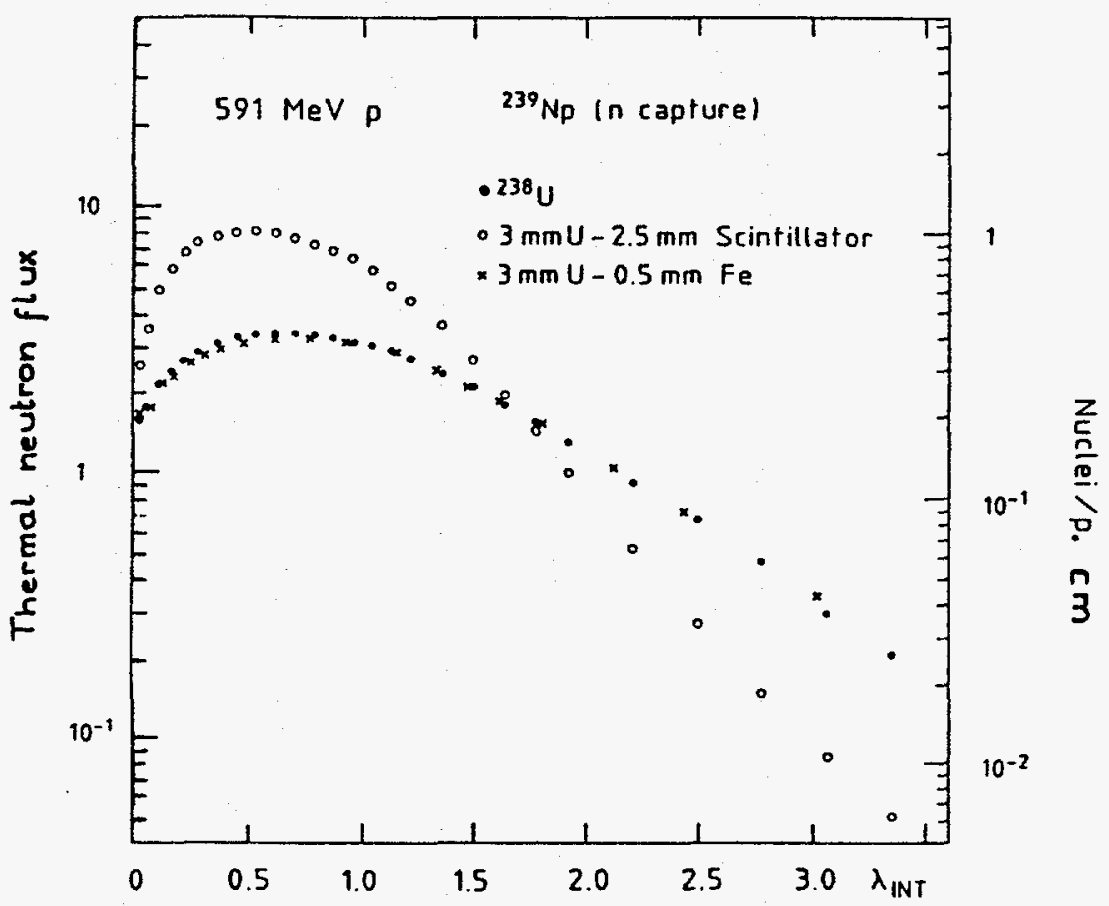

FIG. 5. The longitudinal distribution of ${ }^{239} \mathrm{~Np}$ (from neutron capture by ${ }^{238} \mathrm{U}$ ) produced by $591 \mathrm{MeV}$ protons in various uranium stack configurations. The right hand side gives the number of nuclei produced per beam particle and per $\mathrm{cm}$ of uranium. The left hand scale gives the flux of neutrons producing this nuclide (mainly $E_{n}>10 \mathrm{keV}$ ).

fission ( $0.5 \lambda_{\text {int }}$ vs. 0.3$)$, and the soft neutrons cover a much larger fraction of the calorimeter volume.

The fluxes of neutrons in the $\mathrm{eV}-\mathrm{keV}$ region in lead calorimeters are a factor of 3 lower than in uranium. See Ref. 1 for more details on this point.

\section{Ionizing radiation}

One measurement was done where the dose was explicitly measured with an array of RPL dosimeters at about the shower maximum $\left(1.9 \lambda_{\text {int }}\right)$ for $300 \mathrm{GeV}$ $\pi^{-}$projectiles sent into a massive block of uranium. The result was that $2.3 \times 10^{7}$ incoming beam particles induced $1 \mathrm{~Gy}$ in the core of the shower $\left(1.6 \times 10^{11} \pi \rightarrow\right.$ $700 \mathrm{krad}$ ).

\section{Final remark}

In this note I have tried to interpret the induced radioactivity results in terms of neutron flux and albedo. It should be emphasized that these measurements were not done with this application in mind, and therefore were not optimized in this respect.

However, the profiles of induced radioactivity and the absolute rates of fission 
processes, neutron production etc. given in Ref. 1 should by themselves form an excellent test case for hadronic shower Monte Carlo studies, because of the high degree of accuracy and detail that they provide. I have used them myself very fruitfully in this sense for optimizing my calculations on hadron calorimeter performance (see Table 4 from Ref. 2).

\section{References}

1. C. Leroy, Y. Sirois, and R. Wigmans, Nucl. Instrum. and Meth. A252, 4 (1986).

2. NIM A259, 389 (1987). 



\title{
APPENDIX 19 INCLUSIVE SIMULATION OF HADRONIC AND ELECTROMAGNETIC CASCADES IN THE SSC COMPONENTS
}

\author{
Nikolai V. Mokhov \\ Institute for High Energy Physics, Serpukhov, U.S.S.R.
}

The use of Feynman's ideas concerning the inclusive approach to multiparticle reactions[1] and of statistical weighting methods have resulted in the creation of CASIM[2] and MARS[3], Monte Carlo programs for the inclusive simulation of hadronic cascades. To construct a cascade tree only a fixed number of particles from each vertex are chosen $(1,2$ or 3$)$ and each carries a statistical weight which is equal to the partial mean multiplicity of the particular event (in the simplest case). Energy and momentum are conserved on the average over a number of collisions.

The immediate advantage of such a scheme is that the growth of CPU time per incident particle rises only logarithmically with initial energy, compared with linear rise in the exclusive mode. This opens a door to the multi- $\mathrm{TeV}$ region even well beyond SSC energies[4]. Other motives for such methods are

- in many applications one considers effects due to the simultaneous interactions of a huge number of particles, so to describe the cascade it is sufficient to obtain the first moment of the distribution function using the inclusive cross sections, in the same manner as with Boltzman's equation;

- inclusive spectra are much better known from experimental data than exclusive ones;

- the use of statistical weights allows the production of a given particle type to be enhanced within the phase-space region of interest, a feature which is especially useful in studying rare particle production.

In return for these features comes the impossibility of directly studying fluctuations from cascade to cascade, which is of interest in such problems as exploring the energy resolution of calorimeters.

Both inclusive programs have much in common. The MARS10 program has been developed over a long period of time[5-7]; the present version is described in Refs. 8 and 9. The specific features of this code are

- the hadron production model uses a set of the semi-theoretical formulas for a proton target, coupled with the additive quark model of hadron-nucleus interactions for fast secondaries and a phenomenological model for slow particles[7, 10]; 
- special attention is paid to processes with a small momentum transfer: elastic scattering, diffraction, multiple Coulomb scattering using Moliere's theory with allowance for nuclear size effects, $\delta$-rays, and direct $\mathrm{e}^{+} \mathrm{e}^{-}$production by hadrons[11];

- quasianalog simulation of electromagnetic showers, initiated mainly by $\pi^{0}$ decays with the modified AEGIS program[12];

- multi-media arbitrary geometry with optional distributed superfine structure (e.g. if one wants to examine detail in a small part of a region hundreds of meters long); cascade development in the presence of arbitrary magnetic fields; an iteration-step method with precise localization of boundaries, especially refined near matter-vacuum edges[7, 11, 13];

- statistical fluctuation reduction options: bias techniques, exponential conversion of path length, splitting, Russian roulette, synthesis with analytical solutions;

- scoring of three dimensional distributions of energy deposition, star density, particle fluence and energy spectra;

- particles: $\mathrm{p}, \mathrm{n}, \pi, \mathrm{K}, \mu$, electrons and $\gamma$ 's;

- initial energy: $10 \mathrm{MeV}$ to $30 \mathrm{TeV}$;

- threshold energy: electrons and gammas $0.1 \mathrm{MeV}$, neutrons $10 \mathrm{MeV}$ (and as an option $0.025 \mathrm{eV}$ ), others $2 \mathrm{MeV}$.

These inclusive programs are being used for a wide variety of problems at the new generation accelerators, including the Tevatron, UNK and the SSC. MARS10 has been used to deal efficiently with such problems as radiation heating of targets and beam dumps[6], radiation shielding against hadrons and muons[7, 14, 15], design of the beam abort systems for the Tevatron and UNK[16], radiation heating of superconducting magnets $[17,18]$, minimization of beam losses in the Tevatron and UNK superconducting lattice[19, 20], background in experiments[7], and optimization of the collider detectors[21].

The inclusive approach is very suitable for the SSC design, especially at its present stage. Some results are given in Refs. 8 and 9 and in Section 5. In this Appendix we describe one example relevant to detector design: development of hadronic cascades in a $2 \times 2 \times 2 \mathrm{~m}$ lead absorber which, as was pointed out elsewhere, is very nearly identical to the finely segmented uranium/scintillator calorimeter used as an example throughout this Report. As was shown in Sections 2 and 4, essentially all of the radiation problems in an SSC detector are initiated by hadrons (mainly pions) with energies in the 1 to $100 \mathrm{GeV}$ region (average 20 $\mathrm{GeV}$ ). We have therefore used initial positive pions with momenta $p_{0}=1,3,5$, $10,20,40$ and $100 \mathrm{GeV} / \mathrm{c}$. 


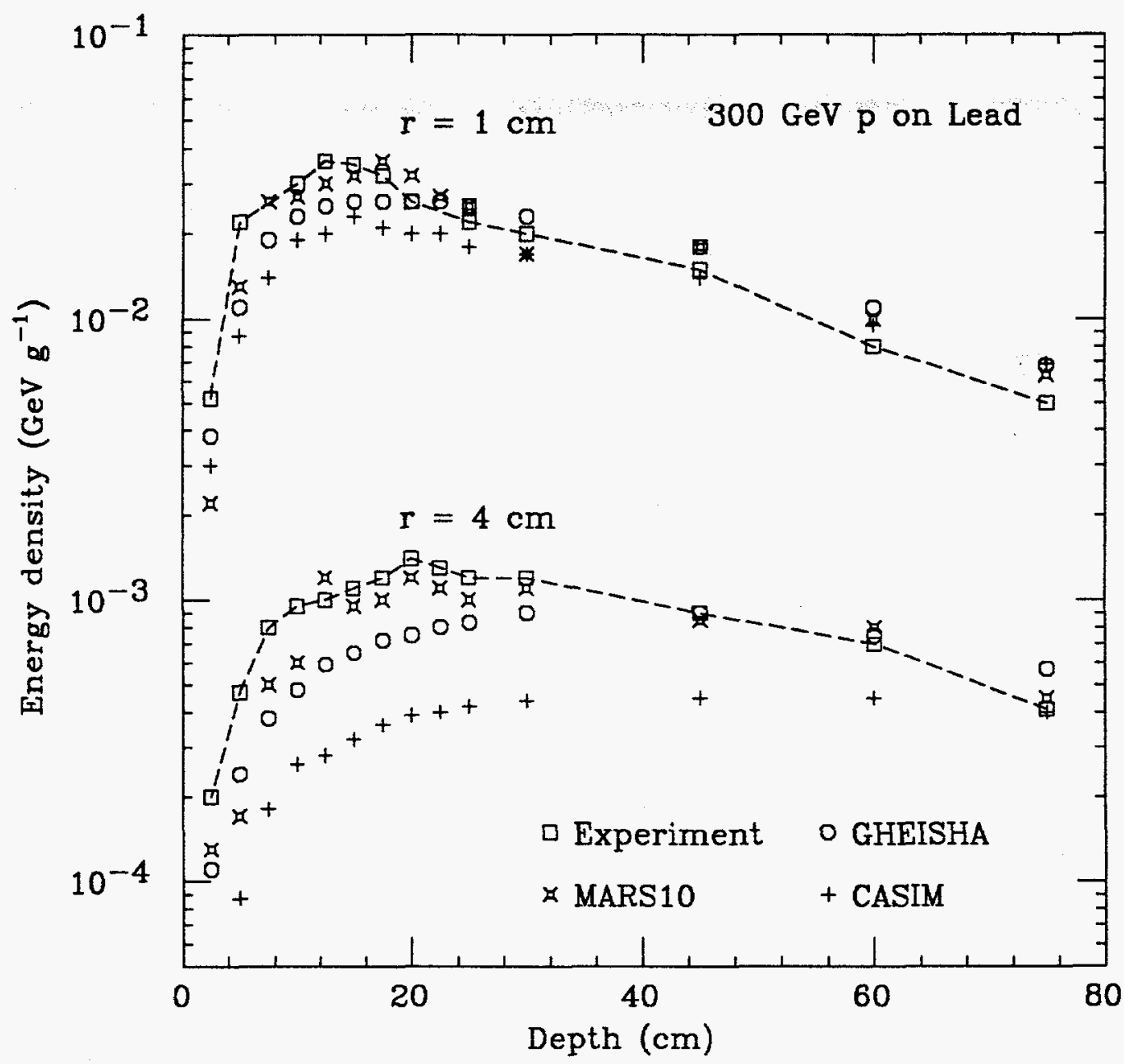

Fig. A19-1. Longitudinal distributions of energy deposition density at two radial distances from the cascade axis in the lead calorimeter[25] as measured and as calculated with three programs.

Fig. 5-7 of the main text illustrates the present situation with the three programs CASIM, MARS10 and the exclusive code FLUKA86[22]. The MARS10 and FLUKA86 results are essentially identical, as was also shown in Ref. 9. The CASIM radial distributions agree well with those produced by the other codes $[8$, 9], but the longitudinal behaviour of energy deposition and star density differs from the others. This was also demonstrated in Refs. 9, 23, and 24.

For example, Fig. A19-1 shows the longitudinal distribution of energy deposition in the lead model calorimeter irradiated by $300 \mathrm{GeV}$ protons as calculated with the various programs and as measured by Muraki et al.[25]. In the experiment, film stacks were place between lead plates to measure the distribution of the energy deposition. A MARS10 simulation of the exact experimental configuration is reported in Ref. 9. The vertical scale in Fig. A19-1 is energy density in 


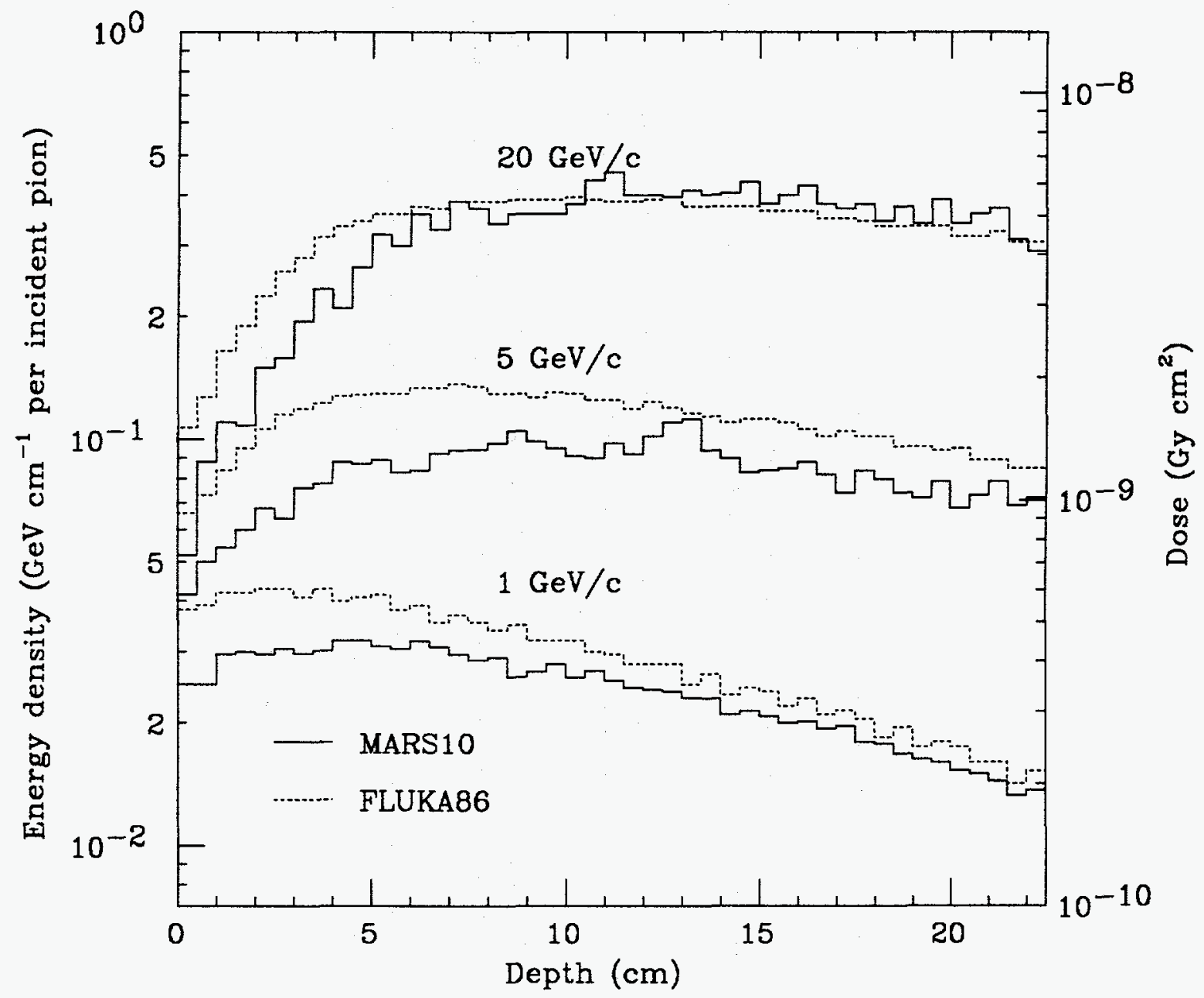

Fig. A19-2. Laterally integrated longitudinal distribution of energy density and dose at small depths in the lead absorber irradiated by pions of various momenta, as calculated with MARS10 and FLUKA86.

emulsion. Because the experimental data given in Ref. 25 are in arbitrary units they have been normalized to the MARS10 results at the point $z=r=0$. The CASIM and GHEISHA[26] results are borrowed from Ref. 24 without changing their normalization with respect to the Muraki data. As is also shown in Fig. $5-7$, CASIM underestimates in the region of the maximum and overestimates in the tails.

A special study has shown that this comes about because of an incorrect $A$-dependence for the leading particle spectrum in CASIM. The thermodynamic model used in CASIM[27] has insufficient suppression of the $\mathrm{p} A \rightarrow \mathrm{p} X$ inclusive cross section in the fragmentation region. As a result, the difference between CASIM results and those of other codes is greater for heavier targets.

Fig. A19-2 illustrates the differences between MARS10 and FLUKA 86 results 


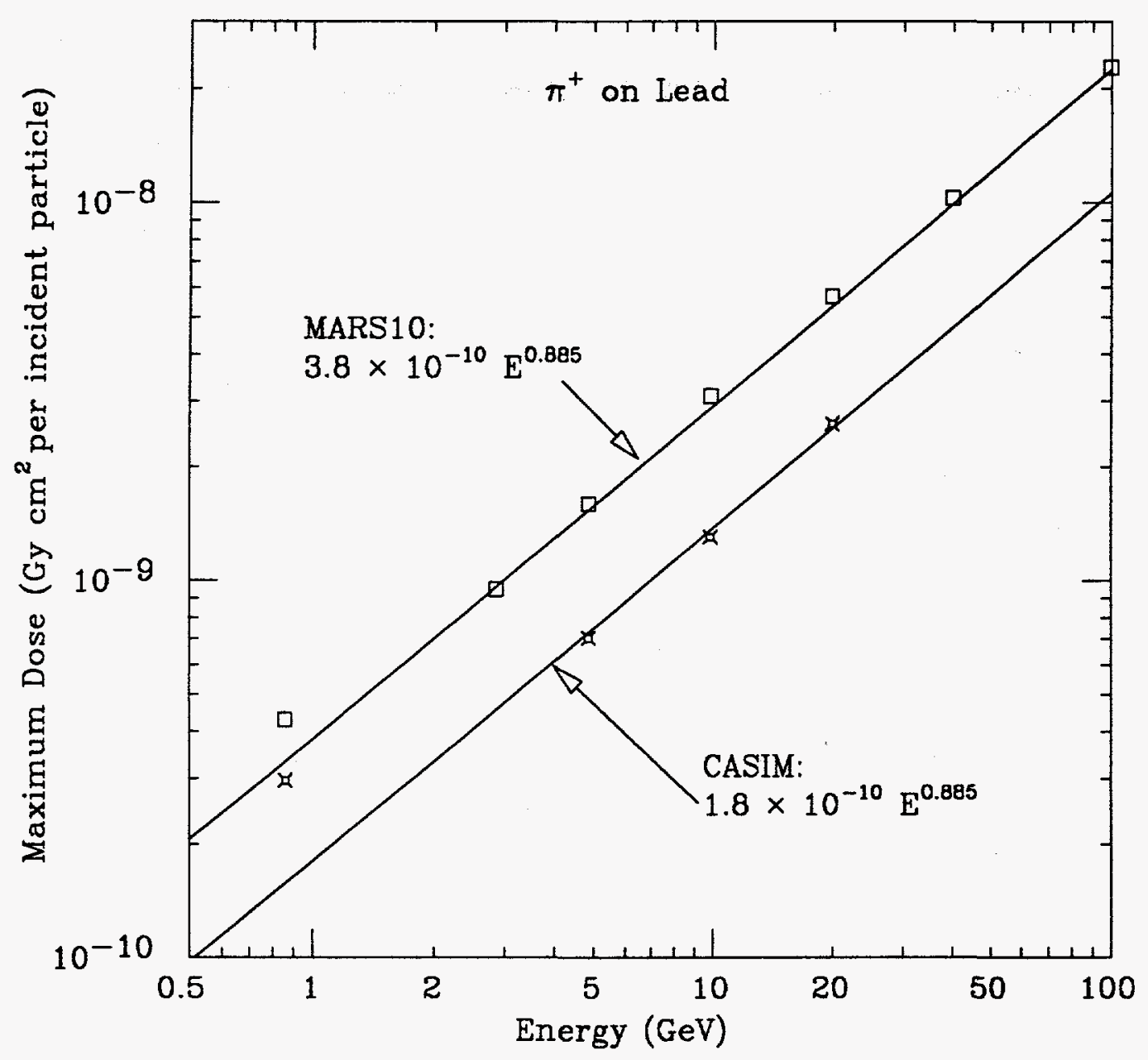

Fig. A19-3. Dependence of maximum dose in lead absorber on incident pion kinetic energy as calculated with MARS10 and CASIM.

for laterally integrated energy deposition at small depths. These appear to come about because of

- differences in threshold energy- $50 \mathrm{MeV}$ in FLUKA86 and $2 \mathrm{MeV}$ in MARS10; we found that higher thresholds can produce overestimates of up to $30 \%$ at small depths and small radii;

- different "grey" particle $(\beta<0.7)$ production schemes; for these particles MARS10 uses a phenomenological model well fitted to experimental data[10]; as has been frequently discussed the intranuclear cascade model which is used in FLUKA86 can overestimate the multiplicity of just the "grey" particles. The discrepancy is greatest at small depths because electromagnetic showers play a greater role deeper into the cascade, particularly for heavy targets. 


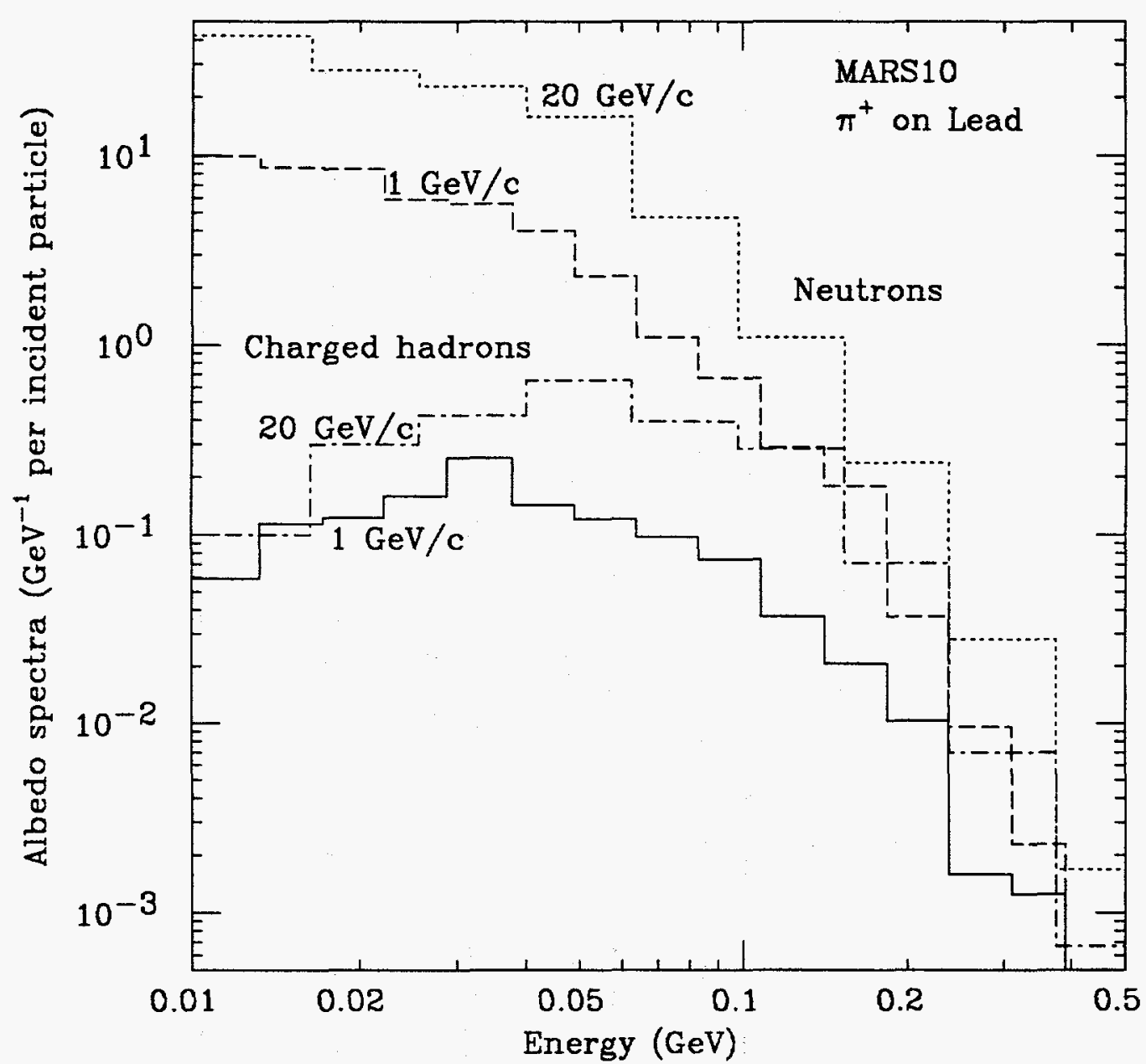

Fig. A19-4. Spectra of neutrons and charge hadrons in back hemisphere of lead absorber irradiated by 1 and $20 \mathrm{GeV} / \mathrm{c}$ pions.

For SSC applications it is important to estimate the maximum dose in a hadronic shower. This is plotted in Fig. A19-3 as a function of the kinetic energy of incident pions. For $2 \mathrm{GeV} \leq E_{\pi} \leq 100 \mathrm{GeV}$ the maximum of the laterally integrated dose (in $\mathrm{Gy} \mathrm{cm}^{2}$ ) is well described by $3.8 \times 10^{-10} E^{0.885}$. The same power law is also consistent with the CASIM data for energies in excess of a few $\mathrm{GeV}$ but with the coefficient $1.8 \times 10^{-10}$. The corresponding exponent for iron at high energies is $\sim 0.8[8]$. The somewhat stronger energy dependence in lead is explained by the growing contribution of electromagnetic showers, which scales as $\sim E$.

The total number of stars in lead initiated by particles in the cascade with $p>300 \mathrm{MeV} / \mathrm{c}$ behaves as $3.7 E^{0.827}$ for $2 \mathrm{GeV}$ to $100 \mathrm{GeV}$ pions. The exponent for iron is between 0.77 and $0.81[7,8]$.

Another concern is the hadronic backscatter (or albedo) considered in several 


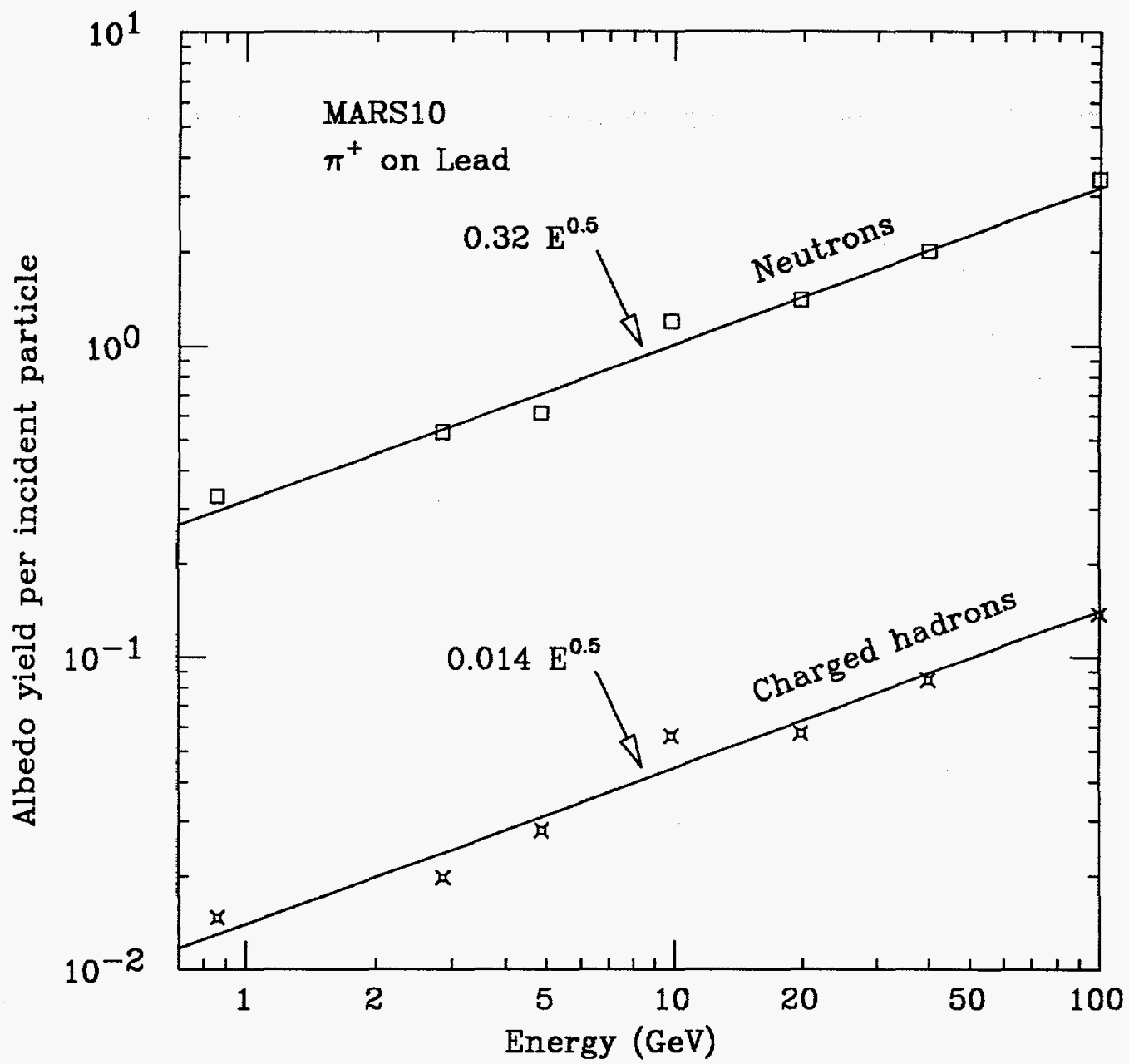

Fig. A19-5. Number of albedo neutrons and charged hadrons of lead as function of kinetic energy of incident pions.

other appendices and discussed in Section 4.3. The MARS10 albedo results are shown in Fig. A19-4 and Fig. A19-5 for neutrons and charged hadrons with energies greater than $10 \mathrm{MeV}$. As one can see, the shape of the spectra are fairly independent of incident momentum, especially for neutrons. The total albedo yield of hadrons with energies in excess of $10 \mathrm{MeV}$ is well described by

$$
N=C E^{k},
$$

where for $1<E<100 \mathrm{GeV}, k=0.5, C=0.32$ for neutrons and $C=0.014$ for charged hadrons. The exponent is in good agreement with the value adopted in Section 4-3, while the coefficient cannot be directly compared because of the comparatively high momentum cutoff in the present case.

There is a broad consensus that the exponent $k$ is about 0.5 for uranium/scintillator calorimeters, as is discussed in Section 4.3. In Appendix 15 the iron/scin- 
tillator case is also simulated, with the result $k \approx 0.45$. MARS6 simulations yield $k \approx 0.45$ and 0.39 for iron and concrete, respectively[7]. In summary, a very rough $A$ dependence can be obtained:

$$
\begin{aligned}
& k=0.50 \text { for } \mathrm{Pb} \text { or } \mathrm{U} / \mathrm{scint} \\
& k=0.45 \text { for } \mathrm{Fe} \text { or } \mathrm{Fe} / \mathrm{scint} ; \\
& k=0.39 \text { for } \mathrm{Al} \text { or concrete. }
\end{aligned}
$$

The author wish to thank Igor Azhgirey for performing the FLUKA 86 calculations for Fig. 5-7 and Fig. A19-2, and Don Groom for introducing him to the TOPDRAWER graphics package.

\section{References}

1. R. P. Feynman, Phys. Rev. Lett., 23, 1415 (1969).

2. A. Van Ginneken. Fermilab FN-272 (1975).

3. N. V. Mokhov, Proc. IV All-Union Conference on Charged Particle Accelerators, Moscow, "Nauka" 2, 222 (1975); N. V. Mokhov and V. V. Phrolov. Atomnaya Energiya, 38, 226 (1975).

4. O. A. Askariyan, B. A. Dolgoshein, A. N. Kalinovsky, and N. V. Mokhov, Nucl. Instrum. and Methods, 164, 267 (1979).

5. I. S. Baishev, S. L. Kuchinin, and N. V. Mokhov, Preprint IHEP 78-2, Serpukhov (1977).

6. N. V. Mokhov and A. Van Ginneken, Fermilab TM-977 (1980); N. V. Mokhov, Fermilab FN-328 (1980); N. V. Mokhov, Preprint IHEP 82-168, Serpukhov (1982).

7. A. N. Kalinovsky, N. V. Mokhov and Yu. P.Nikitin, Penetration of High Energy Particles Through Matter, Moscow, "Energoatomizdat" (1985) (in Russian) (1988-in English).

8. N. V. Mokhov, J. D. Cossairt. Nucl. Instrum. Methods, A244, 349 (1986).

9. N. V. Mokhov, Soviet J. Particles and Nuclei, (Sept.-Oct. 1987), pp. 408-426.

10. N. V. Mokhov, S. I. Striganov, and A. V. Uzunian. Preprint IHEP 87-59, Serpukhov (1987).

11. I. S. Baishev, N. V. Mokhov, and S. I. Striganov, Soviet J. Nucl.Physics 42, 1175 (1985); I. S. Baishev, Preprint IHEP 87-149, Serpukhov (1987).

12. A. Van Ginneken, Fermilab FN-309 (1978).

13. I. S. Baishev, M. A. Maslov, and N. V. Mokhov, Proc. VIII All-Union Conference on Charged Particle Accelerators, Dubna, 2, 167 (1983); M. A. Maslov and N. V. Mokhov, Preprint IHEP 85-8, Serpukhov (1985).

14. J. D. Cossairt, N. V. Mokhov and C. T. Murphy. Nucl. Instrum. and Methods 197, 465 (1982).

15. N. V. Mokhov, G. I. Semenova, and A. V. Uzunian, Nucl. Instrum. and Methods 180, 469 (1981); M. A. Maslov, N. V. Mokhov, and A. V. Uzunian, Nucl. Instrum. and Methods 217, 419 (1983).

16. J. Kidd, N. V. Mokhov, C. T. Murphy, et al., Proc. Particle Accel. Conf. of the IEEE 2, 2774 (1981); I. S. Baishev, V. A. Vasiliev, G. G. Gurov, et al., Proc. VIII All-Union Conference on Charged Particle Accelerators, Dubna 1, 268 (1983). 
17. N. V. Mokhov, Sov. J. Tech. Physics 49, 1254 (1979); M. A. Maslov and N. V. Mokhov, Particle Accelerators 11, 91 (1980); M. A. Maslov and N. V. Mokhov,Proc. III ICFA Workshop, Protvino, p. 3 (1981).

18. R. Dixon, N. V. Mokhov, and A. Van Ginneken. Fermilab FN-327 (1980).

19. I. S. Baishev, V. I. Balbekov, and A. I. Drozhdin et al., Proc. VII All-Union Conference on Charged Particles Accelerators, Dubna, 1, 262 (1983); A. I. Drozhdin, M. A. Maslov, N. V. Mokhov et al., Proc. IX All-Union Conference on Charged Particle Accelerators, Dubna, 2, 368 (1985).

20. A. I. Drozhdin, M. Harrison, and N. V. Mokhov, Fermilab FN-418 (1985).

21. I. L. Azhgirey, N. V. Mokhov, and A. V. Uzunian, Proc. Int. Workshop on Experiments at UNK, Protvino, Sept. 1987; I. L. Azhgirey, A. P. Vorobiev, E. A. Kozlovsky, N. V. Mokhov, Preprint IHEP 87-151, Serpukhov (1987).

22. P. A. Aarnio, A. Fassò, H. J. Möhring, J. Ranft, and G. R. Stevenson, "FLUKA86 User's Guide," CERN TIS-RP/168 (1986).

23. G. R. Stevenson, CERN TIS-RP/183/CF (1987).

24. F. Ebeling, R. Fohrmann, U. Otterpohl, and H. J. Möhring, DESY 87-057, Hamburg (1987).

25. Y. Muraki, K. Kasahara, T. Yuda et al., Nucl. Instrum. and Methods, A236, 47 (1985).

26. H. Fesefeldt, PITHIA 85-02, Aachen (1985).

27. H. Grote, R. Hagedorn, and J. Ranft, "Atlas of Particle Production Spectra," CERN, Geneva (1970). 



\title{
APPENDIX 20 \\ DOSE TO SSC DETECTORS DUE TO p-p COLLISIONS*
}

\author{
Graham R. Stevenson \\ European Organization for Nuclear Research \\ CERN, Geneva, Switzerland
}

Monte Carlo calculations are described in which the energy deposited in shells of different materials surrounding the $p-p$ interactions at the SSC is estimated. These calculations, which are an extension of previous calculations at LHC energies, should help in defining the damage to be expected in particle detectors close to the intersection region.

\section{Introduction}

It was shown in previous calculations [1] made for LHC energies that the intensity of the secondary particles produced in the collisions would be sufficient to cause radiation damage to the detectors of the experiments themselves. In those calculations the source of secondary particles was taken from the programs ABR of Ranft [2] based on the work of Aurenche et al. [3] and PYTHIA [4] and the detector was taken to be a sphere of aluminium with an internal radius of $2 \mathrm{~m}$ and outer radius of $5 \mathrm{~m}$. The transport of the secondary particles was simulated using the FLUKA program [5], and a simplified algorithm was used to transport the electromagnetic showers initiated by incident photons, electrons and pi-zero mesons. In the calculations described in this paper, the source of secondaries was derived from the DTUJET program of Ranft et al. [6] for $\mathrm{p} \overline{\mathrm{p}}$ collisions at $20+20 \mathrm{TeV}$. Spheres of four different materials were considered viz. aluminium, iron, lead and uranium, all of internal radius $2 \mathrm{~m}$ but having approximately the same thickness as $3 \mathrm{~m}$ of aluminium in hadron absorption lengths. Hadron transport in the shells was again simulated with the FLUKA program, but this time the electromagnetic showers were simulated with the program EGS [7].

\section{Calculations}

\section{$2.1 p$ - $p$ collisions}

At the time that these calculations were made only $p \bar{p}$ collisions could be simulated with the DTUJET program. It was not considered that there would be a significant difference between these events and p-p events at $20+20 \mathrm{TeV}$.

A file containing details of particle type and momentum for approximately 40000 secondaries from 250 interactions was written by DTUJET for use in the subsequent analysis. The $\pi^{0}$ mesons were not forced to decay and neutrinos were ignored. The file order had to be randomized before use in the FLUKA simulation

\footnotetext{
* Also available as CERN report TIS-RP/draft, 8 December 1987.
} 
to avoid effects of only taking part of an event or of single events having unique characteristics. A simple break-down of the numbers of particles in each angular interval chosen for the Monte Carlo calculations is given in Table 1. The upper integer numbers $(N)$ are the actual number of particles on the tape; the next numbers $(n)$ are the number per $p-p(p \bar{p})$ event; the third numbers $(E)$ represent the sum of the kinetic energies in $\mathrm{GeV}$ of the particles in that bin per event. Care should be taken when using these numbers since, because of symmetry, forward and backward going particles have been included in the same angular interval.

Table 1

Some statistics on the $20+20 \mathrm{TeV}$ DTU tape. The event tape contains 250 events and 40491 secondaries. Particle types: $1=$ nucleon; $2=$ charged meson; $3=$ charged hyperon; $4=$ electron, positron, photon; $5=$ muon; $6=$ neutrino; $7=$ pizero; $8=$ other.

\begin{tabular}{cccccccccc}
\hline \hline Angular & & \multicolumn{7}{c}{ Particle type } \\
interval & & 1 & 2 & 3 & 4 & 5 & 6 & 7 & 8 \\
\hline $0-1^{\circ}$ & $\mathrm{N}$ & 931 & 9058 & 214 & 557 & 0 & 0 & 4499 & 1147 \\
& $\mathrm{n}$ & 3.72 & 36.23 & 0.86 & 2.23 & 0.00 & 0.00 & 18.00 & 4.59 \\
& $\mathrm{E}$ & 7741.8 & 19294.6 & 1165.5 & 798.0 & 0.0 & 0.0 & 7858.2 & 2750.8 \\
$1-5^{\circ}$ & $\mathrm{N}$ & 327 & 4887 & 110 & 339 & 0 & 0 & 2577 & 607 \\
& $\mathrm{n}$ & 1.31 & 19.55 & 0.44 & 1.36 & 0.00 & 0.00 & 10.31 & 2.43 \\
& $\mathrm{E}$ & 18.2 & 212.5 & 6.1 & 12.5 & 0.0 & 0.0 & 98.2 & 33.0 \\
$5-10^{\circ}$ & $\mathrm{N}$ & 136 & 2068 & 47 & 153 & 0 & 0 & 1168 & 235 \\
& $\mathrm{n}$ & 0.54 & 8.27 & 0.19 & 0.61 & 0.00 & 0.00 & 4.67 & 0.94 \\
& $\mathrm{E}$ & 2.2 & 27.1 & 0.7 & 1.8 & 0.0 & 0.0 & 13.8 & 3.6 \\
$10-20^{\circ}$ & $\mathrm{N}$ & 116 & 2002 & 35 & 156 & 0 & 0 & 1038 & 245 \\
& $\mathrm{n}$ & 0.46 & 8.01 & 0.14 & 0.62 & 0.00 & 0.00 & 4.15 & 0.98 \\
& $\mathrm{E}$ & 0.9 & 12.1 & 0.2 & 0.7 & 0.0 & 0.0 & 5.8 & 1.9 \\
$20-40^{\circ}$ & $\mathrm{N}$ & 92 & 1916 & 28 & 153 & 0 & 0 & 1026 & 170 \\
& $\mathrm{n}$ & 0.37 & 7.66 & 0.11 & 0.61 & 0.00 & 0.00 & 4.10 & 0.68 \\
& $\mathrm{E}$ & 0.4 & 6.4 & 0.1 & 0.4 & 0.0 & 0.0 & 3.0 & 0.7 \\
$40-70^{\circ}$ & $\mathrm{N}$ & 52 & 1620 & 11 & 168 & 0 & 0 & 922 & 179 \\
& $\mathrm{n}$ & 0.21 & 6.48 & 0.04 & 0.67 & 0.00 & 0.00 & 3.69 & 0.72 \\
& $\mathrm{E}$ & 0.1 & 3.4 & 0.0 & 0.4 & 0.0 & 0.0 & 1.6 & 0.4 \\
$70-90^{\circ}$ & $\mathrm{N}$ & 26 & 833 & 4 & 86 & 0 & 0 & 471 & 82 \\
& $\mathrm{n}$ & 0.10 & 3.33 & 0.02 & 0.34 & 0.00 & 0.00 & 1.88 & 0.33 \\
& $\mathrm{E}$ & 0.0 & 1.4 & 0.0 & 0.1 & 0.0 & 0.0 & 0.7 & 0.1 \\
\hline \hline
\end{tabular}




\subsection{Cascade calculation}

Particles from the file produced as described above were used as input to the Monte Carlo Cascade Program FLUKA. Energy densities were scored in spherical shells of aluminium, iron, lead and uranium of inner radius $2 \mathrm{~m}$ centred on the $\mathrm{p}-\mathrm{p}$ collisions. Details of the radial binning are given in Table 2. The properties of the materials used in the calculation are given in Table 3.

Table 2

Radial bins in $\mathrm{cm}$ in the Monte Carlo simulations

\begin{tabular}{cccc}
\hline \hline Aluminum & Iron & Lead & Uranium \\
\hline 200 & 200 & 200 & 200 \\
205 & 202 & 202 & 201 \\
210 & 204 & 204 & 202 \\
215 & 206 & 206 & 203 \\
220 & 208 & 208 & 204 \\
230 & 210 & 210 & 205 \\
240 & 220 & 220 & 210 \\
250 & 240 & 240 & 220 \\
275 & 260 & 260 & 230 \\
300 & 280 & 280 & 240 \\
350 & 300 & 300 & 250 \\
400 & 320 & 320 & 260 \\
450 & 360 & 360 & 280 \\
500 & 400 & 400 & 300 \\
\hline \hline
\end{tabular}

Table 3

Material properties used in the calculations

\begin{tabular}{lcccc}
\hline & Aluminium & Iron & Lead & Uranium \\
\hline Atomic number & 13 & 26 & 82 & 92 \\
Atomic weight & 27.0 & 55.8 & 207.0 & 238.0 \\
Density $\left(\mathrm{g} \mathrm{cm}^{-3}\right)$ & 2.7 & 7.8 & 11.7 & 18.9 \\
D-factor & 0.98 & 1.12 & 1.47 & 1.52 \\
\hline \hline
\end{tabular}

The medium in the inner sphere around the interaction point was assumed to be vacuum. EGS4 was used to treat the electromagnetic part of the cascades. Leading-particle biassing was used in both FLUKA and EGS [5] to avoid wasting excessive time in tracking low energy particles which would not contribute significantly to either cascade propagation or energy deposition. 
The dose in silicon was calculated from the energy deposited in the materials by multiplying the energy densities averaged over the bins by the ratio of the $d E / d x$ of a minimum ionizing particle in the material and in silicon. This is the so-called D-factor in Table 3. Apart from the total energy deposited, the dose from the electromagnetic cascades initiated by photons, electrons and $\pi^{0}$ 's was scored separately. This is called the electromagnetic dose in the following tables and graphs.

Annual doses were calculated on the assumption of an integrated luminosity of $10^{40} \mathrm{~cm}^{-2}$.

\subsection{Results}

The numerical values of the annual dose rates in the spherical shells are given as a function of angle and depth in Tables A1 to A4 in the Appendix. For each angular interval, the maximum value at any depth has been taken and plotted against angle (see Figures 1a-1d). The complete set of values from the tables in the Appendix are shown in Figures 2a-2d for the total dose values and Figures $3 \mathrm{a}-3 \mathrm{~d}$ for the electromagnetic component of the dose.

The peak in the total dose as a function of depth due to the rapid development of the electromagnetic cascade in the heavier materials is evident (see Figures 2c, 2d and 3c, 3d). The electromagnetic component accounts for about half of the dose at smaller angles. This is not surprising given that the energy incident on the spheres at these angles is approximately equal for the electromagnetic and hadronic components, as can be seen from the data of Table 1. At large angles, even though the incident energies are still of the same order of magnitude, the total dose is approximately one order of magnitude higher than the electromagnetic dose. An explanation can be found if one looks at the energy spectrum of the incident particles in the widest angular bins. Such spectra are plotted for pions in Figure 4. It will be seen that the majority of charged pions have energies close to $100 \mathrm{MeV}$ and so will stop in the first depth bins, thus augmenting the fraction of the total energy being deposited by hadrons at these depths.

\section{Conclusions}

The data shown in Figures 1a-1d confirm that in the geometrical conditions chosen for the calculation, i.e. a spherical detector of inner radius $2 \mathrm{~m}$, the dose rates at angles greater than $40^{\circ}$. is sufficient to cause the failure of some solidstate electronic components in less than one year's operation. Certain integrated circuit devices and other solid state electronic devices fail at doses of the order of 100 gray [8].

Scaling of these results to other radial distances can be effected by assuming 
an inverse-square law. Although this is only strictly true for the dose rates in the innermost shells of Figures 2, the inverse square law can be assumed to be valid for the maximum values plotted in Figure 1. This will allow a provisional estimate to be made of the dose to calorimeters and other tracking detectors at distances closer than $2 \mathrm{~m}$ to the interaction point.

It should also be remembered that damage to semiconductor devices depends strongly on the proportion of evaporation energy neutrons in the cascade spectrum. This proportion will be enhanced in a uranium-silicon calorimeter over the situation simulated in this paper since the FLUKA code does not correctly take into account the transport of neutrons below an energy of $50 \mathrm{MeV}$, nor are the fission cross-sections of uranium embedded in the code. The energy of particles below $50 \mathrm{MeV}$ is distributed in an algorithm which approximates to the correct spatial energy distribution without considering the detailed interactions. This is sufficient for most damage predictions around high-energy proton accelerators, but is likely to underestimate the damage to solid-state detectors if the damage is based solely on the dose values given in this paper.

\section{References}

1. G. R. Stevenson, "Dose Due to p-p Collisions in the LHC intersection regions," CERN Divisional Report TIS-RP/188/CF (1987).

2. J. Ranft, private communication (1986).

3. P. Aurenche, F. W. Bopp and J. Ranft, Z. Phys. C23, 67 (1984).

4. H. U. Bengtsson, G. Ingelman and T. Sjöstrand, Pythia version 4.1, "The Lund Monte Carlo for QCD High- $p_{\perp}$ scattering," page 104 of "The LUND Monte Carlo Programs," CERN Long Writeup of Pool Programs W5035, W5045, W5046, W5047 (1986).

5. P. A. Aarnio, A. Fassò, H. J. Möhring, J. Ranft and G. R. Stevenson, "FLUKA86 User's Guide," CERN Divisional Report TIS-RP/168 (1986).

6. J. Ranft, Appendix 3 of this Report.

7. W. R. Nelson, H. Hirayama and D. W. O. Rogers, "The EGS4 Code System," Stanford Linear Accelerator Center Report SLAC-265 (1985).

8. F. Wulf, D. Brauing and W. Gäbler, "Data Compilation of Irradiation-Tested Electronic Components," Vol. 1, 2nd edition, June 1981; Vol. 2, 2nd edition, Jan 1983; Vol. 3, 2nd edition, May 1984; Hahn-Meitner Institute for Nuclear Research (Berlin), Report TN 53/08 (1981). 


\section{APPENDIX}

Tables of dose in silicon in spheres of different materials are given in Tables A1 through A4; these data are plotted in various ways in the figures. An integrated luminosity of $10^{40} \mathrm{~cm}^{-2}$ is assumed.

\section{Table A1}

DTUJET $20+20 \mathrm{TeV}$ source in a spherical aluminium calorimeter. Dose in grays per year to silicon as a function of depth.

A1(a): Total energy density (dose) in Gy/yr

\begin{tabular}{rccccccc}
\hline \hline & & \multicolumn{3}{c}{ Angle } & & \\
Depth (m) & $0^{\circ}-1^{\circ}$ & $1^{\circ}-5^{\circ}$ & $5^{\circ}-10^{\circ}$ & $10^{\circ}-20^{\circ}$ & $20^{\circ}-40^{\circ}$ & $40^{\circ}-70^{\circ}$ & $70^{\circ}-90^{\circ}$ \\
\hline $2.00-2.05$ & $5.63 \times 10^{5}$ & $3.67 \times 10^{4}$ & $4.09 \times 10^{3}$ & $7.16 \times 10^{2}$ & $1.56 \times 10^{2}$ & $5.47 \times 10^{1}$ & $1.62 \times 10^{2}$ \\
$2.05-2.10$ & $1.35 \times 10^{6}$ & $2.83 \times 10^{4}$ & $4.84 \times 10^{3}$ & $1.13 \times 10^{3}$ & $1.43 \times 10^{2}$ & $5.48 \times 10^{1}$ & $9.74 \times 10^{1}$ \\
$2.10-2.15$ & $2.47 \times 10^{6}$ & $4.62 \times 10^{4}$ & $1.11 \times 10^{4}$ & $1.44 \times 10^{3}$ & $2.23 \times 10^{2}$ & $4.91 \times 10^{1}$ & $1.11 \times 10^{2}$ \\
$2.15-2.20$ & $5.56 \times 10^{6}$ & $1.03 \times 10^{5}$ & $7.91 \times 10^{3}$ & $1.72 \times 10^{3}$ & $1.89 \times 10^{2}$ & $3.79 \times 10^{1}$ & $5.14 \times 10^{1}$ \\
$2.20-2.30$ & $1.25 \times 10^{7}$ & $1.20 \times 10^{5}$ & $1.98 \times 10^{4}$ & $3.70 \times 10^{3}$ & $1.93 \times 10^{2}$ & $3.23 \times 10^{1}$ & $4.47 \times 10^{1}$ \\
$2.30-2.40$ & $1.75 \times 10^{7}$ & $2.40 \times 10^{5}$ & $1.24 \times 10^{4}$ & $3.21 \times 10^{3}$ & $2.76 \times 10^{2}$ & $1.98 \times 10^{1}$ & $2.65 \times 10^{1}$ \\
$2.40-2.50$ & $3.24 \times 10^{7} 3.47 \times 10^{5} 3.00 \times 10^{4}$ & $1.94 \times 10^{3}$ & $2.67 \times 10^{2}$ & $4.13 \times 10^{1}$ & $1.23 \times 10^{1}$ \\
$2.50-2.75$ & $6.37 \times 10^{7}$ & $4.36 \times 10^{5}$ & $3.31 \times 10^{4}$ & $4.42 \times 10^{3}$ & $1.22 \times 10^{2}$ & $7.32 \times 10^{\circ}$ & $7.37 \times 10^{0}$ \\
$2.75-3.00$ & $6.42 \times 10^{7}$ & $5.05 \times 10^{5}$ & $4.75 \times 10^{4}$ & $3.64 \times 10^{3}$ & $1.15 \times 10^{2}$ & $3.79 \times 10^{0}$ & $2.07 \times 10^{0}$ \\
$3.00-3.50$ & $4.61 \times 10^{7} 5.24 \times 10^{5} 3.93 \times 10^{4}$ & $3.50 \times 10^{3}$ & $1.00 \times 10^{2}$ & $2.57 \times 10^{0}$ & $5.00 \times 10^{-1}$ \\
$3.50-4.00$ & $2.04 \times 10^{7}$ & $4.11 \times 10^{5}$ & $3.00 \times 10^{4}$ & $1.74 \times 10^{3}$ & $5.84 \times 10^{1}$ & $3.51 \times 10^{-1}$ & $6.16 \times 10^{-2}$ \\
$4.00-4.50$ & $1.13 \times 10^{7}$ & $2.24 \times 10^{5}$ & $1.39 \times 10^{4}$ & $1.28 \times 10^{3}$ & $6.15 \times 10^{1}$ & $9.60 \times 10^{-1}$ & $8.40 \times 10^{-3}$ \\
$4.50-5.00$ & $4.96 \times 10^{6} 1.33 \times 10^{5}$ & $7.29 \times 10^{3} 5.98 \times 10^{2}$ & $7.05 \times 10^{0}$ & $6.34 \times 10^{-3} 9.67 \times 10^{-3}$ \\
\hline \hline
\end{tabular}

A1(b): Electromagnetic energy density (dose) in Gy/yr

\begin{tabular}{|c|c|c|c|c|c|c|c|}
\hline \multirow[b]{2}{*}{ Depth $(\mathrm{m})$} & \multicolumn{7}{|c|}{ Angle } \\
\hline & $0^{\circ}-1^{\circ}$ & $1^{\circ}-5^{\circ}$ & $5^{\circ}-10^{\circ}$ & $10^{\circ}-20^{\circ}$ & $20^{\circ}-40^{\circ}$ & $40^{\circ}-70^{\circ}$ & $70^{\circ}-90^{\circ}$ \\
\hline $2.00-2.05$ & $1.08 \times 10^{5}$ & $9.17 \times 10^{3}$ & $6.73 \times 10^{2}$ & $1.02 \times 10^{2}$ & $3.74 \times 10^{1}$ & $1.27 \times 10^{1}$ & $1.33 \times 10^{1}$ \\
\hline $2.05-2.10$ & $5.09 \times 10^{5}$ & $1.07 \times 1$ & $1.38 \times 10^{3}$ & $2.68 \times 10^{2}$ & $5.38 \times 10^{1}$ & $2.00 \times 10^{1}$ & $1.11 \times 10^{1}$ \\
\hline $2.10-2.15$ & $1.47 \times 10^{6}$ & $1.88 \times$ & $5.14 \times 10^{3}$ & $4.24 \times 10^{2}$ & $6.46 \times 10^{1}$ & $1.85 \times 10^{1}$ & $1.68 \times 10^{1}$ \\
\hline $2.15-2.20$ & $4.42 \times 10^{6}$ & $5.47 \times 10^{4}$ & $3.60 \times 10^{3}$ & $3.66 \times 10^{2}$ & $5.59 \times 10^{1}$ & $1.79 \times 10^{1}$ & $9.64 \times 10^{0}$ \\
\hline $2.20-2.30$ & $1.01 \times 10^{7}$ & $6.25 \times 10^{4}$ & $4.61 \times 10^{3}$ & $5.10 \times 10^{2}$ & $6.37 \times 10^{1}$ & $1.56 \times 10^{1}$ & $7.65 \times 10^{0}$ \\
\hline $2.30-2.40$ & $1.38 \times 10^{7}$ & $1.11 \times 10^{5}$ & $5.85 \times 10^{3}$ & $8.35 \times 10^{2}$ & $5.40 \times 10^{1}$ & $1.13 \times 10^{1}$ & $6.47 \times 10^{0}$ \\
\hline $2.40-2.50$ & $2.92 \times 10^{7}$ & $1.61 \times 10^{5}$ & $7.09 \times 10^{3}$ & $6.16 \times 10^{2}$ & $3.62 \times 10^{1}$ & $9.60 \times 10^{0}$ & $4.38 \times 10^{0}$ \\
\hline $2.50-2.75$ & $6.05 \times 10^{7}$ & $2.43 \times 10^{5}$ & $1.21 \times 10^{4}$ & $7.46 \times 10^{2}$ & $2.67 \times 10^{1}$ & $3.65 \times 10^{0}$ & $2.07 \times 10^{0}$ \\
\hline $2.75-3.00$ & $6.13 \times 10^{7}$ & $3.06 \times 10^{5}$ & $1.77 \times 10^{4}$ & $6.35 \times 10^{2}$ & $1.05 \times 10^{1}$ & $1.50 \times 10^{0}$ & $7.34 \times 10^{-1}$ \\
\hline $3.00-3.50$ & $4.30 \times 10^{7}$ & $2.94 \times 10^{5}$ & $1.27 \times 10^{4}$ & $9.07 \times 10^{2}$ & $3.59 \times 10^{0}$ & $2.63 \times 10^{-1}$ & $1.41 \times 10^{-1}$ \\
\hline $3.50-4.00$ & $1.82 \times 10^{7}$ & $2.43 \times 10^{5}$ & $1.20 \times 10^{4}$ & $2.08 \times 10^{2}$ & $1.84 \times 10^{0}$ & $7.17 \times 10^{-}$ & $2.02 \times 10^{-2}$ \\
\hline $4.00-4.50$ & $1.00 \times 10^{7}$ & $1.16 \times 10^{5}$ & $4.60 \times 10^{3}$ & $1.37 \times 10^{2}$ & $4.47 \times 10^{0}$ & $4.24 \times 10^{-3}$ & $1.85 \times 10^{-5}$ \\
\hline $4.50-5.00$ & $4.26 \times 10^{6}$ & $7.87 \times 10^{4}$ & $2.70 \times 10^{3}$ & $3.69 \times 10^{1}$ & $6.77 \times 10^{-2}$ & $0.00 \times 10^{0}$ & $0.00 \times 10^{0}$ \\
\hline
\end{tabular}


Table A2

DTUJET $20+20 \mathrm{TeV}$ source in a spherical iron calorimeter. Dose in grays per year to silicon as a function of depth.

A2(a): Total energy density (dose) in Gy/yr

\begin{tabular}{rccccccc}
\hline \hline & & Angle & & \\
Depth $(\mathrm{m})$ & $0^{\circ}-1^{\circ}$ & $1^{\circ}-5^{\circ}$ & $5^{\circ}-10^{\circ}$ & $10^{\circ}-20^{\circ}$ & $20^{\circ}-40^{\circ}$ & $40^{\circ}-70^{\circ}$ & $70^{\circ}-90^{\circ}$ \\
\hline $2.00-2.02$ & $1.26 \times 10^{6}$ & $3.70 \times 10^{4}$ & $6.69 \times 10^{3}$ & $6.95 \times 10^{2}$ & $1.43 \times 10^{2}$ & $9.94 \times 10^{1}$ & $1.50 \times 10^{2}$ \\
$2.02-2.04$ & $5.02 \times 10^{6}$ & $8.16 \times 10^{4}$ & $1.20 \times 10^{4}$ & $1.24 \times 10^{3}$ & $1.65 \times 10^{2}$ & $8.29 \times 10^{1}$ & $9.11 \times 10^{1}$ \\
$2.04-2.06$ & $1.19 \times 10^{7}$ & $1.15 \times 10^{5}$ & $1.08 \times 10^{4}$ & $1.27 \times 10^{3}$ & $1.95 \times 10^{2}$ & $7.67 \times 10^{1}$ & $6.27 \times 10^{1}$ \\
$2.06-2.08$ & $2.68 \times 10^{7}$ & $1.97 \times 10^{5}$ & $1.27 \times 10^{4}$ & $1.61 \times 10^{3}$ & $1.89 \times 10^{2}$ & $6.18 \times 10^{1}$ & $9.04 \times 10^{1}$ \\
$2.08-2.10$ & $5.10 \times 10^{7}$ & $2.25 \times 10^{5}$ & $1.41 \times 10^{4}$ & $3.42 \times 10^{3}$ & $1.52 \times 10^{2}$ & $4.49 \times 10^{1}$ & $5.87 \times 10^{1}$ \\
$2.10-2.20$ & $1.45 \times 10^{8} 3.00 \times 10^{5}$ & $1.16 \times 10^{4}$ & $1.82 \times 10^{3}$ & $8.59 \times 10^{1}$ & $2.42 \times 10^{1}$ & $4.37 \times 10^{1}$ \\
$2.20-2.40$ & $1.29 \times 10^{8}$ & $4.67 \times 10^{5}$ & $1.65 \times 10^{4}$ & $1.04 \times 10^{3}$ & $3.43 \times 10^{1}$ & $4.77 \times 10^{\circ}$ & $2.03 \times 10^{1}$ \\
$2.40-2.60$ & $4.92 \times 10^{7} 5.57 \times 10^{5}$ & $1.79 \times 10^{4}$ & $1.26 \times 10^{3}$ & $1.31 \times 10^{1}$ & $1.00 \times 10^{\circ}$ & $3.78 \times 10^{0}$ \\
$2.60-2.80$ & $3.19 \times 10^{7}$ & $4.80 \times 10^{5}$ & $1.92 \times 10^{4}$ & $6.53 \times 10^{2}$ & $4.62 \times 10^{0}$ & $2.99 \times 10^{-1}$ & $8.85 \times 10^{0}$ \\
$2.80-3.00$ & $2.02 \times 10^{7}$ & $3.39 \times 10^{5}$ & $1.22 \times 10^{4}$ & $4.70 \times 10^{2}$ & $3.31 \times 10^{0}$ & $6.51 \times 10^{-2}$ & $2.97 \times 10^{-2}$ \\
$3.00-3.20$ & $9.91 \times 10^{6}$ & $2.11 \times 10^{5}$ & $9.75 \times 10^{3}$ & $4.34 \times 10^{2}$ & $1.24 \times 10^{\circ}$ & $1.11 \times 10^{-3}$ & $1.15 \times 10^{-3}$ \\
$3.20-3.60$ & $4.86 \times 10^{6}$ & $1.06 \times 10^{5}$ & $5.02 \times 10^{3}$ & $1.28 \times 10^{2}$ & $6.06 \times 10^{-1}$ & $0.00 \times 10^{0}$ & $0.00 \times 10^{0}$ \\
$3.60-4.00$ & $1.31 \times 10^{6} 3.87 \times 10^{4}$ & $1.75 \times 10^{3}$ & $7.18 \times 10^{1}$ & $1.28 \times 10^{-1}$ & $9.08 \times 10^{-3}$ & $0.00 \times 10^{\circ}$ \\
\hline \hline
\end{tabular}

A2(b): Electromagnetic energy density (dose) in Gy/yr

\begin{tabular}{cccccccc}
\hline & & Angle & & \\
Depth $(\mathrm{m})$ & $0^{\circ}-1^{\circ}$ & $1^{\circ}-5^{\circ}$ & $5^{\circ}-10^{\circ}$ & $10^{\circ}-20^{\circ}$ & $20^{\circ}-40^{\circ}$ & $40^{\circ}-70^{\circ}$ & $70^{\circ}-90^{\circ}$ \\
\hline $2.00-2.02$ & $5.55 \times 10^{5}$ & $1.02 \times 10^{4}$ & $1.13 \times 10^{3}$ & $2.02 \times 10^{2}$ & $3.83 \times 10^{1}$ & $1.85 \times 10^{1}$ & $1.20 \times 10^{1}$ \\
$2.02-2.04$ & $3.62 \times 10^{6}$ & $3.66 \times 10^{4}$ & $3.41 \times 10^{3}$ & $5.07 \times 10^{2}$ & $9.02 \times 10^{1}$ & $3.11 \times 10^{1}$ & $1.85 \times 10^{1}$ \\
$2.04-2.06$ & $1.03 \times 10^{7}$ & $6.47 \times 10^{4}$ & $6.53 \times 10^{3}$ & $6.99 \times 10^{2}$ & $1.15 \times 10^{2}$ & $2.86 \times 10^{1}$ & $2.01 \times 10^{1}$ \\
$2.06-2.08$ & $2.44 \times 10^{7}$ & $1.14 \times 10^{5}$ & $6.99 \times 10^{3}$ & $6.87 \times 10^{2}$ & $1.00 \times 10^{2}$ & $2.81 \times 10^{1}$ & $1.63 \times 10^{1}$ \\
$2.08-2.10$ & $4.60 \times 10^{7}$ & $1.48 \times 10^{5}$ & $7.26 \times 10^{3}$ & $6.22 \times 10^{2}$ & $9.36 \times 10^{1}$ & $2.20 \times 10^{1}$ & $1.26 \times 10^{1}$ \\
$2.10-2.20$ & $1.39 \times 10^{8}$ & $1.71 \times 10^{5} 3.84 \times 10^{3}$ & $4.38 \times 10^{2}$ & $4.08 \times 10^{1}$ & $1.18 \times 10^{1}$ & $6.23 \times 10^{0}$ \\
$2.20-2.40$ & $1.20 \times 10^{8}$ & $2.25 \times 10^{5}$ & $1.36 \times 10^{3}$ & $7.73 \times 10^{1}$ & $8.51 \times 10^{0}$ & $6.26 \times 10^{-1}$ & $9.21 \times 10^{-1}$ \\
$2.40-2.60$ & $4.17 \times 10^{7}$ & $2.46 \times 10^{5}$ & $1.68 \times 10^{3}$ & $2.69 \times 10^{2}$ & $2.30 \times 10^{0}$ & $7.15 \times 10^{-2}$ & $5.50 \times 10^{-2}$ \\
$2.60-2.80$ & $2.58 \times 10^{7}$ & $1.74 \times 10^{5}$ & $7.27 \times 10^{2}$ & $9.69 \times 10^{0}$ & $6.75 \times 10^{-1}$ & $6.38 \times 10^{-2}$ & $0.00 \times 10^{0}$ \\
$2.80-3.00$ & $1.58 \times 10^{7}$ & $9.33 \times 10^{4}$ & $4.37 \times 10^{2}$ & $2.39 \times 10^{0}$ & $1.01 \times 10^{-1}$ & $0.00 \times 10^{0}$ & $3.29 \times 10^{-3}$ \\
$3.00-3.20$ & $7.03 \times 10^{6}$ & $7.11 \times 10^{4}$ & $2.42 \times 10^{2}$ & $1.13 \times 10^{0}$ & $1.05 \times 10^{-2}$ & $0.00 \times 10^{0}$ & $0.00 \times 10^{0}$ \\
$3.20-3.60$ & $3.33 \times 10^{6} 3.58 \times 10^{4} 4.58 \times 10^{2}$ & $7.40 \times 10^{-2}$ & $0.00 \times 10^{0}$ & $0.00 \times 10^{0}$ & $0.00 \times 10^{0}$ \\
$3.60-4.00$ & $7.22 \times 10^{5} 1.11 \times 10^{4}$ & $2.82 \times 10^{1}$ & $6.96 \times 10^{-1}$ & $0.00 \times 10^{\circ}$ & $0.00 \times 10^{0}$ & $0.00 \times 10^{0}$ \\
\hline \hline
\end{tabular}


Table A3

DTUJET $20+20 \mathrm{TeV}$ source in a spherical lead calorimeter. Dose in grays per year to silicon as a function of depth.

A3(a): Total energy density (dose) in Gy/yr

\begin{tabular}{cccccccc}
\hline & & Angle & & \\
Depth $(\mathrm{m})$ & $0^{\circ}-1^{\circ}$ & $1^{\circ}-5^{\circ}$ & $5^{\circ}-10^{\circ}$ & $10^{\circ}-20^{\circ}$ & $20^{\circ}-40^{\circ}$ & $40^{\circ}-70^{\circ}$ & $70^{\circ}-90^{\circ}$ \\
\hline $2.00-2.02$ & $1.10 \times 10^{7}$ & $1.21 \times 10^{5}$ & $2.89 \times 10^{4}$ & $2.18 \times 10^{3}$ & $3.27 \times 10^{2}$ & $1.98 \times 10^{2}$ & $2.16 \times 10^{2}$ \\
$2.02-2.04$ & $1.20 \times 10^{8}$ & $3.90 \times 10^{5}$ & $2.42 \times 10^{4}$ & $4.26 \times 10^{3}$ & $5.12 \times 10^{2}$ & $7.52 \times 10^{2}$ & $1.85 \times 10^{2}$ \\
$2.04-2.06$ & $3.53 \times 10^{8}$ & $3.47 \times 10^{5}$ & $1.65 \times 10^{4}$ & $1.68 \times 10^{3}$ & $1.84 \times 10^{2}$ & $8.98 \times 10^{1}$ & $8.22 \times 10^{1}$ \\
$2.06-2.08$ & $3.49 \times 10^{8}$ & $1.98 \times 10^{5}$ & $1.51 \times 10^{4}$ & $1.39 \times 10^{3}$ & $1.22 \times 10^{2}$ & $4.42 \times 10^{1}$ & $4.73 \times 10^{1}$ \\
$2.08-2.10$ & $2.90 \times 10^{8}$ & $2.26 \times 10^{5}$ & $1.38 \times 10^{4}$ & $1.36 \times 10^{3}$ & $2.93 \times 10^{2}$ & $1.01 \times 10^{2}$ & $5.25 \times 10^{1}$ \\
$2.10-2.20$ & $1.14 \times 10^{8}$ & $2.70 \times 10^{5}$ & $1.73 \times 10^{4}$ & $1.44 \times 10^{3}$ & $6.03 \times 10^{1}$ & $7.07 \times 10^{1}$ & $6.72 \times 10^{1}$ \\
$2.20-2.40$ & $6.85 \times 10^{7}$ & $3.17 \times 10^{5}$ & $2.89 \times 10^{4}$ & $2.06 \times 10^{3}$ & $2.67 \times 10^{1}$ & $5.20 \times 10^{\circ}$ & $8.87 \times 10^{0}$ \\
$2.40-2.60$ & $4.36 \times 10^{7}$ & $3.59 \times 10^{5}$ & $2.31 \times 10^{4}$ & $1.52 \times 10^{3}$ & $4.46 \times 10^{1}$ & $1.12 \times 10^{\circ}$ & $1.66 \times 10^{0}$ \\
$2.60-2.80$ & $2.64 \times 10^{7} 3.11 \times 10^{5}$ & $1.82 \times 10^{4}$ & $1.15 \times 10^{3}$ & $1.74 \times 10^{1}$ & $1.74 \times 10^{-1}$ & $4.92 \times 10^{-1}$ \\
$2.80-3.00$ & $1.45 \times 10^{7}$ & $2.26 \times 10^{5}$ & $1.77 \times 10^{4}$ & $7.56 \times 10^{2}$ & $2.82 \times 10^{\circ}$ & $2.98 \times 10^{-2}$ & $3.93 \times 10^{-2}$ \\
$3.00-3.20$ & $6.42 \times 10^{6}$ & $1.36 \times 10^{5}$ & $8.08 \times 10^{3}$ & $5.83 \times 10^{2}$ & $4.16 \times 10^{0}$ & $2.00 \times 10^{-3}$ & $9.10 \times 10^{-4}$ \\
$3.20-3.60$ & $2.17 \times 10^{6}$ & $4.65 \times 10^{4}$ & $4.46 \times 10^{3}$ & $1.64 \times 10^{2}$ & $2.51 \times 10^{\circ}$ & $1.17 \times 10^{-3}$ & $9.24 \times 10^{-4}$ \\
$3.60-4.00$ & $7.30 \times 10^{5}$ & $1.70 \times 10^{4}$ & $1.04 \times 10^{3}$ & $3.43 \times 10^{1}$ & $9.85 \times 10^{-2}$ & $0.00 \times 10^{0}$ & $0.00 \times 10^{0}$ \\
\hline \hline
\end{tabular}

A3(b): Electromagnetic energy density (dose) in $\mathrm{Gy} / \mathrm{yr}$

\begin{tabular}{cccccccc}
\hline Depth $(\mathrm{m})$ & $0^{\circ}-1^{\circ}$ & $1^{\circ}-5^{\circ}$ & $5^{\circ}-10^{\circ}$ & $10^{\circ}-20^{\circ}$ & $20^{\circ}-40^{\circ}$ & $40^{\circ}-70^{\circ}$ & $70^{\circ}-90^{\circ}$ \\
\hline $2.00-2.02$ & $1.00 \times 10^{7}$ & $7.51 \times 10^{4}$ & $6.88 \times 10^{3}$ & $1.02 \times 10^{3}$ & $1.84 \times 10^{2}$ & $5.35 \times 10^{1}$ & $4.01 \times 10^{1}$ \\
$2.02-2.04$ & $1.16 \times 10^{8}$ & $2.62 \times 10^{5}$ & $1.40 \times 10^{4}$ & $1.69 \times 10^{3}$ & $1.93 \times 10^{2}$ & $6.38 \times 10^{1}$ & $3.08 \times 10^{1}$ \\
$2.04-2.06$ & $3.49 \times 10^{8}$ & $2.66 \times 10^{5}$ & $1.06 \times 10^{4}$ & $6.54 \times 10^{2}$ & $7.69 \times 10^{1}$ & $2.81 \times 10^{1}$ & $1.85 \times 10^{1}$ \\
$2.06-2.08$ & $3.46 \times 10^{8}$ & $1.04 \times 10^{5}$ & $4.22 \times 10^{3}$ & $5.06 \times 10^{2}$ & $4.24 \times 10^{1}$ & $6.14 \times 10^{0}$ & $3.39 \times 10^{0}$ \\
$2.08-2.10$ & $2.86 \times 10^{8}$ & $1.03 \times 10^{5}$ & $2.39 \times 10^{3}$ & $1.97 \times 10^{2}$ & $1.21 \times 10^{1}$ & $5.00 \times 10^{0}$ & $1.09 \times 10^{0}$ \\
$2.10-2.20$ & $1.09 \times 10^{8} 7.39 \times 10^{4} 9.58 \times 10^{2}$ & $7.68 \times 10^{1}$ & $9.57 \times 10^{\circ}$ & $2.15 \times 10^{0}$ & $1.55 \times 10^{0}$ \\
$2.20-2.40$ & $5.88 \times 10^{7} 5.15 \times 10^{4}$ & $4.31 \times 10^{2}$ & $3.56 \times 10^{1}$ & $4.79 \times 10^{0}$ & $7.18 \times 10^{-1}$ & $2.39 \times 10^{-1}$ \\
$2.40-2.60$ & $3.37 \times 10^{7} 5.00 \times 10^{4}$ & $2.24 \times 10^{2}$ & $7.83 \times 10^{0}$ & $2.84 \times 10^{-1}$ & $1.48 \times 10^{-1}$ & $3.92 \times 10^{-3}$ \\
$2.60-2.80$ & $1.90 \times 10^{7} 4.32 \times 10^{4} 8.45 \times 10^{1}$ & $5.27 \times 10^{0}$ & $2.18 \times 10^{-1}$ & $0.00 \times 10^{0}$ & $0.00 \times 10^{0}$ \\
$2.80-3.00$ & $9.21 \times 10^{6} 2.84 \times 10^{4}$ & $5.13 \times 10^{1}$ & $5.19 \times 10^{0}$ & $2.74 \times 10^{-2}$ & $0.00 \times 10^{0}$ & $0.00 \times 10^{0}$ \\
$3.00-3.20$ & $3.74 \times 10^{6} 1.65 \times 10^{4}$ & $1.12 \times 10^{2}$ & $2.63 \times 10^{-1}$ & $2.43 \times 10^{-2}$ & $0.60 \times 10^{0}$ & $0.00 \times 10^{0}$ \\
$3.20-3.60$ & $1.14 \times 10^{6}$ & $4.13 \times 10^{3}$ & $2.36 \times 10^{1}$ & $8.41 \times 10^{-2}$ & $1.20 \times 10^{-2}$ & $0.00 \times 10^{0}$ & $0.00 \times 10^{0}$ \\
$3.60-4.00$ & $3.30 \times 10^{5} 1.61 \times 10^{3} 3.10 \times 10^{0}$ & $2.19 \times 10^{-1}$ & $4.46 \times 10^{-3}$ & $0.00 \times 10^{0}$ & $0.00 \times 10^{0}$ \\
\hline \hline
\end{tabular}


Table A4

DTUJET $20+20 \mathrm{TeV}$ source in a spherical uranium calorimeter. Dose in grays per year to silicon as a function of depth.

A4(a): Total energy density (dose) in Gy/yr

\begin{tabular}{cccccccc}
\hline \hline & & \multicolumn{5}{c}{ Angle } & \\
Depth $(\mathrm{m})$ & $0^{\circ}-1^{\circ}$ & $1^{\circ}-5^{\circ}$ & $5^{\circ}-10^{\circ}$ & $10^{\circ}-20^{\circ}$ & $20^{\circ}-40^{\circ}$ & $40^{\circ}-70^{\circ}$ & $70^{\circ}-90^{\circ}$ \\
\hline $2.00-2.01$ & $5.56 \times 10^{7}$ & $1.30 \times 10^{5}$ & $1.37 \times 10^{4}$ & $5.48 \times 10^{3}$ & $3.62 \times 10^{2}$ & $1.40 \times 10^{2}$ & $3.65 \times 10^{2}$ \\
$2.01-2.02$ & $8.81 \times 10^{7}$ & $3.44 \times 10^{5}$ & $2.29 \times 10^{4}$ & $2.52 \times 10^{3}$ & $4.15 \times 10^{2}$ & $1.98 \times 10^{2}$ & $3.53 \times 10^{2}$ \\
$2.02-2.03$ & $2.56 \times 10^{8}$ & $3.86 \times 10^{5}$ & $2.44 \times 10^{4}$ & $1.83 \times 10^{3}$ & $2.69 \times 10^{2}$ & $2.68 \times 10^{2}$ & $2.93 \times 10^{2}$ \\
$2.03-2.04$ & $4.32 \times 10^{8}$ & $3.14 \times 10^{5}$ & $1.61 \times 10^{4}$ & $1.74 \times 10^{3}$ & $1.45 \times 10^{2}$ & $1.80 \times 10^{2}$ & $3.28 \times 10^{2}$ \\
$2.04-2.05$ & $3.97 \times 10^{8}$ & $2.56 \times 10^{5}$ & $8.97 \times 10^{3}$ & $1.39 \times 10^{3}$ & $1.03 \times 10^{2}$ & $6.12 \times 10^{1}$ & $4.90 \times 10^{2}$ \\
$2.05-2.10$ & $1.89 \times 10^{8}$ & $2.34 \times 10^{5}$ & $1.80 \times 10^{4}$ & $7.94 \times 10^{2}$ & $6.69 \times 10^{1}$ & $5.95 \times 10^{1}$ & $1.22 \times 10^{2}$ \\
$2.10-2.20$ & $8.91 \times 10^{7} 3.36 \times 10^{5}$ & $1.35 \times 10^{4}$ & $9.12 \times 10^{2}$ & $2.95 \times 10^{1}$ & $1.03 \times 10^{1}$ & $9.30 \times 10^{1}$ \\
$2.20-2.30$ & $5.52 \times 10^{7} 3.74 \times 10^{5}$ & $1.38 \times 10^{4}$ & $5.68 \times 10^{2}$ & $2.55 \times 10^{1}$ & $2.74 \times 10^{0}$ & $3.20 \times 10^{0}$ \\
$2.30-2.40$ & $4.41 \times 10^{7} 4.36 \times 10^{5}$ & $1.63 \times 10^{4}$ & $3.98 \times 10^{2}$ & $4.25 \times 10^{0}$ & $8.24 \times 10^{-1}$ & $4.35 \times 10^{0}$ \\
$2.40-2.50$ & $2.97 \times 10^{7} 3.57 \times 10^{5}$ & $1.13 \times 10^{4}$ & $3.84 \times 10^{2}$ & $9.32 \times 10^{-1}$ & $5.14 \times 10^{-1}$ & $7.87 \times 10^{-1}$ \\
$2.50-2.60$ & $2.02 \times 10^{7}$ & $2.54 \times 10^{5}$ & $1.19 \times 10^{4}$ & $3.30 \times 10^{2}$ & $9.67 \times 10^{-1}$ & $1.40 \times 10^{-1}$ & $1.94 \times 10^{-2}$ \\
$2.60-2.80$ & $9.88 \times 10^{6}$ & $1.64 \times 10^{5}$ & $7.74 \times 10^{3}$ & $4.55 \times 10^{2}$ & $1.85 \times 10^{-1}$ & $2.62 \times 10^{-2}$ & $9.57 \times 10^{-2}$ \\
$2.80-3.00$ & $3.91 \times 10^{6} 6.39 \times 10^{4}$ & $1.83 \times 10^{3}$ & $9.54 \times 10^{1}$ & $4.37 \times 10^{-1}$ & $1.52 \times 10^{-3}$ & $0.00 \times 10^{0}$ \\
\hline \hline
\end{tabular}

A4(b): Electromagnetic energy density (dose) in Gy/yr

\begin{tabular}{cccccccc}
\hline \hline & & Angle & & \\
Depth $(\mathrm{m})$ & $0^{\circ}-1^{\circ}$ & $1^{\circ}-5^{\circ}$ & $5^{\circ}-10^{\circ}$ & $10^{\circ}-20^{\circ}$ & $20^{\circ}-40^{\circ}$ & $40^{\circ}-70^{\circ}$ & $70^{\circ}-90^{\circ}$ \\
\hline $2.00-2.01$ & $5.41 \times 10^{7}$ & $6.62 \times 10^{4}$ & $7.30 \times 10^{3}$ & $1.14 \times 10^{3}$ & $1.78 \times 10^{2}$ & $5.78 \times 10^{1}$ & $3.94 \times 10^{1}$ \\
$2.01-2.02$ & $8.59 \times 10^{7}$ & $2.61 \times 10^{5}$ & $1.48 \times 10^{4}$ & $1.96 \times 10^{3}$ & $2.41 \times 10^{2}$ & $7.13 \times 10^{1}$ & $4.30 \times 10^{1}$ \\
$2.02-2.03$ & $2.53 \times 10^{8}$ & $2.97 \times 10^{5}$ & $1.50 \times 10^{4}$ & $1.27 \times 10^{3}$ & $1.38 \times 10^{2}$ & $4.92 \times 10^{1}$ & $2.67 \times 10^{1}$ \\
$2.03-2.04$ & $4.28 \times 10^{8}$ & $1.93 \times 10^{5}$ & $7.62 \times 10^{3}$ & $5.86 \times 10^{2}$ & $5.60 \times 10^{1}$ & $1.41 \times 10^{1}$ & $7.10 \times 10^{0}$ \\
$2.04-2.05$ & $3.92 \times 10^{8} 1.09 \times 10^{5}$ & $3.75 \times 10^{3}$ & $3.96 \times 10^{2}$ & $2.00 \times 10^{1}$ & $5.04 \times 10^{\circ}$ & $5.89 \times 10^{0}$ \\
$2.05-2.10$ & $1.80 \times 10^{8} 4.14 \times 10^{4}$ & $1.29 \times 10^{3}$ & $1.14 \times 10^{2}$ & $1.42 \times 10^{1}$ & $4.24 \times 10^{0}$ & $1.85 \times 10^{0}$ \\
$2.10-2.20$ & $7.47 \times 10^{7} 2.90 \times 10^{4}$ & $6.50 \times 10^{2}$ & $4.85 \times 10^{1}$ & $6.23 \times 10^{0}$ & $1.04 \times 10^{0}$ & $3.64 \times 10^{-1}$ \\
$2.20-2.30$ & $3.95 \times 10^{7} 2.44 \times 10^{4}$ & $1.43 \times 10^{2}$ & $1.80 \times 10^{1}$ & $8.48 \times 10^{-1}$ & $3.97 \times 10^{-1}$ & $6.76 \times 10^{-2}$ \\
$2.30-2.40$ & $3.02 \times 10^{7} 5.84 \times 10^{4}$ & $8.62 \times 10^{1}$ & $7.31 \times 10^{0}$ & $5.80 \times 10^{-1}$ & $3.94 \times 10^{-2}$ & $1.17 \times 10^{-2}$ \\
$2.40-2.50$ & $1.92 \times 10^{7} 3.35 \times 10^{4}$ & $5.48 \times 10^{1}$ & $6.37 \times 10^{0}$ & $8.78 \times 10^{-2}$ & $4.07 \times 10^{-2}$ & $2.73 \times 10^{-3}$ \\
$2.50-2.60$ & $1.20 \times 10^{7}$ & $2.53 \times 10^{4}$ & $5.48 \times 10^{1}$ & $1.03 \times 10^{0}$ & $2.09 \times 10^{-1}$ & $0.00 \times 10^{0}$ & $0.00 \times 10^{0}$ \\
$2.60-2.80$ & $4.92 \times 10^{6}$ & $1.39 \times 10^{4}$ & $9.48 \times 10^{0}$ & $7.11 \times 10^{-2}$ & $3.06 \times 10^{-3}$ & $1.29 \times 10^{-3}$ & $0.00 \times 10^{0}$ \\
$2.80-3.00$ & $2.11 \times 10^{6} 5.33 \times 10^{3}$ & $7.37 \times 10^{-1}$ & $0.00 \times 10^{\circ}$ & $7.21 \times 10^{-3}$ & $0.00 \times 10^{\circ}$ & $0.00 \times 10^{0}$ \\
\hline \hline
\end{tabular}



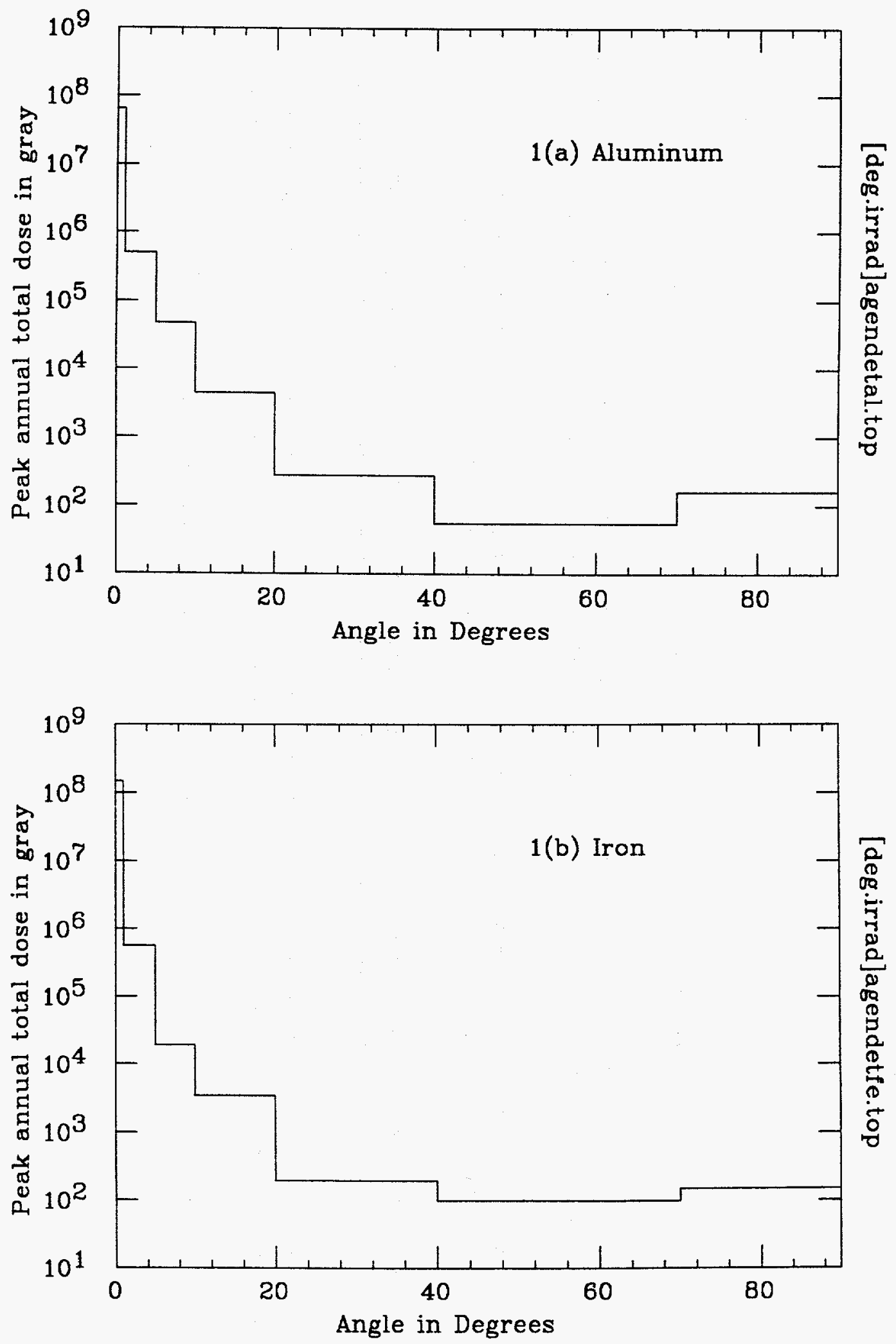

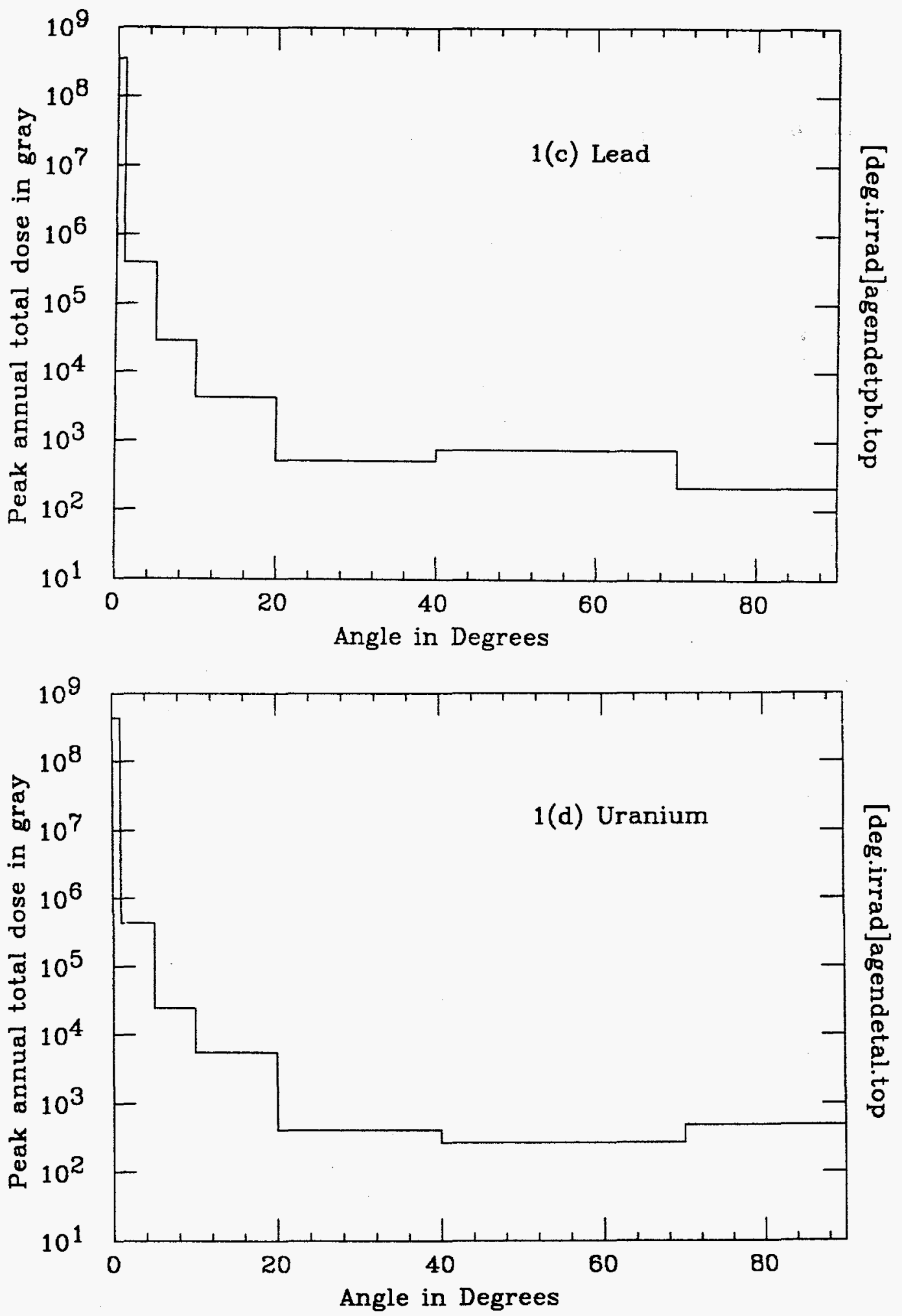

FIG. 1. Variation of the maximum dose rate with angle: (a) Aluminium shell (b) Iron shell (c) Lead shell (d) Uranium shell. Normalization is to an estimated annual integrated luminosity of $10^{40} \mathrm{~cm}^{-2}$ at $20+20 \mathrm{TeV}$. 

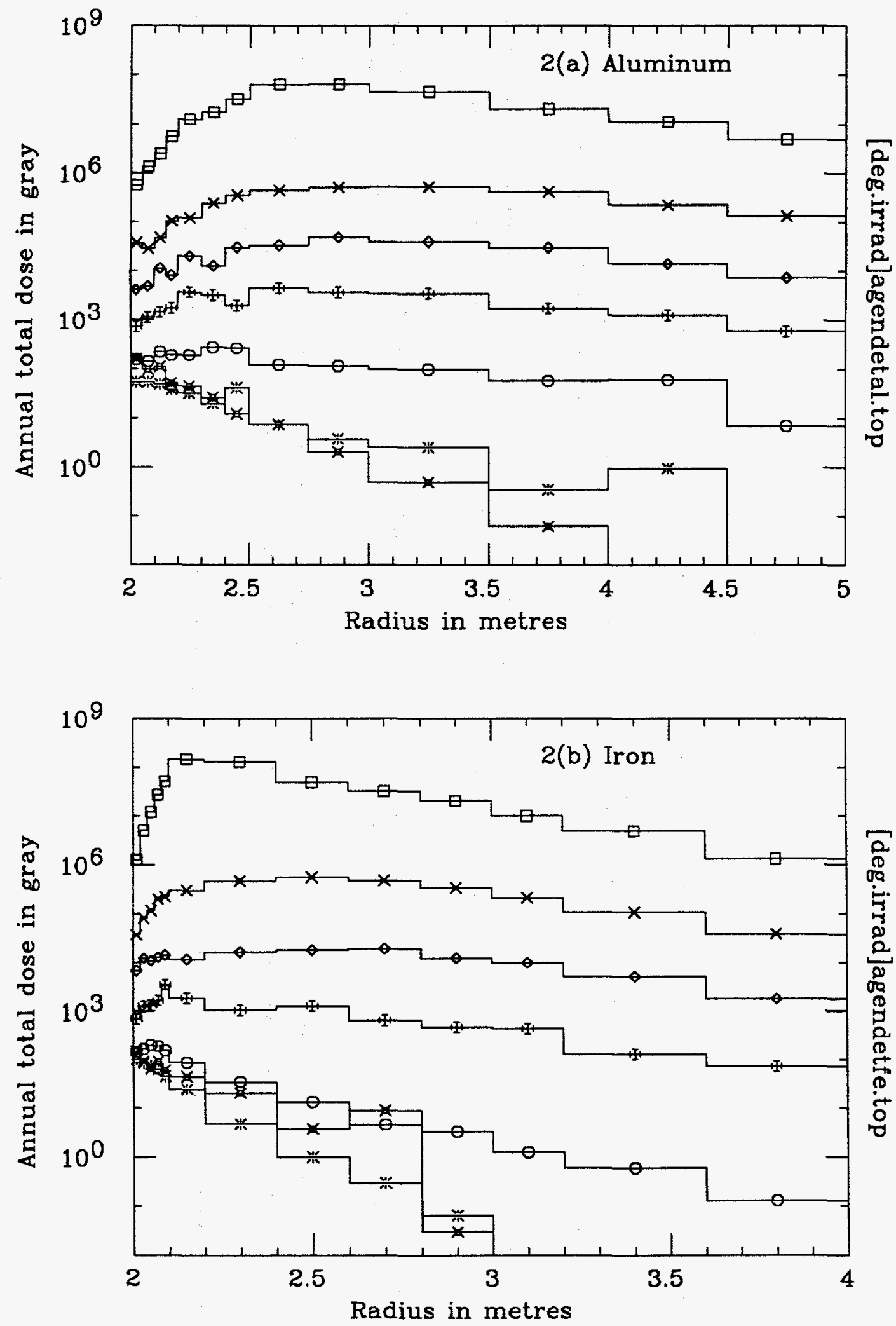


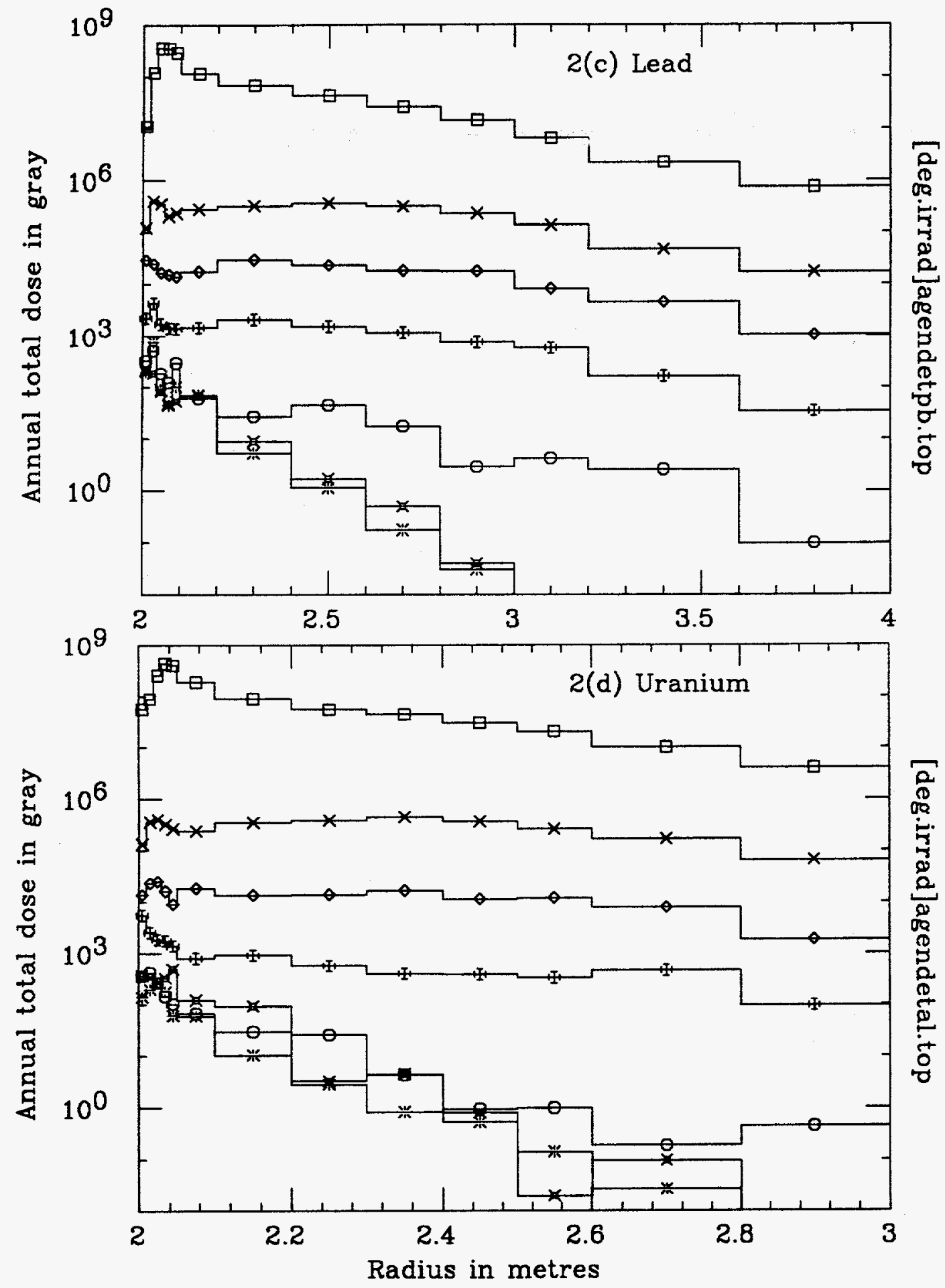

FIG. 2. Variation of the total annual dose rate with depth in the shells of different material for the angular intervals shown: (a) Aluminium shell (b) Iron shell (c) Lead shell (d) Uranium shell. The shells have an inner radius of $2 \mathrm{~m}$. Open squares: $0-1^{\circ}$, diagonal crosses: $1-5^{\circ}$, diamonds: $5-10^{\circ}$, vertical crosses with ears: $10-20^{\circ}$, circles: 20-40 , stars: $40-70^{\circ}$, squares with ears: $70-90^{\circ}$. 

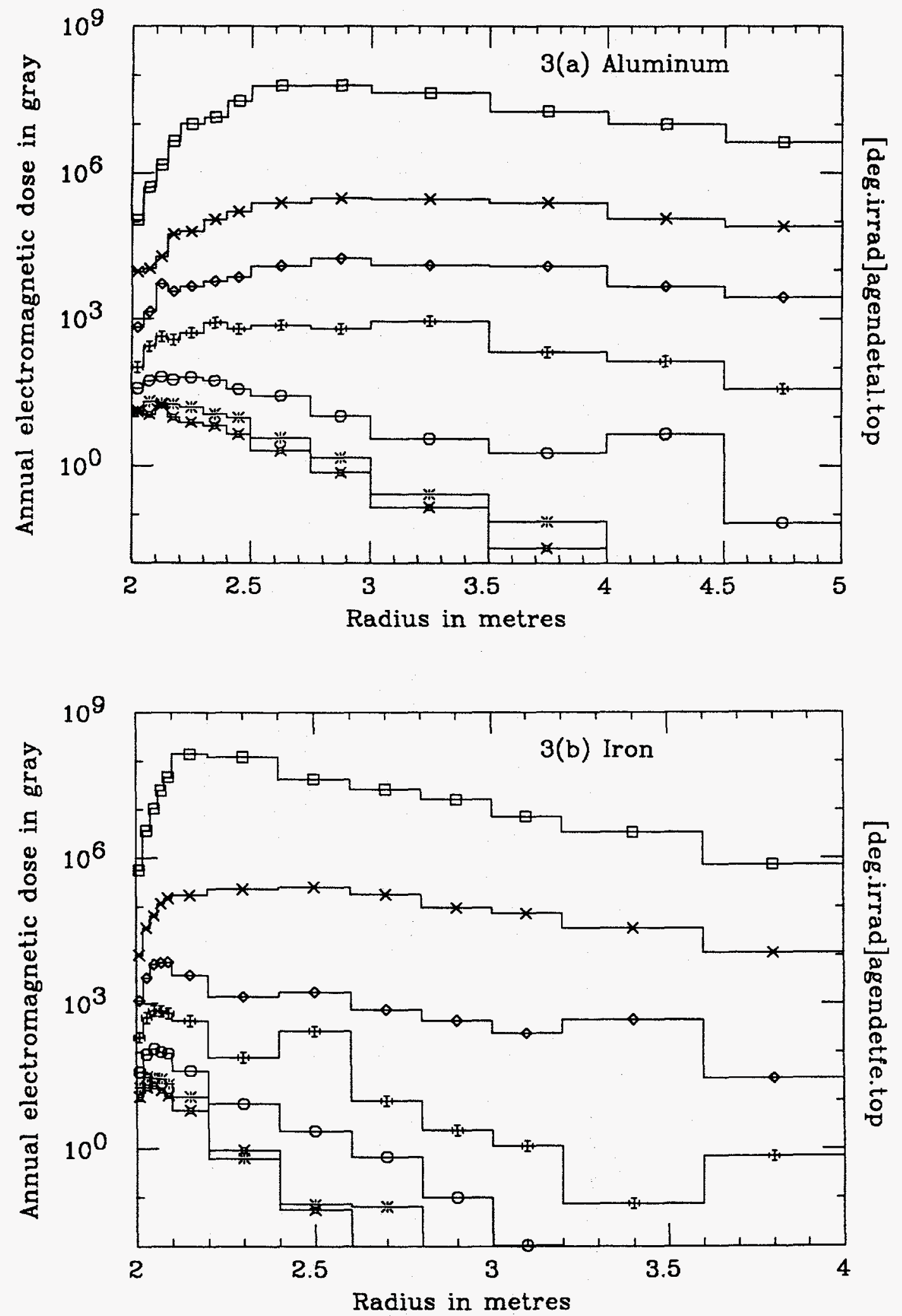


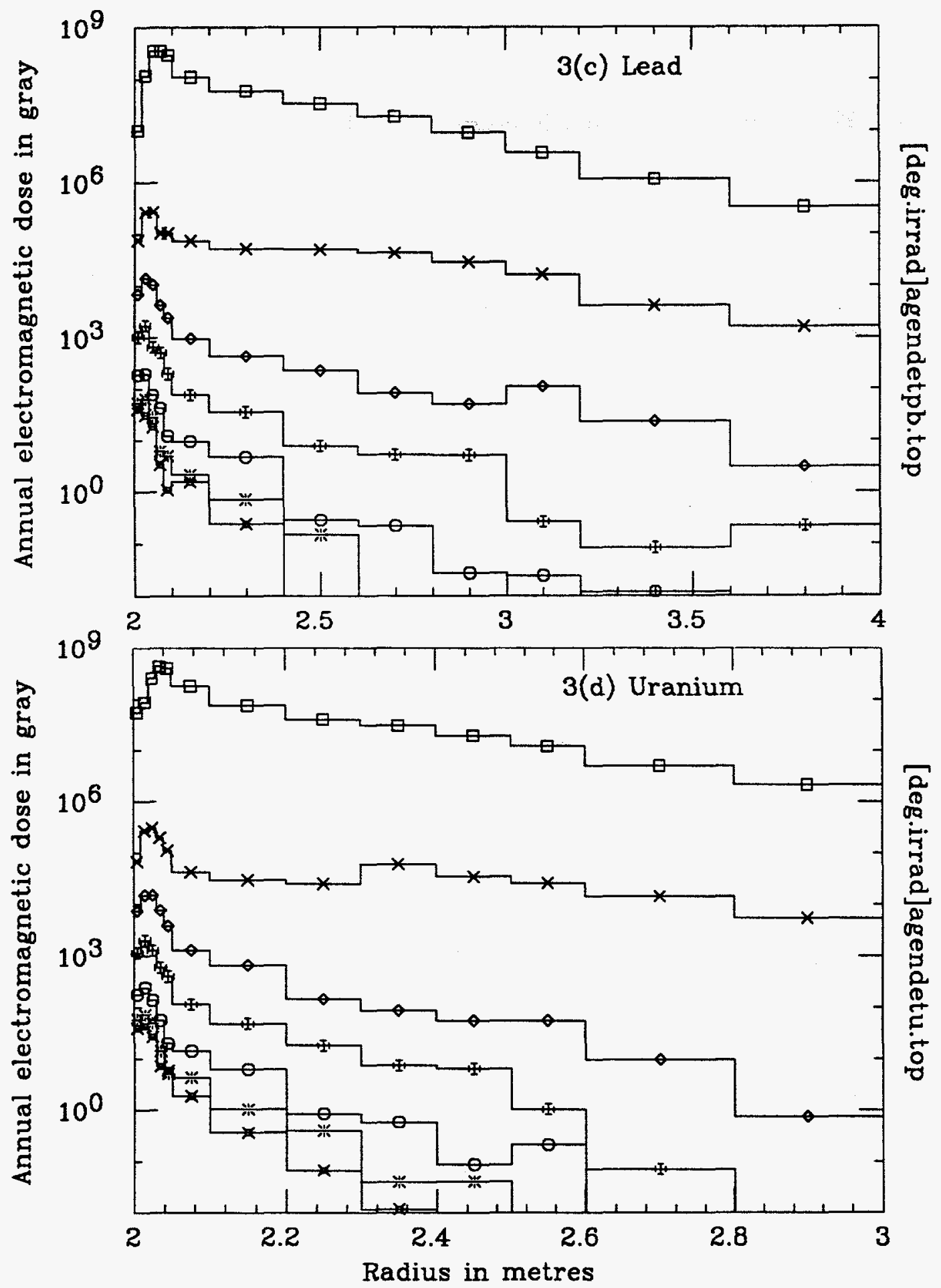

FIG. 3. Variation of the electromagnetic annual dose rate with depth in the shells of different material for the angular intervals shown: (a) Aluminium shell (b) Iron shell (c) Lead shell (d) Uranium shell. The shells have an inner radius of $2 \mathrm{~m}$. Open squares: 0 $1^{\circ}$, diagonal crosses: $1-5^{\circ}$, diamonds: $5-10^{\circ}$, vertical crosses with ears: $10-20^{\circ}$, circles: $20-40^{\circ}$, stars: $40-70^{\circ}$, squares with ears: $70-90^{\circ}$. 


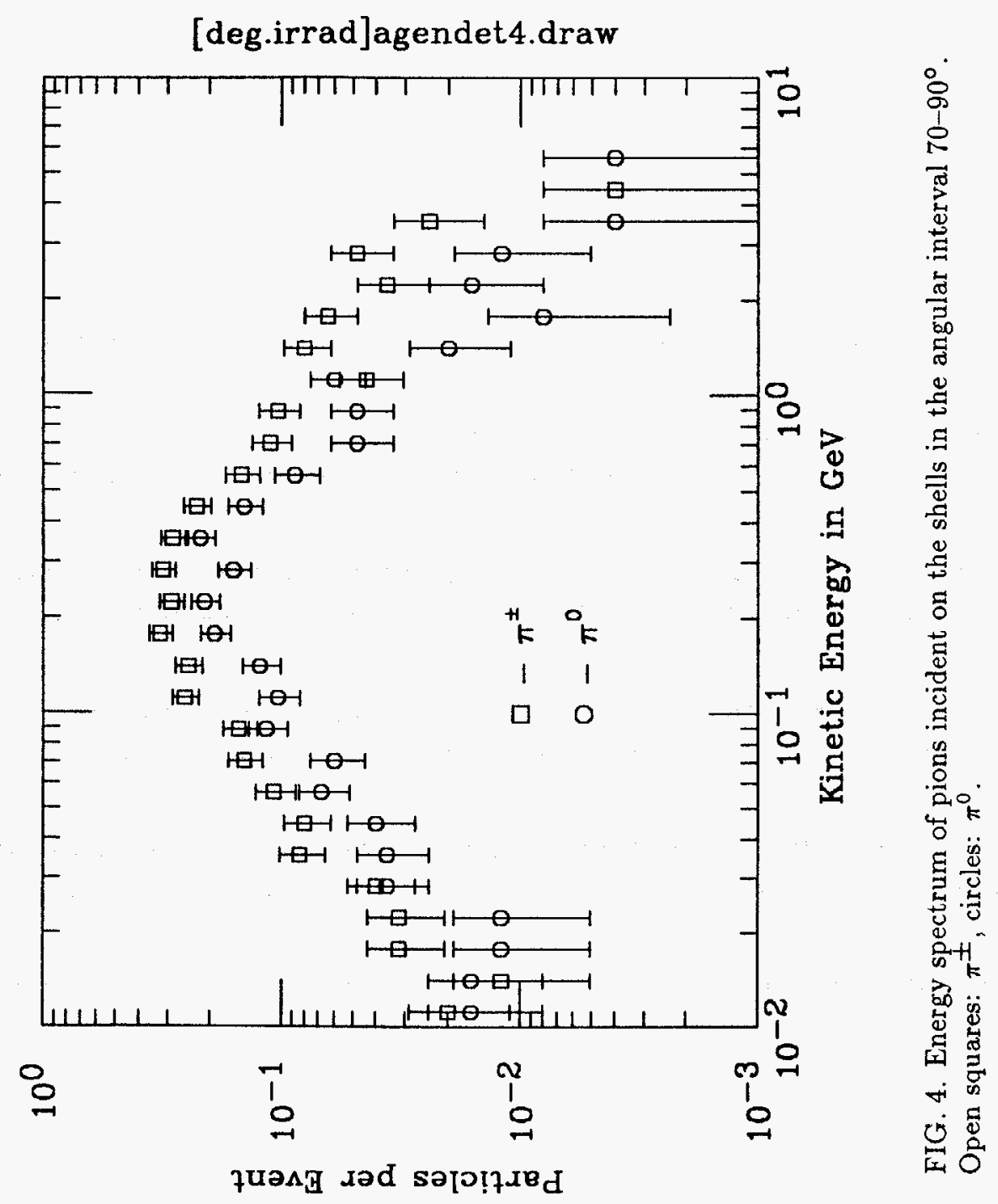




\title{
APPENDIX 21 \\ BACKSCATTER AND LATERAL DIFFUSION OF HADRONIC CASCADES IN A MODEL CALORIMETER
}

\author{
Donald E. Groom \\ SSC Central Design Group, LBL90-4040, Berkeley CA 94720 \\ and Graham R. Stevenson \\ European Organization for Nuclear Research \\ CERN, Geneva, Switzerland
}

In Appendix 20, G. R. Stevenson presented calculations of the dose due to p-p collisions in calorimeters consisting of spherical shells of different materials. The input was a table of particles produced by J. Ranft's Monte-Carlo program DTUJET, and the cascades were simulated using FLUKA87.

Table A21-1

A comparison of input kinetic energy from average DTUJET events with energy deposition scored by FLUKA87 in a model lead calorimeter. Energies are in $\mathrm{GeV}$.

\begin{tabular}{crrrrr}
\hline $\begin{array}{c}\text { Angular } \\
\text { Bin }\end{array}$ & $\begin{array}{r}\text { DTUJET } \\
\text { Input }\end{array}$ & $\begin{array}{c}\text { Pion } \\
\text { mult. }\end{array}$ & \multicolumn{4}{c}{ Energy in angular bins for } \\
calorimeter starting at \\
$0^{\circ}$ & $5.7^{\circ}$ & $70^{\circ}$ \\
\hline $0^{\circ}-1^{\circ}$ & $19,804.5$ & 27.1 & $17,400$. & - & - \\
$1^{\circ}-5^{\circ}$ & 190.3 & 14.9 & $2,770$. & - & - \\
$5^{\circ}-10^{\circ}$ & 24.6 & 6.5 & 619. & 18.8 & - \\
$10^{\circ}-20^{\circ}$ & 10.8 & 6.1 & 150. & 12.8 & - \\
$20^{\circ}-40^{\circ}$ & 5.5 & 5.9 & 15.3 & 7.06 & - \\
$40^{\circ}-70^{\circ}$ & 3.0 & 5.1 & 18.3 & 4.16 & - \\
$70^{\circ}-90^{\circ}$ & 1.1 & 2.6 & 9.77 & 2.18 & 1.66 \\
\hline $110^{\circ}-180^{\circ}$ & - & - & 19.2 & 0.50 & 0.07 \\
\hline Totals & $20,039.7$ & 68.2 & $20,900$. & 45.5 & 1.73 \\
\hline
\end{tabular}

* Since kinetic energies are reported, the tabulated values should be increased by about the available pion mass for comparison with simulation results.

Several problems with energy balance were subsequently noted. In the wideangle bins, the total scored energy was roughly an order of magnitude greater than the total energy of the particles going into the bins. The situation is summarized in Table A20-1. The column headed "DTUJET Input" is obtained from Table 1 of Appendix 20. The column headed " 0 " " is obtained from Table A3(a) 
of that Appendix by summing over radial bins with appropriate weighting for the volume in each bin. As can be seen, the smallest angular bin is deficient by $12 \%$, while all of the others contain more than the input energy. The totals $(20,040 \mathrm{GeV}$ input and $20,900 \mathrm{GeV}$ output from FLUKA87) are in satisfactory agreement.

It eventually became clear to one of us (GP.S) that the problem had to do with hadronic backscatter (or albedo) from the forward parts of the calorimeters. The model calorimeters were closed spheres, and the kinematics are such that more than $10^{4}$ times as much energy goes into the $0^{\circ}-1^{\circ}$ bin as goes into the $70^{\circ}$ $90^{\circ}$ bin. In this Appendix we report further calculations for a lead calorimeter which corroborate this mechanism. *

The model and calculational procedure are the same as those used in Appendix 20, except that the lead was replaced by vacuum in conical regions near the beam line. In one case the half-angle of the holes was $5.7^{\circ}$, corresponding to $|\eta|=3.00$ and representing typical coverage for real detectors under consideration. In the other case the holes subtended half-angles of $70^{\circ}$, so that the dose in the extreme wide-angle bin could be observed without contributions from bins at smaller angles.

In making the calculations, advantage was taken of the forward-backward symmetry of the p-p collision products. The production distributions were folded into the forward hemisphere, and only bins in this hemisphere were considered. In practice, the bin reported as $70^{\circ}-90^{\circ}$ actually extended from $70^{\circ}$ to $110^{\circ}$. Except for this case, the results were appropriately corrected by a factor of two. In addition, energy deposition was scored in a bin extending from $110^{\circ}$ to $180^{\circ}$ which was not discussed in Appendix 20. Since the folding effectively prevented the primary collision particles from going into this hemisphere, the energy deposited here provides another handle on the effect of backscattered hadrons.

The total energy scored in each of the angular regions for the cutout spheres is shown in the last two columns of Table A21. In all five bins the agreement with input energy is very much improved with the $5.7^{\circ}$ cones removed. In the "empty" $110^{\circ}-180^{\circ}$ region the effect is particularly dramatic. The deficiency in the $5^{\circ}-10^{\circ}$ region is due in part to the fact that the cone boundary comes at $5.7^{\circ}$ rather than at $5^{\circ}$, but we interpret most of the loss as due the effects of lateral leakage - the loss of the leakage from the $1^{\circ}-5^{\circ} \mathrm{bin}$, and the presence of leakage

* A. Van Ginneken has pointed out that the radiation and interaction lengths in lead (in $\mathrm{g}^{-1}$ $\mathrm{cm}^{-2}$ ) are nearly identical to those of the finely segmented uranium/scintillator calorimeter (uranium and scintillator sheets with the same thickness) used as an example elsewhere in this Report. Results obtained for solid lead can therefore be compared directly with uranium/scintillator results. 
to the $10^{\circ}-20^{\circ}$ bin. A similar effect can be seen in the change in the $10^{\circ}-20^{\circ}$ bin. In the case of the complete sphere $\left(0^{\circ}\right.$ column $)$ such leakage seems to be responsible for the transfer of energy from the $0^{\circ}-1^{\circ}$ bin to the $1^{\circ}-5^{\circ} \mathrm{bin}$. This is hardly surprising, because the smallest region extends to only about $3.5 \mathrm{~cm}$ from the beam line-far less than the transverse development scale of a hadronic shower, which in this case is about $15 \mathrm{~cm}$.

These features are also evident in the radial distributions of the dose. In the interest of brevity, only the extreme angular regions are shown. The total and electromagnetic dose for the $5^{\circ}-10^{\circ}$ region are shown in Figs. A21-1(a) and A211(b), respectively. The solid histogram is for the closed sphere, and the dashed histogram is for the sphere with the $5.7^{\circ}$ cones removed. Most of the decrease in the total dose comes at great depths, as would be expected for lateral leakage of higher-energy cascades from the $0^{\circ}-1^{\circ}$ region.

Similar data for the $70^{\circ}-90^{\circ}$ region are shown in Figs. A21-2(a) and A21-2(b). In this case the dotted histogram shows the effect of removing the $70^{\circ}$ cones. The electromagnetic dose is not dramatically different for the three cases. The total dose is systematically lower by about an order of magnitude. There is no way to separate the contribution of incident photons and electrons, but, when this is done in a crude way by subtracting the electromagnetic dose from the total dose, there is a strong suggestion of a greater excess at very small depths in the calorimeter, as would be expected for the contribution of low-energy backscatter hadrons.

The maximum dose averaged over each angular bin due to hadronic cascades in the solid spherical-shell lead calorimeter (as calculated from the difference of Tables A3(a) and A3(b) in Appendix 20*) is shown by the solid histogram in Fig. A21-3. Similar results for spheres with $5.7^{\circ}$ and $70^{\circ}$ holes are shown by the dashed and dotted histograms, respectively. The smooth curve was calculated by a totally independent method, and represents the expected maximum dose in the absence of backscatter or lateral leakage. As described in Appendix 19, the MARS10 cascade code was used to obtain longitudinal profiles for cascades induced in a lead beam stop by low-energy charged pions. A power-law fit to the momentum dependence was then folded with the expected production spectrum to yield the plotted curve, which is also shown in Fig. 5-8 (Section 5).

All of the data are summarized in Tables A21-2 through A21-4.

$\star$ This is not quite right, since the electromagnetic part includes electromagnetic energy produced in hadronic cascades. However, it is necessary to subtract the sharp spike produced by the showering of incident photons and electrons near the front of the calorimeter, and this procedure does it more or less correctly. It should be reasonably accurate near the hadronic shower maximum. 
We conclude that the energy balance difficulties in Appendix 20 had two separate physical origins-lateral leakage at small angles, where the intensity gradient with angle was enormous and the dimensions of the regions were not large compared with the transverse dimensions of the cascades, and the backscatter of hadrons from these high-intensity regions to wide-angle regions where the direct contribution was comparatively small. To some extent, these effects are artifacts of the model, since in a real detector the central section will extend to no closer to the beam line than about $5^{\circ}$, with the small-angle regions being covered by comparatively distant endcap calorimeters. However, there are several warnings: Albedo and reflection effects are important for hadrons as well as for neutrons and photons, and lateral cascade development cannot be neglected in places where there is a large transverse intensity gradient in the incident radiation. There are ancillary considerations of occupancy and trigger rate which may be quite important. 

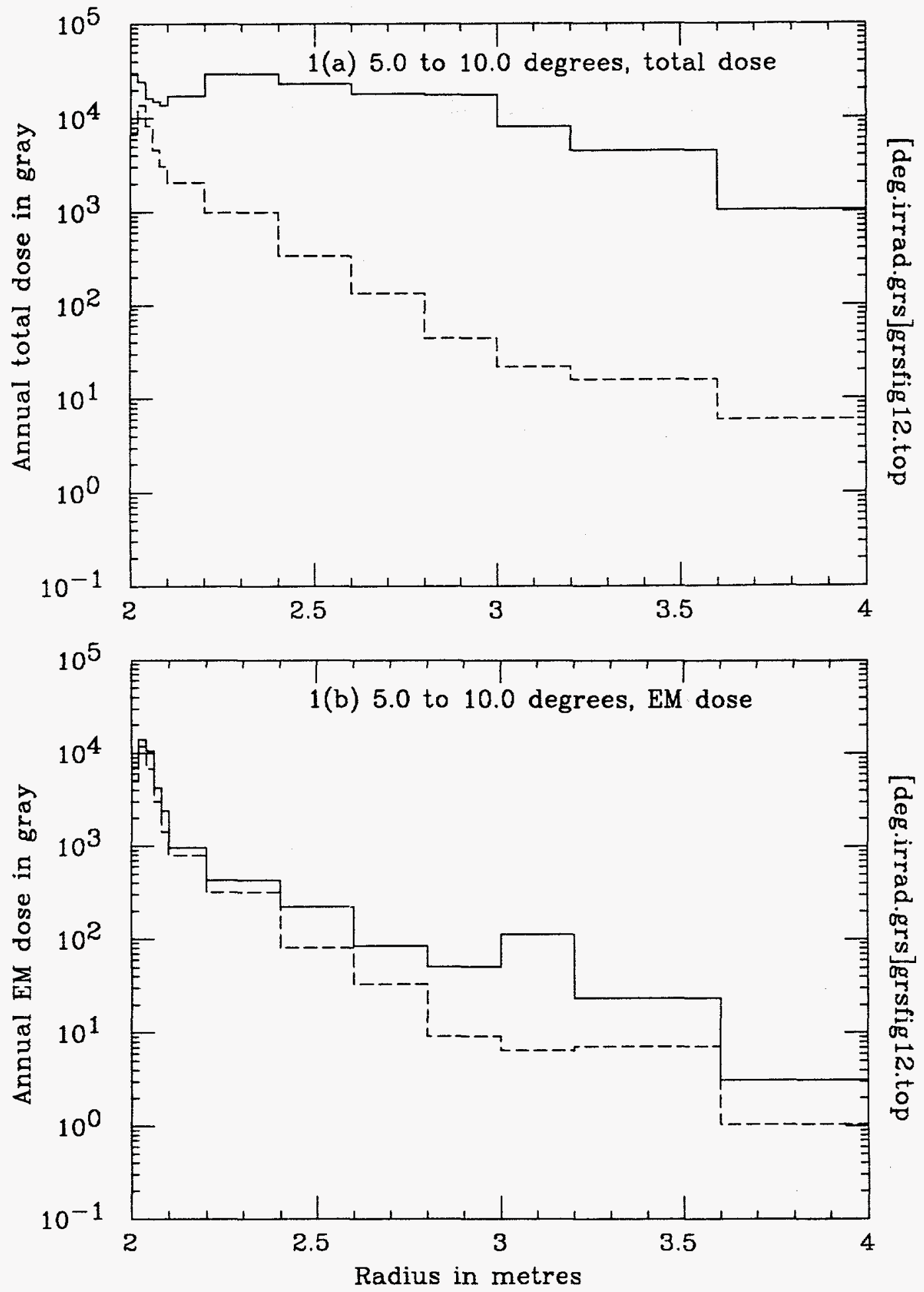

FIG. A21-1. Variation of the total and electromagnetic dose with depth in the angular interval $5^{\circ}-10^{\circ}$ for a spherical shell lead calorimeter with a $2 \mathrm{~m}$ inner radius. The solid histogram is for a complete sphere, and the dashed histogram for a sphere with $5.7^{\circ}$ cones removed near the beam line. 

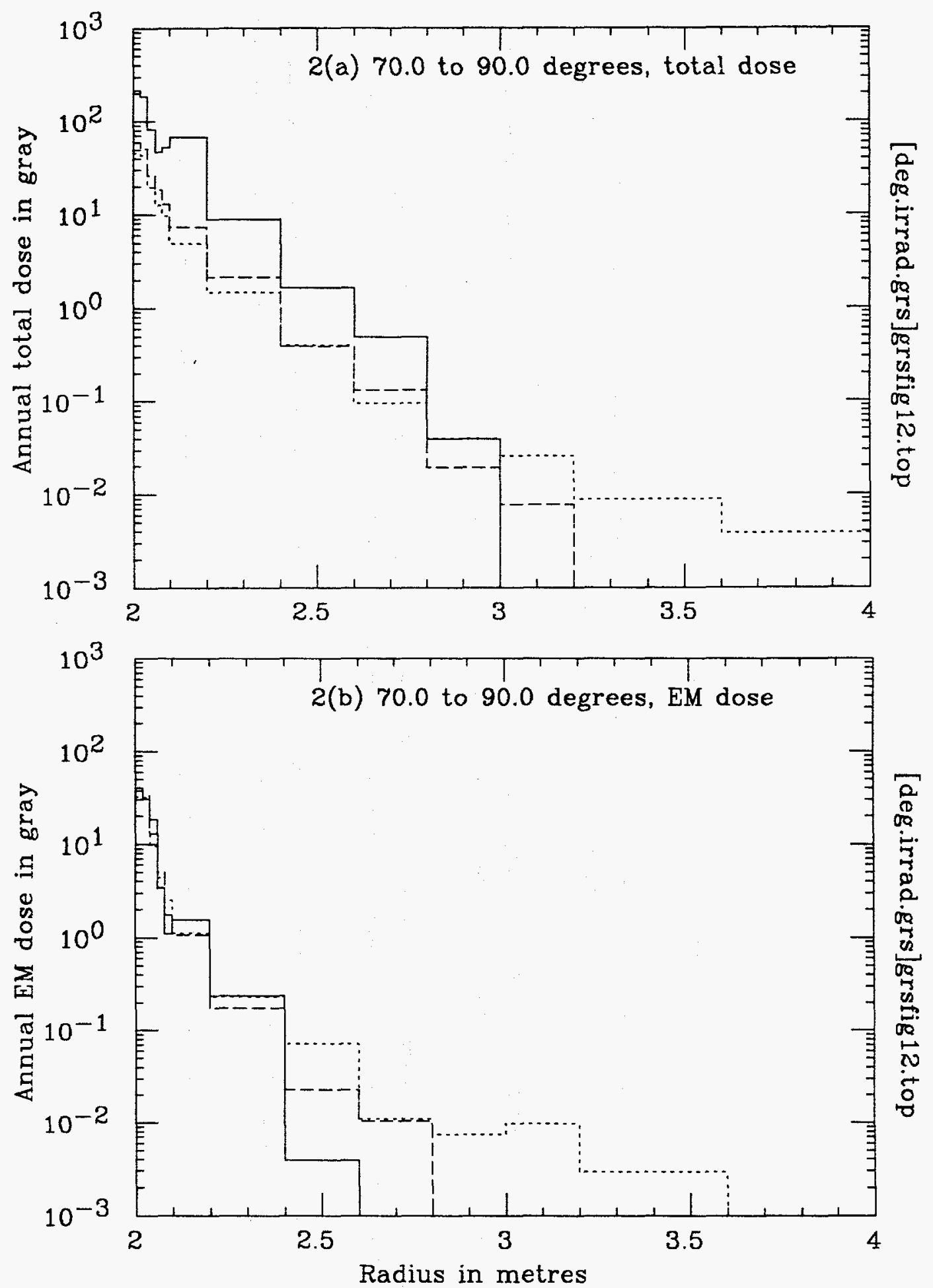

FIG. A21-2. Variation of the total and electromagnetic dose with depth in the angular interval $70^{\circ}-90^{\circ}$ for a spherical shell lead calorimeter with a $2 \mathrm{~m}$ inner radius. The solid histogram is for a complete sphere, the dashed histogram for a sphere with $5.7^{\circ}$ cones removed near the beam line, and the dotted histogram with $70^{\circ}$ cones removed. 


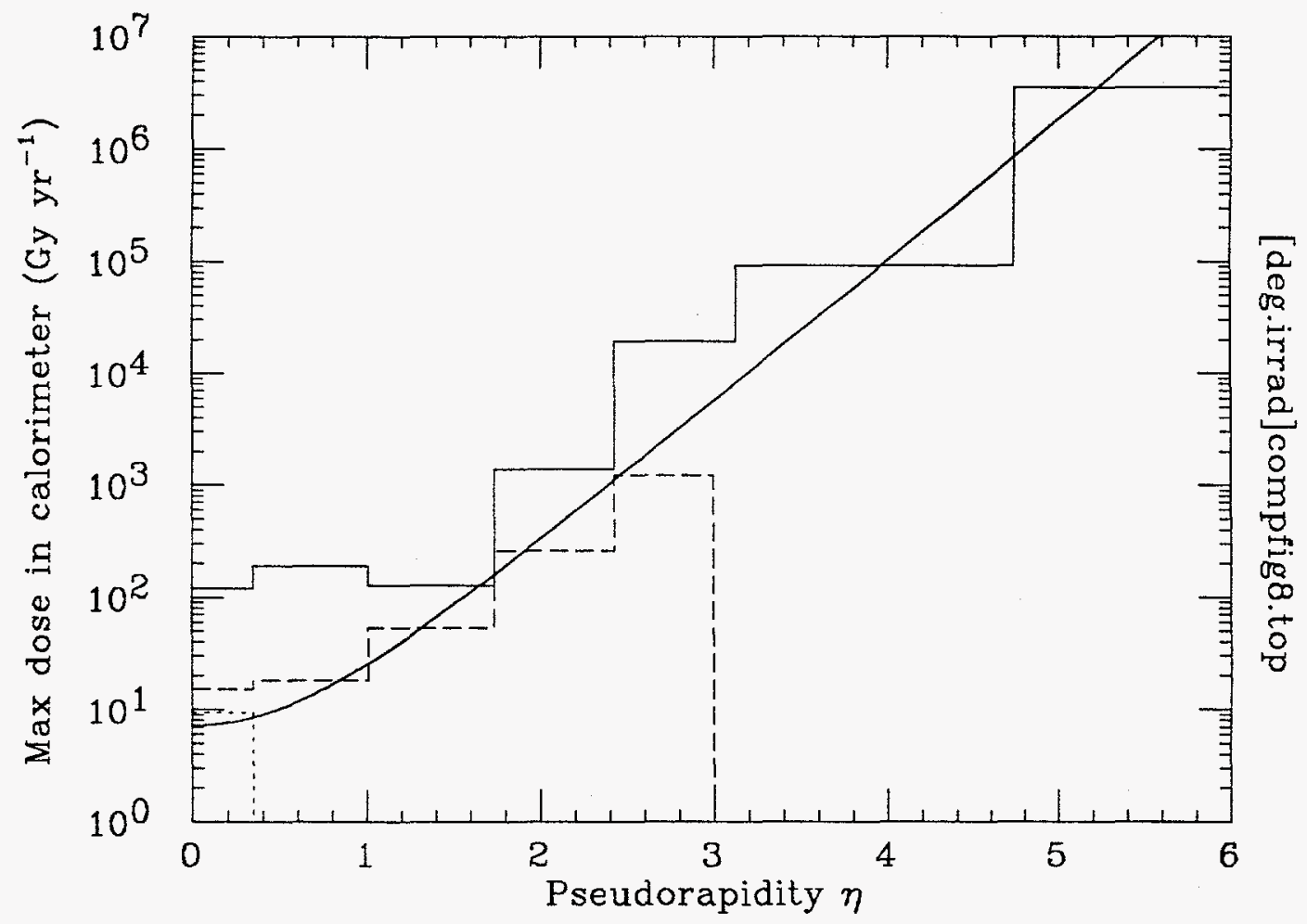

Fig. A21-3. Maximum dose produced by hadronic cascades in a lead calorimeter with an inner radius of $200 \mathrm{~cm}$ (also taken to be the approximate radius at shower maximum). The solid curve is from Fig. 5-8, and is based on the production model of Section 2 and a MARS10 simulation of cascades produced by low-energy pions. The solid histogram is taken from the data given in Tables A3(a) and A3(b) of Appendix 20. The dashed histogram is for a similar calculation with a $5.7^{\circ}$ half-angle hole in either end of the calorimeter, and the dotted entry for openings with $70^{\circ}$ half-angles. 
Table A21-2(a)

DTUJET $20+20 \mathrm{TeV}$ source in a closed spherical lead calorimeter. Dose is in grays per year to silicon as a function of depth, assuming $10^{15}$ collisions per year.

A21-2(a): Total energy density (dose) in Gy/yr

\begin{tabular}{ccccccccc}
\hline \hline & & & \multicolumn{2}{c}{ Angle } \\
Depth $(\mathrm{m})$ & $0^{\circ}-1^{\circ}$ & $1^{\circ}-5^{\circ}$ & $5^{\circ}-10^{\circ}$ & $10^{\circ}-20^{\circ}$ & $20^{\circ}-40^{\circ}$ & $40^{\circ}-70^{\circ}$ & $70^{\circ}-90^{\circ}$ & $110^{\circ}-180^{\circ}$ \\
\hline $2.00-2.02$ & $1.14 \mathrm{E}+7$ & $1.24 \mathrm{E}+5$ & $2.98 \mathrm{E}+4$ & $2.25 \mathrm{E}+3$ & $3.37 \mathrm{E}+2$ & $2.04 \mathrm{E}+2$ & $2.23 \mathrm{E}+2$ & $1.05 \mathrm{E}+2$ \\
$2.02-2.04$ & $1.23 \mathrm{E}+8$ & $4.02 \mathrm{E}+5$ & $2.49 \mathrm{E}+4$ & $4.39 \mathrm{E}+3$ & $5.27 \mathrm{E}+2$ & $7.75 \mathrm{E}+2$ & $1.90 \mathrm{E}+2$ & $6.36 \mathrm{E}+1$ \\
$2.04-2.06$ & $3.64 \mathrm{E}+8$ & $3.57 \mathrm{E}+5$ & $1.71 \mathrm{E}+4$ & $1.73 \mathrm{E}+3$ & $1.90 \mathrm{E}+2$ & $9.25 \mathrm{E}+1$ & $8.48 \mathrm{E}+1$ & $7.42 \mathrm{E}+1$ \\
$2.06-2.08$ & $3.60 \mathrm{E}+8$ & $2.04 \mathrm{E}+5$ & $1.56 \mathrm{E}+4$ & $1.44 \mathrm{E}+3$ & $1.26 \mathrm{E}+2$ & $4.55 \mathrm{E}+1$ & $4.88 \mathrm{E}+1$ & $5.79 \mathrm{E}+1$ \\
$2.08-2.10$ & $2.99 \mathrm{E}+8$ & $2.32 \mathrm{E}+5$ & $1.42 \mathrm{E}+4$ & $1.40 \mathrm{E}+3$ & $3.02 \mathrm{E}+2$ & $1.04 \mathrm{E}+2$ & $5.41 \mathrm{E}+1$ & $1.47 \mathrm{E}+2$ \\
$2.10-2.20$ & $1.18 \mathrm{E}+8$ & $2.78 \mathrm{E}+5$ & $1.78 \mathrm{E}+4$ & $1.48 \mathrm{E}+3$ & $6.21 \mathrm{E}+1$ & $7.29 \mathrm{E}+1$ & $6.92 \mathrm{E}+1$ & $8.19 \mathrm{E}+1$ \\
$2.20-2.40$ & $7.06 \mathrm{E}+7$ & $3.27 \mathrm{E}+5$ & $2.98 \mathrm{E}+4$ & $2.13 \mathrm{E}+3$ & $2.75 \mathrm{E}+1$ & $5.36 \mathrm{E}+0$ & $9.14 \mathrm{E}+0$ & $1.47 \mathrm{E}+1$ \\
$2.40-2.60$ & $4.49 \mathrm{E}+7$ & $3.70 \mathrm{E}+5$ & $2.38 \mathrm{E}+4$ & $1.57 \mathrm{E}+3$ & $4.60 \mathrm{E}+1$ & $1.15 \mathrm{E}+0$ & $1.71 \mathrm{E}+0$ & $1.50 \mathrm{E}+0$ \\
$2.60-2.80$ & $2.72 \mathrm{E}+7$ & $3.21 \mathrm{E}+5$ & $1.87 \mathrm{E}+4$ & $1.19 \mathrm{E}+3$ & $1.80 \mathrm{E}+1$ & $1.79 \mathrm{E}-1$ & $5.07 \mathrm{E}-1$ & $2.03 \mathrm{E}+0$ \\
$2.80-3.00$ & $1.50 \mathrm{E}+7$ & $2.33 \mathrm{E}+5$ & $1.82 \mathrm{E}+4$ & $7.79 \mathrm{E}+2$ & $2.91 \mathrm{E}+0$ & $3.07 \mathrm{E}-2$ & $4.05 \mathrm{E}-2$ & $2.64 \mathrm{E}-3$ \\
$3.00-3.20$ & $6.62 \mathrm{E}+6$ & $1.40 \mathrm{E}+5$ & $8.33 \mathrm{E}+3$ & $6.01 \mathrm{E}+2$ & $4.29 \mathrm{E}+0$ & $2.07 \mathrm{E}-3$ & $9.38 \mathrm{E}-4$ & $1.36 \mathrm{E}-2$ \\
$3.20-3.60$ & $2.24 \mathrm{E}+6$ & $4.79 \mathrm{E}+4$ & $4.59 \mathrm{E}+3$ & $1.69 \mathrm{E}+2$ & $2.59 \mathrm{E}+0$ & $1.21 \mathrm{E}-3$ & $9.52 \mathrm{E}-4$ & $4.41 \mathrm{E}-2$ \\
$3.60-4.00$ & $7.52 \mathrm{E}+5$ & $1.75 \mathrm{E}+4$ & $1.07 \mathrm{E}+3$ & $3.53 \mathrm{E}+1$ & $1.02 \mathrm{E}-1$ & $0.00 \mathrm{E}+0$ & $0.00 \mathrm{E}+0$ & $0.00 \mathrm{E}+0$ \\
\hline \hline
\end{tabular}

A3(b): Electromagnetic energy density (dose) in Gy/yr

\begin{tabular}{rcccccccc}
\hline \hline & & \multicolumn{2}{c}{ Angle } \\
Depth (m) & $0^{\circ}-1^{\circ}$ & $1^{\circ}-5^{\circ}$ & $5^{\circ}-10^{\circ}$ & $10^{\circ}-20^{\circ}$ & $20^{\circ}-40^{\circ}$ & $40^{\circ}-70^{\circ}$ & $70^{\circ}-90^{\circ}$ & $110^{\circ}-180^{\circ}$ \\
\hline $2.00-2.02$ & $1.03 \mathrm{E}+7$ & $7.74 \mathrm{E}+4$ & $7.10 \mathrm{E}+3$ & $1.05 \mathrm{E}+3$ & $1.90 \mathrm{E}+2$ & $5.52 \mathrm{E}+1$ & $4.13 \mathrm{E}+1$ & $8.30 \mathrm{E}+0$ \\
$2.02-2.04$ & $1.19 \mathrm{E}+8$ & $2.70 \mathrm{E}+5$ & $1.44 \mathrm{E}+4$ & $1.74 \mathrm{E}+3$ & $1.99 \mathrm{E}+2$ & $6.58 \mathrm{E}+1$ & $3.18 \mathrm{E}+1$ & $8.09 \mathrm{E}-1$ \\
$2.04-2.06$ & $3.59 \mathrm{E}+8$ & $2.74 \mathrm{E}+5$ & $1.09 \mathrm{E}+4$ & $6.74 \mathrm{E}+2$ & $7.93 \mathrm{E}+1$ & $2.90 \mathrm{E}+1$ & $1.91 \mathrm{E}+1$ & $2.09 \mathrm{E}-1$ \\
$2.06-2.08$ & $3.57 \mathrm{E}+8$ & $1.08 \mathrm{E}+5$ & $4.35 \mathrm{E}+3$ & $5.22 \mathrm{E}+2$ & $4.37 \mathrm{E}+1$ & $6.33 \mathrm{E}+0$ & $3.50 \mathrm{E}+0$ & $5.80 \mathrm{E}-2$ \\
$2.08-2.10$ & $2.95 \mathrm{E}+8$ & $1.06 \mathrm{E}+5$ & $2.46 \mathrm{E}+3$ & $2.03 \mathrm{E}+2$ & $1.25 \mathrm{E}+1$ & $5.15 \mathrm{E}+0$ & $1.12 \mathrm{E}+0$ & $3.38 \mathrm{E}-2$ \\
$2.10-2.20$ & $1.12 \mathrm{E}+8$ & $7.61 \mathrm{E}+4$ & $9.87 \mathrm{E}+2$ & $7.92 \mathrm{E}+1$ & $9.86 \mathrm{E}+0$ & $2.21 \mathrm{E}+0$ & $1.60 \mathrm{E}+0$ & $0.00 \mathrm{E}+0$ \\
$2.20-2.40$ & $6.06 \mathrm{E}+7$ & $5.31 \mathrm{E}+4$ & $4.45 \mathrm{E}+2$ & $3.67 \mathrm{E}+1$ & $4.94 \mathrm{E}+0$ & $7.40 \mathrm{E}-1$ & $2.46 \mathrm{E}-1$ & $0.00 \mathrm{E}+0$ \\
$2.40-2.60$ & $3.47 \mathrm{E}+7$ & $5.15 \mathrm{E}+4$ & $2.31 \mathrm{E}+2$ & $8.07 \mathrm{E}+0$ & $2.92 \mathrm{E}-1$ & $1.53 \mathrm{E}-1$ & $4.04 \mathrm{E}-3$ & $0.00 \mathrm{E}+0$ \\
$2.60-2.80$ & $1.96 \mathrm{E}+7$ & $4.45 \mathrm{E}+4$ & $8.71 \mathrm{E}+1$ & $5.43 \mathrm{E}+0$ & $2.25 \mathrm{E}-1$ & $0.00 \mathrm{E}+0$ & $0.00 \mathrm{E}+0$ & $0.00 \mathrm{E}+0$ \\
$2.80-3.00$ & $9.49 \mathrm{E}+6$ & $2.93 \mathrm{E}+4$ & $5.28 \mathrm{E}+1$ & $5.35 \mathrm{E}+0$ & $2.82 \mathrm{E}-2$ & $0.00 \mathrm{E}+0$ & $0.00 \mathrm{E}+0$ & $0.00 \mathrm{E}+0$ \\
$3.00-3.20$ & $3.85 \mathrm{E}+6$ & $1.71 \mathrm{E}+4$ & $1.15 \mathrm{E}+2$ & $2.71 \mathrm{E}-1$ & $2.50 \mathrm{E}-2$ & $0.00 \mathrm{E}+0$ & $0.00 \mathrm{E}+0$ & $0.00 \mathrm{E}+0$ \\
$3.20-3.60$ & $1.17 \mathrm{E}+6$ & $4.26 \mathrm{E}+3$ & $2.43 \mathrm{E}+1$ & $8.67 \mathrm{E}-2$ & $1.24 \mathrm{E}-2$ & $0.00 \mathrm{E}+0$ & $0.00 \mathrm{E}+0$ & $0.00 \mathrm{E}+0$ \\
$3.60-4.00$ & $3.40 \mathrm{E}+5$ & $1.66 \mathrm{E}+3$ & $3.19 \mathrm{E}+0$ & $2.25 \mathrm{E}-1$ & $4.60 \mathrm{E}-3$ & $0.00 \mathrm{E}+0$ & $0.00 \mathrm{E}+0$ & $0.00 \mathrm{E}+0$ \\
\hline
\end{tabular}




\section{Table A21-3}

DTUJET $20+20 \mathrm{TeV}$ source in a spherical lead calorimeter with $5.7^{\circ}$ half-angle openings along the beam line. Dose is in grays per year to silicon as a function of depth, assuming $10^{15}$ collisions per year.

A21-3(a): Total energy density (dose) in Gy/yr

\begin{tabular}{rcccccccc}
\hline \hline & & \multicolumn{2}{c}{ Angle } \\
Depth $(\mathrm{m})$ & $0^{\circ}-1^{\circ}$ & $1^{\circ}-5^{\circ}$ & $5^{\circ}-10^{\circ}$ & $10^{\circ}-20^{\circ}$ & $20^{\circ}-40^{\circ}$ & $40^{\circ}-70^{\circ}$ & $70^{\circ}-90^{\circ}$ & $110^{\circ}-180^{\circ}$ \\
\hline $2.00-2.02$ & $0.00 \mathrm{E}+0$ & $0.00 \mathrm{E}+0$ & $6.94 \mathrm{E}+3$ & $1.32 \mathrm{E}+3$ & $2.56 \mathrm{E}+2$ & $8.38 \mathrm{E}+1$ & $6.20 \mathrm{E}+1$ & $5.80 \mathrm{E}+0$ \\
$2.02-2.04$ & $0.00 \mathrm{E}+0$ & $0.00 \mathrm{E}+0$ & $1.41 \mathrm{E}+4$ & $1.93 \mathrm{E}+3$ & $3.03 \mathrm{E}+2$ & $8.10 \mathrm{E}+1$ & $5.31 \mathrm{E}+1$ & $4.35 \mathrm{E}+0$ \\
$2.04-2.06$ & $0.00 \mathrm{E}+0$ & $0.00 \mathrm{E}+0$ & $8.56 \mathrm{E}+3$ & $1.17 \mathrm{E}+3$ & $1.66 \mathrm{E}+2$ & $4.63 \mathrm{E}+1$ & $2.74 \mathrm{E}+1$ & $3.50 \mathrm{E}+0$ \\
$2.06-2.08$ & $0.00 \mathrm{E}+0$ & $0.00 \mathrm{E}+0$ & $4.77 \mathrm{E}+3$ & $6.87 \mathrm{E}+2$ & $1.12 \mathrm{E}+2$ & $3.06 \mathrm{E}+1$ & $1.92 \mathrm{E}+1$ & $1.96 \mathrm{E}+0$ \\
$2.08-2.10$ & $0.00 \mathrm{E}+0$ & $0.00 \mathrm{E}+0$ & $3.17 \mathrm{E}+3$ & $5.01 \mathrm{E}+2$ & $8.28 \mathrm{E}+1$ & $1.87 \mathrm{E}+1$ & $1.33 \mathrm{E}+1$ & $1.47 \mathrm{E}+0$ \\
$2.10-2.20$ & $0.00 \mathrm{E}+0$ & $0.00 \mathrm{E}+0$ & $2.13 \mathrm{E}+3$ & $3.55 \mathrm{E}+2$ & $5.67 \mathrm{E}+1$ & $1.15 \mathrm{E}+1$ & $7.49 \mathrm{E}+0$ & $8.70 \mathrm{E}-1$ \\
$2.20-2.40$ & $0.00 \mathrm{E}+0$ & $0.00 \mathrm{E}+0$ & $1.02 \mathrm{E}+3$ & $1.76 \mathrm{E}+2$ & $2.03 \mathrm{E}+1$ & $3.69 \mathrm{E}+0$ & $2.22 \mathrm{E}+0$ & $4.99 \mathrm{E}-1$ \\
$2.40-2.60$ & $0.00 \mathrm{E}+0$ & $0.00 \mathrm{E}+0$ & $3.52 \mathrm{E}+2$ & $5.66 \mathrm{E}+1$ & $5.16 \mathrm{E}+0$ & $9.45 \mathrm{E}-1$ & $4.00 \mathrm{E}-1$ & $7.89 \mathrm{E}-2$ \\
$2.60-2.80$ & $0.00 \mathrm{E}+0$ & $0.00 \mathrm{E}+0$ & $1.38 \mathrm{E}+2$ & $1.86 \mathrm{E}+1$ & $1.27 \mathrm{E}+0$ & $1.75 \mathrm{E}-1$ & $1.36 \mathrm{E}-1$ & $2.91 \mathrm{E}-2$ \\
$2.80-3.00$ & $0.00 \mathrm{E}+0$ & $0.00 \mathrm{E}+0$ & $4.52 \mathrm{E}+1$ & $6.09 \mathrm{E}+0$ & $6.50 \mathrm{E}-1$ & $6.32 \mathrm{E}-2$ & $2.00 \mathrm{E}-2$ & $4.71 \mathrm{E}-4$ \\
$3.00-3.20$ & $0.00 \mathrm{E}+0$ & $0.00 \mathrm{E}+0$ & $2.26 \mathrm{E}+1$ & $1.82 \mathrm{E}+0$ & $2.39 \mathrm{E}-1$ & $3.12 \mathrm{E}-2$ & $7.95 \mathrm{E}-3$ & $2.16 \mathrm{E}-4$ \\
$3.20-3.60$ & $0.00 \mathrm{E}+0$ & $0.00 \mathrm{E}+0$ & $1.64 \mathrm{E}+1$ & $1.06 \mathrm{E}+0$ & $2.08 \mathrm{E}-2$ & $3.50 \mathrm{E}-3$ & $1.23 \mathrm{E}-4$ & $9.57 \mathrm{E}-5$ \\
$3.60-4.00$ & $0.00 \mathrm{E}+0$ & $0.00 \mathrm{E}+0$ & $6.17 \mathrm{E}+0$ & $1.63 \mathrm{E}-1$ & $2.06 \mathrm{E}-3$ & $0.00 \mathrm{E}+0$ & $0.00 \mathrm{E}+0$ & $0.00 \mathrm{E}+0$ \\
\hline \hline
\end{tabular}

A21-3(b): Electromagnetic energy density (dose) in Gy/yr

\begin{tabular}{ccccccccc}
\hline \hline & & & \multicolumn{2}{c}{ Angle } \\
Depth $(\mathrm{m})$ & $0^{\circ}-1^{\circ}$ & $1^{\circ}-5^{\circ}$ & $5^{\circ}-10^{\circ}$ & $10^{\circ}-20^{\circ}$ & $20^{\circ}-40^{\circ}$ & $40^{\circ}-70^{\circ}$ & $70^{\circ}-90^{\circ}$ & $110^{\circ}-180^{\circ}$ \\
\hline $2.00-2.02$ & $0.00 \mathrm{E}+0$ & $0.00 \mathrm{E}+0$ & $5.25 \mathrm{E}+3$ & $1.00 \mathrm{E}+3$ & $1.75 \mathrm{E}+2$ & $5.62 \mathrm{E}+1$ & $3.90 \mathrm{E}+1$ & $2.08 \mathrm{E}+0$ \\
$2.02-2.04$ & $0.00 \mathrm{E}+0$ & $0.00 \mathrm{E}+0$ & $1.23 \mathrm{E}+4$ & $1.54 \mathrm{E}+3$ & $2.28 \mathrm{E}+2$ & $5.81 \mathrm{E}+1$ & $3.44 \mathrm{E}+1$ & $8.13 \mathrm{E}-1$ \\
$2.04-2.06$ & $0.00 \mathrm{E}+0$ & $0.00 \mathrm{E}+0$ & $6.96 \mathrm{E}+3$ & $8.37 \mathrm{E}+2$ & $1.01 \mathrm{E}+2$ & $2.59 \mathrm{E}+1$ & $1.33 \mathrm{E}+1$ & $2.38 \mathrm{E}-1$ \\
$2.06-2.08$ & $0.00 \mathrm{E}+0$ & $0.00 \mathrm{E}+0$ & $3.13 \mathrm{E}+3$ & $3.56 \mathrm{E}+2$ & $5.49 \mathrm{E}+1$ & $1.24 \mathrm{E}+1$ & $5.11 \mathrm{E}+0$ & $5.83 \mathrm{E}-2$ \\
$2.08-2.10$ & $0.00 \mathrm{E}+0$ & $0.00 \mathrm{E}+0$ & $1.47 \mathrm{E}+3$ & $1.63 \mathrm{E}+2$ & $2.80 \mathrm{E}+1$ & $3.80 \mathrm{E}+0$ & $1.79 \mathrm{E}+0$ & $2.68 \mathrm{E}-2$ \\
$2.10-2.20$ & $0.00 \mathrm{E}+0$ & $0.00 \mathrm{E}+0$ & $8.20 \mathrm{E}+2$ & $9.51 \mathrm{E}+1$ & $2.05 \mathrm{E}+1$ & $2.28 \mathrm{E}+0$ & $1.10 \mathrm{E}+0$ & $2.27 \mathrm{E}-3$ \\
$2.20-2.40$ & $0.00 \mathrm{E}+0$ & $0.00 \mathrm{E}+0$ & $3.25 \mathrm{E}+2$ & $4.38 \mathrm{E}+1$ & $4.13 \mathrm{E}+0$ & $6.98 \mathrm{E}-1$ & $1.78 \mathrm{E}-1$ & $0.00 \mathrm{E}+0$ \\
$2.40-2.60$ & $0.00 \mathrm{E}+0$ & $0.00 \mathrm{E}+0$ & $8.36 \mathrm{E}+1$ & $1.15 \mathrm{E}+1$ & $8.24 \mathrm{E}-1$ & $1.61 \mathrm{E}-1$ & $2.33 \mathrm{E}-2$ & $0.00 \mathrm{E}+0$ \\
$2.60-2.80$ & $0.00 \mathrm{E}+0$ & $0.00 \mathrm{E}+0$ & $3.42 \mathrm{E}+1$ & $1.67 \mathrm{E}+0$ & $6.71 \mathrm{E}-2$ & $1.15 \mathrm{E}-2$ & $1.07 \mathrm{E}-2$ & $0.00 \mathrm{E}+0$ \\
$2.80-3.00$ & $0.00 \mathrm{E}+0$ & $0.00 \mathrm{E}+0$ & $9.49 \mathrm{E}+0$ & $1.04 \mathrm{E}+0$ & $4.64 \mathrm{E}-2$ & $1.78 \mathrm{E}-3$ & $0.00 \mathrm{E}+0$ & $0.00 \mathrm{E}+0$ \\
$3.00-3.20$ & $0.00 \mathrm{E}+0$ & $0.00 \mathrm{E}+0$ & $6.67 \mathrm{E}+0$ & $2.73 \mathrm{E}-2$ & $1.08 \mathrm{E}-2$ & $0.00 \mathrm{E}+0$ & $2.66 \mathrm{E}-4$ & $0.00 \mathrm{E}+0$ \\
$3.20-3.60$ & $0.00 \mathrm{E}+0$ & $0.00 \mathrm{E}+0$ & $7.39 \mathrm{E}+0$ & $6.60 \mathrm{E}-2$ & $4.12 \mathrm{E}-1$ & $0.00 \mathrm{E}+0$ & $0.00 \mathrm{E}+0$ & $0.00 \mathrm{E}+0$ \\
$3.60-4.00$ & $0.00 \mathrm{E}+0$ & $0.00 \mathrm{E}+0$ & $1.08 \mathrm{E}+0$ & $0.00 \mathrm{E}+0$ & $0.00 \mathrm{E}+0$ & $0.00 \mathrm{E}+0$ & $0.00 \mathrm{E}+0$ & $0.00 \mathrm{E}+0$ \\
\hline \hline
\end{tabular}


Table A21-4

DTUJET $20+20 \mathrm{TeV}$ source in a spherical lead calorimeter with $70^{\circ}$ half-angle openings along the beam line. Dose is in grays per year to silicon as a function of depth, assuming $10^{15}$ collisions per year.

A21-4(a): Total energy density (dose) in Gy/yr

\begin{tabular}{rcccccccc}
\hline & & & \multicolumn{2}{c}{ Angle } & & & \\
Depth (m) & $0^{\circ}-1^{\circ}$ & $1^{\circ}-5^{\circ}$ & $5^{\circ}-10^{\circ}$ & $10^{\circ}-20^{\circ}$ & $20^{\circ}-40^{\circ}$ & $40^{\circ}-70^{\circ}$ & $70^{\circ}-90^{\circ}$ & $110^{\circ}-180^{\circ}$ \\
\hline $2.00-2.02$ & $0.00 \mathrm{E}+0$ & $0.00 \mathrm{E}+0$ & $0.00 \mathrm{E}+0$ & $0.00 \mathrm{E}+0$ & $0.00 \mathrm{E}+0$ & $0.00 \mathrm{E}+0$ & $4.77 \mathrm{E}+1$ & $2.18 \mathrm{E}+0$ \\
$2.02-2.04$ & $0.00 \mathrm{E}+0$ & $0.00 \mathrm{E}+0$ & $0.00 \mathrm{E}+0$ & $0.00 \mathrm{E}+0$ & $0.00 \mathrm{E}+0$ & $0.00 \mathrm{E}+0$ & $4.50 \mathrm{E}+1$ & $9.40 \mathrm{E}-1$ \\
$2.04-2.06$ & $0.00 \mathrm{E}+0$ & $0.00 \mathrm{E}+0$ & $0.00 \mathrm{E}+0$ & $0.00 \mathrm{E}+0$ & $0.00 \mathrm{E}+0$ & $0.00 \mathrm{E}+0$ & $2.03 \mathrm{E}+1$ & $2.60 \mathrm{E}-1$ \\
$2.06-2.08$ & $0.00 \mathrm{E}+0$ & $0.00 \mathrm{E}+0$ & $0.00 \mathrm{E}+0$ & $0.00 \mathrm{E}+0$ & $0.00 \mathrm{E}+0$ & $0.00 \mathrm{E}+0$ & $1.30 \mathrm{E}+1$ & $3.58 \mathrm{E}-1$ \\
$2.08-2.10$ & $0.00 \mathrm{E}+0$ & $0.00 \mathrm{E}+0$ & $0.00 \mathrm{E}+0$ & $0.00 \mathrm{E}+0$ & $0.00 \mathrm{E}+0$ & $0.00 \mathrm{E}+0$ & $1.00 \mathrm{E}+1$ & $1.04 \mathrm{E}-1$ \\
$2.10-2.20$ & $0.00 \mathrm{E}+0$ & $0.00 \mathrm{E}+0$ & $0.00 \mathrm{E}+0$ & $0.00 \mathrm{E}+0$ & $0.00 \mathrm{E}+0$ & $0.00 \mathrm{E}+0$ & $5.04 \mathrm{E}+0$ & $5.55 \mathrm{E}-2$ \\
$2.20-2.40$ & $0.00 \mathrm{E}+0$ & $0.00 \mathrm{E}+0$ & $0.00 \mathrm{E}+0$ & $0.00 \mathrm{E}+0$ & $0.00 \mathrm{E}+0$ & $0.00 \mathrm{E}+0$ & $1.52 \mathrm{E}+0$ & $1.81 \mathrm{E}-2$ \\
$2.40-2.60$ & $0.00 \mathrm{E}+0$ & $0.00 \mathrm{E}+0$ & $0.00 \mathrm{E}+0$ & $0.00 \mathrm{E}+0$ & $0.00 \mathrm{E}+0$ & $0.00 \mathrm{E}+0$ & $4.14 \mathrm{E}-1$ & $2.05 \mathrm{E}-3$ \\
$2.60-2.80$ & $0.00 \mathrm{E}+0$ & $0.00 \mathrm{E}+0$ & $0.00 \mathrm{E}+0$ & $0.00 \mathrm{E}+0$ & $0.00 \mathrm{E}+0$ & $0.00 \mathrm{E}+0$ & $9.76 \mathrm{E}-2$ & $8.96 \mathrm{E}-4$ \\
$2.80-3.00$ & $0.00 \mathrm{E}+0$ & $0.00 \mathrm{E}+0$ & $0.00 \mathrm{E}+0$ & $0.00 \mathrm{E}+0$ & $0.00 \mathrm{E}+0$ & $0.00 \mathrm{E}+0$ & $4.11 \mathrm{E}-2$ & $4.09 \mathrm{E}-4$ \\
$3.00-3.20$ & $0.00 \mathrm{E}+0$ & $0.00 \mathrm{E}+0$ & $0.00 \mathrm{E}+0$ & $0.00 \mathrm{E}+0$ & $0.00 \mathrm{E}+0$ & $0.00 \mathrm{E}+0$ & $2.65 \mathrm{E}-2$ & $1.57 \mathrm{E}-4$ \\
$3.20-3.60$ & $0.00 \mathrm{E}+0$ & $0.00 \mathrm{E}+0$ & $0.00 \mathrm{E}+0$ & $0.00 \mathrm{E}+0$ & $0.00 \mathrm{E}+0$ & $0.00 \mathrm{E}+0$ & $9.15 \mathrm{E}-3$ & $0.00 \mathrm{E}+0$ \\
$3.60-4.00$ & $0.00 \mathrm{E}+0$ & $0.00 \mathrm{E}+0$ & $0.00 \mathrm{E}+0$ & $0.00 \mathrm{E}+0$ & $0.00 \mathrm{E}+0$ & $0.00 \mathrm{E}+0$ & $3.99 \mathrm{E}-3$ & $0.00 \mathrm{E}+0$ \\
\hline \hline
\end{tabular}

A21-4(a): Electromagnetic energy density (dose) in Gy/yr

\begin{tabular}{rcccccccc}
\hline \hline & & \multicolumn{2}{c}{ Angle } & & \\
Depth $(\mathrm{m})$ & $0^{\circ}-1^{\circ}$ & $1^{\circ}-5^{\circ}$ & $5^{\circ}-10^{\circ}$ & $10^{\circ}-20^{\circ}$ & $20^{\circ}-40^{\circ}$ & $40^{\circ}-70^{\circ}$ & $70^{\circ}-90^{\circ}$ & $110^{\circ}-180^{\circ}$ \\
\hline $2.00-2.02$ & $0.00 \mathrm{E}+0$ & $0.00 \mathrm{E}+0$ & $0.00 \mathrm{E}+0$ & $0.00 \mathrm{E}+0$ & $0.00 \mathrm{E}+0$ & $0.00 \mathrm{E}+0$ & $3.35 \mathrm{E}+1$ & $1.54 \mathrm{E}+0$ \\
$2.02-2.04$ & $0.00 \mathrm{E}+0$ & $0.00 \mathrm{E}+0$ & $0.00 \mathrm{E}+0$ & $0.00 \mathrm{E}+0$ & $0.00 \mathrm{E}+0$ & $0.00 \mathrm{E}+0$ & $3.29 \mathrm{E}+1$ & $5.99 \mathrm{E}-1$ \\
$2.04-2.06$ & $0.00 \mathrm{E}+0$ & $0.00 \mathrm{E}+0$ & $0.00 \mathrm{E}+0$ & $0.00 \mathrm{E}+0$ & $0.00 \mathrm{E}+0$ & $0.00 \mathrm{E}+0$ & $9.87 \mathrm{E}+0$ & $1.06 \mathrm{E}-1$ \\
$2.06-2.08$ & $0.00 \mathrm{E}+0$ & $0.00 \mathrm{E}+0$ & $0.00 \mathrm{E}+0$ & $0.00 \mathrm{E}+0$ & $0.00 \mathrm{E}+0$ & $0.00 \mathrm{E}+0$ & $4.46 \mathrm{E}+0$ & $5.85 \mathrm{E}-2$ \\
$2.08-2.10$ & $0.00 \mathrm{E}+0$ & $0.00 \mathrm{E}+0$ & $0.00 \mathrm{E}+0$ & $0.00 \mathrm{E}+0$ & $0.00 \mathrm{E}+0$ & $0.00 \mathrm{E}+0$ & $2.60 \mathrm{E}+0$ & $2.87 \mathrm{E}-3$ \\
$2.10-2.20$ & $0.00 \mathrm{E}+0$ & $0.00 \mathrm{E}+0$ & $0.00 \mathrm{E}+0$ & $0.00 \mathrm{E}+0$ & $0.00 \mathrm{E}+0$ & $0.00 \mathrm{E}+0$ & $1.14 \mathrm{E}+0$ & $3.86 \mathrm{E}-3$ \\
$2.20-2.40$ & $0.00 \mathrm{E}+0$ & $0.00 \mathrm{E}+0$ & $0.00 \mathrm{E}+0$ & $0.00 \mathrm{E}+0$ & $0.00 \mathrm{E}+0$ & $0.00 \mathrm{E}+0$ & $2.40 \mathrm{E}-1$ & $0.00 \mathrm{E}+0$ \\
$2.40-2.60$ & $0.00 \mathrm{E}+0$ & $0.00 \mathrm{E}+0$ & $0.00 \mathrm{E}+0$ & $0.00 \mathrm{E}+0$ & $0.00 \mathrm{E}+0$ & $0.00 \mathrm{E}+0$ & $7.39 \mathrm{E}-2$ & $0.00 \mathrm{E}+0$ \\
$2.60-2.80$ & $0.00 \mathrm{E}+0$ & $0.00 \mathrm{E}+0$ & $0.00 \mathrm{E}+0$ & $0.00 \mathrm{E}+0$ & $0.00 \mathrm{E}+0$ & $0.00 \mathrm{E}+0$ & $1.13 \mathrm{E}-2$ & $0.00 \mathrm{E}+0$ \\
$2.80-3.00$ & $0.00 \mathrm{E}+0$ & $0.00 \mathrm{E}+0$ & $0.00 \mathrm{E}+0$ & $0.00 \mathrm{E}+0$ & $0.00 \mathrm{E}+0$ & $0.00 \mathrm{E}+0$ & $7.63 \mathrm{E}-3$ & $0.00 \mathrm{E}+0$ \\
$3.00-3.20$ & $0.00 \mathrm{E}+0$ & $0.00 \mathrm{E}+0$ & $0.00 \mathrm{E}+0$ & $0.00 \mathrm{E}+0$ & $0.00 \mathrm{E}+0$ & $0.00 \mathrm{E}+0$ & $1.01 \mathrm{E}-2$ & $0.00 \mathrm{E}+0$ \\
$3.20-3.60$ & $0.00 \mathrm{E}+0$ & $0.00 \mathrm{E}+0$ & $0.00 \mathrm{E}+0$ & $0.00 \mathrm{E}+0$ & $0.00 \mathrm{E}+0$ & $0.00 \mathrm{E}+0$ & $3.02 \mathrm{E}-3$ & $0.00 \mathrm{E}+0$ \\
$3.60-4.00$ & $0.00 \mathrm{E}+0$ & $0.00 \mathrm{E}+0$ & $0.00 \mathrm{E}+0$ & $0.00 \mathrm{E}+0$ & $0.00 \mathrm{E}+0$ & $0.00 \mathrm{E}+0$ & $8.16 \mathrm{E}-4$ & $0.00 \mathrm{E}+0$ \\
\hline \hline
\end{tabular}

